\title{
The atmosphere over mountainous regions
}

Book

Published Version

Creative Commons: Attribution 4.0 (CC-BY)

Open access

Teixeira, M. A. C., Kirshbaum, D. J., Olafsson, H., Sheridan, P. F. and Stiperski, I., eds. (2016) The atmosphere over mountainous regions. Frontiers in Earch Science. Frontiers Media SA, Lausanne, Switzerland, pp162. ISBN 9782889450169 doi: https://doi.org/10.3389/978-2-88945-0169 (Special Issue on the Research Topic "The Atmosphere over Mountainous Regions") Available at https://centaur.reading.ac.uk/66651/

It is advisable to refer to the publisher's version if you intend to cite from the work. See Guidance on citing.

Published version at: http://dx.doi.org/10.3389/978-2-88945-016-9

To link to this article DOI: http://dx.doi.org/10.3389/978-2-88945-016-9

Publisher: Frontiers Media SA

All outputs in CentAUR are protected by Intellectual Property Rights law, including copyright law. Copyright and IPR is retained by the creators or other copyright holders. Terms and conditions for use of this material are defined in the End User Agreement. 


\section{CentAUR}

Central Archive at the University of Reading

Reading's research outputs online 


\section{THE ATMOSPHERE OVER MOUNTAINOUS REGIONS}

EDITED BY: Miguel A. C. Teixeira, Daniel J. Kirshbaum, Haraldur Ólafsson, Peter F. Sheridan and Ivana Stiperski

PUBLISHED IN: Frontiers in Earth Science

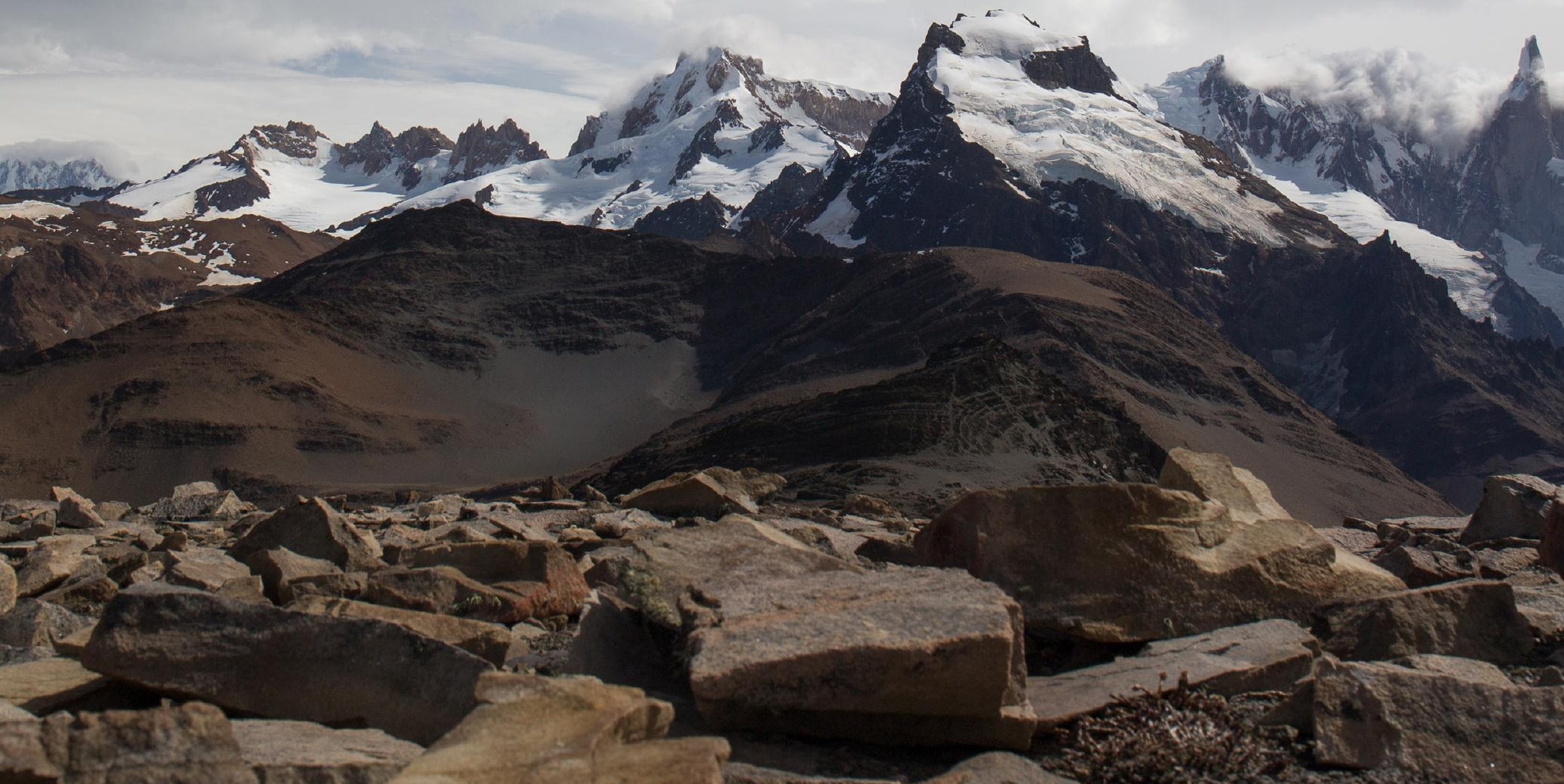

frontiers Research Topics 


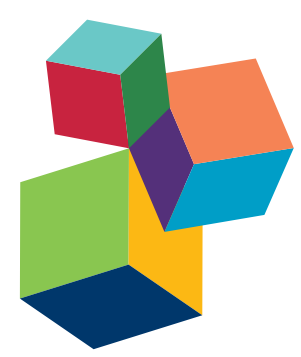

Frontiers Copyright Statement

(c) Copyright 2007-2016 Frontiers Media SA. All rights reserved.

All content included on this site, such as text, graphics, logos, button

icons, images, video/audio clips,

downloads, data compilations and

software, is the property of or is

licensed to Frontiers Media SA

("Frontiers") or its licensees and/or subcontractors. The copyright in the text of individual articles is the property of their respective authors, subject to a license granted to Frontiers.

The compilation of articles constituting

this e-book, wherever published,

as well as the compilation of all other

content on this site, is the exclusive property of Frontiers. For the conditions for downloading and copying of e-books from Frontiers' website, please see the Terms for Website Use. If purchasing Frontiers e-books from other websites or sources, the conditions of the website concerned apply.

Images and graphics not forming part of user-contributed materials may not be downloaded or copied without permission.

Individual articles may be downloaded and reproduced in accordance with the principles of the CC-BY licence subject to any copyright or other notices. They may not be re-sold as an e-book.

As author or other contributor you grant a CC-BY licence to others to reproduce your articles, including any graphics and third-party materials supplied by you, in accordance with the Conditions for Website Use and subject to any copyright notices which you include in connection with your articles and materials.

All copyright, and all rights therein, are protected by national and international copyright laws.

The above represents a summary only. For the full conditions see the Conditions for Authors and the Conditions for Website Use.

ISSN 1664-8714 ISBN 978-2-88945-016-9

DOI 10.3389/978-2-88945-016-9

\section{About Frontiers}

Frontiers is more than just an open-access publisher of scholarly articles: it is a pioneering approach to the world of academia, radically improving the way scholarly research is managed. The grand vision of Frontiers is a world where all people have an equal opportunity to seek, share and generate knowledge. Frontiers provides immediate and permanent online open access to all its publications, but this alone is not enough to realize our grand goals.

\section{Frontiers Journal Series}

The Frontiers Journal Series is a multi-tier and interdisciplinary set of open-access, online journals, promising a paradigm shift from the current review, selection and dissemination processes in academic publishing. All Frontiers journals are driven by researchers for researchers; therefore, they constitute a service to the scholarly community. At the same time, the Frontiers Journal Series operates on a revolutionary invention, the tiered publishing system, initially addressing specific communities of scholars, and gradually climbing up to broader public understanding, thus serving the interests of the lay society, too.

\section{Dedication to Quality}

Each Frontiers article is a landmark of the highest quality, thanks to genuinely collaborative interactions between authors and review editors, who include some of the world's best academicians. Research must be certified by peers before entering a stream of knowledge that may eventually reach the public - and shape society; therefore, Frontiers only applies the most rigorous and unbiased reviews.

Frontiers revolutionizes research publishing by freely delivering the most outstanding research, evaluated with no bias from both the academic and social point of view. By applying the most advanced information technologies, Frontiers is catapulting scholarly publishing into a new generation.

\section{What are Frontiers Research Topics?}

Frontiers Research Topics are very popular trademarks of the Frontiers Journals Series: they are collections of at least ten articles, all centered on a particular subject. With their unique mix of varied contributions from Original Research to Review Articles, Frontiers Research Topics unify the most influential researchers, the latest key findings and historical advances in a hot research area! Find out more on how to host your own Frontiers Research Topic or contribute to one as an author by contacting the Frontiers Editorial Office: researchtopics@frontiersin.org 


\section{THE ATMOSPHERE OVER MOUNTAINOUS REGIONS}

Topic Editors:

Miguel A. C. Teixeira, University of Reading, UK

Daniel J. Kirshbaum, McGill University, Canada

Haraldur Ólafsson, University of Iceland \& Icelandic Met Office, Iceland

Peter F. Sheridan, UK Met Office, UK

Ivana Stiperski, University of Innsbruck, Austria

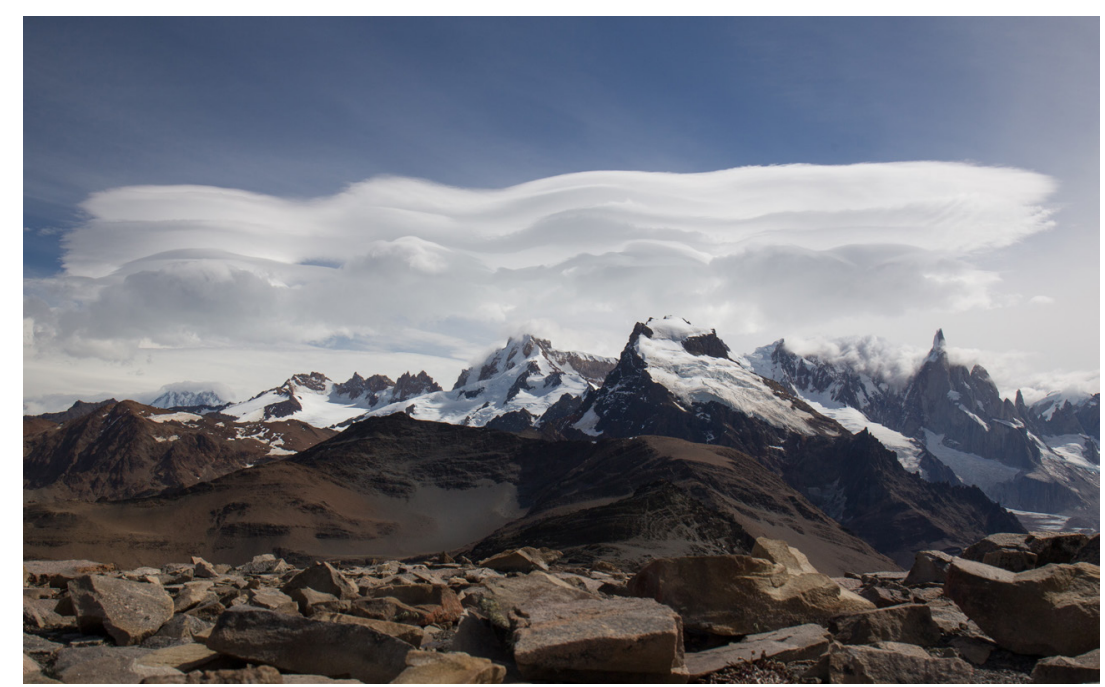

Lenticular clouds formed by flow over mountains in Patagonia.

Image by Ivana Stiperski.

Mountainous regions occupy a significant fraction of the Earth's continents and are characterized by specific meteorological phenomena operating on a wide range of scales. Being a home to large human populations, the impact of mountains on weather and hydrology has significant practical consequences. Mountains modulate the climate and create micro-climates, induce different types of thermally and dynamically driven circulations, generate atmospheric waves of various scales (known as mountain waves), and affect the boundary layer characteristics and the dispersion of pollutants.

At the local scale, strong downslope winds linked with mountain waves (such as the Foehn and Bora) can cause severe damage. Mountain wave breaking in the high atmosphere is a source of Clear Air Turbulence, and lee wave rotors are a major near-surface aviation hazard. Mountains 
also act to block strongly-stratified air layers, leading to the formation of valley cold-air pools (with implications for road safety, pollution, crop damage, etc.) and gap flows. Presently, neither the fine-scale structure of orographic precipitation nor the initiation of deep convection by mountainous terrain can be resolved adequately by regional-to global-scale models, requiring appropriate downscaling or parameterization. Additionally, the shortest mountain waves need to be parameterized in global weather and climate prediction models, because they exert a drag on the atmosphere. This drag not only decelerates the global atmospheric circulation, but also affects temperatures in the polar stratosphere, which control ozone depletion. It is likely that both mountain wave drag and orographic precipitation lead to non-trivial feedbacks in climate change scenarios.

Measurement campaigns such as MAP, T-REX, Materhorn, COLPEX and i-Box provided a wealth of mountain meteorology field data, which is only starting to be explored. Recent advances in computing power allow numerical simulations of unprecedented resolution, e.g. LES modelling of rotors, mountain wave turbulence, and boundary layers in mountainous regions. This will lead to important advances in understanding these phenomena, as well as mixing and pollutant dispersion over complex terrain, or the onset and breakdown of cold-air pools. On the other hand, recent analyses of global circulation biases point towards missing drag, especially in the southern hemisphere, which may be due to processes currently neglected in parameterizations. A better understanding of flow over orography is also crucial for a better management of wind power and a more effective use of data assimilation over complex terrain.

This Research Topic includes contributions that aim to shed light on a number of these issues, using theory, numerical modelling, field measurements, and laboratory experiments.

Citation: Teixeira, M. A. C., Kirshbaum, D. J., Ólafsson, H., Sheridan, P. F., Stiperski, I., eds. (2016). The Atmosphere over Mountainous Regions. Lausanne: Frontiers Media.

doi: 10.3389/978-2-88945-016-9 


\section{Table of Contents}

05 Editorial: The Atmosphere over Mountainous Regions

Miguel A. C. Teixeira, Daniel J. Kirshbaum, Haraldur Ólafsson, Peter F. Sheridan

and Ivana Stiperski

07 Possible observation of horizontal roll vortices over the Adriatic Sea during bora Danijel Belušić, Željko Večenaj and Margaret A. LeMone

14 Lee Waves on the Boundary-Layer Inversion and Their Dependence on FreeAtmospheric Stability

Johannes Sachsperger, Stefano Serafin and Vanda Grubišić

25 Non-linear Topographic Effects in Two-Layer Flows

Peter G. Baines and Edward R. Johnson

35 The Quasi-Steady State of the Valley Wind System

Juerg Schmidli and Richard Rotunno

46 Convective Boundary Layer Heights Over Mountainous Terrain-A Review of Concepts

Stephan F. J. De Wekker and Meinolf Kossmann

68 On the Vertical Exchange of Heat, Mass, and Momentum Over Complex, Mountainous Terrain

Mathias W. Rotach, Alexander Gohm, Moritz N. Lang, Daniel Leukauf, Ivana Stiperski and Johannes S. Wagner

82 Identification and Climatology of Alpine Pumping from a Regional Climate Simulation

Maximilian Graf, Meinolf Kossmann, Kristina Trusilova and Gudrun Mühlbacher

93 The Atmospheric Boundary Layer during Wintertime Persistent Inversions in the Grenoble Valleys

Yann Largeron and Chantal Staquet

112 Energetics of Slope Flows: Linear and Weakly Nonlinear Solutions of the Extended PrandtI Model

Ivan Güttler, Ivana Marinović, Željko Večenaj and Branko Grisogono

125 When Can a High-Resolution Simulation Over Complex Terrain be Called LES? Joan Cuxart

131 Influence of the Details of Topography on Weather Forecast - Evaluation of HARMONIE Experiments in the Sochi Olympics Domain over the Caucasian Mountains

Laura Rontu, Clemens Wastl and Sami Niemelä

147 Effects of Landfall Location and Approach Angle of an Idealized Tropical Cyclone over a Long Mountain Range

Liping Liu, Yuh-Lang Lin and Shu-Hua Chen 


\title{
Editorial: The Atmosphere over Mountainous Regions
}

\author{
Miguel A. C. Teixeira ${ }^{1 *}$, Daniel J. Kirshbaum ${ }^{2}$, Haraldur Ólafsson ${ }^{3}$, Peter F. Sheridan ${ }^{4}$ and \\ Ivana Stiperski ${ }^{5}$ \\ ${ }^{1}$ Department of Meteorology, University of Reading, Reading, UK, ${ }^{2}$ Department of Atmospheric and Oceanic Sciences, \\ McGill University, Montreal, QC, Canada, ${ }^{3}$ Department of Physics, University of Iceland, and Icelandic Met Office, Reykjavik, \\ Iceland, ${ }^{4}$ UK Met Office, Exeter, UK, ${ }^{5}$ Institute of Atmospheric and Cryospheric Sciences, University of Innsbruck, Innsbruck, \\ Austria
}

Keywords: downslope winds, horizontal inhomogeneity, hydraulic jumps, large eddy simulation, mountain waves, orographic precipitation, thermally-driven flows, turbulent fluxes

\section{The Editorial on the Research Topic}

\section{The Atmosphere over Mountainous Regions}

Mountainous regions occupy a significant fraction of the Earth's continents and are characterized by specific meteorological phenomena operating on a wide range of scales. Being a home to large human populations, the impact of mountains on weather and hydrology has significant practical implications. Presently, neither the fine-scale structure of orographic precipitation nor the initiation of convection by mountains can be resolved adequately by regional to globalscale models, requiring appropriate downscaling or parameterization. It is likely that orographic precipitation will be affected by non-trivial feedbacks in climate change scenarios.

Mountains modulate the climate and generate atmospheric waves of various scales. Whilst the

OPEN ACCESS

Edited by:

Luis Gimeno,

University of Vigo, Spain

Reviewed by:

Juan Pedro Montávez,

University of Murcia, Spain

${ }^{*}$ Correspondence:

Miguel A. C. Teixeira m.a.teixeira@reading.ac.uk

Specialty section: This article was submitted to Atmospheric Science, a section of the journal Frontiers in Earth Science

Received: 19 July 2016 Accepted: 26 August 2016 Published: 09 September 2016

Citation:

Teixeira MAC, Kirshbaum DJ, Ólafsson H, Sheridan PF and Stiperski I (2016) Editorial: The Atmosphere over Mountainous Regions. Front. Earth Sci. 4:84. doi: 10.3389/feart.2016.00084 longest mountain waves are adequately resolved in weather prediction models, the shortest still need to be parameterized, as they exert a drag on the atmosphere. This drag not only decelerates the global atmospheric circulation, but also affects temperatures in the polar stratosphere, which control ozone depletion. Locally, strong downslope winds linked to mountain waves (such as the Föhn and Bora) can cause severe damage. Mountain wave breaking in the high troposphere is a source of Clear-Air Turbulence, and large amplitude lee waves, as well as hydraulic jumps, can induce rotors near the surface, which are a major aviation safety hazard.

In this issue, Belušić et al. report a possible occurrence of horizontal roll vortices over the Adriatic Sea during a Bora wind event. Data measured by the National Center for Atmospheric Research Electra aircraft, which sampled the turbulence structure above the Adriatic Sea during the Mesoscale Alpine Program, indicate that horizontal roll vortices with a wavelength of about $1 \mathrm{~km}$ were generated in the strong cold and dry air outbreak associated with the Bora downslope flow.

Using model parameters based on observations taken in the Madeira archipelago, Sachsperger et al. compare several different linear models of two-dimensional interfacial waves developing on a temperature inversion (e.g., the top of the boundary layer), describing how stability in the free atmosphere limits the possible range of lee wavelengths, modulates the length of the stationary wave mode, and strongly constrains the validity of the shallow-water (or long-wave) approximation.

Baines and Johnson theoretically address nonlinear, two-layer, non-Boussinesq, hydrostatic flow with a rigid lid over a long obstacle, where upstream hydraulic jumps can occur, using a new internal hydraulic jump model. Regime diagrams based on the Froude number and the obstacle height show some novel and surprising features, namely in supercritical flow there are situations where hysteresis may occur, with the flow having multiple possible states for the same input conditions.

Mountains induce different types of thermally and dynamically driven circulations (e.g., katabatic and anabatic flows), and affect the boundary layer characteristics and the dispersion of pollutants. Mountains also act to block strongly stratified air layers, leading to the formation 
of gap flows, and cold air-pools (with implications for road safety, pollution, crop damage, etc.).

In this issue, Schmidli and Rotunno analyse the quasisteady-state limit of the diurnal valley wind system over idealized three-dimensional topography, to provide insight into the mass and heat fluxes associated with the along-valley wind. They derive a scaling relation for the quasi-steady-state along-valley mass flux as a function of valley geometry and size, atmospheric stratification, and surface sensible heat flux forcing, and compare it with numerical simulations of the valley wind system.

De Wekker and Kossmann provide a review of the progress made in documenting and understanding the spatio-temporal behavior of dry convective boundary layer (CBL) heights over mountainous terrain. Unlike CBLs over valleys and basins, CBLs over slopes, ridges, or mountain peaks, where advection from multi-scale thermally-driven flows plays an important role, are not well understood, and a universal definition of CBL height over mountains remains unavailable.

Rotach et al. review the role of meso-scale and sub-mesoscale circulations, such as slope and valley winds, in the structure of the boundary layer and turbulent exchanges over complex terrain, with reference to recent idealized numerical simulations. These circulations are ubiquitous in mountainous terrain, develop due to horizontal inhomogeneity in both radiative and dynamical forcing, and are shown to significantly contribute to vertical fluxes of energy and mass between the surface and the atmosphere.

Motivated by its ventilation effect in Munich, Graf et al. investigate Alpine pumping, the thermally driven circulation between the European Alps and the Alpine foreland, using a Regional Climate Simulation to identify the occurrence and describe the spatio-temporal characteristics of this mountainplain wind system, which occurs regularly under clear and calm weather conditions. They estimate the frequency of these circulations, their vertical extent and diurnal variation.

Largeron and Staquet analyse measurements of the atmospheric boundary layer in the Grenoble valleys during persistent inversions in winter, and simulate the most polluted periods using the MesoNH model, focusing on ventilation and flow stagnation effects. They estimate the strength and depth of the inversion, noting that the boundary layer within the inversion layer is decoupled from the synoptic flow, and strongly dependent on the thermal winds, valley topography, and radiative cooling of the ground.

Güttler et al. explore the energetics of the weakly-nonlinear extension to Prandtl's katabatic/anabatic flow model, in terms of the Total Energy (TE) concept, showing that nonlinear effects have a relatively small impact on the variability of the flow solutions, but the maximum of the along-slope wind speed reduces as slope increases. They find that the diffusion, dissipation, and nonlinear interaction terms in the TE budget do not balance perfectly, leading to flow non-stationarity.

Measurement campaigns such as MAP, T-REX, Materhorn, COLPEX, i-Box, provided a wealth of mountain meteorology field data, which is only starting to be explored. Conversely, numerical modeling remains a major method for investigating flow over mountainous terrain. Recent advances in computing power allow numerical simulations of unprecedented resolution, e.g., Large-Eddy Simulations (LES) of rotors, mountain wave turbulence, and boundary layers over mountainous regions.

In this issue, Cuxart discusses the circumstances under which a high-resolution numerical simulations may be classified as LES, noting that aspects such as the complexity of the terrain, and the stability of the atmospheric stratification may render simulations which could be classified as LES in more idealized conditions unable to resolve the inertial sub-range of the turbulence, for example due to the small scale of turbulent eddies in stable stratification.

Rontu et al. use new high-resolution surface elevation data, and new parameterizations of radiation and momentum fluxes, to simulate the weather conditions during the Sochi Winter Olympic Games 2014, using the HARMONIE-AROME-SURFEX numerical model, and validate results against observations from the WMOFROST-2014 program in the Caucasus mountains. Their updated orographic radiation parameterization proves to be crucial to improving the surface temperature forecasts.

Finally, Liu et al. investigate the effects of landfall location and approach angle on track deflection associated with a tropical cyclone passing over idealized and Central Appalachian mountains, using a series of idealized numerical experiments, finding that when the tropical cyclone landfalls on the central portion of the mountain range, its deflection is determined by vorticity dynamics dominated by horizontal vorticity advection, with additional influence from diabatic heating and frictional effects.

Several papers in this special issue were presented at the "Workshop on Advances in Meso- and Micro-meteorology," which took place near Zagreb, Croatia, in November 2015 (http:// www.pmf.unizg.hr/geof/en/research/meteorology/workshop_me so-micrometeology_en).

\section{AUTHOR CONTRIBUTIONS}

All authors listed contributed to this work and approved it for publication.

\section{FUNDING}

MACT acknowledges the financial support of the European Commission, under grant PCIG13-GA-2013-618016. DJK acknowledges the financial support of the Canadian Natural Science and Engineering Research Council, under grant NSERC/RGPIN 418372-12. IS acknowledges the financial support of the Austrian Science Fund (FWF), under grant \#T $781-\mathrm{N} 32$.

Conflict of Interest Statement: The authors declare that the research was conducted in the absence of any commercial or financial relationships that could be construed as a potential conflict of interest.

Copyright (c) 2016 Teixeira, Kirshbaum, Ólafsson, Sheridan and Stiperski. This is an open-access article distributed under the terms of the Creative Commons Attribution License (CC BY). The use, distribution or reproduction in other forums is permitted, provided the original author(s) or licensor are credited and that the original publication in this journal is cited, in accordance with accepted academic practice. No use, distribution or reproduction is permitted which does not comply with these terms. 
OPEN ACCESS

Edited by:

Ivana Stiperski,

University of Innsbruck, Austria

Reviewed by:

Peter F. Sheridan,

Met Office, UK

Gert-Jan Steeneveld,

Wageningen University, Netherlands

*Correspondence:

Danijel Belušić,

School of Earth, Atmosphere and

Environment, Monash University, 9 Rainforest Walk, Clayton Campus, VIC

3800, Melbourne, Australia

danijel.belusic@monash.edu

Specialty section:

This article was submitted to Atmospheric Science,

a section of the journal

Frontiers in Earth Science

Received: 26 March 2015

Accepted: 15 May 2015

Published: 28 May 2015

Citation:

Belušić D, Večenaj Ž and LeMone MA

(2015) Possible observation of

horizontal roll vortices over the Adriatic

Sea during bora.

Front. Earth Sci. 3:23.

doi: 10.3389/feart.2015.00023

\section{Possible observation of horizontal roll vortices over the Adriatic Sea during bora}

\author{
Danijel Belušićc ${ }^{*}$, Željko Večenaj ${ }^{2}$ and Margaret A. LeMone ${ }^{3}$ \\ ${ }^{1}$ School of Earth, Atmosphere and Environment, Monash University, Melbourne, VIC, Australia, ${ }^{2}$ Department of Geophysics, \\ Faculty of Science, University of Zagreb, Zagreb, Croatia, ${ }^{3}$ National Center for Atmospheric Research, Boulder, CO, USA
}

We report a possible occurrence of horizontal roll vortices over the Adriatic Sea during the bora wind. The National Center for Atmospheric Research Electra aircraft measured the turbulence structure above the Adriatic on 07 November 1999 during the Mesoscale Alpine Program. The data indicate that horizontal roll vortices are generated by the strong cold-air outbreak associated with bora. The rolls have a horizontal wavelength of about $1 \mathrm{~km}$ and an aspect ratio of approximately 1.5. There is no indication of the presence of cloud streets typically associated with the rolls, which is a consequence of the dry air advection in the lower troposphere resulting from the downslope flow.

Keywords: downslope windstorm, convection, atmospheric boundary layer, cold-air outbreak, turbulence

\section{Introduction}

The bora wind has been studied for decades by many researchers (for a recent review, see Grisogono and Belušić, 2009). It is a canonical example of a downslope windstorm that is generated by flow over an almost two-dimensional mountain. The Dinaric Alps is a mountain range that extends along the eastern coast of the Adriatic Sea and forms a long barrier that forces the impinging cold north-easterly flow to rise and, to a first approximation, follow a typical two-dimensional hydrauliclike bora flow structure. This is particularly evident for the northern Adriatic bora, which is one of the first real flows that was characterized to a large extent using hydraulic theory of downslope windstorms (Smith, 1985, 1987).

Bora is most frequent in winter and at some locations blows more than 100 days per year (e.g., Yoshino, 1976). A number of complexities arise because of the terrain inhomogeneity, particularly the mountain gaps and peaks along the Dinaric Alps. The major gaps and peaks result in the formation of bora jets and wakes, respectively (e.g., Jiang and Doyle, 2005; Belušić and Klaić, 2006; Signell et al., 2010), which are then related to potential vorticity banners (e.g., Grubišić, 2004) and varying turbulence structure (Večenaj et al., 2012). A number of other features appear within the bora flow, such as lee rotors of different types (e.g., Gohm et al., 2008; Prtenjak and Belušić, 2009; Stiperski et al., 2012) and quasi-periodic pulsations (e.g., Belušić et al., 2007), but they mostly do not extend far over the sea.

Regardless of the large body of bora research studies, the possibility of occurrence of horizontal roll vortices in the bora flow has never been considered. This is rather surprising, since boras are usually strong and relatively cold winds that blow over the warmer Adriatic Sea, creating wellknown conditions for generating roll vortices (e.g., Kelly, 1982; Atlas and Chou, 1983; Walter and Overland, 1984; Brummer, 1999). The most likely reason for this omission is in that the ubiquitous cloud streets associated with rolls do not seem to occur during bora. 
Horizontal rolls are elongated quasi-two-dimensional vortices appearing in the convective atmospheric boundary layer (ABL) (e.g., LeMone, 1973). While they are usually recognized as cloud streets, particularly in satellite imagery (e.g., Young et al., 2002), they have been observed in clear air as well (e.g., Konrad, 1970; Kelly, 1984). They vertically extend to the top of the ABL and their horizontal wavelengths are typically more than two times larger than their vertical size, i.e., their aspect ratio (wavelength/ABL height) is two or larger. The rolls are frequently associated with cold-air outbreaks (e.g., Melfi and Palm, 2012), although they can be generated by a number of mechanisms, including inflection point instability, parallel instability, convective instability and interaction with gravity waves aloft (Etling and Brown, 1993).

Here, we present aircraft observations above the Adriatic Sea that suggest the existence of horizontal roll vortices in bora. We focus on a single bora episode observed during the Mesoscale Alpine Program (MAP) in 1999 (Bougeault et al., 2001).

\section{Data}

The aircraft data over the Adriatic Sea were collected on 7 November 1999 during the MAP Intensive Observation Period 15 (e.g., Bougeault et al., 2001). This was a cyclonic bora case topped by a temperature inversion and a critical level in the upstream wind profile, indicating a shallow bora flow, and one of the stronger and more persistent boras on record (Klaić et al., 2003; Grubišić, 2004). The U. S. National Center for Atmospheric Research (NCAR) Electra aircraft flew three vertically stacked straight and level flight legs from 1347 to 1539 UTC (Figure 1, Table 1). The legs were $\sim 216 \mathrm{~km}$ long and oriented approximately perpendicular to the mean bora direction (e.g., Grubišić, 2004). Six dropsondes were released successfully from approximately $4200 \mathrm{~m}$ above sea level (ASL) along the first flight leg. The aircraft mean speed was $100 \mathrm{~ms}^{-1}$ and the data were sampled at a frequency of $25 \mathrm{~Hz}$. In order to avoid noise at high frequencies, the aircraft data were block-averaged to $5 \mathrm{~Hz}$ prior to the analyses. Further details about the data are given in Večenaj et al. (2012). As a result of the persistence of this bora case, the flow conditions may to a good approximation be considered stationary during the $2 \mathrm{~h}$ period spanning the three flight legs (Klaić et al., 2003; Grubišić, 2004). The analysis of numerical model results corroborates this conclusion (Večenaj et al., 2012).

A right-handed orthogonal coordinate system is chosen with the positive $\mathrm{x}$-axis aligned perpendicular to the flight direction pointing toward the south-west, which is approximately parallel to the mean bora flow. If there are rolls, the $\mathrm{x}$-axis also lies approximately along the roll axis.

\section{Results}

Figure 2 shows the wind components for the lower ABL flight leg ( $370 \mathrm{~m} \mathrm{ASL})$. A region with increased variability occurs around $45^{\circ} \mathrm{N}$, where nearly regular oscillations seem to occur at scales of about $1 \mathrm{~km}$ (Figure 2B).

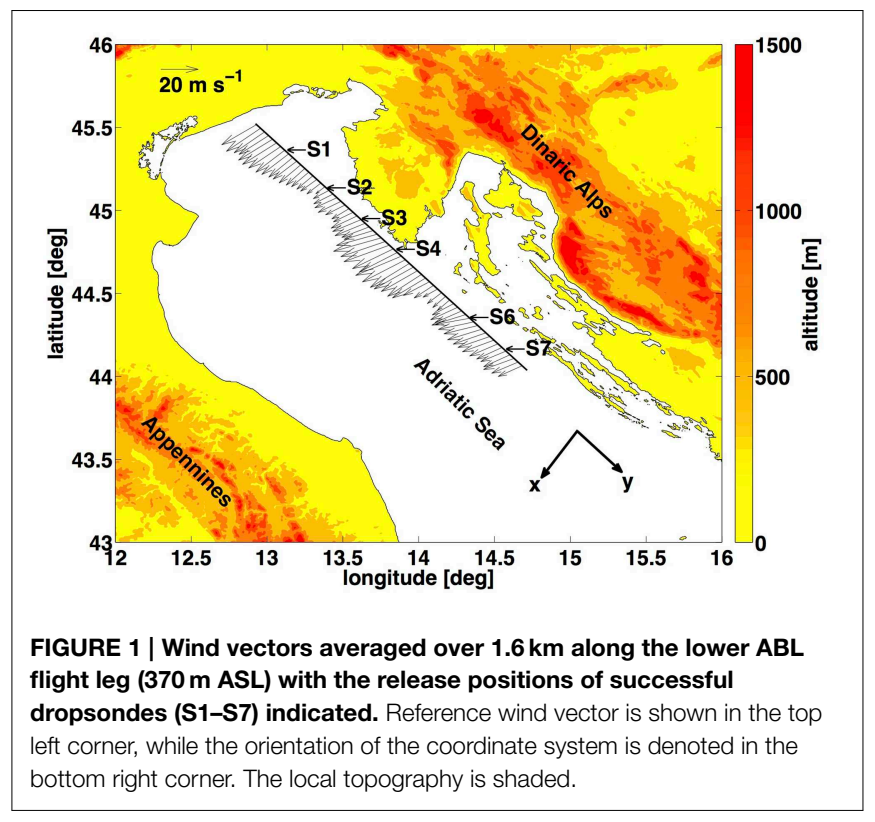

Spectral analysis reveals peaks in the wind component spectra centered at $1 \mathrm{~km}$, at several locations along the two lower flight legs (Figure 3). The dominant peak is evident in all wind components and at both heights for the segment chosen for the analysis $\left(44.90-45.05^{\circ} \mathrm{N}\right)$. The strength and consistency of this peak indicates the most likely location of the roll vortices. Unlike for the lower ABL flight leg, the spectral peak at the upper ABL flight leg does not extend over the entire analyzed segment. The available spectra over the segment show that the peak at the upper ABL flight leg decreases and disappears south of about $44.96^{\circ} \mathrm{N}$.

Data from the dropsonde S3, released closest to the chosen segment, are shown in Figure 4. According to the dropsonde, the ABL height, determined as the base of the low-level temperature inversion, is about $600 \mathrm{~m}$ deep. However, the data measured by the aircraft at the horizontal location of the dropsonde release show that the potential temperature at $680 \mathrm{~m}$ is lower than that recorded by the dropsonde, which probably results from the downwind drift of the dropsonde of approximately $8 \mathrm{~km}$ (estimated form the drift of dropsondes S1 and S2, because S3 did not return GPS data) and the time difference between the dropsonde release and the two ABL flight legs. This suggests that the ABL height decreases downwind, which is unusual for a cold-air outbreak and the development of a convective ABL over the sea. The reason for this is the downslope nature of bora, where the related dynamical subsidence manages to decrease the convective ABL height farther than $100 \mathrm{~km}$ offshore (e.g., Grubišić, 2004). The aircraft-measured potential temperatures at the two lower flight legs on the chosen segment are almost equal north of $44.96^{\circ} \mathrm{N}$, but there is a gradual increase in stability south of $44.96^{\circ} \mathrm{N}$ (Figure 5 and Table 1). This means that the inversion base is most likely just above the upper $\mathrm{ABL}$ flight leg to the north of $44.96^{\circ} \mathrm{N}$, and below to the south. The latter explains the disappearance of the spectral peak at the upper ABL flight leg south of $44.96^{\circ} \mathrm{N}$, since the aircraft is above the ABL there. Hence we estimate the mean ABL height along the chosen segment as 
TABLE 1 | Seventh November 1999 flight legs.

\begin{tabular}{|c|c|c|c|c|c|}
\hline Leg & Time (UTC) & Height (m ASL) & Heading/comments & $\theta_{N} / \theta_{S} / \theta_{D}(K)$ & $r_{N} / r_{S} / r_{D}(g / k g)$ \\
\hline 1 & $1347-1420$ & 4200 & SE/6 dropsondes & - & - \\
\hline 2 & 1429-1501 & 680 & NW/upper ABL leg & 289.0/288.9/289.7 & $4.5 / 4.7 / 4.0$ \\
\hline 3 & 1504-1539 & 370 & SE/lower ABL leg & 289.0/288.5/288.3 & 4.6/4.9/4.5 \\
\hline
\end{tabular}

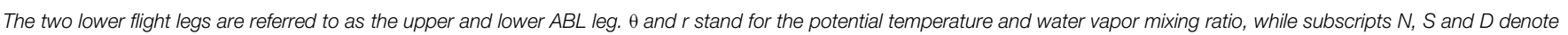
the northern $\left(44.96-45.05^{\circ} \mathrm{N}\right)$ and southern $\left(44.90-44.96^{\circ} \mathrm{N}\right)$ part of the segment chosen for the analysis, and the data from dropsonde S3 at the heights of the two lower flight legs, respectively.

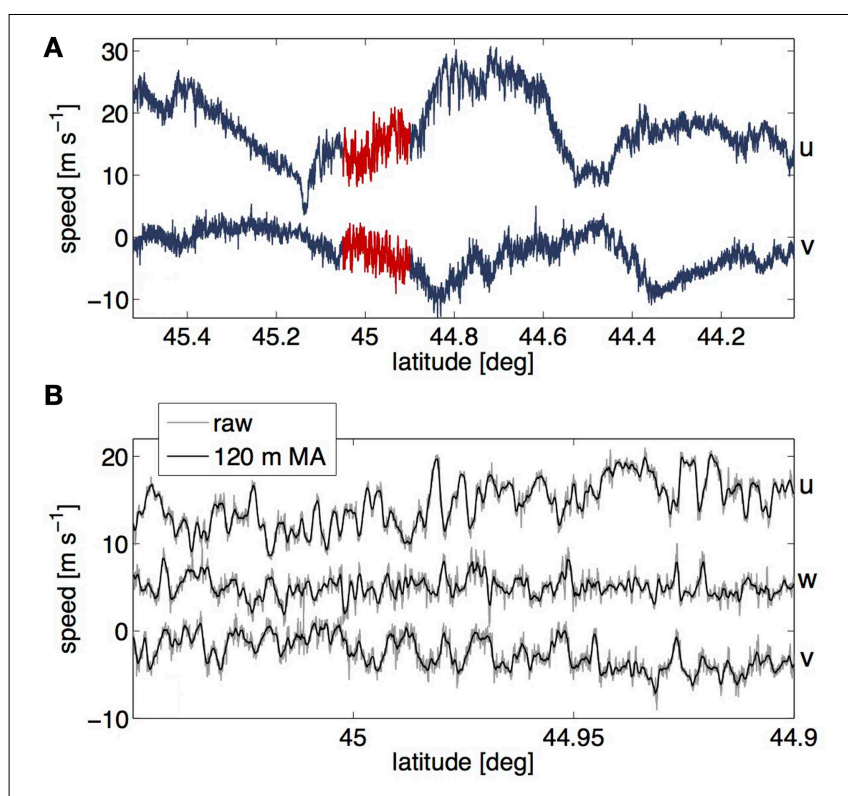

FIGURE 2 | (A) Raw $u$ and $v$ components of the wind velocity along the lower ABL flight leg ( $370 \mathrm{~m} \mathrm{ASL})$ over the Adriatic. The segment chosen for the analysis of possible rolls $\left(44.90-45.05^{\circ} \mathrm{N}\right)$ is shown in red. (B) The three wind components over the analyzed segment; shown are the raw and $120-\mathrm{m}$ moving-average smoothed data. $A 5 \mathrm{~m} \mathrm{~s}^{-1}$ offset has been added to $\mathrm{W}$ for presentation purposes.

the height of the upper ABL flight leg, $\mathrm{zi}=680 \mathrm{~m}$. This leads to the roll aspect ratio of $\mathrm{L} / \mathrm{zi}=1.5$, which is somewhat smaller than those usually reported in the literature (e.g., Young et al., 2002).

The spatial structure of rolls yields specific phase relationships between different wind components (e.g., Hein and Brown, 1988; Alpers and Brummer, 1994). In an idealized system for verticallyupright rolls, the expected phase angles are $\phi(\mathrm{v}, \mathrm{w})= \pm 90^{\circ}$ near the top and bottom, and $\phi(\mathrm{u}, \mathrm{w})= \pm 180^{\circ}$ throughout (Chen et al., 2001; Hein and Brown, 1988). LeMone and Pennell (1976) also show that the longitudinal vortices can be tilted with height, which leads to $\mathrm{v}$ and $\mathrm{w}$ being in phase in the middle of the roll layer (alternatively, $v$ and $w$ could be 180 out of phase, if the tilt is in the opposite direction). The v- (across-roll) component should have maximum amplitude at the top and bottom of the layer, with $\mathrm{w}$ maximum near the middle of the roll layer.

Figures 6, 7 show the coherence and phase angle between the two horizontal wind components and the vertical wind component for our data at the two lower flight legs. The peak in coherence at the wavelength of $1 \mathrm{~km}$ is present for $\mathrm{v}$ and $\mathrm{w}$ at $680 \mathrm{~m}$ (Figure 6) and for all combinations of components at $370 \mathrm{~m}$ (Figure 7). At $370 \mathrm{~m}$, which is approximately in the middle of the roll layer, $\mathrm{u}$ and $\mathrm{w}$ are $180^{\circ}$ out of phase as expected, since $\mathrm{u}$ increases with height (Figure 4), while $\mathrm{v}$ and $\mathrm{w}$ are in phase (Figure 7). This indicates that the rolls are tilted in the crosswind direction. In accordance with the theory, the phase angle between $\mathrm{v}$ and $\mathrm{w}$ changes to $90^{\circ}$ at $680 \mathrm{~m}$ (Figure 6), which is at the top of the roll layer.

While the presented analysis indicates the existence of organized structures with persistent updrafts in the bora ABL, the fact that the related cloud streets have never been reported still calls for an explanation. First, we can use the data from dropsonde S3 (Figure 4) to estimate the lifting condensation level (LCL), which is a reasonable predictor of convective cloud base height. Applying the moistest possible ABL parcel with $r=5 \mathrm{~g}$ $\mathrm{kg}^{-1}$, along with the surface temperature $T=288 \mathrm{~K}$ and surface pressure $p=1000 \mathrm{hPa}$, the LCL is above $1.5 \mathrm{~km}$, which is considerably greater than the $\mathrm{ABL}$, and hence the roll, depth. The dropsonde data also show that the relative humidity remains close to $50 \%$ below $1.5 \mathrm{~km}$, and then increases with height reaching its maximum value of $83 \%$ between 2.5 and $2.8 \mathrm{~km}$. Such vertical distribution is consistent with the bora dynamics and is caused by the descending dry air that is governed by a large-amplitude or breaking mountain wave (e.g., Belušić and Klaić, 2006). The descending dry air usually influences a relatively shallow layer in the lee that does not extend much above $2 \mathrm{~km}$, which is where the roll clouds would appear if sufficient moisture was present. The descending dry air sometimes creates clear areas even with preexisting deeper cloud layers (e.g., Grubišić, 2004).

\section{Discussion and Conclusion}

Aircraft measurements of the bora wind above the Adriatic Sea indicate the existence of horizontal roll vortices with horizontal wavelength of $1 \mathrm{~km}$. The characteristics of the rolls are shown to agree with previous studies, although the wavelength of $1 \mathrm{~km}$ and the corresponding roll aspect ratio (horizontal wavelength/ABL height) of 1.5 are at the lower limit of the previous reports (e.g., Etling and Brown, 1993). There are several specific bora characteristics that could explain such small aspect ratio.

In a modeling study, Müller and Chlond (1996) showed that removing liquid water formation reduces the broadening of convective cells, leading to smaller aspect ratios. The majority of previous studies of rolls, where the aspect ratio is usually larger than two, are based on convective rolls that produced cloud streets, i.e., where liquid water formation was present (e.g., 

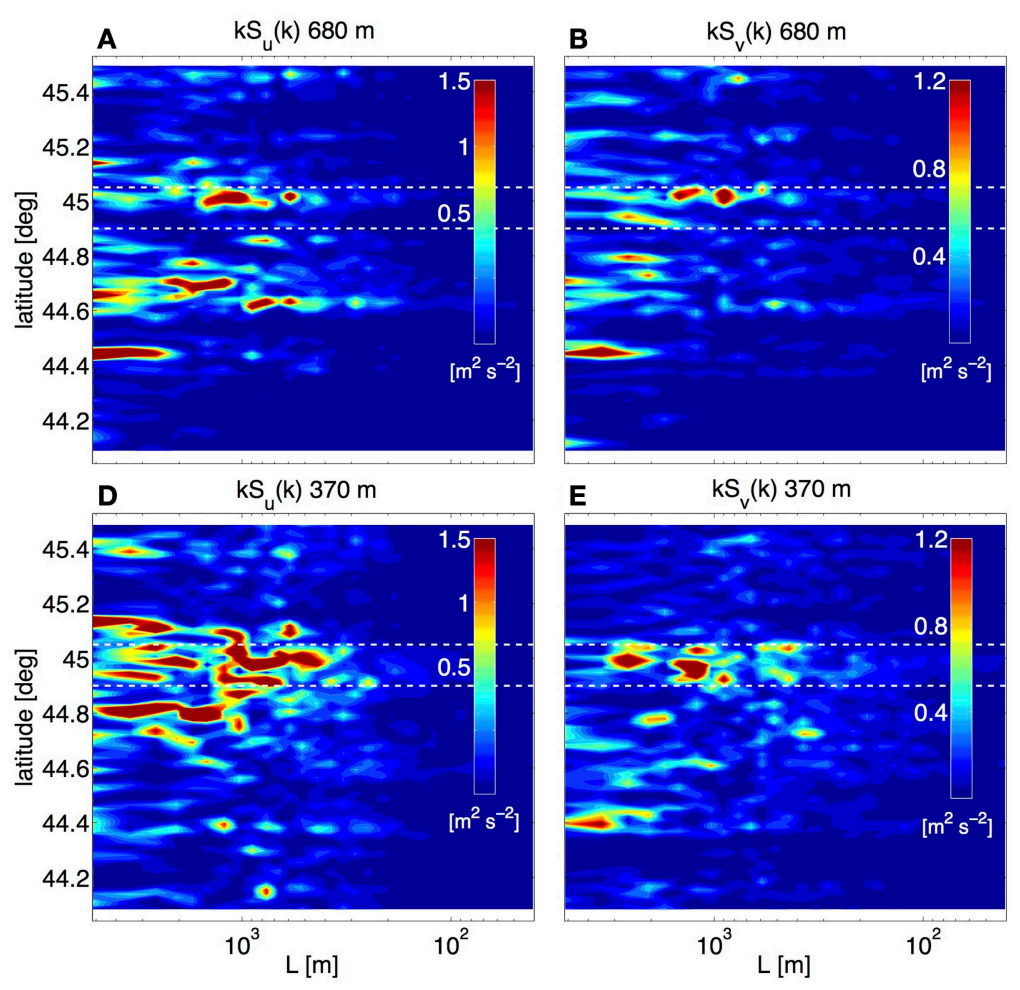
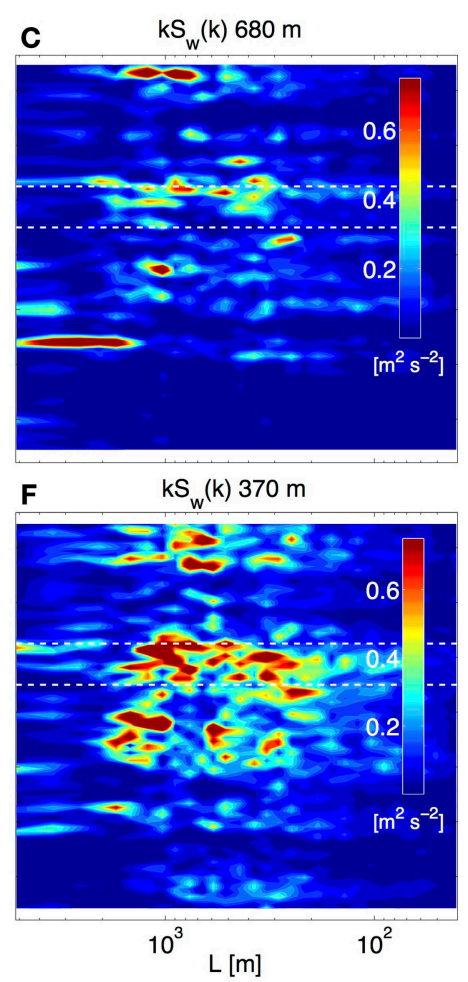

FIGURE 3 | Spatial structure of the weighted energy spectra $k^{\star} \mathbf{S}(\mathbf{k})$ along the entire upper $(A-C)$ and lower (D-F) $A B L$ flight leg for $u$ $(A, D), v(B, E)$ and $\mathbf{w}(C, F)$ wind components. The two white horizontal dashed lines indicate the analyzed segment. A noticeable increase in energy at the horizontal scale of approximately $1 \mathrm{~km}$ is present in all plots around the latitude of $45^{\circ} \mathrm{N}$. The spectra are calculated on overlapping subintervals of 512 data points (about $10 \mathrm{~km}$ long) which slide by 103 data points (about $2 \mathrm{~km}$ ), thus obtaining 100 spectra over the $216-\mathrm{km}$ long flight legs. The $\mathrm{y}$-axis values correspond to the central latitudes of subintervals.
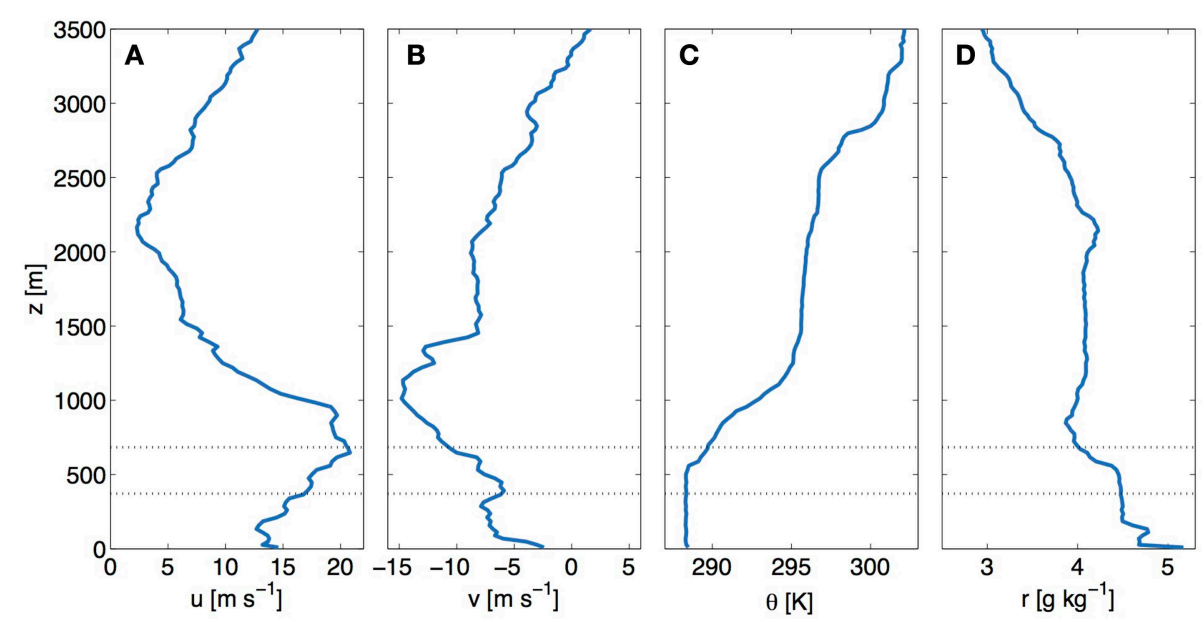

FIGURE 4 | Vertical profiles of the (A) longitudinal $u$ and (B) transverse $v$ wind components, (C) potential temperature $\theta$ and (D) mixing ratio $r$ from the dropsonde $\mathbf{S} 3$ released at $\left(\mathbf{1 3 . 6 3}^{\circ} \mathbf{E}, \mathbf{4 4 . 9 6}^{\circ} \mathbf{N}\right)$. Horizontal dotted lines denote the heights of the two lower flight legs $(370$ and $680 \mathrm{~m})$.

Miura, 1986). However, some studies based on radar or lidar observations, or modeling, of cloud-free rolls report considerably lower aspect ratios, sometimes as low as one (Kelly, 1984; Melfi et al., 1985; Miura, 1986; Mayor et al., 2002). There are no cloud streets in bora due to its downslope nature and the associated advection of dry air to low levels, so the aspect ratio is expected to be smaller. Additionally, it has been shown that the roll aspect ratio increases in the downwind direction on average by about 1 per $110 \mathrm{~km}$ (Miura, 1986). The current bora measurements were taken close to the coast, so larger aspect ratios could 


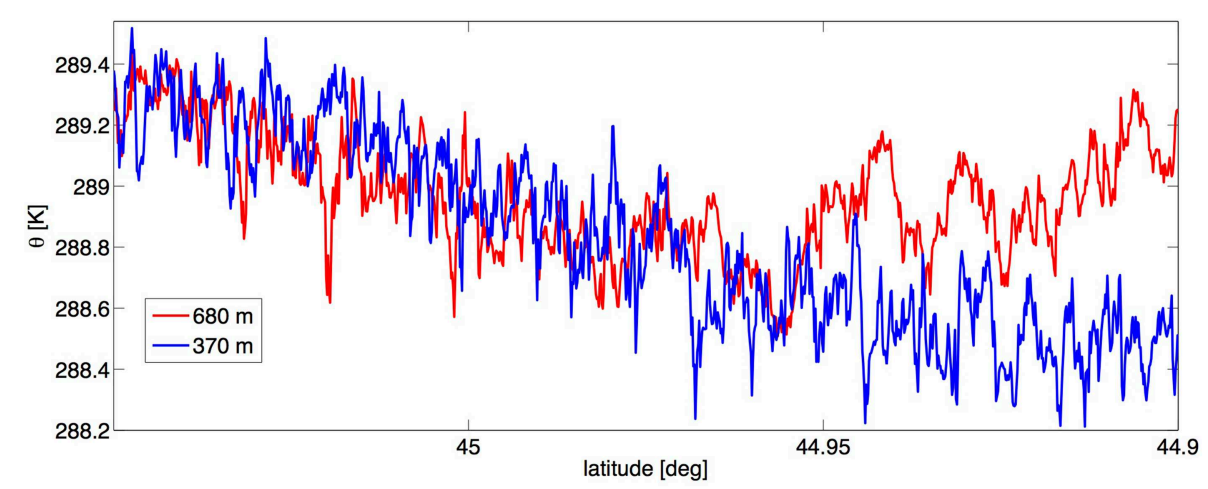

FIGURE 5 | Potential temperature at the two lower flight legs along the analyzed segment.
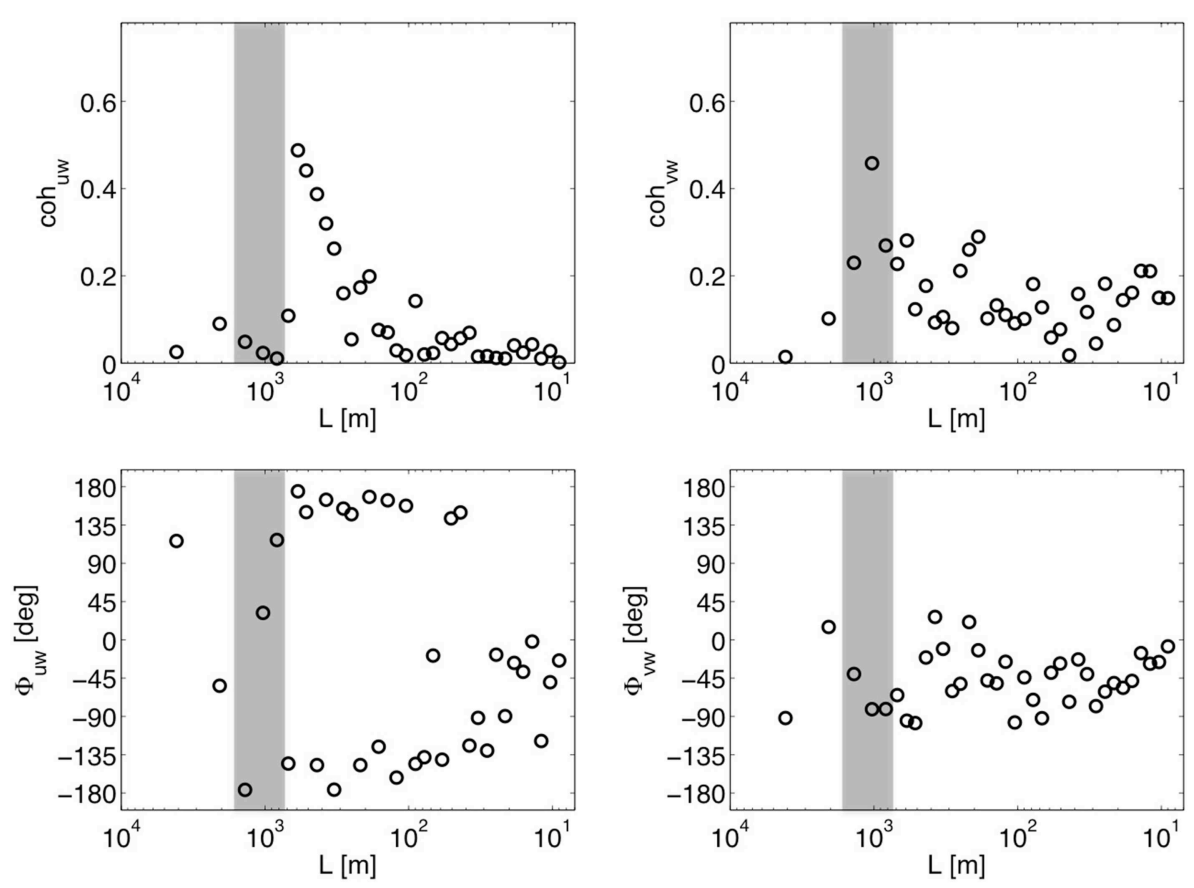

FIGURE 6 | Spectral coherence (top) and phase angle (bottom) between $u$ and $w$ (left) and $v$ and $w$ (right) wind components over the analyzed segment $\left(44.90-45.05^{\circ} \mathrm{N}\right)$ at the upper ABL flight leg $(680 \mathrm{~m})$.

be expected further offshore. Interestingly, Melfi et al. (1985) found in their data that the cell sizes vary even when the PBL height remains almost constant regardless of the downwind distance.

While the evidence supports the presence of rolls, we cannot totally eliminate the possibility that the rolls coexisted with lines of cells (rolls+cells), as is commonly observed in clear-air radar patterns (e.g., Weckwerth et al., 1997), or that we are sampling random cells, although the last possibility is unlikely. The present analysis and the common presence of rolls in other cold-air outbreak studies suggests the presence of rolls or rolls+cells. Also, the strong winds and surface buoyancy flux combined with the unusually shallow ABL depth would likely put the present case in the ABL depth/Obukhov length regime associated with rolls+cells, or less likely, rolls, rather than pure cellular convection (Grossman, 1982). Note that roll-cell patterns are often referred to as rolls in the literature, because they produce well-defined cloud lines if the updrafts on the crosswind sides of the cells merge into clearly-defined bands of updraft air. Indeed, careful analysis of roll+cell radar signatures by Weckwerth et al. (1997) indicate strong two-dimensional signatures, justifying this characterization. The structure could be verified with a combination of cross-wind aircraft legs and legs at a small angle to the expected roll direction, the latter revealing either longwavelength variation in wind direction (as in pure rolls), or, more likely, superposed long wavelengths (rolls) and short wavelengths (cells), as described for such structures being advected past a tower in LeMone (1973). 

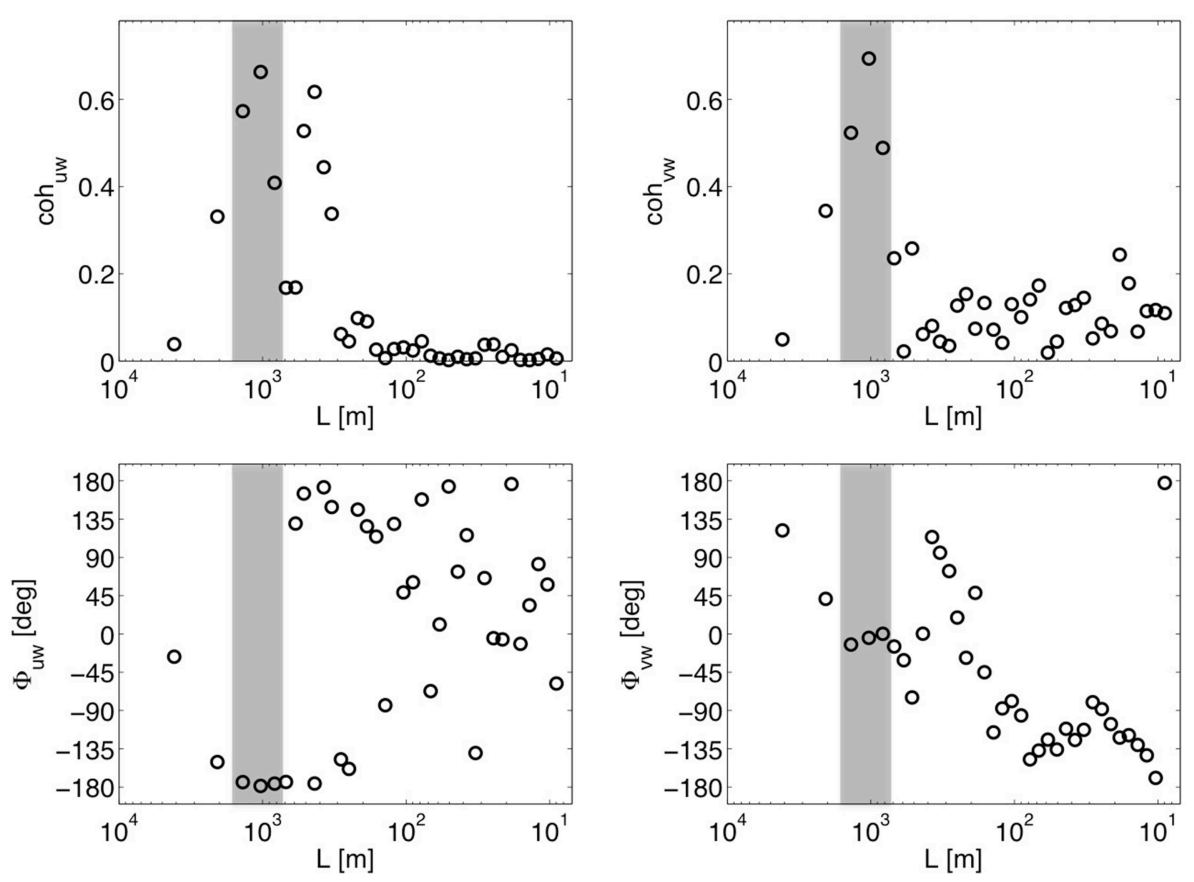

FIGURE 7 | As in Figure 6, except for the lower ABL flight leg (370 m).

As shown by the running spectra (Figure 3), there is another segment with possible occurrence of rolls located around $44.75^{\circ} \mathrm{N}$. However, we focus on the current segment because of the clearest roll-like signatures. Rolls could be present elsewhere, but their structure is masked-or modified-by the presence of gravity waves generated in the shear layers above the PBL. Note that the large shear above the PBL (Figure 4) is consistent with gravity-wave generation. Furthermore, the spatial bora structure in the along-coast direction is complex with several processes interacting at different, although on occasions overlapping scales (e.g., Signell et al., 2010). These processes result in the typical bora jets and wakes and include gap flows, gravity waves, and nonlinear processes such as wave breaking, hydraulic jumps, and the resulting potential vorticity banners. As a result, there are several other peaks in the crosswind wavenumber spectra that may overlap with that of the rolls. This complexity also hinders the determination of relevant scales for calculation of turbulence fluxes, as discussed in Večenaj et al. (2012). The bora jets and wakes, while basically being cold-air outbreaks

\section{References}

Alpers, W., and Brummer, B. (1994). Atmospheric boundary-layer rolls observed by the synthetic aperture radar aboard the ERS-1 satellite. J. Geophys. Res. 99, 12613-12621. doi: 10.1029/94JC00421

Atlas, D., and Chou, S. H. (1983). The influence of coastal shape on winter air-sea interactions. Mon. Weather Rev. 111, 245-252.

Belušić, D., and Klaić, Z. B. (2006). Mesoscale dynamics, structure and predictability of a severe Adriatic bora case. Meteor. Z. 15, 157-168. doi: 10.1127/0941-2948/2006/0116 resulting in a thermodynamically generated convective ABL over the warmer sea, are also dynamically generated phenomena that carry the signatures of their upstream generation mechanisms, particularly gravity waves and the resultant downwind variation of ABL height. Sampling such flow at a single distance from the mountain range does not provide sufficient information for analyzing all the effects of the ABL height and its development on the occurrence of rolls. It is therefore difficult to generalize the current results without additional measurements or highresolution numerical simulations.

\section{Acknowledgments}

We would like to thank the two reviewers for their comments and suggestions. This work has been partially supported by the Croatian Science Foundation, project CATURBO, No. 09/151. LeMone's work is supported by the National Center for Atmospheric Research, which is funded by the U. S. National Science Foundation. 
and wind stress variations. J. Phys. Oceanogr. 31, 174-198. doi: 10.1175/15200485(2001)031<0174:TSOWEP>2.0.CO;2

Etling, D., and Brown, R. A. (1993). Roll vortices in the planetary boundary layer: a review. Boundary Layer Meteor. 65, 215-248. doi: 10.1007/BF00705527

Gohm, A., Mayr, G. J., Fix, A., and Giez, A. (2008). On the onset of bora and the formation of rotors and jumps near a mountain gap. Q. J. R. Meteor. Soc. 134, 21-46. doi: 10.1002/qj.206

Grisogono, B., and Belušić, D. (2009). A review of recent advances in understanding the meso- and microscale properties of the severe Bora wind. Tellus 61A, 1-16. doi: 10.1111/j.1600-0870.2008.00369.x

Grossman, R. L. (1982). An analysis of vertical velocity spectra obtained in the BOMEX fair-weather, trade-wind boundary layer. Bound Layer Meteor. 23, 323-357. doi: 10.1007/BF00121120

Grubišić, V. (2004). Bora-driven potential vorticity banners over the Adriatic. Q. J. R. Meteor. Soc. 130, 2571-2603. doi: 10.1256/qj.03.71

Hein, P. F., and Brown, R. A. (1988). Observations of longitudinal roll vortices during artic cold air outbreaks over open water. Bound Layer Meteor. 45, 177-199. doi: 10.1007/BF00120822

Jiang, Q. F., and Doyle, J. D. (2005). Wave breaking induced surface wakes and jets observed during a bora event. Geophys. Res. Lett. 32:L17807. doi: 10.1029/2005GL022398

Kelly, R. D. (1982). A single Doppler-radar study of horizontal roll convection in a lake-effect snow storm. J. Atmos. Sci. 39, 1521-1531.

Kelly, R. D. (1984). Horizontal roll and boundary-layer interrelationships observed over Lake Michigan. J. Atmos. Sci. 41, 1816-1826.

Klaić, Z. B., Belušić, D., Grubišić, V., Gabela, L., and Ćoso, L. (2003). Mesoscale airflow structure over the northern Croatian coast during MAP IOP 15 - a major bora event. Geofizika 20, 23-61.

Konrad, T. J. (1970). The dynamics of the convective process in clear air as seen by radar. J. Atmos. Sci. 27, 1138-1147.

LeMone, M. A. (1973). The structure and dynamics of horizontal roll vortices in the planetary boundary layer. J. Atmos. Sci. 30, 1077-1091.

LeMone, M. A., and Pennell, W. T. (1976). The relationship of trade wind cumulus distribution to subcloud layer fluxes and structure. Mon. Weather Rev. 104, 524-539.

Mayor, S. D., Spalart, P. R., and Tripoli, G. J. (2002). Application of a perturbation recycling method in the large-eddy simulation of a mesoscale convective internal boundary layer. J. Atmos. Sci. 59, 2385-2395.

Melfi, S. H., Spinhirne, J. D., Chou, S.-H., and Palm, S. P. (1985). Lidar observations of vertically organized convection in the planetary boundary layer over the ocean. J. Climate Appl. Meteor. 24, 806-821.

Melfi, S. H., and Palm, S. P. (2012). Estimating the orientation and spacing of midlatitude linear convective boundary layer features: cloud streets. J. Atmos. Sci. 69, 352-364. doi: 10.1175/JAS-D-11-070.1
Miura, Y. (1986). Aspect ratios of longitudinal rolls and convection cells observed during cold air outbreaks. J. Atmos. Sci. 43, 26-39.

Müller, G., and Chlond, A. (1996). Three-dimensional numerical study of cell broadening during cold-air outbreaks. Boundary Layer Meteor. 81, 289-323. doi: 10.1007/BF02430333

Prtenjak, M. T., and Belušić, D. (2009). Formation of reversed lee flow over the north-eastern Adriatic during bora. Geofizika 26, 145-155.

Signell, R. P., Chiggiato, J., Horstmann, J., Doyle, J. D., Pullen, J., Askari, F., et al. (2010). High-resolution mapping of Bora winds in the northern Adriatic Sea using synthetic aperture radar. J. Geophys. Res. 115:C04020. doi: 10.1029/2009JC005524

Smith, R. B. (1985). On severe downslope winds. J. Atmos. Sci. 42, 2597-2603.

Smith, R. B. (1987). Aerial observations of the Yugoslavian bora. J. Atmos. Sci. 44, 269-297.

Stiperski, I., Ivančan-Picek, B., Grubišić, V., and Bajć, A. (2012). Complex bora flow in the lee of Southern Velebit. Q. J. R. Meteor. Soc. 138, 1490-1506. doi: 10.1002/qj.1901

Večenaj, Ž., Belušić, D., Grubišić, V., and Grisogono, B. (2012). Along-coast features of bora-related turbulence. Boundary Layer Meteor. 143, 527-545. doi: 10.1007/s10546-012-9697-6

Walter, B., and Overland, J. E. (1984). Observations of longitudinal rolls in a near-neutral atmosphere. Mon. Weather Rev. 112, 200-208.

Weckwerth, T. M., Wilson, J. W., Wakimoto, R. M., and Crook, N. A (1997). Horizontal convective rolls: determining the environmental conditions supporting their existence and characteristics. Mon. Weather Rev. 125, 505-526.

Yoshino, M. M. (1976). Local Wind Bora. Tokyo: University of Tokyo Press.

Young, G. S., Kristovich, D. A. R., Hjelmfelt, M. R., and Foster, R. C. (2002). Rolls, streets, waves, and more: a review of quasi-twodimensional structures in the atmospheric boundary layer. Bull. Amer. Meteor. Soc. 83, 997-1001. doi: 10.1175/1520-0477(2002)083<0997:RSWAMA $>2$. 3. $\mathrm{CO} ; 2$

Conflict of Interest Statement: The authors declare that the research was conducted in the absence of any commercial or financial relationships that could be construed as a potential conflict of interest.

Copyright (c) 2015 Belušić, Večenaj and LeMone. This is an open-access article distributed under the terms of the Creative Commons Attribution License (CC BY). The use, distribution or reproduction in other forums is permitted, provided the original author(s) or licensor are credited and that the original publication in this journal is cited, in accordance with accepted academic practice. No use, distribution or reproduction is permitted which does not comply with these terms. 


\section{OPEN ACCESS}

Edited by:

Miguel A. C. Teixeira,

University of Reading, UK

Reviewed by:

Stefano Federico,

Institute of Atmospheric Sciences and

Climate, Italy

Guðrún Nína Petersen, Icelandic Meteorological Office,

Iceland

Qingfang Jiang,

United States Naval Research

Laboratory, USA

*Correspondence:

Johannes Sachsperger johannes.sachsperger@univie.ac.at

Specialty section:

This article was submitted to

Atmospheric Science,

a section of the journal

Frontiers in Earth Science

Received: 21 August 2015 Accepted: 29 October 2015 Published: 18 November 2015

Citation:

Sachsperger J, Serafin S and Grubišić V (2015) Lee Waves on the Boundary-Layer Inversion and Their Dependence on Free-Atmospheric Stability. Front. Earth Sci. 3:70. doi: 10.3389/feart.2015.00070

\section{Lee Waves on the Boundary-Layer Inversion and Their Dependence on Free-Atmospheric Stability}

\author{
Johannes Sachsperger $^{1 *}$, Stefano Serafin ${ }^{1}$ and Vanda Grubišic ${ }^{1,2}$ \\ ${ }^{1}$ Department of Meteorology and Geophysics, University of Vienna, Vienna, Austria, ${ }^{2}$ Earth Observing Laboratory, National \\ Center for Atmospheric Research, Boulder, CO, USA
}

This study examines gravity waves that develop at the boundary-layer capping inversion in the lee of a mountain ridge. By comparing different linear wave theories, we show that lee waves that form under these conditions are most accurately described as forced interfacial waves. Perturbations in this type of flow can be studied with a linear two-dimensional model with constant wind speed and a sharp density discontinuity separating two layers, a neutral one below and a stable one above. Defining the model parameters on the basis of observations taken in the Madeira archipelago, we highlight the impact of upper-level stability on interfacial waves. We demonstrate that stable stratification aloft limits the possible range of lee wavelengths and modulates the length of the stationary wave mode. Finally, we show that the stable stratification aloft strongly constrains the validity of the shallow-water (or long-wave) approximation by permitting only short-wave modes to be trapped at the interface.

Keywords: trapped lee waves, water waves, boundary layer, inversion layer, stratified flow

\section{INTRODUCTION}

Trapped lee waves can be generated in the atmosphere when a mountain perturbs stratified airflow. Unlike other types of mountain waves, trapped waves propagate horizontally (Smith, 1979): their phase lines are vertical and multiple wave crests can extend over several hundreds of kilometers downstream of an obstacle. If the atmosphere contains sufficient moisture, clouds may form at the wave crests (Houze, 2014) and give rise to a characteristic stripe pattern, frequently observed in satellite images close to mountainous areas, e.g., the Alps (Doyle et al., 2002), Pyrenees (Georgelin and Lott, 2001), Rocky Mountains (Ralph et al., 1997), and Pennines (Vosper et al., 2012).

Trapped lee waves can be expected to develop if the atmospheric structure promotes vertical trapping and horizontal propagation of wave energy. This occurs either when the Scorer parameter $l^{2}=\frac{N^{2}}{U^{2}}-\frac{1}{U} \frac{d^{2} U}{d z^{2}}$ (where $N$ is the buoyancy frequency and $U$ the wind speed) decreases with height (Scorer, 1949), or along a density discontinuity, e.g., a temperature inversion. Both of these conditions lead to horizontal propagation of wave energy. In the former case, this happens through wave reflection and superposition in a wave duct; in the latter case, through adjustment to horizontal pressure gradients generated by the deflection of the discontinuity. These two mechanisms have traditionally been regarded as distinct and unrelated. The former has often been highlighted as the primary mechanism that gives rise to trapped lee waves in the atmosphere (e.g., Durran, 1986b; Wurtele et al., 1996; Teixeira, 2014), while the latter has been emphasized mostly in oceanographic investigations, for instance to explain surface water waves and the phenomenon of 
dead water (Gill, 1982). In what follows, we refer to the theories that describe these two processes, respectively, as the internal wave theory and the interfacial wave theor $y^{1}$.

The internal gravity wave framework, where density is assumed to be a continuous function of height, was used by Scorer (1949) to develop a theory for lee waves in a twolayer atmosphere. Scorer attributed the origin of trapped lee waves to a discontinuity in the parameter $l^{2}$, defined above. For $l$ higher in the lower layer, horizontal wave propagation results from the linear superposition of two internal gravity wave modes, an upward-propagating one, excited by the flow over the mountain, and a downward-propagating one arising through wave reflection at the interface between the two layers.

Scorer (1949) also applied interfacial wave theory to atmospheric lee waves that form on a density discontinuity. However, that part of his work has received only little attention. After Scorer's, probably the first study explicitly addressing interfacial lee waves was made by Vosper (2004). Seeking for the most favorable environment for the formation of lee-wave rotors and low-level hydraulic jumps, Vosper (2004) analyzed the conditions that lead to wave trapping at a boundary-layer inversion underneath a continuously-stratified atmosphere. For such a density profile, interfacial waves along the inversion and vertically-propagating wave modes in the free atmosphere may occur at the same time. Teixeira et al. (2013) investigated the magnitude of wave drag, generated by both interfacial and vertically propagating waves in this scenario, and determined the state of maximum total drag. Although these two studies shed light on important aspects of interfacial lee waves, an assessment of how their wavelength (the most striking and immediate feature of the phenomenon, as visible in satellite images) depends on the environmental conditions in the free atmosphere still seems to be lacking. Therefore, the first objective of this paper is to improve the current understanding of the impact of the stratified free atmosphere on the dynamics - and the wavelength in particular-of interfacial lee waves that form at the boundarylayer inversion.

A related theme is the applicability of hydraulic analogies to the quantitative description of layered atmospheric flows. The possibility of a hydraulic-type response in two-layer mountain flow (with discontinuous $l^{2}$ ) was first demonstrated by Durran (1986a), who suggested that transition to supercritical flow at the top of a mountain is an effective mechanism to generate downslope windstorms. The applicability of hydraulic theory, however, depends on stratification in the free atmosphere, which affects the validity of two of the basic assumptions, namely: (i) the presence of a passive upper layer, i.e., one that causes no horizontal pressure gradient force on the lower layer (Jiang and Smith, 2001), and (ii) hydrostatic conditions, which imply the validity of the shallow-water (long-wave) approximation. Relationships between free-atmospheric stratification and the validity of the passive layer assumption have been explored by Jiang (2014). A similar study focusing on the long-wave

\footnotetext{
${ }^{1}$ Interfacial wave theory is in turn related to hydraulic theory. Hydraulic theory is based on the analogy between the flow of a compressible gas and the flow of a shallow liquid, and is inherently hydrostatic. In contrast, no a priori assumption about hydrostaticity is made in interfacial wave theory.
}

approximation, however, still seems to be lacking. Hence, the second aim of this study is to explain how static stability in the free atmosphere affects the accuracy of the shallow-water theory when applied to low-level flow below an inversion.

We begin by reviewing the existing theories of trapped lee waves in Section 2. By taking representative values of freeatmospheric stability and inversion strength from an observed lee-wave event (Section 3), we describe how different linear analytical models lead to different estimates of lee wavelength for the same conditions (Section 4). Recognizing the important role played by free-atmospheric stratification, in Section 5 we discuss its impact on lee wavelength and on the applicability of shallow-water theory. Conclusions are drawn in Section 6.

\section{REVIEW OF LEE WAVE THEORIES}

As briefly discussed in the Introduction, the simplest models of trapped lee waves consider uniform wind speed and a vertical discontinuity, either in the Scorer parameter $l$ or in potential temperature $\theta$. Such discontinuities are responsible for different wave-trapping mechanisms, which may co-exist for a vertical potential temperature profile like the one shown in Figure 1. The defining parameters of this type of flow are the buoyancy frequencies in the lower and upper layer ( $N_{1}$ and $N_{2}$ respectively), the potential temperature jump across the interface $(\Delta \theta)$, the potential temperature at the ground $\left(\theta_{0}\right)$ and the height of the lower layer $\left(h_{1}\right)$.

In general, the dynamics of wave propagation can be concisely described by a frequency dispersion relationship (FDR), i.e., a relation between the intrinsic frequency of the wave, $\Omega$, and the wavenumber, $k$. For simplicity, we focus on flows with uniform background wind speed $U$. The Scorer parameter simplifies to $l^{2}=\frac{N^{2}}{U^{2}}$ in this case. Since lee waves are typically stationary, we consider only wave modes that do not move in a reference frame attached to the mountain, thus $\Omega / k-U=0$. All wave modes $k$ that satisfy this relation are stationary with respect to the mountain.

For the stability profile in Figure 1, the FDR of trapped lee waves can be derived by assuming that wavelike solutions to the governing equations exist in each of the two layers. The derivation, presented in the Appendix, leads to:

$$
U^{2}=\frac{g^{\prime}}{i m_{1} \operatorname{coth}\left(i m_{1} h_{1}\right)-i m_{2}}
$$

Here, $h_{1}$ is the finite thickness of the lower layer (the upper one being instead infinitely deep), $g^{\prime}=g \cdot \Delta \theta / \theta_{0}$ is the reduced gravity at the interface and $m_{1,2}=\left(l_{1,2}^{2}-k^{2}\right)^{1 / 2}$ are the vertical wave-numbers in the lower (subscript 1 ) and in the upper layer (subscript 2) respectively.

Equation (1) summarizes the behavior of four wave types that may occur in common atmospheric conditions:

- Internal interface waves, developing at a density discontinuity between two neutrally stratified fluid layers $(\Delta \theta \neq 0$, $N_{1,2}=0$ ). 


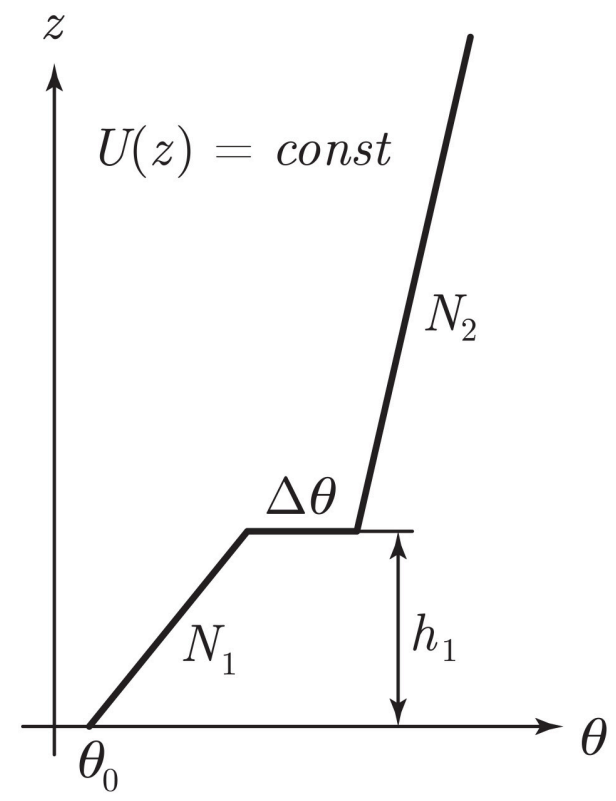

FIGURE 1 | Vertical potential temperature $(\theta)$ profile of a generic two-layer flow with a density interface. The wind profile $U(z)$ is assumed to be constant with height (not shown).

- Free interface waves, propagating along a density discontinuity between a lower neutral layer and an upper stably stratified passive layer $\left(\Delta \theta \neq 0, N_{1}=0, m_{2}=0\right)$.

- Forced interface waves, similar to the previous case, but with an upper stably stratified active layer $\left(\Delta \theta \neq 0, N_{1}=0, m_{2} \neq 0\right)$.

- Resonant trapped waves, developing at a discontinuity in the Scorer parameter $\left(\Delta \theta=0, N_{1}>N_{2}>0\right)$.

These wave types, and the conditions under which they may form, are described in greater detail in what follows.

\subsection{Internal Interface Waves}

If both fluid layers are neutrally stratified, wave energy is concentrated at the density discontinuity between them, because all wave modes are evanescent below and above it $\left(m_{1,2}^{2}<0\right)$. If the source of the wave energy is at the surface, as is the case with mountain waves, higher altitudes of the interface will result in lower lee wave amplitudes. This happens because evanescent wave modes decay with increasing distance from the wave source. Assuming $N_{1,2}=0$ in Equation (1), the FDR of stationary interfacial waves becomes (Turner, 1973):

$$
U^{2}=\frac{g^{\prime}}{k \operatorname{coth}\left(k h_{1}\right)+k}
$$

The coth $\left(k h_{1}\right)$ term in Equation (2) is non-periodic in $k$, because the argument is a real number. Thus, only a single stationary wave mode can exist on the density discontinuity. The FDR in Equation (2) describes interfacial waves at internal boundaries with a non-passive upper layer, which is the case for relatively short wave modes $\left(\partial p_{2}^{\prime} / \partial x \neq 0\right.$ if $k>0, p_{2}^{\prime}$ being the pressure perturbation in the upper layer; see e.g., Nappo, 2012).

Equation (2) is well-known in its long-wave approximation form, where the condition $k h_{1} \ll 1$ leads to the non-dispersive FDR:

$$
U=\left(g^{\prime} h_{1}\right)^{1 / 2}
$$

Another limiting case of Equation (2), that gained less attention in meteorological literature, can be derived by adopting the short-wave approximation, $k h_{1} \gg 1$ :

$$
U=\left(g^{\prime} / 2 k\right)^{1 / 2}
$$

Unlike Equation (3), Equation (4) retains the dispersive character of Equation (2) and can be solved for $k$ to estimate the wavelength of a stationary internal interface wave. The FDRs in Equations (3) and (4) are similar to those of shallow- and deep-water external waves, which correspond to the limit $g^{\prime} \rightarrow g$. However, since Equations (3) and (4) apply to flows where the upper layer has non-negligible density, they still describe internal interface wave modes.

\subsection{Free Interface Waves}

Interfaces that are not subject to stress exerted by the upper fluid are referred to as free surfaces. The passive layer assumption, $\partial p_{2}^{\prime} / \partial x=0$, applies in this case. Special cases of free surfaces are those where the density difference between the two fluid layers is very large, e.g., between water and air. The corresponding theory was developed by Airy (1841), who firstly derived the universal FDR of (external) water waves (Craik, 2004):

$$
U^{2}=\frac{g}{k \operatorname{coth}\left(k h_{1}\right)} .
$$

This equation is not generally valid in atmospheric flows, which are typically characterized by very small density discontinuities. However, even in the atmosphere it is possible for a density discontinuity (e.g., the capping inversion of the convective boundary layer) to act as a free surface. This is the case if $N_{1}=0$ and $m_{2}=0$. The second constraint means that any internal gravity wave mode in the stably-stratified upper layer must have vertical phase lines at the interface. In this case, Equation (1) becomes:

$$
U^{2}=\frac{g^{\prime}}{k \operatorname{coth}\left(k h_{1}\right)} .
$$

Although this wave type occurs in the interior of a fluid, the interface behaves as a free surface. The upper layer is passive because of the condition $m_{2}=0$, which implies that wave perturbations in the pressure field, and consequently the horizontal pressure gradient, must vanish therein, as can be demonstrated from the polarization relationships of internal gravity waves (see, e.g., Nappo, 2012). The same applies to wave perturbations in the horizontal wind speed. The FDR of these (internal) free interface waves is similar to that of (external) water waves in Equation (5), except for reduced gravity $g^{\prime}$ instead of $g$. 


\subsection{Forced Interface Waves}

Interfacial waves conform to Equation (6) only when their horizontal wavenumber is equal to the Scorer parameter in the atmosphere aloft (in fact, $m_{2}=0$ implies $k=l_{2}$ ). In general, however, $k \neq l_{2}$. In this case the upper layer is not passive, i.e., waves propagating along the interface are subject to forcing from internal waves above. The FDR describing these (internal) forced interface waves is obtained from Equation (1) by requiring $N_{1}=0$ and maintaining a wave-permitting layer with $N_{2}>0$ aloft (Scorer, 1997):

$$
U^{2}=\frac{g^{\prime}}{k \operatorname{coth}\left(k h_{1}\right)+\sqrt{k^{2}-l_{2}^{2}}} .
$$

Equation (7) is the general form of the FDR considered by Vosper (2004), which is the corresponding short-wavelength $\left(k h_{1} \gg 1\right)$ approximation. All wave modes satisfying $k^{2}<l_{2}^{2}\left(m_{2}^{2}>0\right)$ can propagate into the upper stratified layer, hence they are not trapped at the interface. Consequently, the condition $k^{2}=$ $l_{2}^{2}$ defines a critical wavenumber, i.e., the lowest wavenumber (longest wavelength) for which wave trapping is possible. This condition transforms Equation (7) into Equation (6), i.e., into the special case of a free interfacial wave (Section 2.2), and generates the maximum total wave drag (Teixeira et al., 2013).

Equation (7) shows that, given $N_{2}$ and $U$, the critical wavenumber depends on the inversion strength $\Delta \theta$ (through $g^{\prime}$ ) and on the lower layer depth $h_{1}$. Critical values for $\Delta \theta$ and $h_{1}$ can be determined inserting $k=l_{2}=N_{2} / U$ into Equation (7). Solving for $\Delta \theta$ or $h_{1}$ then gives:

$$
\begin{aligned}
\Delta \theta_{\text {crit }} & =\frac{N_{2} U \theta_{0}}{g} \cdot \operatorname{coth}\left(\frac{N_{2} h_{1}}{U}\right) \\
h_{1 \text { crit }} & =\frac{U}{N_{2}} \cdot \operatorname{acoth}\left(\frac{g^{\prime}}{N_{2} U}\right)
\end{aligned}
$$

Inversions with strength $\Delta \theta \geq \Delta \theta_{\text {crit }}$, or located at height $h_{1} \geq$ $h_{1 \text { crit }}$, cause wave trapping because they make stationary waves become evanescent in the layer aloft.

\subsection{Resonant Trapped Waves $\left(g^{\prime}=0\right)$}

Setting $g^{\prime}=0$ (that is, $\Delta \theta=0$ ) in Equation (1) simplifies it to (Scorer, 1949):

$$
\operatorname{coth}\left(i m_{1} h_{1}\right)=\sqrt{\frac{l_{2}^{2}-k^{2}}{l_{1}^{2}-k^{2}}} .
$$

Therefore, wave trapping occurs even in absence of a density discontinuity if a wave mode $k$ that satisfies Equation (10) exists. Scorer (1949) showed that this is the case if

$$
\begin{aligned}
& l_{1}^{2}>k^{2}>l_{2}^{2} \\
& l_{1}^{2}-l_{2}^{2}>\frac{\pi^{2}}{4 h_{1}^{2}} .
\end{aligned}
$$

In these conditions, an internal gravity wave mode that propagates vertically in the lower layer is reflected at the discontinuity of $l^{2}$, above which it becomes evanescent. The superposition of the reflected and primary mode generates a stationary wave with vertical phase lines, trapped in the layer below the interface. We refer to this wave type as a resonant trapped wave. In contrast to the FDRs for interfacial waves [Equations (2), (6), and (7)], the argument of the cotangent function in Equation (10) is imaginary. Since the roots of $\operatorname{coth}\left(i m_{1} h_{1}\right)$ are periodic, multiple resonant modes may exist. The amplitude of these modes depends essentially on the interface height and on the mountain shape. In general, the mode with the wavelength closest to the mountain half-width $L$ is dominant.

\subsection{Bridging the Theories of Interfacial and Resonant Trapped Lee Waves}

Wave propagation in stratified fluids with a more complex vertical structure, i.e., with more than two layers, has been studied with analytical models in the past. For instance, Baines (1995, p. 189) formulated a non-hydrostatic three-layer model, that can be used to estimate the horizontal wavenumber of a lee wave trapped along a finite-thickness inversion. In this case, the wavenumber $k$ satisfies the following FDR:

$$
\begin{aligned}
& {\left[\tan \left(m_{1} h_{1}\right)-\frac{i m_{1}}{m_{2}}\right]\left(m_{2}-m_{3}\right)=e^{-2 i m_{2} d}\left[\tan \left(m_{1} h_{1}\right)+\frac{i m_{1}}{m_{2}}\right]} \\
& \left(m_{2}+m_{3}\right) \text {. }
\end{aligned}
$$

Here, subscripts $j=1,2,3$ denote the fluid layers, numbered from below; $m_{j}=\left(N_{j}^{2} / U^{2}-k^{2}\right)^{1 / 2}$ is the vertical wavenumber in each of the layers; $h_{1}$ is the thickness of the lowest layer and $d$ that of the middle one (the inversion layer). The third layer represents the free atmosphere, which is assumed to be infinitely deep. Equation (13) is transcendental, because it has no closedform solutions for $k$. Moreover, it remains transcendental even after adopting the long- $\left(k h_{1} \ll 1\right)$ or short-wave $\left(k h_{1} \gg 1\right)$ approximations, in contrast to the two-layer models introduced in Sections 2.1-2.3 above.

In the strict limit of the middle layer depth being zero $(d=$ 0 ), the inversion disappears and the FDR in Equation (13) approaches the FDR for resonant waves (Equation 10). This is to be expected as the Baines (1995) model assumes a continuous density profile.

For small but finite values of the inversion depth and the finite value of $\Delta \theta$ across the inversion, one would expect the solution of Equation (13) to converge to that of the most general twolayer model (Equation 1), assuming that all other parameters are the same. We show this to be true in the following section by numerically solving Equation (13) for a specific case (cf. Figure 3).

\section{WAVES ALONG THE BOUNDARY-LAYER INVERSION}

The typical thermal structure of the convective boundary layer $\left(N_{1} \approx 0, N_{2}>0, \Delta \theta>0\right)$ is such that it supports forced interface waves (Section 2.3). Since similar conditions are often 
present above ocean surfaces, or at daytime during the warm season over land, it is likely that wave trapping at the boundary layer inversion is a common mechanism for lee waves.

To confirm this hypothesis, we study a lee wave event that occurred on 24 December 2013 downstream of the Desertas islands. These islands are part of the Madeira archipelago, located in the subtropical Atlantic $\sim 800 \mathrm{~km}$ south-west of mainland Portugal. The Desertas are elongated in the N-S direction and reach an altitude of $300 \mathrm{~m}$ MSL. Sufficient data is available in this area to estimate the wavelength (from high-resolution satellite images) and the vertical structure of the atmosphere (from operational radiosonde observations in Funchal, Madeira's capital).

The satellite image in Figure 2A shows a characteristic cloudstripe pattern, generated by a trapped wave leeward of the Desertas islands. The lee wave train is not visibly influenced by the prominent wake of Madeira (Grubišić et al., 2015) and extends over a distance of $40 \mathrm{~km}$ with approximately nine wave crests, hence $\lambda \approx 4.4 \mathrm{~km}$.

The vertical structure of the atmosphere during this event, as measured in nearby Funchal, is shown in Figure 2B. It consists of a neutrally stratified lower layer $\left(N_{1}=0\right)$ with $\theta_{0}=291 \mathrm{~K}$, a boundary-layer inversion with $\Delta \theta=8 \mathrm{~K}$ at $h_{1}=1100 \mathrm{~m}$ and a continuously stratified free atmosphere with $N_{2}=0.010 \mathrm{~s}^{-1}$ aloft. The flow is westerly with almost no directional shear. A representative value of $U=10 \mathrm{~m} \mathrm{~s}^{-1}$ for the wind speed is determined from the wind profile in Figure $\mathbf{2 C}$ as the average in the layer below $z=2 h_{1}$. The altitude of the inversion coincides with the layer of largest relative humidity (gray shading, $R H=97 \%$ at $h_{1}=1100 \mathrm{~m}$ ), implying that the cloud pattern in Figure 2A likely corresponds to an interfacial wave. Further evidence for the interfacial wave character of the disturbance is provided by the Scorer parameter profiles in Figure 2D. The observed wave mode (dashed black) is evanescent $\left(k^{2}>l^{2}\right)$ below and above the inversion, indicating that the disturbance can only propagate along it. The flow configuration determined from Figure $\mathbf{2 B}$ is similar to that causing forced interfacial waves (Section 2.3).

Given its small thickness, the inversion layer can be approximated as a density discontinuity. This is supported by a comparison between the solutions of Equation (1), which is derived from the discontinuous two-layer model, and Equation (13), which is derived from the continuous threelayer model introduced in Section 2.5. Both equations are solved numerically for $\lambda=2 \pi / k$, imposing the sounding parameters presented above and letting $d$ in the three-layer model vary in the range between 0 and $1000 \mathrm{~m}$. The results in Figure 3 reveal a difference of $\sim 5 \%$ in the wavelength estimates for $d=150 \mathrm{~m}$, which corresponds to the observed profile. This level of accuracy is sufficient for our needs. Hence, from now on, we only consider two-layer models, with the benefit that an analytical solution for $k$ is possible (if the short-wave approximation is adopted).

In what follows, we use observations from the Desertas islands to provide numerical values for the parameters of the linear models described in Section 2, and thereby explore the impact of free-atmospheric stratification on interfacial waves.

\section{LINEAR THEORY RESULTS}

Our two-dimensional, two-layer linear model is described in detail in the Appendix. We solve it for the perturbation velocity fields $u^{\prime}(x, z)$ and $w^{\prime}(x, z)$ as a function of the flow profile
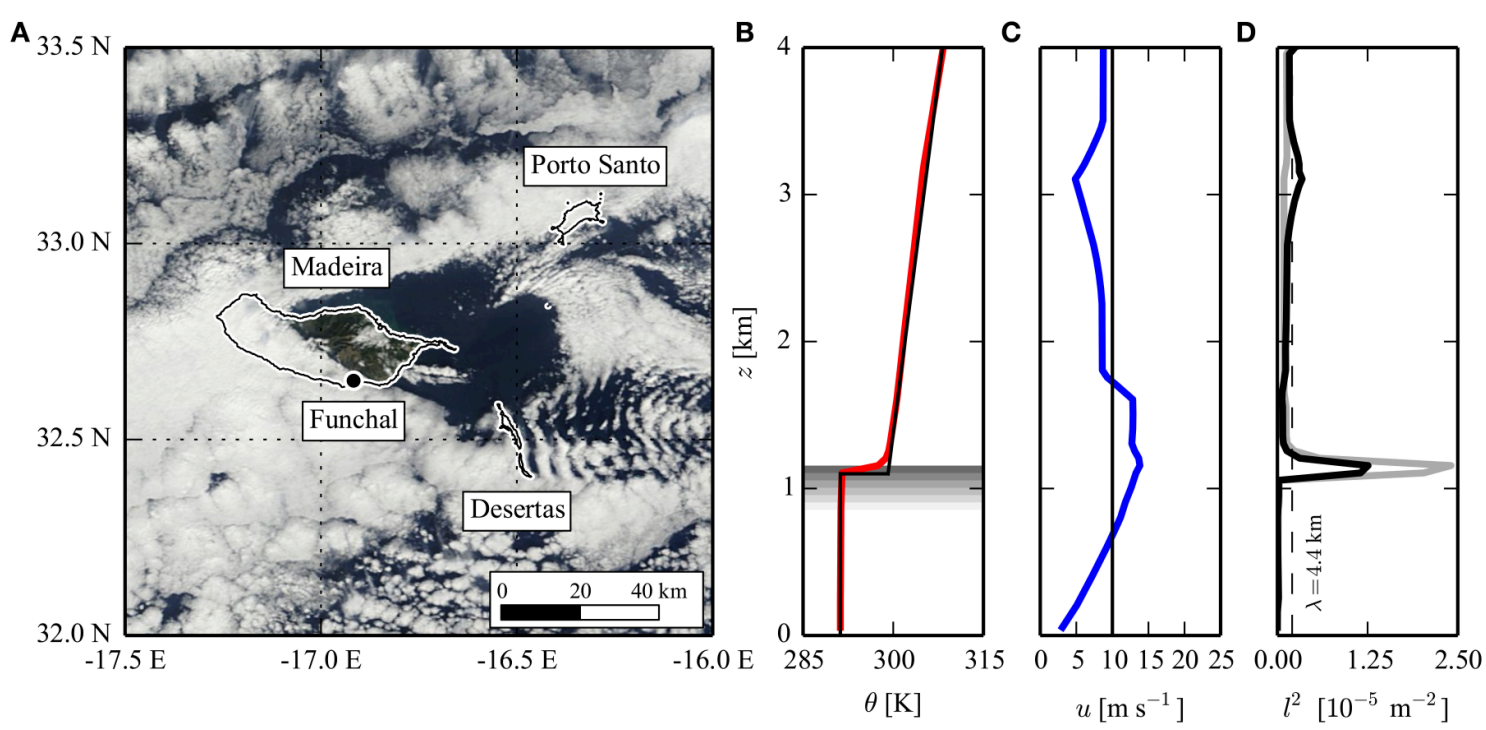

FIGURE 2 | (A) MODIS Terra visible satellite image on 24 December 2013, showing signature of trapped lee waves downwind of the Desertas islands in the Madeira archipelago. Coastlines are shown with solid white-black contours. The location of the sounding site (Funchal) is marked with a black dot. Flow is from left to right. Satellite image from NASA Earthdata and SRTM topography data from Jarvis et al. (2008). (B-D) Funchal operational sounding at 1200 UTC 24 December 2013. The panels show vertical profiles of $\mathbf{( B )}$ potential temperature, $(\mathbf{C})$ wind speed projected in lee wave direction and (D) Scorer parameter $I^{2} \approx[N / U(z)]^{2}(\mathrm{black}), I^{2} \approx[N / U]^{2}$ with $U=10 \mathrm{~m} \mathrm{~s}^{-1}$ (gray) and observed wavenumber $k^{2}$ (dashed). The solid black lines in (B,C) show the approximated idealized sounding that defines the parameters listed in Table 1. Gray shaded areas in (B) indicate regions where relative humidity $R H>90 \%$. 
TABLE 1 | Flow parameters determined from the sounding in Figure 2.

\begin{tabular}{ccccc}
\hline & FIW & IIW & Free IIW & \\
\hline$N_{1}$ & 0 & 0 & 0 & $\mathrm{~s}^{-1}$ \\
$N_{2}$ & 0.010 & 0 & 0.010 & $\mathrm{~s}^{-1}$ \\
$\Delta \theta$ & 8 & 8 & $\Delta \theta_{\text {crit }}=3.7$ & $\mathrm{~K}$ \\
$h_{1}$ & 1100 & 1100 & 1100 & $\mathrm{~m}$ \\
$U$ & 10 & 10 & 10 & $\mathrm{~m} \mathrm{~s}^{-1}$ \\
\hline
\end{tabular}

FIW (forced interface wave) represents the observed flow configuration, IIW (internal interface wave) assumes $N_{2}=0$ and Free IIW (free internal interface wave) assumes $\Delta \theta=\Delta \theta_{\text {crit. }}$

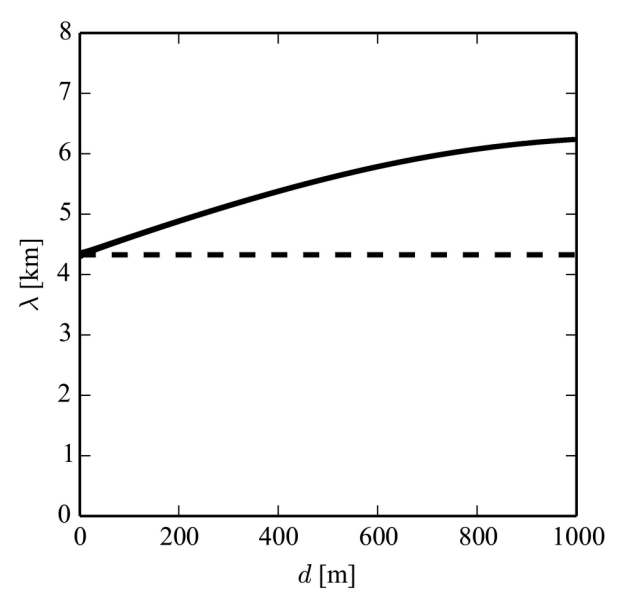

FIGURE 3 | Comparison of the wavelength $\lambda$ estimated from the FDR of forced interfacial waves (Equation 7, dashed) and from the Baines (1995) three-layer model (Equation 13, solid).

$\left(N_{1}, N_{2}, U, \Delta \theta, h_{1}\right)$ and the topography $h_{0}(x)$. The latter is specified with a cosine function, in analogy to Vosper (2004):

$h_{0}(x)= \begin{cases}H / 2+H / 2 \cdot \cos \left[\frac{\pi}{L}\left(x-x_{0}\right)\right] & \text { if } x_{0}-L<x<x_{0}+L \\ 0 & \text { elsewhere }\end{cases}$

Here, $H=300 \mathrm{~m}$ denotes the mountain height and $L=2$ $\mathrm{km}$ its half-width, while $x_{0}$ is the location of the mountain top. The model parameters $\left(N_{1}, N_{2}, U, \Delta \theta, h_{1}\right)$ are estimated from the idealized sounding in Figures 2B,C and are listed in Table $\mathbf{1 .}$

Figure 4 shows the wave field predicted by linear theory in two-layer flow over the mountain for two combinations of parameters, one reflecting the observations $\left(N_{2}=0.010 \mathrm{~s}^{-1}\right.$, Figures 4A,D) and the other disregarding stratification in the free atmosphere $\left(N_{2}=0\right.$, Figures $\left.4 \mathrm{~B}, \mathbf{E}\right)$. For completeness, in Figures $4 \mathbf{C}, \mathbf{F}$ we also show the wave field for the special case $\Delta \theta=\Delta \theta_{\text {crit }}$, i.e., when the interface acts as a free surface (Section 2.2).

Two wave branches are evident in the wave perturbations $u^{\prime}$ and $w^{\prime}$ in Figures 4A,D: a vertically propagating internal gravity wave and an interfacial wave. The mountain wave branch consists of those wave modes that can propagate into the continuously stratified upper layer $\left(k^{2}<l_{2}^{2}\right)$. The interfacial disturbance, in contrast, consists of a single stationary wave mode, described by Equation (7). The wavelength of this stationary mode ( $\lambda=$ $4.2 \mathrm{~km})$ is close to the observations $(\lambda=4.4 \mathrm{~km})$ in the satellite image in Figure 2A. Both $u^{\prime}$ and $w^{\prime}$ reach their maximum amplitude at the interface height. While $w^{\prime}$ is continuous across the interface, $u^{\prime}$ is discontinuous and changes sign as a consequence of the incompressibility constraint.

The wave structure in flow with a neutral upper layer in Figures $4 \mathrm{~B}, \mathrm{E}$ is different. As expected, the wave branch in the upper layer vanishes due to the absence of stratification in the free atmosphere. The wavelength of the disturbance $(\lambda=$ $5.0 \mathrm{~km}$ ), determined from Equation (2), increases slightly (by $19 \%)$ compared to that in Figure 4A. This suggests that freeatmospheric stratification does not impact the lee wavelength significantly for the case examined in this paper. This aspect is discussed in more detail in Section 5.

In the special case where $\Delta \theta=\Delta \theta_{\text {crit }}$, the wave field consists of a free interfacial disturbance with a wavelength of $\lambda=2 \pi / l_{2}$ (Figures 4C,F). In this case, the perturbations $u^{\prime}$ necessarily vanish right above the inversion, as discussed in Section 2.2. In fact, $u^{\prime}$ is confined entirely in the layer below the inversion in Figure 4F, similarly to what happens in water waves. The vertical wind speed $w^{\prime}$, in contrast to $u^{\prime}$, is continuous across the interface. In this case, $\lambda=6.3 \mathrm{~km}$.

Given the relatively good agreement between observations and linear model results in the Desertas lee-wave case, we use linear theory to explore the dependence of the lee wavelength $(\lambda=2 \pi / k)$ on the flow parameters $\left(\Delta \theta, h_{1}, N_{2}, U\right)$. We impose $N_{1}=0$, because convective boundary layers are typically neutrally stratified.

We proceed by solving the FDRs of internal interface waves (IIW, Equation 2) and of forced interface waves (FIW, Equation 7), varying systematically one of the four parameters mentioned above while keeping all others unchanged. We consider the IIW $\left(N_{2}=0\right)$ and FIW $\left(N_{2} \neq 0\right)$ flow models because the difference between their solutions allows us to appreciate the impact of free-atmospheric stratification on the lee wavelength. The lee wavelengths, $\lambda_{I I W}$ and $\lambda_{F I W}$, are obtained numerically, because the FDRs are transcendental and cannot be solved analytically.

The results of this elaboration are shown in the four panels of Figure 5, which consider $\Delta \theta \in[0,15] \mathrm{K}, h_{1} \in[0,5000] \mathrm{m}, N_{2} \in$ $[0,0.03] \mathrm{s}^{-1}$, and $U \in[0,15] \mathrm{m} \mathrm{s}^{-1} ; \lambda_{I I W}$ and $\lambda_{F I W}$ are shown in all panels as dashed and solid lines respectively. White dots in Figures $\mathbf{5 A}, \mathbf{B}$ mark the critical values $\Delta \theta_{\text {crit }}$ (Equation 8) and $h_{1 \text { crit }}$ (Equation 9). The behavior of $\lambda_{I I W}$ and $\lambda_{F I W}$ is generally similar: they increase with increasing wind speed $U$, while they decrease with increasing $\Delta \theta$ and $h_{1}$. While $\lambda_{F I W}$ decreases with $N_{2}, \lambda_{I I W}$ is inherently independent of it, because $N_{2}=0$ in the IIW model.

Since $N_{2}$ is the only parameter that differs between the IIW $\left(N_{2}=0\right)$ and the FIW model $\left(N_{2} \neq 0\right)$, large differences between $\lambda_{I I W}$ and $\lambda_{F I W}$ indicate that interfacial waves are heavily affected by the free-atmospheric stratification. This is the case for: weak inversions (Figure 5A, maximum deviation 38\%), high wind speeds (Figure 5D, maximum deviation 27\%) and for strong stratification (Figure 5C, maximum deviation 117\%). Conversely, $\lambda_{I I W}$ and $\lambda_{\text {FIW }}$ both become independent of the layer 

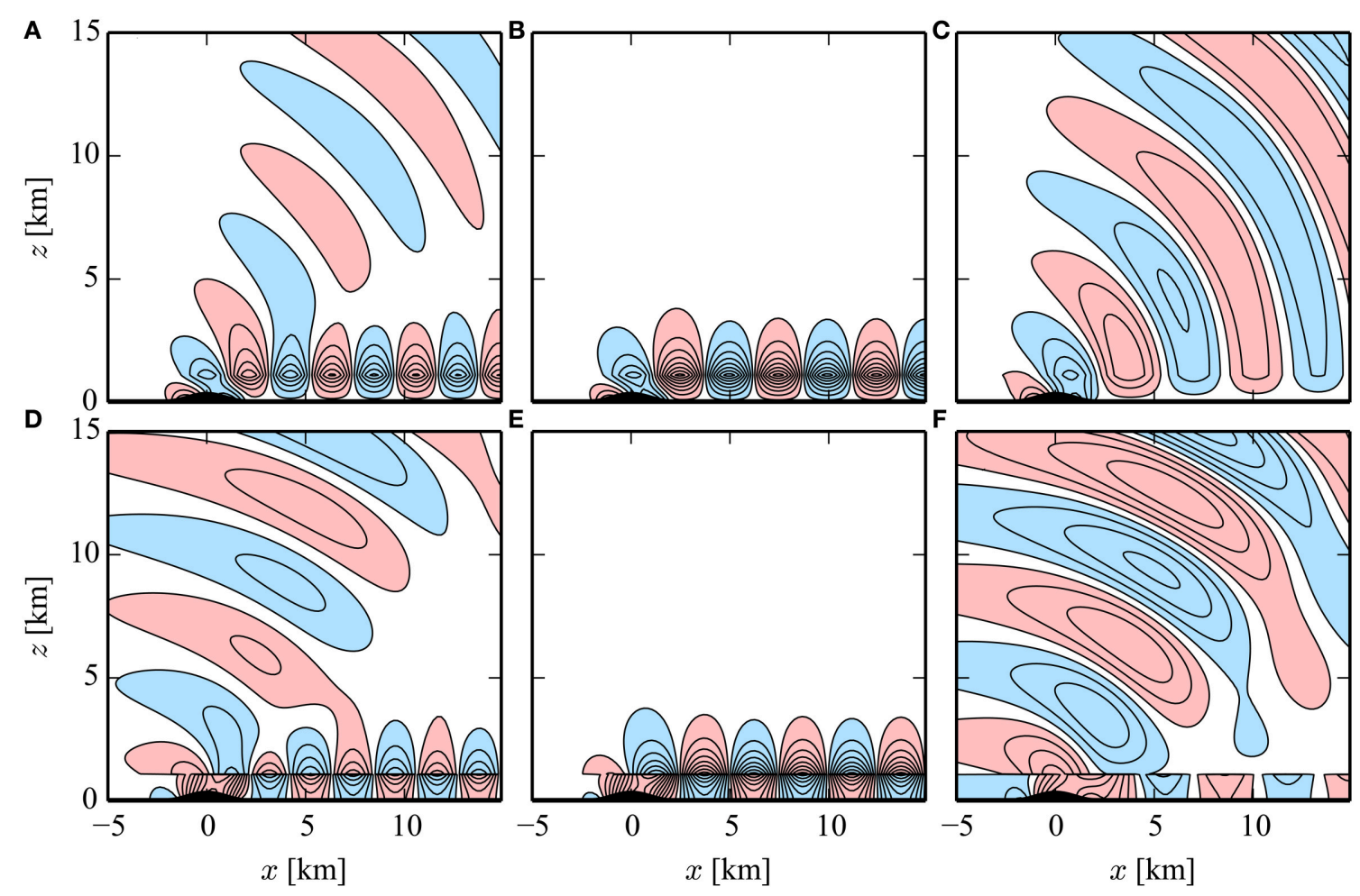

FIGURE 4 | Wave perturbations $\boldsymbol{w}_{\mathbf{1 , 2}}^{\prime}$ (top) and $\boldsymbol{u}_{\mathbf{1 , 2}}^{\prime}$ (bottom) determined from linear model solutions (Equations A2 and A3). Model parameters are listed in Table $\mathbf{1}$ and correspond respectively to: (A-D) forced interface waves; (B-E) internal interface waves; (C-F) free interface waves. Red and blue shading denote respectively positive and negative areas. The contour interval is $0.5 \mathrm{~m} \mathrm{~s}^{-1}$.

height $h_{1}$ for values beyond $h_{1}>1100 \mathrm{~m}$ (Figure 5B). This indicates that the short-wave (or deep-water) approximation is valid for the Desertas lee-wave case examined (the FDR is then independent of $h_{1}$, see Equation 4).

\section{DISCUSSION}

The results described in Section 4 suggest that stratification in the free atmosphere can indeed have a significant influence on the length of interfacial trapped lee waves. In Section 5.1 below, we determine this impact more precisely after simplifying the FDRs of the FIW and IIW models with the short-wave approximation.

Besides controlling lee wavelength, a continuously-stratified free atmosphere limits the range of possible trapped modes on the interface, because it allows the longest ones to propagate vertically through it (as shown in Section 2). This circumstance can impact the validity of the shallow-water approximation, which is inherently applicable only for long wave modes. We discuss this aspect in Section 5.2.

\subsection{Impact of Free-atmospheric Stratification on Lee Wavelength}

The results shown in Figure 5 suggest that differences between IIW and FIW predictions depend mostly on $g^{\prime}, N_{2}$, and $U$.
The relative difference between $\lambda_{I I W}$ and $\lambda_{F I W}$ cannot be derived analytically from the corresponding FDRs, Equations (2) and (7), because these equations are transcendental with respect to $k$. Therefore, we simplify them adopting the short-wave approximation $\operatorname{coth}\left(k h_{1}\right) \approx 1$ (when $k h_{1} \gg 1$ ) to obtain analytical expressions for the wave number $k$. Equations (2) and (7) simplify to:

$$
\begin{aligned}
& k_{I I W}=\frac{g^{\prime}}{2 U^{2}} \\
& k_{F I W}=\frac{g^{\prime}}{2 U^{2}}+\frac{N_{2}^{2}}{2 g^{\prime}} .
\end{aligned}
$$

Clearly, the second term on the right-hand side in Equation (15) represents the influence of free-atmospheric stratification on interfacial waves. The relative magnitude of the second term in Equation (15) can be expressed as:

$$
\sigma=\frac{k_{F I W}-k_{I I W}}{k_{I I W}}=\frac{N_{2}^{2}}{2 g^{\prime}} / \frac{g^{\prime}}{2 U^{2}}=\left(l_{2} / \frac{g^{\prime}}{U^{2}}\right)^{2}=\left(\frac{N_{2} U}{g^{\prime}}\right)^{2} .
$$

The parameter $\sigma$ is the ratio between the Scorer parameter in the free atmosphere, $l_{2}$, and the coefficient $g^{\prime} / U^{2}$, which quantifies the strength of the $\mathrm{BL}$ inversion and appears in the dynamic boundary condition imposed at that interface (see Appendix). According to Jiang (2014), $\sqrt{\sigma}$ represents the ratio 

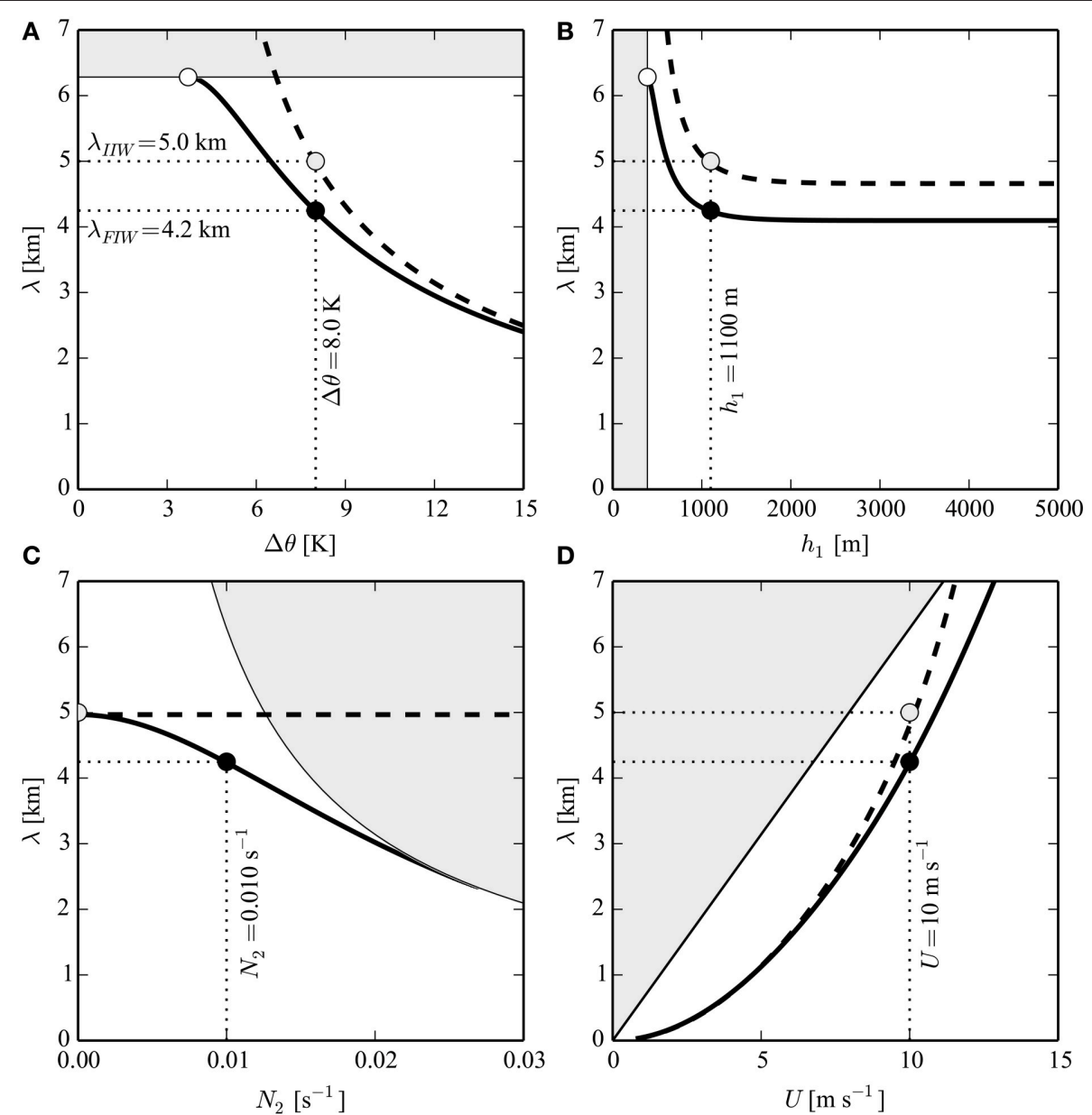

FIGURE 5 | Dependence of the lee wavelength $\lambda$ on: (A) inversion strength, $\Delta \theta$; (B) inversion height, $h_{1}$; (C) free-atmospheric stratification, $N_{2}$; and (D) wind speed, $\boldsymbol{U}$. Solid and dashed lines correspond respectively to $\lambda_{F I W}$ and $\lambda / / W$. Black dots represent the wavelength estimated from the observed flow profile (see Figure 2); gray dots indicate the corresponding wavelength when $N_{2}=0$; and white dots mark the critical values $\Delta \theta_{\text {crit }}$ and $h_{1}$ crit at the corresponding wavelength $\lambda=2 \pi U / N_{2}$. Shaded areas indicate the propagating regime in the upper layer, i.e., where wave trapping on the interface is not possible.

between the perturbation pressure associated with waves above the inversion (proportional to $N_{2} U$ ) and the density jump across the inversion (proportional to $g^{\prime}$ ). If $\sigma$ is small, inversion effects dominate over those introduced by the wave-permitting layer above, and interfacial waves are unaffected by stratification aloft. The passive layer assumption can then be applied if the flow is hydrostatic (Jiang, 2014). However, it generally does not hold for non-hydrostatic flow, even when $\sigma=0$. This is because interfacial trapped waves excite evanescent short-wavelength pressure perturbations $p_{2}^{\prime}$ into the upper layer, consequently $\partial p_{2}^{\prime} / \partial x \neq 0$ (Section 2.1). The dependence of $\sigma$ on $l_{2}$ and $g^{\prime} / U^{2}$ is shown in Figure 6: $\sigma$ increases with increasing $l_{2}$, but decreases with increasing $g^{\prime}$. This behavior is consistent with the curves representing the full FDRs in Figure 5.

The considerations expressed above are rigorously valid only if $k h_{1} \gg 1$. If this condition applies, then $\sigma$ (and hence the difference between $\lambda_{I I W}$ and $\left.\lambda_{F I W}\right)$ is independent of the layer depth $h_{1}$. However, this is not the case if the layer depth is relatively shallow (Figure 5B).
For the case in exam here, the layer depth can be shown to have only a marginal impact on the lee wavelength. With the deep-water approximation (that is, neglecting the influence of the lower layer depth), Equation (16) leads to $\sigma=0.14$. Without the deep-water approximation (that is, preserving the influence of the lower layer depth), $\sigma$ can be estimated on the basis of the relative difference between $\lambda_{I I W}$ and $\lambda_{F I W}$ from any panel in Figure 5: $\sigma=\left(\lambda_{I I W}-\lambda_{\text {FIW }}\right) / \lambda_{\text {FIW }} \approx 0.8 / 4.2=$ 0.19 . This means that the wavenumber of the interfacial wave increases due to stratification in the free atmosphere by $19 \%$ (white dot in Figure 6). The close agreement with the estimate from Equation (16) ( $\sigma=14 \%)$ indicates that the deep-water approximation is valid in this case.

Given the good agreement between wavelength estimates that are subject to and independent of the short-wavelength approximation, we expect that using $\sigma$ to assess the importance of stratification effects on interfacial waves beneath the stable layer will give accurate results for most interfacial lee wave observations. 


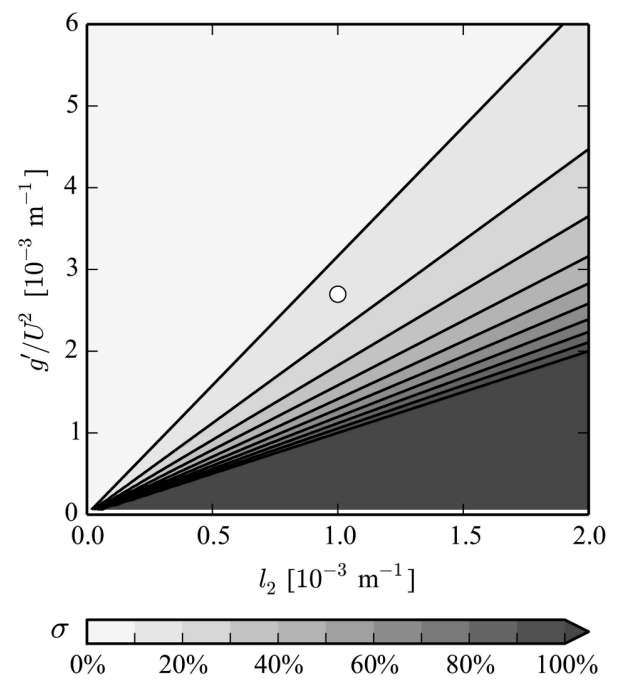

FIGURE 6 | Estimation of the stratification impact on interfacial deep-water waves. Gray shading represents the values of $\sigma=\left[I_{2} /\left(g^{\prime} / U^{2}\right)\right]^{2}$. The white dot shows the flow configuration of the observed lee wave case.

\subsection{Impact of Free-atmospheric Stratification on the Shallow-water Approximation}

Mountains excite a continuous spectrum of verticallypropagating wave modes if the atmosphere is continuously stratified. In two-layer flow, as shown above, part of these modes become trapped at the interface between the fluid layers. In particular, only relatively short modes satisfying $k>l_{2}$ are trapped. In what follows, we use this trapping criterion to investigate the validity of the long-wave approximation, which is a fundamental assumption in shallow-water theory, in the presence of a stably stratified upper layer.

Multiplying the terms in the inequality above by $h_{1}$ yields $k h_{1}>l_{2} h_{1}$. On the other hand, the long-wave (or shallow-water) approximation, $\operatorname{coth}\left(k h_{1}\right) \approx\left(k h_{1}\right)^{-1}$, is only valid when $k h_{1} \ll 1$. Scale analysis suggests that the two conditions $k h_{1}>l_{2} h_{1}$ (under which interfacial waves exist) and $k h_{1} \ll 1$ (under which they can be treated with the shallow-water approximation) can be incompatible.

Typical values for the order of magnitude of the Scorer parameter and the layer depth are respectively $\mathcal{O}\left(l_{2}\right)=10^{-3}$ and $\mathcal{O}\left(h_{1}\right)=10^{3}$, hence $\mathcal{O}\left(l_{2} h_{1}\right)=1$. In this case, the shallowwater approximation is clearly inappropriate. The range of values of $l_{2}$ and $h_{1}$ for which the approximation remains valid can be estimated from the ratio between the transcendental term in Equation (7), coth $\left(k h_{1}\right)$, and its shallow-water approximation form, $\left(k h_{1}\right)^{-1}$. This ratio is equal to unity if the approximation is accurate. The inaccuracy is hence quantified by the deviation of the ratio from unity, considering the longest possible trapped mode $\left(k=l_{2}\right)$ :

$$
\epsilon=1-\left(l_{2} h_{1}\right) \cdot \operatorname{coth}\left(l_{2} h_{1}\right)
$$

The dependence of $\epsilon$ on $l_{2}$ and $h_{1}$ is shown in Figure 7. Clearly, stratification aloft $\left(l_{2}\right)$ can cause large deviations from ideal

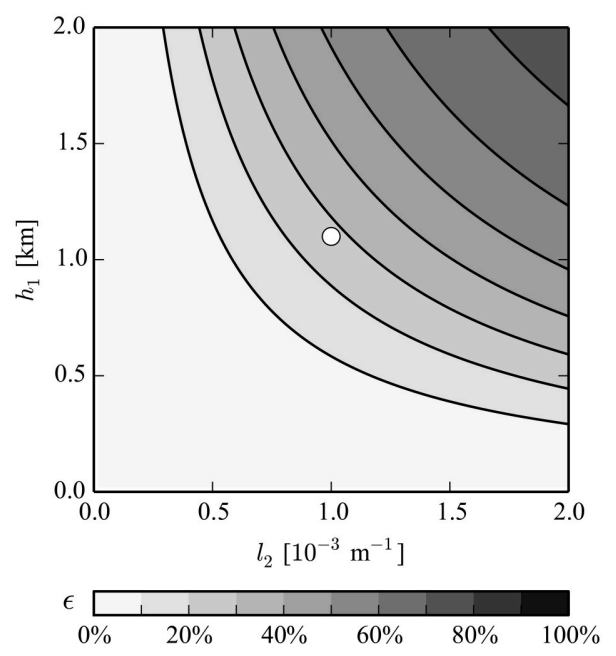

FIGURE 7 | Estimation of the error due to stratification effects in the shallow-water approximation. Gray shading and contours represent the values of $\epsilon=1-\left(I_{2} h_{1}\right) \cdot \operatorname{coth}\left(I_{2} h_{1}\right)$. The white dot shows the flow configuration of the observed lee wave case.

shallow-water behavior, easily exceeding $\epsilon=50 \%$ for fairly typical atmospheric conditions. For the Desertas case, $\epsilon \approx$ $30 \%$. We conclude that the non-hydrostatic linear frameworks presented in Section 2 are far more accurate than the shallowwater framework in describing the propagation properties of interfacial waves. This is especially the case, when the overlying free atmosphere is stably stratified.

\section{SUMMARY AND CONCLUSIONS}

Lee-wave cloud patterns, frequently observed downstream of islands, are often attributed to the presence of resonant gravity waves. Rigorously, resonant waves are expected to develop if the potential temperature profile is continuous and includes a stable layer below a neutral (or less stable) one. In the case of the marine boundary layer, one instead finds a nearly neutral layer capped by a sharp inversion, which can be thought of as a discontinuity in the potential temperature profile. Lee waves may be more appropriately interpreted as interfacial waves in this case.

Using the results of a linear two-layer flow model to explain the lee wavelength in observations taken in the Madeira archipelago, we highlight the large impact that free-atmospheric stability has on interfacial waves at the boundary-layer capping inversion. Through use of linear theory, we demonstrate that interfacial waves are affected by stable stratification aloft (i.e., by an overlying wave-permitting layer) in two ways:

- First, stratification in the upper layer limits the possible range of lee wavelengths. This happens because wave modes with $k^{2}<l_{2}^{2}$ propagate vertically in the upper layer, without being trapped at the interface (Vosper, 2004). From this criterion, we derived the critical (minimum) values of inversion strength $\left(\Delta \theta_{\text {crit }}\right.$, Equation 8) and height $\left(h_{1 \text { crit }}\right.$, Equation 9) for wave trapping. We showed that waves on an inversion where $\Delta \theta=$ $\Delta \theta_{\text {crit }}$, or equivalently $h_{1}=h_{1 \text { crit }}$, are dynamically identical to 
surface water waves (see Section 2.2), i.e., they are described by identical FDRs. This equivalence is only possible if the atmosphere above the inversion is stably stratified.

- Second, the wavelength of interfacial disturbances decreases with increasing stratification in the upper layer. In the short-wave limit $\left(k h_{1} \gg 1\right)$, the parameter $\sigma=\left[l_{2} /\left(g^{\prime} / U^{2}\right)\right]^{2}$ quantifies the relative difference between the length of interfacial waves in flow with a neutral and a stratified upper layer. If $\sigma$ is small, stratification in the free atmosphere has a negligible impact on interfacial waves riding along the inversion.

Furthermore, we show that the shallow-water (or long-wave) approximation $\left(k h_{1} \ll 1\right)$ may give an inaccurate description of interfacial waves in atmospheric flows when the upper layer is stably stratified. This is because inversions only trap short wave modes $\left(k^{2}>l_{2}^{2}\right)$. In fact, the two criteria $k^{2}>l_{2}^{2}$ and $k h_{1} \ll 1$ are generally incompatible for typical values of $h_{1}$ and $l_{2}$ in the atmosphere. Conversely, interfacial waves in such conditions are more properly described with the non-hydrostatic linear frameworks presented in Section 2. The deep-water approximation is more appropriate in this case.

\section{REFERENCES}

Airy, G. B. (1841). "Tides and waves," in Encyclopaedia Metropolitana (1817-1845), Mixed Sciences, Vol. 3, eds H. J. Rose, H. J. Rose, and E. Smedley (London: B Fellowes), 241-396.

Baines, P. G. (1995). Topographic Effects in Stratified Fluids. Cambridge: Cambridge University Press.

Craik, A. D. (2004). The origins of water wave theory. Annu. Rev. Fluid Mech. 36, 1-28. doi: 10.1146/annurev.fluid.36.050802. 122118

Doyle, J. D., Volkert, H., Dörnbrack, A., Hoinka, K. P., and Hogan, T. F. (2002). Aircraft measurements and numerical simulations of mountain waves over the central Alps: a pre-MAP test case. Q. J. R. Meteorol. Soc. 128, 2175-2184. doi: $10.1256 / 003590002320603601$

Durran, D. R. (1986a). Another look at downslope windstorms. Part I: the development of analogs to supercritical flow in an infinitely deep, continuously stratified fluid. J. Atmos. Sci. 43, 2527-2543. doi: 10.1175/15200469(1986)043<2527:ALADWP> 2.0.CO;2

Durran, D. R. (1986b). "Mountain waves," in Mesoscale Meteorology and Forecasting, ed P. S. Ray (Boston, MA: American Meteorological Society), 472-492.

Georgelin, M., and Lott, F. (2001). On the transfer of momentum by trapped lee waves: case of the IOP 3 of PYREX. J. Atmos. Sci. 58, 3563-3580. doi: 10.1175/1520-0469(2001)058<3563:OTTOMB $>$ 2.0.CO;2

Gill, A. E. (1982). Atmosphere-Ocean Dynamics. London: Academic Press.

Grubišić, V., Sachsperger, J., and Caldeira, R. M. (2015). Atmospheric wake of Madeira: first aerial observations and numerical simulations. J. Atmos. Sci. doi: 10.1175/JAS-D-14-0251.1. [Epub ahead of print].

Houze, R. A. (2014). Cloud Dynamics. Oxford: Academic Press.

Jarvis, A., Reuter, H. I., Nelson, A., and Guevara, E. (2008). Hole-filled SRTM for the Globe Version 4. Available from the CGIAR-CSI SRTM 90m Database. Available online at: http://srtm.csi.cgiar.org

Jiang, Q. (2014). Applicability of reduced-gravity shallow-water theory to atmospheric flow over topography. J. Atmos. Sci. 71, 1460-1479. doi: 10.1175/JAS-D-13-0101.1

Jiang, Q., and Smith, R. B. (2001). Ideal shocks in 2-layer flow. Part II: under a passive layer. Tellus A 53, 146-167. doi: 10.1034/j.1600-0870.2001. 00097.x
A major limitation of this study is that it is restricted to flows with constant background wind speed $U$. Vertical variability in the horizontal wind profile is known to affect wave propagation by creating wave ducts in regions with high curvature, $(1 / U)\left(d^{2} U / d z^{2}\right)$, which typically coincide with jets. Furthermore, sharp wind speed gradients, typically occurring across temperature inversions, favor the onset of shear instability (e.g., Kelvin-Helmholtz billows). We note here that the FDR of Kelvin-Helmholtz waves in a two-layer discontinuous density profile (see, e.g., Nappo, 2012) converges to that of internal interface waves (Equation 2), for vanishing wind speed difference across the interface. An accurate investigation of the impact of wind speed variability on interfacial waves is, however, outside the scope of this work, and is left for future investigation.

\section{ACKNOWLEDGMENTS}

We thank the reviewers of this paper for their comments and suggestions. This study was supported by the FWF (Austrian Science Fund) grant P24726-N27 to the University of Vienna. NCAR is sponsored by the National Science Foundation.

Klemp, J., and Lilly, D. (1975). The dynamics of wave-induced downslope winds. J. Atmos. Sci. 32, 320-339. doi: 10.1175/1520-0469(1975)032<0320:TDOWID> 2.0.CO;2

Nappo, C. J. (2012). An Introduction to Atmospheric Gravity Waves, 2 Edn. London: Academic Press.

Ralph, F. M., Neiman, P. J., Keller, T. L., Levinson, D., and Fedor, L. (1997). Observations, simulations, and analysis of nonstationary trapped lee waves. J. Atmos. Sci. 54, 1308-1333. doi: 10.1175/1520-0469(1997)054<1308: Osaaon $>2.0 . \mathrm{Co} ; 2$

Scorer, R. S. (1949). Theory of waves in the lee of mountains. Q. J. R. Meteorol. Soc. 75, 41-56. doi: 10.1002/qj.49707532308

Scorer, R. S. (1997). Dynamics of Meteorology and Climate. Chichester: Wiley.

Smith, R. B. (1979). The influence of mountains on the atmosphere. Adv. Geophys. $21,87-230$.

Teixeira, M. A. (2014). The physics of orographic gravity wave drag. Front. Phys. 2:43. doi: 10.3389/fphy.2014.00043

Teixeira, M. A., Argaín, J. L., and Miranda, P. M. (2013). Orographic drag associated with lee waves trapped at an inversion. J. Atmos. Sci. 70, 2930-2947. doi: 10.1175/JAS-D-12-0350.1

Turner, J. S. (1973). Buoyancy Effects in Fluids. Cambridge: Cambridge University Press.

Vosper, S. B. (2004). Inversion effects on mountain lee waves. Q. J. R. Meteorol. Soc. 130, 1723-1748. doi: 10.1256/qj.03.63

Vosper, S. B., Wells, H., Sinclair, A., and Sheridan, P. F. (2012). A climatology of lee waves over the UK derived from model forecasts. Meteorol. Appl. 20, 466-481. doi: $10.1002 /$ met.1311

Wurtele, M., Sharman, R., and Datta, A. (1996). Atmospheric lee waves. Annu. Rev. Fluid Mech. 28, 429-476.

Conflict of Interest Statement: The authors declare that the research was conducted in the absence of any commercial or financial relationships that could be construed as a potential conflict of interest.

Copyright (c) 2015 Sachsperger, Serafin and Grubišić. This is an open-access article distributed under the terms of the Creative Commons Attribution License (CC BY). The use, distribution or reproduction in other forums is permitted, provided the original author(s) or licensor are credited and that the original publication in this journal is cited, in accordance with accepted academic practice. No use, distribution or reproduction is permitted which does not comply with these terms. 


\section{APPENDIX}

\section{Frequency Dispersion Relationship for Trapped Lee Waves}

The Taylor-Goldstein equation can be derived from the two-dimensional Euler equations (subject to the Boussinesq approximation), the first law of thermodynamics and the incompressible mass continuity equation after linearization and by postulating the existence of wavelike solutions (see, e.g., Nappo, 2012):

$$
\frac{d^{2} \hat{w}}{d z^{2}}+\left[l^{2}-k^{2}\right] \hat{w}=0
$$

We solve this equation in two fluid layers by assuming that wave solutions are stationary in both the lower (subscript 1 ) and the upper one (subscript 2):

$$
\begin{aligned}
& \hat{w}_{1}=A_{1} e^{i m_{1} z^{\prime}}+B_{1} e^{-i m_{1} z^{\prime}} \\
& \hat{w}_{2}=A_{2} e^{i m_{2} z^{\prime}}+B_{2} e^{-i m_{2} z^{\prime}} .
\end{aligned}
$$

Here $\hat{w}=\left[\rho_{s} / \rho_{0}(z)\right]^{-1 / 2} \cdot \tilde{w}$, where $\tilde{w}$ denotes a Fourier component of the vertical wind speed perturbation, and $\rho_{s}$ and $\rho_{0}$ the surface density and the unperturbed density profile respectively; $z^{\prime}=z-h_{1}$, where $h_{1}$ is the thickness of the lower layer.

Four boundary conditions (BCs) are required to close the system. The first two are the parallel flow condition at the surface and a radiation condition in the upper layer. For simplicity, we assume flat terrain with constant interface height $\left(z^{\prime}=0\right)$, a realistic scenario for a lee wave that propagates above a flat surface after being excited by a mountain. The corresponding BCs become then

$$
\begin{aligned}
\hat{w}_{1}\left(z^{\prime}=-h_{1}\right) & =0 \\
B_{2} & =0 .
\end{aligned}
$$

The other two conditions are the kinematic and the dynamic BC at the interface $\left(z^{\prime}=0\right)$, which consists of a potential temperature jump with reduced gravity $g^{\prime}=g \cdot \Delta \theta / \theta_{0}$. The former condition ensures continuous vertical wind speed, while the latter requires continuous pressure across the $\theta$ discontinuity. Following Klemp and Lilly (1975) and Vosper (2004), the kinematic and dynamic BCs are respectively:

$$
\begin{aligned}
\hat{w}_{1} & =\hat{w}_{2} \\
\frac{d \hat{w}_{1}}{d z}-\hat{w}_{1} \frac{g^{\prime}}{U^{2}} & =\frac{d \hat{w}_{2}}{d z} .
\end{aligned}
$$

Imposing the constraints (A4)-(A7) on Equations (A2) and (A3) and solving for $A_{1}$ and $B_{1}$ leads to:

$$
\begin{aligned}
A_{1} & =\frac{A_{2}}{1-e^{-2 i m_{1} h_{1}}} \\
B_{1} & =\frac{A_{2}}{1-e^{2 i m_{1} h_{1}}} .
\end{aligned}
$$

Both coefficients depend on the amplitude $A_{2}$ of the interfacial disturbance. Substituting Equations (A5)-(A9) in Equations (A2) and (A3) and eliminating $A_{2}$ results finally in a condition that relates the wind speed with the vertical wave numbers $m_{1,2}$

$$
U^{2}=\frac{g^{\prime}}{i m_{1} \operatorname{coth}\left(i m_{1} h_{1}\right)-i m_{2}} .
$$

Equation (A10) is the frequency dispersion relationship that describes interfacial waves on an inversion between two continuously stratified fluid layers.

\section{Two-dimensional Trapped Lee Wave Model}

The two-dimensional wave fields in Figure 4 are obtained by determining the coefficients $A_{1}, A_{2}, B_{1}$ and $B_{2}$ in Equations (A2) and (A3) after imposing a parallel flow condition along topography. Equation (A4) is therefore replaced by:

$$
\hat{w}_{1}\left(z^{\prime}=-h_{1}\right)=U \frac{d H}{d x}=i \hat{h} k U e^{i k x},
$$

where $H$ is the height and $\hat{h}$ is the Fourier-series expansion of the terrain profile. Using again (A5)-(A7), and (A11) in Equations (A2) and (A3) yields:

$$
\begin{aligned}
& A_{1}=\hat{w}_{1\left(z^{\prime}=-h_{1}\right)} e^{i m_{1} h_{1}}-B_{1} e^{2 i m_{1} h_{1}} \\
& B_{1}=\hat{w}_{1\left(z^{\prime}=-h_{1}\right)} \frac{\alpha \cdot e^{i m_{1} h_{1}}}{\beta+\alpha \cdot e^{2 i m_{1} h_{1}}}
\end{aligned}
$$

where $\alpha=i\left(m_{1}-m_{2}\right)-\frac{g^{\prime}}{U^{2}}$ and $\beta=i\left(m_{1}+m_{2}\right)+\frac{g^{\prime}}{U^{2}}$. In the upper layer,

$$
\begin{aligned}
& A_{2}=\hat{w}_{1\left(z^{\prime}=-h_{1}\right)} \cdot e^{i m_{1} h_{1}}+B_{1} \cdot\left(1-e^{2 i m_{1} h_{1}}\right) \\
& B_{2}=0 \text {. }
\end{aligned}
$$

The full solution in a domain of $n$ horizontal grid points and a length $D=n \Delta x$ is computed independently at each level by summing up all Fourier components:

$$
w_{1,2}^{\prime}(x, z)=\left[\rho_{s} / \rho_{0}(z)\right]^{1 / 2} \cdot \operatorname{Re}\left[\frac{1}{n} \sum_{k=0}^{k=2 \pi / D} \hat{w}_{1,2}(x, z)\right] \text {. }
$$

Horizontal wind speed perturbations $u_{1,2}^{\prime}$ are determined in a similar fashion, using the incompressibility constraint $\hat{u}_{1,2}=$ $-\left(m_{1,2} / k\right) \cdot \hat{w}_{1,2}$.

Since the model described above is solved numerically in spectral space, the boundary conditions are necessarily periodic. This is a critical aspect for trapped lee wave solutions, which are asymmetric with respect to the mountain and do not decay with distance downstream of it. We solve this problem by including an artificial ghost-mountain in the domain, downstream of the first. The distance between the two mountains is set so as to achieve complete destructive interference of the lee wave train. The flow field becomes then symmetric. Figure 4 only shows the left half of the domain. 


\section{OPEN ACCESS}

Edited by:

Miguel A. C. Teixeira,

University of Reading, UK

Reviewed by:

Paulina Wong,

The University of Hong Kong,

Hong Kong

Qingfang Jiang,

United States Naval Research

Laboratory, USA

Vanda Grubisic

National Center for Atmospheric

Research, USA

*Correspondence:

Edward R. Johnson

e.johnson@ucl.ac.uk

Specialty section:

This article was submitted to

Atmospheric Science,

a section of the journal

Frontiers in Earth Science

Received: 31 August 2015

Accepted: 15 January 2016

Published: 09 February 2016

Citation:

Baines PG and Johnson ER (2016) Non-linear Topographic Effects in Two-Layer Flows.

Front. Earth Sci. 4:9.

doi: 10.3389/feart.2016.00009

\section{Non-linear Topographic Effects in Two-Layer Flows}

\author{
Peter G. Baines ${ }^{1}$ and Edward R. Johnson ${ }^{2 *}$ \\ ${ }^{1}$ Department of Infrastructure Engineering, The University of Melbourne, Melbourne, VIC, Australia, ${ }^{2}$ Department of \\ Mathematics, University College London, London, UK
}

We consider the nature of non-linear flow of a two-layer fluid with a rigid lid over a long obstacle, such that the flow may be assumed to be hydrostatic. Such flows can generate hydraulic jumps upstream, and the model uses a new model of internal hydraulic jumps, which results in corrections to flows that have been computed using earlier models of jumps that are now known to be incorrect. The model covers the whole range of ratios of the densities of the two fluids, and is not restricted to the Boussinesq limit. The results are presented in terms of flow types in various regions of a Froude number-obstacle height $\left(F_{0}-H_{m}\right)$ diagram, in which the Froude number $F_{0}$ is based on the initial flow conditions. When compared with single-layer flow, and some previous results with two layers, some surprising and novel patterns emerge on these diagrams. Specifically, in parts of the diagram where the flow may be supercritical $\left(F_{0}>1\right)$, there are regions where hysteresis may occur, implying that the flow may have two and sometimes three multiple flow states for the same conditions (i.e., values of $F_{0}$ and $H_{m}$ ).

\section{Keywords: 2-layer flow, non-Boussinesq, topography, Froude number, hydraulic jump}

\section{INTRODUCTION}

Some phenomena in the lower atmosphere may be approximately described by motion of a dense lower layer surmounted by a deep upper layer of approximately uniform density. The processes that occur in such flows can be described by the analysis of flows consisting of two layers of uniform density and velocity with a rigid upper surface. This constitutes the simplest form of density-stratified flow, and contains a number of phenomena that are prominent in more complex flows, such as hydraulic jumps. Here we report results of a study of two-layer stratified flow over isolated topography that incorporate some recent results on hydraulic jumps, namely improved (and justifiably correct) models of such jumps, for all density ratios. The Boussinesq approximation (namely, that the fluid density is assumed uniform except where multiplied by $g$ ) is not made here. This enables a better appreciation of the effects of density variation, particularly in hydraulic jumps. The presence of the upper boundary provides some simplifications, and while not being particularly relevant to the atmosphere, can be removed to an arbitrarily high level.

A general description of two-layer flows over topography is given in Chapter 3 (specifically, Section 3.6 of Baines, 1995, 1998), using models of hydraulic jumps that were prevalent at that time. The cases considered were those of two-layer flow with uniform velocity that commenced from a state of rest over an isolated, long obstacle, and concentrated on the "Boussinesq limit" where the density difference is very small. The resulting flows were described in terms of the parameters $r, F_{0}$, and $H_{m}$, where (generalizing to all densities)

$$
r=\frac{d_{10}}{D}, H_{m}=\frac{h_{m}}{d_{10}}, F_{0}=\frac{U}{c_{0}}, c_{0}^{2}=\frac{\Delta \rho g}{\frac{\rho_{1}}{d_{10}}+\frac{\rho_{2}}{d_{20}}},
$$


where $\rho_{1}$ and $\rho_{2}$ denote the lower and upper layer densities respectively, $\Delta \rho=\rho_{1}-\rho_{2}, U$ is the initial fluid velocity in the frame of the obstacle, $D$ the total depth, $g$ gravity, $d_{10}$ and $d_{20}=D-d_{10}$ the initial undisturbed lower and upper layer thicknesses, and $h_{m}$ is the height of the highest point of the obstacle. $c_{0}$ denotes the speed of long waves in two-layer fluid at rest. A definition sketch with velocities given in the frame of the topography is given in Figure 1. If the lower layer is thinner than the upper layer, the sudden onset of flow generates an hydraulic jump upstream, and the appropriate solutions for the flow depend on the dynamical properties of such jumps.

Section Hydraulic Jumps in Two-layer Flows gives a summary of the properties of such jumps based on recent analysis by Borden and Meiburg (2013) for Boussinesq flows, which have been generalized to all densities by the first author of this paper in Baines (2015). A study of jumps in continuously stratified fluids in the Boussinesq limit has been described by White and Helfrich (2014) using the Dubreil-Jacotin-Long equations, which provides an interesting contrast to the two-layer model employed here.

The basic equations for time-dependent non-linear two-layer flow are presented in Section Equations for Time-Dependent Non-linear Two-Layer Flow, and using the new model of hydraulic jumps, the methodology for computing the properties of flow over a single obstacle is presented in Section Two-Layer Flow over a Single Obstacle. The results are given in terms of $F_{0}-H_{m}$ diagrams in Section Results, and the conclusions are summarized in Section Conclusions.

Flows close to the Boussinesq limit are more relevant to applications to the atmosphere and ocean, where (potential) densities do not vary greatly, and these have been more closely studied in the past, but non-Boussinesq flows show some interesting differences. Flows where the lower layer is thinner than the upper are perhaps the most relevant to atmospheric and oceanic situations, and these are found to be the more complex in the non-Boussinesq case. The new hydraulic jump model may be extended to include mixing within (or downstream of) the jump, and hence also applied to flow over topography, but this has not been done here.

\section{HYDRAULIC JUMPS IN TWO-LAYER FLOWS}

This section summarizes the results given in Baines (2015). An hydraulic jump in two-layer fluids is here defined to be a

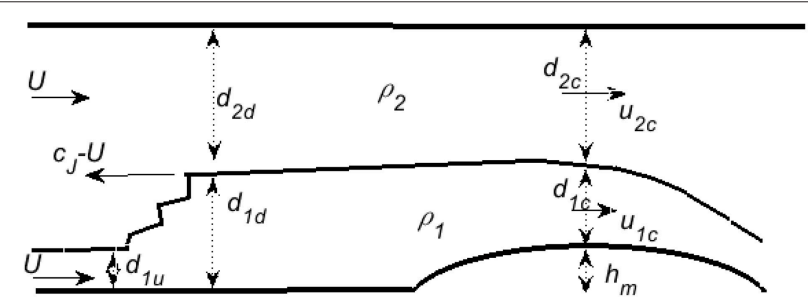

FIGURE 1 | A representative diagram showing the nature of the flows considered in this analysis. These are hydrostatic two-layer flows with a rigid lid, forced by flow over a long obstacle. See text for notation. flow structure that represents a transition between two steady uniform two-layer flow states (this may be extended to include an undular bore that leaves a downstream wavetrain, in which case the downstream flow state is taken to be the average layer thickness and velocities). A definition sketch for jumps is shown in Figure 2, which shows variables and velocities in the frame of the jump: subscripts 1 and 2 denote the lower and upper layers, subscripts " $u$ " and "d" denote upstream and downstream, and $u$ and $d$ with subscripts denote layer velocities and thicknesses (see Figure 1). $p_{s u}$ and $p_{s d}$ denote the pressure at the upper surface upstream and downstream respectively. Our objective is to obtain the flow properties downstream of the jump in terms of its amplitude, where the latter is measured by the increment in lower-layer thickness. We make the following assumptions: (1) the jump is steady in a reference frame moving with it; (2) the top and bottom surfaces are horizontal through the jump, with no surface stress, and (3) the layers maintain their identity through the jump, with negligible exchange of fluid between them. With these assumptions we have

$$
d_{1 u}+d_{2 u}=D=d_{1 d}+d_{2 d}, d_{i d} u_{i d}=d_{i u} u_{i u}, i=1,2 .
$$

and from assumption (2) we have that the momentum flux $S$ must be uniform (see Baines, 1995, 1998), so that

$$
\begin{aligned}
S & =\int_{0}^{D}\left(p+\rho u^{2}\right) \mathrm{d} z=\mathrm{constant} \\
& =p_{s u} D+\frac{1}{2} \Delta \rho g d_{1 u}^{2}+\rho_{1} u_{1 u}^{2} d_{1 u}+\rho_{2} u_{2 u}^{2} d_{2 u}+\frac{1}{2} \rho_{2} g D^{2}
\end{aligned}
$$

which is equal to the same expression on the downstream side. This equation introduces an additional variable, $p_{s u}-p_{s d}$, which needs to be determined by other considerations:

$$
\begin{aligned}
p_{s u}-p_{s d}= & \frac{\Delta \rho g}{2 D}\left(d_{1 d}^{2}-d_{1 u}^{2}\right)+\rho_{1}\left(u_{1 d}^{2} d_{1 d}-u_{1 u}^{2} d_{1 u}\right) / D \\
& +\rho_{2}\left(u_{2 d}^{2} d_{2 d}-u_{12}^{2} d_{2 u}\right) / D
\end{aligned}
$$

There have been essentially three previous attempts which have involved assumptions about the dynamics to give expressions for

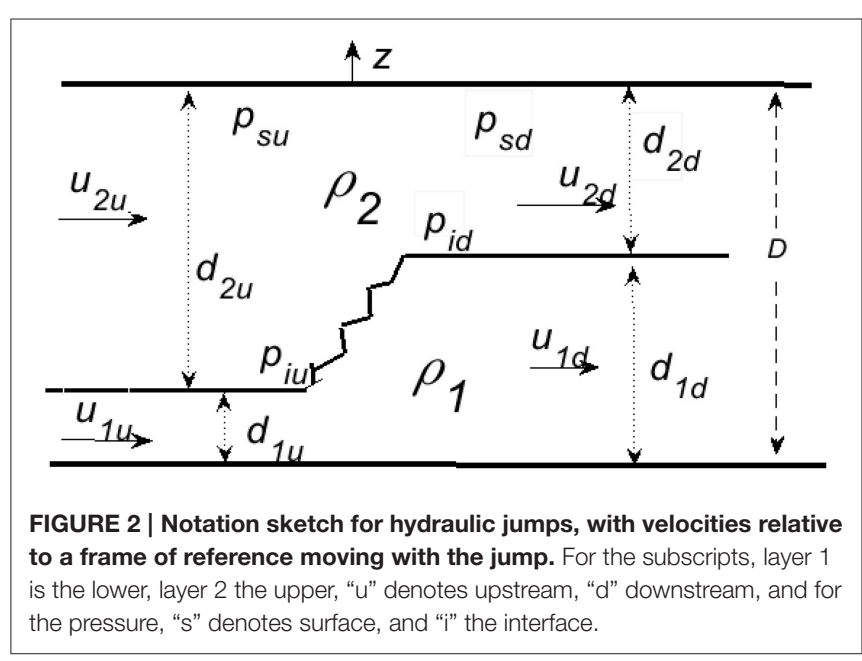


$p_{s u}-p_{\text {sd. }}$ The first was by Yih and Guha (1955) (the problem is 60 years old!), who assumed that the flow within the jump was hydrostatic, and that $p_{\mathrm{s}}$ varies linearly with $d_{2}$; the second was by Chu and Baddour (1977) and Wood and Simpson (1984), who assumed that all the energy dissipation in the jump was contained within the expanding (lower) layer, and the third was by Klemp et al. (1997), who assumed that all the energy dissipation was contained in the contracting (upper) layer. These assumptions gave different results, and with hindsight, none of them is correct, though as it turns out, the model of Klemp et al. is a good approximation in the Boussinesq limit. See Baines (2015) for details.

The novel approach from Borden and Meiburg (2013) was to consider a vorticity budget of the jump, and show, for the Boussinesq case, that the vorticity generation is independent of the pressure difference across the jump, and Equation (2.3) is not needed. In the non-Boussinesq case (Baines, 2015), the vorticity generated is determined by this pressure difference, and provides an expression for it. Specifically, if the vorticity equation (without friction) is written in the form

$$
\text { u. } \nabla \omega=-\nabla x\left(\frac{1}{\rho} \nabla p\right) \text {, }
$$

and integrated over the area $A$ of the jump, using the Gauss and Stokes theorems it reduces to

$$
\int \omega \mathbf{u}_{\bullet} d \mathbf{S}=-\int \frac{1}{\rho} \nabla p \cdot d \mathbf{l},
$$

where $\mathbf{S}$ denotes the normal vector to the boundary of $A$, and $\mathbf{1}$ denotes the vector along it, in the anti-clockwise sense. Since the density is uniform in each layer, and if $u_{1 u}=u_{2 u}$, this equation reduces to

$$
\begin{aligned}
\frac{1}{2}\left(u_{2 d}^{2}-u_{1 d}^{2}\right) & =\left[\frac{1}{\rho_{2}}-\frac{1}{\rho_{1}}\right]\left(p_{i u}-p_{i d}\right) \\
& =\frac{\Delta \rho}{\rho_{1}} \frac{p_{s u}-p_{s d}}{\rho_{2}}+\frac{\Delta \rho g}{\rho_{1}}\left(d_{1 d}-d_{1 u}\right)
\end{aligned}
$$

where $p_{\text {id }}$ and $p_{\text {iu }}$ denote the pressures at the downsteam and upstream interfaces respectively, which may be related hydrostatically to the surface pressures. This analysis obviates the necessity for the various different assumptions made or proposed by Yih and Guha (1955), Wood and Simpson (1984), Klemp et al. (1997) and Li and Cummins (1998).

From Equations (2.3) and (2.6) we may eliminate $p_{s u}-p_{s d}$, and obtain the expression for the speed $c_{J}$ of an hydraulic jump (see Figure 1) moving into fluid at rest:

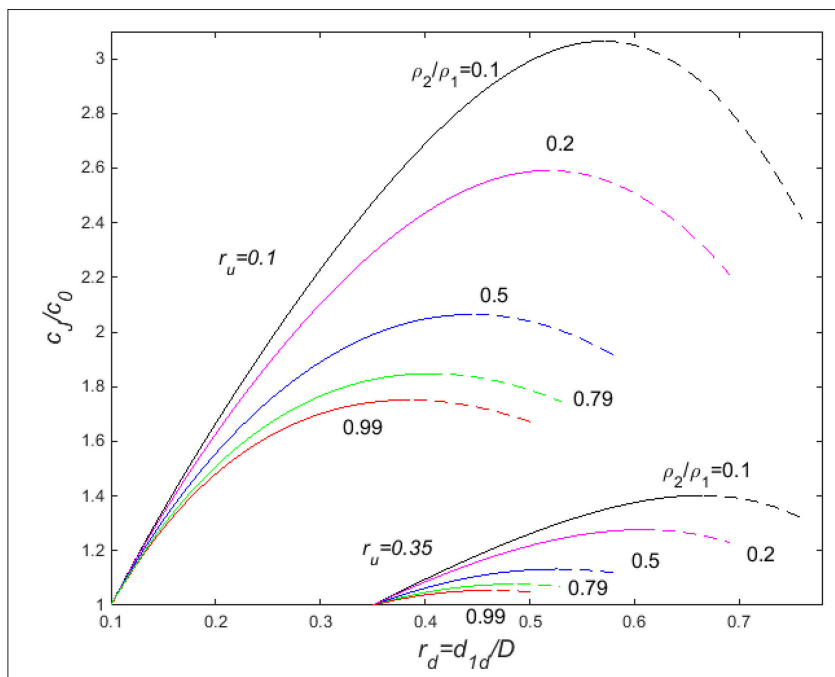

FIGURE 3 | Some representative jump speeds as a function of jump amplitude, moving into fluid at rest, for a range of density ratios $\left(\rho_{2} / \rho_{1}=0.1,0.2,0.5,0.79,0.99\right)$. The upstream lower layer thicknesses are given by $r_{u}=d_{1 u} / D=0.1$ and 0.35 . The dashed portions of the curves denote the regions where jump speed decreases with increasing amplitude. This makes them potentially unstable. Further, for these dashed curves, the flow is supercritical (relative to the jump) on the downstream side. This means that disturbances from downstream cannot propagate up to the jump and increase (or affect) it. In particular, jumps with amplitude greater than that of maximum speed cannot be generated from downstream, so that only the solid parts of the curves represent enduring jumps generated by flow over topography.

where $c_{0}$ is the linear wavespeed as in Equation (1.1) and $r_{u}=$ $d_{1 u} / D, r_{d}=d_{1 d} / D$. Borden and Meiburg (2013) termed their Boussinesq model for the jump speed the VS, or "Vortex Sheet" model, so that it is appropriate to term this model the FVS, or "Full Vortex Sheet" model.

Some representative plots for $c_{J} / c_{0}$ are shown in Figure 3, as a function of $r_{\mathrm{d}}$, for a range of density ratios. Note that all of these curves attain a maximum speed, but exist at larger amplitude, up to a maximum amplitude where the energy loss in each layer vanishes. For each curve, the maximum energy loss in each layer within the jump coincides with the point of maximum speed. Further, relative to the reference frame of the jump, the flow immediately downstream is subcritical for jump amplitudes $r_{\mathrm{d}}$ less than that of maximum speed (denoted $r_{\mathrm{dms}}$ ), but is supercritical for jumps with amplitudes greater than or equal to that maximum speed $\left(r_{\mathrm{d}} \geq r_{\mathrm{dms}}\right)$. This means that, on the dashed part of the curves, disturbances from downstream cannot propagate up to these jumps and affect them. Such jumps, if they did exist, would also be expected to be unstable, and degenerate to a jump of maximum speed. For these reasons, the jumps with $r_{\mathrm{d}} \geq r_{\mathrm{dms}}$ are shown dashed in Figure 3, and are not significant in the following analysis. More details are given in Baines (2015).

$$
\left(\frac{c_{J}}{c_{0}}\right)^{2}=\frac{r_{d}^{2}\left(1-r_{d}\right)^{2}\left[1-r_{u}\left(1-\frac{\rho_{2}}{\rho_{1}}\right)\right]\left[1+\left(\frac{\rho_{1}}{\rho_{2}}-1\right) \frac{\left(r_{u}+r_{d}\right)}{2}\right]}{r_{u}\left(1-r_{u}\right)\left[\frac{\left(r_{u}+r_{d}\right)}{2}-r_{u} r_{d}+\left(\frac{\rho_{1}}{\rho_{2}}-1\right) r_{u} r_{d}\left(1-r_{d}\right)^{2}-\left(1-\frac{\rho_{2}}{\rho_{1}}\right) r_{d}^{2}\left(1-r_{u}\right)\left(1-r_{d}\right)\right]},
$$


Observations of the speeds of hydraulic jumps in two-layer flows with water and kerosene $\left(\rho_{2} / \rho_{1}=0.79\right)$ forced by flow over (under) an obstacle were measured by Baines (1984), and at the time were compared with the model of Yih and Guha (1955). The agreement was somewhat disappointing. Comparisons between the same observations and jump speeds from the current model described above are shown in Figure 4, and the agreement is much more satisfying (see Baines, 2015 for a comparison with Yih and Guha). This lends support to the notion that this new model is indeed the correct formulation for two-layer hydraulic jumps.

\section{EQUATIONS FOR TIME-DEPENDENT NON-LINEAR TWO-LAYER FLOW}

We now consider two-layer flow over a long obstacle for which the horizontal length scale L and the time scale of the motion are sufficiently large for the equation for vertical motion to be hydrostatic, apart from the possible presence of hydraulic jumps. Under these conditions the equations of motion for the two layers may be written

$$
\begin{gathered}
\frac{\partial u_{1}}{\partial t}+\frac{\partial}{\partial x}\left[\frac{1}{2} u_{1}^{2}+g\left(h+d_{1}+\frac{\rho_{2}}{\rho_{1}} d_{2}\right)+\frac{p_{s}}{\rho_{1}}\right]=0, \\
\frac{\partial u_{2}}{\partial t}+\frac{\partial}{\partial x}\left[\frac{1}{2} u_{2}^{2}+g\left(h+d_{1}+d_{2}\right)+\frac{p_{s}}{\rho_{2}}\right]=0,
\end{gathered}
$$

where $u_{1}, u_{2}, d_{1}, d_{2}$ denote the velocities and thicknesses of layers 1 and 2, and $h(x)$ is the height of any bottom topography on the lower surface. If the upper layer has a rigid upper surface, and we assume that the total flow of fluid through the system is constant, we have, at all locations $x$,

$$
h(x)+d_{1}+d_{2}=D, d_{1} u_{1}+d_{2} u_{2}=Q,
$$

where $Q$ is the nett volume flux through the system, presumed constant after some initial starting time $t=t_{0}$. This system of equations governs the internal or baroclinic mode, and may be reduced to two equations for two convenient perturbation variables $v$ and $\eta$, defined by

$$
v=u_{1}-\frac{\rho_{2}}{\rho_{1}} u_{2}, d_{1}+h=d_{10}+\eta, d_{2}=d_{20}-\eta
$$

where $d_{10}$ and $d_{20}$ denote a reference state for the thicknesses $d_{1}$ and $d_{2}$. If the unknown surface pressure $p_{\mathrm{s}}$ is eliminated by subtraction, from the above we may obtain equations for $v$ and $\eta$ in the form where

$$
\delta=\frac{\Delta \rho}{\rho_{1}}\left(d_{10}-h+\eta\right) .
$$

These equations describe time-dependent motions in this twolayer system, which involve waves on the interface. There are two waves, propagating in opposite directions relative to the mean motion of the local fluid, and these waves may be described by the characteristic equations as follows (e.g., Whitham, 1974).

Equations (3.6-7) may be expressed as

$$
\frac{\partial \eta}{\partial t}+a_{11} \frac{\partial \eta}{\partial x}+a_{12} \frac{\partial v}{\partial x}=b_{1} \frac{\partial h}{\partial x}, \frac{\partial v}{\partial t}+a_{21} \frac{\partial \eta}{\partial x}+a_{22} \frac{\partial v}{\partial x}=b_{2} \frac{\partial h}{\partial x},
$$

where $a_{11}(\eta, v)$ etc. are given in the Appendix, and in the region where $h=0$, we may (trivially) write, for any arbitrary factors $l_{1}, l_{2}$,

$l_{1}\left(\frac{\partial \eta}{\partial t}+a_{11} \frac{\partial \eta}{\partial x}+a_{12} \frac{\partial v}{\partial x}\right)=0, l_{2}\left(\frac{\partial v}{\partial t}+a_{21} \frac{\partial \eta}{\partial x}+a_{22} \frac{\partial v}{\partial x}\right)=0$.

If we may write

$$
\begin{array}{r}
l_{1} a_{11}+l_{2} a_{21}=l_{1} c, l_{1} a_{12}+l_{2} a_{22}= \\
l_{2} c, \text { which requires } \\
\operatorname{det}\left[a_{i j}-c I\right]=0,(3.1
\end{array}
$$

for real values of $c$, adding the two equations in Equation (3.9) gives

$$
l_{1}\left(\frac{\partial \eta}{\partial t}+c \frac{\partial \eta}{\partial x}\right)+l_{2}\left(\frac{\partial v}{\partial t}+c \frac{\partial v}{\partial x}\right)=0, \text { i.e., } l_{1} \frac{\mathrm{d} \eta}{\mathrm{d} t}+l_{2} \frac{\mathrm{d} v}{\mathrm{~d} t}=0
$$

where

$$
\frac{\mathrm{d}}{\mathrm{d} t}(\eta, v)=\frac{\partial}{\partial t}(\eta, v)+c \frac{\partial}{\partial x}(\eta, v) .
$$

There are two solutions for $c$, denoted $c_{+}$and $c_{-}$, for waves propagating rightward (with the stream $c_{+}$), and leftward, against it $\left(c_{-}\right)$. It follows that the time-dependent motion at a given point is the sum of the leftward- and rightward-propagating motions with speeds given by Equation (3.10), which are

$$
c_{+}, c_{-}=\frac{1}{2}\left(a_{11}+a_{22}\right) \pm \frac{1}{2}\left[\left(a_{11}-a_{22}\right)^{2}+4 a_{21} a_{12}\right]^{1 / 2} \text {. }
$$

\section{TWO-LAYER FLOW OVER A SINGLE OBSTACLE}

We consider motions governed by the above equations, with the initial conditions:

$$
\begin{gathered}
\frac{\partial \eta}{\partial t}+\frac{\partial}{\partial x}\left[\frac{\left(d_{20}-\eta\right)\left(d_{10}-h+\eta\right) v+\frac{1}{2} Q\left(\eta-d_{20}+\frac{\rho_{2}}{\rho_{1}}\left(d_{10}-h+\eta\right)\right)}{D-h-\delta}\right]=0, \\
\frac{\partial v}{\partial t}+\frac{\partial}{\partial x}\left[\frac{\Delta \rho g \eta}{\rho_{1}}+\frac{\left[\left(d_{20}-\eta\right)^{2}-\frac{\rho_{2}}{\rho_{1}}\left(d_{10}-h+\eta\right)^{2}\right] v^{2}+\frac{\rho_{2}}{\rho_{1}}\left[2 Q v(D-h)-\left(1-\frac{\rho_{2}}{\rho_{1}}\right) Q^{2}\right]}{2(D-h-\delta)^{2}}\right]=0,
\end{gathered}
$$




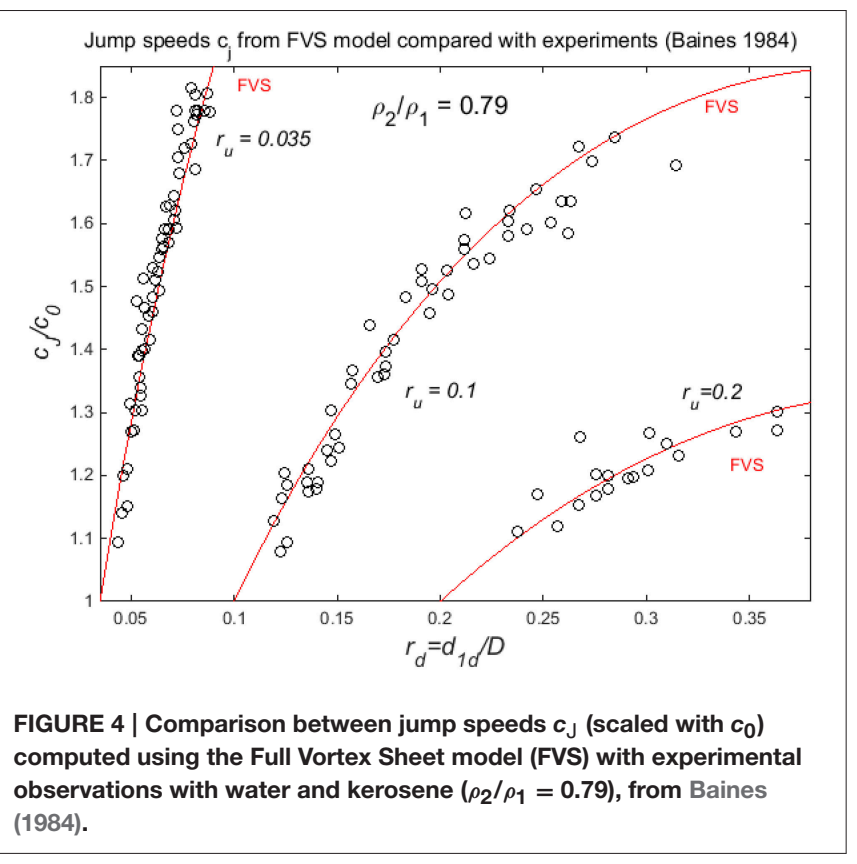

$u_{1}=u_{2}=U, d_{1}=d_{10}+h(x), d_{2}=d_{20}-h(x), a t t=0$,

after which $U$ and $h(x)$ remain constant. This corresponds to the sudden introduction of the obstacle into a uniform twolayer stream (the result is similar to that for the sudden onset of a uniform two-layer stream over a stationary obstacle). Our objective is to describe the properties of the resulting flow in terms of the parameters given in Equation (1.1).

For a sufficiently small obstacle, the commencement of conditions Equation (4.1) causes two transient disturbances, each having the form of the topography. One of these propagates against the flow, and the other with it, as described in Section Equations for Time-Dependent Non-linear Two-layer Flow, leaving a locally steady solution in the vicinity of the obstacle. This steady solution is given by steady forms of Equations (3.5) and (3.6), where the square-bracket terms are equal to their values where $h, \eta$, and $v=0$. They may also be manipulated to give the "hydraulic alternative," namely that

$$
\frac{\mathrm{d}}{\mathrm{d} x}(\eta, v)=0, \text { or } c_{-}=0, \text { where } \frac{\mathrm{d} h}{\mathrm{~d} x}=0 .
$$

If $c_{-}<0$ the flow is subcritical, and if both $c_{+}, c_{-}>0$ the flow is supercritical, and the steady solution applies provided $c_{-} \neq 0$, which implies $F_{0} \neq 1$.

For subcritical flows where $r_{u}=d_{10} / D$ is small, as $H_{m}$ is increased the steady solution is valid until a point is reached at which $c_{-}=0$ at the peak of the obstacle, i.e.,

$$
c_{-}=\frac{1}{2}\left(a_{11}+a_{22}\right)-\frac{1}{2}\left[\left(a_{11}-a_{22}\right)^{2}+4 a_{21} a_{12}\right]^{1 / 2},
$$

where $a_{i j}$ are given in the Appendix with

$$
d_{1}=d_{11}, d_{2}=d_{21}, \eta=\eta_{c}, v=v_{c}, h=h_{m},
$$

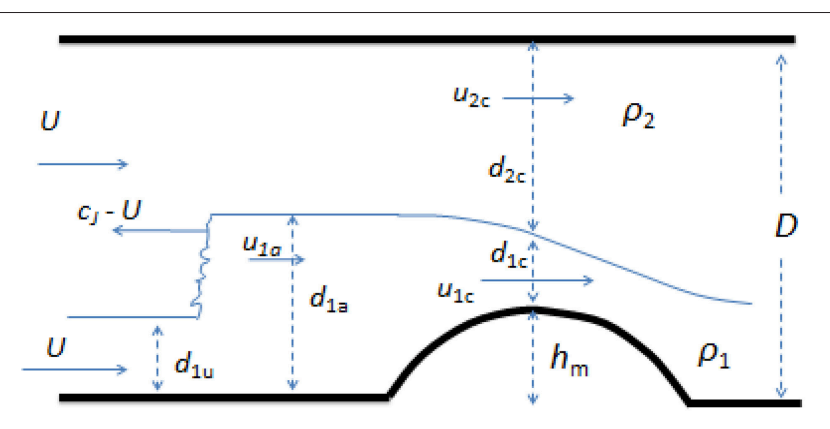

FIGURE 5 | Definition sketch for notation for flow over an obstacle with an upstream jump. All velocities are relative to a frame of reference at rest with the obstacle. Here the velocities and thickness immediately downstream of the jump (section "a") are the same as those immediately upstream of the obstacle.

$\eta_{c}$ and $v_{c}$ denoting the values of $\eta$ and $v$ at the critical section at the peak of the obstacle.

For larger heights the steady solution is not valid, and the fluid responds by sending a disturbance upstream, which effectively alters the upstream flow conditions. The flow over the obstacle is then no longer symmetric about the highest point, and for the resulting steady flow, the fluid must adjust so that the second alternative $-c_{-}=0$ applies there. Further increases in the obstacle height beyond this point cause yet faster disturbances to propagate upstream, with the result that the flow forms upstream hydraulic jumps. When this happens, it is necessary to take into account the properties of hydraulic jumps, and assuming steady flow over the obstacle, to link this to the condition $c_{-}=0$ at the obstacle crest. The appropriate variables and notation are shown in Figure 5. The new (correct) formulation for hydraulic jumps described in Section Hydraulic Jumps in Two-layer Flows above is used here. The simplest procedure is to assume a given upstream jump height, and then take the conditions downstream of the jump as input to flow over the topography, where the critical condition at the obstacle crest together with the steady-state forms of Equations (3.5) and (3.6) determines the obstacle height. From this one may construct regions of the $F_{0}-H_{m}$ diagram. For subcritical flows with small $r_{u}$, the above phenomena are sufficient to describe the upstream flow properties up to the point where the lower layer becomes blocked by the topography, and is locally absent on the downstream side.

The above procedure is essentially the same as that for singlelayer flows over obstacles (e.g., Baines, 1995, 1998, Section 2.3). But for larger values of $r_{u}$, and supercritical flows, increasing $H_{m}$ may cause the upstream jump to reach its maximum speed. At this point, the flow immediately downstream of the upstream jump becomes supercritical, as described in Section Hydraulic Jumps in Two-layer Flows. This means that, although larger-amplitude jumps are theoretically possible, they cannot be built up by disturbances approaching it from the downstream side, and hence the flow must respond in some other way. Increasing $H_{m}$ causes more upstream disturbances that increase the lower layer thickness and progressively travel 


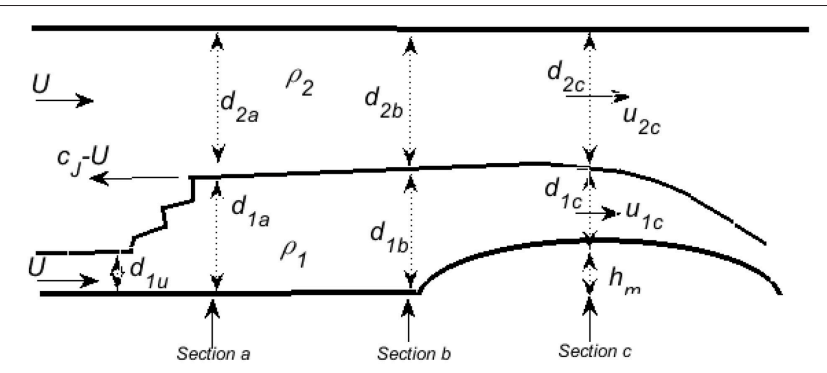

FIGURE 6 | The same as Figure 5, except for larger obstacle heights where the flow contains a rarefaction. Here the jump has the maximum speed, and section "b" immediately upstream of the obstacle has larger lower-layer thickness than section "a," immediately downstream of the jump.

more slowly, so that the resulting upstream disturbance becomes more spread out as it propagates, rather than steepening to form an hydraulic jump. Such a disturbance is termed a rarefaction, because the disturbance itself is becoming more rarefied with time (see Chapter 3 of Baines, 1995, 1998). The resulting upstream motions are governed by Equations (3.8-12), and the flow has the form shown in Figure 6. The flow eventually reaches a quasi-steady form, with the distance between the jump and the obstacle progressively increasing, but with a dynamical link between them manifested in the interfacial waves. The only way the jump can influence the flow downstream is via the rightward-propagating waves (with speed $c_{+}$).

The rightward propagating waves commence with the conditions at section $a$ of Figure 6, immediately downstream of the jump. With $c=c_{+}$, Equation (3.11) provides the relation

$$
\frac{\mathrm{d} v}{\mathrm{~d} \eta}=-\frac{l_{1}}{l_{2}}=-\frac{a_{11}-a_{22} \pm\left[\left(a_{11}-a_{22}\right)^{2}+4 a_{21} a_{12}\right]^{1 / 2}}{2 a_{12}}
$$

where the plus sign is taken. This gives a relation between $v$ and $\eta$ for these downstream propagating waves. The expressions for $a_{11}(\eta, v)$ etc. are those given in the Appendix, with $d_{1}=d_{11}$, $d_{2}=d_{21}$, and the initial conditions of section $a$ :

$$
\eta=0, v=u_{11}-\frac{\rho_{2}}{\rho_{1}} u_{21}
$$

where $u_{11}, u_{21}$ are the velocities at Section Introduction in the frame of the obstacle, and are determined by the properties of the jump. Integrating Equation (4.3) then gives a relation between $v$ and $\eta$ that applies up to section $b$ of Figure 6, immediately upstream of the obstacle.

The jump equations give the conditions ( $v$ and $\eta$ ) downstream of the jump at section $a$, and Equation (4.3) links these conditions with those upstream of the obstacle at section $b$. The steadystate forms of Equations (3.5) and (3.6) and the critical condition at the obstacle crest then give the obstacle height, as before. For clarity, we here describe this process in a little more detail.
In general, Equation (4.3) must be integrated numerically using the expressions in the Appendix, where $d_{1}, d_{2}$ denote the layer thicknesses immediately downstream of the jump. In the notation of Figure 5, the initial conditions at section $a$ are therefore

$$
d_{1}=d_{1 a}, d_{2}=d_{2 a}, \eta_{a}=0, v_{a}=u_{1 a}-\frac{\rho_{2}}{\rho_{1}} u_{2 a}
$$

and Equation (4.5) gives a relationship between $v$ and $\eta$. It should be noted that Section Introduction is not at a fixed location, but the velocities in Equation (4.7) are relative to the reference frame of the obstacle, as in Figure 5.

In the Boussinesq limit, the $a_{i j}$ take the form

$$
\begin{aligned}
& a_{11}=\frac{v\left(d_{2}-d_{1}+h-2 \eta\right)+Q}{D-h}, \\
& a_{12}=\frac{\left(d_{2}-\eta\right)\left(d_{1}-h+\eta\right)}{D-h}, \\
& a_{21}=\frac{\Delta \rho g}{\rho_{1}}-\frac{v^{2}}{D-h}, a_{22}=a_{11},
\end{aligned}
$$

so that Equation (4.5) can be expressed as

$$
\begin{array}{r}
\frac{\mathrm{d} v}{\mathrm{~d} \eta}=-\left(\frac{a_{21}}{a_{12}}\right)^{1 / 2}=-\left(\frac{g^{\prime} D-v^{2}}{\left(d_{2 a}-\eta\right)\left(d_{1 a}+\eta\right)}\right)^{1 / 2} \\
\text { where } g^{\prime}=\frac{\Delta \rho g}{\rho_{0}}
\end{array}
$$

Equation (4.8) can then be integrated directly to give the "Riemann invariant"

$$
\begin{aligned}
& \arcsin \left(\frac{2 \eta+d_{1 a}-d_{2 a}}{D}\right)+\arcsin \left(\frac{v}{\left(g^{\prime} D\right)^{1 / 2}}\right) \\
& =\arcsin \left(\frac{d_{1 a}-d_{2 a}}{D}\right)+\arcsin \left(\frac{u_{1 a}-u_{2 a}}{\left(g^{\prime} D\right)^{1 / 2}}\right) .
\end{aligned}
$$

This gives a relationship between $v$ and $\eta$ that applies immediately upstream of the topography (denoted section $b$ in Figure 5, with variables $\left.d_{1 b}, d_{2 b}, u_{1 b}, u_{2 b}, \eta_{b}, v_{b}\right)$. The flow over the obstacle may be assumed to be steady after sufficient time has elapsed. We may therefore use the steady-state versions of Equations (3.5) and (3.6) to link the flow immediately upstream of the obstacle to the flow at the obstacle crest (denoted section $c$ in Figure 5), which for the general case give. 


$$
\begin{array}{r}
\frac{\left(d_{2 a}-\eta_{b}\right)\left(d_{1 a}+\eta_{b}\right) v_{b}+\frac{1}{2} Q\left(\eta_{b}-d_{2 a}+\frac{\rho_{2}}{\rho_{1}}\left(d_{1 a}+\eta_{b}\right)\right)}{D-\delta_{b}}= \\
\frac{\left(d_{2 a}-\eta_{c}\right)\left(d_{1 a}-h_{m}+\eta_{c}\right) v_{c}+\frac{1}{2} Q\left(\eta_{c}-d_{2 a}+\frac{\rho_{2}}{\rho_{1}}\left(d_{1 a}-h_{m}+\eta_{c}\right)\right)}{D-h_{m}-\delta_{c}}, \\
g^{\prime} \eta_{b}+\frac{\left[\left(d_{2 a}-\eta_{b}\right)^{2}-\frac{\rho_{2}}{\rho_{1}}\left(d_{1 a}+\eta_{b}\right)^{2}\right] v_{b}^{2}+\frac{\rho_{2}}{\rho_{1}}\left[2 Q v_{b} D-\left(1-\frac{\rho_{2}}{\rho_{1}}\right) Q^{2}\right]}{2\left(D-\delta_{b}\right)^{2}}= \\
g^{\prime} \eta_{c}+\frac{\left[\left(d_{2 a}-\eta_{c}\right)^{2}-\frac{\rho_{2}}{\rho_{1}}\left(d_{1 a}-h_{m}+\eta_{c}\right)^{2}\right] v_{c}^{2}+\frac{\rho_{2}}{\rho_{1}}\left[2 Q v_{c}\left(D-h_{m}\right)-\left(1-\frac{\rho_{2}}{\rho_{1}}\right) Q^{2}\right]}{2\left(D-h_{m}-\delta_{c}\right)^{2}},
\end{array}
$$

where $\delta_{b}=\frac{\Delta \rho}{\rho_{1}}\left(d_{1 a}+\eta_{b}\right), \delta_{c}=\frac{\Delta \rho}{\rho_{1}}\left(d_{1 a}-h_{m}+\eta_{c}\right)$.

The other remaining equation is the critical condition at the obstacle crest, as in Equation (4.3), which takes the form

$$
\begin{aligned}
& c_{-}=\frac{1}{2}\left(a_{11}\left(\eta_{c}, v_{c}, h_{m}\right)+a_{22}\left(\eta_{c}, v_{c}, h_{m}\right)\right) \\
& -\frac{1}{2}\left[\left(a_{11}\left(\eta_{c}, v_{c}, h_{m}\right)-a_{22}\left(\eta_{c}, v_{c}, h_{m}\right)\right)^{2}\right. \\
& \left.+4 a_{21}\left(\eta_{c}, v_{c}, h_{m}\right) a_{12}\left(\eta_{c}, v_{c}, h_{m}\right)\right]^{1 / 2}=0
\end{aligned}
$$

using the expressions for $a_{i j}$ in the Appendix, with $d_{1}=d_{1 a}$, $d_{2}=d_{2 a}$. We therefore have four equations for the four variables $\eta_{b}, v_{b}, \eta_{c}, v_{c}$, for an upstream jump of given amplitude and hence known values of $d_{1 a}, d_{2 a}, u_{1 a}, u_{2 a}, v_{a}$, (with $\eta_{a}=0$ ) which enables us to determine the appropriate value of $h_{m}$. Hence one may construct diagrams of flow properties in the $F_{0}-H_{m}$ plane, for various values of the density ratio $\rho_{2} / \rho_{1}$. For the situations analyzed for this paper (shown in Figures 9, 10), the regions in $F_{0}-H_{m}$ space where rarefactions occur are small.

\section{RESULTS}

With the above analysis, we may determine the nature of the two-layer flow over an obstacle, and its variation with the relevant parameters. We are concerned with flows that are (or are equivalent to being) started from a state of rest, and after a short starting period are maintained at constant conditions. This includes, for example, an obstacle that is towed through stationary fluid, or a stationary obstacle situated in a two-layer stream where the latter is suddenly forced into uniform motion.

An effective way of getting an overall view of the types of flow that may occur is through the $F_{0}-H_{m}$ diagram. Here, given uniform initial flow velocity, we have two additional parameters: $r_{u}=d_{10} / \mathrm{D}$, the initial relative thickness of the lower layer, and $\rho_{2} / \rho_{1}$, the ratio of the densities of the fluids in the two layers. We present some representative examples of such diagrams which indicate some surprising and novel features of the flow of two fluids. This includes the phenomenon of "hysteresis," or multiple steady flow states, in parameter ranges that include the state of fully supercritical flow over the obstacle.
As a point of reference and subsequent comparison, Figure 7 shows the $F_{0}-H_{m}$ diagram for a single homogeneous fluid layer (from Baines and Davies, 1980; Baines, 1995, 1998). This shows parameter ranges where the ultimate steady flow is wholly subcritical or supercritical over the obstacle, where the fluid is blocked by the topography, and where the flow over the obstacle forces an upstream jump that alters the approaching flow, which is governed by a critical condition at the crest of the obstacle. There is also a parameter range within which a hysteresis phenomenon may occur. Here the flow over the obstacle may be entirely supercritical, or partially blocked (with an upstream hydraulic jump) with critical flow at the crest: subcritical on the upstream side, and supercritical on the downstream side. Which of these two states occurs in practice will depend on how the flow is set up. One may then make the transition from one state to the other by slowly varying the basic flow parameters $F_{0}$ and $H_{m}$ in a quasi-static manner-hence the term "hysteresis."

One may expect similar types of behavior to occur for two-layer flow over topography. Some examples have been

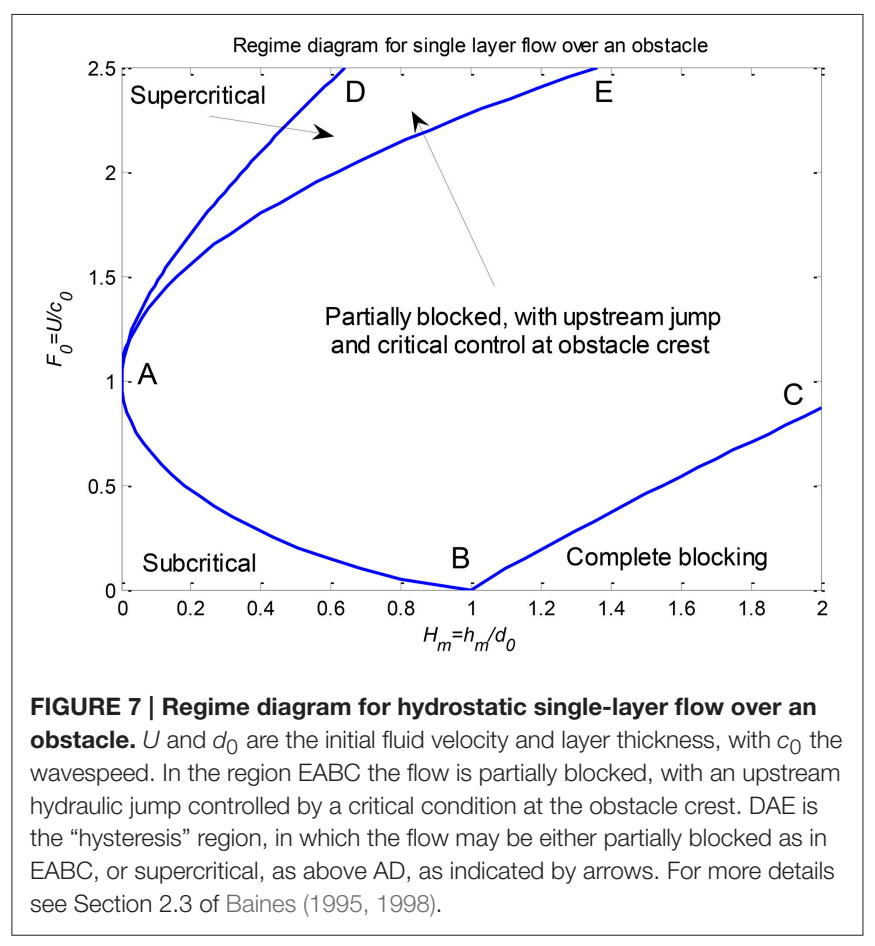




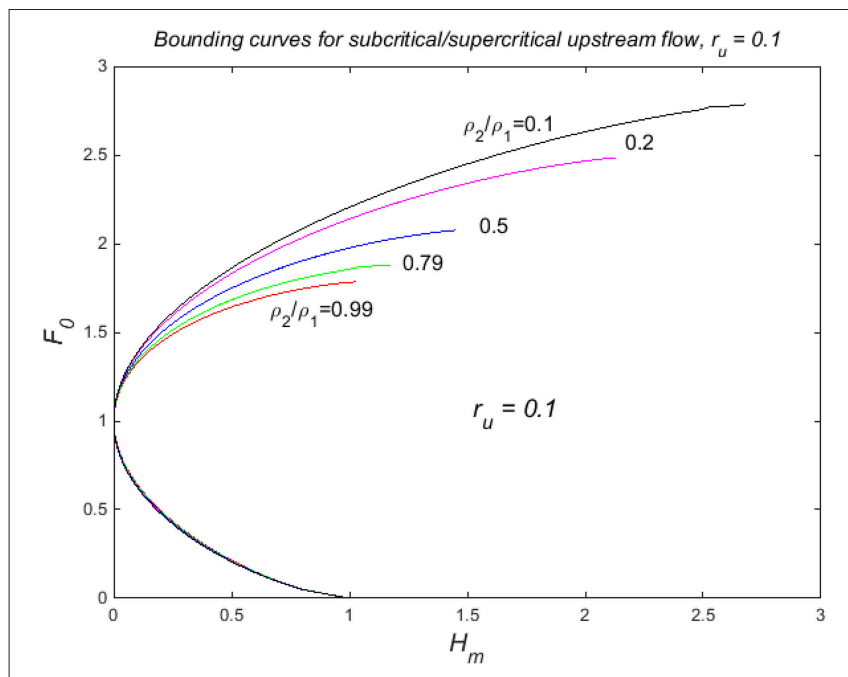

FIGURE 8 | Curves for the onset of critical flow at the obstacle crest as $\boldsymbol{H}_{\boldsymbol{m}}$ increases from zero, with no upstream jump, for a range of density ratios, for $\boldsymbol{r}_{\boldsymbol{u}}=\mathbf{0 . 1}$. For $F_{0}$ values larger (or $H_{m}$ less) than those on the upper part of these curves, the steady flow is everywhere supercritical, and for $F_{0}$ (or $H_{m}$ ) less than the lower part (where these curves effectively coincide), the steady flow is subcritical.

given by Baines (1984) for the special case of water and kerosene, where a range of parameters with two states was observed experimentally. However, computations for flows in the Boussinesq limit (Baines, 1995, 1998) using the Yih-Guha hydraulic jump model (which seemed to be the best available at the time) did not give any multiple states. Here we present two parameter diagrams in which $r_{u}=0.1$, and is representative of thin lower layers. In one of these, $\rho_{2} / \rho_{1}$ is small $(0.1)$, and in the other, 0.99 , which is very close to the Boussinesq limit. The contrast between these two cases is surprising and instructive. But first, Figure 8 shows the curves for the boundary of wholly subcritical (for $F_{0}<1$ ) or wholly supercritical (for $F_{0}>1$ ) flow as the obstacle height is increased from zero in a stream with given $F_{0}$, for a range of density ratios, for $r_{u}=0.1$. The positions and shapes of these curves are generally similar in form to that for a single layer, which is curve BAE in Figure 7. The main difference is that single-layer curve BAE is unlimited, whereas all the two-layer curves must necessarily terminate.

Figure 9 shows the $F_{0}-H_{m}$ diagram for $r=r_{u}=0.1$, $\rho_{2} / \rho_{1}=0.99$, near the Boussinesq limit. Here curve BAE denotes the critical flow condition, with subcritical/supercritical flow to the left if $F_{0}<1 / F_{0}>1$, and partially blocked lower-layer flow with an upstream jump to the right (as in Figure 7). The magnitude of this jump is given by the plotted values of $r_{\mathrm{a}}=d_{1 \mathrm{a}} / D$. This part of the diagram extends to the line where $r_{\mathrm{a}}=0.385$, which is the value for the maximum jump speed. The curves terminate at the boundary where the lower layer is completely blocked, and for larger $F_{0}$, there is a small region where larger upstream motions occur through rarefactions. This is limited by the flow immediately upstream of the obstacle becoming critical, which occurs where $r_{\mathrm{b}}$ is very close to 0.45 . For larger values of $F_{0}$, or $H_{m}$, the flow over the obstacle is supercritical.

This figure is similar to Figure 3.12a of (Baines, 1995, 1998), which uses Yih and Guha, as described above except that the

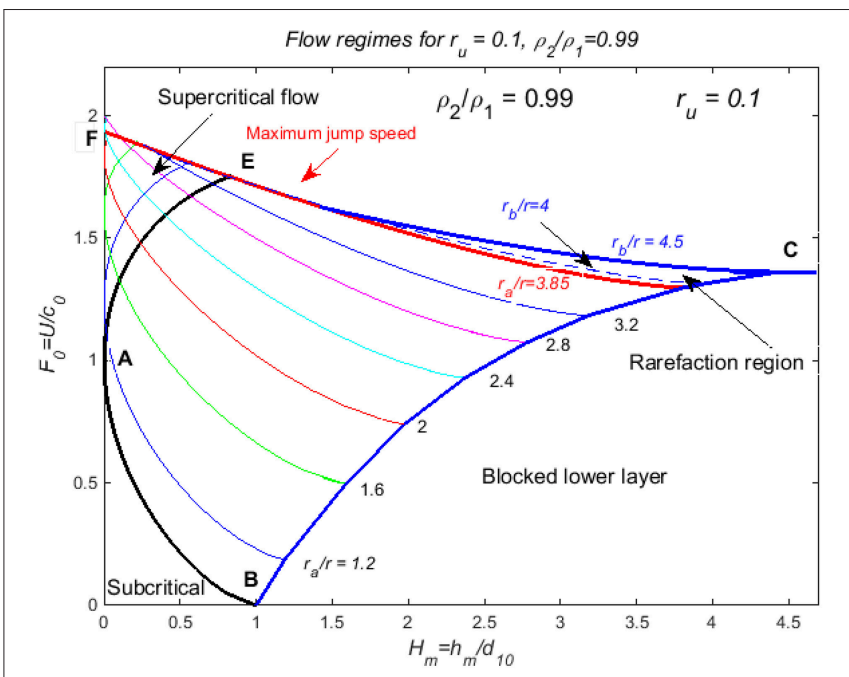

FIGURE 9 | Flow properties for steady-state flow over an obstacle, depicted on the $F_{0}-H_{m}$ diagram for $r=r_{u}=0.1, \rho_{2} / \rho_{1}=0.99-$ the near-Boussinesq case. The flow is subcritical below $A B$, and upstream hydraulic jumps occur to the right of EAB below EC; the lower layer is completely blocked to the right of $\mathrm{BC}$. Rarefactions that increase the upstream disturbance above that due to the jump of maximum speed occur in the small region immediately below $\mathrm{EC}$, above the curve with $r_{\mathrm{a}} / \mathrm{r}_{u}=3.85$. In the region AEFA, there are three possible flow states: supercritical flow, and two states with different upstream jump heights, given by the cross-over of the two curves passing through any given point.

curves for the amplitudes of upstream jumps extend beyond AE. Here, because some of these curves "double back" after touching $H_{m}=0$, there are two additional solutions for the upstream flow, in the region below the curve for maximum jump speed. Given that the flow in this region may also be supercritical, there are three possible solutions in the region AEF-a jump at small amplitude, a jump at somewhat larger amplitude, and no jump at all (supercritical flow, as indicated by the arrow). It is possible that one or more of these jumps may be unstable, but this has not been investigated.

The $F_{0}-H_{m}$ diagram for $r=0.1, \rho_{2} / \rho_{1}=0.1$ is shown in Figure 10. Here the overall pattern is the same as for Figure 9, with the exception that the bounding curve BADE for supercritical flow extends above the boundary line FDC for partially blocked flows. In particular there is a region above FD where partially blocked or completely supercritical flows are possible, between $\mathrm{DE}$ and the axis $H_{m}=0$. This is an upward extension of the curves that commence from the line BC. Hence, there are six different regions in this diagram. The first four are: subcritical flow below $A B$, blocked lower layer below $\mathrm{BC}$, supercritical flow above GEDC, and partially blocked flow with upstream jump in DABCD; in these regions the flow state for given $\left(F_{0}, H_{m}\right)$ is unique. In the region ADFA there are three possible flow states: supercritical flow, and two possible critically controlled flows with different upstream jumps, given by the intersecting contours of jump height at any particular point. In section DEGFD the same applies, except that there is only one possible upstream jump. This latter region is curious because, when compared with the diagram for single-layer flows (Figure 7), the region with multiple states is to the left of curve $\mathrm{ADE}$, whereas for single-layer flows it is to the right of it. 


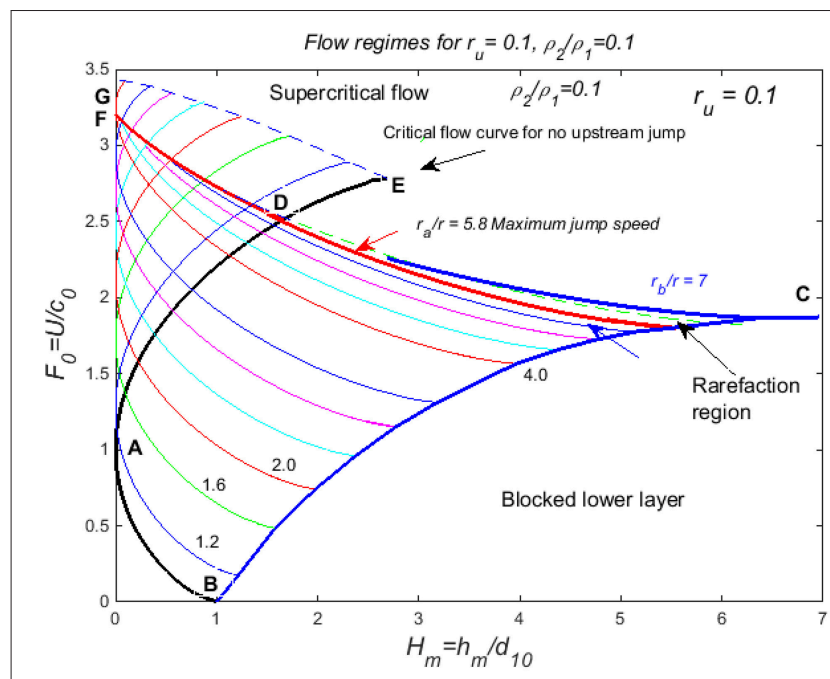

FIGURE 10 | As for Figure 9, but for the parameters $r_{u}=\mathbf{0 . 1}$, $\rho_{\mathbf{2}} / \rho_{1}=\mathbf{0 . 1}$. The properties are essentially the same, except for the region DEGF, within which the flow has two possible flow states: supercritical flow, or critical flow at the obstacle crest with an upstream jump.

A corresponding diagram for $r_{u}=0.1, \rho_{2} / \rho_{1}=0.5$ (not shown) displays the same features as in Figure 10, but the region ADFA is less pronounced, so that the diagram is closer to that of Figure 9, as might be expected.

\section{CONCLUSIONS}

We have used a new (and within its limitations, essentially correct) formulation of two-layer hydraulic jumps to determine the properties of non-linear flow over long obstacles. This hydraulic jump formulation uses a vorticity balance to infer the pressure differences across the jump, and avoids assumptions made in earlier models that are now seen to be incorrect. The model can be (and has been) applied to a full range of upstream conditions and density ratios, and hence is not restricted to the

\section{REFERENCES}

Baines, P. G., and Davies, P. A. (1980). "Laboratory studies of topographic effects in rotating and/or stratified fluids," in Orographic Effects in Planetary Flows, GARP Publication No. 23 (World Meteorological Organisation/Ingernational Council of Scientific Unions), 233-299.

Baines, P. G. (1984). A unified description of two-layer flow over topography. J. Fluid Mech. 146, 127-167.

Baines, P. G. (1995, 1998). Topographic Effects in Stratified Flows. Cambridge, UK: Cambridge University Press.

Baines, P. G. (2015). Internal hydraulic jumps in two-layer systems. J. Fluid Mech. 787, 1-15. doi: 10.1017/jfm.2015.662

Borden, Z., and Meiburg, E. (2013). Circulation-based models of Boussinesq internal bores. J. Fluid Mech. 276, R1, doi: 10.1017/jfm.2013.239

Chu, V. H., and Baddour, R. E. (1977). Surges, waves and mixing in two-layer density stratified flow. Proc. Int. Assn. Hydraul. Res. 1, 303-310.

Klemp, J. B., Rotunno, R., and Skamarock, W. C. (1997). On the propagation of internal bores. J. Fluid Mech. 331, 81-106.

Li, M., and Cummins, P. F. (1998). A note on hydraulic theory of internal bores. Dyn. Atmos. Oceans 28, 1-7.
Boussinesq limit. For obstacles moving relative to fluid at rest, there are four main dimensionless parameters involved: the lower layer thickness ratio $r_{u}=d_{10} / D$, the density ratio $\rho_{2} / \rho_{1}$, the initial Froude number $F_{0}$, and the obstacle height ratio $H_{m}=$ $h_{m} / d_{10}$.

The nature of the resulting flows (steady over the obstacle) has features similar to those obtained with a single layersub- or supercritical flow for small obstacle heights for small values of $H_{m}$, and an upstream hydraulic jump followed by a thicker lower layer and governed by a critical condition at the obstacle crest, for larger $H_{m}$. The hydraulic jumps, however, have a maximum speed, and when this is reached, larger amplitude jumps cannot be forced from the topography. Instead, the upstream disturbances forced by larger obstacle heights have the form of rarefactions, which become elongated and lag behind the jump. For $r_{u}$ values $\geq 0.5$, only rarefactions are present, and previous analysis without jumps is still valid (e.g., Figure $3.12 \mathrm{~d}$ of Baines, 1995, 1998).

The use of the new hydraulic jump formulation produces some surprises that are best seen in the flow types in the various regions of the $F_{0}-H_{m}$ plane diagrams of Figures 9, 10. These are very different from previous versions (see for example Figures 3.12a,b of Baines, 1995, 1998) which used the jump formulation of Yih and Guha (1955). Figure 9 shows results for $\rho_{2} / \rho_{1}=0.99$, near the Boussinesq limit. Here the surprise is in the "supercritical" region AEF, where in addition to possibly being supercritical, the flow may have two other flow states with upstream jumps, of different heights. In Figure 10, the same phenomenon is seen in region $\mathrm{ADF}$, but now there is an additional region FDEG in which there are two possible statessupercritical flow, or an upstream jump with critical flow at the crest. This region FDEG is vanishingly small for $\rho_{2} / \rho_{1}=0.99$, but increases in size as $\rho_{2} / \rho_{1}$ decreases toward zero.

\section{AUTHOR CONTRIBUTIONS}

This work relies heavily on earlier work by PGB who initiated the present study. ERJ contributed subsequently. Both authors approved it for publication.

White, B. L., and Helfrich, K. R. (2014). A model for internal bores in continuous stratification. J. Fluid Mech. 761, 282-304. doi: 10.1017/jfm. 2014.599

Whitham, G. B. (1974). Linear and Non-Linear Waves. New York, NY: J. Wiley \& Sons. 636.

Wood, I. R., and Simpson, J. E. (1984). Jumps in layered miscible fluids. J. Fluid Mech. 140, 329-342.

Yih, C.-S., and Guha, C. R. (1955). Hydraulic jump in a uid system of two layers. Tellus 7, 358-366.

Conflict of Interest Statement: The authors declare that the research was conducted in the absence of any commercial or financial relationships that could be construed as a potential conflict of interest.

Copyright (C) 2016 Baines and Johnson. This is an open-access article distributed under the terms of the Creative Commons Attribution License (CC BY). The use, distribution or reproduction in other forums is permitted, provided the original author(s) or licensor are credited and that the original publication in this journal is cited, in accordance with accepted academic practice. No use, distribution or reproduction is permitted which does not comply with these terms. 


\section{APPENDIX}

The functions $a_{i j}, b_{i}$ in Equation (3.8) take the following general forms. The variable $\eta$ is relative to layer thicknesses $d_{1}, d_{2}$, for the lower and upper layers respectively, where $d_{1}+d_{2}+$ $h=D$. With $d_{1}$ and $d_{2}$ as reference values, for $a_{i j}(\eta, v)$ we have

$a_{11}=\frac{v\left(d_{2}-d_{1}+h-2 \eta\right)+\frac{Q}{2}\left(1+\frac{\rho_{2}}{\rho_{1}}\right)}{D-h-\delta}+\frac{\Delta \rho}{\rho_{1}} \frac{T_{1}}{(D-h-\delta)^{2}}$,

where

$$
\begin{gathered}
T_{1}=v\left(d_{2}-\eta\right)\left(d_{1}-h+\eta\right) \\
+\frac{Q}{2}\left(\eta-d_{2}+\frac{\rho_{2}}{\rho_{1}}\left(d_{1}-h+\eta\right)\right), \\
\delta=\frac{\Delta \rho}{\rho_{1}}\left(d_{1}-h+\eta\right), \\
a_{12}=\frac{\left(d_{2}-\eta\right)\left(d_{1}-h+\eta\right)}{D-h-\delta}, \\
a_{21}=\frac{\Delta \rho g}{\rho_{1}}-\frac{v^{2}\left(d_{2}-\eta+\frac{\rho_{2}}{\rho_{1}}\left(d_{1}-h+\eta\right)\right)}{(D-h-\delta)^{2}}+\frac{\Delta \rho}{\rho_{1}} \frac{T_{2}}{(D-h-\delta)^{3}},
\end{gathered}
$$

where

$$
\begin{array}{r}
T_{2}=v^{2}\left[\left(d_{2}-\eta\right)^{2}-\frac{\rho_{2}}{\rho_{1}}\left(d_{1}-h+\eta\right)^{2}\right] \\
+\frac{\rho_{2}}{\rho_{1}} Q\left(2 v D-\left(1-\frac{\rho_{2}}{\rho_{1}}\right) Q\right),
\end{array}
$$

$$
a_{22}=\frac{\left[v\left(\left(d_{2}-\eta\right)^{2}-\frac{\rho_{2}}{\rho_{1}}\left(d_{1}-h+\eta\right)^{2}\right)+\frac{\rho_{2}}{\rho_{1}} Q(D-h)\right]}{(D-h-\delta)^{2}} .
$$

If one can make the assumption

$$
\eta \frac{\Delta \rho}{\rho_{1}}<<D-\frac{\Delta \rho}{\rho_{1}} d_{1}-h\left(1-\frac{\Delta \rho}{\rho_{1}}\right)
$$

which implies $\delta \approx \frac{\Delta \rho}{\rho_{1}}\left(d_{1}-h\right)$, and if $h=0$, these reduce to

$$
\eta \frac{\Delta \rho}{\rho_{1}}<<D-\frac{\Delta \rho}{\rho_{1}} d_{1} \text {, and } \delta \approx \frac{\Delta \rho}{\rho_{1}} d_{1},(A .8)
$$

the above expressions simplify to

$$
\begin{gathered}
a_{11}=\frac{v\left(d_{2}-d_{1}+h-2 \eta\right)+\frac{Q}{2}\left(1+\frac{\rho_{2}}{\rho_{1}}\right)}{D-h-\delta}, \\
a_{12}=\frac{\left(d_{2}-\eta\right)\left(d_{1}-h+\eta\right)}{D-h-\delta}, \\
a_{21}=\frac{\Delta \rho g}{\rho_{1}}-\frac{v^{2}\left(d_{2}-\eta+\frac{\rho_{2}}{\rho_{1}}\left(d_{1}-h+\eta\right)\right)}{(D-h-\delta)^{2}}, \\
a_{22}=\frac{\left[v\left(\left(d_{2}-\eta\right)^{2}-\frac{\rho_{2}}{\rho_{1}}\left(d_{1}-h+\eta\right)^{2}\right)+\frac{\rho_{2}}{\rho_{1}} Q(D-h)\right]}{(D-h-\delta)^{2}},
\end{gathered}
$$

If we further make the Boussinesq approximation $\left(\rho_{2}=\rho_{1}\right.$, except for the first term in $a_{21}$ ) we have

$$
\begin{gathered}
a_{11}=\frac{v\left(d_{2}-d_{1}+h-2 \eta\right)+Q}{D-h}, \\
a_{12}=\frac{\left(d_{2}-\eta\right)\left(d_{1}-h+\eta\right)}{D-h}, \\
a_{21}=\frac{\Delta \rho g}{\rho_{1}}-\frac{v^{2}}{D-h}, a_{22}=a_{11} .
\end{gathered}
$$




\title{
The Quasi-Steady State of the Valley Wind System
}

\author{
Juerg Schmidli ${ }^{1,2 *}$ and Richard Rotunno ${ }^{3}$ \\ ${ }^{1}$ Institute for Atmospheric and Climate Science, ETH Zurich, Zurich, Switzerland, ${ }^{2}$ Institute for Atmospheric and \\ Environmental Sciences, Goethe-University Frankfurt, Frankfurt, Germany, ${ }^{3}$ National Center for Atmospheric Research, \\ Boulder, Colorado
}

The quasi-steady-state limit of the diurnal valley wind system is investigated over idealized three-dimensional topography. Although this limit is rarely attained in reality due to ever-changing forcings, the investigation of this limit can provide valuable insight, in particular on the mass and heat fluxes associated with the along-valley wind. We derive a scaling relation for the quasi-steady-state along-valley mass flux as a function of valley geometry, valley size, atmospheric stratification, and surface sensible heat flux forcing. The scaling relation is tested by comparison with the mass flux diagnosed from numerical simulations of the valley wind system. Good agreement is found. The results also provide insight into the relation between surface friction and the strength of the along-valley pressure gradient.

OPEN ACCESS

Edited by:

Ivana Stiperski,

University of Innsbruck, Austria

Reviewed by:

Ernesto Dos Santos Caetano Neto, National Autonomous University of

Mexico, Mexico

Dino Zardi,

University of Trento, Italy Johannes Wagner, Institute of Atmospheric Physics,

Germany

*Correspondence: Juerg Schmidll

schmidli@iau.uni-frankfurt.de

Specialty section:

This article was submitted to Atmospheric Science,

a section of the journal

Frontiers in Earth Science

Received: 27 June 2015 Accepted: 23 November 2015 Published: 16 December 2015

Citation:

Schmidli J and Rotunno R (2015) The

Quasi-Steady State of the Valley Wind System. Front. Earth Sci. 3:79. doi: 10.3389/feart.2015.00079
Keywords: quasi-steady state, valley wind system, along-valley wind, mass and heat fluxes, scaling relation, valley geometry

\section{INTRODUCTION}

Thermally forced diurnal along-valley winds are a prominent phenomenon in many mountain areas and they are particularly pronounced in larger valleys. They are not only an important element of the near-surface climate in mountain regions, but they also influence the horizontal transport and vertical exchange of heat, mass, moisture, and pollutants over complex terrain. They contribute to the formation of clouds and precipitation and the interaction with the larger-scale atmospheric flow (Banta, 1990; Whiteman, 1990; Zardi and Whiteman, 2013). Due to their importance they have been intensively investigated in the past using conceptual models (e.g., Wagner, 1938; Steinacker, 1984; Vergeiner and Dreiseitl, 1987; Egger, 1990) and more recently using idealized numerical simulations (e.g., Rampanelli et al., 2004; Schmidli and Rotunno, 2010; Schmidli, 2013; Wagner et al., 2015a).

In the present work we return to a basic question: can one estimate a-priori the (maximum) strength of the along-valley wind and the associated mass and heat fluxes? More specifically, given a valley, a specific atmospheric environment, and a specified forcing, can one estimate the maximum along-valley mass flux, and hence also the (maximum) heat and mass exchange of the valley with its surroundings induced by the along-valley flow.

One approach would be to use simple linear and non-linear conceptual models such as those described in Vergeiner (1987) and Egger $(1987,1990)$. Although these models are capable of predicting the time evolution of the along-valley wind, their results are very sensitive to key parameters such as the eddy-diffusivity coefficients for heat and momentum or some empirical time scales. In addition they are only applicable to simple valley geometries.

Here we take a different approach. We investigate the quasi-steady-state limit of the valley wind system. In order to be able to investigate this limit also for large valleys, we assume a time-independent forcing of the valley-plain system. In the quasi-steady-state limit the thermal 
gradients, the resulting pressure gradients, and the along-valley wind are approximately constant in time, only the temperature of the atmosphere is slowly changing (e.g., Wyngaard, 2010, p. 204). As long as the temperature gradients are not changing, the slow temperature change does not have a significant influence on the dynamics of the along-valley wind. Hence the relevant parts of the system are in an approximate steady state. Although this limit is rarely attained for large valleys due to everchanging forcings, the analysis of this limit can provide valuable insight, in particular into the mass and heat fluxes associated with the alongvalley circulation. In contrast to the simple conceptual models, the current approach is valid for arbitrary valley geometries. As with other conceptual models our approach provides insight into the basic governing principles of the along-valley wind.

The accuracy of the quasi-steady-state approximation for a particular situation depends primarily on the valley size and the background stratification, as these two quantities determine the linear response time of the along-valley wind. According to Egger (1990), the phase speed of the linear solution is $c=\frac{1}{2} H N$, where $H$ is the valley depth and $N$ is the Brunt-Väisälä frequency, resulting in a characteristic response time $\tau_{c}=L / c$, where $L$ is the length of the valley. Assuming constant forcing, the along-valley wind attains its steady state value after about $3 \tau_{c}$. For typical static stabilities, the response time varies from less than an hour for small valleys to several hours for a large Alpine valley. These time scales have to be compared with the time scales of the forcings. In undisturbed conditions the relevant forcing time scale is given by the diurnal motion of the sun. Thus, it is clear that the valley wind in a smaller valley will be closer to the quasi-steady-state limit than the valley wind in a large valley. Even if the quasisteady-state is not reached in large valleys, the present analysis can provide useful estimates on the maximum strength of the valley wind and associated fluxes.

Our goal is to derive scaling relations for the along-valley mass flux and the associated advective heat flux in the quasisteady state limit as a function of valley geometry, valley size, atmospheric stratification, surface sensible heat flux forcing, and surface friction. Our derivation highlights the key balances associated with the along-valley circulation. If we consider the valley volume argument (e.g., Whiteman, 1990) to represent a zeroth-order model of the along-valley wind, our approach represents a consistent first-order model, as it takes the implicit advective tendencies into account (e.g., advective cooling during daytime up-valley wind conditions). The mere existence of a thermally induced along-valley wind implies corresponding advective tendencies. The scaling relations are then tested by comparison with the mass and heat fluxes diagnosed from numerical model simulations of the valley wind system. The analysis of the quasi-steady state highlights the essential role of the valley heat budget in determining the strength of the along-valley wind.

The paper is organized as follows. In Section 2, we derive the scaling relations for the along-valley mass flux and the associated net advective heat flux. The numerical setup and the set of simulations are introduced in Section 3. The scaling relations are evaluated in Section 4, and the conclusions are drawn in Section 5 .

\section{THEORY}

We consider an arbitrary valley-plain system as illustrated in Figure 1. Our goal is to derive a diagnostic expression for the along-valley mass flux in the quasi-steady state limit. For this we proceed in two steps: (i) analysis of the net heat exchange of the valley with its surroundings, (ii) determination of the resulting steady-state along-valley mass flux from the steady-state advective heat flux. Using additional assumptions the diagnostic expression can be used for the a-priori estimation of the alongvalley mass flux and the strength of the along-valley wind.

\subsection{Heat Budget and Advective Heat Transport}

We first derive an expression for the net heat exchange of the valley with its surroundings due to advection. The bulk heat budget for an arbitrary control volume $V$ can be expressed as

$$
\begin{aligned}
\underbrace{\int_{V} \rho c_{p} \frac{\partial \theta}{\partial t} d V}_{m^{i} \dot{\theta}^{i}} & =\underbrace{\int_{A_{s}} H_{s} d S+\int_{V} \nabla \cdot \mathbf{R} d V}_{F_{d b}^{i}} \\
& +\underbrace{\int_{V} \rho c_{p} \mathbf{v} \cdot \nabla \theta d V}_{F_{a}^{i}}+\underbrace{\int_{A_{a}} H d S}_{F_{t}^{i}},
\end{aligned}
$$

where $H_{s}$ is the turbulent sensible heat flux normal to the land surface $A_{s}, \mathbf{R}$ is the radiation flux, and $H$ is the turbulent sensible heat flux normal to the surface $A_{a}$. The surface $A_{a}$ is the atmospheric component of the surface of the control volume $V$. For a conventional control volume the last term simplifies to the integral of the vertical turbulent sensible heat flux across the top surface of the control volume. Equation (1) is derived by integration of the potential-temperature equation and by using Gauss' theorem to convert the resulting volume integral of the turbulent heat flux divergence into a surface integral and by decomposing the resulting surface integral into a land surface part $A_{s}$ and an atmospheric part $A_{a}$ (as in Schmidli and Rotunno, 2010). In other words, the net change of heat content in an arbitrary volume $V^{i}\left(m^{i} \dot{\theta}^{i}\right)$ is equal to the sum

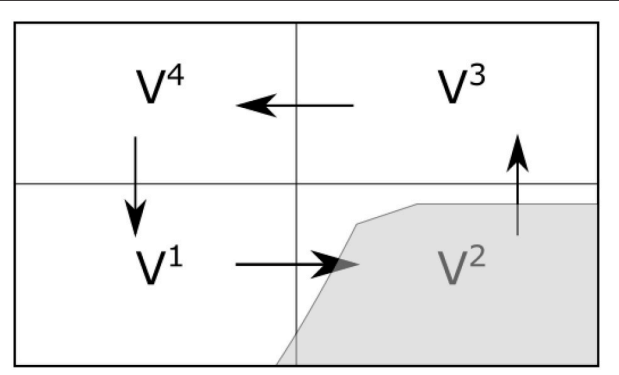

FIGURE 1 | Schematic of the three-dimensional valley-plain system indicating the four control volumes and the mean along-valley circulation during the up-valley wind phase. The arrows indicate the net mass flux across the interfaces between adjacent control volumes. See Figure $\mathbf{3}$ for a top view of the valley-plain system. 
of the contributions due to diabatic processes $\left(F_{d b}^{i}\right)$, mean-flow advection $\left(F_{a}^{i}\right)$, and turbulent heat flux through $A_{a}\left(F_{t}^{i}\right)$. Here $m^{i}$ refers to the mass of the air in the volume $V^{i}$, multiplied by the specific heat of the air, that is $m^{i}=c_{p} \int_{V^{i}} \rho d V$, and $\dot{\theta}^{i}$ refers to the density-weighted volume-averaged temperature tendency.

Now let us return to the valley-plain system, as illustrated in Figure 1. Our goal is to determine net heat exchange due to advection $F_{a}^{2}$ in the valley volume $V^{2}$. Assuming no diabatic heating and turbulent exchange in the upper plain volume $V^{4}$, the bulk heat budgets for the four control volumes are

$$
\begin{aligned}
& m^{1} \dot{\theta}^{1}=F_{d b}^{1}+F_{a}^{1}, \\
& m^{2} \dot{\theta}^{2}=F_{d b}^{2}+F_{a}^{2}+F_{t}^{2}, \\
& m^{3} \dot{\theta}^{3}=F_{d b}^{3}+F_{a}^{3}+F_{t}^{3}, \\
& m^{4} \dot{\theta}^{4}=F_{a}^{4} .
\end{aligned}
$$

Assuming equal heating rates in the quasi-steady state, $\dot{\theta}^{1}=\dot{\theta}^{2}$ and $\dot{\theta}^{3}=\dot{\theta}^{4}$, and introducing

$$
\begin{aligned}
& F_{d b} \equiv F_{d b}^{2}, \quad F_{a} \equiv-F_{a}^{2}, \quad F_{t} \equiv-F_{t}^{2}, \\
& \beta_{d b} \equiv F_{d b}^{3} / F_{d b}^{2}, \quad \beta_{a} \equiv F_{a}^{3} / F_{a}^{2},
\end{aligned}
$$

where the sign is chosen such that $F_{a} \geq 0$ and $F_{t} \geq 0$ during the daytime heating period, and noting that $F_{t}^{3}=-F_{t}^{2}$, the above equation system can be written as

$$
\begin{aligned}
m^{2}\left(F_{d b}^{1}+F_{a}^{1}\right) & =m^{1}\left(F_{d b}-F_{a}-F_{t}\right) \\
m^{4}\left(\beta_{d b} F_{d b}-\beta_{a} F_{a}+F_{t}\right) & =m^{3} F_{a}^{4} .
\end{aligned}
$$

Next, the net advective fluxes over the plain are related to those over the valley:

$$
F_{a}^{1}+F_{a}^{4}=\left(1+\beta_{a}\right) F_{a}+F_{a}^{n},
$$

where $F_{a}^{n}$ is the net advective exchange of the entire valley-plain system with its surroundings (later on we will assume that $F_{a}^{n}=$ 0 ). Solving (8) for $F_{a}^{1}$ and (9) for $F_{a}^{4}$ and substituting into (10) one obtains

$$
\begin{array}{r}
\frac{m^{1}}{m^{2}}\left(F_{d b}-F_{a}-F_{t}\right)-F_{d b}^{1}+\frac{m^{4}}{m^{3}}\left(\beta_{d b} F_{d b}-\beta_{a} F_{a}+F_{t}\right) \\
=\left(1+\beta_{a}\right) F_{a}+F_{a}^{n} .
\end{array}
$$

Defining the mass (volume) ratios $\tau_{v} \equiv \frac{m^{1}}{m^{2}}, \tau_{u} \equiv \frac{m^{4}}{m^{3}}$, and the turbulent exchange ratio $\gamma_{t} \equiv F_{t} / F_{d b}$ and factoring out $F_{a}$ yields

$$
\begin{aligned}
F_{a}\left(1+\beta_{a}+\tau_{v}+\tau_{u} \beta_{a}\right) & =\tau_{v}\left(1-\gamma_{t}\right) F_{d b}-F_{d b}^{1} \\
& +\tau_{u}\left(\beta_{d b}+\gamma_{t}\right) F_{d b}-F_{a}^{n}
\end{aligned}
$$

Assuming equal diabatic forcing per unit area, the diabatic heat flux in the plain volume $V^{1}$ can be expressed by the diabatic heat flux in the valley volume by $F_{d b}^{1}=\tau_{A} F_{d b}$, where $\tau_{A}$ is the ratio of the areas of the upper control surfaces of the plain and valley volume. [Under typical topographic amplification factor considerations (Whiteman, 1990), these surface areas would be assumed to be of equal size, hence $\left.\tau_{A}=1\right]$. Substituting $F_{d b}^{1}$ into (12) yields

$$
f_{a} \equiv \frac{F_{a}}{F_{d b}}=\frac{\tau_{v}-\tau_{A}+\beta_{d b} \tau_{u}-\gamma_{t}\left(\tau_{v}-\tau_{u}\right)-\delta_{a}}{\tau_{v}+1+\beta_{a}\left(\tau_{u}+1\right)},
$$

where $f_{a}$ represents the ratio of the advective to the diabatic forcing of the valley control volume and $\delta_{a} \equiv F_{a}^{n} / F_{d b}$ represents the net advective exchange of the entire valley-plain system with its surroundings. Note that (13) follows directly from the first law of thermodynamics and the three assumptions of quasisteady state, equal diabatic forcing per unit area, and no diabatic or turbulent heating of the upper plain volume. It is valid for arbitary valley geometries. It can be seen that the advective heat flux increases if there is diabatic heating of the upper valley volume and it decreases if there is significant turbulent exchange. It also decreases if there is advective heating of the upper valley volume (i.e., $\beta_{a}>0$ ). If one assumes that the valley-plain system is closed to external influences, i.e., negligible larger-scale flows, then $\delta_{a}=0$. Application of (13) requires diagnosis or estimation of the ratios $\beta_{d b}, \gamma_{t}$, and $\beta_{a}$, and the geometric factors $\tau_{v}, \tau_{A}$, and $\tau_{u}$. In general, not all of these parameters are known a-priori.

As our goal is to derive scaling relations that can be applied a-priori, or with minimal additional assumptions, we assume that the flow-dependent parameters $\beta_{a}$ and $\gamma_{t}$ are approximately zero. In other words, assuming only diabatic heating of the upper valley volume, (13) reduces to

$$
f_{a}=\frac{\tau_{h}-1}{\tau_{h}+1 / \tau_{A}}+\frac{\beta_{d b} \tau_{u}}{\tau_{v}+1},
$$

where $\tau_{h} \equiv \tau_{v} / \tau_{A}=h / \bar{h}_{v}$ is the ratio of the mean depth of the plain to that of the valley control volume. For the standard case of equal upper control surfaces, $\tau_{h}$ corresponds to the topographic amplification factor (TAF). Returning to the heat budgets, it can be seen that in this limit, the diabatic heating of the upper valley volume is matched by a corresponding advective heating of the upper plain volume.

To provide further insight, suppose that $\beta_{d b}=0$ and that the surface areas of the valley and plain control volumes are identical, as is often done in TAF considerations (e.g., Whiteman, 1990), then $\tau_{A}=1$, and the advective heating ratio becomes

$$
f_{a}=\frac{\tau_{h}-1}{\tau_{h}+1} .
$$

For illustration consider a valley with triangular cross section with $\tau_{h}=2$, then $f_{a}=1 / 3$ for the closed valley-plain system.

\subsection{The Along-Valley Mass Flux}

Using the advective ratio $f_{a}$, it is now possible to derive an a-priori estimate of the strength of the mean up-valley wind at the valley entrance. First note that the net advective heat flux out of the valley volume can be expressed as

$$
F_{a}=c_{p} M \Delta \theta,
$$

where $M$ is the mass flux through the valley volume and $\Delta \theta=$ $\theta_{\text {out }}-\theta_{\text {in }}$ is the difference in the mean potential temperature 
of the outflow and the inflow. Combining (16) and (13), the up-valley mass flux at the valley entrance is

$$
M=\frac{f_{a} F_{d b}}{c_{p} \Delta \theta}=\frac{f_{d b}}{c_{p} c_{\theta} \gamma_{\theta}} \cdot \frac{L W}{h} \cdot f_{a},
$$

where $f_{d b}$ is the diabatic heating rate per unit area, $\gamma_{\theta}$ is the potential temperature lapse rate, $c_{\theta}$ is an empirical constant relating the temperature difference $\Delta \theta$ to the lapse rate, $L$ is the valley length, and $W$ is the valley width.

Assuming that the inflow is through the valley entrance and the outflow is through the valley top, the mass flux is related to the mean along-valley wind through $M=\bar{v} \bar{\rho} A_{\text {in }}$. Substituting into (17), using (14) with $\beta_{d b}=0$, and rearranging, the mean up-valley wind at the valley entrance is

$$
\bar{v}=\frac{f_{d b}}{c_{p} c_{\theta} \gamma_{\theta} h \bar{\rho}} \cdot \frac{L}{\bar{h}_{v}} \cdot \frac{\tau_{h}-1}{\tau_{h}+1 / \tau_{A}},
$$

where $A_{\text {in }}=W \bar{h}_{v}$ has been used. It can be seen that the valley wind strength is proportional to the valley length, independent of the valley width, and inversely proportional to the square of the valley depth. It increases monotonically with increasing TAF $\left(\tau_{h}\right)$.

\subsection{Parameter Estimation}

Several parameters are required in order to estimate the up-valley mass flux using (17) and the advective heating ratio using (14). Some of these parameters, such as $\tau_{h}, \beta_{d b}$, and $\tau_{v}$, depend on the choice of the control volumes, in particular on the height $h$ of the upper control surfaces of the valley and plain volume. They can be calculated directly from the given topography as a function of the height $h$, once the horizontal location of the control volumes has been fixed, as illustrated in Figure 2. It can be seen that the TAF, $\tau_{h}$, increases with decreasing height of the control volume,

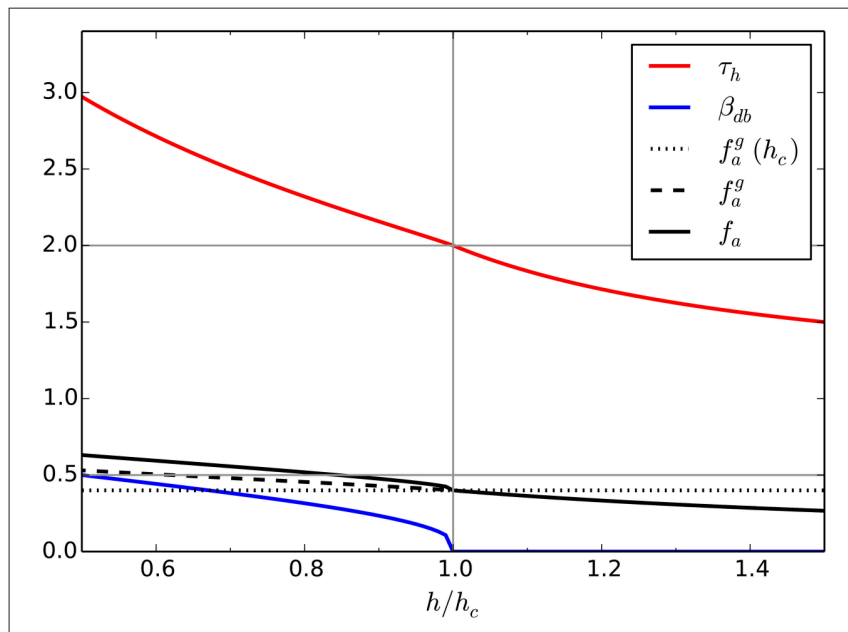

FIGURE 2 | Dependence of the parameters $\tau_{h}, \beta_{d b}$ and the different approximations of the advective ratio $f_{a}$ (see main text) on the height of the upper control surface (depth of the control volume), for the topography to be introduced in Section 3 and illustrated in Figure 3. as has been pointed out by Steinacker (1984). If $h$ is smaller than the crest height $h_{c}$, the diabatic ratio $\beta_{d b}$ becomes nonzero and increases with decreasing $h$. Assuming uniform diabatic forcing per unit area, $\beta_{d b}$ is equal to the area fraction occupied by topography on the lower surface of the upper control volume (for $h>h_{c}$, this area fraction is zero).

Two choices for estimating the parameters are considered: first, $h$ equal to the height of the mountain crest, $h_{c}$; second, $h$ equal to the height of the "equilibrium" level, $h_{e}$, that is the level of zero mean along-valley flow. The first choice corresponds to the standard application of the TAF argument; all required parameters, except $c_{\theta}$, can be calculated a-priori. The second choice better represents the true flow geometry, in particular the depth of the up-valley flow layer. If $h_{e}$ is known, then the required parameters can be calculated.

As we are interested in the a-priori scaling of the valley wind, a simplified estimate of the advective ratio is introduced. Assuming that $\beta_{d b} \approx 0$, one obtains

$$
f_{a}^{g}=\frac{\tau_{h}-1}{\tau_{h}+1 / \tau_{A}}
$$

It can be seen that $f_{a}^{g}$ depends only on parameters related to the valley geometry and can thus be calculated a-priori, if $h$ is given.

In summary, three different approximations of the up-valley mass flux and the advective heating ratio will be compared in comparison to the numerical simulations to be introduced next. The simplest approximation is based on the parameters evaluated with $h=h_{c}$. The next is based on (19) using $h=h_{e}$, and the final, and potentially most accurate estimate, is based on (14) and also using $h=h_{e}$. For the latter $\beta_{d b}>0$ if $h_{e}<h_{c}$.

\section{NUMERICAL SETUP}

The numerical setup of the simulations is similar to the one described in Schmidli and Rotunno (2010, 2012) (hereafter SR10 and SR12) and detailed information can be found there. For convenience we summarize the main aspects of the setup and point out the key differences with respect to SR10. To investigate the quasi-steady state of the valley wind system, we introduce a valley-plain configuration with a horizontal valley floor as shown in Figure 3. Except for the east-west orientation, the topography is identical to the periodic configuration introduced in SR12, and the corresponding analytic expression for the topography can be found in SR12. To save computing resources, the cross-valley extension of the computational domain is reduced from 120 to $20 \mathrm{~km}$. For the periodic configuration this does not influence the results.

As in SR10, the simulations are initialized from an atmosphere at rest with a constant stratification. The main difference with respect to SR10 is in the thermal forcing of the valley wind system. As we are interested in the quasi-steady state, the wind system is forced by a constant surface sensible heat flux. In contrast to the previous simulations, the land surface model is turned off. Momentum transfer is determined by a constant momentum drag coefficient, corresponding to a neutral surface layer and a given momentum roughness length. While the 


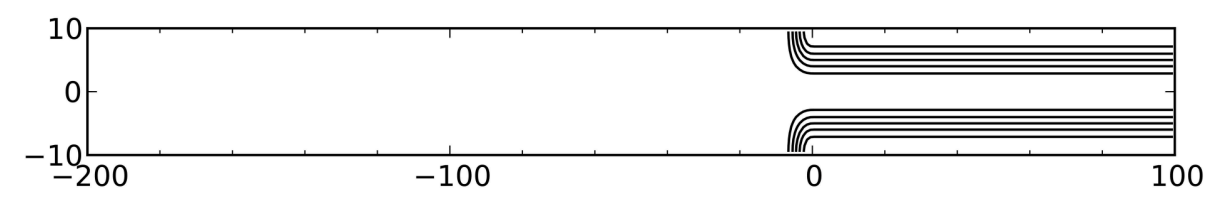

FIGURE 3 | Computational domain adopted in this paper. The lines denote the height contours of the topography (contour interval = $250 \mathrm{~m}$ ).

TABLE 1 | Summary of the numerical experiments.

\begin{tabular}{|c|c|c|c|}
\hline Experiment & Setup & $h_{\mathrm{e}}$ & $f_{\mathrm{a}}^{\mathrm{sim}}$ \\
\hline REF & Reference & 1.41 & 0.43 \\
\hline L40 & $L=40 \mathrm{~km}$ & 1.40 & 0.50 \\
\hline L60 & $L=60 \mathrm{~km}$ & 1.40 & 0.48 \\
\hline W30 & $W=30 \mathrm{~km}$ & 1.50 & 0.44 \\
\hline W40 & $W=40 \mathrm{~km}$ & 1.53 & 0.46 \\
\hline H1000 & $H=1000 \mathrm{~m}$ & 1.22 & 0.34 \\
\hline H2O00 & $H=2000 \mathrm{~m}$ & 1.69 & 0.54 \\
\hline LR2 & $L R=2 \mathrm{Kkm}^{-1}$ & 1.56 & 0.37 \\
\hline LR4 & $L R=4 \mathrm{Kkm}^{-1}$ & 1.35 & 0.49 \\
\hline LR6 & $L R=6 \mathrm{Kkm}^{-1}$ & 1.30 & 0.58 \\
\hline $\mathrm{SH} 25$ & $S H=25 \mathrm{Wm}^{-2}$ & 1.31 & 0.54 \\
\hline $\mathrm{SH} 100$ & $S H=100 \mathrm{Wm}^{-2}$ & 1.60 & 0.32 \\
\hline zOm2 & $z_{0}=0.01 m$ & 1.39 & 0.44 \\
\hline zOm0 & $z_{0}=1 m$ & 1.44 & 0.42 \\
\hline
\end{tabular}

The abbreviations refer to valley length $(L)$, valley width $(W)$, valley depth $(H)$, potential temperature lapse rate $(\mathrm{LR})$, surface sensible heat flux $(\mathrm{SH})$, and momentum roughness length $\left(z_{0}\right)$.

simulations are integrated for $14 \mathrm{~h}$, our main focus is on the scaling properties after $9 \mathrm{~h}$, when the simulation is close to a steady-state.

To investigate the scaling of the quasi-steady state, a set of simulations are carried out. The set is created by varying the valley dimensions, the lapse rate of the initial state, the thermal forcing in terms of the prescribed surface sensible heat flux, and the momentum roughness length. The reference simulation comprises a valley with a length of $100 \mathrm{~km}$, a crest-to-crest width of $20 \mathrm{~km}$, and a depth of $1500 \mathrm{~m}$, a potential temperature lapse rate of $+3 \mathrm{~K} \mathrm{~km}^{-1}$, a surface sensible heat flux of $50 \mathrm{~W} \mathrm{~m}^{-2}$, and a momentum roughness length of $0.1 \mathrm{~m}$. All experiments are created by varying one parameter in comparison to the reference simulation (see Table 1).

As in SR10, the numerical simulations have been carried out using the Advanced Regional Prediction System (ARPS) model (Xue et al., 2000, 2001). The horizontal grid spacing is $1 \mathrm{~km}$ and the vertical grid spacing varies from $20 \mathrm{~m}$ near the surface to a maximum of $200 \mathrm{~m}$ above $2 \mathrm{~km}$. The lateral boundary conditions are periodic in the cross-valley direction and free-slip wall conditions are imposed in the along-valley direction. This choice minimizes the required computational resources, as doubly periodic boundary conditions would require a computational domain of double the current size in the alongvalley direction. In order to reduce the implicit diffusivity of the integration, horizontal and vertical advection of momentum and scalars is carried out by a 4th-order advection scheme (in SR10, vertical advection is carried out by a 2 nd-order scheme). Thus, the time step had to be reduced from 12 to $6 \mathrm{~s}$. Vertical mixing is parameterized with a TKE-based PBL scheme using a non-local turbulence length scale (Sun and Chang, 1986). All moist processes are turned off (moist $=0$ ). The current setup was chosen in order to be able to do many simulations. Although large-eddy simulations for two-dimensional valley sections are now fairly common (e.g., Catalano and Moeng, 2010; Serafin and Zardi, 2010; Schmidli, 2013; Wagner et al., 2014), and even isolated examples of large-domain simulations of the along-valley wind exist (Schmidli, 2013; Wagner et al., 2015a,b), for the purpose of the present study simulations using parameterized turbulence are sufficient. We have found that for well-resolved valleys mean and bulk quantities obtained with the current setup compare well with large-eddy simulation results (see also Wagner et al., 2014).

\section{RESULTS}

Next, we test the formula for the advective heating ratios and the along-valley mass flux for the set of numerical simulations introduced above. The section starts with a brief discussion of the evolution of the reference simulation, followed by the presentation of the main results on the net advective heat flux (heat export out of the valley control volume) and the alongvalley mass flux.

\subsection{The Reference Simulation}

Snapshots of the evolution of the valley wind system are shown in Figure 4 after 3, 6, and $9 \mathrm{~h}$ of integration. The figure illustrates the evolution of the along-valley flow, both in an along-valley plane located at the valley center and in a crossvalley plane located $20 \mathrm{~km}$ up valley from the valley entrance, the cross-valley circulation and the thermal structure of the atmosphere. The basic characteristics of the flow are very similar to previous simulations with a time-dependent diurnal forcing. The symmetric cross-valley circulations develop rapidly, while the along-valley circulation takes longer to develop fully. It can be seen that while the up-valley component of the along-valley circulation (on the valley center plane) is already close to a steady state after $6 \mathrm{~h}$, the upper-level return flow is still increasing between hours 6 and 9. Note also the suppression of the growth of the convective boundary layer within the valley, which is clearly visible in the along-valley sections. For a more detailed discussion of the evolution of the valley wind system, see SR10 and SR12. As already noted in SR12, the disturbances in the along-valley flow 

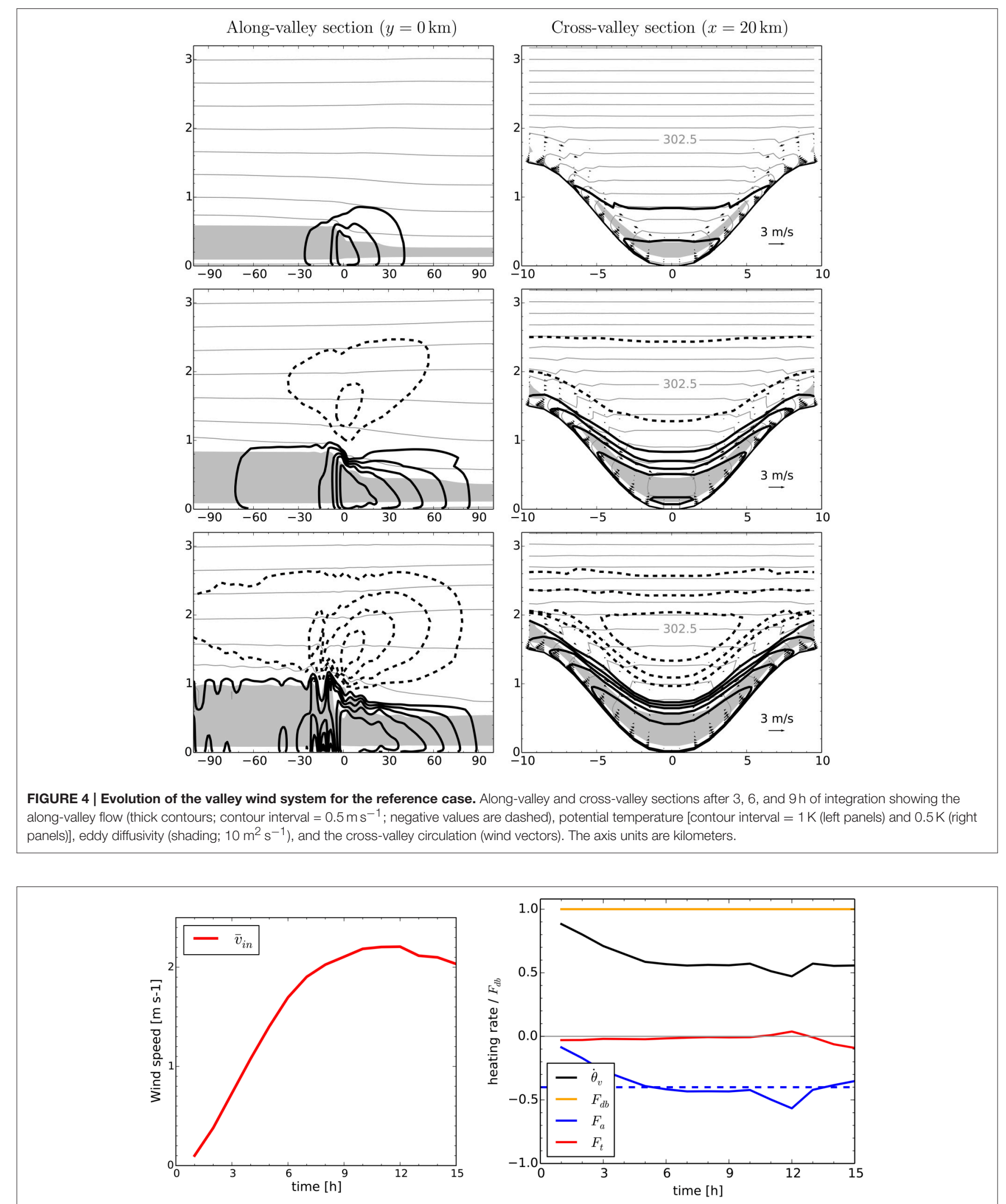

FIGURE 5 | Time evolution of the mean up-valley wind at the valley entrance (left panel) and the heat budget tendencies for the valley control volume (right panel). $\bar{v}_{\text {in }}$ denotes the average of the along-valley wind over the inflow layer, that is up to $h_{e}$. The dashed line indicates the a-priori estimate, $f_{a}\left(h_{C}\right)$, of the steady-state advective heating rate. 


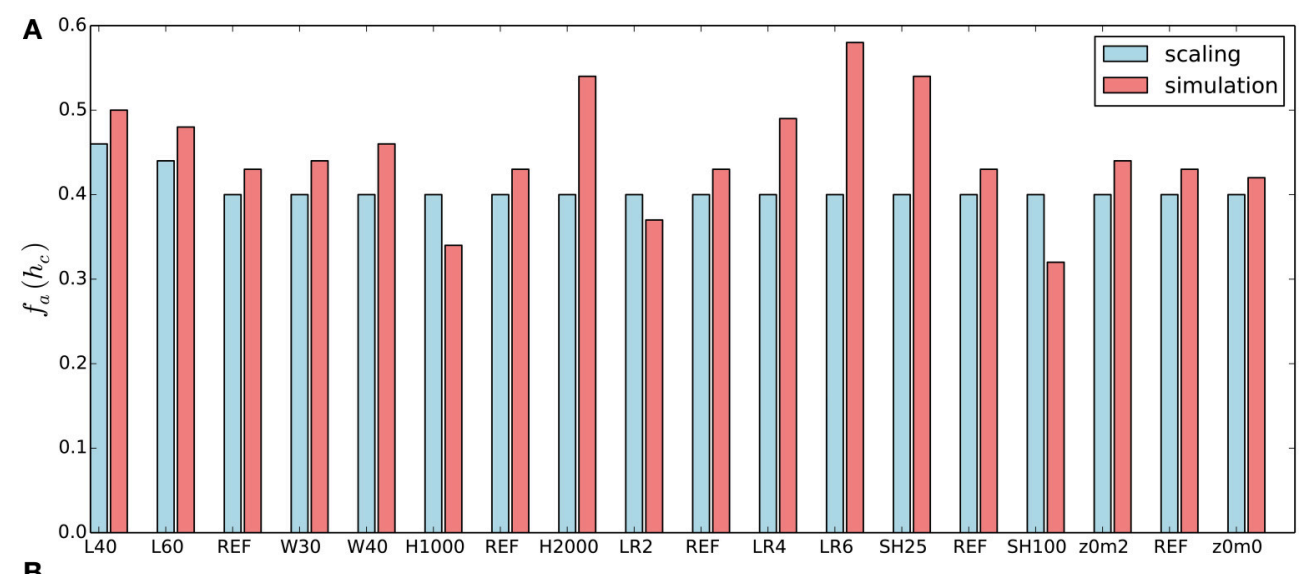

B

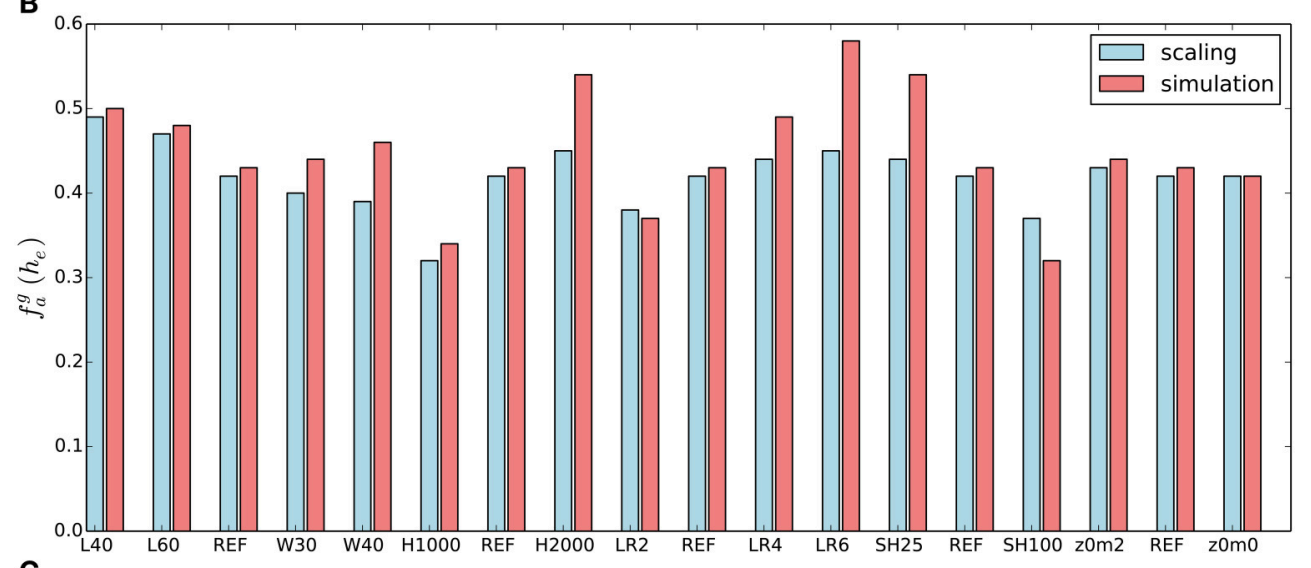

C

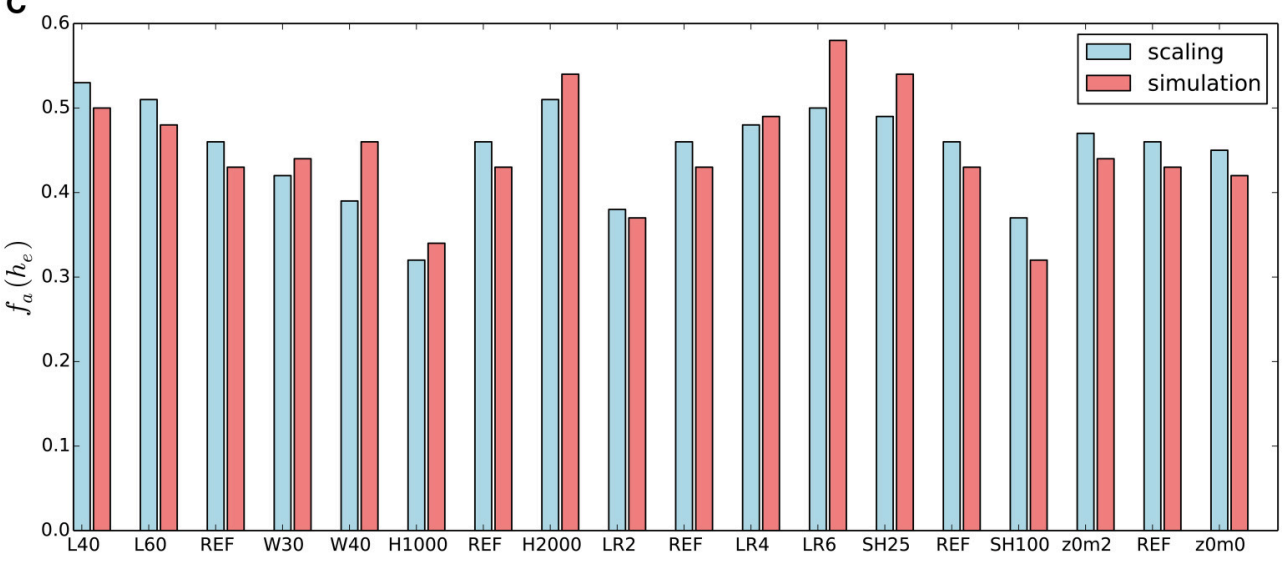

FIGURE 6 | Scaling the advective heat flux: comparison of different approximations of $f_{a}$ with the diagnosed value from the numerical simulations. (A) basic approximation: $f_{a}=f_{a}\left(h_{C}\right)$, (B) improved estimate with $f_{a}=f_{a}^{g}\left(h_{e}\right)$, (C) improved estimate with $f_{a}=f_{a}\left(h_{e}\right)$.

pattern near the valley entrance seen after $9 \mathrm{~h}$ are likely related to the ARPS turbulence scheme and unresolved cellular motions in the convective boundary layer (Ching et al., 2014). Other models do not show such quasi-periodic patterns (Schmidli et al., 2011). Toward the end of the simulation these patterns may dominate the flow. Luckily they do not significantly influence the early stages of the quasi-steady state which is the focus of our study.

The approach to the quasi-steady state is depicted in Figure 5 in terms of the evolution of the mean up-valley wind (at the valley entrance) and the valley heat budget. During the first 6 $\mathrm{h}$, the mean along-valley wind increases approximately linearly. Thereafter, as the valley wind system approaches the quasi-steady state, the increase is much reduced and the mean along-valley wind reaches its maximum value of about $2.2 \mathrm{~m} \mathrm{~s}^{-1}$ after $10 \mathrm{~h}$. In terms of the heat budget, and in particular the net advective heat flux, the components are already steady after 6-7 h. The simulated cooling rate due to advection is close to the a-priori estimate indicated by the dashed line. Note that the net heat exchange due to turbulence is close to zero for the reference simulation. This is due to the combination of a relatively deep 
valley, sufficient stratification and relatively weak surface forcing. It can be seen that after $10 \mathrm{~h}$ of integration there are deviations from the steady-state values. This is due to the ARPS-specific disturbances discussed above. In order to avoid issues related to these disturbances we will focus on hour 9 for the analysis of the quasi-steady state.

\subsection{Net Advective Heat Flux}

The quasi-steady state predictions of the net advective heat flux out of the valley control volume are compared with the diagnosed value from the numerical simulations in Figure 6. The scaling predictions correspond to the three approximations introduced in Section 2.3. The most basic approximation corresponds to the assumption that the top of the valley control volume corresponds to the height of the mountain ridges [i.e., $f_{a}=f_{a}\left(h_{c}\right)$ ]. While this assumption results in reasonable estimates for some of the valleys, it fails to represent the variations associated with changes in the valley depth, the atmospheric stratification, and the surface sensible heat flux. Further analysis shows that this failure is partly related to the varying depth of the up-valley wind layer. For the simulation with strong stability, for example, the up-valley wind layer is restricted to a depth of less than $1 \mathrm{~km}$ and the upper-level return flow occurs already at heights well below the mountain ridge height (not shown).

In order to improve the estimate of $f_{a}$, the true flow geometry needs to be considered. From the cross-valley averaged alongvalley circulation at the valley entrance, one can estimate the height of the mean inflow, the mean outflow, and the level of zero net along-valley flow. For the reference simulation the zeroflow height corresponds to $1.41 \mathrm{~km}$. The results for the other simulations are tabulated in Table $\mathbf{1}$ and visualized in Figure 7. Improved estimates of $f_{a}$ can now be obtained by setting the top of the lower control volumes to the zero-flow height. These are denoted by $f_{a}^{g}\left(h_{e}\right)$ and $f_{a}\left(h_{e}\right)$. The former represents only the more accurate consideration of the flow geometry, while the latter also includes the effect of a non-zero diabatic factor $\beta_{d b}$. Consideration of the flow geometry leads to an overall improvement of the estimates. The correspondance between the analytic estimates and the diagnosed values from the numerical simulations is further increased by including the effect of the non-zero diabatic factor, except for the simulations with different valley widths. Much of the remaining differences (e.g., for LR6, SH25) are likely due to larger deviations from the quasi-steadystate assumption for the particular simulations (not shown).

In summary, the a-priori estimation of the net advective heat flux out of the valley volume is successful if the zero-flow height is close to the valley ridge height, or if the corresponding zero-flow height can be estimated (a-priori), provided the circulation is approximately in a quasi-steady state.

\subsection{The Along-Valley Mass Flux}

Once the net advective heat flux out of the valley control volume is known, it is possible to estimate the along-valley mass flux and thus the strength of the mean along-valley wind at the valley entrance. The key idea is that the net advective heat flux is proportional to the mass flux through the valley control volume and the difference in the mean potential temperature of the mean inflow into the valley volume and the mean outflow out of the valley volume, see (16).

The scaling of the along-valley mass flux according to the (a-priori) quasi-steady state estimates and the numerical simulations is depicted in Figure 8, for the same three approximations discussed above. Note that both the estimated and the diagnosed values are scaled with respect to the reference case, i.e., REF $=1$. Even for the most basic approximation, $M\left(h_{c}\right)$, the scaling of the along-valley mass flux works quite well. Larger differences are only found for H1000, LR2, and SH100, the three cases for which the inflow layer extends above the crest height (i.e., $h_{e}>h_{c}$, see Table 1). This indicates that the a-priori scaling is less accurate if the valley is shallow in comparison to the surface forcing and the stratification. If the valley is too shallow, that is if the convective boundary layer extends well beyond the crest height, the scaling does not work (additional simulations with shallower valleys were undertaken, but are not shown).

The lower two panels show the scaling of the up-valley mass flux, that is of the along-valley mass flux up to the equilibrium height $h_{e}$, for the two approximations of $f_{a}$. Overall the performance of the scaling is quite similar for both cases,

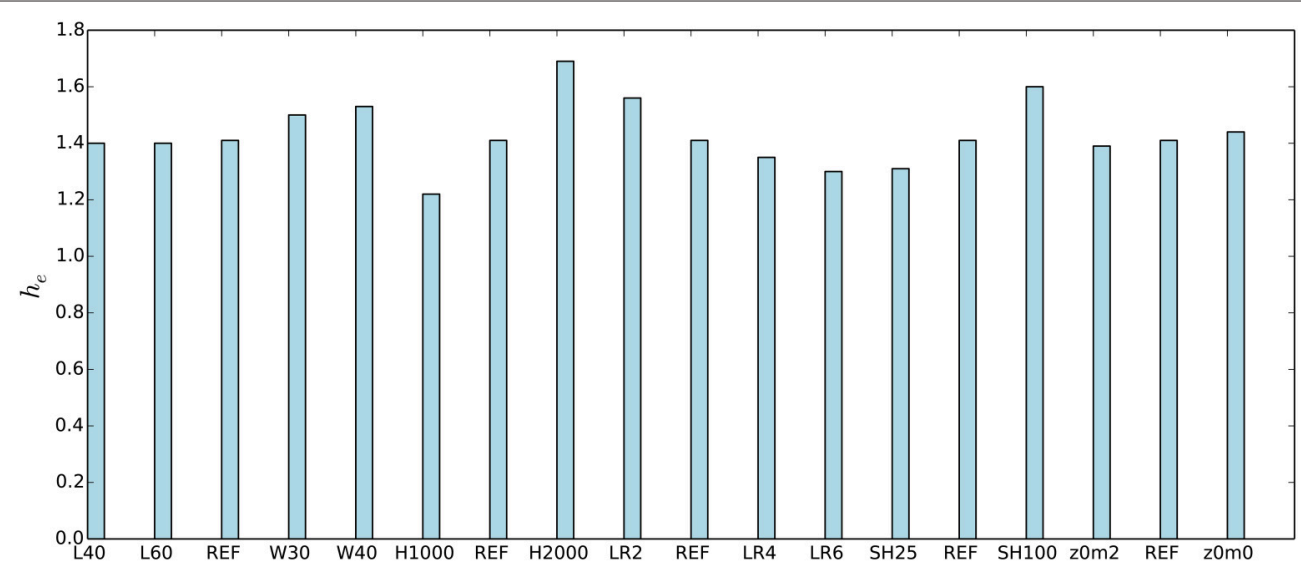

FIGURE 7 | Diagnosed depth of the inflow layer, $h_{e}$. 


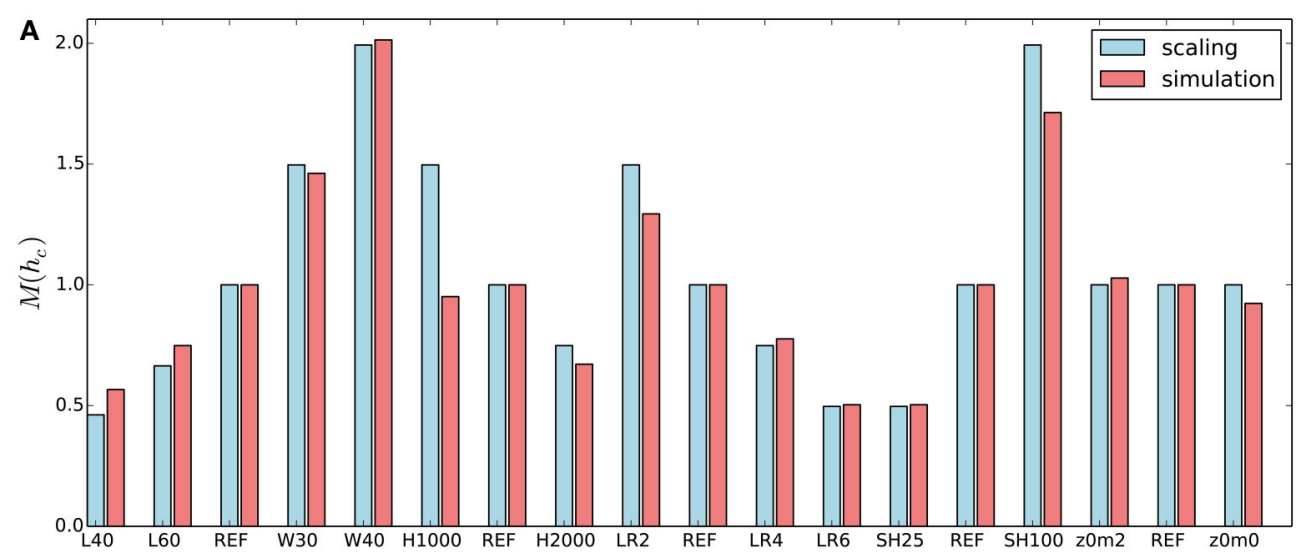

B

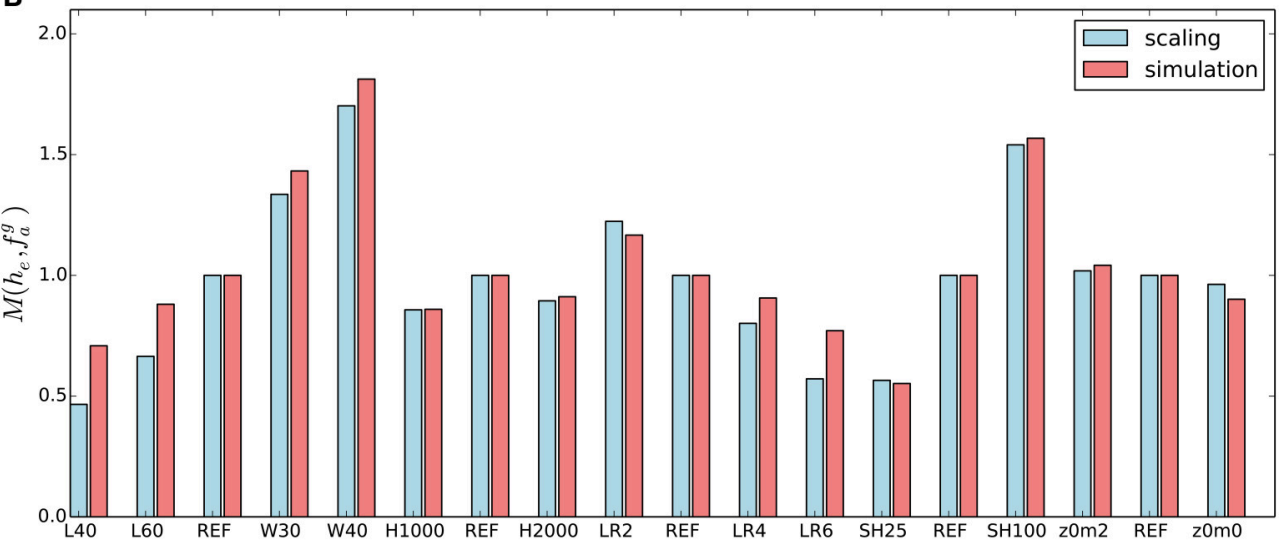

C

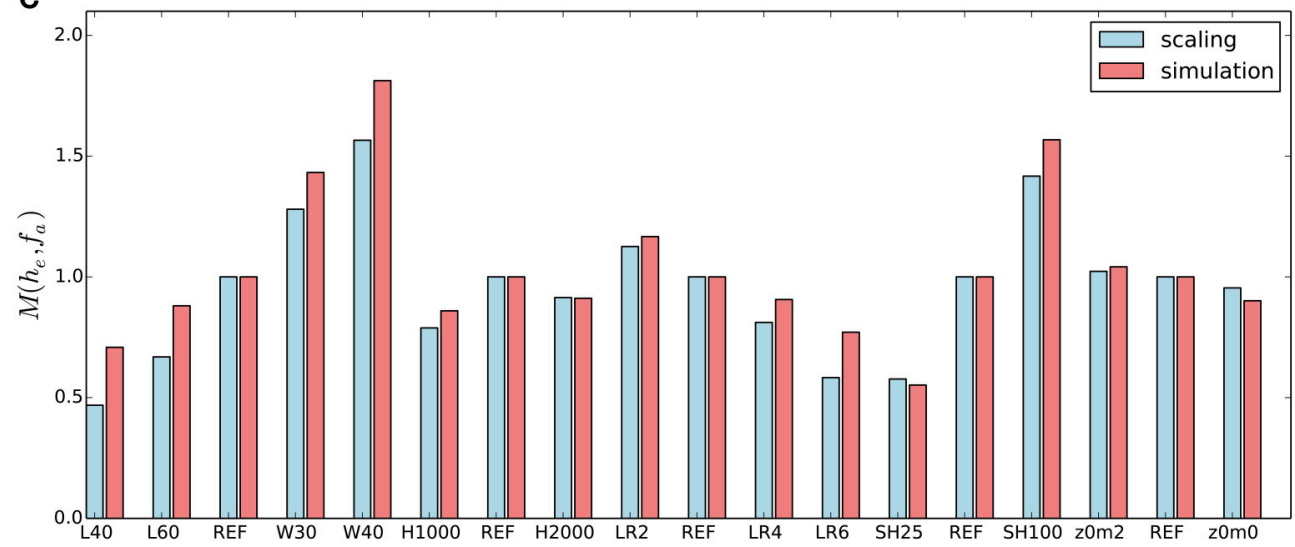

FIGURE 8 | Scaling the along-valley mass flux $M$ : comparison of different a-priori estimates of $M$ with the corresponding diagnosed value from the numerical simulations. (A) Basic approximation $M=M\left(h_{C}\right)$, (B) improved estimate with $M=M\left(h_{e}, f_{a}^{g}\right)$, (C) improved estimate with $M=M\left(h_{e}, f_{a}\right)$. The former estimate is compared to the simulated along-valley flux up to ridge height, the latter two estimates are compared to the total simulated up-valley flux, that is to the height $h_{e}$.

with a slightly higher overall skill for the simpler estimate $f_{a}^{g}$. Comparing the simpler estimate (middle panel) with the a-priori scaling (top panel), the skill is clearly improved for variations of the valley depth and of the surface sensible heat flux.

According to the analysis presented in Section 2, the steady-state along-valley wind and the associated mass and heat fluxes should be independent of the surface roughness (friction). Comparing the three simulations with different surface roughnesses $(0.01,0.1$, and $1.0 \mathrm{~m})$, it is found that this is indeed approximately the case. The pressure gradient forcing adjusts itself in order to support roughly the same along-valley mass flux. Note that the near-surface wind speed is of course reduced for large roughness lengths, but this has only a minor effect on the integrated along-valley mass flux (not shown). 
In summary, the scaling of the along-valley mass flux (and hence of the along-valley wind) with respect to variations of the valley geometry, atmospheric stratification, and surface sensible heat flux is successful, provided the up-valley wind circulation (and hence the convective boundary layer) does not extend significantly above the height of the surrounding mountain ridges. This implies that the valleys need to be sufficiently deep, given the specific combination of surface forcing and atmospheric stratification.

\section{CONCLUSIONS}

The quasi-steady-state limit of the diurnal valley wind system has been examined in order to contribute to an improved understanding of the along-valley winds and the associated transport processes. A scaling relation for the steady-state along-valley wind speed and the associated advective heat flux as a function of valley geometry, valley size, atmospheric stratification, and surface sensible heat flux forcing has been derived. In contrast to results from simple dynamical models (e.g., Vergeiner, 1987), the steady-state scaling is also valid for complex valley geometries. The formula can be used as a diagnostic tool or for the a-priori estimation of the along-valley mass flux and the valley-wind strength in a given situation. The a-priori estimation requires either additional closure assumptions or the use of an approximate formula.

It is found that the steady-state along-valley wind speed increases linearly with the magnitude of the surface forcing and the strength of the valley volume effect $\left(\tau_{h}-1\right)$, these two factors correspond to the extra heating factor in simple linear models (Egger, 1990). Also as in the linear case, the wind speed decreases with increasing atmospheric stability. In contrast to the results from simple dynamical models, the steady-state wind speed increases with increasing valley length and it is largely independent of the surface roughness. This may at first seem counterintuitive, but can easily be understood. The initial evolution of the along-valley wind is of course restrained by surface friction, but in the steady state the plain-temperature contrast will increase until the along-valley pressure gradient is strong enough to support the along-valley wind required by the steady-state valley heat budget. Also the longer valley, the larger the excess heat that needs to be exported out of the valley to maintain a steady-state heat budget. In summary, the strength

\section{REFERENCES}

Banta, R. M. (1990). "The role of mountain flows in making clouds", in Atmospheric Processes Over Complex Terrain, Meteorological Monographs, Vol. 23, ed W. Blumen (Boston, MA: American Meteorological Society), 229-283.

Catalano, F., and Moeng, C.-H. (2010). Large-eddy simulation of the daytime boundary layer in an idealized valley using the weather research and forecasting numerical model. Bound. Layer Meteorol. 137, 49-75. doi: 10.1007/s10546-0109518-8

Ching, J., Rotunno, R., LeMone, M., Martilli, A., Kosovic, B., Jimenez, P. A., et al. (2014). Convectively induced secondary circulations in fine-grid mesoscale of the steady-state valley wind is primarily controlled by the diabatic forcing and the atmospheric stability, and not by surface friction. This scaling prediction is confirmed by the numerical simulations.

The scaling relation for the steady-state mass and heat flux is tested by comparison with the corresponding diagnosed fluxes from numerical simulations of the along-valley wind system for idealized three-dimensional topographies. In general, good agreement is found. The scaling of the along-valley mass flux with respect to variations of the valley geometry, atmospheric stratification, and surface sensible heat flux works well, provided the valley is sufficiently deep, such that the convective boundary layer (and hence the up-valley flow layer) does not extend significantly above the height of the surrounding mountain ridges.

The quasi-steady state scaling relations can be used for the a-priori estimation of the mass and heat fluxes associated with the along-valley circulation. Two different approximations of the scaling relations have been developed and tested. The first one assumes that the budgeting volumes extend to the top of the mountain ridge; the second one sets the height of the budgeting volumes to the top of the inflow layer. While the former approximation can be calculated a-priori, the latter requires the height of the inflow layer as an input. The results show that this height is strongly correlated with variations of the valley depth and the atmospheric stratification. This opens up the possibility for the development of a parameterization of the depth of the inflow layer and thus of using also the more complete scaling relations in an a-priori setup. This requires further investigation. In any event it points to the most important parameter dependencies for estimating the alongvalley mass flux. The a-priori scaling relations could be used as a basis to parameterize the larger-scale effects of the thermallyinduced along-valley circulations in coarse-resolution weather and climate models.

\section{ACKNOWLEDGMENTS}

The numerical simulations have been performed on the CRAY XE6 at the Swiss National Supercomputing Centre (CSCS) using the Advanced Regional Prediction System (ARPS) developed by the Center for Analysis and Prediction of Storms, University of Oklahoma. The figures have been produced using matplotlib (Hunter, 2007). numerical weather prediction models. Mon. Weather Rev. 142, 3284-3302. doi: 10.1175/MWR-D-13-00318.1

Egger, J. (1987). Simple models of the valley-plain circulation. Part I: minimum resolution model. Meteorol. Atmos. Phys. 36, 231-242. doi: 10.1007/BF01045151

Egger, J. (1990). "Thermally forced flows: theory," in Atmospheric Processes Over Complex Terrain, Meteorological Monographsm, Vol. 23, ed W. Blumen (Boston, MA: American Meteorological Society), 43-58.

Hunter, J. D. (2007). Matplotlib: a 2D graphics environment. Comput. Sci. Eng. 9, 90-95. doi: 10.1109/MCSE.2007.55

Rampanelli, G., Zardi, D., and Rotunno, R. (2004). Mechanisms of up-valley winds. J. Atmos. Sci. 61, 3097-3111. doi: 10.1175/JAS-3354.1 
Schmidli, J. (2013). Daytime heat transfer processes over mountainous terrain. J. Atmos. Sci. 70, 4041-4066. doi: 10.1175/JAS-D-13-083.1

Schmidli, J., Billings, B., Chow, F. K., De Wekker, S. F. J., Doyle, J., Grubisic, V., et al. (2011). Intercomparison of mesoscale model simulations of the daytime valley wind system. Mon. Weather Rev. 139, 1389-1409. doi: 10.1175/2010MWR3523.1

Schmidli, J., and Rotunno, R. (2010). Mechanisms of along-valley winds and heat exchange over mountainous terrain. J. Atmos. Sci. 67, 3033-3047. doi: 10.1175/2010JAS3473.1

Schmidli, J., and Rotunno, R. (2012). Influence of the valley surroundings on valley-wind dynamics. J. Atmos. Sci. 69, 561-577. doi: 10.1175/JAS-D-11-0129.1

Serafin, S., and Zardi, D. (2010). Daytime heat transfer processes related to slope flows and turbulent convection in an idealized mountain valley. J. Atmos. Sci. 67, 3739-3756. doi: 10.1175/2010JAS3428.1

Steinacker, R. (1984). Area-height distribution of a valley and its relation to the valley wind. Contrib. Atmos. Phys. 57, 64-71.

Sun, W.-Y., and Chang, C.-Z. (1986). Diffusion model for a convective layer. Part I: numerical simulation of convective boundary layer. J. Clim. Appl. Meteorol. 25 , 1445-1453. doi: 10.1175/1520-0450(1986)025<1445:DMFACL $>2$. $0 . \mathrm{CO} ; 2$

Vergeiner, I. (1987). An elementary valley wind model. Meteorol. Atmos. Phys. 36, 264-286. doi: 10.1007/BF01045154

Vergeiner, I., and Dreiseitl, E. (1987). Valley winds and slope winds observations and elementary thoughts. Meteorol. Atmos. Phys. 36, 264-286. doi: 10.1007/BF01045154

Wagner, A. (1938). Theorie und beobachtung der periodischen gebirgswinde. Gerlands Beitr. Geophys. 52, 408-449.

Wagner, J. S., Gohm, A., and Rotach, M. W. (2014). Impact of horizontal model grid resolution on the boundary layer structure over an idealized valley. Mon. Weather Rev. 142, 3446-3465. doi: 10.1175/MWR-D-14-00002.1

Wagner, J. S., Gohm, A., and Rotach, M. W. (2015a). The impact of valley geometry on daytime thermally driven flows and vertical transport processes. Q. J. R. Meteorol. Soc. 141, 1780-1794. doi: 10.1002/ qj. 2481
Wagner, J. S., Gohm, A., and Rotach, M. W. (2015b). Influence of along-valley terrain heterogeneity on exchange processes over idealized valleys. Atmos. Chem. Phys. 15, 6589-6603. doi: 10.5194/acp-15-6589-2015

Whiteman, C. D. (1990). "Observations of thermally developed wind systems in mountainous terrain," in Atmospheric Processes over Complex Terrain, Meteorological Monographs, Vol. 23, ed W. Blumen (Boston, MA: American Meteorological Society), 5-42.

Wyngaard, J. C. (2010). Turbulence in the Atmosphere. Cambridge: Cambridge University Press.

Xue, M., Droegemeier, K. K., and Wong, V. (2000). The advanced regional prediction system (ARPS)-A multi-scale non-hydrostatic atmospheric simulation and prediction model. Part I: Model dynamics and verification. Meteor. Atmos. Phys. 75, 161-193. doi: 10.1007/s007030070003

Xue, M., Droegemeier, K. K., Wong, V., Shapiro, A., Brewster, K., Carr, F., et al. (2001). The advanced regional prediction system (ARPS) - A multiscale nonhydrostatic atmospheric simulation and prediction model. Part II: Model physics and applications. Meteor. Atmos. Phys. 76, 143-165. doi: $10.1007 / \mathrm{s} 007030170027$

Zardi, D., and Whiteman, C. D. (2013). "Observations of thermally developed wind systems in mountainous terrain," in Mountain Weather Research and Forecasting - Recent Progress and Current Challenges, Springer Atmospheric Sciences, eds F. K. Chow, S. F. J. DeWekker, and B. Snyder (Berlin: Springer), $35-122$.

Conflict of Interest Statement: The authors declare that the research was conducted in the absence of any commercial or financial relationships that could be construed as a potential conflict of interest.

Copyright $\odot 2015$ Schmidli and Rotunno. This is an open-access article distributed under the terms of the Creative Commons Attribution License (CC BY). The use, distribution or reproduction in other forums is permitted, provided the original author(s) or licensor are credited and that the original publication in this journal is cited, in accordance with accepted academic practice. No use, distribution or reproduction is permitted which does not comply with these terms. 


\title{
Convective Boundary Layer Heights Over Mountainous Terrain-A Review of Concepts
}

\author{
Stephan F. J. De Wekker ${ }^{1 *}$ and Meinolf Kossmann ${ }^{2}$ \\ ${ }^{1}$ Department of Environmental Sciences, University of Virginia, Charlottesville, VA, USA, ${ }^{2}$ Climate and Environment \\ Consultancy, Deutscher Wetterdienst, Offenbach am Main, Germany
}

\section{OPEN ACCESS}

Edited by:

Miguel A. C. Teixeira,

University of Reading, UK

Reviewed by:

Charles Chemel,

University of Hertfordshire, UK

Norbert Kalthoff,

Karlsruher Institut für Technologie,

Germany

${ }^{*}$ Correspondence:

Stephan F. J. De Wekker

dewekker@virginia.edu

Specialty section:

This article was submitted to

Atmospheric Science,

a section of the journal

Frontiers in Earth Science

Received: 19 August 2015 Accepted: 19 November 2015 Published: 17 December 2015

Citation:

De Wekker SFJ and Kossmann M (2015) Convective Boundary Layer Heights Over Mountainous Terrain -A

Review of Concepts.

Front. Earth Sci. 3:77. doi: 10.3389/feart.2015.00077
Mountainous terrain exerts an important influence on the Earth's atmosphere and affects atmospheric transport and mixing at a wide range of temporal and spatial scales. The vertical scale of this transport and mixing is determined by the height of the atmospheric boundary layer, which is therefore an important parameter in air pollution studies, weather forecasting, climate modeling, and many other applications. It is recognized that the spatio-temporal structure of the daytime convective boundary layer $(\mathrm{CBL})$ height is strongly modified and more complex in hilly and mountainous terrain compared to flat terrain. While the CBL over flat terrain is mostly dominated by turbulent convection, advection from multi-scale thermally driven flows plays an important role for the CBL evolution over mountainous terrain. However, detailed observations of the CBL structure and understanding of the underlying processes are still limited. Characteristics of CBL heights in mountainous terrain are reviewed for dry, convective conditions. CBLs in valleys and basins, where hazardous accumulation of pollutants is of particular concern, are relatively well-understood compared to CBLs over slopes, ridges, or mountain peaks. Interests in the initiation of shallow and deep convection, and of budgets and long-range transport of air pollutants and trace gases, have triggered some recent studies on terrain induced exchange processes between the CBL and the overlying atmosphere. These studies have helped to gain more insight into CBL structure over complex mountainous terrain, but also show that the universal definition of CBL height over mountains remains an unresolved issue. The review summarizes the progress that has been made in documenting and understanding spatio-temporal behavior of CBL heights in mountainous terrain and concludes with a discussion of open research questions and opportunities for future research.

Keywords: convective boundary layer height, thermally driven winds, mountains, vertical transport and mixing, exchange processes between boundary layer and free troposphere

\section{INTRODUCTION}

The effect of mountains on the Earth's atmosphere has long been recognized and studied (Atkinson, 1981; Barry, 1992; Whiteman, 2000; Chow et al., 2013). Almost 50\% of the Earth's land surface is covered by hilly and mountainous terrain (Meybeck et al., 2001) and the effect of mountains on weather and climate is oftentimes felt far away from the mountains. 
Mountains exert an influence on the atmosphere in two basic ways that can be described as passive and active effects (Geiger, 1961). Passive effects involve momentum exchange between the surface and the atmosphere and occur when a flow is modified by the presence of mountains. Examples include flow blocking, flow channeling, and lee waves. There are many review articles on the passive effects when air flows over or around mountains (e.g., Queney, 1948; Smith, 1979; Wood, 2000; Jackson et al., 2013; Richner and Hächler, 2013). Active effects involve thermal and radiative energy exchange processes and occur when the terrain interacts with the diurnal heating and cooling cycle. Examples include thermally driven wind systems generated by horizontal temperature and pressure differences. These effects are most pronounced in cloudless conditions and weak synoptic pressure gradients. Several review articles exist on thermally driven wind systems in mountainous terrain (Whiteman, 1990; Zardi and Whiteman, 2013). At any moment, passive and active effects play an important role in determining the dynamical and thermodynamical atmospheric structure, with their individual roles depending on factors such as wind speed, cloud cover, and atmospheric stability. The current review paper focuses on the height of the atmospheric boundary layer during daytime, dry, convective conditions when active effects are dominant.

The atmospheric boundary layer is usually defined as the atmospheric layer that interacts directly with the Earth's surface on a time scale of a few hours or less (e.g., Stull, 1988). During daytime convective conditions, the atmospheric boundary layer is also called the convective boundary layer (CBL). Knowledge of CBL height and structure is useful or required in many applications such as air-quality studies, aerial spraying- and fire weather operations, regional climatology investigations, and weather forecasting.

In flat, homogeneous terrain, the CBL has been investigated intensively (e.g., Stull, 1988). The CBL grows by upward sensible heat flux at the surface and downward sensible heat flux (entrainment) at the top of the CBL. The convergence of the sensible heat flux causes the CBL to warm and grow in depth. The CBL growth rate is affected primarily by the characteristics of the surface-based temperature inversion at sunrise, the strength of the elevated temperature inversion, the free-atmospheric stability, and by the upward sensible heat flux at the surface. Subsidence at the top of the CBL will slow its growth rate.

Over mountainous terrain, the atmospheric structure becomes much more complicated. Transport and mixing processes such as those related to mountain waves (Smith et al., 2007), rotors (Doyle and Durran, 2007), and thermally-driven wind systems (Zardi and Whiteman, 2013) may affect CBL variability. One of the first attempts at describing the thermal structure of the CBL over mountainous terrain was made by Ekhart (1948). Ekhart analyzed radiosonde data and found that, compared to flat terrain, the diurnal amplitude of temperature in mountainous regions not only increases toward the surface, but is also significant at higher elevations. Ekhart concluded that the atmosphere above the mountain ridges is very different and independent from the valley atmosphere. Based on analyses of the diurnal temperature range and the winds at various locations, Ekhart makes a distinction between four different mountain

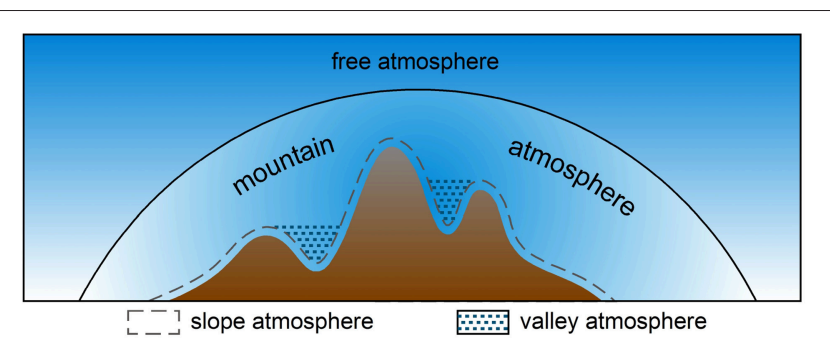

FIGURE 1 | Structure of the lower atmosphere over a mountain. The solid line indicates the boundary between the free atmosphere and the atmosphere influenced by the mountain. The dashed line indicates the top of the slope atmosphere, while the hatched pattern indicates the valley atmosphere (after Ekhart, 1948).

atmospheres (Figure 1): the slope atmosphere with a depth of at most a couple of $100 \mathrm{~m}$; a valley atmosphere, characterized by along valley flows; a large-scale mountain atmosphere which is influenced by the active (thermal) and passive (dynamical) effects of the entire mountain range; and the free atmosphere which is the unperturbed atmosphere at great horizontal and vertical distance from the mountain chain. Our major objectives of this review paper are to highlight some important aspects of CBL structure in mountainous terrain, to provide an overview of the current concepts, and to identify fruitful areas of future research.

The structure of this review paper will follow Ekhart's notion of a slope, valley, and mountain atmosphere with each of the "atmospheres" discussed separately. After defining CBL height and its determination, we will discuss the CBL height over slopes, valleys, basins, mountain tops and plateaus, and entire mountain ranges. Current notions concerning exchange processes between the $\mathrm{CBL}$ and the free atmosphere in mountainous terrain are also reviewed. We will conclude this review paper with a discussion of possible areas for future research.

\section{DEFINITION AND DETERMINATION OF CBL HEIGHTS}

One of the challenges of describing the spatio-temporal variability of the CBL height is the lack of consensus regarding its definition and determination. This is especially true for complex, mountainous terrain. While the term CBL is used throughout this review, other terms are used in the literature such as convective mixed layer or simply mixed or mixing layer (AMS, 2000). The term "mixing layer" is also used for the nocturnal boundary layer with high levels of shear-generated turbulence. In the $\mathrm{CBL}$, convectively generated turbulence dominates over shear generated turbulence to produce vigorous mixing which tends to make the potential temperature, moisture, tracer concentrations, and momentum independent of height in a major portion of the CBL.

At the top of the CBL, there is usually an increase in potential temperature and wind speed and a sharp decrease in humidity and pollutant concentration (Figure 2). A layer in which potential temperature increases with height or in which 
the temperature lapse rate is less than the dry adiabatic lapse rate is considered a stable layer. Such a layer could be surface-based or elevated. The top of the CBL is often defined as the base of an elevated stable layer. This criterion is used as the working definition in many of the examples provided in this paper. It should be recognized however, that other definitions exist that are using either air pollution (Maughan et al., 1982; Baxter, 1991; Myrick et al., 1994; Seibert et al., 2000) or turbulence parameters (Holzworth, 1972; Norton and Hoidale, 1976; Deardorff et al., 1980; Cai and Steyn, 1993; Vogelezang and Holtslag, 1996; Beyrich, 1997; Adler and Kalthoff, 2014; Dai et al., 2014) to indicate the CBL top. For example, Seibert et al. (2000) define the $\mathrm{CBL}$ top as "the height of the layer adjacent to the ground over which pollutants or any constituents emitted within this layer or entrained into it become vertically dispersed by convection or mechanical turbulence within a time scale of about an hour." They note that this definition corresponds to the top of the entrainment zone; a zone at the top of the CBL that is not well-mixed and where turbulence intensity weakens. In their definition, the CBL top is therefore not necessarily located at the base of an elevated stable layer.

Conventionally, CBL heights have been determined most frequently from vertical temperature profiles. In recent years, the development and availability of remote sensors such as sodars (sound detection and ranging) and lidars (light detection and ranging) have provided alternatives to this conventional method and have enabled the documentation of the daily variation of the CBL height at large temporal and spatial resolution. Sodars detect temperature fluctuations (turbulence), winds, and wind shear, while lidars detect vertical aerosol distribution. Particularly, downward looking airborne lidars have proven useful for the investigation of the spatial variability of CBL heights (e.g., Melfi et al., 1985; Kiemle et al., 1995; Hayden et al., 1997; Hägeli et al., 2000; De Wekker et al., 2004). An elevated maximum in the return signal from sodar emitted acoustic waves is caused by sharp vertical changes in turbulence and temperature stratification and usually indicates the height of the CBL top. The CBL top is also characterized by a vertical gradient in aerosol concentrations that can be determined by a sharp change in the range corrected return signal from lidar emitted laser pulses (Figure 2). An overview of the determination of CBL heights from remote sensing is given by Emeis et al. (2008). Several studies over flat terrain have shown a good correspondence between estimates of the CBL height from lidar and temperature profiles (Coulter, 1979; Van Pul et al., 1994; Marsik et al., 1995; Hayden et al., 1997; Hennemuth and Lammert, 2006) with maximum differences on the order of a few hundred meters in deep afternoon CBLs. Figure 2 provides a visual summary of the various methods discussed above to estimate the CBL top over flat and homogeneous terrain. Over mountainous terrain, several issues arise with the comparability of the various methods. For example, presence of upper-level turbulence induced by thermally driven flows can make the turbulence-based methods impractical or inapplicable (De Wekker, 2008; Catalano and Moeng, 2010). Also, mountain venting processes can cause aerosols and humidity to be transported above the CBL top (De Wekker et al., 2004; Steyn et al., 2013; Adler and Kalthoff, 2014). We will further discuss these processes and the implications for determining CBL heights over mountainous terrain in Section "Vertical Transport and Mixing Between the CBL and the Free Troposphere".

We are making a distinction between CBL depth and CBL height. In applications where the volume of air in which e.g., pollutants are mixed is considered, or for scaling arguments, it is important to use CBL depth $\mathrm{z}_{\mathrm{i}}$ (or thickness) which is the height of the CBL top above ground level (AGL). On the other hand, for

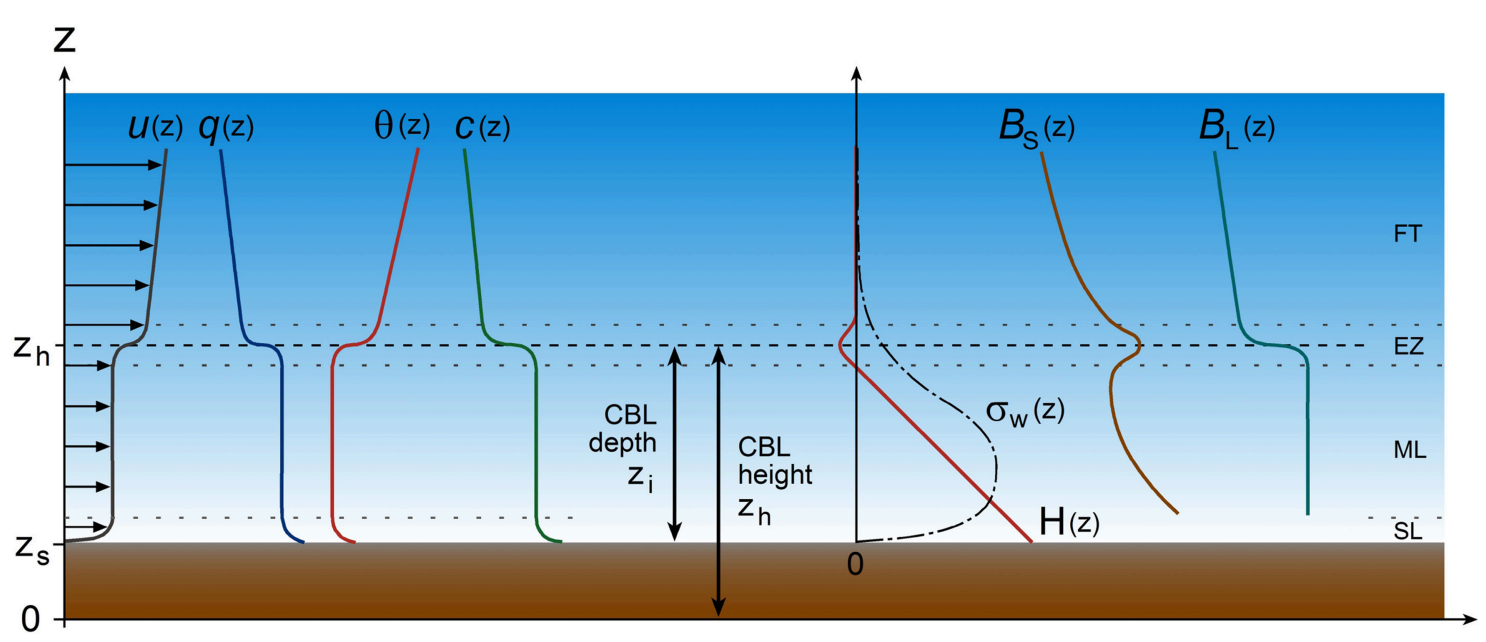

FIGURE 2 | Idealized vertical structure of the lower troposphere under daytime convective conditions over flat and homogeneous terrain, subdivided into the surface layer (SL), the mixed layer (ML), the entrainment zone (EZ), and the free troposphere (FT). The vertical profiles represent wind velocity $u(z)$, specific humidity $q(z)$, potential temperature $\theta(z)$, air pollutant concentration $c(z)$, vertical turbulent sensible heat flux $\mathrm{H}(\mathrm{z})$, standard deviation of turbulent vertical velocity fluctuations $\sigma_{\mathrm{W}}(\mathrm{z})$, and backscatter signal intensities $\mathrm{B}_{\mathrm{S}}(\mathrm{z})$ from a sodar and $\mathrm{B}_{\mathrm{L}}(\mathrm{z})$ from a lidar. $\mathrm{z}_{\mathrm{S}}$ is the terrain height while $\mathrm{z}_{\mathrm{i}}$ and $\mathrm{z}_{\mathrm{h}}$ are the depth and the height of the $\mathrm{CBL}$, respectively. 
investigating the extent to which the CBL follows the terrain, it is appropriate to use the CBL height $z_{h}$, which is the height of the CBL top above mean sea level (MSL). If the terrain elevation $\mathrm{z}_{\mathrm{s}}$ is known, CBL height and CBL depth can be easily converted (Figure 2).

\section{CBL HEIGHT IN MOUNTAINOUS TERRAIN}

While the focus of this review is on the thermal structure of the $\mathrm{CBL}$ in mountainous terrain, the accompanying flow structure is of critical importance for the investigation of CBL heights. Flows that interact with CBL heights over hilly and mountainous terrain are often thermally driven and occur at a wide range of spatial and temporal scales (Whiteman, 1990; Zardi and Whiteman, 2013). Diurnal winds in valleys generally blow up the valley during daytime and down the valley during nighttime, termed upvalley and downvalley flows, respectively. Similarly, flows blowing up the slope during daytime and down the slope during nighttime are termed upslope and downslope flows, respectively. Large-scale thermally driven wind systems in mountainous areas are also known as mountain-plain wind systems and have been given various names for basin and plateau topographies. The mountain-plain wind system with near surface winds directed from a plain toward a plateau has been called a plain-to-plateau wind (Mannouji, 1982). Similarly, a mountain-plain wind system carrying air from the plains over a mountain range into a basin has been called a plainto-basin wind (Kimura and Kuwagata, 1993). The importance of these thermally driven wind systems for CBL heights over mountainous terrain will be demonstrated throughout the next sections that consecutively discuss CBL heights over slopes, valleys, basins and plateaus, and entire mountain ranges. Many of the reviewed observational studies that have contributed to recent understanding of CBL heights in mountainous terrain did not employ vertical soundings of turbulence parameters. Conceptual diagrams of CBL top behavior shown for these terrain settings therefore use the base of the elevated stable layer (or 'capping inversion') as commonly available criterion for CBL height detection.

\section{Slope CBL}

In the spectrum of atmospheres present over mountainous terrain, the slope atmosphere constitutes the smallest spatial scales (Figure 1). However, many of the CBL structures observed over mountainous terrain originate in the slope CBL which therefore, deserves much attention. The most striking feature of the CBL over slopes during daytime fair weather conditions is the development of thermally induced upslope winds. These upslope flows interact with turbulent convection and affect CBL heights, not only over slope locations but also over adjacent plain, valley, basin, plateau, and ridge locations. The importance of the slope atmosphere in affecting other parts of the mountain atmosphere makes the slope CBL an appropriate starting point in this review paper. To this end, we consider an idealized timeheight cross section of the various wind systems at a particular location over a slope on a fair weather day (Figure 3). Vertical potential temperature profiles at selected times of the day are

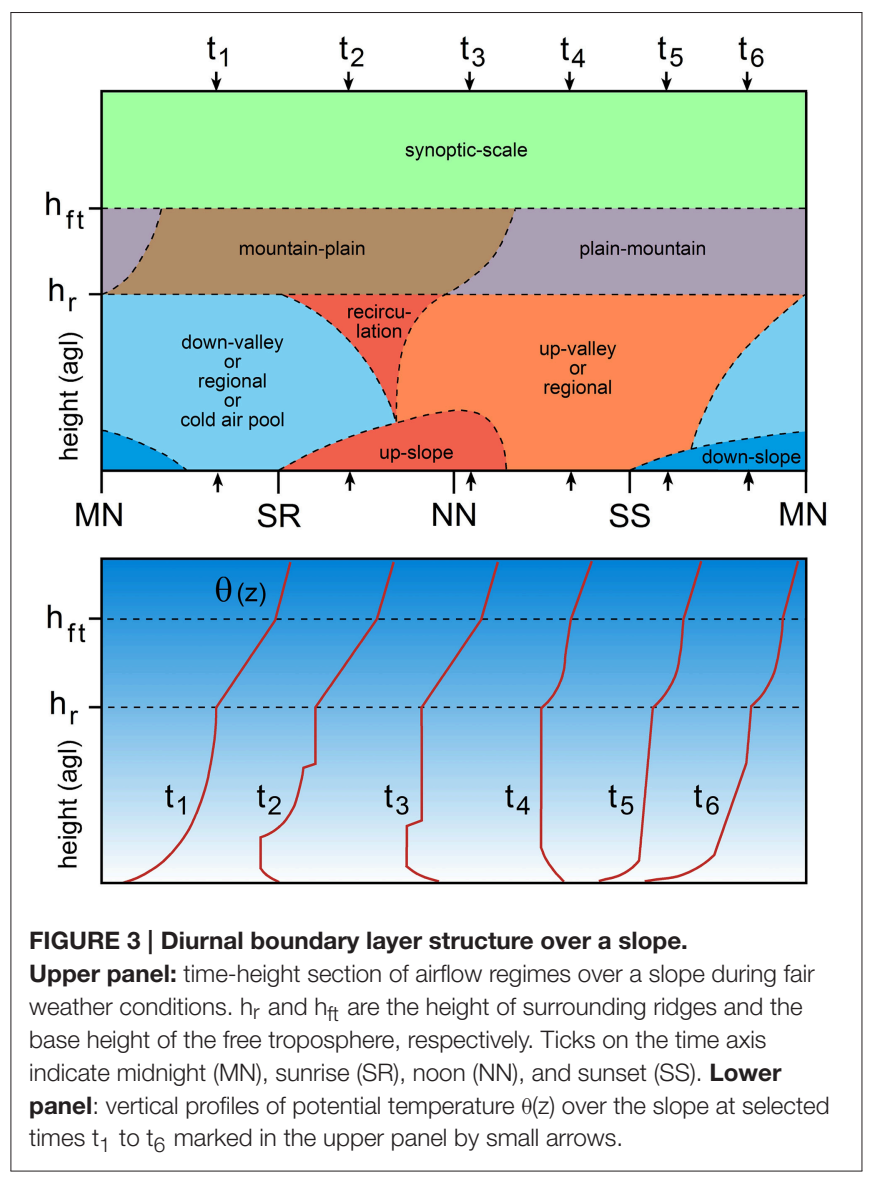

also shown to illustrate boundary layer structure evolution. Note that these profiles are idealized, with, for example, the presence of an elevated stable layer at ridge height limiting slope CBL growth to greater heights. Just before sunrise, the near surface airflow over slopes is typically down-valley (in draining valleys), but stagnant conditions could also occur (in pooling valleys or enclosed basins). Downslope flows typically become weaker during the course of the night and are often imperceptible just before sunrise (e.g., Whiteman, 1990). The importance of these nighttime downslope winds for daytime CBL development arises because of the associated overnight accumulation of cold air in valleys and basins. The strong stability in these cold air pools leads to rather slow CBL growth rates over valley and basin floors and lower sidewalls after sunrise. In contrast, only shallow and weak inversions are usually found over upper slopes and mountain tops at sunrise, since most of the cold air forming during the night drained to lower terrain. In Figure 3, we assume that toward the end of the night $\left(t_{1}\right.$ in Figure 3$)$ the surface based inversion over the valley center or the adjacent plain exceeds the height of the slope location. If the valley inversion top were below the slope location, downslope winds would still be present at the surface and the average stability up to the ridge height $h_{r}$ would be much smaller.

After sunrise an upslope wind layer develops over insolated slopes with a return circulation toward the valley center aloft $\left(t_{2}\right.$ in Figure 3). After erosion of the nocturnal valley inversion 
layer, up-valley winds or other regional wind systems become dominant in the neutrally or weakly stably stratified layer between the upslope wind layer and ridge top. For the common situation with a stably stratified ambient atmosphere, upslope wind conditions are associated with an along-slope gradient in potential temperature in the slope wind layer. The upslope winds are therefore characterized by cold air advection which often leads to the phenomenon of undercutting (Kossmann et al., 1998), also known from cold air advection associated with other thermally induced wind systems such as sea breezes (e.g., Blumenthal et al., 1978; McElroy and Smith, 1991; Lu and Turco, 1994). The cold air advection counteracts heating due to the turbulent and radiative heat flux convergences over the slopes. This negative feedback mechanism limits the intensity of the daytime slope wind circulation (Prandtl, 1942) and represents a possible reason for non-stationary upslope flow behavior (Vergeiner and Dreiseitl, 1987; Hunt et al., 2003; Bastin and Drobinski, 2005). The along-slope advection of cold air ( $t_{3}$ in Figure 3) leads to the formation of a stable layer near the top of the slope wind layer (Kossmann et al., 1998). Consequently, the CBL depth over slopes often coincides with the depth of the slope wind layer (e.g., Segal et al., 1987; Ye et al., 1987; Kuwagata and Kondo, 1989), similar to how the internal boundary layer depth in coastal areas coincides with the depth of the sea-breeze layer (Johnson and O'Brien, 1973; Mizzi and Pielke, 1984; Physick et al., 1989). Non-stationary upslope flows, in their turn, result in highly variable CBL depths over slopes, with the possible occurrence of sudden jumps to higher or lower values (Kossmann et al., 1998).

During the course of the afternoon, slope flows become less well-defined and are often overpowered by the up-valley or regional winds due to downward turbulent momentum transfer ( $t_{4}$ in Figure 3 ). Vigorous mixing induced by thermals originating at the surface can also make horizontal temperature gradients and resulting advective transport less important than turbulence-induced convective transport. This convective mixing results in a deepening of the $\mathrm{CBL}$ over the slopes similar to flat terrain.

After sunset, surface cooling leads to the formation of downslope winds in the developing surface inversion layer, while up-valley and/or regional winds still prevail aloft in a residual layer ( $t_{5}$ in Figure 3). Later, up-valley winds will cease and may be replaced by down-valley winds ( $t_{6}$ in Figure 3 ). The mountain atmosphere above ridge height is characterized by the presence of a large scale, diurnally reversing mountain-plain wind system which is often masked by strong synoptic flows and therefore may be too weak to be observed. In the presence of significant heating of the elevated terrain, the atmosphere at ridge top height becomes destabilized during the day $\left(t_{4}-t_{6}\right.$ in Figure 3 ) as was already observed by Ekhart (1948).

Observations and models have shown many different scenarios for the behavior of thermal and dynamical structures over sloping surfaces (Figure 4). The classical daytime slope flow regime in valleys by Wagner (1932) is shown in Figure 4A, where the upslope flows detach from the slope without preference to any particular along-slope location. This leads to a more or less continuous detrainment from the slope flow layer and
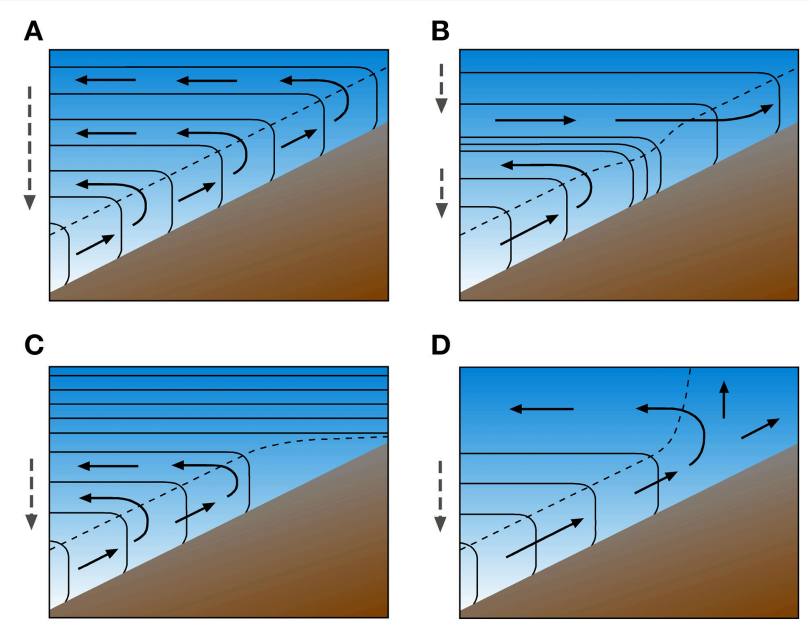

D

E
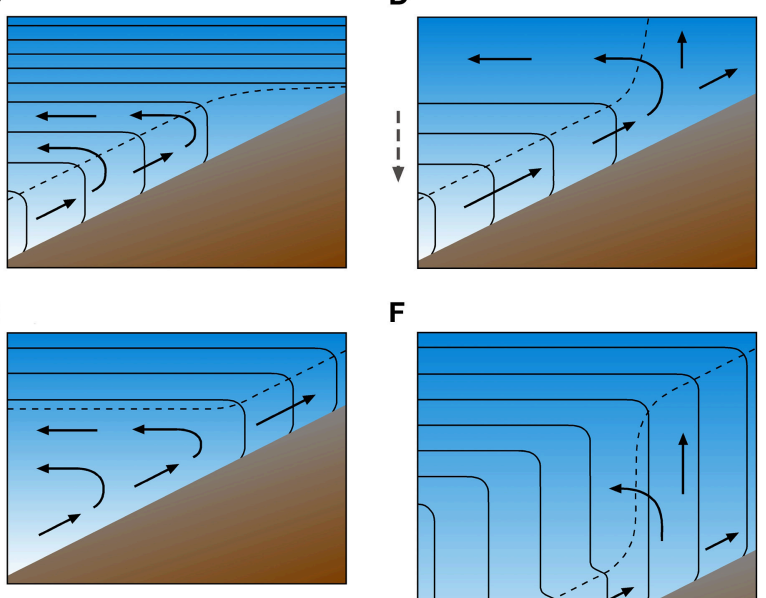

G

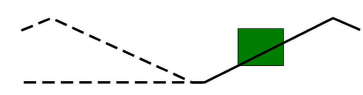

$\mathbf{F}$

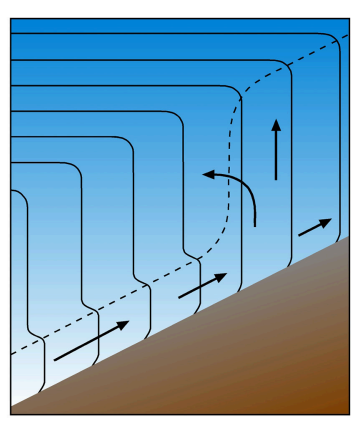

FIGURE 4 | Conceptual models of slope wind circulation and CBL structure over a slope on a fair weather day. The green box in (G) on the lower left shows the location of the depicted cross section over a slope facing a valley or a plain. Thin solid and dashed lines represent isentropes and the top of the turbulent $\mathrm{CBL}$, respectively. Solid and dashed arrows indicate the airflow and the compensating subsidence over the valley center or over the plain. (A) Slope wind circulation with homogeneously stratified atmosphere over the valley center or plain. (B) Two staggered slope wind circulations separated by a strongly stratified layer. (C) Slope wind circulation limited to lower slope due to strongly stratified layer aloft. (D) Slope wind venting into neutrally stratified layer aloft. (E) Recirculation of slope winds within the turbulent CBL. (F) Jump in $\mathrm{CBL}$ depth due to erosion of the inversion above the upslope wind layer and a deep neutrally stratified layer aloft.

compensating subsidence over the valley center. This conceptual model was extended by Vergeiner (1982). Based on energetic considerations he concluded that upslope winds prefer to detach from the slope at elevations where particularly strong inversions are present in the valley atmosphere (Figure 4B). This process favors the existence of two or more stacked slope wind circulations and associated entrainment and detrainment to and from the slope wind layer. A recent modeling study by Wagner et al. (2014) showed that in deeper valleys, stacked slope wind circulations are more likely to occur. Furthermore, in laboratory experiments, Princevac and Fernando (2008) show that the upslope flow is forced to detach from the slope as the flow approaches an extremely strong inversion (Figure 4C). In a conceptual model of valley inversion breakup by Whiteman (1982), the upslope winds vent into a neutral or weakly stratified layer above the valley inversion built up during the previous 
night (Figure 4D). This means that slope flow detachment in this conceptual model occurs at elevations above the (subsiding) valley inversion. Reuten et al. (2005) provide observational evidence that scenarios where the return circulation penetrates into the inversion capping the upslope wind layer, might be typical only for cases with shallow CBLs in the valley or over the adjacent plain. Later in the day, when the CBL in the valley or over the plain exceeds a critical height (about half the height of the nearby ridges), the return circulation occurs within the turbulent valley/slope CBL (Figure 4E). In these situations, turbulent vertical momentum transfer within the CBL mixes the upslope wind layer with the return circulation aloft, possibly weakening or destroying the organized slope wind circulation. An upslope flow re-circulation contained within the CBL has also been found in numerical model simulations (e.g., Demko and Geerts, 2010). Another type of CBL behavior observed over a slope is described by Kossmann et al. (1998) for cases with a terrain following CBL top or residual layer top (Figure 4F). In such situations with near- neutral stratification above the slope flow layer, any weakening of the along-slope cold air advection due to disturbances in the upslope flow can lead to turbulent erosion of the inversion above the slope wind layer. This results in a sudden increase of CBL height from the top of the slope wind layer to the top of the near-neutral layer. Re-intensification of the upslope winds might then re-establish an inversion above the slope wind layer due to undercutting. During the course of the day, these mechanisms can lead to significant oscillations in CBL depth above slopes and in the associated vertical convergence of turbulent sensible heat fluxes (Kossmann et al., 1998). In summary, a wide range of different slope flow regimes exists that interact in various ways with the $\mathrm{CBL}$ height. Investigation of these interactions and the relevant processes is difficult, due in large part to the presence of ambient synoptically-driven flows which mask the weak return flows.

The characteristics of the upslope flow circulation have strong implications for the dispersion of air pollutants. Return flows within the turbulent CBL cause trapping of pollutants within the valley (Reuten et al., 2005). On the other hand, air pollutants transported by return circulations above the turbulent CBL can be re-entrained into the valley $\mathrm{CBL}$ due to subsidence over the valley (Whiteman and McKee, 1978), or vented into the free troposphere (see Section "Summary and Directions for Future Research"). Subsidence induced by the return flow can also result in a decrease of the CBL depth near a mountain base (Section "Mountain Range CBL") and affects CBL development in valleys as illustrated in the next section.

\section{Valley CBL}

CBL evolution can be notably different from valley to valley depending on the relative importance of surface heating, subsidence, and other processes in valley atmospheres. A schematic illustration of vertical potential temperature profiles at four consecutive times ( $\mathrm{t} 0-\mathrm{t} 3$ ) observed over valley floors under a variety of conditions is shown in Figure 5. Note that these profiles
A

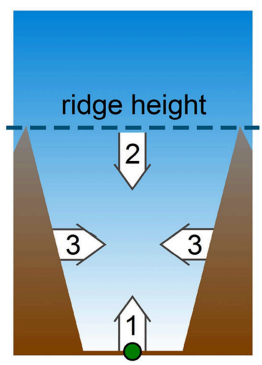

E

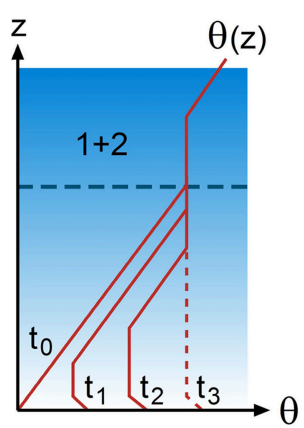

B

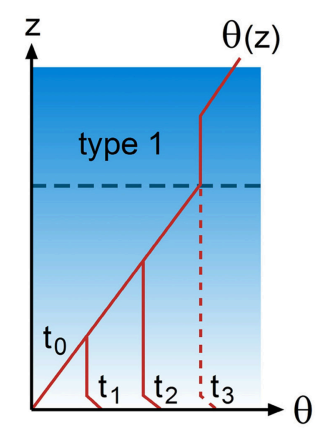

F

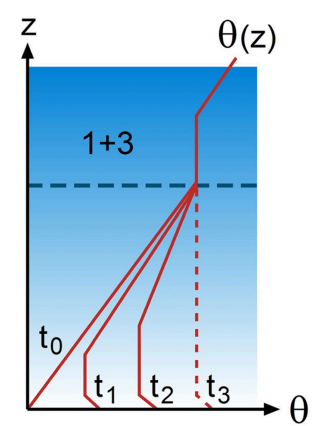

C

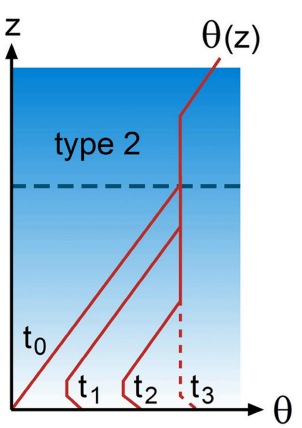

G

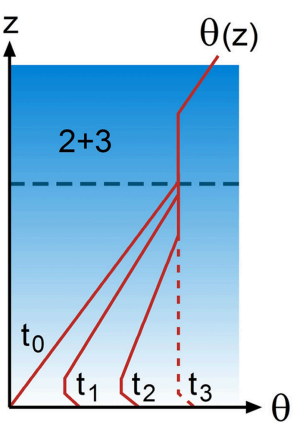

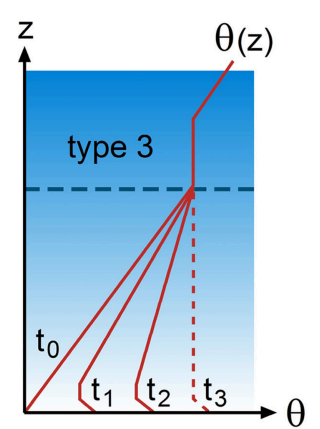

H

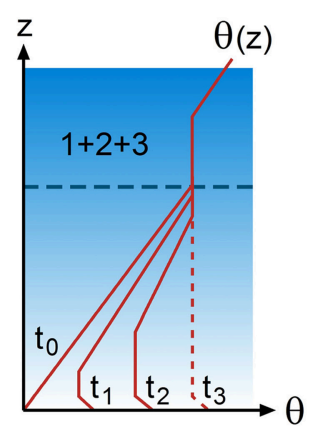

FIGURE 5 | Conceptual models of CBL development in the center of a valley. The location of the idealized vertical profiles of potential temperature $\theta(z)$ at times $t_{0}-t_{3}$ is shown by the green dot in (A). (B-D) in the top row show three basic types of CBL development, while (E-H) in the bottom row show four combinations (mixed types) of the three basic types. The main direction of heat supply and erosion of the valley inversion for the three basic types are illustrated by numbered arrows in (A): arrow 1: turbulent heat flux from the valley floor (type 1); arrow 2: subsiding valley inversion (type 2); and arrow 3: heating from valley side walls by slope wind recirculation (type 3 ). The dashed line at $t_{3}$ indicates that a well-mixed potential temperature profile throughout the entire valley atmosphere does not necessarily occur. 
are idealized with the assumption that nighttime cooling takes place up to ridge height while a neutrally stratified layer capped by an inversion is present above the valley atmosphere. Three basic patterns of CBL development in valleys have been observed in which the patterns are dominated by either surface heating (type 1, Figure 5B), subsidence heating (type 2; Figure 5C), or horizontal heat transfer from the heated slope surface (type 3; Figure 5D). The first two patterns are based on simultaneous observations of the wind- and temperature structure in various deep Colorado valleys primarily during the morning transition period (Whiteman, 1982; Whiteman and McKee, 1982). In type 1 , CBL growth is similar to that over flat terrain while in type 2 the CBL height remains at a rather constant level until the top of the early morning temperature inversion descends to the top of the underlying CBL. The rate of subsidence of the temperature inversion over the valley center is determined by the rate of withdrawal of air mass from the valley by upslope flows (e.g., as indicated in Figure 4D). The subsiding valley inversion contributes locally to the heating of the valley atmosphere (Brehm and Freytag, 1982; Whiteman, 1982; Kuwagata and Kimura, 1995) and thus has an important impact on valley CBL evolution. Type 2 typically occurs in valleys during winter time or in situations with small surface sensible heat fluxes at the valley floor when the stability in the valley atmosphere remains weakly stable throughout the day. Many of the valleys studied by Whiteman (1982) showed a CBL evolution that resembled a combination of the type 1 and type 2 patterns.

Upslope flows play a different role in the daytime heating of the boundary layer over valleys compared to the heating of the slope atmosphere. While upslope flows indirectly enhance local heating of the atmosphere in the valley center by subsidence, these flows also counteract daytime heating over slopes as a result of along-slope cold air advection (see previous section). However, both cold air advection and subsidence heating tend to counteract $\mathrm{CBL}$ growth. Type 3 (Figure 5D) illustrates a situation with surface heating but without subsidence. This pattern without a deep CBL reaching ridge height is often observed in Alpine valleys (e.g., Freytag, 1985; Furger et al., 2000; Rotach et al., 2004) and valleys in the Andes mountains (Kalthoff et al., 2002; Khodayar et al., 2008). Note that a wellmixed potential temperature profile throughout the entire valley atmosphere, as indicated by the dashed lines at $t_{3}$ in Figure 5, does not necessarily occur, especially if surface heating is weak. The absence of well-mixed potential temperature profiles in the valley atmosphere has been attributed to heat transfer from the valley sidewall surfaces to the valley atmosphere (De Wekker, 2002; Serafin and Zardi, 2010a) and to strong along-valley advection of cold air, due to e.g., a lake or coast near the valley entrance (Bischoff-Gauss et al., 2008; Khodayar et al., 2008; Laiti et al., 2014). Model simulations by Wagner et al. (2014, 2015) show that, compared to wide reference valleys, daytime along-valley cold air advection is stronger for deeper valleys, for valleys narrowing in the up-valley direction, and for valleys with a strongly inclined valley floor. During the calm period in the morning transition phase between the erosion of downvalley winds in the remaining elevated valley inversion and the onset of up-valley winds (with associated cold air advection), a relatively deep valley CBL can develop for a short period of time (Eigenmann et al., 2009).

Horizontal transport and mixing processes away from the slope surface were already hypothesized by Wagner (1932) and evidence of these processes can be seen, for example, in recent observations and simulations of aerosol transport in the Inn Valley (Gohm et al., 2009; Lehner and Gohm, 2010). Horizontal heat transfer from the slope to the valley center can weaken or strengthen stability in the valley inversion, depending on the along-slope variations of the slope wind re-circulation intensity, of the heat supply provided by valley sidewall heating, and of the valley air volume with height (De Wekker et al., 2005; Schmidli, 2013). Observations and model results indicate that the amount of sensible heat available for heating the slope boundary layer generally increases with elevation up to the snow line (Brehm, 1986; Mannstein, 1989; Wenzel et al., 1997; De Wekker et al., 1998; Noppel and Fiedler, 2002) which can be attributed to decreasing soil moisture availability with elevation. Although the diabatic heating from the slopes increases with elevation, the volume of air to be heated in a horizontal layer of the valley atmosphere also increases with elevation. This volume or topographic amplification factor (TAF) effect depends on the area height distribution of the valley sidewalls (Steinacker, 1984; Müller and Whiteman, 1988; Liechti and Neininger, 1994) and can contribute to a decreasing heating rate with increasing elevation in the valley atmosphere. Recent model studies confirm the influence of the volume effect, but also find that the magnitude of the heating rate of the valley atmosphere strongly depends on along-valley cold air advection and slope wind induced export of heat from the valley to heights above ridge level (Schmidli and Rotunno, 2010; Schmidli, 2013; Leukauf et al., 2015).

Type 1 CBL development in valleys is characterized by a steady growth in $\mathrm{CBL}$ depth during erosion of the valley inversion, while types 2 and 3 exhibit a slow growth in the initial phase, followed by a sudden increase in CBL depth at the time when erosion of the inversion is completed ( $t_{3}$ in Figure 5$)$. In many cases however, the three basic processes described above act simultaneously and over a range of spatial and temporal scales which can lead to any of the other patterns sketched in Figures 5E-H. In the absence of surface heat flux observations and multiple soundings in time and/or space, it is difficult to isolate the underlying processes of importance in a certain situation. The actual time from $t_{0}$ to $t_{3}$ in Figure 5 can be highly variably as well, depending on the atmospheric situation, valley surface characteristics, and season. In dry and broad valleys, $t_{3}$ in type 1 may be reached in a couple of hours while in deep valleys with abundance of vegetation and moisture, the vertical temperature structure may remain relatively stationary with a change from $t_{0}$ to $t_{1}$ that takes most of the day.

In contrast to the temporal evolution of CBLs in valleys, the spatial structure of CBL height and the associated wind and temperature fields along and across a valley have not been given much attention. In many studies, such as those where mass budgets are calculated, it is assumed that temperature- and wind structure is horizontally homogeneous across the valley and that the along-valley atmospheric structure is simple, with 
monotonically increasing/decreasing or constant flows along the valley (Freytag, 1987). Hewson and Gill (1944) presented temperature data on a cross section of the Columbia Valley near Trail, BC. Their aircraft observations showed that crossvalley differences in temperature of about $3-4^{\circ} \mathrm{C}$ can occur on a constant height surface due to unequal heating of the slopes. Similar observations were made by Hennemuth (1985) in a deep Swiss valley. Depending on the orientation of the valley, an asymmetric removal of temperature inversions in a high mountain valley can occur as observed by Kelly (1988). One would expect non-horizontal CBL heights across the valley in these situations (Figure 6A).

Along-valley CBL height structure is affected by areas of horizontal flow divergence or convergence and resulting compensating vertical motions along the valley. Freytag (1987) concluded from valley air mass budgets that compensating lifting and subsidence are strongest at the valley head and the valley mouth, respectively. Bianco et al. (2011) indeed found deeper valley CBLs near the valley head associated with deceleration of the thermally driven up-valley winds (Figure 6B). They hypothesized that these deeper CBLs were caused by lifting due to the convergence in the horizontal wind field in the valley, as was already observed by Freytag (1987). Similarly, compensating subsidence as a result of divergence at the valley mouth represents a mechanism for shallower CBLs at the valley mouth than at the valley head. Model simulations by Rampanelli et al. (2004) show that CBLs can be significantly deeper over the plain than in the valley where along-valley cold air advection counteracts CBL growth (Figure 6B). Spatial variability in cold air advection and varying heating rates along the valley as a result of the complex flow pattern also cause irregularities in CBL heights along the valley (De Wekker, 2002; Weigel et al., 2006). In other situations however, observed afternoon CBL heights are horizontally homogeneous along and across the valley despite the presence of complex and asymmetric flow patterns (Kuwagata and Kimura, 1995, 1997; De Wekker et al., 2005).

\section{A morning}

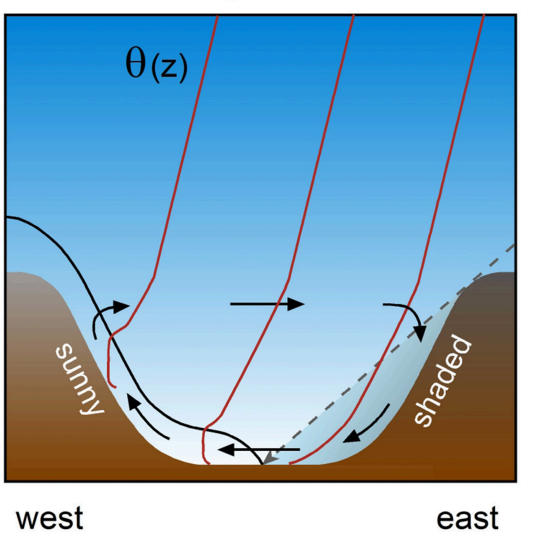

B early afternoon

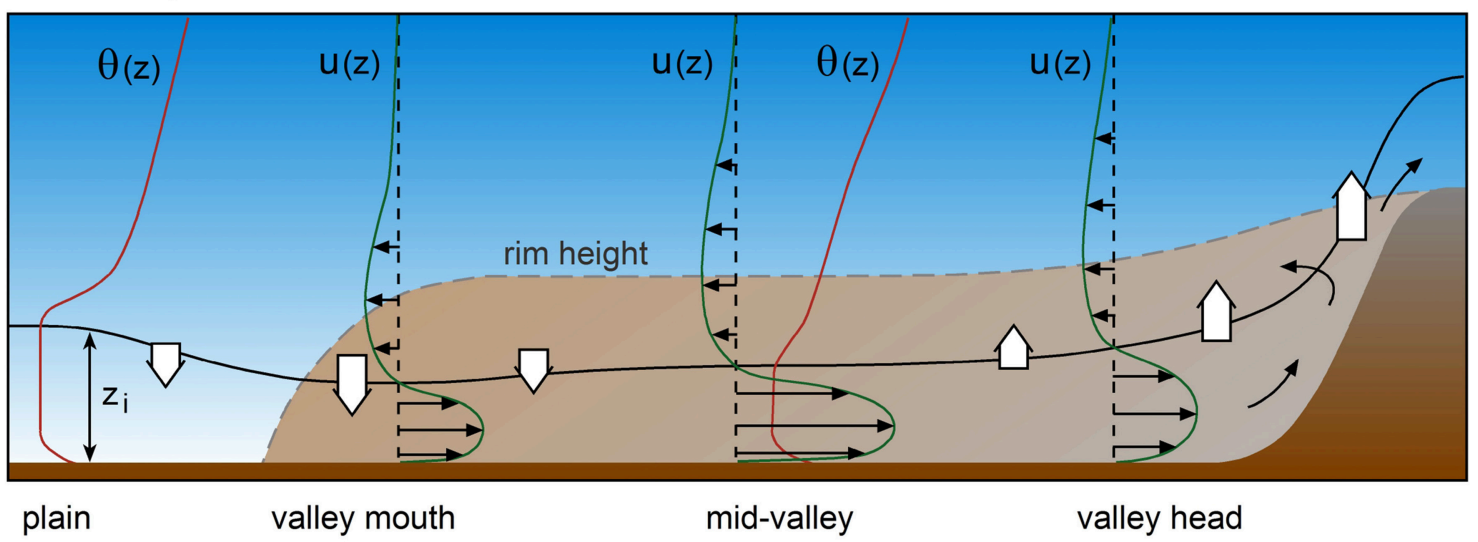

FIGURE 6 | Vertical cross sections of thermal wind circulation and CBL structure in a valley for (A) a cross valley section during the morning transition and $(\mathbf{B})$ an along valley section around noon or mid-afternoon. The solid black line indicates CBL depth $\mathrm{z}_{\mathrm{i}}$. Thin solid vectors indicate airflow components in the cross section direction. Red solid lines indicate vertical profiles of potential temperature $\theta(\mathbf{z})$. The dashed line in (A) indicates a sun ray which separates the sunny and shaded parts of the valley. Green lines in (B) indicate vertical profiles of the horizontal along-valley wind component with the horizontal black vectors depicting the direction of the flow. Curved black vectors and vertically pointing white arrows indicate subsidence or lifting at the CBL top caused by horizontal divergence or convergence of the along-valley airflow. 
Not only thermally driven flows but also dynamically driven flows can have an effect on CBL heights in a valley. For example accelerating/decelerating flows related to the varying intensity of channeled synoptic winds along a valley may be associated with decreasing/increasing CBL heights in along-valley direction due to effects of air mass conservation (Frank, 1997). In valleys with a curved valley axis, flows can cause asymmetric CBL heights across the valley (Weigel and Rotach, 2004). Also, channeling effects in these curved valleys can induce along-valley confluence or diffluence with along-valley differences in vertical motions that can enhance or suppress CBL growth (Kossmann and Sturman, 2003). Mountain waves traveling across the valley can also modulate the CBL height in valleys (Worthington, 2002).

\section{Basin and Plateau CBLs}

Basins and plateaus are common land forms in many parts of the world, influencing atmospheric motions that extend in scale from the local scale to thousands of kilometers. CBL development shows some degree of similarity in basins, valleys and over plateaus. The similarity with plateaus holds in particular for basins with an elevated basin floor and justifies a simultaneous discussion of basin and plateau CBLs.

Basins are concave land forms that, in contrast to valleys, are characterized by no or only a small number of low lying exits to surrounding terrain. Hence, upvalley flows are suppressed or non-existent in basins. The distinction between basins and valleys, however, can be somewhat arbitrary and there are basins where the low lying exits appear as side valleys where up and down valley flows are observed.

Any of the valley CBL development patterns (Figure 5) can also occur in basin atmospheres. In the absence of nighttime downvalley flows however, the early morning stability of the nearsurface air layer in basins may be enhanced compared to valleys. Cold air drainage from the surrounding slopes accumulates and stagnates in the basin (Wolyn and McKee, 1989; Whiteman et al., 2001). These cold air pools can delay CBL growth in basins compared to valleys. During daytime, the basin atmosphere warms up more than the surrounding plains due to the TAF effect. This basin warming can promote a plain-to-basin flow in the afternoon which crosses the mountain ridges and saddles surrounding the basin (Kimura and Kuwagata, 1993; Doran and Zhong, 1994; De Wekker et al., 1998; Regmi et al., 2003). The cold air advection associated with these thermally driven flows can have a large impact on the heat balance of the basin's atmosphere (Whiteman et al., 2000; Kossmann et al., 2002) and has been hypothesized to reduce or level off CBL growth in the afternoon (Muñoz and Undurraga, 2010). Especially in situations when basin CBLs are deep and extend above the surrounding ridges (Figure 7A), a sudden reduction in CBL height on the basin-side slope can occur after the onset of the plain-to-basin wind in the late afternoon (Figure 7B). The air quality in large cities that are situated in basin-like environments may be affected considerably by the plain-to-basin winds and their effect on thermal structure and CBL heights (Fast and Zhong, 1998; Kitada and Regmi, 2003; de Foy et al., 2005, 2006; Panday et al., 2009).

While plain-to-basin winds can potentially affect CBL heights in basins, CBL heights themselves could play a role in the initiation of these winds. Kimura and Kuwagata (1993) concluded from their observations and numerical modeling that plain-to-basin winds do not occur when the CBL height over the plains is lower than the mountain height. In contrast, De Wekker et al. (1998) showed that the CBL over the plains does not necessarily have to grow above the mountain height to generate plain-to-basin winds. The ratio of the CBL height over the plains to the mountain height may affect the plain-to-basin wind by modifying the horizontal temperature gradient above mountain height, but this ratio is not a critical factor for the development of the plain-to-basin wind. Even if the CBL height over the plains is lower than the mountain height, air can still be transported from the plains to the basin atmosphere.

The processes described above occur for basins with floors ranging in height between the surrounding plains height and the height of the surrounding ridges. As the basin floor becomes more elevated compared to the surrounding plains height, the elevated heating source (Hafner et al., 1987; Kuwagata and Sumioka, 1991) increases the horizontal temperature gradient between the plains and the basin, and plain-to-basin winds are expected to occur earlier. For basins, upslope winds over the inner slopes of the basin sidewalls counteract the regional-scale plain-to-basin winds (Figure 7A). With increasing basin floor height, the topography approaches that of a plateau and the plainto-basin wind becomes a plain-to-plateau wind. It is sometimes difficult to distinguish between elevated basins and plateaus. While some geographical regions are denoted as "plateau" they should probably be classified meteorologically as elevated broad valleys or basins. Studies such as those by Yang et al. (2004) over the Tibetan Plateau in Asia or by Egger et al. (2005) over the Altiplano of South America may therefore not necessarily be representative of "pure" plateau CBLs. On the other hand, the Mexico "basin" may be classified meteorologically as an elevated basin or even as a plateau (Bossert, 1997; Whiteman et al., 2000). Surface heating over plateaus is able to generate a relatively uniform CBL that extends over the elevated flat terrain. An observational study by Chen et al. (2013) confirms the occurrence of particularly deep CBLs over a large and high plateau (the Tibetan Plateau). For a distinct plateau CBL to develop, the horizontal scale of the plateau needs to be much larger than the typical CBL height. Plateau-like topographic features such as mesas and buttes may be too small to form their own CBLs as expected for large plateaus.

While observational studies of CBL development over welldefined plateaus are sparse, several numerical model studies have investigated the daytime evolution of CBLs over idealized plateaus (Mannouji, 1982; Gaertner et al., 1993; Bossert and Cotton, 1994; Zängl and Gonzalez Chico, 2006; Zawar-Reza and Sturman, 2006). These studies show that plateau CBLs often develop to a significantly greater depth than over the surrounding plains. Deep CBLs over plateaus represent an elevated heat source and are promoted by various effects such as the lower air density at higher elevations (higher heating rate of the air for a given heat flux convergence); weaker initial temperature stratification in the morning (cold air drains along the slopes away from the plateau surface toward the adjacent plains); meso-scale lifting caused by flow convergence of upslope winds developing over the slopes 
A

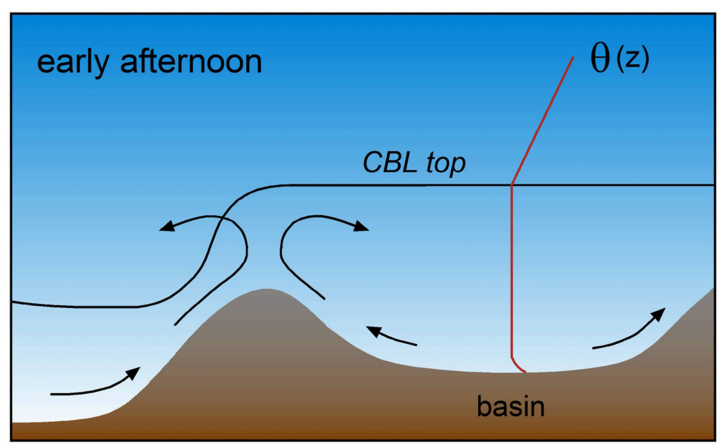

C

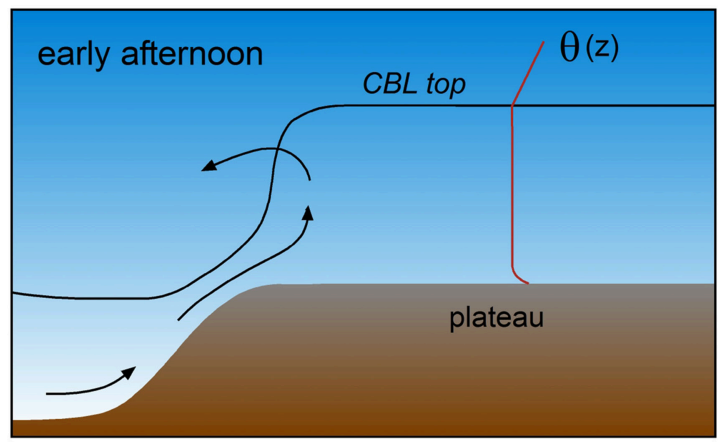

B

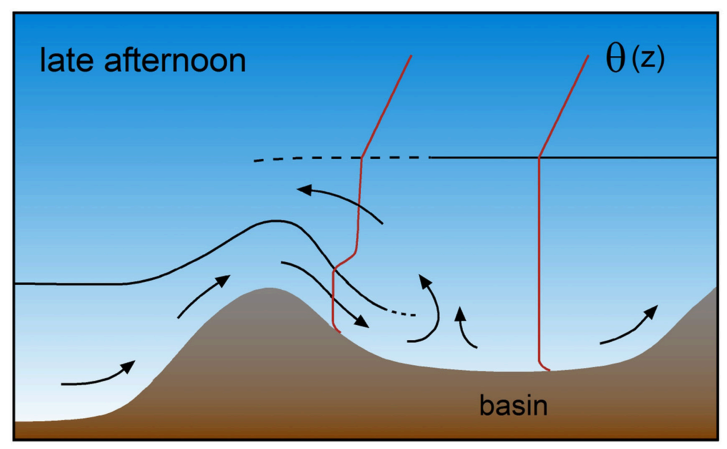

D

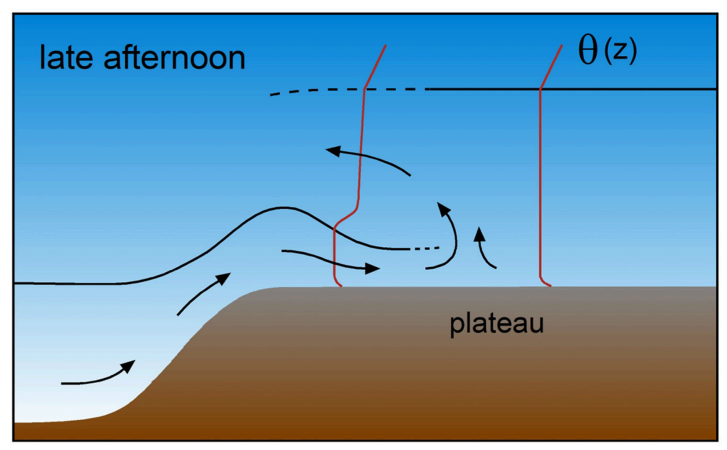

FIGURE 7 | CBL evolution in the afternoon over a mountain basin (A,B) and over a plateau (C,D). Airflow and vertical profiles of potential temperature $\theta$ are shown by arrows and red lines, respectively. The intrusion of cold air in the late afternoon leads to shallower CBLs over both basins and plateaus.

around the plateau; and sparse vegetation on high plateaus (high Bowen ratio).

The daytime evolution of plateau CBLs and the formation of a plain-to-plateau circulation are associated with the formation of a heat low over the plateau. The interaction of the heat low and the plateau-induced thermal circulation is important for the development of moist convection and strongly influences the onset of the summer monsoon over the Tibetan Plateau (Yanai et al., 1992). Model results by Zängl and Gonzalez Chico (2006) suggest that, despite the strong pressure gradients related to the presence of the heat low over the plateau, upslope winds entering the plateau ("inflow") only slowly propagate over the plateau (Figure 7C). During daytime, the organized structure of the upslope winds arriving on the plateau may be destroyed by strong turbulence over the plateaus, causing no or very slow propagation of the inflow. The combination of greater CBL depths and the higher terrain elevation over plateaus leads to strong horizontal temperature gradients and spatial variability in $\mathrm{CBL}$ height between the plateau and the surrounding plains (Figure 7C). The CBL height variability depends on factors such as the CBL height over the plains relative to plateau height, and the strength of the upslope flows and the plain-plateau circulation (Zängl and Gonzalez Chico, 2006). In the late afternoon when turbulent boundary layer convection weakens, the upslope winds turn into a density current which quickly propagates over the plateau (Figure 7D) and possibly collides with density currents generated at the opposing end of the plateau. Similar transitions from thermally driven winds under convective conditions to density currents in the nocturnal stable boundary layer have been observed in other topographic settings (Taylor et al., 2005).

\section{Mountain Range CBL}

While attempts have been made to describe the CBL height over valleys, slopes, basins, and plateaus individually, many observations show the integrative effect of the various processes responsible for the $\mathrm{CBL}$ height over any of these landscape features. The resulting CBL behavior over a mountain range can vary considerably in space and time. An intriguing question that is difficult to answer relates to the extent to which the CBL height follows the terrain. This question is important, for example, for air pollution transport over mountain ridges and for designing research aircraft flight strategies where mean and turbulence measurements are needed within the CBL.

A conceptual model based on observations of diurnal CBL behavior over an extended mountainous area with multiple ridges and valleys is shown in Figure 8. In the night and early morning before sunrise, cold air accumulates in the valleys resulting in an atmospheric stability in the valleys that is larger than over the mountain ridges (Figure 8A). After sunrise, the larger stability in the valleys leads to a slower growth of the CBL in the valleys than over the mountain ridges (Figure $\mathbf{8 B}$ ). After the valley inversion breaks up, the CBL develops faster in the valleys 


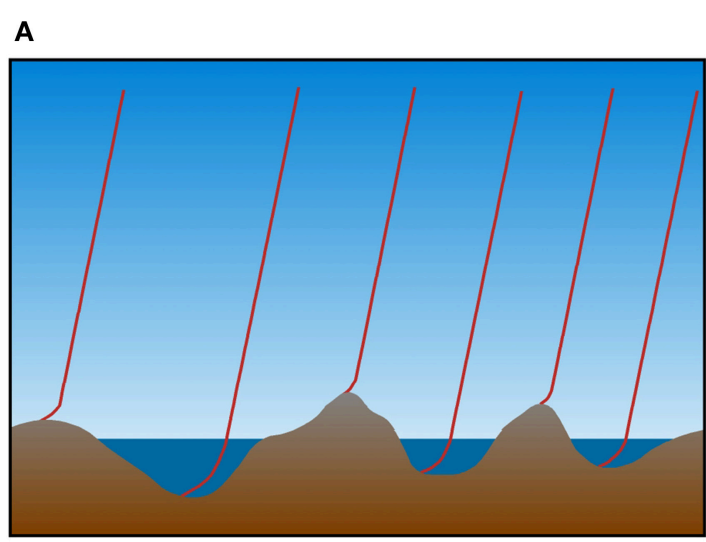

C

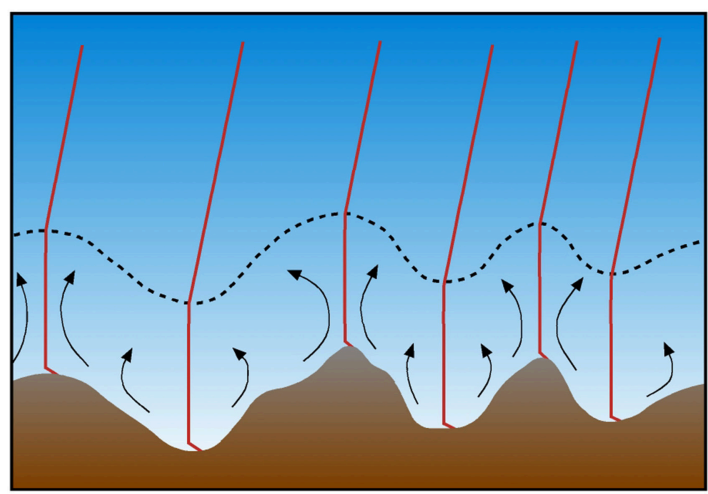

cold pool
B

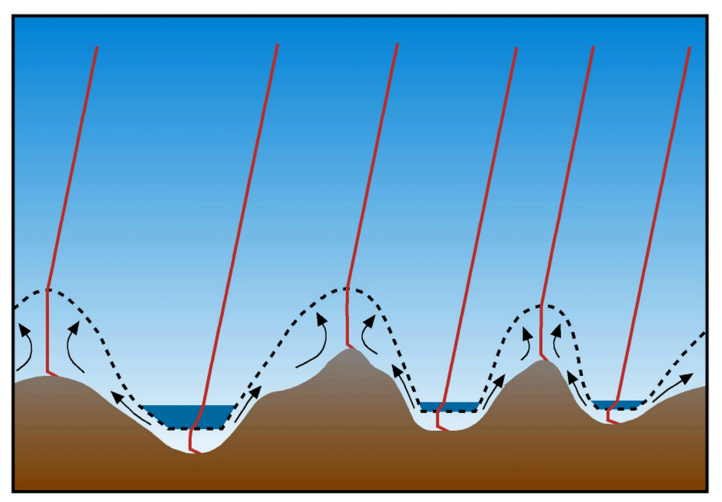

D

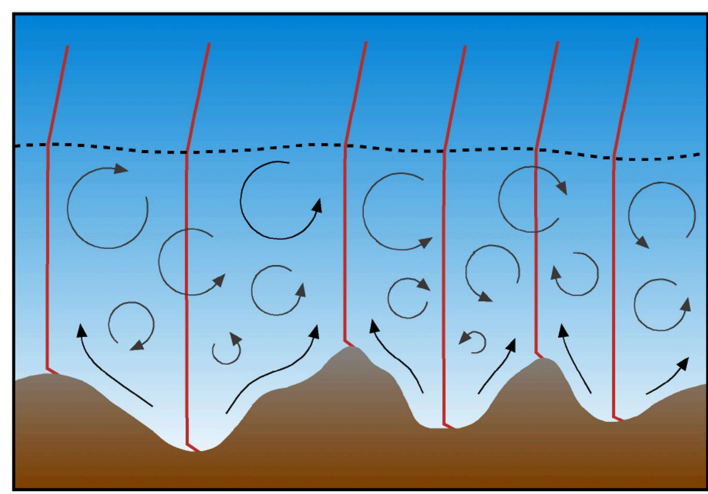

CBL top

airflow

FIGURE 8 | Conceptual model of the CBL development over mountainous terrain on a fair weather day (A) before sunrise, (B) in the early morning, (C) at noon, and (D) in the late afternoon. Dark blue shading indicates strongly stable stratification in nocturnal cold air pools. Arrows indicate thermal circulation and turbulent mixing. The dashed lines indicate the top of the turbulent CBL. Vertical profiles of potential temperature $\theta$ are shown in red at several locations along the cross section (after Fiedler et al., 1987).

than over the mountain ridges. Thus, in the early afternoon, the CBL top more or less follows the underlying terrain (Figure 8C). If the CBL in the valleys keeps growing faster there than over the mountain ridges, the top of the CBL eventually becomes level (Figure 8D). Under certain conditions, (almost) terrain following CBL tops as in Figure 8C will be the final status of CBL development before the onset of the evening transition with its stable boundary layer formation. The development of level CBL tops as in Figure 8D is more likely on long summer days with weak early morning stratification, and strong surface heating.

Studies that have alluded to the question of terrain-following vs. level CBL heights found the general pattern of CBL behavior described above from theoretical and numerical investigations (Stull, 1992; Walko et al., 1992; De Wekker et al., 1997; Gopalakrishnan et al., 2000; De Wekker, 2002; Tian and Parker, 2002; Tian et al., 2003; Catalano and Moeng, 2010) and from observations (Lenschow et al., 1979; Banta, 1982, 1984; Fiedler et al., 1987; Dayan et al., 1988; De Wekker, 1995; Binder, 1997; Kalthoff et al., 1998; Kossmann et al., 1998). In the observational studies, CBL heights were derived mostly from in-situ aircraft and radiosonde measurements during intensive measurement campaigns. Climatological analyses using radiosonde data have also been performed (Dayan et al., 1988; Liu and Liang, 2010) and show a variety of CBL height behaviors, from terrain-following to more level CBL heights.

While the studies mentioned above used vertical profiles of thermodynamic variables to determine CBL heights, other studies have used airborne backscatter lidar to derive information on CBL height variability over mountain ranges. From these lidar observations, Nyeki et al. (2000) and De Wekker et al. (2004) concluded that aerosol layer (AL) heights did not follow the underlying topography. However, while AL heights were initially equated with CBL heights, numerical simulations by De Wekker et al. (2004) showed large differences between CBL and $\mathrm{AL}$ heights over a large region in the Swiss Alps. In contrast to $\mathrm{AL}$ heights, $\mathrm{CBL}$ heights tended to follow the terrain but differences between AL and CBL heights became smaller in the course of the day. Differences in $\mathrm{AL}$ and $\mathrm{CBL}$ heights 
have been explained by the presence of orographically induced transport and mixing processes between the turbulent CBL and the overlying atmosphere which will be discussed in the next Section.

Closely related to the question of terrain-following vs. level CBL heights is how the CBL evolves over individual mountain tops and ridges and their direct surroundings. Various advective effects illustrated in Figure 9 are known to influence CBL heights in these terrain locations. As was pointed out in the conceptual model (Figure 8), a relatively deep CBL can exist over the mountain top early in the day because cold air forming during nighttime drains to lower terrain which results in shallower and weaker surface-based temperature inversions at sunrise over mountain ridges and peaks than over surrounding valleys or plains. Such "bulging" CBLs over mountain tops and ridges have been observed in several field studies that were mostly performed to investigate dry convection over mountain tops and ridges preceding moist convection later in the day (e.g., Braham and Draginis, 1960; Fosberg, 1967, 1969; Raymond and Wilkening, 1980, 1982; Demko and Geerts, 2010). Many of these studies do not explicitly address variability of CBL heights but from their published data, some information about CBL heights can be derived. In general, the studies show that the bulging of the CBL over the highest elevations is caused by heating of the slopes and ridgetops and a resulting "convection core". Lifting generated by upslope winds can enhance this bulging CBL height behavior (Figure 9A), while background winds forcing the convective core to move downwind of isolated mountain tops can also cause a wavelike pattern of the CBL height (Fosberg, 1969). Banta (1982) and Kirshbaum (2011) observed and modeled a lee side convergence zone induced by upslope flows on the leeside of the mountains. These convergence zones can result in an increased spatial variability of CBL height with increased heights at the convergence zones. While convective cores appear to be attached to terrain features, Banta (1982) speculated the transient (non-stationary and short-lived) nature of these increased CBL heights at the convergence zones. Transient CBL height features associated with thermal activity over mountains and sinking air motions adjacent to them, have also been found in the form of relatively low $\mathrm{CBL}$ heights (depressed CBL heights) near mountain bases (Figure 9B; De Wekker, 2008; Serafin and Zardi, 2010b).

Other observations indicate a CBL over the mountain top that is thinner than the CBL over adjacent regions, indicating a $\mathrm{CBL}$ that does not follow the terrain but is rather level. Asimakopoulos et al. (1980, 1994) used data from sodar measurements and radiosonde measurements on a $1000 \mathrm{~m}$ high isolated mountain top location to show that there is minimal or no effect of the orography on the height of the CBL in some situations (i.e., a level CBL top) while in other situations the capping inversion over the mountain top decreases in height. They argued that during convective conditions with $\mathrm{CBL}$ heights exceeding the mountain height and with moderate wind speeds, a decrease in CBL height can be explained by a speed-up of the winds over the mountain top due to the Bernoulli effect (Figure 9C). Relatively shallow CBLs can also exist over
A

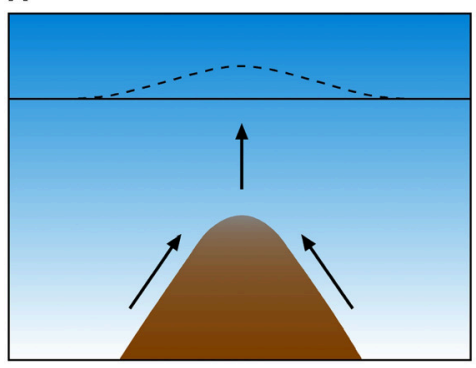

D

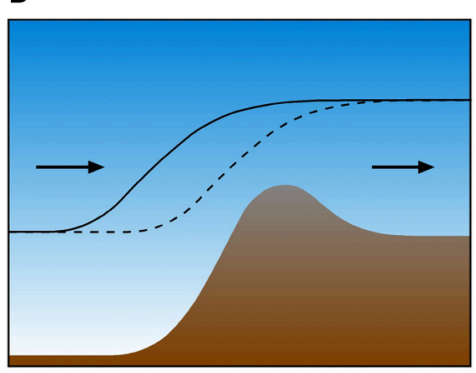

B

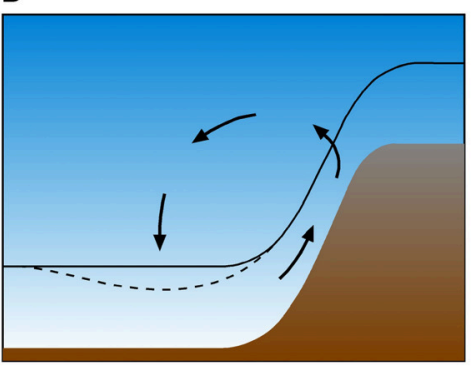

E
C

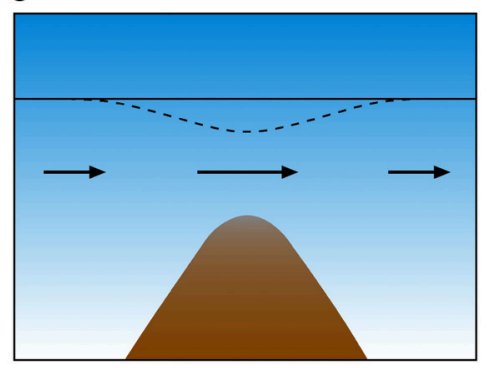

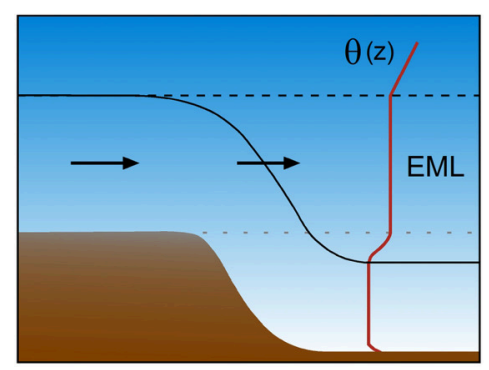

FIGURE 9 | Schematic illustration of advective effects on CBL structure over mountainous terrain. (A) Lifted CBL top over a ridge due to convergence of upslope winds, (B) depressed CBL at a mountain base due to subsiding return circulation of a slope wind system, (C) lowered CBL top over a ridge due to Bernoulli effect, (D) depressed CBL over a ridge due to horizontal advection of a tilted CBL top, and (E) formation of an elevated mixed layer (EML) over a valley due to horizontal advection of a CBL formed over high windward terrain. The solid and dashed black lines indicate the top of the CBL before and after considering the advective effect. Arrows illustrate the airflow. 
mountain tops because of the exposure of mountain tops to cold air advection due to upslope winds and because of the advection of a capping inversion by the mean horizontal wind (Figure 9D; Kossmann et al., 1998). Furthermore, shallow mountain top CBLs are favored by the small terrain surface area at mountain top height, which is limiting the total surface heat flux available for CBL growth at the mountain top (De Wekker, 1995).

Over mountain ranges in dry climates such as over the continental mountainous terrain in western North America, deep and level afternoon CBLs exceeding 2-3 km in depth are frequently present (Cramer and Lynott, 1961; Holzworth, 1964; Cramer, 1972; Norton and Hoidale, 1976; Raymond and Wilkening, 1980). Occasionally, the warm air produced in these deep CBLs above a mountain range can be advected off the mountains into the surrounding atmosphere. These advected CBLs can sometimes be detected over the adjacent plains (Figure 9E) where they form elevated mixed or residual layers that can affect air quality (Steinbacher et al., 2004) and the development of afternoon thunderstorms (Arritt et al., 1992; Stensrud, 1993).

In summary, a range of spatial CBL top behaviors is observed which can be organized in four distinct patterns (Figure 10): bulging $\mathrm{CBL}$ heights over mountain tops and ridges and suppressed CBL growth in valleys, also referred to as hyper terrain following (Figure 10A), terrain-following (Figure 10B) and level (Figure 10C) CBL heights, and lower CBL heights over mountain tops and ridges than over adjacent terrain, also referred to as contra terrain following (Figure 10D).

General conclusions regarding circumstances under which different behaviors occur have seldom been made, but factors such as atmospheric stability, synoptic wind speed, and vertical and horizontal scales of the orography are found to be important. A theoretical study on factors determining the spatial behavior of the CBL top was conducted by Stull (1992). In this study, an equation for the tendency of the CBL top to become more horizontal in the course of the day was derived by applying the mass conservation equation for a CBL over hilly terrain. Advection, entrainment and friction were found to make the CBL follow the terrain while gravitational forces tend to make the top of the CBL more level. Stull's theory shows further that deeper CBLs are less terrain following than shallower ones and that the mixed layer top is less level over orographical features that have a larger horizontal extent. These latter implications of the theory are generally corroborated by the observations mentioned earlier and were also quantified by Kalthoff et al. (1998) using a scale analysis.

The role of advection in making the CBL height more or less terrain following is not entirely clear but its potential importance is recognized (e.g., Kossmann et al., 1998). Figure 9 showed that there is a strong interaction between the flows that are responsible for the horizontal advection and the CBL height. For example, the shallow $\mathrm{CBL}$ depth over a mountain ridge illustrated in Figure 9D is caused by advective effects and will tend to make the CBL height more level (De Wekker, 1995; Kossmann et al., 1998). However, Stull (1992) assumes isentropic flows which makes the top of the CBL follow the orography more closely with

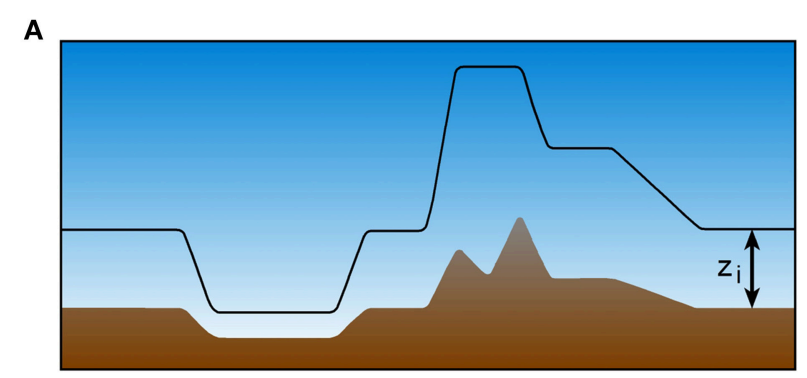

B

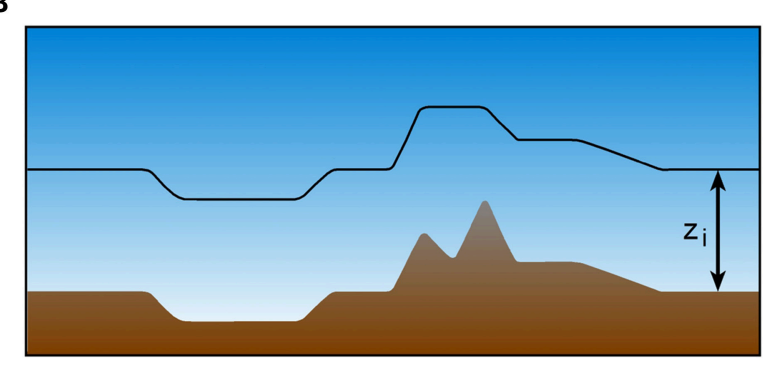

C

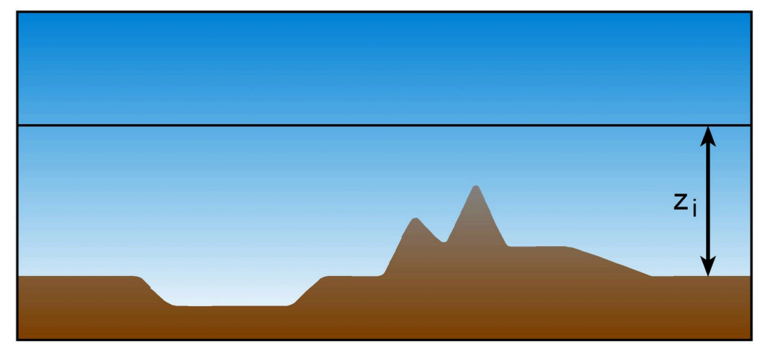

D

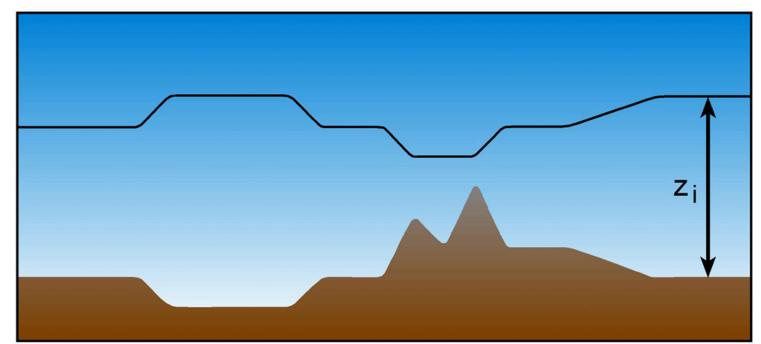

FIGURE 10 | Schematic illustration of four types of CBL top behavior. (A) Hyper terrain following, (B) terrain following, (C) level, (D) contra-terrain following (after Stull, 1992). The solid black line indicates CBL depth $z_{i}$.

increasing wind speed. Stull's (1992) theoretical model excludes effects of thermally driven circulations and therefore is unable to describe the under-cutting by thermal circulations that was shown previously (Section "Slope CBL") to play an important role in CBL height behavior over slopes.

De Wekker (1995) and Binder (1997) proposed the use of an effective sensible heat flux for use in growth rate models that implicitly takes into account the effects of advection, orography shape and other effects that influence CBL growth over mountain ridges. The magnitude of the calculated effective sensible heat flux that would lead to comparable CBL growth over homogeneous terrain, was only about $20 \%$ of the sensible heat flux observed 
at the surface but the relative contributions of individual effects were unclear.

Even though several studies have now investigated meteorological and terrain effects on CBL growth over mountain ridges, conclusive and general statements are still difficult to make.

\section{VERTICAL TRANSPORT AND MIXING BETWEEN THE CBL AND THE FREE TROPOSPHERE}

Understanding vertical transport and mixing of air pollution between the CBL and the free troposphere (FT) and the effects of these processes on air quality represent a major application of the knowledge gained in more basic studies of CBL behavior over mountainous terrain. Addressing vertical transport and mixing is also important with respect to the determination of CBL height by lidar measurements that rely on the presence of air pollution, or more specifically, aerosols that scatter the radiation emitted by a laser. Aerosols are only a subset of air pollutants but the physical transport and mixing processes acting on them are the same as on other air pollutants.

The diurnal boundary layer cycle over flat terrain has two ways of vertically transporting air pollutants in the atmospherepollutants are mixed upward by convection through a deepening CBL during daytime, while pollutants in the residual layer (the elevated remnant of the previous day's $\mathrm{CBL}$ ) are entrained downward into the growing CBL. It is frequently assumed that constituents within the CBL are well-mixed and capped by an inversion. The inversion acts as a lid and limits the transport of constituents to the atmosphere above. As pointed out in Section "Definition and Determination of CBL Heights," these characteristics are often used to define the top of the CBL. There is increasing observational support now that pollutants emitted within the CBL over complex terrain are often transported upward into atmospheric layers above the CBL. In other words, they cannot be considered to be confined within the turbulent CBL. This vertical transport of air pollutants from the CBL to the free atmosphere often manifests itself as elevated aerosol layers (e.g., Wakimoto and McElroy, 1986; McKendry et al., 1997; Tyson and D'Abreton, 1998; Carnuth and Trickl, 2000; McKendry and Lundgren, 2000; Millan et al., 2002; Nyeki et al., 2002; Frioud et al., 2003; Emeis et al., 2007) and/or a deepening of the surface-based aerosol layer, transforming into a residual layer after sunset (De Wekker et al., 2004; Henne et al., 2004).

Some of the hypothesized mechanisms that transport air pollutants between the $\mathrm{CBL}$ and the FT in mountainous terrain are summarized in a conceptual diagram in Figure 11. Rotach et al. (2015) also discusses some of these mechanisms in some more detail. Figure 11 highlights the generation of multi-layer temperature and air pollution profiles and also the close connection of spatial $\mathrm{CBL}$ and $\mathrm{AL}$ structure with vertical transport and mixing mechanisms. The dotted line depicts the AL height which can be considered the maximum vertical extent of aerosol transport while the dashed line depicts the CBL height, defined as the base of a capping inversion that limits the depth of penetrative convection. While AL and CBL heights are approximately equal over flat terrain, mountainous terrain exerts a profound influence on aerosol distribution in the atmosphere: mixing and transport processes are often enhanced compared to those over flat and horizontally homogeneous terrain and the equality of $\mathrm{AL}$ and $\mathrm{CBL}$ heights breaks down. CBL heights are considerably lower than AL heights, show more spatial variability, and tend to follow the terrain more than AL heights, although the extent to which the CBL height follows the terrain decreases during the day (De Wekker et al., 2004). De Wekker et al. (2004) pointed out that simultaneous measurements of

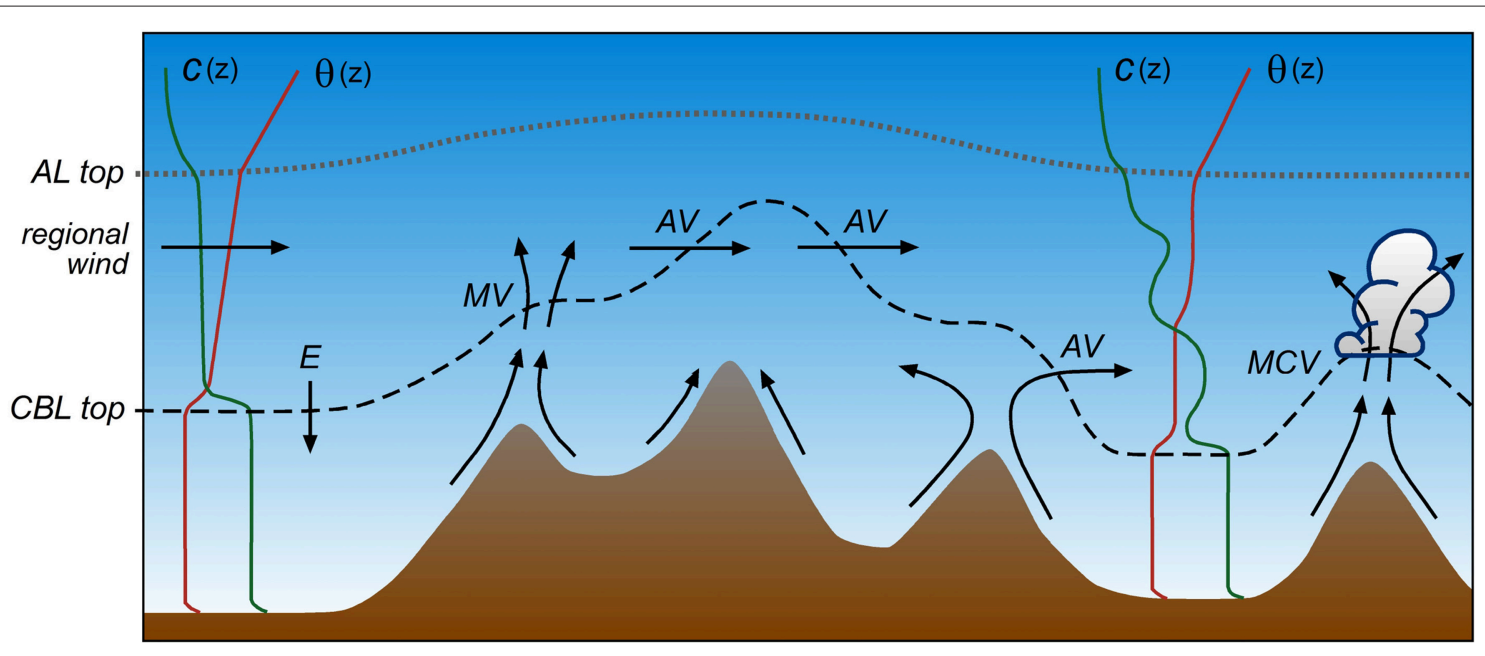

FIGURE 11 | Schematic illustration of mountain induced exchange processes between the convective boundary layer and the overlying atmosphere. $\mathrm{E}$, entrainment; AV, advective venting; MV, mountain venting; and MCV, mountain-cloud venting. Vectors indicate airflow while $\mathrm{c}(\mathrm{z})$ and $\theta(\mathrm{z})$ indicate vertical profiles of pollutant concentration and potential temperature, respectively. The dotted and dashed line indicate the top of the aerosol layer (AL) and the CBL, respectively (after Kossmann et al., 1999; De Wekker, 2002; De Wekker et al., 2004). 
atmospheric temperature structure and AL height are rarely available and that conclusions about mixing processes and $\mathrm{CBL} / \mathrm{AL}$ correspondence are mostly based on modeling results.

If CBL heights follow the terrain and ambient winds or slope flow re-circulations are quasi-horizontal, advective venting can occur, a process that has been quantified for case studies in the Black Forest region in Germany during the TRACT (Transport of Air pollutants over Complex Terrain) field study (Kossmann et al., 1999) and for climatological periods over the Alps (Henne et al., 2005). Transport by slope flows is one of the mechanisms by which heat and mass can be transported from the $\mathrm{CBL}$ to the $\mathrm{FT}$ in mountainous terrain (Wakimoto and McElroy, 1986; Lu and Turco, 1994; Fast and Zhong, 1998; Lugauer et al., 1998; Kossmann et al., 1999; Fiedler et al., 2000; Sasaki et al., 2004; Gohm et al., 2009; Harnisch et al., 2009; Langford et al., 2010). The slope flow mechanism has been given several names including "chimney effect" (Lu and Turco, 1994) and mountain venting (Kossmann et al., 1999; De Wekker et al., 2004; Henne et al., 2005). This effect can be particularly strong when slope flows converge over a mountain summit or when there is an additional sea breeze flow in coastal terrain (McKendry et al., 1997). Compensating lifting and CBL to FT transport are also triggered by along-valley flow convergence (Kossmann and Sturman, 2003; Henne et al., 2004; Weigel et al., 2007), or by terrain-associated convergence zones on the lee sides of isolated mountains or mountain ranges (Edinger and Helvey, 1961). Regional scale plain-to-mountain winds combine with these slope and valley flow mechanisms to lead to a transport of boundary layer air to the FT over the entire mountain range (Henne et al., 2005; Lang et al., 2015), a mechanism that has been termed "alpine pumping" (Lugauer and Winkler, 2005). Vertical transport and mixing by urban heat island-induced circulations is an additional process that can play an important role in the venting of pollutants in urbanized valleys (Rendón et al., 2014, 2015).

Mountain cloud venting (MCV) is a particularly effective CBL-FT exchange process induced by the initiation of moist convection in hilly and mountainous terrain (Kalthoff et al., 2013). MCV originates from cloud venting, a process that has been studied extensively over flat terrain (Ching et al., 1988; Cotton et al., 1995). During the summer, cumulus convection occurs almost daily over the mountain ranges of the interior western United States (e.g., Banta and Schaaf, 1987). Moist convection typically develops in a deep CBL, capped by a weakly stably layer, and under weak winds. Several studies have been conducted in the last decade that focus on convection initiation and associated vertical exchange processes. These studies include VERTIKATOR (Vertical Exchange and Orography, Barthlott et al., 2006; Hasel, 2006) and COPS (Convective and Orographical Induced precipitation Study) in the Black Forest in southwestern Germany (Kottmeier et al., 2008; Wulfmeyer et al., 2008), CUPIDO (Cumulus Photogrammetric, in-situ and Doppler Observations) in the southwestern US (Damiani et al., 2008; Geerts et al., 2008; Demko and Geerts, 2010), BLLAST (Boundary-Layer Late Afternoon and Turbulence) in southern France (Lothon et al., 2014), and as part of HyMeX (Hydrological cycle in the Mediterranean
eXperiment; Drobinski et al., 2014) on the island of Corsica (Adler and Kalthoff, 2014). Thermally driven wind systems are important for the initiation of convective clouds and therefore of MCV, because slope winds often generate convergence zones over the mountain ridges, which are therefore favored areas for the onset of convection (Orville, 1965; Raymond and Wilkening, 1980; Holroyd, 1982; Klitch et al., 1985; Toth and Johnson, 1985; Pielke and Segal, 1986; Tripoli and Cotton, 1989; Tian and Parker, 2002; Stein, 2004; Barthlott et al., 2006; Kottmeier et al., 2008). Forecasting of local convection in mountainous terrain and thus of MCV processes is extremely challenging and relies on an accurate simulation of CBL growth and structure prior to moist convection onset. Even numerical weather prediction models that have sufficient resolution to resolve the thermally driven circulations have difficulties simulating the surface fluxes and CBL growth over complex terrain and accurately predicting the timing and intensity of moist convection (e.g., Trentmann et al., 2009).

The various venting processes described above and in Figure 11 are closely related to $\mathrm{CBL}$ top levelness illustrated in Figure 10. Mountain (cloud) venting processes that act predominantly in the vertical direction would tend to make the CBL top less level, while advective venting processes can make the CBL top more level.

\section{SUMMARY AND DIRECTIONS FOR FUTURE RESEARCH}

This review discussed current knowledge of $\mathrm{CBL}$ heights in mountainous terrain. Much progress has been made in understanding CBL heights from observational, numerical, theoretical, and laboratory studies. A major barrier to improving our present understanding arises from the multitude of processes acting simultaneously over a range of spatial and temporal scales. These difficulties often hamper a thorough explanation of all the observed and modeled features of CBLs in mountainous terrain and cause difficulties in isolating the effect of individual factors on CBL height. In situ and remote sensing observations are crucial for advancing our knowledge of boundary layers in mountainous terrain. Remote sensors such as lidars and sodars have been very helpful in identifying the multilayered structure and in continuously observing the spatiotemporal structure of the mountain atmosphere. These measurements have helped alleviate the limitations of sporadic radiosonde profiles in a spatially varying CBL. However, there are also challenges related to the use of different measured variables for the determination of CBL height. Thermodynamic measurements have traditionally been used in CBL height determination based on the importance of surface based convection in turbulent mixing and transport. Remote sensing measurements frequently observe the integrated result of different mixing and transport mechanisms (not limited to surface based convection) and have added insight but also complexity to understanding CBL heights over complex terrain such as those related to advective effects from thermally driven flows. In particular the use of lidar-derived AL heights as a proxy for CBL heights always needs to be assessed for individual cases. 
There is observational and numerical evidence that lidar-derived AL heights frequently exceed CBL heights over mountainous terrain. This consideration is becoming more important as an increasing number of ground-based ceilometer and lidar networks and space-borne lidar measurements are used to extract information about CBL heights at regional and larger scales. To reduce the level of uncertainty and ambiguity of CBL height observations, multiple instruments at several strategically chosen locations across a mountain range with a variety of in-situ and remote sensors need to be deployed.

From the basic concepts and understanding of the separate slope, valley, and mountain atmospheres, including basins and plateaus, we arrived at a general understanding of boundary layer structure over mountainous terrain. Complex terrain wind systems and CBL evolution are closely connected. Examples include the lowering of the CBL top due to subsiding motions that compensate for upslope flows on the sidewalls and the undercutting of the CBL due to cold air advection from upslope and upvalley flows, producing internal boundary layers and multilayered thermal structures. Boundary layer evolution in valleys is relatively well-understood compared to the evolution over slopes, plateaus, and ridges. Several approaches have been developed to describe the CBL height quantitatively, and in particular the reduced growth in valley boundary layers in the morning hours. These models could be extended to situations with cross-valley flow due to asymmetric heating in the morning, non-homogeneous conditions in the along-valley direction, and the presence of tributary valleys. Boundary layers over slopes and ridges have received less attention than boundary layers over valleys and are complicated by additional advective effects related to thermally driven flows and their interaction with synoptic flows. Some initial approaches have been developed to quantify the leveling of the CBL height over mountainous terrain that need to be tested and further expanded. It is generally observed that CBLs become more level in the late afternoon and during the evening transition period. Models describing these observations can help, for example, to explain the spatio-temporal variability of air quality measurements at mountain top locations and to determine the spatial representativeness of these measurements. While meteorological and chemical measurements are often made at mountain top locations, CBL heights over mountain tops are rarely studied. It is often assumed that the mountain top does not develop a CBL and free tropospheric background air is sampled. While this may be true for mountain tops at elevations high above the adjacent valley or plains CBL, this assumption is not valid when the valley or plains CBL exceeds the mountain top height (Lugauer et al., 1998; De Wekker et al., 2009; Ketterer et al., 2014; Lee et al., 2015).

Daytime thermally driven flows have historically received less attention than their nighttime counterparts, particularly for the case of upslope flows (Atkinson, 1981; Whiteman, 2000; Zardi and Whiteman, 2013). This is partly due to observational limitations; the deep daytime boundary layer requires the utilization of sounding systems with large vertical range making it more difficult to sample the daytime than the nighttime mountain boundary layer. Furthermore, vigorous mixing within the CBL and coupling with larger-scale synoptic flows make it difficult to study the effects of or even observe the presence of upslope flows. Many potentially important effects of upslope flow on CBL height behavior remain unexplored, including the importance of along-slope cold air advection.

The spatial distribution and patchiness of the vegetation and surface cover in hilly and mountainous terrain can have a strong impact on CBL evolution and its thermodynamic structure. Changes of the surface energy budget resulting from heterogeneous land cover directly influence the surface turbulent fluxes of moisture, momentum and heat, which act as the link between the atmosphere and the underlying soil-vegetation system. No studies have been performed to investigate the combined impact of land cover heterogeneity and orography on CBL heights. Knowledge is also limited on the effects of the geographical location (mid-latitude, tropical, etc), season, and scale of the terrain features (shallow vs. deep valleys, gentle vs. steep slopes, etc) on CBL heights. Numerical simulations can be helpful in these situations to correctly distinguish between orography and other effects on the variability of observed CBL heights.

CBL height behavior is closely coupled to vertical transport and mixing processes in mountainous terrain (Rotach et al., 2015) and the transport of pollutants over regional and even global spatial scales (Steyn et al., 2013). It is the spatial CBL height variability that promotes venting processes (e.g., via slope wind recirculation) between the turbulent boundary layer and the overlying atmosphere. Studies suggest that it is useful for mountainous terrain to distinguish between the height of the turbulent $\mathrm{CBL}$ and the height to which pollutants (released from the surface) can reach. Over flat terrain, these heights are identical but over mountainous terrain the thermally driven flows can contribute to the transport of pollutants across the CBL top (Section "Vertical Transport and Mixing Between the CBL and the Free Troposphere") which challenges the traditional concept of a "mixing height" over mountainous terrain. De Wekker et al. (2004) suggested that because pollutants are transported and/or mixed in mountainous areas to the AL height, the $\mathrm{AL}$ height is a more relevant parameter for air pollution studies than the CBL height in mountainous terrain and may be more closely identified with the commonly used term "mixing height" (Seibert et al., 2000). One may also refer to the layer that is influenced by mountain-induced circulations and venting processes, and therefore more relevant to exchange studies, as the mountain CBL (De Wekker, 2002; Adler and Kalthoff, 2014; Adler et al., 2015). Such a mountain CBL would be similar to the conceptual model of a "mountain atmosphere" that Ekhart (1948) introduced (Figure 1). Whether layers above the base of the inversion (capping the highly turbulent layer above the surface) should be considered part of the CBL when influenced by thermally driven flows will depend on the application of the CBL height data. For example, the layer above the inversion base should not be considered part of the CBL in cases where CBL height data are needed to parameterize turbulent boundary layer fluxes. In contrast, the layer influenced by thermally driven flows above the inversion base should be considered part of the CBL when CBL height data are used to determine the possible effects of (valley) boundary layer air on background air pollution 
monitoring at mountain tops or in exchange studies. In recent years several projects have been initiated to study the effects of thermally-driven flows on exchange processes between the atmospheric boundary layer and the overlying free atmosphere (Section "Vertical Transport and Mixing Between the CBL and the Free Troposphere").

CBL heights and closely related vertical transport and mixing processes have more recently gained attention in explaining the uncertainty in estimating air pollution and greenhouse gas budgets (e.g., Sun and de Wekker, 2011; Rotach et al., 2014). The global transport models that are used for carbon budget estimates, for example, are too coarse to take into account vertical exchange processes due to spatial heterogeneity in CBL height over complex terrain. More research is needed to parameterize these effects in these coarse models and their impact on the greenhouse gas budgets.

While it is acknowledged that there is a relationship between convection initiation and CBL height behavior over complex terrain, details of this relationship remain unclear. For example, do shallow CBLs over ridges decrease or increase the likelihood of moist convection? Are terrain-following or level CBL tops more favorable for the occurrence of moist convection? How important is the advection of mountain CBLs over the adjacent plains for the generation of severe weather? What is the relationship between the height of the dry (cloud free) CBL top and the lifting condensation level? Answering these questions requires collaborative field experiments that collect data on both dry CBLs and moist convection processes.

The investigation of CBL heights over mountainous terrain can benefit from recent technological advances in measurement techniques including the use of mobile vertical sounding systems (such as from airborne downward looking Doppler lidars, carborne upward-looking Doppler lidars, and unmanned aerial systems). Networks of ceilometers and aerosol lidars that have been set up to monitor cloud ceiling and aerosol structure, and satellite-based measurements using lidar and radio occultation can provide regional to global coverage of $\mathrm{CBL}$ heights and provide opportunities to investigate the effect of mountain regions on these larger scales. Cup anemometers traditionally used in operational surface station networks are increasingly replaced by ultra-sonic anemometers-thermometers. This will

\section{REFERENCES}

Adler, B., and Kalthoff, N. (2014). Multi-scale transport processes observed in the boundary layer over a mountainous island. Bound. Layer Meteorol. 153, 515-537. doi: 10.1007/s10546-014-9957-8

Adler, B., Kalthoff, N., Kohler, M., Handwerker, J., Wieser, A., Corsmeier, U., et al. (2015). The variability of water vapour and pre-convective conditions over the mountainous island of Corsica. Q. J. R. Meteorol. Soc. doi: 10.1002/qj.2545. [Epub ahead of print].

Arritt, R. W., Wilczak, J. M., and Young, G. S. (1992). Observations and numerical modelling of an elevated mixed layer. Mon. Weather Rev. 120, 2869-2880.

AMS (2000). Glossary of Meteorology, American Meteorological Society. Available online at: http://glossary.ametsoc.org make measurements of variances (turbulence intensities) and covariances (e.g., surface sensible heat flux) from which CBL height estimates can be made, more commonly available in coming years.

Numerical simulations at fine (micro- to mesoscale) resolutions will continue to be important for investigating processes underlying specific CBL height behavior and parameterizing these processes in global models at coarse resolutions. Systematic simulations of $\mathrm{CBL}$ development over idealized terrain features can also be used to investigate, for example, the separate effects of orography and land use on CBL height behavior. Also, future land-use changes in mountainous terrain due to reforestation, deforestation, urbanization, melting glaciers, shifting treelines, changing vegetation/precipitation patterns, and other anthropogenic and natural activities can have profound impacts on CBL dynamics and closely related air pollution transport processes in many parts of the world. Small-scale laboratory studies can play a role in identifying and quantifying processes which are not well-represented by numerical models due to simplifications or inappropriate parameterizations.

$\mathrm{CBL}$ height investigations over mountainous terrain remain important and challenging despite recent progress. The combination of different scientific approaches, such as observational studies, numerical or physical modeling studies, and theoretical studies will continue to close existing gaps in understanding of the relevant processes. New instrumental and computational techniques combined with new generations of scientists will likely lead to new findings that question some of the established views, and to revisions of existing conceptual models of $\mathrm{CBL}$ structure in mountainous terrain that were reviewed in this paper.

\section{ACKNOWLEDGMENTS}

This research was funded by NSF award ATM-1151445, Office of Naval Research award N00014-11-1-0709, and by NOAA award NA13OAR4310065. We would like to thank Dave Whiteman, Mathias Rotach, Norbert Kalthoff, Bianca Adler, Frank Beyrich, and Charles Chemel for providing comments and suggestions that improved the manuscript. 
Banta, R. M. (1984). Daytime boundary-layer evolution over mountainous terrain: Part I. observations of dry circulations. Mon. Weather Rev. 112, 340-356.

Barry, R. G. (1992). Mountain Weather and Climate, 2nd Edn. London: Routledge. Barthlott, C., Corsmeier, U., Meißner, C., Braun, F., and Kottmeier, C. (2006). The influence of mesoscale circulation systems on triggering convective cells over complex terrain. Atmos. Res. 81, 150-175. doi: 10.1016/j.atmosres.2005.11.010

Bastin, S., and Drobinski, P. (2005). Temperature and wind velocity oscillations along a gentle slope during sea breeze events. Bound. Layer Meteorol. 114, 573-594. doi: 10.1007/s10546-004-1237-6

Baxter, R. A. (1991). Determination of mixing heights from data collected during the 1985 SCCCAMP field program. J. Appl. Meteorol. 30, 598-606.

Beyrich, F. (1997). Mixing height estimation from sodar data - a critical discussion. Atmos. Environ. 31, 3941-3953. doi: 10.1016/S1352-2310(97)00231-8

Bianco, L., Djalalova, I. V., King, C. W., and Wilczak, J. M. (2011). Diurnal evolution and annual variability of boundary-layer height and its correlation to other meteorological variables in California's Central Valley. Bound. Layer Meteorol. 140, 491-511. doi: 10.1007/s10546-011-9622-4

Binder, H.-J. (1997). Tageszeitlich und Räumliche Entwicklung der Konvektiven Grenzschicht über Stark Gegliedertem Gelände. Dissertation, Faculty of Physics, University of Karlsruhe.

Bischoff-Gauss, I., Kalthoff, N., Khodayar, S., Fiebig-Wittmaack, M., and Montecinos, S. (2008). Model simulations of the boundary-layer evolution over an arid andes valley. Bound. Layer Meteorol. 128, 357-379. doi: 10.1007/s10546008-9293-y

Blumenthal, D. L., White, W. H., and Smith, T. B. (1978). Anatomy of a Los Angeles smog episode: pollutant transport in the daytime sea breeze regime. Atmos. Environ. 12, 893-907. doi: 10.1016/0004-6981(78)90028-8

Bossert, J. E. (1997). An investigation of flow regimes affecting the Mexico City region. J. Appl. Meteor. 36, 119-140. doi: 10.1175/1520-0450(1997)036<0119: AIOFRA > 2.0.CO;2

Bossert, J. E., and Cotton, W. R. (1994). Regional-scale flows in mountainous terrain. Part II: simplified numerical experiments. Mon. Weather Rev. 122, 1472-1489.

Braham, R. R., and Draginis, H. (1960). Roots of orographic cumuli. J. Meteorol. $17,214-226$

Brehm, M., and Freytag, C. (1982). Erosion of night-time thermal circulation in an Alpine valley. Arch. Meteorol. Geoph. Biokl. B31, 331-352. doi: 10.1007/BF02263439

Brehm, M. (1986). Experimentelle und Numerische Untersuchungen der Hangwindschicht und Ihrer Rolle bei der Erwärmung von Tälern. Wiss. Mitt. Meteor Inst. München.

Cai, X.-M., and Steyn, D. G. (1993). Mesoscale Meteorological Modelling Study of the Lower Fraser Valley, B.C., Canada from July 17 to 20, 1985. Department of Geography Occasional Paper No. 40. University of British Columbia, Vancouver, BC.

Carnuth, W., and Trickl, T. (2000). Transport studies with the IFU three wavelength aerosol lidar during the VOTALP Mesolcina experiment. Atmos. Environ. 34, 1425-1434. doi: 10.1016/S1352-2310(99)00423-9

Catalano, F., and Moeng, C.-H. (2010). Large-eddy simulation of the daytime boundary layer in an idealized valley using the weather research and forecasting numerical model. Bound. Layer Meteorol. 137, 49-75. doi: 10.1007/s10546-0109518-8

Chen, X., Añel, J. A., Su, Z., de la Torre, L., Kelder, H., van Peet, J., et al. (2013). The deep atmospheric boundary layer and its significance to the stratosphere and troposphere exchange over the Tibetan Plateau. PLOS ONE 8:e56909. doi: 10.1371/journal.pone.0056909

Ching, J. K. S., Shipley, S. T., and Browell, E. V. (1988). Evidence for cloud venting of mixed layer ozone and aerosols. Atmos. Environ. 22, 225-242. doi: 10.1016/0004-6981(88)90030-3

Chow, F. K., De Wekker, S. F. J., and Snyder, B., Eds. (2013). Mountain Weather Research and Forecasting. Recent Progress and Current Challenges. Berlin: Springer.

Cotton, W. R., Alexander, G. D., Hertenstein, R., Walko, R. L., McAnelly, R. L., and Nicholls, M. (1995). Cloud venting - A review and some new global annual estimates. Earth Sci. Rev. 39, 169-206. doi: 10.1016/0012-8252(95)0 0007-0

Coulter, R. L. (1979). A comparison of three methods for measuring mixing-layer height. J. Appl. Meteorol. 8, 1495-1499.
Cramer, O. P., and Lynott, R. E. (1961). Cross-section analysis in the study of windflow over mountainous terrain. Bull. Am. Meteorol. Soc. 42, 693-702.

Cramer, O. P. (1972). Potential temperature analysis for mountainous terrain. J. Appl. Meteorol. 11, 44-50.

Dai, C., Wang, Q., Gao, J.-Z., and Zhou, M. (2014). Determining boundary-layer height from aircraft measurements. Bound. Layer Meteor. 152, 277-302. doi: 10.1007/s10546-014-9929-z

Damiani, R., Zehnder, J., Geerts, B., Demko, J., Haimov, S., Petti, J., et al. (2008). Cumulus photogrammetric, In-situ and doppler observations: the CuPIDO 2006 experiment. Bull. Am. Meteorol. Soc. 89, 57-73. doi: 10.1175/BAMS89-1-57

Dayan, U., Shenhav, R., and Graber, M. (1988). The spatial and temporal behavior of the mixed layer in Israel. J. Appl. Meteorol. 27, 1382-1394.

de Foy, B., Caetano, E., Magana, V., Zitacuaro, A., Cardenas, B., Retama, A., et al. (2005). Mexico City basin wind circulation during the MCMA-2003 field campaign. Atmos. Chem. Phys. 5, 2267-2288. doi: 10.5194/acp-5-2267-2005

de Foy, B., Clappier, A., Molina, L. T., and Molina, M. J. (2006). Distinct wind convergence patterns in the Mexico City basin due to the interaction of the gap winds with the synoptic flow. Atmos. Chem. Phys. 6, 1249-1265. doi: 10.5194/acp-6-1249-2006

De Wekker, S. F. J., Ameen, A., Song, G., Stephens, B. B., Hallar, A. G., and McCubbin, I. (2009). A preliminary investigation of boundary layer effects on daytime atmospheric $\mathrm{CO} 2$ concentrations at a mountaintop location in the Rocky Mountains. Acta Geophys. 57, 904-922. doi: 10.2478/s11600-0090033-6

De Wekker, S. F. J., Kossmann, M., and Fiedler, F. (1997). "Observations of daytime mixed layer heights over mountainous terrain during the TRACT field campaign," 12th AMS Symposium on Boundary Layers and Turbulence, 28 July - 1 August 1997, Vancouver, BC.

De Wekker, S. F. J., Steyn, D. G., Fast, J. D., Rotach, M. W., and Zhong, S. (2005). The performance of RAMS in representing the convective boundary layer structure in a very steep valley. Environ. Fluid Mech. 5, 35-62. doi: 10.1007/s10652-005-8396-y

De Wekker, S. F. J., Steyn, D. G., and Nyeki, S. (2004). A comparison of aerosol-layer and convective boundary-layer structure over a mountain range during STAAARTE'97. Bound. Layer Meteorol. 113, 249-271. doi: 10.1023/B:BOUN.0000039371.41823.37

De Wekker, S. F. J., Zhong, S., Fast, J. D., and Whiteman, C. D. (1998). A numerical study of the thermally driven plain-to-basin wind over idealized basin topographies. J. Appl. Meteorol. 37, 606-622. doi: 10.1175/15200450(1998)037<0606:ANSOTT>2.0.CO;2

De Wekker, S. F. J. (1995). The Behaviour of the Convective Boundary Layer Height Over Orographically Complex Terrain. MS thesis University of Karlsruhe, Agricultural University, Germany/Wageningen.

De Wekker, S. F. J. (2002). Structure and Morphology of the Convective Boundary Layer in Mountainous Terrain. Ph.D. dissertation, University British Columbia, British Columbia, BC.

De Wekker, S. F. J. (2008). Observational and numerical evidence of depressed convective boundary layer heights near a mountain base. J. Appl. Meteorol. Clim. 47, 1017-1026. doi: 10.1175/2007JAMC1651.1

Deardorff, J. W., Willis, G. E., and Stockton, B. H. (1980). Laboratory studies of the entrainment zone of a convectively mixed layer. J. Fluid Mech. 100, 41-64. doi: $10.1017 /$ S0022112080001000

Demko, J. C., and Geerts, B. (2010). A numerical study of the evolving convective boundary layer and orographic circulation around the Santa Catalina Mountains in Arizona. Part I: circulation without deep convection. Mon. Weather Rev. 138, 1902-1922. doi: 10.1175/2009MWR3098.1

Doran, J. C., and Zhong, S. (1994). Regional drainage flows in the Pacific Northwest. Mon. Weather Rev. 122, 1158-1167.

Doyle, J. D., and Durran, D. R. (2007). Rotor and sub-rotor dynamics in the lee of three dimensional terrain. J. Atmos. Sci. 64, 4202-4221. doi: 10.1175/2007JAS2352.1

Drobinski, P., Ducrocq, V., Alpert, P., Anagnostou, E., Béranger, K., Borga, M., et al. (2014). HyMeX, a 10-year multidisciplinary program on the Mediterranean water cycle. Bull. Am. Meteorol. Soc. 95, 1063-1082. doi: 10.1175/BAMS-D-12-00242.1

Edinger, J., and Helvey, R. (1961). The San Fernando convergence zone. Bull. Am. Meteorol. Soc. 42, 626-635. 
Egger, J., Blacutt, L., Ghezzi, F., Heinrich, R., Kolb, P., Lämmlein, S., et al. (2005). Diurnal circulation of the Bolivian Altiplano. Part I: observations. Mon. Weather Rev. 133, 911-924. doi: 10.1175/MWR2894.1

Eigenmann, R., Metzger, S., and Foken, T. (2009). Generation of free convection due to changes of the local circulation system. Atmos. Chem. Phys. 9, 8587-8600. doi: 10.5194/acp-9-8587-2009

Ekhart, E. (1948). De la structure thermique de l'atmosphere dans la montagne [On the thermal structure of the mountain atmosphere]. La Meteorologie 4, 3-26. [English translation: Whiteman, C.D., and Dreiseitl, E. (1984). Alpine Meteorology: Translations of Classic Contributions by A.Wagner, E. Ekhart and F. Defant. PNL-5141/ASCOT-84-3. Pacific Northwest Laboratory, Richland, Washington, 121 pp, Available online at: http://www.osti.gov/bridge/ product.biblio.jsp?osti_id=6665518]

Emeis, S., Jahn, C., Münkel, C., Münsterer, C., and Schäfer, K. (2007). Multiple atmospheric layering and mixing-layer height in the Inn valley observed by remote sensing. Meteorol. Z. 16, 415-424. doi: 10.1127/0941-2948/2007/0203

Emeis, S., Schäfer, K., and Münkel, C. (2008). Surface-based remote sensing of the mixing-layer height-A review. Meteorol. Z. 17, 621-630. doi: 10.1127/09412948/2008/0312

Fast, J. D., and Zhong, S. (1998). Meteorological factors associated with inhomogeneous ozone concentrations within the Mexico City basin. J. Geophys. Res. D103, 18927-18946. doi: 10.1029/98JD01725

Fiedler, F., Adrian, G., and Hugelmann, C.-P. (1987). Beobachtete Phänomene während des Tulla-Experimentes, 3rd PEF Statuskolloquium 10.-12.3.1987, Bd. 2. Karlsruhe: KfK-PEF 12.

Fiedler, F., Bischoff-Gauß, I., Kalthoff, N., and Adrian, G. (2000). Modeling of the transport of a tracer in the Freiburg-Schauinsland area. J. Geophys. Res. D105, 1599-1610. doi: 10.1029/1999JD900911

Fosberg, M. A. (1967). Numerical analysis of convective motions over a mountain ridge. J. Appl. Meteorol. 6, 889-904.

Fosberg, M. A. (1969). Airflow over a heated coastal mountain. J. Appl. Meteorol. $8,436-442$.

Frank, H. P. (1997). "Simulation of the convective mixed layer over Athens," in Proceedings EURASAP Workshop on the Determination of the Mixing Height Progress and Problems (Roskilde: Riso National Laboratory), 145-148. Report Riso-R-997(EN).

Freytag, C. (1985). MERKUR-results: aspects of the temperature field and the energy budget in a large Alpine valley during mountain and valley wind. Contrib. Atmos. Phys. 58, 458-476.

Freytag, C. (1987). Results from the MERKUR experiment: mass budget and vertical motions in a large valley during mountain and valley wind. Meteorol. Atmos. Phys. 37, 129-140. doi: 10.1007/BF01040843

Frioud, M., Mitev, V., Matthey, R., Haberli, C. H., Richner, H., Werner, R., et al. (2003). Elevated aerosol stratification above the Rhine Valley under strong anticyclonic conditions. Atmos. Environ. 37, 1785-1797. doi: 10.1016/S13522310(03)00049-9

Furger, M., Dommen, J., Graber, W. K., Poggio, L., Prévôt, A., Emeis, S., et al. (2000). The VOTALP Mesolcina Valley Campaign 1996 - concept, background, and some highlights. Atmos. Environ. 34, 1395-1412. doi: 10.1016/S13522310(99)00377-5

Gaertner, M. A., Fernandez, C., and Castro, M. (1993). A two-dimensional simulation of the Iberian summer thermal low. Mon. Weather Rev. 121, 2740-2756.

Geerts, B., Miao, Q., and Demko, J. C. (2008). Pressure perturbations and upslope flow over a heated, isolated mountain. Mon. Weather Rev. 136, 4272-4288. doi: 10.1175/2008MWR2546.1

Geiger, R. (1961). Das Klima der bodennahen Luftschicht. 4th Edn. Braunschweig: Vieweg Verlag.

Gohm, A., Harnisch, F., Vergeiner, J., Obleitner, F., Schnitzhofer, R., Hansel, A., et al. (2009). Air pollution transport in an alpine valley: results from airborne and ground-based observations. Bound. Layer Meteorol. 131, 441-463. doi: 10.1007/s10546-009-9371-9

Gopalakrishnan, S. G., Baidya Roy, S., and Avissar, R. (2000). An evaluation of the scale at which topographical features affect the convective boundary layer using large eddy simulations. J. Atmos. Sci. 57, 334-351.

Hafner, T. A., Reinhard, M. E., Weisel, E. L., and Fimpel, H. P. (1987). Boundary layer aspects and elevated heat source effects of the Alps. Meteorol. Atmos. Phys. 31, 61-73. doi: 10.1007/BF01045140
Hägeli, P., Steyn, D. G., and Strawbridge, K. B. (2000). Spatial and temporal variability of mixed-layer depth and entrainment zone thickness. Bound. Layer Meteorol. 97, 47-71. doi: 10.1023/A:1002790424133

Harnisch, F., Gohm, A., Fix, A., Schnitzhofer, R., Hansel, A., and Neininger, B. (2009). Spatial distribution of aerosols in the Inn Valley atmosphere during wintertime. Meteorol. Atmos. Phys. 103, 215-228. doi: 10.1007/s00703-0080318-3

Hasel, M. (2006). Strukturmerkmale und Modelldarstellung der Konvektion über Mittelgebirgen. Dissertation, Faculty of Physics, University of Karlsruhe.

Hayden, K. L., Anlauf, K. G., Hoff, R. M., Strapp, W. J., Bottenheim, J. W., Wiebe, H. A., et al. (1997). The vertical chemical and meteorological structure of the boundary layer in the Lower Fraser Valley during Pacific'93. Atmos. Environ. 31, 2089-2106. doi: 10.1016/S1352-2310(96)00300-7

Henne, S., Furger, M., Nyeki, S., Steinbacher, M., Neininger, B., de Wekker, S. F. J., et al. (2004). Quantification of topographic venting of boundary layer air to the free troposphere. Atmos. Chem. Phys. 4, 497-509. doi: 10.5194/acp-4-497-2004

Henne, S., Furger, M., and Prévôt, A. S. H. (2005). Climatology of mountain venting induced moisture layers in the lee of the Alps. J. Appl. Meteorol. 44, 620-633. doi: 10.1175/JAM2217.1

Hennemuth, B., and Lammert, A. (2006). Determination of the atmospheric boundary layer height from radiosonde and lidar backscatter. Bound. Layer Meteorol. 120, 181-200. doi: 10.1007/s10546-005-9035-3

Hennemuth, B. (1985). Temperature field and energy budget in a small Alpine valley. Contrib. Atmos. Phys. 58, 545-559.

Hewson, E. W., and Gill, G. C. (1944). Meteorological Investigations in the Columbia River Valley near Trail, B.C. United States Department of Interior, Bureau of Mines, Bulletin.

Holroyd, E. W. (1982). Some observations on the mountain-generated cumulonimbus rainfall on the Northern Great Plains. J. Appl. Meteorol. 21, 560-565

Holzworth, G. C. (1964). Estimates of mean maximum mixing depths in the contiguous U.S. Mon. Weather Rev. 92, 235-242.

Holzworth, G. C. (1972). Mixing Heights, Wind Speeds, and Potential for Urban Air Pollution Throughout the Contiguous United States. North Carolina, NC: Environmental Protection Agency; Office of Air Programs Publication AP-101; Research Triangle Park.

Hunt, J. C. R., Fernando, H. J. S., and Princevac, M. (2003). Unsteady thermally driven flows on gentle slopes. J. Atmos. Sci. 60, 2169-2182.

Jackson, P. L., Mayr, G., and Vosper, S. (2013). "Dynamically-driven winds," in Mountain Weather Research and Forecasting: Recent Progress and Current Challenges, eds F. Chow, S. F. J. De Wekker, and B. Synder (New York, NY: Springer), 121-218.

Johnson, A. Jr., and O’Brien, J. J. (1973). A study of an Oregon sea breeze event. J. Appl. Meteorol. 12, 1267-1283

Kalthoff, N., Binder, H.-J., Kossmann, M., Vögtlin, R., Corsmeier, U., Fiedler, F., et al. (1998). Temporal evolution and spatial variation of the boundary layer over complex terrain. Atmos. Environ. 32, 1179-1194. doi: 10.1016/S13522310(97)00193-3

Kalthoff, N., Bischoff-Gauß, I., Fiebig-Wittmaack, M., Fiedler, F., Thürauf, J., Novoa, E., et al. (2002). Mesoscale wind regimes in Chile at 30 S. J. Appl. Meteorol. 41, 953-970. doi: 10.1175/15200450(2002)041 <0953:MWRICA > 2.0.CO;2

Kalthoff, N., Träumner, K., Adler, B., Späth, S., Behrendt, A., Wieser, A., et al. (2013). Dry and moist convection in the boundary layer over the Black Forest - a combined analysis of in situ and remote sensing data. Meteorol. Z. 22, 445-461. doi: 10.1127/0941-2948/2013/0417

Kelly, R. D. (1988). Asymmetric removal of temperature inversions in a high mountain valley. J. Appl. Meteorol. 27, 664-673.

Ketterer, C., Zieger, P., Bukowiecki, N., Collaud Coen, M., Maier, O., Ruffieux, D., et al. (2014). Investigation of the planetary boundary layer in the Swiss Alps using remote sensing and in situ measurements. Bound. Layer Meteorol. 151, 317-334. doi: 10.1007/s10546-013-9897-8

Khodayar, S., Kalthoff, N., Fiebig-Wittmaack, M., and Kohler, M. (2008). Evolution of the atmospheric boundary layer structure of an arid Andes valley. Meteorol. Atmos. Phys. 99, 181-198. doi: 10.1007/s00703-007-0274-3

Kiemle, C., Kästner, M., and Ehret, G. (1995). The convective boundary layer structure from lidar and radiosonde measurements during the EFEDA'91 campaign. J. Atmos. Ocean. Technol. 12, 771-782. 
Kimura, F., and Kuwagata, T. (1993). Thermally induced wind passing from plain to basin over a mountain range. J. Appl. Meteorol. 32, 1538-1547

Kirshbaum, D. J. (2011). Cloud-resolving simulations of deep convection over a heated mountain. J. Atmos. Sci. 68, 361-378. doi: 10.1175/2010JAS3642.1

Kitada, T., and Regmi, R. P. (2003). Dynamics of air pollution transport in late wintertime over Kathmandu Valley, Nepal, as revealed with numerical simulation. J. Appl. Meteorol. 42, 1770-1798.

Klitch, M. A., Weaver, J. F., Kelly, F. P., and Vonder Haar, T. H. (1985). Convective cloud climatologies constructed from satellite imagery. Mon. Weather Rev. 113, 326-337.

Kossmann, M., Corsmeier, U., De Wekker, S. F. J., Fiedler, F., Vögtlin, R., Kalthoff, N., et al. (1999). Observations of handover processes between the atmospheric boundary layer and the free troposphere over mountainous terrain. Contrib. Atmos. Phys. 72, 329-350.

Kossmann, M., and Sturman, A. P. (2003). Pressure-driven channeling effects in bent valleys. J. Appl. Meteorol. 42, 151-158. doi: 10.1175/15200450(2003)042<0151:PDCEIB > 2.0.CO;2

Kossmann, M., Sturman, A. P., Zawar-Reza, P., McGowan, H. A., Oliphant, A. J., Owens, I. F., et al. (2002). Analysis of the wind field and heat budget in an alpine lake basin during summertime fair weather conditions. Meteorol. Atmos. Phys. 81, 27-52. doi: 10.1007/s007030200029

Kossmann, M., Vögtlin, R., Corsmeier, U., Vogel, B., Fiedler, F., Binder, H.-J., et al. (1998). Aspects of the convective boundary layer structure over complex terrain. Atmos. Environ. 32, 1323-1348. doi: 10.1016/S1352-2310(97)00271-9

Kottmeier, C., Kalthoff, N., Barthlott, C., Corsmeier, U., Van Baelen, J., Behrendt, A., et al. (2008). Mechanisms initiating deep convection over complex terrain during COPS. Meteorol. Z. 17, 931-948. doi: 10.1127/0941-2948/2008/0348

Kuwagata, T., and Kimura, F. (1995). Daytime boundary layer evolution in a deep valley. Part I: observations in the Ina Valley. J. Appl. Meteorol. 34, 1082-1091.

Kuwagata, T., and Kimura, F. (1997). Daytime boundary layer evolution in a deep valley. Part II: numerical simulation of the cross-valley circulation. J. Appl. Meteorol. 36, 883-895.

Kuwagata, T., and Kondo, J. (1989). Observation and modeling of thermally induced upslope flow. Bound. Layer Meteorol. 49, 265-293. doi: 10.1007/BF00120973

Kuwagata, T., and Sumioka, M. (1991). The daytime heating process over complex terrain in central Japan under fair and calm weather conditions. Part III: daytime thermal low and nocturnal thermal high. J. Meteorol. Soc. Japan 69, 91-103.

Laiti, L., Zardi, D., de Franceschi, M., Rampanelli, G., and Giovannini, L. (2014). Analysis of the diurnal development of a lake-valley circulation in the Alps based on airborne and surface measurements. Atmos. Chem. Phys. 14, 9771-9786. doi: 10.5194/acp-14-9771-2014

Lang, M. N., Gohm, A., and Wagner, J. S. (2015). The impact of embedded valleys on daytime pollution transport over a mountain range, Atmos. Chem. Phys. 15, 11981-11998. doi: 10.5194/acp-15-11981-2015

Langford, A. O., Senff, C. J., Alvarez, R. J., Banta, R. M., and Hardesty, R. M. (2010). Long-range transport of ozone from the Los Angeles Basin: a case study. Geophys. Res. Lett. 37. doi: 10.1029/2010GL042507

Lee, T. R., De Wekker, S. F. J., Pal, S., Andrews, A., and Kofler, J. (2015). Meteorological controls on the diurnal variability of carbon monoxide mixing ratio at a mountaintop monitoring site in the Appalachian Mountains. Tellus $B$ 67:25659. doi: 10.3402/tellusb.v67.25659

Lehner, M., and Gohm, A. (2010). Idealised simulations of daytime pollution transport in a steep valley and its sensitivity to thermal stratification and surface albedo. Bound. Layer Meteorol. 134, 327-351. doi: 10.1007/s10546-009$9442-y$

Lenschow, D. H., Stankov, B. B., and Mahrt, L. (1979). The rapid morning boundary-layer transition. J. Atmos. Sci. 36, 2108-2124.

Leukauf, D., Gohm, A., Rotach, M., and Wagner, J. S. (2015). The impact of temperature inversion breakup on the exchange of heat and mass in an idealized valley: sensitivity to radiative forcing. J. Appl. Meteorol. Climatol. 54, 2199-2216. doi: 10.1175/JAMC-D-15-0091.1

Liechti, O., and Neininger, B. (1994). ALPTHERM: a PC-based model for atmospheric convection over complex topography. Tech. Soaring 18, 73-78.

Liu, S., and Liang, X.-Z. (2010). Observed diurnal cycle climatology of planetary boundary layer height. J. Clim. 23, 5790-5809. doi: 10.1175/2010JCLI 3552.1
Lothon, M., Lohou, F., Pino, D., Couvreux, F., Pardyjak, E. R., Reuder, J., et al. (2014). The BLLAST field experiment: boundary-layer late afternoon and turbulence. Atmos. Chem. Phys. 14, 10931-10960. doi: 10.5194/acpd-14-107892014

Lu, R., and Turco, R. P. (1994). Air pollutant transport in a coastal environment. Part I: two-dimensional simulations of sea-breeze and mountain effects. J. Atmos. Sci. 51, 2285-2308.

Lugauer, M., Baltensperger, U., Furger, M., Gäggeler, H. W., Jost, D. T., Schwikowski, M., et al. (1998). Aerosol transport to the high Alpine sites Jungfraujoch (3454 m asl) and Colle Gnifetti (4452 m asl). Tellus B 50, 76-92.

Lugauer, M., and Winkler, P. (2005). Thermal circulation in South Bavaria climatology and synoptic aspects. Meteorol. Z. 14, 15-30. doi: 10.1127/09412948/2005/0014-0015

Mannouji, N. (1982). A numerical experiment on the mountain and valley winds. J. Meteorol. Soc. Japan 60, 1085-1105.

Mannstein, H. (1989). Die radiometrisch bestimmte Oberflächentemperatur im Gebirge und die Ermittlung des Stroms fühlbarer Wärme. Dissertation, University of Munich.

Marsik, F. J., Fischer, K. W., McDonald, T. D., and Samson, P. J. (1995). Comparison of methods for estimating mixing height used during the 1992 Atlanta Field Intensive. J. Appl. Meteorol. 34, 1802-1814.

Maughan, R. A., Spanton, A. M., and Williams, M. L. (1982). An analysis of the frequency distribution of sodar derived mixing heights classified by atmospheric stability. Atmos. Environ. 16, 1209-1218. doi: 10.1016/00046981(82)90209-8

McElroy, J. L., and Smith, T. B. (1991). Lidar descriptions of mixing-layer thickness characteristics in a complex terrain/coastal environment. J. Appl. Meteorol. 30, 585-597.

McKendry, I. G., and Lundgren, J. (2000). Tropospheric layering of ozone in regions of urbanized complex and/or coastal terrain: a review. Prog. Phys. Geogr. 24, 329-354. doi: 10.1177/030913330002400302

McKendry, I. G., Steyn, D. G., Lundgren, J., Hoff, R. M., Strapp, W., Anlauf, K., et al. (1997). Elevated ozone layers and vertical down-mixing over the Lower Fraser Valley. Atmos. Environ. 31, 2135-2146. doi: 10.1016/S13522310(96)00127-6

Melfi, S. H., Spinhirne, J. D., Chou, S.-H., and Palm, S. P. (1985). Lidar observation of vertically organized convection in the planetary boundary layer over the ocean. J. Clim. Appl. Meteorol. 24, 806-821.

Meybeck, M., Green, P., and Vörösmarty, C. J. (2001). A new typology for mountains and other relief classes: an application to global continental water resources and population distribution. Mt. Res. Dev. 21, 34-45. doi: 10.1659/0276-4741(2001)021[0034:antfma]2.0.co;2

Millan, M. M., Sanz, M. J., Salvador, R., and Mantilla, E. (2002). Atmospheric dynamics and ozone cycles related to nitrogen deposition in the western Mediterranean. Environ. Pollut. 10, 167-186. doi: 10.1016/S0269-7491(01)00311-6

Mizzi, A. P., and Pielke, R. A. (1984). A numerical study of the mesoscale atmospheric circulation observed during a coastal upwelling event on 23 August 1972. Part I: sensitivity studies. Mon. Weather Rev. 112, 76-90.

Müller, H., and Whiteman, C. D. (1988). Breakup of a nocturnal temperature inversion in the Dischma Valley during DISKUS. J. Appl. Meteorol. 27, 188-194.

Muñoz, R. C., and Undurraga, A. A. (2010). Daytime mixed layer over the Santiago Basin: description of two years of observations with a lidar ceilometer. J. Appl. Meteorol. Climatol. 49, 1728-1741. doi: 10.1175/2010JAMC 2347.1

Myrick, R. H., Sakiyama, S. K., Angle, P. R., and Sandhu, A. S. (1994). Seasonal mixing heights and inversions at Edmonton, Alberta. Atmos. Environ. 28A, 723-729. doi: 10.1016/1352-2310(94)90049-3

Noppel, H., and Fiedler, F. (2002). Mesoscale heat transport over complex terrain by slope winds - A conceptual model and numerical simulations. Bound. Layer Meteorol. 104, 73-97. doi: 10.1023/A:1015556228119

Norton, C. L., and Hoidale, G. B. (1976). The diurnal variation of mixing height by season over White Sands missile range, New Mexico. Mon. Weather Rev. 104, $1317-1320$.

Nyeki, S., Eleftheriadis, K., Baltensperger, U., Colbeck, I., Fiebig, M., Fix, A., et al. (2002). Airborne lidar and in-situ aerosol observations of an elevated layer, leeward of the European Alps and Apennines. Geophys. Res. Lett. 29, 1852. doi: $10.1029 / 2002$ GL014897 
Nyeki, S., Kalberer, M., Colbeck, I., De Wekker, S. F. J., Furger, M., Gäggeler, H. W., et al. (2000). Convective boundary layer evolution to $4 \mathrm{~km}$ asl over high-alpine terrain: airborne lidar observations in the Alps. Geophys. Res. Lett. 27, 689-692. doi: 10.1029/1999GL010928

Orville, H. D. (1965). A numerical study of the initiation of cumulus clouds over mountainous terrain. J. Atmos. Sci. 22, 684-699.

Panday, A. K., Prinn, R. G., and Schär, C. (2009). Diurnal cycle of air pollution in the Kathmandu Valley, Nepal: 2. Modeling results. J. Geophys. Res. 114, D21308. doi: $10.1029 / 2008 j \mathrm{~d} 009808$

Physick, W. L., Abbs, D. J., and Pielke, R. A. (1989). Formulation of the thermal internal boundary layer in a mesoscale model. Bound. Layer Meteorol. 49, 99-111. doi: 10.1007/BF00116407

Pielke, R. A., and Segal, M. (1986). "Mesoscale circulations forced by differential terrain heating," in Mesoscale Meteorology and Forecasting, ed P. Ray (Boston, MA: American Meteorological Society), 516-548.

Prandtl, L. (1942). Führer durch die Strömungslehre. Braunschweig: Vieweg-Verlag. Princevac, M., and Fernando, H. J. S. (2008). Morning breakup of cold pools in complex terrain. J. Fluid Mech. 616, 99-109. doi: 10.1017/S0022112008004199

Queney, P. (1948). The problem of air flow over mountains: a summary of theoretical results. Bull. Am. Meteorol. Soc. 29, 16-26.

Rampanelli, G., Zardi, D., and Rotunno, R. (2004). Mechanisms of up-valley winds. J. Atmos. Sci. 61, 3097-3111. doi: 10.1175/JAS-3354.1

Raymond, D. J., and Wilkening, M. (1980). Mountain-induced convection under fair weather conditions. J. Atmos. Sci. 37, 2693-2700.

Raymond, D. J., and Wilkening, M. H. (1982). Flow and mixing in New Mexico mountain cumuli. J. Atmos. Sci. 39, 2211-2228.

Regmi, R. P., Kitada, T., and Kurata, G. (2003). Numerical simulation of late wintertime local flows in Kathmandu Valley, Nepal: implication for air pollution transport. J. Appl. Meteorol. 42, 389-403. doi: 10.1175/15200450(2003)042<0389:NSOLWL>2.0.CO;2

Rendón, A. M., Salazar, J. F., Palacio, C. A., and Wirth, V. (2015). Temperature inversion breakup with impacts on air quality in urban valleys influenced by topographic shading. J. Appl. Meteorol. Climatol. 54, 302-321. doi: 10.1175/JAMC-D-14-0111.1

Rendón, A. M., Salazar, J. F., Palacio, C. A., Wirth, V., and Brötz, B. (2014). Effects of urbanization on the temperature inversion breakup in a mountain valley with implications for air quality. J. Appl. Meteorol. Climatol. 53, 840-858. doi: 10.1175/JAMC-D-13-0165.1

Reuten, C., Steyn, D. G., Strawbridge, K. B., and Bovis, P. (2005). Observations of the relationship between the upslope flows and the convective boundary layer in steep terrain. Bound. Layer Meteorol. 116, 37-61. doi: 10.1007/s10546-0047299-7

Richner, H., and Hächler, P. (2013). "Understanding and forecasting alpine foehn,” Mountain Weather Research and Forecasting: Recent Progress and Current Challenges, eds F. Chow, S. F. J. De Wekker, and B. Synder (New York, NY: Springer), 219-260.

Rotach, M. W., Calanca, P., Vogt, R., Steyn, D. G., Graziani, G., Andretta, M., et al. (2004). The turbulence structure and exchange processes in an Alpine valley: the Riviera project. Bull. Am. Soc. 85, 1367-1385. doi: 10.1175/BAMS-85-91367

Rotach, M. W., Gohm, A., Lang, N., Leukauf, D., Stiperski, I., and Wagner, S. (2015). On the vertical exchange of heat, mass and momentum over complex, mountainous terrain. Front. Earth Sci. 3:76. doi: 10.3389/feart.2015.00076

Rotach, M. W., Wohlfahrt, G., Hansel, A., Reif, M., Wagner, J., and Gohm, A. (2014). The world is not flat: implications for the global carbon balance. Bull. Am. Meteorol. Soc. 95, 1021-1028. doi: 10.1175/BAMS-D-13-00109.1

Sasaki, T., Wu, P., Mori, S., Hamada, J.-I., Tauhid, Y. I., Yamanaka, M. D., et al. (2004). Vertical moisture transport above the mixed layer around the mountains in western Sumatra. Geophys. Res. Lett. 31, L08106. doi: 10.1029/2004GL019730

Schmidli, J., and Rotunno, R. (2010). Mechanisms of along-valley winds and heat exchange over mountainous terrain. J. Atmos. Sci. 67, 3033-3047. doi: 10.1175/2010JAS3473.1

Schmidli, J. (2013). Daytime heat transfer processes over mountainous terrain. J. Atmos. Sci. 70, 4041-4066. doi: 10.1175/JAS-D-13-083.1

Segal, M., Ookuchi, Y., and Pielke, R. A. (1987). On the effect of steep slope orientation on the intensity of daytime upslope flow. J. Atmos. Sci. 44, 3587-3592.
Seibert, P., Beyrich, F., Gryning, S.-E., Joffre, S., Rasmussen, A., and Tercier, P. (2000). Review and intercomparison of operational methods for the determination of the mixing height. Atmos. Environ. 34, 1001-1027. doi: 10.1016/S1352-2310(99)00349-0

Serafin, S., and Zardi, D. (2010a). Structure of the atmospheric boundary layer in the vicinity of a developing upslope flow system: a numerical model study. J. Atmos. Sci. 67, 1171-1185. doi: 10.1175/2009JAS3231.1

Serafin, S., and Zardi, D. (2010b). Daytime heat transfer processes related to slope flows and turbulent convection in an idealized mountain valley. J. Atmos. Sci. 67, 3739-3756. doi: 10.1175/2010JAS3428.1

Smith, R. B., Doyle, J. D., Jiang, Q., and Smith, S. A. (2007). Alpine gravity waves: lessons from MAP regarding mountain waves generation and braking. Q. J. R. Meteorol. Soc. 133, 917-936. doi: 10.1002/qj.103

Smith, R. B. (1979). The influence of mountains on the atmosphere. Adv. Geophys. 21, 87-230. doi: 10.1016/S0065-2687(08)60262-9

Stein, J. (2004). Exploration of some convective regimes over the Alpine orography. Q. J. R. Meteorol. Soc. 130, 418-502. doi: 10.1256/qj.02.220

Steinacker, R. (1984). Area-height distribution of a valley and its relation to the valley wind. Contrib. Atmos. Phys. 57, 64-71.

Steinbacher, M., Henne, S., Dommen, J., Wiesen, P., and Prevot, A. S. (2004). Nocturnal trans-alpine transport of ozone and its effects on air quality on the Swiss Plateau. Atmos. Environ. 36, 4539-4550. doi: 10.1016/j.atmosenv.2004.05.008

Stensrud, D. J. (1993). Elevated residual layers and their influence on surface boundary-layer evolution. J. Atmos. Sci. 50, 2284-2293.

Steyn, D. G., De Wekker, S. F. J., Kossmann, M., and Martilli, A. (2013). "Boundary Layers and Air Quality in Mountainous Terrain," in Chapter 5 Mountain Weather Research and Forecasting, Recent Progress and Current Challenges, eds F. K.Chow, S. F. J. De Wekker, and B. Snyder (Berlin: Springer), 261-290.

Stull, R. B. (1988). An Introduction to Boundary Layer Meteorology. Dordrecht: Kluwer Academic Publishers.

Stull, R. B. (1992). "A theory for mixed-layer-top levelness over irregular topography," in Proceedings of the 10th AMS Symposium on Turbulence and Diffusion (Portland).

Sun, J., and de Wekker, S. F. J. (2011). "Atmospheric carbon dioxide transport over complex terrain," in Mountain Ecosystems: Dynamics, Management and Conservation, ed K. E. Richards (New York, NY: Nova Science Publishers), 101-121.

Taylor, J. R., Kossmann, M., Low, D. J., and Zawar-Reza, P. (2005). Summertime easterly surges in southeastern Australia: a case study of thermally forced flow. Aust. Meteorol. Mag. 54, 213-223.

Tian, W., and Parker, D. J. (2002). Two-dimensional simulation of orographic effects on mesoscale boundary-layer convection. Q. J. R. Meteorol. Soc. 128, 1929-1952. doi: 10.1256/003590002320603476

Tian, W., Parker, D. J., and Kilburn, C. A. D. (2003). Observations and numerical simulation of atmospheric cellular convection over mesoscale topography. Mon. Weather Rev. 131, 222-235. doi: 10.1175/1520-0493(2003)131<0222:OANSOA > 2.0.CO;2

Toth, J., and Johnson, R. H. (1985). Summer surface flow characteristics over northeast Colorado. Mon. Weather Rev. 113, 1779-1800.

Trentmann, J., Keil, C., Salzmann, M., Barthlott, C., Bauer, H.-S., Schwitalla, T., et al. (2009). Multi-model simulations of a convective situation in mountainous terrain. Meteorol. Atmos. Phys. 103, 95-103. doi: 10.1007/s00703-0080323-6

Tripoli, G. J., and Cotton, W. R. (1989). Numerical study of an observed orogenic mesoscale convective system: Part 1 . Simulated genesis and comparison with observations. Mon. Weather Rev. 117, 272-304.

Tyson, P. D., and D'Abreton, P. C. (1998). Transport and recirculation of aerosols off southern Africa - Macroscale plume structure. Atmos. Environ. 32, 1511-1524.

Van Pul, W. A. J., Holtslag, A. A. M., and Swart, D. P. J. (1994). A comparison of $\mathrm{ABL}$ heights inferred routinely from lidar and radiosondes at noontime. Bound. Layer Meteorol. 68, 173-191. doi: 10.1007/BF00712670

Vergeiner, I., and Dreiseitl, E. (1987). Valley winds and slope winds observations and elementary thoughts. Meteorol. Atmos. Phys. 36, 264-286. doi: 10.1007/BF01045154

Vergeiner, I. (1982). Eine energetische Théorie der Hangwinde. 17 Internationale Tagung fiir Alpine Météorologie, Berchtesgaden. Ann. Meteor. 19, 189-191. 
Vogelezang, D. H. P., and Holtslag, A. A. M. (1996). Evaluation and model impacts of alternative boundary-layer height formulations. Bound. Layer Meteorol. 81, 245-269. doi: 10.1007/BF02430331

Wagner, A. (1932). Neue Theorie des Berg- und talwindes [New theory of the mountain and valley wind]. Meteorol. Z. 49, 329-341.

Wagner, J. S., Gohm, A., and Rotach, M. W. (2014). The impact of valley geometry on daytime thermally driven flows and vertical transport processes. Q. J. R. Meteorol. Soc. 141, 1780-1794. doi: 10.1002/qj.2481

Wagner, J. S., Gohm, A., and Rotach, M. W. (2015). Influence of along-valley terrain heterogeneity on exchange processes over idealized valleys. Atmos. Chem. Phys. Discuss. 15, 415-451. doi: 10.5194/acpd-15-415-2015

Wakimoto, R. M., and McElroy, J. L. (1986). Lidar observations of elevated pollution layers over Los Angeles. J. Clim. Appl. Meteorol. 25, 1583-1599.

Walko, R. L., Cotton, W. R., and Pielke, R. A. (1992). Large eddy simulation of the effects of hilly terrain on the convective boundary layer. Bound. Layer Meteorol. 58, 133-150. doi: 10.1007/BF00120755

Weigel, A. P., Chow, F. K., and Rotach, M. (2007). The effect of mountainous topography on moisture exchange between the "surface" and the free atmosphere. Bound. Layer Meteorol. 125, 227-244. doi: 10.1007/s10546-0069120-2

Weigel, A. P., Chow, F. K., Rotach, M. W., Street, R. L., and Xue, M. (2006). High-resolution large-eddy simulations of flow in a steep Alpine valley. Part II: Flow structure and heat budgets. J. Appl. Meteorol. Clim. 45, 87-107. doi: 10.1175/JAM2323.1

Weigel, A. P., and Rotach, M. W. (2004). Flow structure and turbulence characteristics of the daytime atmosphere in a steep and narrow Alpine valley. Q. J. R. Meteorol. Soc. 130, 2605-2627. doi: 10.1256/qj.03.214

Wenzel, A., Kalthoff, N., and Fiedler, F. (1997). On the variation of the energybalance components with orography in the Upper Rhine Valley. Theor. Appl. Climatol. 57, 1-9. doi: 10.1007/BF00867973

Whiteman, C., Zhong, S., Bian, X., Fast, J., and Doran, J. C. (2000). Boundary layer evolution and regional-scale diurnal circulations over the Mexican plateau. J. Geophys. Res. D105, 10081-10102. doi: 10.1029/2000JD900039

Whiteman, C. D., and McKee, T. B. (1978). Air pollution implications of inversion descent in a mountain valley. Atmos. Environ. 12, 2151-2158. doi: 10.1016/0004-6981(78)90170-1

Whiteman, C. D., and McKee, T. B. (1982). Breakup of temperature inversions in deep mountain valleys: Part II. thermodynamic model. J. Appl. Meteorol. 21, 290-302.

Whiteman, C. D., Zhong, S., Shaw, W. J., Hubbe, J. M., Bian, X., and Mittelstadt, J. (2001). Cold pools in the Columbia Basin. Weather Forecast. 16, 432-447. doi: 10.1175/1520-0434(2001)016<0432:CPITCB > 2.0.CO;2

Whiteman, C. D. (1982). Breakup of temperature inversions in deep mountain valleys. Part I: observations. J. Appl. Meteorol. 21, 270-289.

Whiteman, C. D. (1990). "Observations of thermally developed wind systems in mountainous terrain," in Atmospheric Processes Over Complex Terrain. Meteorological Monographs, Vol. 23, ed W. Blumen (Boston, MA: American Meteorological Society), 5-42.
Whiteman, C. D. (2000). Mountain Meteorology - Fundamentals and Applications. New York, NY: Oxford University Press.

Wolyn, P. G., and McKee, T. B. (1989). Deep stable layers in the intermountain western United States. Mon. Weather Rev. 117, 461-472.

Wood, N. (2000). Wind flow over complex terrain: a historical perspective and the prospect for large-eddy modelling. Bound. Layer Meteorol. 96, 11-32. doi: 10.1023/A:1002017732694

Worthington, R. M. (2002). Mountain waves launched by convective activity within the boundary layer above mountains. Bound. Layer Meteorol. 103, 469-491. doi: 10.1023/A:1014965029602

Wulfmeyer, V., Behrendt, A., Bauer, H.-S., Kottmeier, C., Corsmeier, U., Blyth, A., et al. (2008). The convective and orographically-induced precipitation study: a research and development project of the World Weather Research Program for improving quantitative precipitation forecasting in low-mountain regions. Bull. Am. Meteorol. Soc. 89, 1477-1486. doi: 10.1175/2008BAMS 2367.1

Yanai, M., Li, C., and Song, Z. (1992). Seasonal heating of the Tibetan Plateau and its effects on the evolution of the Asian summer monsoon. J. Meteorol. Soc. Japan 70, 319-351.

Yang, K., Koike, T., Fujii, H., Tamura, T., Xu, X., Bian, L., et al. (2004). The daytime evolution of the atmospheric boundary layer and convection over the Tibetan Plateau: observations and simulations. J. Meteorol. Soc. Japan 82, 1777-1792. doi: $10.2151 /$ jmsj.82.1777

Ye, Z. J., Segal, M., and Pielke, R. A. (1987). Effects of atmospheric thermal stability and slope steepness on the development of daytime thermally induced upslope flow. J. Atmos. Sci. 44, 3341-3354.

Zängl, G., and Gonzalez Chico, S. (2006). The thermal circulation of a grand plateau: sensitivity to the height, width, and shape of the plateau. Mon. Weather Rev. 134, 2581-2600. doi: 10.1175/MWR 3207.1

Zardi, D., and Whiteman, C. D. (2013). "Diurnal mountain wind systems," in Mountain Weather Research and Forecasting: Recent Progress and Current Challenges, eds F. Chow, S. F. J. De Wekker, and B. Synder (New York, NY: Springer), 35-119.

Zawar-Reza, P., and Sturman, A. P. (2006). Two-dimensional numerical analysis of a thermally generated mesoscale wind system observed in the Mackenzie Basin, New Zealand. Aust. Meteorol. Mag. 55, 19-34.

Conflict of Interest Statement: The authors declare that the research was conducted in the absence of any commercial or financial relationships that could be construed as a potential conflict of interest.

Copyright (C) 2015 De Wekker and Kossmann. This is an open-access article distributed under the terms of the Creative Commons Attribution License (CC BY). The use, distribution or reproduction in other forums is permitted, provided the original author(s) or licensor are credited and that the original publication in this journal is cited, in accordance with accepted academic practice. No use, distribution or reproduction is permitted which does not comply with these terms. 


\section{On the Vertical Exchange of Heat, Mass, and Momentum Over Complex, Mountainous Terrain}

\section{OPEN ACCESS}

Edited by:

Peter F. Sheridan

Met Office, UK

Reviewed by:

Moacyr Cunha De Araujo,

Universidade Federal de Pernambuco,

Brazil

Ke Wei,

Institute of Atmospheric Physics, Chinese Academy of Sciences, China

*Correspondence: Mathias W. Rotach

mathias.rotach@uibk.ac.at

Specialty section:

This article was submitted to

Atmospheric Science,

a section of the journal

Frontiers in Earth Science

Received: 29 August 2015 Accepted: 17 November 2015 Published: 21 December 2015

Citation:

Rotach MW, Gohm A, Lang MN Leukauf D, Stiperski I and Wagner JS (2015) On the Vertical Exchange of

Heat, Mass, and Momentum Over Complex, Mountainous Terrain.

Front. Earth Sci. 3:76.

doi: 10.3389/feart.2015.00076

\author{
Mathias W. Rotach ${ }^{1 *}$, Alexander Gohm ${ }^{1}$, Moritz N. Lang ${ }^{2}$, Daniel Leukauf ${ }^{1}$, \\ Ivana Stiperski ${ }^{1}$ and Johannes S. Wagner ${ }^{3}$
}

${ }^{1}$ Institute of Atmospheric and Cryospheric Sciences, University of Innsbruck, Innsbruck, Austria, ${ }^{2}$ Zentralanstalt für Meteorologie und Geodynamik, Vienna, Austria, ${ }^{3}$ Institut für Physik der Atmosphäre, Deutsches Zentrum für Luft und Raumfahrt, Oberpfaffenhofen, Germany

The role of the atmospheric boundary layer (ABL) in the atmosphere-climate system is the exchange of heat, mass, and momentum between "the earth's surface" and the atmosphere. Traditionally, it is understood that turbulent transport is responsible for this exchange and hence the understanding and physical description of the turbulence structure of the boundary layer is key to assess the effectiveness of earth-atmosphere exchange (EAE). This understanding is rooted in the (implicit) assumption of a scale separation or spectral gap between turbulence and mean atmospheric motions, which in turn leads to the assumption of a horizontally homogeneous and flat (HHF) surface as a reference, for which both physical understanding and model parameterizations have successfully been developed over the years. Over mountainous terrain, however, the ABL is generically inhomogeneous due to both thermal (radiative) and dynamic forcing. This inhomogeneity leads to meso-scale and even sub-meso-scale flows such as slope and valley winds or wake effects. It is argued here that these (sub)meso-scale motions can significantly contribute to the vertical structure of the boundary layer and hence vertical exchange of heat and mass between the surface and the atmosphere. If model grid resolution is not high enough the latter will have to be parameterized (in a similar fashion as gravity wave drag (GWD) parameterizations take into account the momentum transport due to gravity waves in large-scale models). In this contribution we summarize the available evidence of the contribution of (sub)meso-scale motions to vertical exchange in mountainous terrain from observational and numerical modeling studies. In particular, a number of recent simulation studies using idealized topography will be summarized and put into perspective-so as to identify possible limitations and areas of necessary future research.

Keywords: mountain meteorology, boundary layers, horizontal inhomogeneity, thermally driven flows, gravity waves

\section{INTRODUCTION}

According to Stull (1988) the Atmospheric Boundary Layer (ABL) is defined "as that part of the troposphere that is directly influenced by the presence of the earth's surface, and responds to surface forcing with a timescale of about an hour or less." Stull adds that "These forcings 
include frictional drag, evaporation and transpiration, heat transfer, pollutant emission, and terrain induced flow modification." Due to these surface forcings (mainly friction and heat exchange) ABL flows are generally turbulent, and hence turbulent transport plays an important role and determines to a large extent the exchange efficiency of energy, mass, and momentum between the surface and the atmosphere. One may, therefore, also define, the ABL as that atmospheric layer through which the earth-atmosphere exchange (EAE) is effectuated. The so-called "boundary layer approximation" assumesmostly for the purpose of ABL parameterizations in numerical models-that turbulent transport exceeds that associated with the mean flow, especially in the vertical direction. Hence a "single column" (vertical) approach is usually employed in ABL parameterizations for models with grid-scales larger than typical ABL heights (order $1 \mathrm{~km}$ ). For horizontally homogeneous and flat (HHF) surfaces this indeed covers the entire vertical exchange so that at the time when numerical atmospheric modeling emerged-with large-scale, low-resolution models where topography is smoothed and horizontal gradients are correspondingly small-successful one-dimensional ABL parameterizations were developed. These are formulated based on similarity theory using the properties of different "scaling regimes" (e.g., Holtslag and Nieuwstadt, 1986).

Since the world is not flat (Rotach et al., 2014) and-maybe with the exception of the oceans-not generally horizontally homogeneous, the efficiency of the exchange between the "surface" and the "free atmosphere" is not controlled by vertical turbulent transport in the $\mathrm{ABL}$ alone. The effect of horizontal inhomogeneity on the ABL structure and thus EAE has been studied using the concept of "internal boundary layers" (Garratt, 1990; Savelyev and Taylor, 2005) and its consequences on "vertical" transport. However, little research has been done concerning the impact of non-flat surfaces on the earthatmosphere exchange (EAE). As "non-flat" intrinsically means horizontally inhomogeneous (Rotach and Zardi, 2007), i.e., "non-HHF" the purpose of this paper is to summarize current understanding concerning net exchange of energy, mass, and momentum between the topographically shaped surface on the one hand and the atmosphere on the other hand in the presence of substantial topography. By "substantial" we mean that the influencing topography is characterized by length scales which are on the same order or even larger than the ABL height of the hypothetical "undisturbed far upstream" boundary layer. We note that this exceeds what is usually denoted as EAE, i.e., the turbulent fluxes at the surface. Rather, this corresponds to identifying the entire $\mathrm{ABL}$ in mountainous terrain as the "agent" of EAE and requires identification of (sub-) meso-scale processes contributing to this exchange (Section Exchange Processes Over Complex, Mountainous Topography). In Section Observed Evidence for Earth-Atmosphere Exchange Over Mountainous Terrain we review the sparse evidence for EAE over mountainous terrain based on real-terrain atmospheric studies (both experimental and modeling) and in Section Idealized-Terrain Numerical Simulations we focus on results from high-resolution numerical modeling using idealized terrain. Finally, in Section Outlook we identify the critical gaps in knowledge concerning EAE over mountainous terrain thereby suggesting future research directions.

\section{EXCHANGE PROCESSES OVER COMPLEX, MOUNTAINOUS TOPOGRAPHY}

Exchange processes can generally be identified as having dynamic or thermodynamic origin, even if the dynamically forced processes also depend on the thermodynamic state (background stratification) of the atmosphere.

\section{Dynamically Forced Exchange Processes Over Mountainous Terrain}

Under the condition of a stably stratified background flow, topography (variability in surface elevation) can produce gravity waves at all scales (Baines, 1995; Nappo, 2002), which transport energy and momentum, in a direction normal to the phase propagation (Holton and Hakim, 2013). In numerical models, the effect of subgrid-scale topography in extracting momentum from the mean flow has long been recognized at global (hemispheric) scales (Palmer et al., 1986) and corresponding "gravity-wave drag parameterizations" have been developed and implemented. Nevertheless, for stable background flow Lott and Miller (1997) noted that the linear gravity wave response may not be sufficient to fully cover the effect of sub-grid scale topography on the momentum budget, and therefore proposed to add some "low level drag" below the level of blocked flow (which depends on the stratification and flow conditions). Their work demonstrates that such low level drag can be of equal magnitude as the gravitywave drag alone. Applying the sum of the two contributions as "orographic drag parameterization" compares favorably to direct drag measurements as performed during PYREX (Bougeault et al., 1993) and also improves model predictions (using the ECMWF model at T103 and T206 resolution at the time) of mean flow characteristics.

Even under neutral conditions, the state of the $\mathrm{ABL}$ is modified due to topography. Based on the work of Jackson and Hunt (1975), Hunt et al. (1988a) have developed a linearized theoretical framework for the boundary layer structure over low topography (with a vertical extension of the boundary layer being much smaller than the half-width of the hill so as to allow for linearization) that has later been extended to stratified flows (Hunt et al., 1988b) and is comprehensively summarized by Belcher and Hunt (1998). This framework allows estimation of the additional drag a "hilly" surface exerts on the flow (Belcher et al., 1993), thus giving rise to an additional impact of sub-grid scale topography on the flow. Essentially a "wavy surface" will transport more momentum toward the surface than the boundary layer parameterization alone will determine, and thus slows down the flow. The need for an additional parameterization at least for global NWP and climate models has been recognized and introduced into model parameterizations (e.g., Wood and Mason, 1993; Wood et al., 2001). However, the extent to which sub-meso scale terrain variability may contribute to momentum exchange in high-resolution NWP models remains to be investigated. Belcher and Hunt (1998) 
further note that over low hills modification of mean flow and turbulence structure in the boundary layer (Raupach et al., 1992) also affect the exchange of mass and energy, but to a lesser extent than momentum transfer is affected.

\section{Thermally Forced Exchange Processes Over Mountainous Terrain}

Exchange between earth and atmosphere is associated with flows induced by differential heating in relation to topography, occurring at a range of horizontal scales due to varying slope angles and exposition. Thermally forced flows, driven by radiative heating have a clear daily cycle and occur preferentially during clear-sky conditions with weak synoptic forcing. Under overcast conditions spatial variability of diabatic heating is essentially zero even in complex topography (Matzinger et al., 2003) and if the synoptic forcing is considerable it may easily mask the thermal flow. Mechanisms and examples have been discussed extensively in the literature-most recently in a comprehensive review by Zardi and Whiteman (2013) that updates Whiteman (2000). We therefore concentrate on the vertical exchange associated with these flows (see Sections Observed Evidence For Earth-Atmosphere Exchange Over Mountainous Terrain and Idealized-Terrain Numerical Simulations) and very briefly characterize their properties relevant for this.

At the meso- $\alpha$ scale (conventionally taken to range up to some $200 \mathrm{~km}$; Orlanski, 1975) a mountain range, or isolated mountain, is often associated with a thermal low, thus causing flow toward the topography during the day. Different names have been coined for this phenomenon depending on location and application. Figure 1 seeks to summarize these flows graphically.

- Plain-to-mountain flow (Kleinschmidt, 1922; De Wekker et al., 1998; Weissmann et al., 2005) is the classical, idealized situation where the difference in temperature between equal heights over the plain and over the mountain leads to the establishment of a thermally driven flow. In Figure 1, this would typically be flow normal to the mountain or the mountain range. These flows can transport air pollutants from major cities in the plain, or moisture from the humid boundary layer over the plain

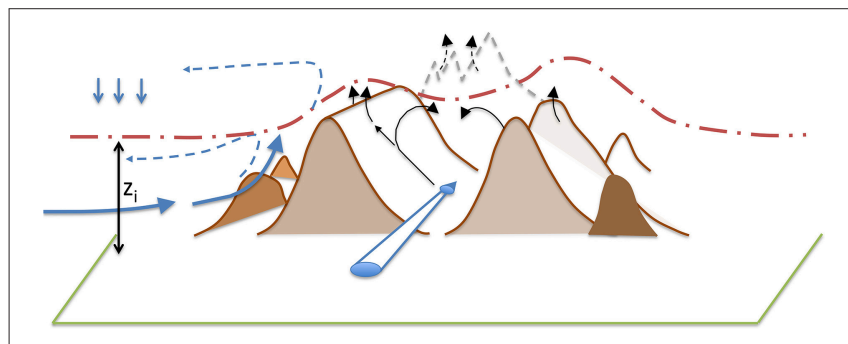

FIGURE 1 | Conceptual sketch of thermally driven flows in mountainous terrain. " $z_{i}$ " denotes the Mixed layer height (red dash-dotted line) over the adjacent plain. Blue arrows, plain-to- mountain circulation and valley wind circulation; black arrows, slope-flow and mountain venting. Situation during daytime. into the mountains (or vice versa). A plain situated at the entrance of a major valley running normal to the mountain range, can result in plain-to-mountain flow being the driver for the valley wind system (see below, meso- $\beta$ scale). Viewed as part of a mountain-plain circulation (Weissmann et al., 2005) a plain-to-mountain flow is, in both concept and simulations, associated with a return flow either within (e.g., Demko et al., 2009) or above (dashed arrows in Figure 1) the boundary layer. Return flows are, of course, difficult to experimentally document since they are often masked by (even weak) synoptic flow (Weissmann et al., 2005).

- Mountain venting (Kossmann et al., 1999; De Wekker et al., 2004; Henne et al., 2005) refers to the vertical exchange associated with the plain-to-mountain flow or mountain-plain circulation. While the mechanism for this vertical exchange has sometimes been attributed to boundary layer growth beyond the top of the mountains (e.g., Nyeki et al., 2000), realterrain (De Wekker et al., 2004) and idealized (e.g., Wagner et al., 2015b) simulations indicate that the exchange is not the result of a uniform mixing process (i.e., "boundary layer growth") but preferably occurs through the slope flows (meso$\gamma$ scale, see below), i.e., through organized vertical motions (Kossmann et al., 1999). De Wekker and Kossmann (this volume) discuss in more detail the difference between the boundary layer height and the "mixing height." Kossmann et al. (1999) further distinguish two different venting types, what they call "mountain venting" and "advective venting." Both are (thermo-)dynamically driven meso-scale flows that export boundary layer to the free atmosphere, the former by vertically crossing the $\mathrm{ABL}$ top and the latter if the meso-scale flow crosses an inclined ABL top (e.g., over a slope).

- Alpine pumping: this expression has specifically been introduced (Lugauer and Winkler, 2005) for the mountainplain circulation in the alpine foreland north of the Alps and in relation to the development of convection.

At the meso- $\beta$ scale (20-200 km), temperature and hence pressure differences between "valley" and "plain" (see Figure 1) give rise to a valley wind system directed up-valley during the day and down-valley during the night. There has been some debate in the literature concerning the origin of the elevated daytime (reduced nighttime) temperature in a valley as compared to the reference plain. We refer to the discussion of results from (idealized) numerical simulations (Section Idealized-Terrain Numerical Simulations) to address this question. The valley wind system contributes in two ways to vertical exchange. Firstly, if the valley narrows up-valley the "geometric effect" leads to a net export (import) of mass for up-valley (down-valley) flows during daytime (nighttime) and hence exchange of trace constituents such as moisture or pollutants (Weigel et al., 2007). Secondly, turbulent transport through a lid separating the valley air from the "free tropospheric air"-or more generally through the valley boundary layer top (see De Wekker and Kossmann, this volume)-constitutes another possible mechanism for EAE in mountainous terrain. Again, when understood as a circulation pattern arising from a locally baroclinic circulation in alongvalley direction, the valley wind system is associated with a return 
flow aloft. Similar to the plain-to-mountain scale this return flow is evident from idealized numerical simulations (e.g., Rampanelli et al., 2004) but hard to document experimentally, even if some evidence seems to be available (see Zardi and Whiteman, 2013 and references therein).

At the meso- $\gamma$ scale $(2-20 \mathrm{~km})$ the difference between the nearsurface temperature above a slope and that at some horizontal distance from the slope leads to a pressure gradient toward (away from) the slope and hence the establishment of an up-slope (down-slope) flow during the day (night). For these flows to establish, physically a sloped terrain is sufficient and models such as the classical Prandtl model (Prandtl, 1942, 1952), extended over the years to include e.g., rotational effects (Stiperski et al., 2007), weak non-linearity (Grisogono et al., 2015) or temporal variations in the forcing (Zardi and Serafin, 2015) have been devised for their description. In mountainous terrain, slopes are often associated with some symmetry in a valley so that a locally baroclinic stratification across the valley leads to a slope-flow circulation (see Figure 1). In numerical simulations (when employing over idealized terrain) this circulation is usually symmetric while in reality this is not necessarily the case (e.g., Weigel and Rotach, 2004; Gohm et al., 2009; see also below, Figure 5). Both, isolated slope flows (in Figure 1 this might refer to the "outer slope" of a mountain range or to the slope of an isolated mountain) and cross-valley circulations are associated with transport of mass and energy in the slope-wind layer (generally: the boundary layer on the slope) and thus contribute to EAE over mountainous terrain. Its efficiency depends on slope (valley) geometry and atmospheric forcing conditions as will be discussed in more detail in Section Idealized-Terrain Numerical Simulations.

At the micro-scale, comprising terrain features with horizontal scales $<2 \mathrm{~km}$, EAE is mostly, or perhaps exclusively affected under (very) stable conditions through so-called sub-meso motions. According to Mahrt (2014) very stable conditions are particularly likely to occur "in basins, local depressions and valleys with weak down-valley slopes." Under these conditions of strong stability the well-known theoretical similarity framework for turbulent exchange no longer holds (i.e., the flow is not fully turbulent) and exchange processes occur through complicated and hitherto poorly understood interactions between intermittent turbulence, wave activity, small-scale (submeso) organized motions, and a wealth of other features. Relatively little is known at present concerning these flows, nothing to say about their interaction or exchange efficiency. Mahrt (2014) provides a number of emerging research topics and requirements in order to better understand and formally treat these situations.

\section{OBSERVED EVIDENCE FOR EARTH-ATMOSPHERE EXCHANGE OVER MOUNTAINOUS TERRAIN}

Evidence for EAE over mountainous terrain may arise from large observational campaigns such as the Mesoscale Alpine Programme (MAP; Bougeault et al., 2001), and/or through real-terrain numerical modeling. In this section we summarize such evidence separately for momentum, energy and mass.

\section{Momentum Exchange}

For momentum transport over topography due to both gravity waves and modified boundary layer structure (Belcher and Hunt, 1998) indirect evidence can be sought by comparing near-surface wind climatologies from large-scale numerical models with those from observational networks. This is used to confirm that the "westerly bias" (too strong and too westerly surface winds, especially in the winter $\mathrm{NH}$ ) has been correctly alleviated by the use of a gravity wave drag (GWD) parameterization (e.g., Palmer et al., 1986; Lott and Miller, 1997). It should be noted, however, that this "westerly bias" is not the only model deficiency for which remedy is sought by introducing a GWD parameterization in a global atmospheric model, and also that mountains are not the only source of gravity waves (see Kim et al., 2013). Kim et al. (2013) also discuss the difficulty of reliably measuring momentum flux (and even more so, its divergence) in order to directly compare a GWD parameterization with observations. Again, as some sort of indirect evidence, high-resolution meso-scale numerical modeling of a particular case study (e.g., Doyle and Shapiro, 2000) can-when successfully validated against observationsbe used to diagnose momentum transport and thus evaluate a parameterization.

Milton and Wilson (1996) analyzed the momentum budget of the Unified Model of the UK MetOffice. For the GWD parameterization used at that time, the largest deviations in momentum were found in the lower troposphere (boundary layer) over major mountain ranges. Besides improving the GWD parameterization Milton and Wilson (1996) introduced another parameterization for orographic drag (form drag due to sub-grid scale orography) thus substantially reducing the inconsistency in the momentum balance and the "westerly bias" in mid latitudes by up to $2 \mathrm{~ms}^{-1}$.

Parameterizations of orographic drag are often based on introducing an additional contribution to the roughness length due to subgrid-scale orography, yielding a so-called "effective roughness length” (Taylor et al., 1989; Wood and Mason, 1993). Good correspondence of observed effective roughness lengths with those parameterized (e.g., Wood and Mason, 1993) using the additional drag as a source for momentum transport is another means of indirect evidence for the importance of this process.

The observational study of Turnipseed et al. (2004) identifies gravity waves as the main drivers for the exchange of momentum and energy in stable boundary layers. Up to $50 \%$ of the total scalar variance can be accounted for by low frequency waves. They also point to the rare, but significant, influence of meso-scale gravity waves on boundary layer characteristics. Over moderate terrain, Medeiros and Fitzjarrald (2014) attribute the missing drag to averaging over their non-homogeneous terrain. Focusing on the small-scale end of the gravity wave spectrum, Sun et al. (2015) give a detailed review of the wave-turbulence interaction in the stable boundary layer. 


\section{Energy (Heat) Exchange}

The introduction of GWD parameterizations in numerical models provides an improved distribution of global temperatures (e.g., Kim et al., 2013-even if this effect is the result of an adjustment process to the thermal wind balance) and hence indirect evidence for modified energy exchange due to processes related to topography. Still, it is quite difficult to experimentally assess the effect of thermally forced meso-scale circulations (as described in Section Thermally Forced Exchange Processes Over Mountainous Terrain) for a real-terrain case study or a regional climatology over mountainous terrain. For heat exchange, therefore, there is little direct experimental evidence available-and only very limited indirect evidence. The difficulty with measuring the effect of energy exchange between the surface and the atmosphere in complex terrain may be best illustrated by the results of Kossmann et al. (1999). While clearly documenting the exchange of, e.g., $\mathrm{NO}_{2}$ through mountain venting, the attribution to exchange of, e.g., moisture (nothing to say about sensible heat) turned out to be difficult due to complicated interaction between different processes and comparably small vertical gradients. A rare example of indirect evidence for vertical heat exchange may be the study of Weigel and Rotach (2004) in the Riviera Valley in southern Switzerland (Rotach et al., 2004). On several days with weak synoptic forcing ("pure" thermally driven flow) a Mixed Layer initially started to develop in the morning but-despite continuing surface forcing-the valley atmosphere re-stabilized during the early afternoon. This re-stabilization was attributed to a "secondary circulation" in addition to the primary symmetric valley wind circulation, transporting potentially warmer air from aloft into the valley and thus heating it from above. This hypothesized circulation was attributed to valley curvature and later (Weigel et al., 2006) supported by high-resolution numerical simulations. Indeed the modeled valley atmospherecorresponding to the observations-was characterized by a single circulation pattern transporting heat down the primarily heated eastern slope thus leading to re-stabilization in the (early) afternoon.

While the impact of topographically-modified boundary layer structure on momentum transport-even if restricted to small slopes due to linearization (Belcher and Hunt, 1998)-has been, at least indirectly, experimentally confirmed (see Section Momentum Exchange), its role in scalar exchange has, to the knowledge of the authors, not been demonstrated in the field. Some findings according to linear theory are discussed in Raupach and Finnigan (1997) and extended to the non-linear regime by conducting "preliminary" (as the authors denote it) wind tunnel experiments. Results indicate that surface fluxes (sensible and latent heat, as well as their sum, i.e., available energy) exhibit significant spatial and temporal differences between flat and complex terrain, but when regionally averaged (using a simple slab model for the convective boundary layer) this difference vanishes. Whether this is due to the highly idealized setting, the symmetry of the topography used, or the total insignificance of heat transport due to complex topography remains to be clarified.

\section{Mass Exchange}

The most intuitive examples of mass exchange are related to cloud formation over an isolated mountain due to mountain venting (note that since we are interested in un-resolved subgridscale exchange processes we are not referring here to orographic precipitation, (e.g., Rotunno and Houze, 2007), where the largescale synoptic flow impinges on a mountain (ridge) and gives rise to cloud formation and precipitation). Geerts et al. (2008) present some 2 months of data around Santa Catalina Mountains (with a diameter of about $30 \mathrm{~km}$ ) to document the plain-to-mountain flow (convergence) due to the frequent development of a heat low over the topography that is associated with moist boundary layer air being transported to the mountain, and cloud (thunderstorm) formation over the peak. Further experimental (Demko et al., 2009) and modeling (Demko and Geerts, 2010) evidence from the same mountains reveals that-at least over this very mountain range-the convergent flow is solenoidal and largely confined to the convective boundary layer.

Henne et al. (2004) used different kinds of airborne and surface-based instrumentation, in combination with interpolation schemes and transport modeling to estimate EAE for a relatively small, steep and convergent valley (Leventina Valley) in southern Switzerland. For their 3 example days in summer, experiencing fair weather, and thermally forced conditions, their rough estimates of the mass fluxes in and out of the valley atmosphere indicated that the "valley boundary layer air" was replaced about three times per day, thus giving rise to pollutant exchange (either export of in-valley emissions or vice versa). In a subsequent study in similar weather conditions Henne et al. (2005) used different surface networks (including meso-net and soundings) to attribute enhanced moisture content in an elevated layer in the lee of the Alps (2500-3500 m above sea level, respective lee side, depending on flow conditions) to study mountain venting. Its efficiency was estimated as $30 \%$ of the boundary layer air being exchanged per hour during daytime. This lee side upper level moisture excess was detectable even when averaged over the entire summer season (JJA).

Similarly, using moisture as a tracer, Weigel et al. (2007) modeled the various contributions of exchange processes to the moisture exchange in and out of the Riviera Valley in southern Switzerland, again for a few fair weather days in summer. Results from this non-hydrostatic modeling, run at $350 \mathrm{~m}$ horizontal grid spacing, were previously subject to rigorous validation (Chow et al., 2006; Weigel et al., 2006) so that we treat the simulation results from this real-case study as "observation." Comparison of EAE with the valley resolved or completely unresolved in the model indicates that contributions of individual exchange processes vary widely in time, and the cumulated daytime exchange was underestimated by a factor of about three when the valley was not resolved at all.

Mass exchange related to a possibly super-critical flow pattern (Zangl et al., 2001) was hypothesized to contribute to the extraordinarily strong valley wind in one of the deepest valleys on Earth, the Kali Kandaki Valley in Nepal (Egger et al., 2000). Even if the numerical simulations of Zangl et al. (2001) suggested a strong impact of humidity and hence moisture exchange, this 
could not be substantiated in a second field campaign (Egger et al., 2002).

All these examples concerning moisture exchange over complex topography are limited to a few usually fair weather, weak synoptic forcing days at a particular location. For these conditions they demonstrate the potential of primarily (valley) geometry and thermally driven flows to substantially contribute to mass (and hence moisture) exchange between the mountainous surface and the free atmosphere. To what degree other flow conditions and topographic landforms (e.g., basins) contribute to such exchange will have to be subject of further research.

$\mathrm{CO}_{2}$ is another potential tracer for mass exchange, but the low resolution of global $\mathrm{CO}_{2}$ inversion models is insufficient to resolve the impact of meso-scale (thermal) circulations on $\mathrm{CO}_{2}$ exchange processes (Pérez-Landa et al., 2007). A combination of high-resolution numerical modeling and experimental data from a 2-week campaign in the coastal region of Valencia (E) influenced by profound topography, was used by Pérez-Landa et al. (2007) to assess the impact of non-resolved meso-scale circulations. While encountering a number of difficulties-e.g., the "correct" attribution of source (sink) strength, timing and localization to the "tracer" $\left(\mathrm{CO}_{2}\right)$-as usual in a study with pioneering character such as this one, the authors could clearly demonstrate the importance of thermal circulations (dominantly sea breeze in their case, but in combination with topographic forcing) on the regional budget of $\mathrm{CO}_{2}$. Similarly concerned with the quality of $\mathrm{CO}_{2}$ budget modeling using coarse-grid inversion technique, Pillai et al. (2011) present evidence for the impact of thermally driven circulations on the flux-tower site "Ochsenkopf" situated in the "Fichtelgebirge," a moderately high-some $500 \mathrm{~m}$ elevation with respect to surroundingspre-alpine region in southern Germany. By demonstrating that "the effects of meso-scale transport such as mountain-valley circulations and mountain-wave activities on atmospheric $\mathrm{CO}_{2}$ distributions are reproduced remarkably well in the highresolution models" they indirectly confirm the importance of these exchange processes in mountains terrain. Sun et al. (2010), in a multi-scale and multi-disciplinary study using a wealth of different observational and modeling techniques, investigated the processes influencing the regional $\mathrm{CO}_{2}$ budget over the Rocky Mountains (CO). Within the mountain boundary layer, $\mathrm{CO}_{2}$ was found to be transported effectively by drainage (nighttime) and up-slope flows thereby largely affecting the local net ecosystem exchange at one of the rare (Rotach et al., 2014) mountainous "flux tower sites." At a regional scale, they quite successfully and for the first time applied the "regional budget" approach to assess the flux tower's representativeness for larger scale fluxes of $\mathrm{CO}_{2}$. As an overall conclusion they state that even if the distribution of $\mathrm{CO}_{2}$ over mountains terrain, and hence the exchange processes contributing to it, is complicated, it is "predictable" if sufficient high-resolution information and modeling capacity is available. Using the same database, Sun and De Wekker (2011) investigated two detailed case studies through which they demonstrated the interplay between slope flow and convective boundary layer mixing on the slope of the Rocky Mountains. Even some experimental evidence for the return flow aloft (see Figure 1) could be observed.

Exchange of aerosols is a readily observable example for the exchange of mass in mountainous terrain. Nyeki et al. (2000) have documented elevated aerosol layers-reaching over the tops of the Alps, i.e., up to some $4200 \mathrm{~m}$ a.s.l. (above sea level, see Figure 2)-and attributed this to the growth of the convective boundary layer up to this level. More recently, simulations of De Wekker et al. (2004) have shown that the height of the "aerosol layer" does not correspond to the "mixed layer" (or convective boundary layer) height (see discussion in Section Thermally Forced Exchange Processes Over Mountainous Terrain). Similar conclusions had already been drawn by De Wekker (2002), based on simulations of the Riviera Valley (Rotach et al., 2004) with the meteorological part of the simulations validated using observations (De Wekker et al., 2005), but the aerosols (particles) artificially being introduced into the domain. However, the elevated aerosol concentrations as in Figure 2 can be taken as experimental evidence for (i) the efficiency of mass exchange over truly complex topography and (ii) the fact that different processes, i.e., turbulent exchange within the mountain boundary layer and meso-scale processes (mountain venting), are coresponsible for the total EAE over mountainous terrain. The relative importance of these (and geometrical, i.e., terrain related)
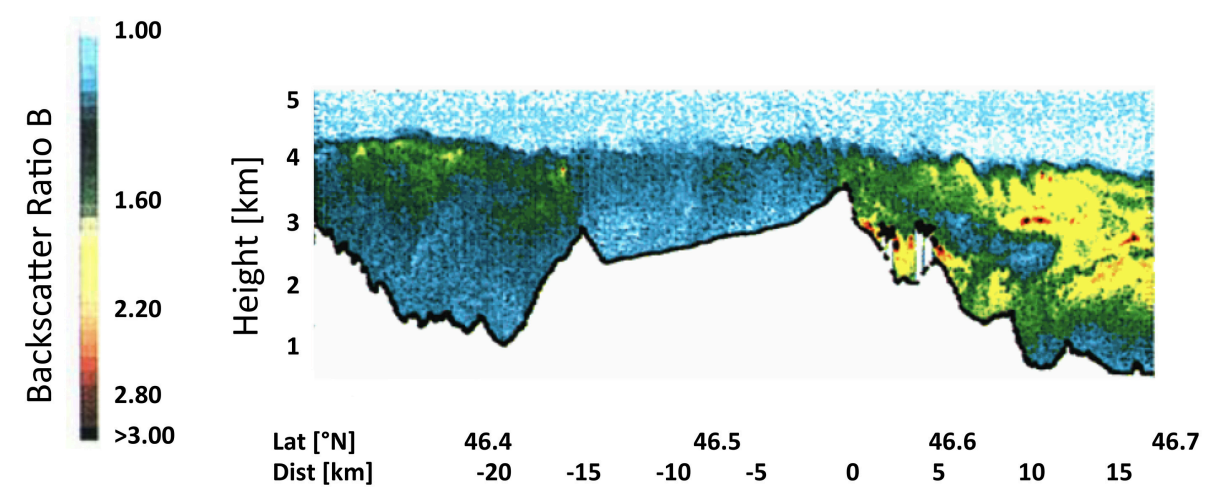

FIGURE 2 | Backscatter ratio as measured with airborne lidar over a transect across the Alps on July 30 1997, 16.20 LST (reproduced with permission from Nyeki et al., 2000). The highest visible peak (at horizontal distance 0) is Jungfraujoch. The Rhone Valley at approximately -20 km. 
processes, to EAE is likely dependent on several external factors and cannot be determined experimentally. The next section on idealized modeling will address some of these questions.

A particular form of mass exchange over a mountainous area occurs over the western Tibetan Plateau where the ABL can grow in late winter very high (up to $5000 \mathrm{~m}$ a.s.l., e.g., Chen et al., 2013). Together with the surface elevation of some $4000 \mathrm{~m}$ above sea level this means that the boundary layer top can reach the neighborhood of the tropopause. While the reasons for these deep boundary layers are under debate (Chen et al., under review and references therein) it is clear that what is usually termed stratosphere-troposphere exchange under such conditions results in exchange of mass (e.g., ozone) from the stratosphere directly into the near-surface region thus potentially having profound implications on atmospheric chemistry (e.g., Škerlak et al., 2014).

\section{IDEALIZED-TERRAIN NUMERICAL SIMULATIONS}

Most of the work dedicated to the derivation of parameterizations for orographic drag or boundary layer modification over low hills is based on idealized simulations in concert with the respective theoretical framework (e.g., Smith, 1979, 2007; Teixeira, 2014). Since the results of these simulations are already widely employed in large-scale numerical models and comprehensive reviews are available (e.g., Kim et al., 2013) we only briefly refer here to more recent efforts based on idealized modeling to assess the interaction of gravity waves with boundary layer processes.

Most of the research effort studying the interaction between boundary layers and gravity waves in recent years focused on the influence of the boundary layer in modifying meso-scale mountain wave characteristics (e.g., Jiang et al., 2006, 2008; Smith, 2007; Jiang and Doyle, 2008; Stiperski and Grubišič, 2011). The majority of these studies used 2D idealized terrain profiles. The theoretical framework of Smith (2007) and idealized simulations of Jiang et al. $(2006,2008)$ and Jiang and Doyle (2008) have shown that mountain waves are absorbed within the boundary layer, thus the wave drag and wave momentum flux above the boundary layer are reduced and wave patterns shifted upstream. Boundary layer height, stability and non-hydrostacity were found to be among the most important parameters. The convective boundary layer was found to be most effective in reducing wave amplitude and drag (Jiang and Doyle, 2008). The effect is less pronounced for narrow mountains and very nonlinear flow (e.g., high mountains) where the boundary layer height is small compared to flow depth. Vosper and Brown (2007) noted the significant reduction in total drag due to smallscale hills. On the other hand, Steeneveld et al. $(2008,2009)$, investigating the effect of small-scale terrain on the stable boundary layer characteristics, found that drag caused by smallscale gravity waves can be on the same order as the turbulent drag and accounts for the missing drag in standard drag parameterizations.

In a recent investigation Kirshbaum (2013) studied the dynamic response of an idealized heated mountain (i.e., a "mountainlike heat source") using a linearized scaling of the
Boussinesq set of equations in a two-layer approach. Three different regimes are identified exhibiting distinctly different patterns with respect to vertical exchange between the lower (boundary) layer and the troposphere (upper layer). Among these regimes, the one representing a convective boundary layer flow under weak wind forcing produced the strongest and most localized updrafts and thus vertical exchange. Clearly, simulations of this kind are constrained by the necessity to restrict the slope angles (linearization) but they provide insight in the theoretical understanding of what has been termed plain-tomountain flows in Section Thermally Forced Exchange Processes Over Mountainous Terrain.

Over the last decade or so a number of modeling studies were performed using idealized terrain (and idealized atmospheric conditions, too) to study exchange processes due to thermally driven flows over complex terrain (Noppel and Fiedler, 2002; Rampanelli et al., 2004; Catalano and Cenedese, 2010; Catalano and Moeng, 2010; Schmidli and Rotunno, 2010, 2012; Serafin and Zardi, 2010a,b, 2011; Schmidli, 2013; Wagner et al., 2014, 2015a,b; Lang et al., 2015; Leukauf et al., 2015). Results clearly demonstrate that energy and mass is exchanged as the result of thermally driven (turbulent) flows (meso-scale circulations) due to the presence of topography. They may be summarized as follows.

\section{Heating Mechanism of a Valley}

Most often, the Topographic Amplification Factor (TAF; Steinacker, 1984; based on Wagner, 1932) or so-called valley volume effect is employed in order to explain the elevated (reduced) temperature in a valley, as compared to a neighboring plain, leading to the thermally forced valley flow. Based on the argument that a smaller air volume is heated (cooled) by the same amount of solar heating (cooling) than over the plain the TAF is not dependent on (mean) vertical motions. Rather it considers the valley as a closed system without net fluxes at its top. This seems to be at odds with the apparent vertical motions associated with the cross-valley circulation (meso- $\gamma$ scale; see also the black arrows in Figure 1) and hence the argument (e.g., Rampanelli et al., 2004; Serafin and Zardi, 2010b, 2011) that the excess valley daytime-heating be predominantly due to subsidence heating in the middle of the valley thus bringing high-potential temperature air from the free atmosphere aloft down into the valley. Schmidli (2013), in a comprehensive budgeting study showed that indeed the vertical contribution to the advection term in the heat budget equation can locally be positive (i.e., downward, thus heating the valley from above). This is the case in the center of the valley and for elevated levels. However, other components of the advection term (most prominently the along-slope component), which form the crossvalley circulation together with the subsidence in the center of the valley, lead to substantial cooling. Consequently, if the heat budget for the bulk of the valley is considered, Schmidli (2013) demonstrated that the net effect of the cross-valley circulation (subsidence in the middle of the valley plus slope flow advection) is to cool the bulk valley volume. When averaged over the entire valley depth the contribution of subsidence is negligible. Hence, the excess heating according to the TAF actually constitutes an 
upper limit for the heat exchange into (out of) a valley that is somewhat reduced by the cross-valley circulation. Overall, idealized simulations show that the cross-valley circulation contributes to energy exchange between the valley and the free troposphere. Subsidence heating-while locally being relevant as a heat source in the center of the valley (Schmidli, 2013) is not the dominant factor of the enhanced daily temperature amplitude in valleys (as compared to a reference plain).

\section{Exchange is Dependent on Geometry}

Most numerical idealized-terrain simulations use one predefined terrain configuration that corresponds to an "average valley" (often straight, and symmetric with respect to the valley axis, often 1500 or $2000 \mathrm{~m}$ deep, using a trapezoid or cosine-type cross-valley shape and usually uniform in surface characteristics and, if three-dimensional at all, then without along-valley slope). Wagner et al. (2015a) systematically varied the height-to-width ratio and length of an idealized (straight) valley attached to a plain to study the impact on EAE efficiency. While relatively low (and/or wide) valleys exhibit the "classical" picture of a single slope-flow circulation ranging up to higher-than-ridge top around noon (Figure 3), relatively steeper and narrower valleys exhibit stronger slope flows giving rise to the formation of two
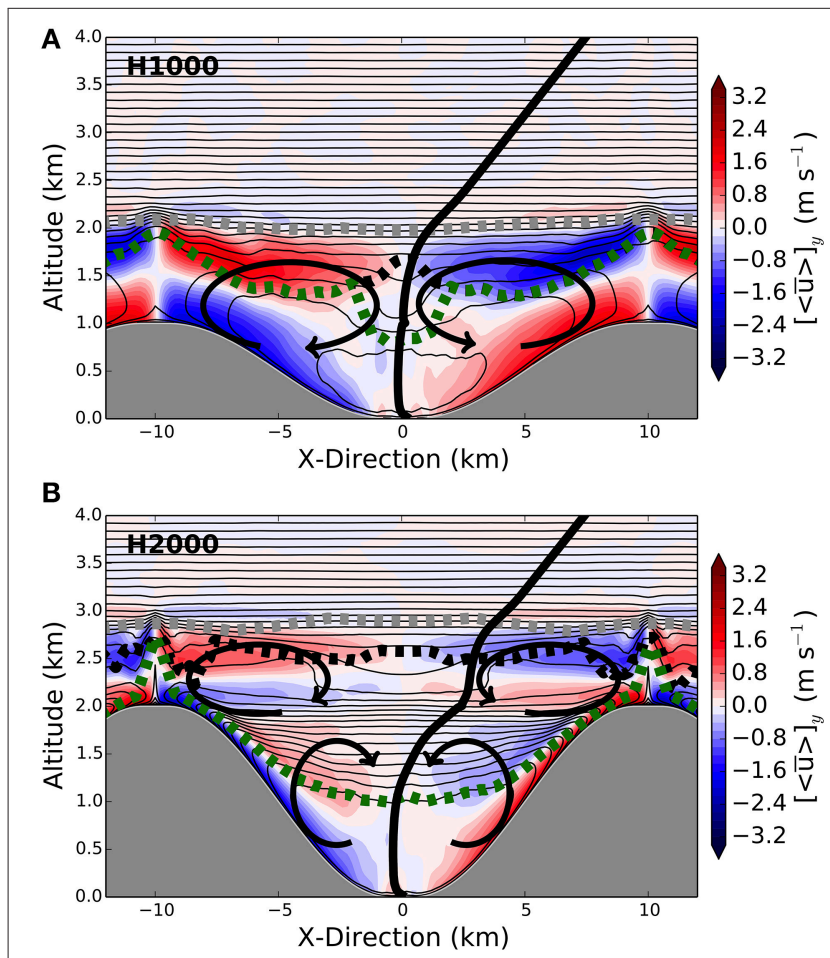

FIGURE 3 | Effect of height-to-width ratio on the thermally forced cross-valley circulation. Potential temperature (thin contour lines), cross-valley wind speed (color shading), and three different boundary layer heights are shown for a $1000 \mathrm{~m}$ (A) and $2000 \mathrm{~m}$ (B) deep valley with identical forcing and after $6 \mathrm{~h}$ of simulation. See Wagner et al. (2015a) for the definition of the boundary layer heights and the simulations. The bold black line denotes the valley-mean potential temperature profile. vertically stacked circulation cells within the valley. Serafin and Zardi (2010b) similarly found a stronger cross-valley circulation in the narrower valley (of their two configurations) and two stacked circulation cells can be distinguished (even if they are not explicitly discussed). However, the development of the associated plain-to-mountain flow is also dependent on the height-towidth ratio of the valley system. These mean flow conditions have a profound impact on the vertical exchange associated with the valley and slope flow circulations. Comparing EAE for the different valley-plain systems to that of a plain alone suggests a mass exchange (as based on numerical tracer release experiments) that is three to four times more efficient over the mountain-plain system. Introducing further inhomogeneity in the valley simulation, Wagner et al. (2015b) found that both, along-valley slope as well as narrowing, substantially increase the strength of the valley wind system. Associated with this, narrowing of the valley cross-section enhances the vertical exchange of mass (as measured with a released tracer at different positions) by a factor between 1.8 and 2.8 while introducing an along-valley slope increases vertical exchange by a factor of about 1.2. Clearly, these numbers are dependent on the detailed choices concerning topography (e.g., introducing along-valley slope) and original set-up (e.g., type of cross-section) and idealization. Still, they give a rough estimate of how important geometrical aspects can be on the exchange over complex mountainous terrain.

Lang et al. (2015) have extended the geometry as used by all the above studies where "the valley" is usually made up by two parallel ridges of equal heights (but slightly different crosssections in the different studies). They have embedded a valley with variable depth into a "mountain range" (Figure 4). Releasing a passive tracer at different near-surface locations they studied mass transfer to the free atmosphere influenced by the combined effect of plain-to-mountain flow and slope circulations. For a deep embedded valley the slope wind regime was found to establish within large parts of the valley while an elevated (less deep) valley allowed the plain-to-mountain flow to descend into the valley thus replacing the opposing slope flow on the upwind side of the valley. Comparing the "aerosol layer depth" (i.e., the height up to which the aerosol mixes) with the different estimates of CBL depth (Figures 4B,D) indicates the fraction of tracer that has been mixed up by processes other than turbulent mixing. Overall, a more than three-fold enhancement of tracer EAE was found for the various mountain ridge topographies when compared to the reference plain simulation (for comparison, in the example of Figure 4 the difference between the deep and the shallower valley amounts to some $20 \%$ ).

Schmidli and Rotunno (2012) did not change the geometry of the valley itself, but its surrounding (periodic valleys, vs. one isolated valley on a plain vs. valley in an elevated plateau). They found considerable differences in the strength of the valley wind system that could be attributed to the upperlevel pressure distribution and advective momentum transport between the valley and its surroundings while direct impact of the surroundings on the valley heat budget was relatively minor. All the idealized studies cited above were conducted with zero synoptic pressure difference (purely thermally driven flows), indicating a potential limitation of idealized simulations 

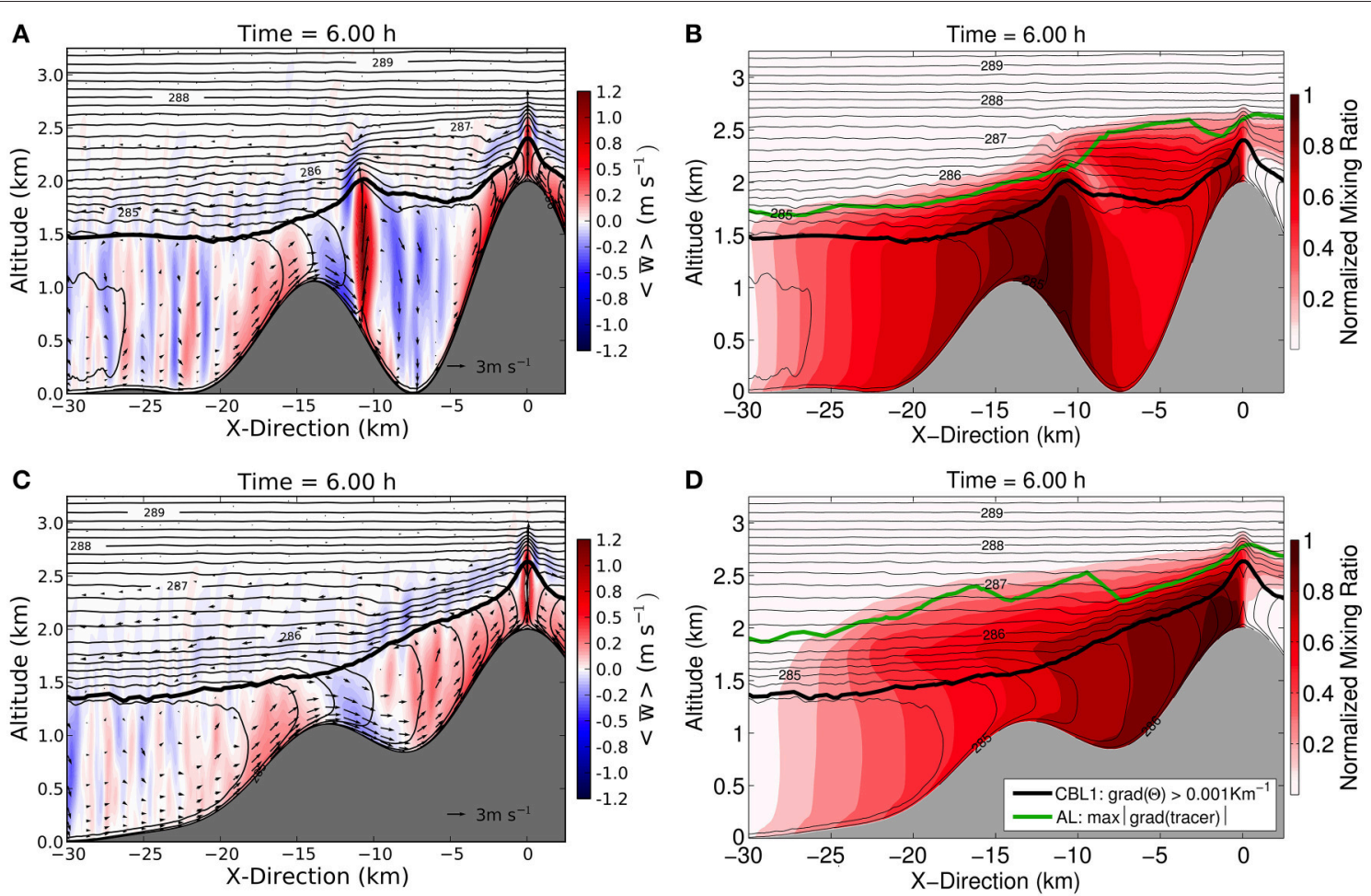

FIGURE 4 | Flow and temperature fields, (A) and (C) and tracer concentration, (B) and (D) from high-resolution numerical simulations of a deep embedded valley (upper row) and a shallower embedded valley (lower row) in a mountain range. Thin solid lines: potential, temperature, arrows $x-Z$ wind vector, color shading vertical velocity, (A) and (C), and tracer mixing ratio, (B) and (D). The black bold solid line corresponds to the Convective Boundary Layer height (diagnosed from first height where the gradient of potential temperature exceeds $0.001 \mathrm{~km}^{-1}$ when searching from the below), green line: "aerosol layer height" (diagnosed from maximum gradient in mixing ratio). The tracer is released across the entire mountain range at the lowest eight grid points (about $110 \mathrm{~m}$ from ground) at constant rate. Figure reassembled from different figures in Lang et al. (2015). See this paper for details of the simulations.

carried out so far. Even a minor along-valley pressure gradient at upper levels will likely influence the valley wind system through momentum exchange between the valley and the atmosphere and hence the efficiency of the EAE in truly complex terrain through the geometric factors discussed above. The degree to which this will be effective, however, will require some additional research even if real-terrain simulations (e.g., Zangl, 2009) seem to indicate that valley flow characteristics are quite robust features.

\section{Exchange is Dependent on Atmospheric Forcing}

Similar to valley geometry other factors like the spatial distribution of surface properties such as roughness length and soil moisture, and the strength of the solar forcing (i.e., season, latitude) can be expected to modify topographic flows and EAE in mountainous terrain. In the simulations discussed above, net radiation at the surface forces the thermal valley flow either through an assumed spatially homogeneous but time-dependent surface heat flux (e.g., Rampanelli et al., 2004; Wagner et al., 2015a,b) or temperature (Catalano and Cenedese, 2010), or through a full surface exchange scheme (e.g., Serafin and Zardi, 2010a,b, 2011; Schmidli and Rotunno, 2010, 2012). The latter setup then corresponds to placing an east-west running valley to the equator and choosing an equinox so as to ensure symmetry along the valley axis. Clearly, in a real valley (at least off the equator) solar forcing (net radiation) is largely different in timing and magnitude at different positions within a valley (e.g., Matzinger et al., 2003), leading to a correspondingly similar spatial spatiotemporal variability of surface heat fluxes (Rotach et al., 2008). Figure 5 shows the impact of the spatial variability of solar forcing on the instantaneous flow and stratification. As can be seen, the actual flow structure deviates from symmetric with potentially important consequences on pollutant redistribution within a valley (e.g., Gohm et al., 2009). However, when it comes to the overall exchange characteristics between the valley and the atmosphere, for example the total mass exchanged over daytime, or the estimated impact on the valley heat budget, results are quite insensitive to those asymmetries.

The studies cited above all used dry or low soil moisture conditions-in order to avoid the additional "complication" of cloud formation-and some medium time-dependent surface heat flux (200-300 $\mathrm{W} \mathrm{m}^{-2}$ amplitude) as a thermal forcing. Leukauf et al. (2015) systematically varied this forcing by using a maximum short-wave net radiation in a parameterization of the daily cycle between 150 and $850 \mathrm{~W} \mathrm{~m}^{-2}$ (corresponding to surface heat flux with amplitudes of 5-450 $\mathrm{W} \mathrm{m}^{-2}$ ). While their analysis was basically motivated by studying the breakup of the nighttime cold pool, it additionally provides insight 


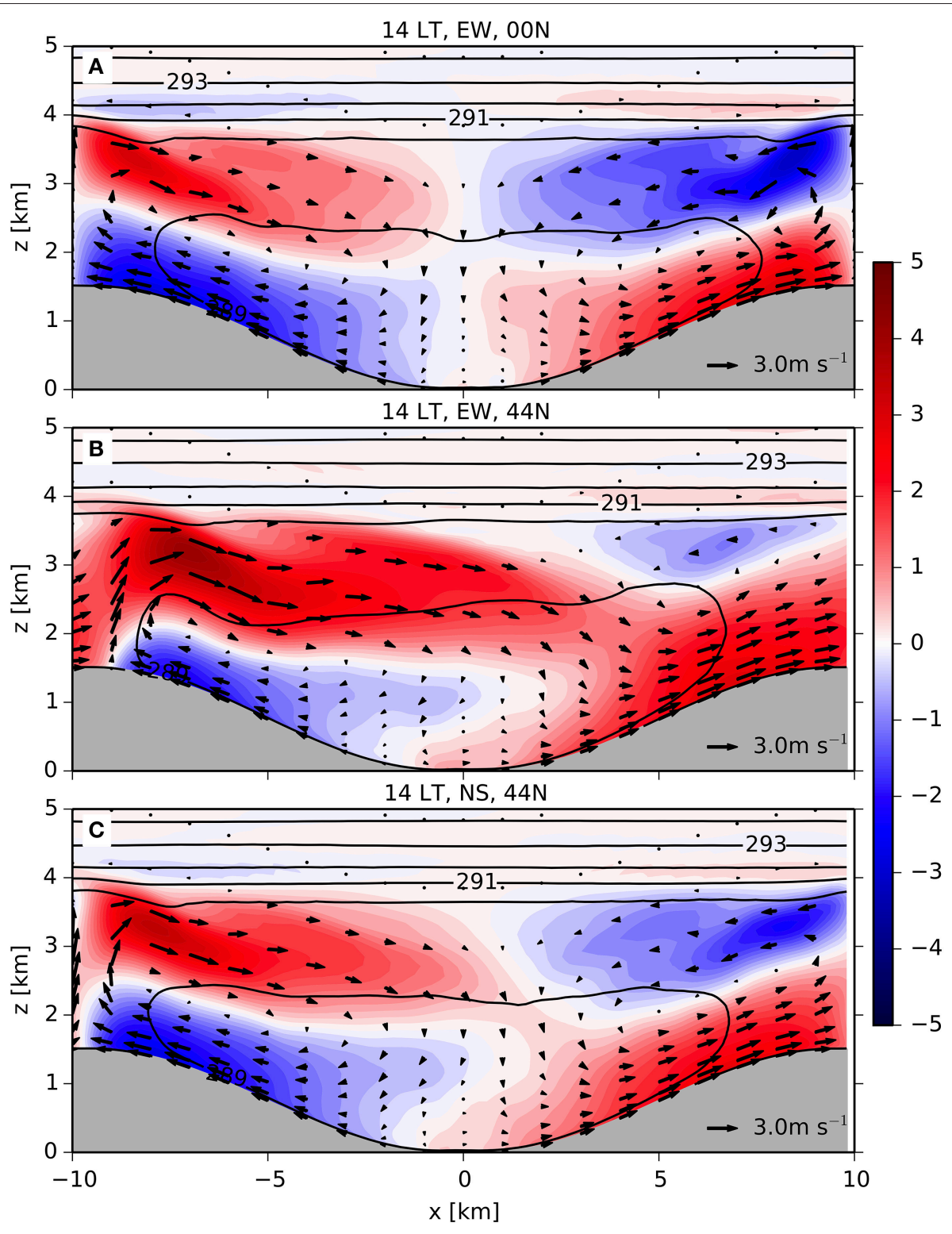

FIGURE 5 | Flow and temperature fields from high-resolution simulation of a $1.5 \mathrm{~km}$ deep symmetric valley, (A) at the equator, east-west orientation; (B) at $44^{\circ} \mathrm{N}$, east-west orientation; and (C) at $44^{\circ} \mathrm{N}$, north-south orientation. Solid lines are isentropes, arrows xz-wind vectors, the color code for the $x$-component of the wind $\left[\mathrm{ms}^{-1}\right]$. The panels show the situation on the second day of the simulation, $2 \mathrm{pm}$ local time. The solar forcing in all three panels corresponds to the case of $550 \mathrm{Wm}^{-2}$ amplitude (roughly $240 \mathrm{Wm}^{-2}$ amplitude in surface heat flux) of Leukauf et al. (2015). For details of the simulations see Leukauf et al. (2015) or Wagner et al. (2015a,b).

into the efficiency of EAE over their idealized valley system. Not surprisingly, the complete break up of the cold pool required a minimum net short-wave forcing (an amplitude of $450 \mathrm{~W} \mathrm{~m}^{-2}$ for the given geometry). Similarly, the energy and mass exchange between the valley and the atmosphere was found to be strongly dependent on this fact. While a surprisingly consistent factor of "about three" had previously been reported for the number of times the "valley atmosphere was exchanged" over a convective day (Henne et al., 2004 from observations; Weigel et al., 2007 for real valley simulations; Wagner et al., 2015a; Lang et al., 2015 for idealized geometry simulations), this figure reduces to some $30 \%$ (i.e., a factor of 1.3) for a net short wave forcing just not strong enough to entirely break up the cold pool $\left(400 \mathrm{~W} \mathrm{~m}^{-2}\right.$ amplitude in solar forcing). For the strongest solar forcing, on the other hand, more than five times the valley atmosphere is exported during the daytime period. Bearing in mind that this study again used a symmetric valley configuration the geometryrelated amplifications (such as for sloping valley axis, narrowing valley, etc.) as discussed above will similarly modify these numbers. 
Overall, idealized simulations have tremendously advanced our understanding of the impact of gravity waves under conditions of stable stratification, as well as purely thermally driven valley-atmosphere exchange processes. Despite this, the degree of idealization is substantial, and imposes some limitations on the applicability of the findings. Idealized studies of mass-fluxes over topography for dynamically forced flows are awaiting their realization. Similarly, we are not aware of idealized simulations concerned with the interaction of the purely thermally driven flows with an overlying (possibly only weak) synoptic flow. As far as surface properties are concerned, real-terrain simulations have shown soil moisture (e.g., Chow et al., 2006; Szintai et al., 2010) to modify the flow characteristics but there seems to be no systematic idealized study to put these results into context and estimate the impact on EAE. Processes related to moisture in the atmosphere such as cloud formation (or even precipitation) will certainly have an impact of valleyatmosphere exchange that will have to be investigated using idealized modeling.

\section{OUTLOOK}

Topography as a particular form of surface condition to the atmospheric flow is apparently responsible for various processes leading to modifications of EAE when compared to the pure boundary layer turbulent transport over flat terrain. In this contribution we have attempted to identify these processes and give examples from observational studies and numerical simulations using real and idealized atmospheric and terrainconditions. Today's modeling capabilities appear to be advanced enough to capture these processes, or at least allow for an estimate of their exchange efficiency, provided that the model's spatial resolution is sufficiently high. Wagner et al. (2014) concluded that the grid scale needs to be some 10-20 times smaller than the relevant topographic scale in order to properly resolve these processes. Many numerical models for global numerical weather prediction (NWP) or climate scenario simulations, however, still have grid spacing far from this requirement and this is likely to remain so for the decades to come even if computing power continues to increase at its present pace. Thus, these models would need parameterizations to cover EAE due to unresolved (sub-grid scale) topography. While such parameterizations are in operation for momentum exchange due to gravity wave and orographic drag for some time now (and of course still have room for improvement, see Kim et al., 2013 and Teixeira, 2014), they are still missing for energy and mass exchange. Most likely, their (missing) impact is somewhere "buried" in tuned parameters of the boundary layer and surface exchange schemes-thus giving rise to acceptable verification scores for the mean flow variables at large scales. While this was sufficient (and important) to reproduce the large-scale dynamical fields for many years and has led to an impressive improvement of forecast quality over the last decades (Bauer et al., 2015), even large-scale atmospheric models progressively extend their scope. Most notably, many NWP and climate models are being developed into Earth-System simulators (e.g., Palmer, 2012;
Flato et al., 2013) thus adding-among others-atmospheric constituents (beyond water vapor) as prognostic variables. This evidently makes it necessary to also capture processes relevant for mass exchange from and to the surface, and this also over mountainous terrain. One may debate whether taking into account unresolved processes should be achieved by increasing resolution ad infinitum or by more rigorously moving to ensemble approaches (Palmer, 2012) - but they should be taken into account.

We have, in the previous sections, pointed to a number of missing pieces required on the way of establishing an extended boundary layer parameterization for topography and it seems that the community is still some distance from proposing a blueprint for such an endeavor. Still, a number of potentially useful future research directions can be summarized as follows.

$>$ First of all, our understanding of the boundary layer structure and associated processes of turbulent transport over mountainous terrain is still very limited (Rotach and Zardi, 2007). Thus, in order to establish a better assessment of the relative importance of turbulent exchange vs. mesoscale transport the former must be understood in much more depth. Recent efforts in which numerical modeling and observations are combined for limited (e.g., Fernando et al., 2015) or extended (e.g., Stiperski et al., 2012) periods of time go in this direction.

> Sensitivity studies for idealized simulations need to be extended. Notably, the community seems to agree on an initial background stability (of $0.003 \mathrm{Km}^{-1}$ ) and often only the 2nd day is analyzed in order to minimize effects of initialization. While this is certainly sufficient for the boundary layer processes themselves, the growth of the boundary layer is known to be sensitive to overlying stability (e.g., Chen et al., under review), which in these idealized configurations will be given by the initial stratification.

> Other interesting sensitivities for idealized simulations would include further geometrical effects such as curvature (Weigel and Rotach, 2004), asymmetry of ridge top height (as initiated in Lang et al., 2015) or introduction of side valleys; and surface properties such as characteristic distribution of soil moisture or surface parameters.

> The rigorous budgeting approach of Schmidli (2013) should be applied to real-terrain simulations in order to assess the degree to which results can be transferred. Most likely this would require inclusion of background flow (such as in Zangl, 2009) even if weak. This would also allow, or even necessitate investigation of the interaction between purely thermally driven flows (as currently in the idealized simulations) and dynamically forced flows.

> Systematic observations in order to evaluate the numerical models in complex terrain are required. When assessing the extent to which turbulent exchange and meso-scale circulations contribute to total exchange in very highresolution simulations, the former is rarely "verified." This requires the ability to reliably measure near-surface turbulence in mountainous terrain (see e.g., Stiperski and 
Rotach, 2015) and, probably even more so, the assessment of turbulence profiles in such terrain (e.g., Adler and Kalthoff, 2014).

$>$ Verifying actual exchange processes often suffers from the fact that a one-to-one attribution is hampered by the abundance of the investigated tracer sources (most obvious for heat, but similar for water vapor or $\mathrm{CO}_{2}$ ). Tracers which are more specific for one particular surface type would be better suited to establishing unique relations between elevated

\section{REFERENCES}

Adler, B., and Kalthoff, N. (2014). Multi-scale transport processes observed in the boundary layer over a mountainous island. Boundary Layer Meteorol. 153, 515-537. doi: 10.1007/s10546-014-9957-8

Baines, P. G. (1995). Topographic Effects in Stratified Flows. Cambridge, UK: Cambridge Univ. Press.

Bauer, P., Thorpe, A., and Brunet, G. (2015). The quiet revolution of numerical weather prediction. Nature 525, 47-35. doi: 10.1038/nature 14956

Belcher, S. E., and Hunt, J. R. C. (1998). Turbulent flows over hills and waves. Ann. Rev. Fluid Mech. 30, 507-538. doi: 10.1146/annurev.fluid.30. 1.507

Belcher, S. E., Newley, T. M. J., and Hunt, J. C. R. (1993). The drag on an undulating surface due to the flow of a turbulent boundary layer. J. Fluid Mech. 249, 557-596. doi: 10.1017/S0022112093001296

Bougeault, P., Binder, P., Buzzi, A., Dirks, R., Houze, R., Kuettner, J., et al. (2001). The MAP special observing period. Bull. Amer. Meteorol. Soc. 82, 433-462. doi: 10.1175/1520-0477(2001)082<0433:TMSOP > 2.3.CO;2

Bougeault, P., Jansa, A., Attie, J. L., Beau, I., Benech, B., Benoit, R., et al. (1993). The atmospheric momentum budget over a major mountain range: first results of the PYREX field program. Ann. Geophys. 11, 395-341.

Catalano, F., and Cenedese, A. (2010). High-resolution numerical modeling of thermally driven slope winds in a valley with strong capping. J. Appl. Meteorol. Climatol. 49, 1859-1880. doi: 10.1175/2010JAMC2385.1

Catalano, F., and Moeng, C. (2010). Large-eddy simulation of the daytime boundary layer in an idealized valley using the weather research and forecasting numerical model. Boundary Layer Meteorol. 137, 49-75. doi: 10.1007/s10546010-9518-8

Chen, X., Añel, J. A., Su, Z., de la Torre, L., Kelder, H., van Peet, J., et al. (2013). The deep atmospheric boundary layer and its significance to the stratosphere and troposphere exchange over the Tibetan Plateau. PLoS ONE 8:e56909. doi: 10.1371/journal.pone.0056909

Chow, F. K., Weigel, A. P., Street, R. L., Rotach, M. W., and Xue, M. (2006). High-resolution large-eddy simulations of flow in a steep Alpine valley. Part I: methodology, verification, and sensitivity studies. J. Appl. Meteorol. Climatol. 45, 63-86. doi: 10.1175/JAM2322.1

Demko, J., and Geerts, B. (2010). A numerical study of evolving convective boundary layer and oro- graphic circulation around the Santa Catalina Mountains in Arizona. Part I: circulation without deep convection. Mon. Wea. Rev. 138, 1902-1922. doi: 10.1175/2009MWR3098.1

Demko, J., Geerts, B., and Miao, Q. (2009). Boundary layer transport and cumulus development over a heated mountain: an observational study. Mon. Wea. Rev. 137, 447-468. doi: 10.1175/2008MWR2467.1

De Wekker, S. F. J. (2002). Structure and Morphology of the Convective Boundary Ayer Over Mountainous Terrain. Ph.D. thesis, Univ British Columbia.

De Wekker, S. F. J., Steyn, D. G., Fast, J. D., Rotach, M. W., and Zhong, S. (2005). The performance of RAMS in representing the convective boundary layer structure in a very steep valley. Environ. Fluid Mech. 5, 35-62. doi: 10.1007/s10652-005-8396-y

De Wekker, S. F. J., Steyn, D. G., and Nyeki, S. (2004). A comparison of aerosol layer- and convective boundary layer structure over a mountain range during STAAARTE'97. Boundary Layer Meteor. 113, 249-271. doi: 10.1023/B:BOUN.0000039371.41823.37 or non-zero concentrations aloft and the corresponding surface sources so as to demonstrate the efficiency of the hypothesized exchange processes. While such tracers are easily implemented in a numerical model they first need to be identified (which surface type exclusively emits which marker substance?) and second the measurement of their concentration and turbulent exchange must be possible. Suitable strategies should be worked out in order to overcome this difficulty.

De Wekker, S. F. J., Zhong, S., Fast, J. D., and Whiteman, C. D. (1998). A numerical study of the thermally driven plain-to-basin wind over idealized basin topographies. J. Appl. Meteor. 37, 606-622

Doyle, J. D., and Shapiro, M. A. (2000). A multi-scale simulation of an extreme downslope wind-storm over Norway. Meteorol. Atmos. Phys. 74, 83-101. doi: $10.1007 /$ s007030070027

Egger, J., Bajrachaya, S., Egger, U., Heinrich, R., Reuder, J., Shakya, P., et al. (2000). Diurnal winds in the Himalayan Kali Gandaki valley. Part I: observations. Mon. Wea. Rev. 128, 1106-1122. doi: 10.1175/15200493(2000)128<1106:DWITHK>2.0.CO;2

Egger, J., Bajrachaya, S., Heinrich, R., Kolb, P., Lammlein, S., Mech, M., et al. (2002). Diurnal winds in the Himalayan Kali Gandaki valley. Part III: remotely piloted aircraft soundings. Mon. Wea. Rev. 130, 2042-2058. doi: 10.1175/15200493(2002) $130<2042$ :DWITHK > 2.0.CO;2

Fernando, H., Pardyjak, E., Di Sabatino, S., Chow, F., De Wekker, S., Hoch, S., et al. (2015). The MATERHORN - unraveling the intricacies of mountain weather. Bull. Am. Meteor. Soc. doi: 10.1175/bams-d-13-00131.1. [Epub ahead of print].

Flato, G., Marotzke, J., Abiodun, B., Braconnot, P., Chou, S. C., Collins, W., et al. (2013). "Evaluation of climate models," in Climate Change 2013: The Physical Science Basis, Contribution of Working Group I to the Fifth Assessment Report of the Intergovernmental Panel on Climate Change, eds T. F. Stocker, D. Qin, G.-K. Plattner, M. Tignor, S. K. Allen, J. Boschung, A. Nauels, Y. Xia, V. Bex, P. M. Midgley (Cambridge; New York, NY: Cambridge University Press).

Garratt, J. R. (1990). The internal boundary layer - a review. Boundary Layer Meteorol. 50, 171-203. doi: 10.1007/BF00120524

Geerts, B., Miao, Q., and Demko, J. (2008). Pressure perturbations and upslope flow over a heated, isolated mountain. Mon. Wea. Rev. 136, 4272-4288. doi: 10.1175/2008MWR2546.1

Gohm, A., Harnisch, F., Vergeiner, J., Obleitner, F., Schnitzhofer, R., Hansel, A., et al. (2009). Air pollution transport in an alpine valley: results from airborne and ground-based observations. Boundary Layer Meteorol. 131, 441-463. doi: $10.1007 /$ s10546-009-9371-9

Grisogono, B., Jurlina, T., Vecenaj, Z., and Guttler, I. (2015). Weakly nonlinear Prandtl model for simple slope flows. Q. J. R. Meteorol Soc. 141, 883-892. doi: $10.1002 /$ qj.2406

Henne, S., Furger, M., Nyeki, S., Steinbacher, M., Neininger, B., De Wekker, S. F. J., et al. (2004). Quantification of topographic venting of boundary layer air to the free troposphere. Atmos. Chem. Phys. 4, 497-509. doi: 10.5194/acp-4-497-2004

Henne, S., Furger, M., and Prévôt, A. S. H. (2005). Climatology of mountain venting induced moisture layers in the lee of the Alps. J. Appl. Meteorol. 44, 620-633. doi: 10.1175/JAM2217.1

Holton, J. R., and Hakim, G. J. (2013). An Introduction to Dynamic Meteorology. Waltham, MA; Oxford, UK: Academic Press.

Holtslag, A. A. M., and Nieuwstadt, F. T. M. (1986). Scaling the atmospheric boundary layer. Boundary Layer Meteorol. 36, 201-209. doi: $10.1007 / \mathrm{BF} 00117468$

Hunt, J. R. C., Leibovich, S., and Richards, K. J. (1988a). Turbulent shear flows over low hills. Q. J. R. Meteorol. Soc. 114, 1435-1470.

Hunt, J. R. C., Richards, K. J., and Brighton, P. W. M. (1988b). Stratified flows over low hills. Q. J. R. Meteorol. Soc. 114, 859-868.

Jackson, P. S., and Hunt, J. R. C. (1975). Turbulent wind low over a low hill. Q. J. R. Meteorol. Soc. 101, 929-955. doi: 10.1002/qj.49710143015

Jiang, Q., and Doyle, J. D. (2008). On the diurnal variation of mountain waves. J. Atmos. Sci. 65, 1360-1377. doi: 10.1175/2007JAS2460.1 
Jiang, Q., Doyle, J. D., and Smith, R. B. (2006). Interaction between trapped waves and boundary layers. J. Atmos. Sci. 63, 617-633. doi: 10.1175/JAS3640.1

Jiang, Q., Smith, R. B., and Doyle, J. D. (2008). Impact of the atmospheric boundary layer on mountain waves. J. Atmos. Sci. 65, 592-608. doi: 10.1175/2007JAS2376.1

Kim, Y.-J., Eckermann, S. D., and Chun, H.-Y. (2013). An overview of the past, present and future of gravity - wave drag parametrization for numerical climate and weather prediction models. Atmos. Ocean 41, 65-98. doi: $10.3137 /$ ao. 410105

Kirshbaum, D. J. (2013). On thermally forced circulations over heated terrain. J. Atmos. Sci. 70, 1690-1709. doi: 10.1175/JAS-D-12-0199.1

Kleinschmidt, E. (1922). Der taegliche Gang des Windes in der freien Atmosphaere und auf Berggipfeln (The diurnal course of the wind in the free atmosphere and on mountain tops). Beitr. Phys. Atmos. 10, 1-15.

Kossmann, M., Corsmeier, U., De Wekker, S. F. J., Fiedler, F., Voegtlin, S., Kalthoff, N., et al. (1999). Observations of handover processes between the atmospheric boundary layer and the free troposphere over mountainous terrain. Contrib. Atmos. Phys. 72, 329-350.

Lang, M. N., Gohm, A., and Wagner, J. S. (2015). The impact of embedded valleys on daytime pollution transport over a mountain range, Atmos. Chem. Phys. Discuss. 15, 14315-14356. doi: 10.5194/acpd-15-14315-2015

Leukauf, D., Gohm, A., Rotach, M. W., and Wagner, J. S. (2015). The impact of the temperature inversion breakup on the exchange of heat and mass in an idealized valley: sensitivity to the radiative forcing. J. Appl. Meteorol. Climatol. 54, 2199-2216. doi: 10.1175/jamc-d-15-0091.1

Lott, F., and Miller, M. J. (1997). A new subgrid-scale orographic drag parametrization: its formulation and testing. Q. J. R. Meteorol. Soc. 123, 101-127. doi: 10.1002/qj.49712353704

Lugauer, M., and Winkler, P. (2005). Thermal circulation in South Bavaria climatology and synoptic aspects. Meteor. Z. 14, 15-30. doi: 10.1127/09412948/2005/0014-0015

Mahrt, L. (2014). Stably stratified atmospheric boundary layers. Ann. Rev. Fluid Mech. 46, 23-45. doi: 10.1146/annurev-fluid-010313-141354

Matzinger, N., Andretta, M., van Gorsel, E., Vogt, R., Ohmura, A., and Rotach, M. W. (2003). Surface radiation budget in an Alpine valley. Q. J. R. Meteorol. Soc. 129, 877-895. doi: $10.1256 /$ qj.02.44

Medeiros, L. E., and Fitzjarrald, D. E. (2014). Stable boundary layer in complex terrain. Part I: linking fluxes and intermittency to an average stability index. J. Appl. Meteorol. Cimatol. 53, 2196-2215. doi: 10.1175/JAMC-D13-0345.1

Milton, S. F., and Wilson, C. A. (1996). Impact of parameterised subgrid-scale orographic forcing on systematic errors in a global NWP model. Mon. Wea. Rev. 124, 2023-2045.

Nappo, C. J. (2002). An Introduction to Atmospheric Gravity Waves. San Diego, CA: Academic Press.

Noppel, H., and Fiedler, F. (2002). Mesoscale heat transport over complex terrainby slope winds. A conceptual model and numerical simulations. Boundary Layer Meteorol. 104, 73-97. doi: 10.1023/A:1015 556228119

Nyeki, S., Kalberer, M., Colbeck, I., De Wekker, S. F. J., Furger, M., Gäggeler, H. W., et al. (2000). Convective boundary layer evolution to $4 \mathrm{~km}$ ASL over highalpine terrain: airborne lidar observations in the Alps. Geophys. Res. Lett. 27, 689-692. doi: 10.1029/1999GL010928

Orlanski, I. (1975). A rational subdivision of scales for atmospheric processes. Bull. Am. Meteorol. Soc. 56, 527-530.

Palmer, T. N. (2012). Towards the probabilistic earth-system simulator: a vision for the future of climate and weather prediction. Q. J. R. Meteorol. Soc. 138, 841-861. doi: 10.1002/qj.1923

Palmer, T. N., Shutts, G. J., and Swinbank, R. (1986). Alleviation of a systematic westerly bias in general circulation and numerical weather prediction models through an orographic gravity wave drag parameterization. Q. J. R. Meteorol. Soc. 112, 1001-1039. doi: 10.1002/qj.49711247406

Pérez-Landa, G., Ciais, P., Sanz, M. J., Giol, B., Miglietta, F., Palau, J. L., et al. (2007). Mesoscale circulations over complex terrain in the Valencia coastal region, Spain-Part 2: modeling $\mathrm{CO} 2$ transport using idealized surface fluxes. Atmos. Chem. Phys. 7, 1851-1868. doi: 10.5194/acp-7-1851-2007

Pillai, D., Gerbig, C., Ahmadov, R., Rodenbeck, C., Kretschmer, R., Koch, T., et al. (2011). High-resolution simulations of atmospheric CO2 over complex terrain - representing the Ochsenkopf mountain tall tower. Atmos. Chem. Phys. 11, 7445-7464. doi: 10.5194/acp-11-7445-2011

Prandtl, L. (1942). Fuehrer Durch Die Stroeungslehre. Braunschweig: Viehweg and Son.

Prandtl, L. (1952). Mountain and valley winds in stratified air. Essentials Fluid Dyn. $422-425$.

Rampanelli, G., Zardi, D., and Rotunno, R. (2004). Mechanisms of up-valley winds. J. Atmos. Sci. 61, 3097-3111. doi: 10.1175/JAS-3354.1

Raupach, M. R., and Finnigan, J. J. (1997). The influence of topography on meteorological variables and surface-atmosphere interactions. J. Hydrol. 190, 182-213. doi: 10.1016/S0022-1694(96)03127-7

Raupach, M. R., Weng, W. S., Carruthers, D. J., and Hunt, J. C. R. (1992). Temperature and humidity fields and fluxes over low hills. Q. J. R. Meteorol. Soc. 118, 191-225. doi: 10.1002/qj.49711850403

Rotach, M. W., Andretta, M., Calanca, P., Weigel, A. P., and Weiss, A. (2008). Boundary layer characteristics and turbulent exchange mechanisms in highly complex terrain. Acta Geophysicae 56, 194-219. doi: 10.2478/s11600007-0043-1

Rotach, M. W., Calanca, P., Graziani, G., Gurtz, J., Steyn, D. G., Vogt, R., et al. (2004). Turbulence structure and exchange processes in an Alpine Valley: the Riviera project. Bull. Am. Meteorol. Soc. 85, 1367-1385. doi: 10.1175/BAMS85-9-1367

Rotach, M. W., Wohlfahrt, G., Hansel, A., Reif, M., Wagner, J., and Gohm, A. (2014). The world is not flat - implications for the global carbon balance. Bull. Am. Meteor. Soc. 95, 1021-1028. doi: 10.1175/BAMS-D-13-00109.1

Rotach, M. W., and Zardi, D. (2007). On the boundary layer structure over highly complex terrain: key findings from MAP. Q. J. R. Meteorol. Soc. 133, 937-948. doi: $10.1002 /$ qj.71

Rotunno, R., and Houze, R. (2007). Lessons on orographic precipitation from the Mesoscale Alpine Programme. Q. J. R. Meteorol. Soc. 133, 811-830. doi: $10.1002 /$ qj.67

Savelyev, S. A., and Taylor, P. A. (2005). Internal boundary layers: I. Height formulae for neutral and diabatic flows. Boundary Layer Meteorol. 115, 1-25. doi: $10.1007 / \mathrm{s} 10546-004-2122-\mathrm{z}$

Schmidli, J. (2013). Daytime heat transfer processes over mountainous terrain. J. Atmos. Sci. 70, 4041-4066. doi: 10.1175/JAS-D-13-083.1

Schmidli, J., and Rotunno, R. (2010). Mechanisms of along-valley winds and heat exchange over mountainous terrain. J. Atmos. Sci. 67, 3033-3047. doi: 10.1175/2010JAS3473.1

Schmidli, J., and Rotunno, R. (2012). Influence of the valley surroundings on valley wind dynamics. J. Atmos. Sci. 69, 561-579. doi: 10.1175/JAS-D-11-0129.1

Serafin, S., and Zardi, D. (2010a). Structure of the atmospheric boundary layer in the vicinity of a developing upslope flow system: a numerical model study. J. Atmos. Sci. 67, 1171-1185. doi: 10.1175/2009JAS3231.1

Serafin, S., and Zardi, D. (2010b). Daytime heat transfer processes related to slope flows and turbulent convection in an idealized mountain valley. J. Atmos. Sci. 67, 3739-3756. doi: 10.1175/2010JAS3428.1

Serafin, S., and Zardi, D. (2011). Daytime development of the boundary layer over a plain and in a valley under fair weather conditions: a comparison by means of idealized numerical simulations. J. Atmos. Sci. 68, 2128-2141. doi: 10.1175/2011JAS3610.1

Škerlak, B., Sprenger M., and Wernli, H. (2014). A global climatology of stratosphere-troposphere exchange using the ERA-Interim dataset from 1979 to 2011. Atmos. Chem. Phys. 14, 913-917. doi: 10.5194/acp-14913-2014

Smith, R. B. (1979). The influence of mountains on the atmosphere. Adv. Geophys. 33, 87-230. doi: 10.1016/S0065-2687(08)60262-9

Smith, R. B. (2007). Interacting mountain waves and boundary layers. J. Atmos. Sci. 64, 594-607. doi: 10.1175/JAS3836.1

Steeneveld, G. J., Holtslag, A. A. M., Nappo, C. J., van de Wiel, B. J. H., and Mahrt, L. (2008). Exploring the role of small-scale terrain drag on stable boundary layers over land. J. Appl. Meteorol. Climatol. 47, 2518-2530. doi: 10.1175/2008JAMC1816.1

Steeneveld, G. J., Nappo, C. J., and Holtslag, A. A. M. (2009). Estimation of orographically induced wave drag in the stable boundary layer during CASES99. Acta Geophys. 57, 857-881. doi: 10.2478/s11600-009-0028-3

Steinacker, R. (1984). Area-height distribution of a valley and its relation to the valley wind. Contrib. Atmos. Phys. 57, 64-71. 
Stiperski, I., and Grubišič, V. (2011). Trapped lee wave interference in the presence of surface friction. J. Atmos. Sci. 68, 918-936. doi: 10.1175/2010JAS3495.1

Stiperski, I., Kavčič, I., Grisogono, B., and Durran, D. R. (2007). Including Coriolis effects in the Prandtl model for katabatic flow. Q. J. R. Meteorol. Soc. 133, 101-106. doi: 10.1002/qj.19

Stiperski, I., and Rotach, M. W. (2015). On the measurement of turbulent fluxes over complex mountainous topography. Boundary Layer Meteorol. doi: 10.1007/s10546-015-0103-z. [Epub ahead of print].

Stiperski, I., Rotach, M. W., and Gohm, A. (2012). Boundary layer measurements in complex terrain: innsbruck-box, session AS2.1, Air-Land Interactions. Geophys. Res. Abstr. 2012, EGU2012-8863.

Stull, R. B. (1988). An Introduction to Boundary Layer Meteorology. Dordrecht: Kluwer. doi: 10.1007/978-94-009-3027-8

Sun, J., and De Wekker, S. F. J. (2011). "Atmospheric carbon dioxide transport over complex terrain," in Mountain Ecosystems: Dynamics, Management and Conservation, ed K. E. Richards (New York, NY: Nova Science Publishers), 101-121.

Sun, J., Nappo, C. J., Mahrt, L., Belušić, D., Grisogono, B., Stauffer, D. R., et al. (2015). Review of wave-turbulence interactions in the stable atmospheric boundary layer. Rev. Geophys. 53. doi: 10.1002/2015rg000487

Sun, J., Oncley, S. P., Burns, S. P., Stephens, B. B., Lenschow, D. H., Campos, T., et al. (2010). A multiscale and multidisciplinary investigation of ecosystematmosphere $\mathrm{CO} 2$ exchange over the Rocky Mountains of Colorado. Bull. Am. Meteor. Soc. 91, 209-230. doi: 10.1175/2009BAMS2733.1

Szintai, B., Kaufmann, P., and Rotach, M. W. (2010). Simulation of pollutant transport in complex terrain with a numerical weather prediction-particle dispersion model combination. Boundary Layer Meteorol. 137, 373-396. doi: 10.1007/s10546-010-9541-9

Taylor, P. A., Sykes, R. I., and Mason, P. J. (1989). On parameterisation of drag over small-scale topography in neutrally-stratified boundarylayer flow. Boundary Layer Meteorol. 48, 409-422. doi: 10.1007/BF00123062

Teixeira, M. A. C. (2014). The physics of orographic gravity wave drag. Front. Phys. 2:43. doi: $10.3389 /$ fphy.2014.00043

Turnipseed, A. A., Anderson, D. E., Burns, S. P., Blanken, P. D., and Monson, R. K. (2004). Airflows and turbulent flux measurements in mountainous terrain: Part 2: mesoscale effects. Agric. Forest Meteorol. 125, 187-205. doi: 10.1016/j.agrformet.2004.04.007

Vosper, S. B., and Brown, A. R. (2007). Numerical simulations of sheltering in valleys: the formation of nighttime cold-air pools. Boundary Layer Meteorol. 127, 429-448. doi: 10.1007/s10546-008-9272-3

Wagner, A. (1932). Neue theorie der berg- und talwinde [New theory of mountain and valley winds]. Meteorol. Z. 49, 329-341.

Wagner, J. S., Gohm, A., and Rotach, M. W. (2014). The impact of horizontal model grid resolution on the boundary layer structure over an idealized valley. Mon. Wea. Rev. 142, 3446-3465. doi: 10.1175/MWR-D-14-00002.1

Wagner, J. S., Gohm, A., and Rotach, M. W. (2015b). Influence of along-valley terrain heterogeneity on exchange processes over idealized valleys. Atmos. Chem. Phys. 15, 6589-6603; 15, 415-451.

Wagner, J. S., Gohm, A., and Rotach, M. W. (2015a). The impact of valley geometry on daytime thermally driven flows and vertical transport processes. Q. J. R. Meteorol. Soc. doi: 10.1002/qj.2481. [Epub ahead of print].
Weigel, A. P., Chow, F. K., and Rotach, M. W. (2007). The effect of mountainous topography on moisture exchange between the "surface" and the free atmosphere. Boundary Layer Meteorol. 125, 227-244. doi: 10.1007/s10546-0069120-2

Weigel, A. P., Chow, F. K., Rotach, M. W., Street, R. L., and Xue, M. (2006). High-resolution large-eddy simulations of flow in a steep Alpine valley. Part II: flow structure and heat budgets. J. Appl. Meteorol. Clim. 45, 87-107. doi: 10.1175/JAM2323.1

Weigel, A. P., and Rotach, M. W. (2004). Flow structure and turbulence characteristics of the daytime atmosphere in a steep and narrow Alpine valley. Q. J. R. Meteorol. Soc. 130, 2605-2627. doi: 10.1256/qj.03.214

Weissmann, M., Braun, F., Gantner, L., Mayr, G., Rham, S., and Reitebuch, O. (2005). The alpine mountain plaincirculation: airborne doppler lidar measurements and numerical simulations. Mon. Wea. Rev. 133, 3095-3109. doi: 10.1175/MWR3012.1

Whiteman, C. D. (2000). Mountain Meteorology: Fundamentals and Applications. New York, NY: Oxford University Press.

Wood, N., Brown, A. R., and Hewer, F. E. (2001). Parameterizing the effects of orography on the boundary layer: an alternative to effective roughness lengths. Q. J. R. Meteorol. Soc. 127, 759-777. doi: 10.1002/qj.497127 57303

Wood, N., and Mason, P. J. (1993). The pressure force induced by neutral turbulent flow over hills. Q. J. R. Meteorol. Soc. 119, 1233-1267. doi: 10.1002/qj.49711951402

Zangl, G. (2009). The impact of weak synoptic forcing on the valley-wind circulation in the Alpine Inn Valley. Meteorol. Atmos. Phys. 105, 37-53. doi: 10.1007/s00703-009-0030-y

Zangl, G., Egger, J., and Wirth, V. (2001). Diurnal winds in the Himalayan Kali Gandaki valley. Part II: modeling. Mon. Wea. Rev. 129, 1062-1078. doi: 10.1175/1520-0493(2001)129<1062:DWITHK>2.0.CO;2

Zardi, D., and Serafin, S. (2015). An analytic solution for time-periodic thermally driven slope flows. Q. J. R. Meteorol. Soc. 141, 1968-1974. doi: 10.1002/qj.2485

Zardi, D., and Whiteman, C. D. (2013). "Diurnal mountain wind systems," in Mountain Weather Research and Forecasting, eds F. K. Chow, S. F. J. De Wekker, and B. J. Snyder (Dordrecht: Springer Netherlands), 35-119.

Conflict of Interest Statement: The authors declare that the research was conducted in the absence of any commercial or financial relationships that could be construed as a potential conflict of interest.

The Guest Associate Editor Peter Sheridan declares that, despite having collaborated with author Ivana Stiperski, the review process was handled objectively.

Copyright (c) 2015 Rotach, Gohm, Lang, Leukauf, Stiperski and Wagner. This is an open-access article distributed under the terms of the Creative Commons Attribution License (CC BY). The use, distribution or reproduction in other forums is permitted, provided the original author(s) or licensor are credited and that the original publication in this journal is cited, in accordance with accepted academic practice. No use, distribution or reproduction is permitted which does not comply with these terms. 


\section{OPEN ACCESS}

Edited by:

Peter F. Sheridan,

Met Office, UK

Reviewed by:

Julia Hidalgo,

Laboratoire Interdisciplinaire, Solidarités, Sociétés, Territoires,

France

Bijoy Vengasseril Thampi, Science Systems and Applications,

Inc., USA

*Correspondence:

Meinolf Kossmann

meinolf.kossmann@dwd.de

Specialty section:

This article was submitted to

Atmospheric Science,

a section of the journal

Frontiers in Earth Science

Received: 20 August 2015

Accepted: 11 January 2016

Published: 02 February 2016

Citation:

Graf M, Kossmann M, Trusilova K and Mühlbacher G (2016) Identification and Climatology of Alpine Pumping from a Regional Climate Simulation

Front. Earth Sci. 4:5.

doi: 10.3389/feart.2016.00005

\section{Identification and Climatology of Alpine Pumping from a Regional Climate Simulation}

\author{
Maximilian Graf ${ }^{1,2}$, Meinolf Kossmann ${ }^{1 *}$, Kristina Trusilova $^{1}$ and Gudrun Mühlbacher ${ }^{3}$ \\ ${ }^{1}$ Deutscher Wetterdienst, Department of Climate and Environment Consultancy, Offenbach, Germany, ${ }^{2}$ Faculty of \\ Mathematics and Geography, Katholische Universität Eichstätt-Ingolstadt, Eichstätt, Germany, ${ }^{3}$ Deutscher Wetterdienst, \\ Department Climate and Environment Consultancy, München, Germany
}

The thermally driven circulation between the European Alps and the Alpine foreland-named Alpine pumping-occurs regularly under clear and calm weather conditions. While previous studies focused on the impact of Alpine pumping on moist convection and transport of air pollutants, this study was motivated by its ventilation effect for Munich, located about $50 \mathrm{~km}$ north of the Alps in undulating and only slightly inclined terrain, where local thermal circulations are weak. Hourly data from a reanalysis driven regional climate simulation with COSMO-CLM model for the period 1989-2008 were analyzed to identify days with Alpine pumping and to determine the mean diurnal characteristics of this regional thermal circulation. Four literature derived combinations of meteorological criteria were tested to identify days favorable for Alpine pumping from COSMO-CLM results. The first criterion selects days with a daily sum of solar radiation $\geq 20 \mathrm{MJ} / \mathrm{m}^{2}$ and has been used in an earlier observational study. On average 60 day/year are fulfilling the criterion in the model simulation, which compares well to the 67 day/year determined from observations. The other three criteria combinations consider a maximum wind velocity at $850 \mathrm{hPa}$, a maximum daily precipitation sum, and/or a maximum mean cloud cover. The mean annual number of selected days is lower for these criteria combinations and ranges between 20 and 52. Diurnal wind reversals occur on $77-81 \%$ of the selected days, depending on the criteria combination. The daily solar radiation sum of $20 \mathrm{MJ} / \mathrm{m}^{2}$ is only exceeded during April to September, while days satisfying the criteria combinations without the radiation threshold occur all year round. In agreement with observations, the simulated regional thermally driven wind field extends up to $\sim 100 \mathrm{~km}$ north of the Alps with average near-surface wind speeds of $0.5-1.5 \mathrm{~m} / \mathrm{s}$ in the Munich area. With increasing distance from the Alps, the diurnal cycle of Alpine pumping is delayed by up to $3 \mathrm{~h}$. The simulated mean depth of the daytime inflow layer ranges between 500 and $1500 \mathrm{~m}$, whereas the depth of the nocturnal outflow layer typically reaches up to a few hundred meters.

Keywords: COSMO CLM, mountain-plain wind system, valley wind systems, slope wind systems, urban climate 


\section{INTRODUCTION}

Data from simultaneous aerological soundings in central Europe became increasingly available during the first decades of the twentieth century and led to the discovery of a regional, thermally driven wind system between the European Alps and its surroundings (Burger and Ekhart, 1937). The diurnally reversing mountain-plain wind system occurs under fair weather conditions due to the formation of a nocturnal thermal high and a daytime thermal low over the Alps that can be attributed to the energy exchanges at the elevated Alpine terrain surfaces (Hafner et al., 1987; Sturman and Wanner, 2001; Zardi and Whiteman, 2013). Due to its spatial extension the mountain-plain wind system reveals considerable inertia. E.g., onset times of wind reversals in the areas south of Munich are typically delayed up to a few hours relative to sunrise and sunset (Prügel, 1942).

The field campaign VERTIKATOR ("Vertikaler Austausch und Orographie") was conducted to study the physical processes and the climatology of mountain-plain wind systems in the northern Black Forest (Barthlott et al., 2006; Meißner et al., 2007) and in the German Alpine area (Corsmeier et al., 2003; Lugauer and Winkler, 2005; Weissmann et al., 2005). In the pre-alpine area of Bavaria (Germany), it was found that the mountain-plain wind system propagates about $100 \mathrm{~km}$ into the Alpine foreland (Lugauer and Winkler, 2005). The depth of the inflow layer often reaches $1000 \mathrm{~m}$ or more during the afternoon (Corsmeier et al., 2003; Weissmann et al., 2005), whereas the depth of the nocturnal outflow layer is much shallower. Over Alpine slopes and in valleys the regional-scale thermal circulation is enhanced by local slope wind and valley wind systems. The thermal circulation that results from the superposition of these three thermal circulation systems has been named "Alpine pumping” (Lugauer and Winkler, 2005). Spatial scales and patterns of the thermal circulations that contribute to Alpine pumping are shown schematically in Figure $\mathbf{1}$ for both daytime and nighttime. Lifting of the inflowing air during daytime and sinking of the outflowing air during nighttime is favored over slopes, valley heads and over the main divide of the Alps. Anti-winds (recirculation) aloft are much weaker than winds in the surface bound branches of Alpine pumping and often obscured by synoptic scale winds (McGowan, 2004).

In other mountain ranges around the globe similar regional scale mountain-plain wind systems have been observed. Examples are the Rocky Mountains (Zardi and Whiteman, 2013), Central Japan (Kurita et al., 1990; Kuwagata et al., 1990), and the Southern Alps of New Zealand (Sturman and Wanner, 2001). The regional scale mountain-plain wind systems in these areas have been studied to investigate the transport of air pollutants, boundary layer growth, and thunderstorm development (Kurita et al., 1990; Kuwagata et al., 1990; Lugauer and Winkler, 2005). This study investigates the characteristics and occurrence frequency for a better understanding of Alpine pumping and its importance for the urban environment of Munich, which is located about $50 \mathrm{~km}$ north of the European Alps in a slightly undulating terrain. During synoptically calm high pressure conditions, Alpine pumping can potentially alleviate the urban heat island by advection of cool rural air into the city. This is illustrated in Figure 2 which shows surface observations taken during fair weather conditions on 18 July 2014 in Munich City and at two rural stations located about $30 \mathrm{~km}$ north (Munich Airport) and south (Königsdorf, at a glider airfield) of Munich (see Figure 3). The stations in Munich City (WMO ID\# 10865, $515 \mathrm{~m}$ asl) and at Munich Airport (WMO ID\# 10870, $445 \mathrm{~m}$ asl) belong to the operational German weather station network, while the station at the glider airfield in Königsdorf $(600 \mathrm{~m}$ asl) is only temporarily operated. Air temperature measurements at all 3 stations are taken at $2 \mathrm{~m}$ above ground level. Wind measurements in Munich City are taken at $28.5 \mathrm{~m}$ above ground level with wind sensors mounted on a mast $9 \mathrm{~m}$ above the roof. The Munich wind observations show a clear diurnal cycle of Alpine pumping with an abrupt transition to northeasterly winds in the morning, a more gradual transition to southeasterly in the evening, and southwesterly winds later in the night. Wind speed varied between 1.5 and $2.5 \mathrm{~m} / \mathrm{s}$ during daytime and between 0.5 and $1.5 \mathrm{~m} / \mathrm{s}$ during nighttime.

Potential temperatures calculated for the height of the station in Munich City $(515 \mathrm{~m}$ asl) reveal a maximum urban heat island (UHI) intensity of about $5 \mathrm{~K}$ during the night while the daytime UHI intensity varied from $+2 \mathrm{~K}$ to slightly negative values at noon (Figure 2). The cooling potential of Alpine pumping can be estimated using the temperature advection term $\Delta \mathrm{T} / \Delta \mathrm{t}=-\mathrm{v} \Delta \mathrm{T} / \Delta \mathrm{y}$, where $\mathrm{v}$ is the typical southerly wind component near the surface, $\Delta \mathrm{T}$ is the maximum UHI intensity and $\Delta y$ is the approximate south-north distance of $5 \mathrm{~km}$ from the urban fringe to the city center. Applying the daytime values of $|\mathrm{v}|=2 \mathrm{~m} / \mathrm{s}$ and $|\Delta \mathrm{T}|=2 \mathrm{~K}$ and night time values of $|\mathrm{v}|=1 \mathrm{~m} / \mathrm{s}$ and $|\Delta \mathrm{T}|=5 \mathrm{~K}$ yields a magnitude of advective cooling of $\sim 1.5 \mathrm{~K}$ per hour during daytime and $\sim 3.5 \mathrm{~K}$ per hour during nighttime.

Motivated by this abundant impact of Alpine pumping on thermal conditions in Munich, this study uses a 20-year regional climate simulation to determine the characteristics and occurrence frequency of Alpine pumping in the northern foreland of the Alps. Section "Data and Methods" briefly describes the regional climate simulation used for this study and also the literature based criteria used for identification of days favorable for Alpine pumping. Model outputs for the identified days are used in Section "Results-Climatological Analysis of Alpine Pumping Days" to investigate the temporal and spatial characteristics at the surface and the vertical structure of Alpine pumping in the region around Munich. Section "Summary and Discussion" concludes with the main findings of the study.

\section{DATA AND METHODS}

\section{Model Setup COSMO-CLM}

The mesoscale non-hydrostatic regional climate model COSMOCLM (CCLM) (Rockel et al., 2008) used in this study was developed by an open international network of scientists of the Climate Limited-area Modeling-Community (CLMcommunity, www.clm-community.eu) from the original weather predicting model COSMO (Steppeler et al., 2003) of the German Meteorological Service (Deutscher Wetterdienst). 


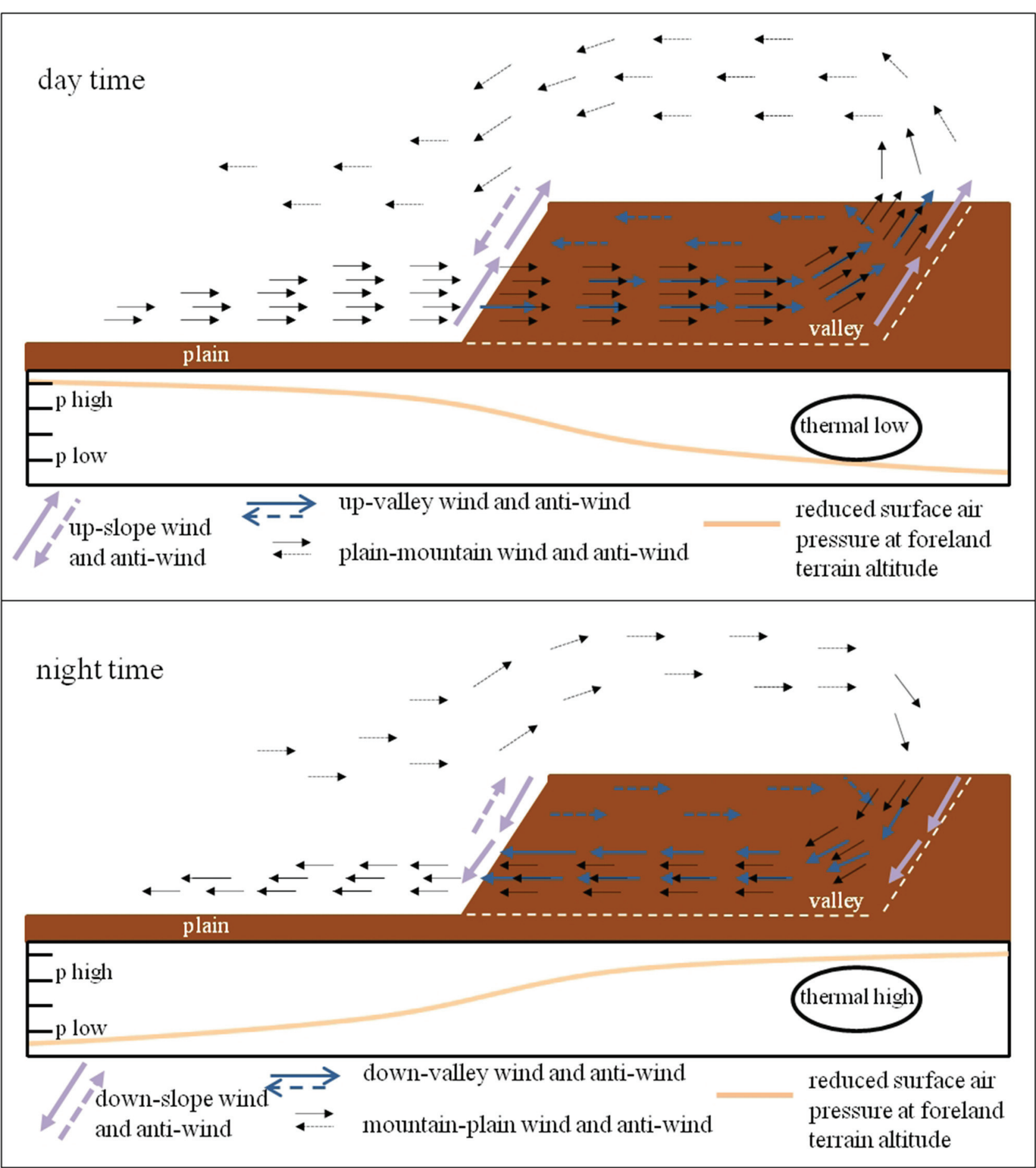

FIGURE 1 | Schematic figure of the different wind systems combining to Alpine pumping with an idealized line of surface air pressure reduced to the altitude of the foreland.

For simulations we use the well-tested reference version 4.8_clm19 of CCLM and setup a chain of two model domainsdomain EUROPE with the spatial resolution of $0.11^{\circ}(\sim 12 \mathrm{~km})$ with 40 vertical levels and domain MUNICH with the spatial resolution of $0.025^{\circ}(\sim 2.8 \mathrm{~km})$ with 50 vertical levels and $110 \times$
112 horizontal grid cells nested into the domain EUROPE as shown Figure 3. The MUNICH domain extends from the foothills of the Swabian Mountains and the Bavarian Forest in the north to the southern side of the Alpine divide. The lowest model level is $20 \mathrm{~m}$ thick in both model domains. The period of 

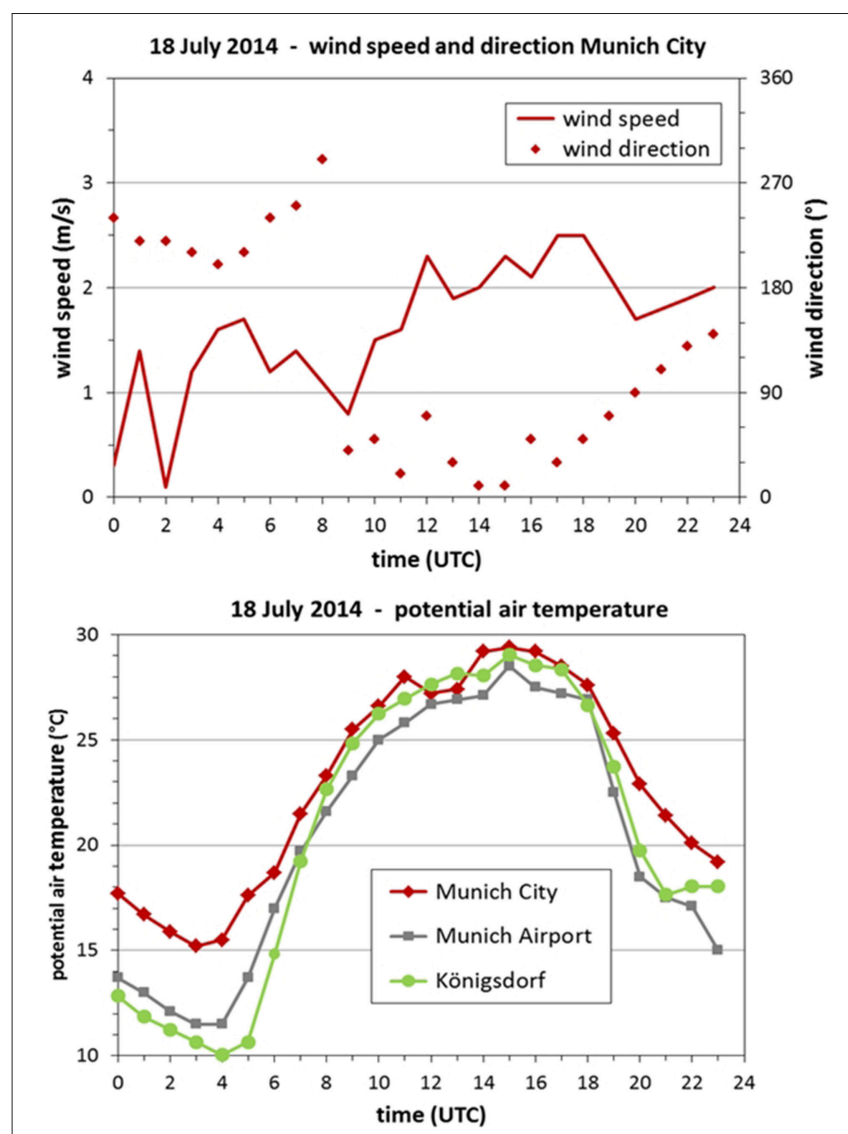

FIGURE 2 | Surface station data measured on 18 July 2014. Wind speed and direction above roof level at the station Munich City located northwest of the historic downtown area (top) and potential air temperature $2 \mathrm{~m}$ above ground level at Munich City and at the two rural stations Munich Airport and Königsdorf (bottom). The station locations are shown in Figure 3.

simulation is 1989-2008. The initialization and constraining of the model domain EUROPE at the lateral boundaries is done with the ERA Interim Reanalysis dataset (Dee et al., 2011) available from the European Centre for Medium-Range Weather Forecasts (www.ecmwf.int). The nested domain MUNICH is forced at its boundaries by the output of the simulation for the domain EUROPE. For both model domains, the two-time level RungeKutta split-explicit scheme and the land surface model TERRA (Doms et al., 2011) with nine soil layers between 0.5, 2.5, 7, 16, $34,70,142,286,574$, and $1150 \mathrm{~cm}$ are used. The land cover data classification of GLC2000 (Fritz et al., 2003) is used. Several large lakes are located south of Munich and at the foothills of the Alps. Land cover in this part of the domain is dominated by forest (see surface roughness map in Supplementary Material). The presented analysis of model results is limited to the central part of the nested domain (Figure 3).

\section{Identification of Alpine Pumping Days Criteria for Identification}

Four literature-based combinations of meteorological criteria were tested to identify days favorable for Alpine pumping from
COSMO-CLM results (Table 1). The data needed for application of all criteria are available from the hourly COSMO-CLM simulation output and in principle also from routine climate network observations. Radiation is seen as the main driving mechanism of Alpine pumping (Zaitchik et al., 2007). Therefore, a spatial mean daily sum of solar radiation $\geq 20 \mathrm{MJ} / \mathrm{m}^{2}$ in the model output analysis domain is defined as the first identification criterion (SR criterion). This threshold has previously been used in an observational study by Lugauer and Winkler (2005).

The second criteria combination also applies the SR criterion but excludes days with upper level wind speeds greater or equal to $6 \mathrm{~m} / \mathrm{s}$ at 00,12 or $23 \mathrm{UTC}$ at $850 \mathrm{hPa}$ (UW criterion). High ambient wind speeds (e.g., due to large-scale pressure systems), impede the evolution of the thermally driven winds (Kossmann et al., 2002; Stewart et al., 2002). The simulated $850 \mathrm{hPa}$ wind velocity is extracted from model grid cells at Oberschleißheim (Coordinates: $48,17^{\circ} \mathrm{N} ; 11,56^{\circ} \mathrm{E}$ ) where radiosondes are operationally released by the German Meteorological Service twice a day at 12 and 00 UTC.

The other two criteria combinations are independent of radiation and can be seen as excluding criteria. During precipitation events, forcing of thermally driven winds is suppressed due to reduced sensible heat fluxes at the surface (Geiger et al., 1995). The third criteria combination interrelates the UW criterion with the exclusion of days with spatially averaged precipitation sum in the analysis domain $>1 \mathrm{~mm}$ (PR criterion).

The fourth criteria combination applies a maximum cloud coverage threshold (CC criterion) in addition to the UW and PR criteria, as a proxy for solar radiation. The value of the total cloud coverage threshold averaged over the analysis domain was set to $5 / 8$ in order to exclude days with a daily sum of solar radiation lower than $75 \%$ of the theoretical maximum radiation (Schafer et al., 1996).

In an alternative approach, a wind oscillation criterion, was applied to select days with outflow wind direction (between $120^{\circ}$ and $240^{\circ}$ ) at 03:00 UTC, inflow wind direction (between $300^{\circ}$ and $60^{\circ}$ ) at 13:00 UTC, and again outflow direction at 23:00 UTC. The regional climate model output for an area south of Munich $(8 \times 8$ grid cells $)$ was averaged over all days selected by the wind oscillation criterion. Results showed a mean daily solar radiation sum of $\sim 18 \mathrm{MJ} / \mathrm{m}^{2}$, a mean $850 \mathrm{hPa}$ wind velocity over Munich of about $6.5 \mathrm{~m} / \mathrm{s}$, a daily precipitation sum of about $1.5 \mathrm{~mm}$, and a mean cloud cover between $4 / 8$ and 5/8. These results give some confidence that the literature-based threshold values listed in Table $\mathbf{1}$ are appropriate to define favorable conditions for the Alpine pumping days. The wind oscillation criterion was also applied to the 4 subsets of days that fulfill the identification criteria combinations in Table 1 to determine the number (or percentage) of favorable days that individually reveal the diurnal wind reversal of interest.

\section{Frequencies of Days Favorable for Alpine Pumping}

On average 60 days per year fulfill the SR criterion in the model simulation (Table 1). This compares well to 67 days per year determined from observations by Lugauer and Winkler (2005). The mean annual number of selected days from the other three 


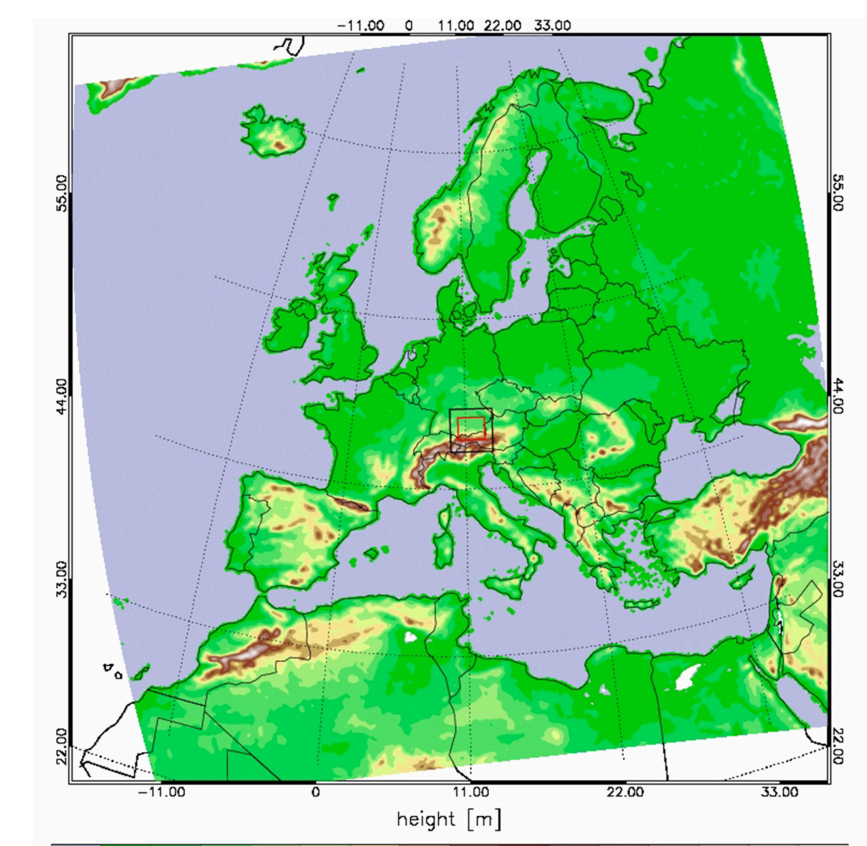

$\begin{array}{lllllllllllllllll}-20 & 0 & 200 & 400 & 600 & 800 & 1000 & 1200 & 1400 & 1600 & 1800 & 2000 & 2200 & 2400 & 2600 & 2800 & 3000\end{array}$

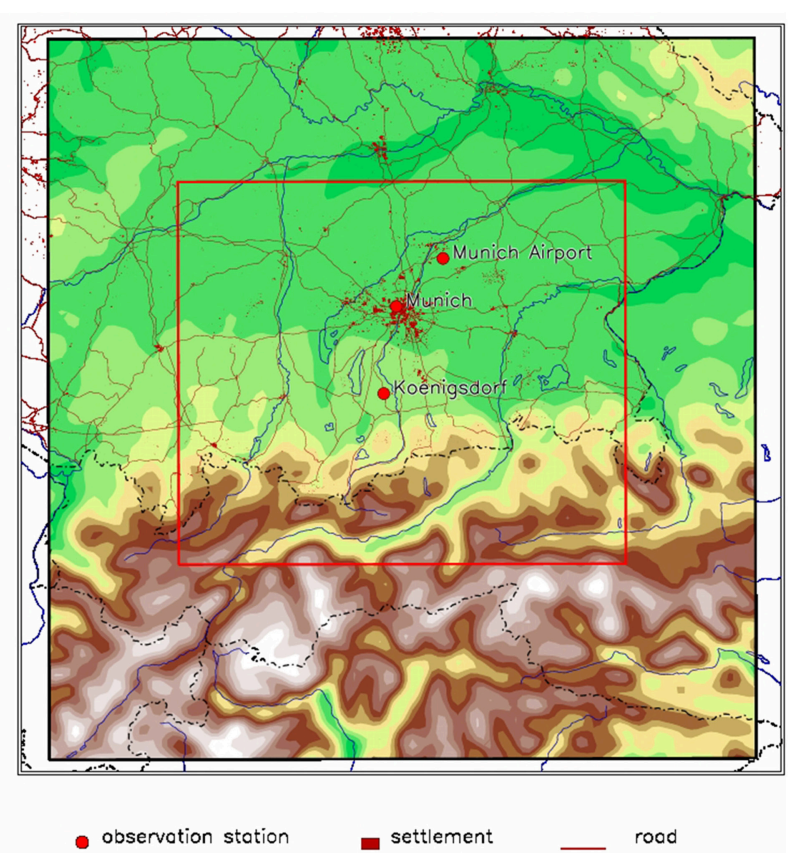

observation station settlement rood

FIGURE 3 | Model domains for EUROPE (left) and for MUNICH (right) nested into the Europe domain (location of the nest is shown by the black frame).

The domain for the analysis of model results is shown by the red frame.

TABLE 1 | Identification criteria combinations with threshold values and average yearly number of days identified from the COSMO-CLM simulation results for the period 1989-2008.

\begin{tabular}{|c|c|c|c|}
\hline No. & $\begin{array}{l}\text { Name of individual } \\
\text { criteria } \\
\text { combinations }\end{array}$ & $\begin{array}{l}\text { Corresponding threshold values } \\
\text { for meteorological criteria }\end{array}$ & $\begin{array}{l}\text { Identified } \\
\text { days per } \\
\text { year }\end{array}$ \\
\hline 1 & SR & Daily sum solar radiation ${ }^{\#} \geq 20 \mathrm{MJ} / \mathrm{m}^{2}$ & 60.0 \\
\hline 2 & SR_UW & $\begin{array}{l}\text { Upper level windspeed* }<6 \mathrm{~m} / \mathrm{s} \text { and } \\
\text { daily sum solar radiation } \geq 20 \mathrm{MJ} / \mathrm{m}^{2}\end{array}$ & 20.4 \\
\hline 3 & PR_UW & $\begin{array}{l}\text { Daily sum precipitation }{ }^{\#}<1 \mathrm{~mm} \text { and } \\
\text { upper level windspeed }{ }^{*}<6 \mathrm{~m} / \mathrm{s}\end{array}$ & 52.1 \\
\hline 4 & PR_UW_CC & $\begin{array}{l}\text { Daily sum precipitation }{ }^{\#}<1 \mathrm{~mm} \text { and } \\
\text { upper level windspeed }{ }^{*}<6 \mathrm{~m} / \mathrm{s} \text { and } \\
\text { daily mean cloud cover }{ }^{\#}<5 / 8\end{array}$ & 38.9 \\
\hline
\end{tabular}

\# Averaged over the evaluation domain.

* Wind speeds at $850 \mathrm{hPa}$ for 00, 12, 23 UTC over Munich/Oberschleissheim.

criteria combinations is lower and ranges between 20 and 52 . Figure 4 illustrates how many criteria combinations select the same day during the 20-year period of the model simulation. Hit rates for all criteria combinations show a strong interannual variability, with years of high frequencies (e.g., 2003) and years with low frequencies (e.g., 1993) of days with favorable conditions for Alpine pumping. Overall, 25.9\% of all days are selected by at least one of the four criteria combinations, but only about half of these days are selected by two, three or four criteria combinations.

On one hand, the daily solar radiation sum of $20 \mathrm{MJ} / \mathrm{m}^{2}$ is only exceeded during the months from April to September with highest incidence frequencies from May to August. When the SR criterion is combined with the UW criterion, only one third of the SR criterion fulfilling days are selected (see Table 1). This finding indicates that on two thirds of the high-radiation days, the thermal wind systems are affected by moderate or strong synoptic winds.

On the other hand, days fulfilling the criteria combinations without a radiation threshold occur all year round. With fewer Alpine pumping days per year, these two criteria combinations appear stricter than the SR criterion, particularly during the warm season. Basically radiation-dependent criteria appear suitable to study the influences of Alpine pumping on urban heat load or photochemical smog events, while radiation-independent criteria combinations may be recommended to study its influence on air pollution problems that arise all year round (e.g., urban ventilation).

The hit rate of the wind oscillation criterion applied to all days and to the four subsets of days that fulfill the identification criteria combinations reveals a strong spatial variability. For the days preselected by the four identification criteria combinations, high hit rates between 75 and $85 \%$ are found in the area between Munich and the Alpine foothills. Analysis of $8 \times$ 8 grid cells between Munich and the Alpine foothills (see Figure 5) shows that the wind oscillation criterion is fulfilled on $81 \%$ of the days that also fulfill the radiation criterion $\mathrm{S}$ or the combined S_UW criterion. For the days selected by the precipitation and upper level wind combination PR_UW and by the extended PR_UW_CC combination it is found that somewhat smaller fractions of 77 and $78 \%$ fulfill the wind oscillation criterion, respectively. When applied to all 


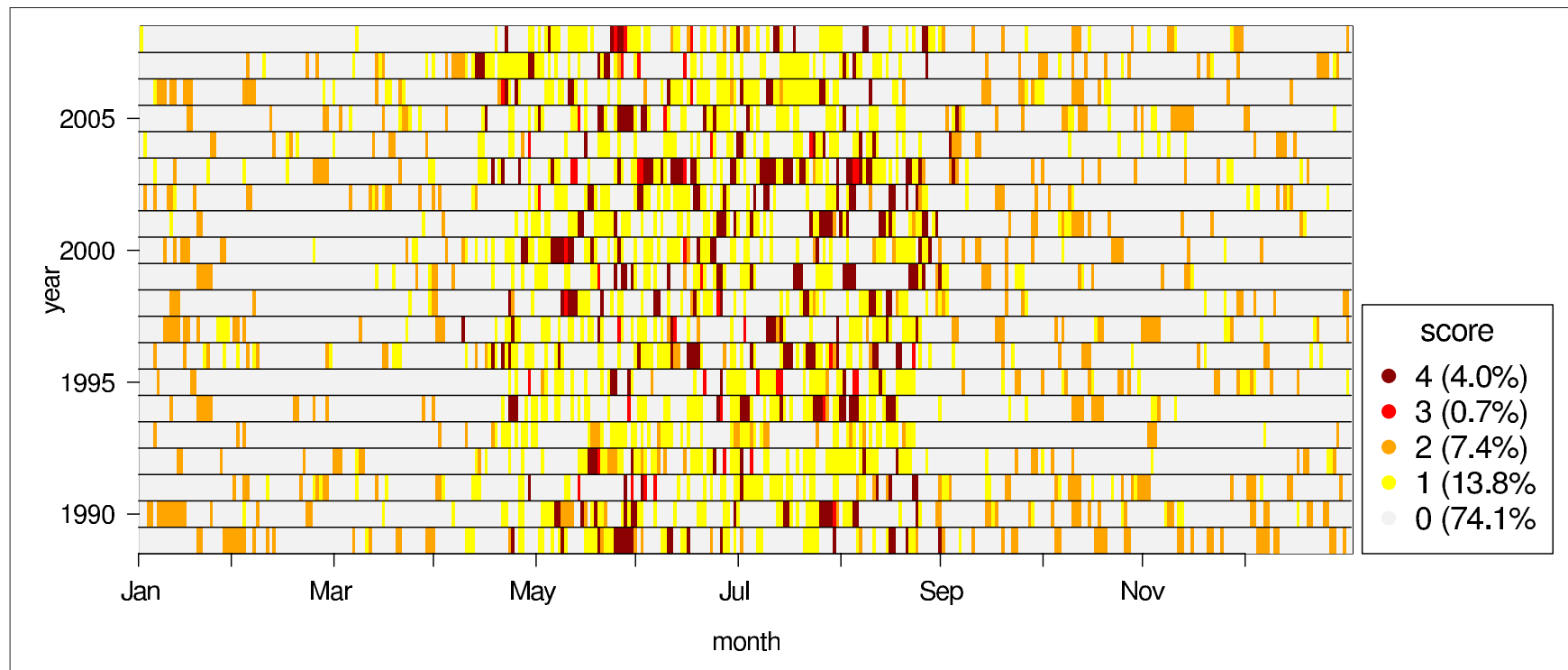

FIGURE 4 | Daily score of the four criteria combinations defined in Table 1.

days, the hit rate of the wind oscillation criterion is 62 days per year.

The relatively high hit rates of the wind oscillation criterion suggest that Alpine pumping wind signatures have been largely captured by the four identification criteria combinations. In Section "Results-Climatological Analysis of Alpine Pumping Days," the wind data from the selected days are used to analyze the mean temporal and spatial characteristics of Alpine pumping. Possible reasons for the gap between the high hit rates and 100\% are discussed in Section "Summary and Discussion."

\section{RESULTS-CLIMATOLOGICAL ANALYSIS OF ALPINE PUMPING DAYS}

Hourly model outputs are used to calculate average wind fields for days selected by the four criteria combinations introduced in Section "Identification of Alpine Pumping Days." Alpine pumping is found to be quite pronounced in the mean wind fields of all four criteria combinations, and its diurnal cycle has been identified by visual inspection of mean hourly surface wind maps and of mean hourly vertical cross sections of the wind field through Munich (Graf, 2014). Table 2 summarizes the mean diurnal phases of inflow, outflow, and flow transition. In agreement with Lugauer and Winkler (2005), the inflow phase is found to be shorter than the outflow phase, even for the radiation-dependent criteria that selects only days with longer daytime than nighttime duration. Due to the stronger variation in times of sunrise and sunset, the wind climatology for the radiation-independent criteria combinations reveals longer transition phases between inflow and outflow regimes.

Because of the similarity of the main findings and for brevity, detailed presentation and analysis of the mean wind fields in Sections "Temporal and Spatial Characteristics Near the Surface" and "Vertical Structure of Alpine Pumping" are limited to the SR criterion and the PR_UW criteria combination as representatives for radiation-dependent and radiation-independent selection criteria.

\section{Temporal and Spatial Characteristics Near the Surface}

Mean vectors of the wind field at $10 \mathrm{~m}$ above ground level (agl) at 00:00 UTC and 12:00 UTC are shown in Figure 5 for the days selected during the simulated 20 -year period by the SR criterion and by the PR_UW criteria combination. Furthermore, latitudetime sections of the southerly surface wind component at the longitude of Munich $\left(11.58^{\circ} \mathrm{E}\right)$, averaged over the selected days and 5 grid cells (ca. $14 \mathrm{~km}$ ) in zonal direction are shown in Figure 6.

In agreement with observations, the simulated circulation extends up to the Danube Valley, located about $100 \mathrm{~km}$ north of the Alps (at the northern end of domain MUNICH). In the area of Munich $\left(\sim 48.15^{\circ} \mathrm{N}\right)$, average southerly wind speeds reach $0.5-1.0 \mathrm{~m} / \mathrm{s}$ during nighttime and up to $\sim 1.5 \mathrm{~m} / \mathrm{s}$ during daytime (Figure 6). The magnitudes of these simulated wind speeds are similar to those observed on SR criterion days by Lugauer and Winkler (2005). Due to effects of local surface roughness and local orography the measured wind speeds exhibit a stronger spatial variability than wind speeds simulated on the $2.8 \mathrm{~km}$ model grid. The observational case study shown in Figure 2 suggests that wind speeds on days with ideal conditions can be at least $1 \mathrm{~m} / \mathrm{s}$ higher than mean wind speeds for all selected days.

For the PR_UW criteria combination, Figure 6 shows that during nighttime southerly winds north of Munich are very weak (below $0.5 \mathrm{~m} / \mathrm{s}$ ), which is probably caused by stronger surface temperature inversions forming in the Danube valley during the cooler seasons at night time (Zängl, 2005). Daytime wind direction in the areas north of Munich gradually turns 

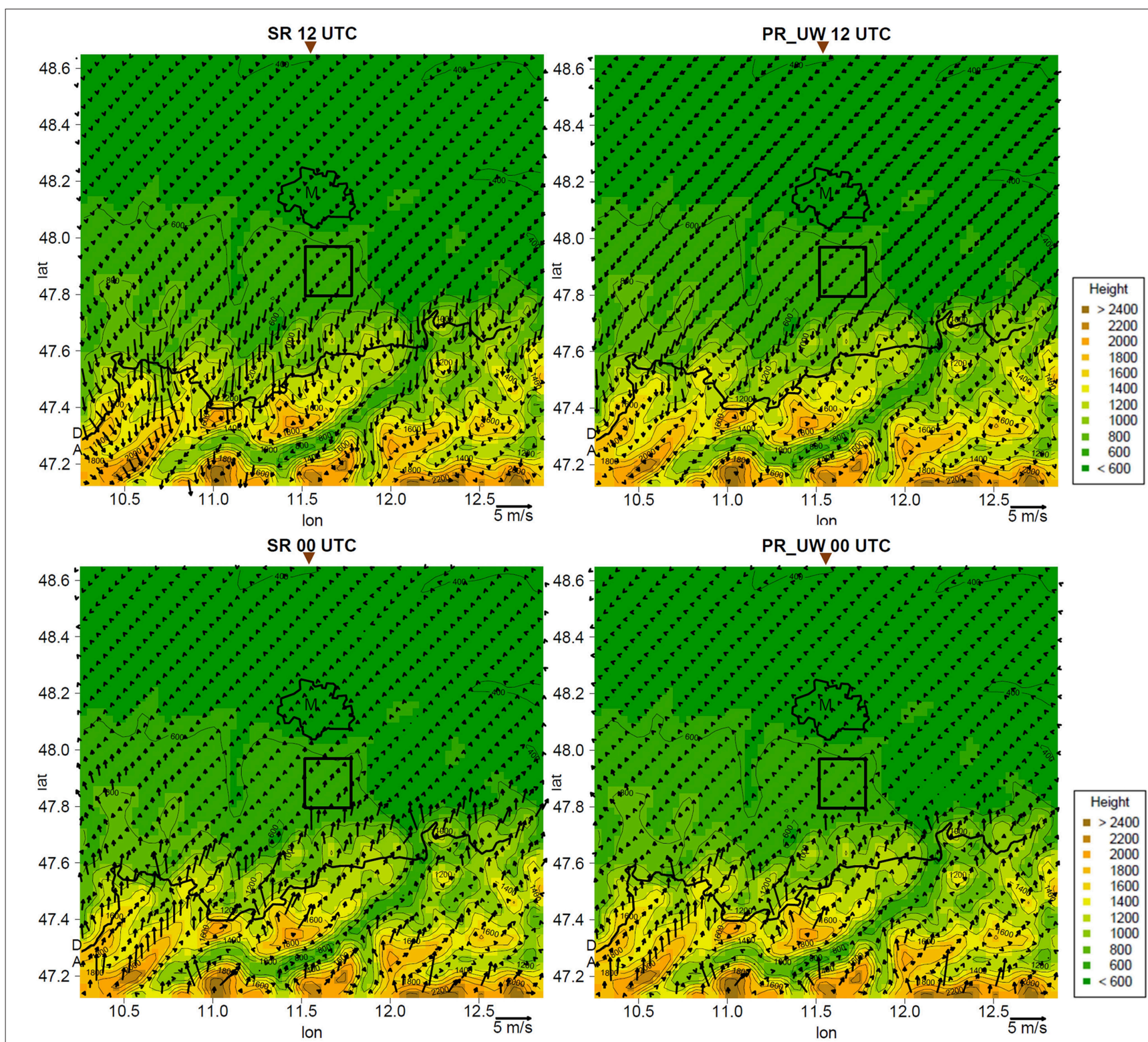

FIGURE 5 | Averaged surface wind maps of the horizontal wind vector at $10 \mathrm{~m}$ agl for SR criterion (left) and PR_UW criteria combination (right) at $\mathbf{1 2}$ UTC (top) and 00 UTC (bottom). Bold black lines show the urban area of Munich (indicated by the letter M) and the German-Austrian border, while thin black contour lines represent the terrain height. $8 \times 8$ model grid points within the black rectangular frame (south of Munich) are used to calculate the wind oscillation criterion. The triangles at the top of the panels indicate the geographical longitude of the meridional cross sections shown in Figure 7.

TABLE 2 | Average times of inflow (red), outflow (blue), and transition (brown) phases of Alpine pumping derived from the mean hourly wind fields for the different criteria combinations.

\begin{tabular}{|c|c|c|c|c|c|c|c|c|c|c|c|c|c|c|c|c|c|c|c|c|c|c|c|c|}
\hline UTC & 1 & 2 & 3 & 4 & 5 & 6 & 7 & 8 & 9 & 10 & 11 & 12 & 13 & 14 & 15 & 16 & 17 & 18 & 19 & 20 & 21 & 22 & 23 & 0 \\
\hline SR & & & & & & & & & & & & & & & & & & & & & & & & \\
\hline SR_UW & & & & & & & & & & & & & & & & & & & & & & & & \\
\hline PR_UW & & & & & & & & & & & & & & & & & & & & & & & & \\
\hline PR_UW_CC & & & & & & & & & & & & & & & & & & & & & & & & \\
\hline
\end{tabular}


to northeasterly directions for both the SR criterion and the PR_UW criteria combination.

At the foothills and at valley exits of the Alps, the regional scale mountain-plain wind system is superimposed upon slope and valley wind systems. The simulated mean wind speeds are therefore stronger, locally reaching about $2.0 \mathrm{~m} / \mathrm{s}$ during daytime and $2.5-3.0 \mathrm{~m} / \mathrm{s}$ during nighttime (Figure 5). This behavior has also been analyzed by Lugauer and Winkler (2005), although local terrain effects on wind speed and direction at the measurement stations in this area are stronger than in the Alpine foreland.

With increasing distance from the Alps, the diurnal cycle of Alpine pumping is delayed by up to $3 \mathrm{~h}$, as shown by the latitude-time sections of the southerly surface wind component in Figure 6. While the extension, surface wind velocity, and wind direction of Alpine pumping in the Alpine foreland agree well with observations by Lugauer and Winkler (2005), the quality of the simulated slope and valley wind systems is limited by the horizontal model resolution of $2.8 \mathrm{~km}$.

\section{Vertical Structure of Alpine Pumping}

The vertical structure of the Alpine pumping layer is strongly related to the distance to the Alps and the time of the day (Figure 7). Analysis of meridional vertical cross sections of the simulated wind field through Munich show higher wind velocities close to the Alps (discussed in the previous Section "Temporal and Spatial Characteristics Near the Surface") also extend to higher levels. Furthermore, the vertical cross sections confirm that wind reversals between the daytime and nighttime phases of Alpine pumping occur earlier in this region compared to the foreland. With greater distance from the Alps, the increasing time lag in wind reversal is evident in the entire inflow and outflow layer. The depth of the surface bound inflow and outflow layers are found to increase from north to south. Averaged winds above $2500 \mathrm{~m}$ reflect weak synoptic scale winds blowing mostly from northerly directions during days selected by the SR criterion and the PR_UW combination (Figure 7).
Due to this general upper level wind direction, the depth of the outflow layer can be detected more easily than the depth of inflow layer (Figure 7). At 00:00 UTC, the mean depth of the outflow layer reaches up to $400 \mathrm{~m}$ and wind speed in the outflow layer near the Alps reaches up to $3 \mathrm{~m} / \mathrm{s}$ for both SR and PR_UW combination. High outflow wind velocities occur in two zones separated by weaker winds in between. One zone is located over the steepest north facing slopes of the Alps, where the regional scale mountain-plain winds are strongly enhanced by local downslope winds. The second zone is located further north, about halfway between the Alps and Munich. Later in the night this second zone with high wind speeds moves northward and ceases before sunrise.

The mean simulated depth of the daytime inflow layer at 12:00 UTC is about $400 \mathrm{~m}$ for the PR_UW combination. With the SR combination, the mean depth is about $1000 \mathrm{~m}$. The maximum depth can reach up to $700 \mathrm{~m}$ (PR_UW) and $1500 \mathrm{~m}$ (SR) respectively in the following 3 hours. For both combinations, the strongest winds occur over the foothills, and for the SR criterion, strong winds occur also over the north facing slopes of the Alps. Overall, stronger average insolation causes stronger inflow for the SR criterion with wind speeds up to $3 \mathrm{~m} / \mathrm{s}$, while the mean inflow velocity for the PR_UW combination is limited to about $1.5 \mathrm{~m} / \mathrm{s}$ due to the inclusion of days with low insolation from the colder seasons.

The surface branch of the thermal circulation reveals a jet-like structure in wind velocity during both day and night. Jet heights are mostly between 30 and $150 \mathrm{~m}$, but inflow jet heights for the SR criterion are somewhat higher, locally reaching 200-300 m agl.

\section{SUMMARY AND DISCUSSION}

Hourly and daily data from a reanalysis-driven regional climate simulation with COSMO-CLM model for the period of 19892008 were analyzed to identify days with Alpine pumping and to determine the mean diurnal variation of the direction, intensity,
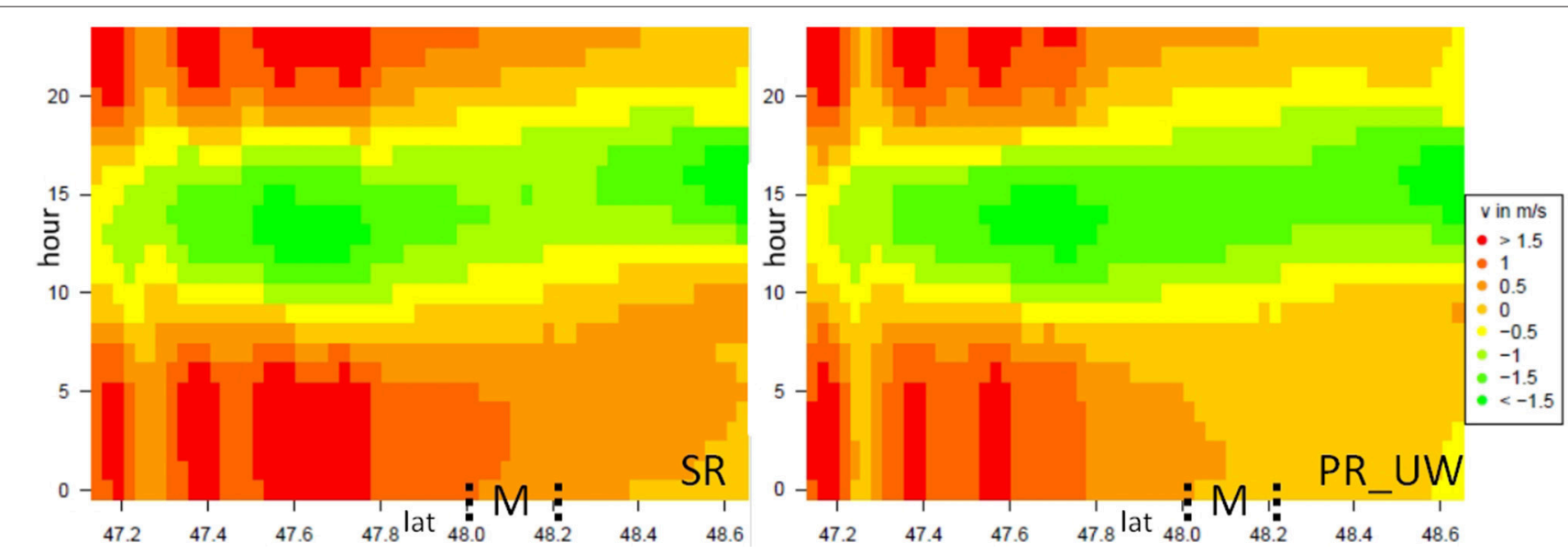

FIGURE 6 | Latitude-time sections of the southerly surface wind component at the longitude of Munich, averaged over 5 grid cells in zonal direction for the SR criterion (left) and the PR_UW combination (right). 

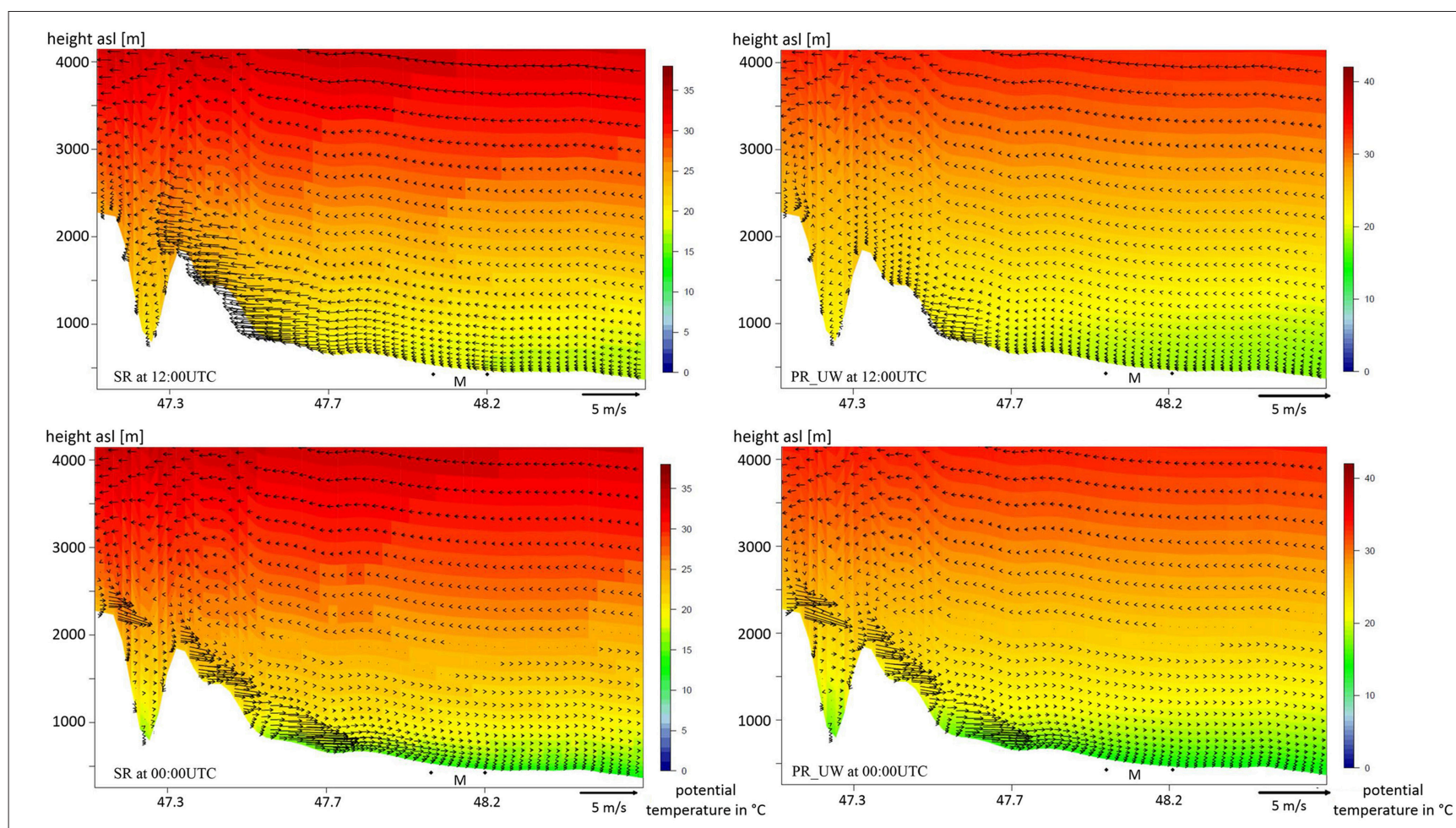

FIGURE 7 | Averaged $v$ and $w$ wind components along a meridional vertical cross section through Munich at 12:00 UTC (top) and 00:00 UTC (bottom) for the SR criterion (left) and PR_UW combination (right), with potential temperature shown by color shading in the background. The two black dots mark the northern and southern border of Munich.

and extension of the regional thermal circulation. The main findings are:

- The mean annual number of days with favorable conditions for Alpine pumping estimated by four criteria combinations varies between 20 and 60 days. A simple criterion requiring inflow and outflow conditions at certain times of the day identifies 62 days per year on average.

- For all four criteria combinations, the wind fields averaged over the selected days clearly reveal the expected diurnal cycle of Alpine pumping, with weak upper level (synoptic scale) winds from northeasterly directions.

- The days selected by the four identification criteria combinations were individually tested for occurrence of diurnal wind reversals using the wind oscillation criterion. For the area between Munich and the Alpine foothills, it is found that diurnal wind reversal occurs on $77-81 \%$ of the selected days, depending on the identification criteria combination.

- The mean annual number of 60 days found for the SR criterion compares well to the 67 days per year determined by the observational study of Lugauer and Winkler (2005).

- In agreement with Lugauer and Winkler (2005), the simulated regional thermal circulation extends from the Alps to the Danube Valley (located ca. $100 \mathrm{~km}$ north of the Alps).

- The simulated mean surface wind speed of Alpine pumping in the Munich area varies between 0.5 and $1.0 \mathrm{~m} / \mathrm{s}$ during nighttime and reaches up to about $1.5 \mathrm{~m} / \mathrm{s}$ during daytime. At the foothills and at valley exits of the Alps, the regional mountain-plain wind system is enhanced by slope and valley wind systems. Here the mean wind speed locally reaches about $2.0 \mathrm{~m} / \mathrm{s}$ during daytime and $2.5-3.0 \mathrm{~m} / \mathrm{s}$ during nighttime.

- Overall, the mean near surface wind speeds on selected days from the observational analysis (Lugauer and Winkler, 2005) and from the model results are of comparable magnitude. A more quantitative comparison is desirable in future studies, but requires the availability of regionalized wind observations, where effects of local surface roughness and local orography at the measurement stations have been removed or up-scaled to model grid resolution.

- In the Alpine foreland, the onset times for the inflow and outflow phases of Alpine pumping depend on the distance from the Alps. In comparison to locations at the foothills of the Alps the diurnal cycle north of Munich is delayed by up to $3 \mathrm{~h}$.

- The simulated mean depth of the daytime inflow layer derived from meridional vertical cross sections of the wind field through Munich ranges between 500 and $1500 \mathrm{~m}$, while the depth of the nocturnal outflow layer typically reaches up to a few hundred meters.

- The vertical cross sections reveal a jet structure in the thermal circulation between the Alps and the foreland with the highest wind speeds mostly at heights between 30 and $150 \mathrm{~m}$ agl, and 
higher daytime inflow jet heights of up to $200-300 \mathrm{~m}$ agl for the SR criterion.

From these findings it can be concluded that Alpine pumping is a frequently occurring phenomenon in the study area that provides significant ventilation under synoptically calm conditions. Cities located in the Alpine foreland strongly benefit from the advection of cold rural air provided by Alpine pumping under heat load conditions during both daytime and nighttime (see observational case study in Section "Introduction").

In subsequent research, the presented COSMO-CLM model results will be used to drive simulations with the local scale urban climate model MUKLIMO_3 (Früh et al., 2011) under conditions with and without Alpine pumping. The model's horizontal grid spacing of $100 \mathrm{~m}$ and the detailed representation of urban boundary layer processes will allow a detailed analysis of the influences of Alpine pumping on summertime thermal conditions in Munich.

Despite the interesting outcomes, there are several limitations to the present study, and additional investigations are needed to improve understanding and climatological knowledge of Alpine pumping. E.g., not all the days identified by the four criteria combinations fulfill the wind oscillation criterion and the gap to $100 \%$ indicates that the data stratification used in this study for the identification of Alpine pumping days is clearly not perfect. Furthermore, the stricter criteria combinations of S_UW and PR_UW_CC do not provide higher hit rates for the wind oscillation criterion than the corresponding $S$ and PR_UW criteria although they are showing a stronger signal of Alpine pumping in the mean wind fields. A reason for this could be that the applied wind oscillation criterion is too simplistic for the identification of the diurnal wind reversals of Alpine pumping and/or that the refinement of the identification criteria through upper level wind or cloud cover filters needs improvement. E.g., upper level wind effects are likely to be dependent on wind direction and upper level wind data at the $850 \mathrm{hPa}$ level are possibly too high above the surface wind regime during night time when decoupling of surface winds often occurs at a few hundred meters above the surface.

\section{REFERENCES}

Barthlott, C., Corsmeier, U., Meißner, C., Braun, F., and Kottmeier, C. (2006). The influence of mesoscale circulation systems on triggering convective cells over complex terrain. Atmos. Res. 81, 150-175. doi: 10.1016/j.atmosres. 2005.11.010

Burger, A., and Ekhart, E. (1937). Über die tägliche Zirkulation der Atmosphäre im Bereich der Alpen. Gerl. Beitr. Geophys. 49, 341-367.

Corsmeier, U., Kottmeier, C., Winkler, P., Lugauer, M., Reitebuch, O., and Dobrinski, P. (2003). "Flow modification and mesoscale transport caused by "Alpine Pumping": a VERTIKATOR case study," in Proceedings of the International Conference on Alpine Meteorology and Mesoscale Alpine Project Meeting (Brigg), Vol. A., 138-140. Available online at: http://www.map. meteoswiss.ch/icam2003/744.pdf

Dee, D. P., Uppala, S. M., Simmons, A. J., Berrisford, P., Poli, P., Kobayashi, S., et al. (2011). The ERA-Interim reanalysis: configuration and performance of the data assimilation system. Quart. J. R. Meteorol. Soc. 137, 553-597. doi: 10.1002/qj.828
There will be days when Alpine pumping is present but weak and hardly distinguishable from synoptic scale wind characteristics. Our study did not aim at identifying these days but rather intended to select days with moderate to strong intensities of the multi-scale mountain induced thermal circulation in the study area. Alternative approaches of data processing such as weather type and cluster analysis (e.g., Kaufmann and Weber, 1996; Hidalgo et al., 2014) could be promising to gain further insight to climatological occurrence and behavior of Alpine pumping. In a further step, reanalysis driven regional climate simulations could be complemented by regional climate projections for different climate change scenarios. The application of suitable identification criteria would allow estimation of changes in the frequencies of Alpine pumping days to be expected under possible future climate conditions.

\section{ACKNOWLEDGMENTS}

The presented study was initiated by a cooperation between the Deutscher Wetterdienst and the Landeshauptstadt München on urban climate adaptation. The COSMO-CLM model simulation for the domain EUROPE was provided by Klaus Keuler, Brandenburgische Technische Universität Cottbus-Senftenberg, Germany. This simulation was done according to guidelines of the Coordinated Downscaling Experiment-European Domain (EURO-CORDEX, www.euro-cordex.net), which is the European branch of CORDEX international program sponsored by the World Climate Research Program (WRCP). We thank the reviewers and the editor for their helpful comments on the manuscript and also express our sincere thanks to Saskia Buchholz for her arrangements to the collaboration behind this work and Tobias Heckmann for his critical supervision of the thesis this paper is based on.

\section{SUPPLEMENTARY MATERIAL}

The Supplementary Material for this article can be found online at: http://journal.frontiersin.org/article/10.3389/feart. 2016.00005

Doms, G., Förstner, J., Heise, E., Herzog, H.-J., Mironov, D., Raschendorfer, M., et al. (2011). A Description of the Nonhydrostatic Regional COSMO Model. Part II: Physical Parameterization. Offenbach: Deutscher Wetterdienst. 1-154. Available online at: http://www.cosmo-model.org/content/model/ documentation/core/cosmoPhysParamtr.pdf

Fritz, S., Bartholome, E. M. C., Belward, A. S., Hartley, A., Stibig, H.-J., Eva, H. D., et al. (2003). Harmonisation, Mosaicing and Production of the Global Land Cover 2000 Database. Ispra: Joint Research Centre. Available online at: http:// publications.jrc.ec.europa.eu/repository/handle/JRC26168

Früh, B., Becker, P., Deutschländer, T., Hessel, J., Kossmann, M., Mieskes, I., et al. (2011). Estimation of climate-change impacts on the urban heat load using an urban climate model and regional climate projections. J. Appl. Meteorol. Climatol. 50, 167-184. doi: 10.1175/2010jamc2377.1

Geiger, R., Aron, R. H., and Todhunter, P. (1995). The Climate Near the Ground. Cambridge: Harvard University Press.

Graf, M. (2014). Identifizierung und Klimatologie des Alpinen Pumpens aus einer Regionalen Klimasimulation. Bachelor thesis, Katholische Universität Eichstätt, 56. doi: 10.13140/RG.2.1.4263.1209 
Hafner, T. A., Reinhard, M. E., Weisel, E. L., and Fimpel, H. P. (1987). Boundary layer aspects an elevated heat source effects of the Alps. Meteorol. Atmos. Phys. 36, 61-73. doi: 10.1007/BF01045140

Hidalgo, J., Masson, V., and Baehr, C. (2014). From daily climatic scenarios to hourly atmospheric forcing fields to force Soil-Vegetation-Atmosphere transfer models. Front. Environ. Sci. 2:40. doi: 10.3389/fenvs.2014.00040

Kaufmann, P., and Weber, R. O. (1996). Classification of mesoscale wind fields in the MISTRAL field experiment. J. Appl. Meteorol. 35, 1963-1979.

Kossmann, M., Whiteman, C. D., and Bian, X. (2002). "Dynamic airflow channelling over the Snake River Plain, Idaho," in Proceedings of the 10th Conference on Mountain Meteorology (Park City: Utah. The American Meteor Society), 360-363.

Kurita, H., Ueda, H., and Mitsumoto, S. (1990). Combination of local wind systems under light gradient wind conditions and its contribution to the long-range transport of air pollutants. J. Appl. Meteorol. 29, 331-348.

Kuwagata, T., Sumioka, M., Masuko, N., and Kondo, J. (1990). The daytime heating process over complex terrain in central Japan under fair and calm weather conditions, Part I: mesoscale circulation and the PBL heating rate. J. Meteorol. Soc. Jpn. 68, 625-638.

Lugauer, M., and Winkler, P. (2005). Thermal circulation in South Bavaria climatology and synoptic aspects. Meteorol. Z. 14, 15-13. doi: 10.1127/09412948/2005/0014-0015

McGowan, H. (2004). Observations of anti-winds in a deep Alpine valley, Lake Tekapo, New Zealand. Arct. Antarc. Alp. Res. 36, 495-501. doi: 10.1657/15230430(2004)036[0495:ooaiad]2.0.co;2

Meißner, C., Kalthoff, N., Kunz, M., and Adrian, G. (2007). Initiation of shallow convection in the Black Forest mountains. Atmos. Res. 86, 42-60. doi: 10.1016/j.atmosres.2007.03.003

Prügel, H. (1942). Der "Mitternachtswind" auf den oberbayrischen Seen. Meteorol. Z. 59, 97-98

Rockel, B., Will, A., and Hense, A. (2008). The regional climate model COSMO-CLM (CCLM). Meteorol. Z. (N.F.) 17, 347-348. doi: 10.1127/09412948/2008/0309

Schafer, J. S., Saxena, V. K., Wenny, B. N., Barnard, W., and De Luisi, J. J. (1996). Observed influence of clouds in ultraviolet-B radiation. Res. Lett. 23, 2625-2628. doi: 10.1029/96GL01984
Steppeler, J., Doms, G., Schättler, U., Bitzer, H. W., Gassmann, A., Damrath, U., et al. (2003). Meso-gamma scale forecasts using the nonhydrostatic model LM. Meteor. Atmos. Phys. 82, 75-96. doi: 10.1007/s00703-001-0592-9

Stewart, J. Q., Whiteman, C. D., Steenburgh, W. J., and Bian, X. (2002). A climatological study of thermally driven wind systems of the U.S. Intermountain West. Bull. Amer. Meteor. Soc. 83, 699-708. doi: 10.1175/15200477(2002)083<0699:ACSOTD > 2.3.CO;2

Sturman, A. P., and Wanner, H. (2001). A comparative review of the weather and climate of the Southern Alps of New Zealand and the European Alps. Mt. Res. Dev. 21, 359-369. doi: 10.1659/0276-4741(2001)021[0359:ACROTW]2.0.CO;2

Weissmann, M., Braun, F. J., Gantner, L., Mayr, G. J., Rahm, S., and Reitebuch, O. (2005). The alpine mountain-plain circulation: airborne doppler lidar measurements and numerical simulations. Monthly Weather Rev. 133, 3095-3109. doi: 10.1175/MWR3012.1

Zaitchik, B. F., Evans, J. P., and Smith, R. B. (2007). Regional impact of an elevated heat source: the Zagros Plateau of Iran. J. Climate 20, 4133-4146. doi: 10.1175/ JCLI4248.1

Zängl, G. (2005). Wintertime cold-air pools in the Bavarian Danube valley basin: data analysis and idealized numerical simulations. J. Appl. Meteorol. 44, 1950-1971. doi: 10.1175/JAM2321.1

Zardi, D., and Whiteman, C. D. (2013). "Diurnal mountain wind systems," in Mountain Weather Research and Forecasting - Recent Progress and Current Challenges, eds F. K. Chow, S. F. J. de Wekker, and B. J. B. Snyder (New York, NY: Springer Publishing), 35-119.

Conflict of Interest Statement: The authors declare that the research was conducted in the absence of any commercial or financial relationships that could be construed as a potential conflict of interest.

Copyright (๑) 2016 Graf, Kossmann, Trusilova and Mühlbacher. This is an openaccess article distributed under the terms of the Creative Commons Attribution License (CC BY). The use, distribution or reproduction in other forums is permitted, provided the original author(s) or licensor are credited and that the original publication in this journal is cited, in accordance with accepted academic practice. No use, distribution or reproduction is permitted which does not comply with these terms. 


\section{OPEN ACCESS}

Edited by:

Haraldur Ólafsson,

University of Bergen, Norway/University of Iceland and Icelandic Meteorological Office, Iceland

Reviewed by: Valéry Masson, Météo-France, France Joan Cuxart,

University of the Balearic Islands,

Spain

*Correspondence: Yann Largeron yann.largeron@meteo.fr

Specialty section: This article was submitted to Atmospheric Science, a section of the journal Frontiers in Earth Science

Received: 23 February 2016 Accepted: 06 June 2016 Published: 21 July 2016

Citation: Largeron Y and Staquet C (2016) The Atmospheric Boundary Layer during Wintertime Persistent Inversions in the Grenoble Valleys.

Front. Earth Sci. 4:70 doi: 10.3389/feart.2016.00070

\section{The Atmospheric Boundary Layer during Wintertime Persistent Inversions in the Grenoble Valleys}

\author{
Yann Largeron ${ }^{1 *}$ and Chantal Staquet ${ }^{2}$ \\ ${ }^{1}$ Centre National de Recherches Météorologiques, UMR 3589, Météo-France/CNRS, Toulouse, France, ${ }^{2}$ Université \\ Grenoble Alpes, CNRS, LEGl, F-38000, Grenoble, France
}

This study addresses the atmospheric boundary layer dynamics in the Grenoble valleys during persistent inversions, for 5 months during the 2006-2007 winter. During a persistent inversion, the boundary layer contains a layer with a positive vertical temperature gradient over a few days. Temperature data recorded on the valley sidewalls are first used. A bulk measure of the boundary layer stability, based upon the temperature difference between the valley top and the valley bottom, is introduced and a criterion is proposed to detect persistent inversions. We show that this criterion is equivalently expressed in terms of the heat deficit inside the boundary layer. Nine episodes are detected and coincide with the $\mathrm{PM}_{10}$-polluted periods of the 2006-2007 winter. Secondly, the five strongest and longest persistent inversions are simulated using the MesoNH model. Focus is made on the stagnation stage of the episode, during which the inversion exhibits a diurnal cycle that does not significantly evolve from day to day. Whatever the episode, the inversion develops from the ground over a height of about $1200 \mathrm{~m}$, with a nighttime temperature strength reaching $20 \mathrm{~K}$. The boundary-layer dynamics within the inversion layer are fully decoupled from the (anticyclonic, weak) synoptic flow, independent from the synoptic-wind direction and similar whatever the episode. This implies that these dynamics are controlled by thermal winds and solely depends upon the geometry of the topography and upon the radiative cooling of the ground. Finally, a 2-day high-resolution simulation is made for the strongest case, representative of any persistent inversion. The flow pattern displays a well-defined spatial structure, with a vertical layering resulting from the superposition of the down-valley winds flowing from the different valleys surrounding Grenoble. This pattern persists all day long over a shallow convective layer of about $50 \mathrm{~m}$ forming above the ground during the reduced daytime period. Within this shallow layer, convection triggers a weak up-valley wind. Ventilation and stagnation areas in the surface layer are also computed, providing insights for air quality studies. The main characteristics of these persistent inversions are comparable to the most extreme wintertime inversions recorded in the Grand Canyon, Arizona.

Keywords: atmospheric boundary layer, stable wintertime conditions, Grenoble, Alpine valleys, down-valley wind, persistent inversion 


\section{INTRODUCTION}

A temperature inversion consists in a layer of positive temperature gradient. Urbanized sites are very sensitive to temperature inversions forming in the atmospheric boundarylayer (ABL), as poor air quality is then induced (Figure 1A). Pollutants emitted by traffic, industry and biomass burning from wood combustion are indeed trapped into the inversion layer and induce well-documented health impact (e.g., Pope et al., 2002). Many world urban centers are located in complex terrain, such as valleys and basins (Fernando, 2010). In valleys, inversions are also called cold-air pools and form as a result of both cooling of the ground due to long-wave radiation and nocturnal down-slope winds (Whiteman, 2000). They usually extend from the bottom of the valley up to the boundary-layer top. In winter, inversions can last for a few days to several weeks, being triggered and maintained by anticyclonic conditions at synoptic scale (Reeves and Stensrud, 2009). We then talk about persistent inversions, as opposed to nocturnal inversions that form even in summer because of the boundary-layer diurnal cycle but are destroyed each day by thermal convection in the surface layer (f.i., Whiteman, 1982; Neff and King, 1989; Whiteman et al., 2008). During persistent inversions, a warm air mass surmounts the region at mid-altitude which results in a quasi-decoupling of the ABL within the valley or basin and the free troposphere, and the buildup of the inversion layer (Largeron and Staquet, 2016, and the present study). This accounts for the sustained increase of pollutants in urbanized complex terrain all along an inversion period (Silcox et al., 2012).

The ABL in complex terrain during persistent inversions has therefore very specific features compared to the flat $A B L$, since slope and valley winds control the flow dynamics in the former case. As a result, data stemming from field campaigns or numerical experiments over flat terrain (see Mahrt, 2014; Sun et al., 2015, for a review) cannot be used to get insight into the ABL dynamics in complex terrain.

Several field campaigns dedicated to the study of persistent inversions in complex terrain have been conducted, with gradual focusing on smaller scales and growing importance of numerical studies for planning and conducting the campaigns and analysing the data. Campaigns were held in the Colorado basin (Wolyn and McKee, 1989; Whiteman et al., 1999a) or Columbia basin (Whiteman et al., 2001; Zhong et al., 2001) and first aimed at studying the formation, stagnation and destruction of the inversions (or cold air pools). Recent programs also include air quality concerns, such as PCAPS (Persistent Cold-Air Pool Study) in the Salt Lake valley (Silcox et al., 2012; Lareau et al., 2013; Wei et al., 2013). In Europe, the COLPEX program (COLd air Pooling and fog formation over complEX terrain), led by the Met Office in the UK, involved field and highresolution numerical studies in a region of small hills in the UK (Price et al., 2011; Vosper et al., 2014). In the Alps, the first wintertime field campaign aiming at studying persistent inversions was held in the Chamonix-Mont-Blanc valley in January-February 2015, motivated by the anomalously high pollution level frequently recorded there; both meteorological and chemical data were collected during the campaign (Chemel et al., 2016).

In valleys, during persistent inversions, several mechanisms can significantly impact the concentration and the transport of pollutants, among which the slope and valley winds (Gohm et al., 2009). Down- and up-slope winds can drive the spatial distribution of pollutants which can be strongly heterogeneous inside the valley ABL (Schnitzhofer et al., 2007; Harnisch et al., 2009). Interactions between the cold-air pool and the valley winds are frequent (Banta et al., 2004; Cuxart et al., 2007) and the local specificities of a given site need to be investigated to know whether these valley-scale processes can explain the observed evolution of pollutant concentrations.

In France, the city of Grenoble is very critical in terms of pollution due to both its deep and landlocked orography and to the high-occupancy of the territory (Figure 1A): the city counts over half a million inhabitants and many industries. Moreover, the region of Grenoble is a strategic traffic crossroads, as a transit point and a necessary access to many ski resorts. Pollutant emissions, such as $\mathrm{PM}_{10}$ (particulate matter of diameter less than $10 \mu \mathrm{m}$ ) are then important and often concentrated on specific periods which coincides with periods when the boundary layer is the most stable. These emissions thus tend to result in acute episodes of local pollution, which makes Grenoble stand among the most polluted cities in France (Air Rhône-Alpes, 2011). Despite the strong impact of wintertime ABL dynamics on local air quality, field campaigns held in the Grenoble valleys up to now have focused on the summertime ABL to account for ozone pollution (Couach et al., 2003) and on the chemistry of aerosols from urban ground stations (Favez et al., 2010). No wintertime field campaign investigating the ABL dynamics has been performed yet. The present study, mostly numerical, is the first attempt to identify the circulation and thermal structure in the Grenoble valleys during wintertime persistent inversions, thus providing insights into the surface layer ventilation during such events. More details can be found in Largeron (2010). As we shall show, some conclusions of the present study apply to valleys with similar deep and complex orography, implying that they could be generalizable to many other world valleys. In the following, focus is made on the persistent stage of the inversion, between formation and destruction. Time series of 2-m air temperature data recorded at different altitudes around the city, and simulation results from high-resolution mesoscale simulations are used. In this study, we used the MesoNH numerical model (Lafore et al., 1998) that has already been successfully used to model stable ABL (e.g., Cuxart and Jiménez, 2007; Martínez et al., 2010) and valley or basin winds (e.g., Flamant et al., 2002; Cuxart et al., 2007; Drobinski et al., 2007).

In Section 2, we use the temperature data to identify the persistent inversion of the 2006-2007 winter in the Grenoble area. Nine such inversions are identified between November and February, which all coincide with very poor air quality. Numerical simulations of the five longest and strongest persistent inversions are then carried out, to investigate the general flow organization inside the valley and its possible coupling with the free troposphere (Section 3). A detailed study of one such 
A

\section{Grenoble valleys overview}

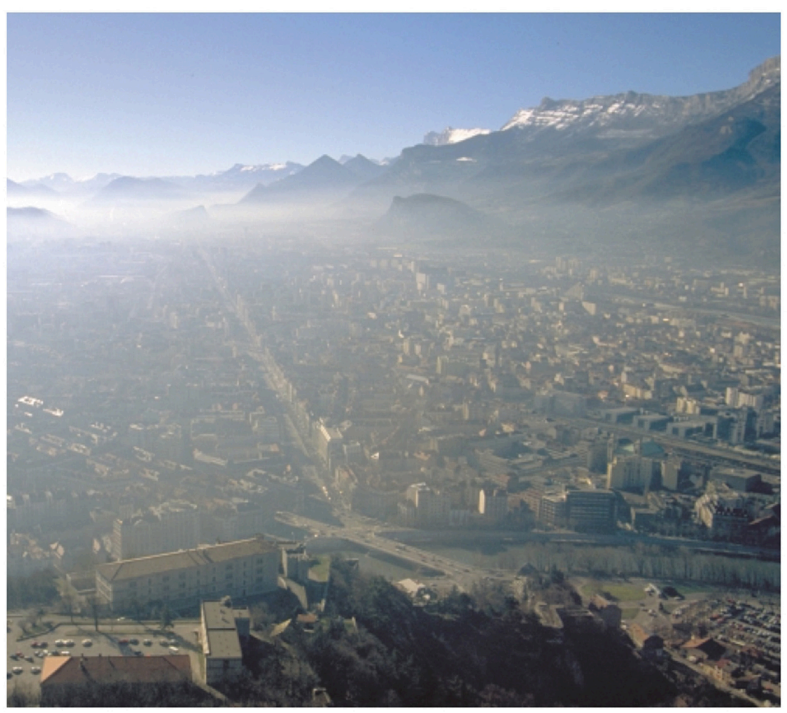

B

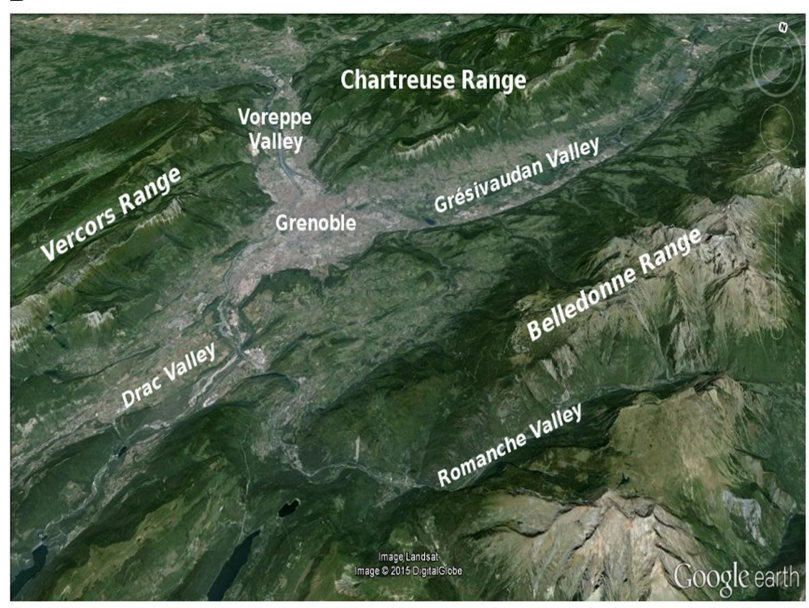

C

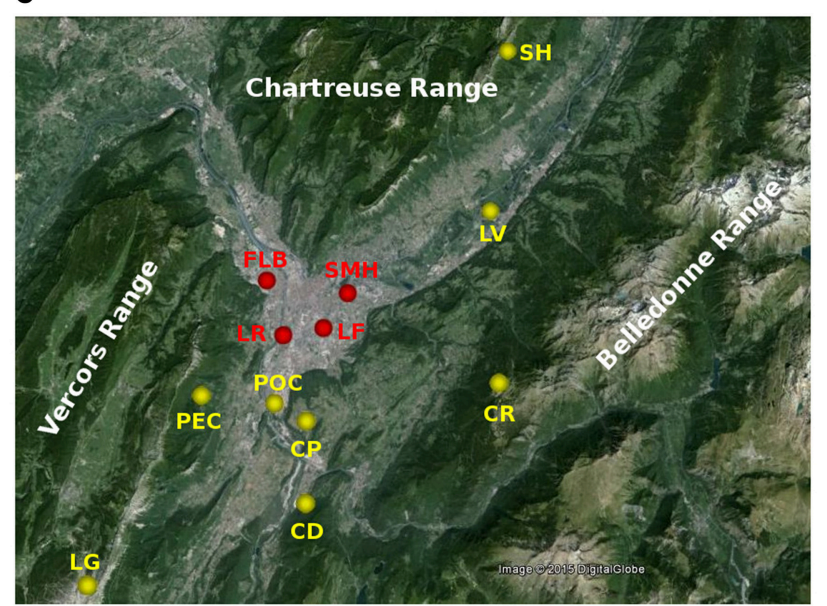

FIGURE 1 | (A) Particulate Matter pollution during wintertime inversions over Grenoble (Photography: courtesy of Eric Chaxel). (B) View of the mountain ranges and Grenoble valleys discussed in the paper. (C) Locations of the ground-based Automatic Weather Stations (yellow) and air-quality stations (red, not used in the present study); the acronyms are defined in Table 1. Extracted and adapted from Largeron and Staquet (2016) with background maps taken from Google Earth.

inversion is next conducted, using a higher vertical resolution. Results are reported in Section 4 for the wind pattern and in Section 5 for the thermal structure of the persistent inversion. Conclusions are drawn in Section 6.

\section{DETECTION OF PERSISTENT INVERSIONS}

\subsection{The Grenoble Valleys and the Ground-Based Observations \\ 2.1.1. The Grenoble Valleys}

The city of Grenoble is located in the French Alps and lies at the junction of three valleys, the Grésivaudan valley on the northeast, the Voreppe valley in the north-west and the Drac valley in the south (see Figure 1B). These valleys have a width comprised between 3 and $5 \mathrm{~km}$ and are of several tens of kilometers long. They separate three mountain ranges, the Vercors range on the west of the city, the Chartreuse range on the north and the Belledonne range on the east part. The highest altitude of the two former ranges is $2000 \mathrm{~m}$ ASL (above sea level) while the Belledonne range peaks at $3000 \mathrm{~m}$ ASL. The site also involves a fourth valley, the Romanche valley located south-east of Grenoble, which is narrower, deeper and higher in altitude than the other three valleys. The area comprising these four valleys and the city of Grenoble will be referred to as the Grenoble valleys hereafter. The flat part of the valley around and including Grenoble, will be referred to as the Grenoble basin; the largest width of this basin is about $6.5 \mathrm{~km}$, and its altitude is $210 \mathrm{~m}$ ASL. The city of Grenoble has severe wintertime $\mathrm{PM}_{10}$ pollution issues. 
TABLE 1 | Description of the ground-based Automatic Weather Stations in the Grenoble valleys.

\begin{tabular}{llll}
\hline Station & Acronym & Elevation & Elevation category \\
\hline Le Versoud & LV & $220 \mathrm{~m}$ & Low \\
Champ sur Drac & CD & $230 \mathrm{~m}$ & Low \\
Pont de Claix & POC & $237 \mathrm{~m}$ & Low \\
Champagnier & CP & $363 \mathrm{~m}$ & Low \\
Peuil de Claix & PEC & $935 \mathrm{~m}$ & Medium \\
Le Gua & LG & $1600 \mathrm{~m}$ & High \\
Saint Hilaire & SH & $1700 \mathrm{~m}$ & High \\
Chamrousse & CR & $1730 \mathrm{~m}$ & High \\
\hline
\end{tabular}

The low-elevation stations are located at the valley bottom.

The number of days where the daily-average $\mathrm{PM}_{10}$ concentration exceeds the warning threshold of $50 \mu \mathrm{g} \mathrm{m}^{-3}$ has been of 50 in average per year over the 2007-2010 period, therefore exceeding the threshold of 35 per year set by the French legislation (Air Rhône-Alpes, 2011).

\subsubsection{The Ground-Based Observations}

The Grenoble valleys are equipped with ground-based Automatic Weather Stations measuring 2-m air temperature, and 10-m wind speed and direction every hour. These stations, which are either routinely operated by Meteo-France, the French weather forecast service, or by Air Rhône-Alpes, the air quality agency of Région Rhône-Alpes, are located at different places and altitudes in the Grenoble valleys. Time series of the temperature at eight stations have been used for the present study, whose name and elevations are displayed in Table 1, and locations indicated in Figure 1C. As can be seen from Table 1, the stations can be broadly categorized as low elevation, medium elevation and high elevation stations.

\subsection{Methodology}

We developed a simple method to detect persistent inversions of the 2006-2007 winter using the temperature records from the eight ground-based meteorological stations listed in Table 1. The principle of this method, detailed in Largeron and Staquet (2016), is to compute a bulk measure of the stability of the ABL from which persistent inversions are detected when this bulk measure exceeds a given threshold.

Figure 2A displays the 2-m air temperature recorded at the stations LV and CR during the month of December. Two points should be noticed: (i) the temperature difference between these two stations exhibits a high variability over the month; (ii) two main persistent inversion episodes occur, around mid-december and at the end of the month, during which the temperature at the valley bottom can be lower by $10 \mathrm{~K}$ than that at the high-elevation station.

Inside the valley $\mathrm{ABL}$, temperature records show that the temperature field is approximately horizontally homogeneous. Temperature time series at stations of similar elevations are indeed strongly correlated $\left(r^{2}=0.97\right)$, as can be seen for the two low-elevation stations of LV and POC (Figure 2B), or similarly for the two high-elevations stations of CR and LG (Figure 2C), for the whole month of December. On the opposite, high-elevation stations display a very distinct behavior than low-elevation stations (see Figure 2A) with a low correlation $\left(r^{2}=0.36\right)$. It can be shown that the temperature difference between two stations belonging to the same category is two orders of magnitude smaller than the temperature difference between a high- and a low-elevation station, on average over the five considered months (November 2006 to March 2007). It follows that the temperature difference between the top and the bottom of the ABL is well estimated by the difference in temperature between any high-elevation station and any lowelevation station, and that the latter may be indicative of the $\mathrm{ABL}$ static stability. This is consistent with the results of Whiteman et al. (2004) who have shown that temperature vertical profiles can be well-approximated by pseudovertical profiles obtained from temperature stations on the sidewalls, in strongly stable and low synoptic wind conditions.

In valleys, inversion layers often fill the entire ABL up to the valley top (Whiteman, 1982). In the present case, the valley top is about $1500 \mathrm{~m}$, which implies that the high-elevation stations are located just above the inversion. As shown below, this statement is consistent with results from numerical simulations of the Grenoble valleys.

A persistent inversion can therefore be detected by considering the temperature difference between a highand a low-elevation station, divided by their elevation difference, $(\Delta T / \Delta z)_{i}$, where the index $i$ refers to any couple of stations. We compute the average over 4 such couples of stations of the temperature difference across the $\mathrm{ABL}$, which we refer as the bulk temperature gradient hereafter and denote $\Delta T / \Delta z$.

The detection of persistent inversions is then made using the following criterion:

$\langle\Delta T / \Delta z\rangle_{24 \mathrm{~h}}>\langle\Delta T / \Delta z\rangle_{\text {winter }}$ for more than 72 consecutive

hours (1)

where $<>_{24} \mathrm{~h}$ and $<>_{\text {winter }}$ represent a 24 -h-running average and the winter average (from November to March), respectively. For the winter of 2006-2007, $<\Delta T / \Delta z>_{\text {winter }}$ is about $-3 \mathrm{~K}$.

The 24-h-running average bulk temperature gradient $\langle\Delta T / \Delta z\rangle_{24} \mathrm{~h}$ is displayed over the whole winter in Figure 3, along with the constant value of $-3 \mathrm{~K}$. Using criterion (1), nine persistent inversions are detected, which are referred to as $\mathrm{I}_{\mathrm{k}}, 1 \leq \mathrm{k} \leq 9$. The December inversions are also indicated in Figure 2A. The characteristics of these nine inversions are displayed in Table 2, namely their period of occurrence with respect to criterion (1), the corresponding duration and an estimate of their strength as measured by the bulk temperature gradient averaged over their respective duration. The longest and strongest inversion is $I_{6}$, on which we shall mainly focus in the following.

Criterion (1) can be equivalently expressed in terms of the heat deficit $H$ inside the inversion layer (Largeron and Staquet, 2016). The heat deficit is the amount of heat which should be provided to bring the temperature gradient to the adiabatic gradient, namely to fully mix the fluid column (Whiteman et al., 1999a,b). The 24-h-running average of the heat deficit is also displayed in Figure 3. As shown in this figure, the $-3 \mathrm{~K}$ threshold 


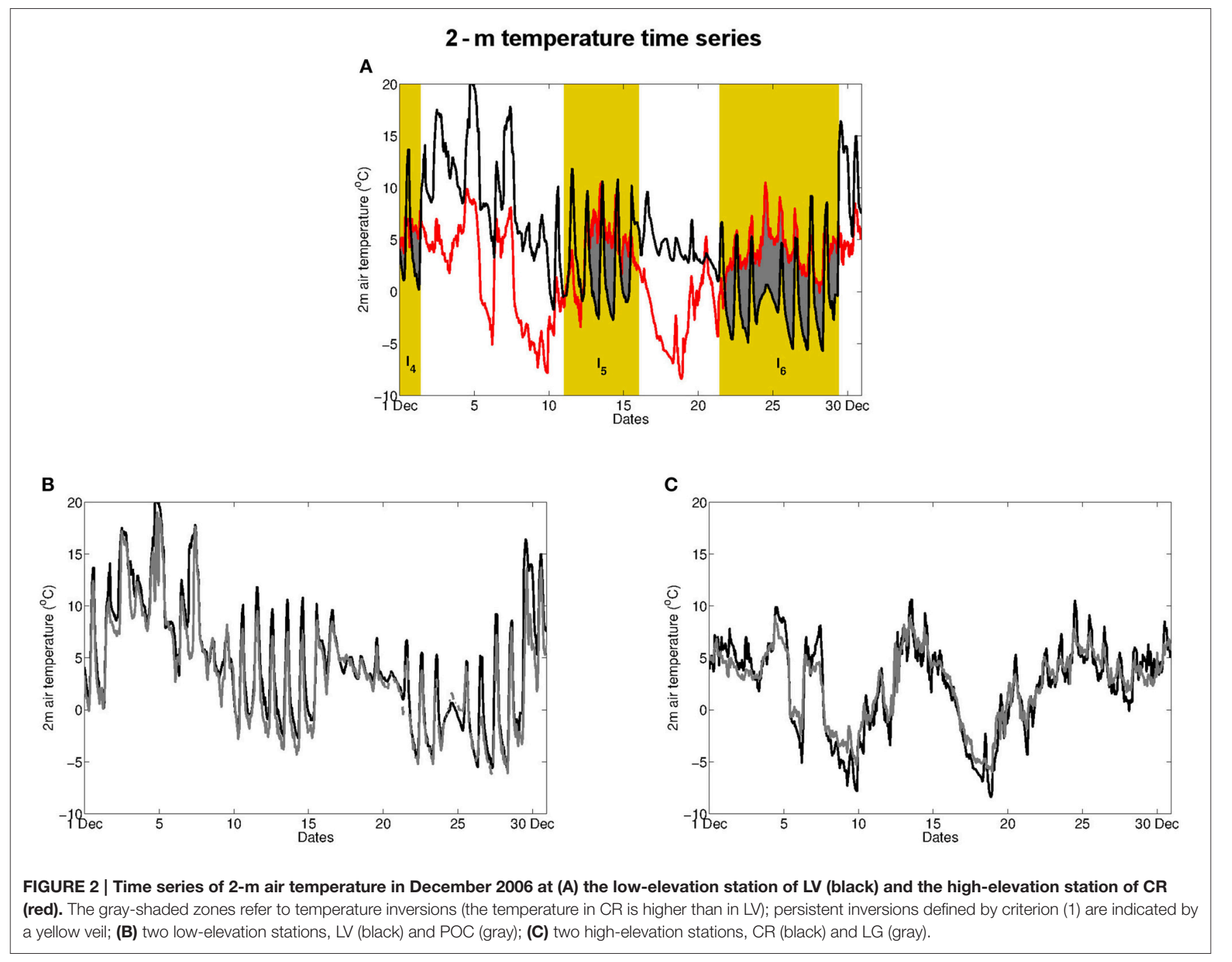

for the winter-average bulk temperature gradient is equivalent to a threshold value of $10 \mathrm{MJ} \mathrm{m}^{-2}$ for the winter-average heat deficit.

It can also be shown (Largeron and Staquet, 2016) that (i) the nine detected episodes are strongly polluted, in the sense that the 24-h-running average concentration of $\mathrm{PM}_{10}$ exceeds the air-quality legal threshold of $50 \mathrm{\mu g} \mathrm{m}^{-3}$ for at least three consecutive days during each episode; (ii) all persistent inversion periods reported in Table 2 occur during and are triggered by anticyclonic conditions; (iii) all persistent inversions are generated by a mid-level warming and destroyed by a mid-level cooling.

\section{THE NOCTURNAL WIND CIRCULATION IN THE GRENOBLE VALLEYS DURING PERSISTENT INVERSIONS}

Figure 3 and Table 2 show that, among the nine persistent inversions of the considered winter, four of them $\left(\mathrm{I}_{2}, \mathrm{I}_{3}, \mathrm{I}_{4}\right.$, and
I9) last for a short duration (though greater than 3 days) and are of lower intensity. On the opposite, the five other persistent inversions last longer and are associated to stronger inversion strengths. These five episodes all involve a stagnation stage (see Largeron and Staquet, 2016), between the formation and the destruction of the inversion, during which the inversion displays similar features from one day to the next. The stagnation stage will also be referred to as the heart of the episode hereafter. In the following, focus is made on these five episodes for which numerical simulations are made, in order to investigate the main characteristics of the wind circulation in the Grenoble valleys during persistent inversions. We briefly describe the numerical model below, stressing the aspects relevant to the present study, before analyzing the wind circulation in the valleys. More details are provided in Largeron (2010).

\subsection{Description of the Numerical Simulations}

We performed high-resolution mesoscale simulations (according to the terminology defined by Cuxart, 2015) of the five episodes 


\section{Heat deficit and bulk temperature gradient}

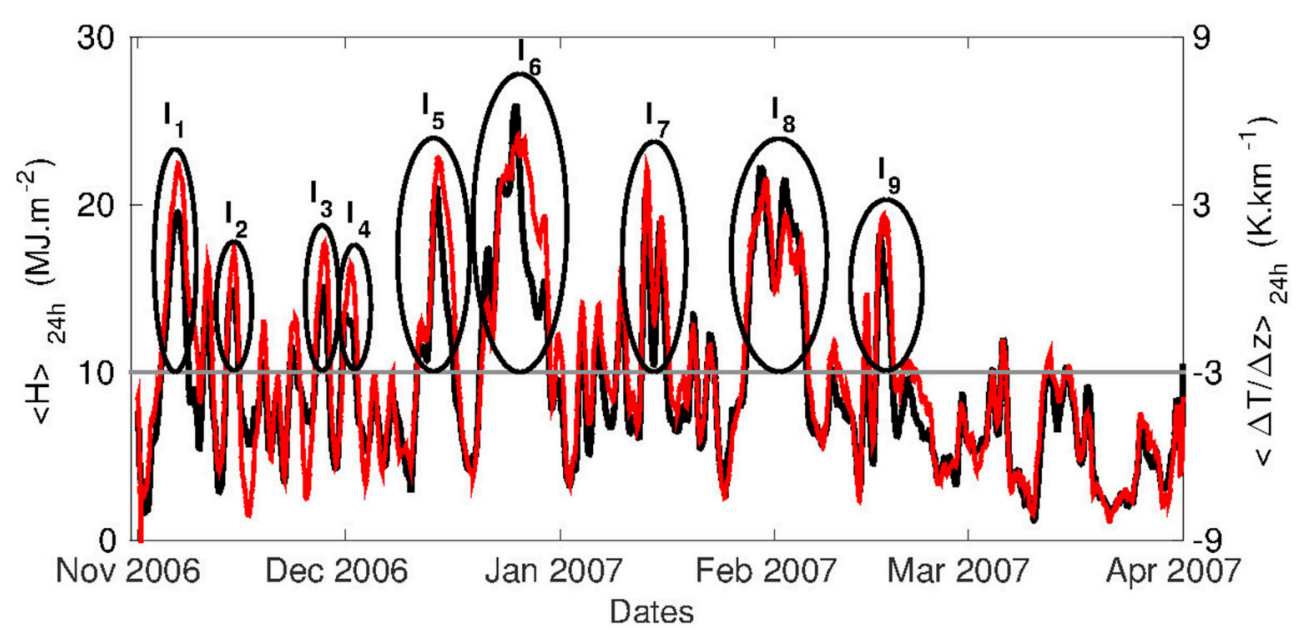

FIGURE 3 | Temporal evolution of the 24-h-running average of the bulk temperature gradient $\Delta T / \Delta z$ (red) and heat deficit $\boldsymbol{H}$ (black) during the 2006-2007 winter. The 9 inversions $I_{k}, 1 \leq k \leq 9$, detected by criterion (1) are circled in black. The persistence threshold $\langle\Delta T / \Delta z\rangle_{\text {winter }}$ is indicated with a gray line. Extracted and adapted from Largeron and Staquet (2016).

$I_{1}, I_{5}, I_{6}, I_{7}$, and $I_{8}$ using the Meso-NH numerical model developed by Meteo-France and the Laboratory of Aerology (Lafore et al., 1998).

The numerical model Meso-NH solves the Navier-Stokes equations in the anelastic approximation, thus filtering sound waves while allowing the density to vary in space and time. The model uses a terrain-following coordinate system along the vertical direction. The atmospheric model is coupled with the externalized land and ocean surface model SURFEX (Masson et al., 2013), to account for the surface fluxes and the evolution of four types of surfaces: nature, town, inland water and ocean with a large variety of ground covers. It can include a specific parameterization scheme for the urban energy budget (Masson, 2000). A full three-dimensional turbulence scheme that uses a prognostic equation for the turbulent kinetic energy is activated (Cuxart et al., 2000). The model may also include microphysical schemes for mixed-phase clouds and warm clouds and other specific parameterization for chemical species and aerosols, which we did not use in the present study.

As is now standard, a nesting of domains is used, allowing to capture the dynamical features of a wide range of scales and to focus on small scale processes in the innermost domain, which has the highest resolution (e.g., Zhou and Chow, 2013). The vertical resolution in each domain is the same, the size of the horizontal grid cells being decreased -in the present case by a factor of 3-from one outer domain to the inner one. For the present study, four nested domains are used, which are all centered on the Grenoble valleys. The largest domain $\mathrm{D}_{1}$ has a horizontal resolution of $9 \mathrm{~km}$ and an extension of $675 \times 675 \mathrm{~km}$; and is forced at the boundary with 6-hourly fields from ECMWF operational analyses. The innermost domain is of size $30 \times$ $30 \mathrm{~km}$, with a horizontal grid size of $0.333 \mathrm{~km}$. The databases Gtopo30 for the orography, with a resolution of approximately
TABLE 2 | Persistent inversions detected using criterion (1).

\begin{tabular}{|c|c|c|c|}
\hline $\begin{array}{l}\text { Episode } \\
\text { number }\end{array}$ & $\begin{array}{l}\text { Persistent inversion } \\
\text { period }\end{array}$ & $\begin{array}{c}\text { Number of } \\
\text { successive days }\end{array}$ & $\begin{array}{c}\langle\Delta T / \Delta Z\rangle_{\text {episode }} \\
\left(\mathrm{K} \mathrm{km}^{-1}\right)\end{array}$ \\
\hline$I_{1}$ & 04 Nov 06 - 09 Nov 06 & 6.5 & +0.79 \\
\hline $\mathrm{I}_{2}$ & 13 Nov 06 - 15 Nov 06 & 3 & -1.16 \\
\hline$l_{3}$ & 26 Nov 06 - 28 Nov 06 & 3.3 & +0.36 \\
\hline $\mathrm{I}_{4}$ & 30 Nov 06 - 02 Dec 06 & 3.2 & -1.16 \\
\hline$I_{5}$ & 11 Dec 06 - 17 Dec 06 & 6.9 & +1.29 \\
\hline$I_{6}$ & 20 Dec 06 - 30 Dec 06 & 11 & +1.86 \\
\hline$I_{7}$ & 12 Jan 07 - 16 Jan 07 & 5.2 & +0.29 \\
\hline$l_{8}$ & 27 Jan 07 - 05 Feb 07 & 10.5 & +0.82 \\
\hline lg & 15 Feb 07 - 18 Feb 07 & 3.7 & -1.41 \\
\hline
\end{tabular}

The duration of each episode is indicated in columns 2 and 3 and a measure of the intensity of the episode, $\langle\Delta T / \Delta Z\rangle_{\text {episode, }}$, is given in column 4 .

$1 \mathrm{~km}$, and Ecoclimap 2 (Masson et al., 2003) for the ground cover are used, and are interpolated in the innermost domain to $1 / 3 \mathrm{~km}$. Two configurations are considered, denoted $C_{1}$ and $C_{2}$ in Table 3 below, which mainly differ in the vertical resolution and duration of the computation. In configuration $C_{1}, 60$ levels are used in all domains, the vertical resolution at the ground being $15 \mathrm{~m}$. In configuration $\mathrm{C}_{2}$, a finer vertical resolution is used, of $4 \mathrm{~m}$ close to the ground, with 90 levels in total. The grid size is stretched along the vertical, the resolution being equal to $600 \mathrm{~m}$ at the top of the domain in each configuration. The duration of the computation is $10 \mathrm{~h}$ in $\mathrm{C}_{1}$ and $53 \mathrm{~h}$ in $\mathrm{C}_{2}$. The main features of the simulations are summarized in Table 3 . The influence of these numerical choices (vertical resolutions and duration of the simulations) is investigated in the following Section 3.2 and in the Supplementary Material. 
TABLE 3 | Main parameters of the numerical simulations.

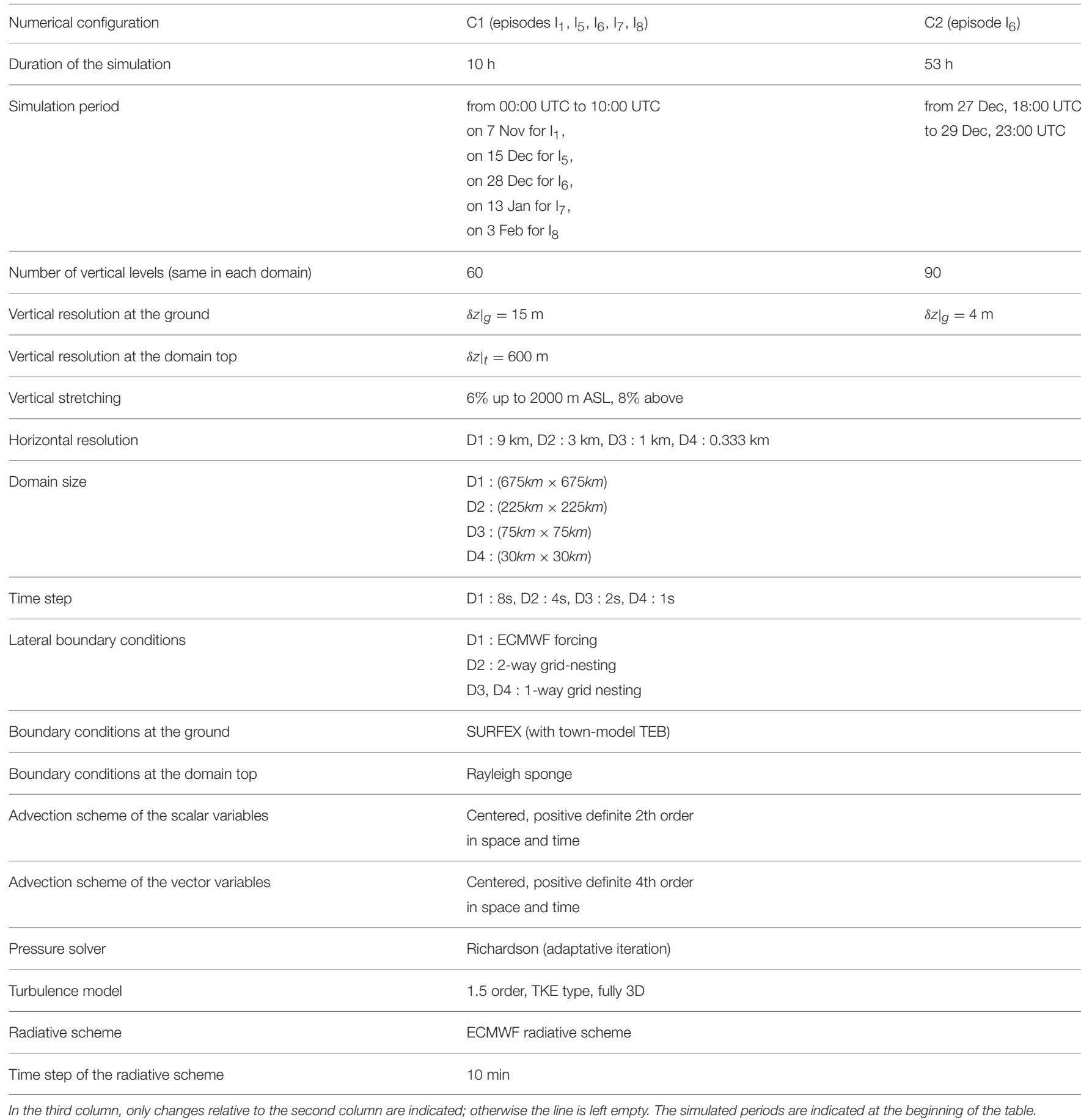

\subsection{Sensitivity Analysis of the Numerical Simulations}

A first sensivity analysis has been made to investigate the numerical spin-up in the case of episode $\mathrm{I}_{6}$ and configuration $\mathrm{C}_{1}$. Starting the run at different times $(0 \mathrm{~h} \mathrm{UTC}$ on December 27,18 $\mathrm{h}$ UTC on December 27 and $0 \mathrm{~h}$ UTC on December 28), results were compared at the end of the night (06:00 UTC) on December 28. This test, which is not presented here, leads to the conclusion that the numerical spin-up is about $4 \mathrm{~h}$, namely, if comparison is made at the same time at least $4 \mathrm{~h}$ after the beginning of all the simulations, then the temperature and wind fields inside the valley are similar (see Largeron, 2010, for details). This result shows that the nocturnal quasi-steady circulation at valley-scale at the end of the night and in early morning (on which we focus in the following Section 3.3) can be analyzed by means of simulations starting at $0 \mathrm{~h}$ UTC in the $\mathrm{C} 1$ configuration. This 
choice appears facilitated by the presence of a persistent inversion which implies that an elevated inversion is already present and seen by the outer domain at the beginning of the simulations at $0 \mathrm{~h}$ UTC.

A second sensitivity analysis has been performed to investigate the effect of the vertical resolution, in the case of episode $\mathrm{I}_{6}$, during the night from $18 \mathrm{~h}$ UTC on December 27 to $8 \mathrm{~h}$ UTC on December 28. This analysis is reported in the Supplementary Material. The main conclusion is that the vertical resolution close to the ground should be at least $\left.\delta z\right|_{g}=15 \mathrm{~m}$ for the general features of the $\mathrm{ABL}$ dynamics and temperature profiles to have converged. Hence, a vertical resolution of $\left.\delta z\right|_{g}=15$ $\mathrm{m}$ can therefore be considered as sufficient to model these features.

However, comparisons with ground observations of 2-m air temperature data show that in a very shallow layer close to the ground, the temperature is only well reproduced with a fine resolution of $\left.\delta z\right|_{g}=4 \mathrm{~m}$ in high-elevation stations, and still has a hot bias at the low-elevation stations.

For $\left.\delta z\right|_{g}>4 \mathrm{~m}$, simulations present a systematic hot bias at the bottom of the valley, and a systematic cold bias in altitude, which otherwise formulated, corresponds to a too weak temperature inversion compared to the observations. In this case $\left(\left.\delta z\right|_{g}>4 \mathrm{~m}\right)$, surface fluxes suffer from a misrepresentation that in turn leads to a too weak cooling of the near-surface air in altitude and to an underestimated drainage of cold-air by katabatic winds toward the bottom of the valley.

In the following, consistently with these findings, the analysis made with the $\mathrm{C}_{1}$ configuration $\left(\left.\delta z\right|_{g}=15 \mathrm{~m}\right)$ then only deals with the general ABL dynamics for which a finest resolution leads to the same results. Fine-scale details of the near-surface ventilation and temperature gradient, reported in Sections 4 and 5 , rely on the $\mathrm{C}_{2}$ configuration $\left(\left.\delta z\right|_{g}=4 \mathrm{~m}\right)$ instead.

\subsection{Overall Nocturnal Circulation of the Five Episodes}

Focus is made on one night of the five episodes $\mathrm{I}_{1}, \mathrm{I}_{5}, \mathrm{I}_{6}, \mathrm{I}_{7}$, and $\mathrm{I}_{8}$, each simulation starting at 00:00 UTC and lasting for $10 \mathrm{~h}$ (see configuration $\mathrm{C} 1$ in Table 3 ). These nights are indicated in Table 3.

\subsubsection{Synoptic Regime}

As noted at the end of Section 2, each episode occurs under anticyclonic conditions, with low synoptic wind speed, of about 4 to $5 \mathrm{~m} \mathrm{~s}^{-1}$. As for the wind direction, taken at the $600 \mathrm{hPa}$ geopotential height (which is about $4200 \mathrm{~m} \mathrm{ASL}$ in the present case of strong anticyclone), it hardly varies over the $10 \mathrm{~h}$ of the simulation but varies from one episode to the other: it flows either from the south-east $\left(\mathrm{I}_{1}, \mathrm{I}_{5}\right)$, north-west $\left(\mathrm{I}_{6}\right)$, west $\left(\mathrm{I}_{7}\right)$ or from the east $\left(\mathrm{I}_{8}\right)$. The synoptic regime during each simulated period is very stable, the altitude of the 600 -hPa geopotential height over the area varying by less than $15 \mathrm{~m}$ for $\mathrm{I}_{1}, \mathrm{I}_{6}, \mathrm{I}_{7}, \mathrm{I}_{8}$ and by $35 \mathrm{~m}$ for $\mathrm{I}_{5}$.

These features of the synoptic wind computed by the model are consistent with those recorded from radio-soundings at the Lyon Saint Exupery airport, located about $80 \mathrm{~km}$ from the Grenoble site, which is a small distance compared to the synoptic scale. Wind recorded over $3000 \mathrm{~m}$ ASL there, the altitude of the highest summits of the Grenoble area, is representative of the synoptic wind over this site.

\subsubsection{Valley Wind Circulation at the End of the Night}

The key feature of the valley-scale circulation in the Grenoble valleys is that it consists in the superposition of several valley winds flowing from the different mountain ranges and valleys of the site. This circulation slightly varies from one episode to the other but common dominant features can be extracted, which are illustrated in Figure 4 by maps of the wind vector field at different altitudes between 350 and $3000 \mathrm{~m}$ ASL, for episode $\mathrm{I}_{6}$. A sketch of this circulation is displayed in Figure 5 for each of the five episodes.

During these five episodes, the circulation in the Grenoble valleys consists in four main winds which flow almost horizontally at different altitudes, resulting in a vertical layering in the ABL:

- The Grésivaudan wind flows along the bottom of the Grésivaudan valley from the north-east (the Grésivaudan valley has a very gentle slope descending toward Grenoble). This is a cold wind, which extends vertically over a layer of thickness 150-250 m, namely between the ground and about $500 \mathrm{~m}$ ASL (as shown in Section 4). It splits into two mass fluxes over the city, the major part flowing into the Voreppe valley toward the north-west, while a smaller part flows southward (Figure 4A and white colour current in Figure 5).

- The second wind comes from the Trieves plateau located south of Grenoble (Figure 4B and red colour current in Figure 5). We refer to this wind as the Trieves wind. It flows between about $500 \mathrm{~m}$ ASL, therefore above the Grésivaudan wind, to an altitude of 1000 to $1200 \mathrm{~m}$ ASL depending upon the episode. This wind is then channelled into the Voreppe valley whatever the episode. A small part flows into the Grésivaudan valley for episodes $I_{1}$ and $I_{6}$, in the direction opposite to that wind (and above it), while it recirculates as a large scale vortex over the city in $\mathrm{I}_{5}$ (Figure 4B). A smaller vortex structure also forms along the flank of the Belledonne range, between 500 and $700 \mathrm{~m}$ ASL (as is visible in Figure 4B).

- The third wind flows from the Romanche valley and reaches the Grenoble basin from the south-east (Figure 4C and blue color current in Figure 5). This cold wind, referred to as the Oisans wind, meets the Trieves wind over the city between 600 and $1000 \mathrm{~m}$ ASL. A part of the mass flux is then channeled into the Voreppe valley and flows along with the Trieves wind $\left(\mathrm{I}_{1}, \mathrm{I}_{5}, \mathrm{I}_{6}\right)$. Another part recirculates as a large-scale vortex in the Grenoble basin and in the Grésivaudan valley $\left(\mathrm{I}_{1}, \mathrm{I}_{6}\right)$. The thickness of the Oisans wind varies from one episode to the other, ranging from 500 and $1800 \mathrm{~m} \mathrm{ASL}$, which are roughly the lowest and highest altitude of the Romanche valley, respectively.

- Between 750 and 850 m ASL, a fourth wind, the Belledonne wind is sometimes observed $\left(\mathrm{I}_{5}\right.$ and $\left.\mathrm{I}_{6}\right)$, which flows from the Belledonne range into the Grésivaudan valley while oscillating. Several large scale vortex structures are then visible in the latter valley. 

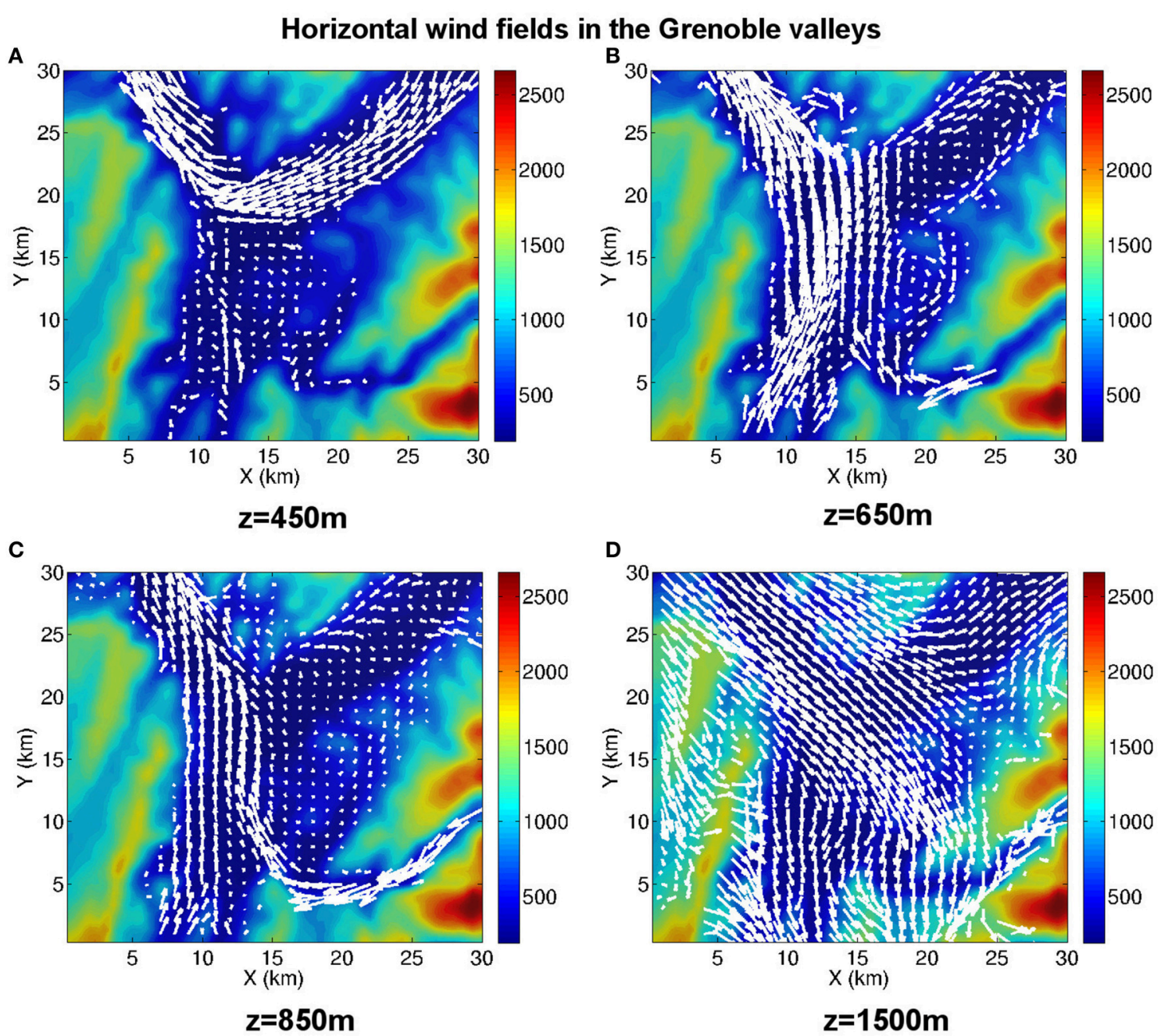

FIGURE 4 | Maps of the wind at a given altitude during episode $\mathrm{I}_{6}$ at 06:00 UTC on December 28 . (A) $z=450 \mathrm{~m}$ ASL; (B) $z=650 \mathrm{~m} \mathrm{ASL;} \mathrm{(C)} z=850 \mathrm{~m}$ $\mathrm{ASL} ;$ (D) $z=1500 \mathrm{~m}$ ASL.

From $1500 \mathrm{~m} \mathrm{ASL}$, which is the inversion altitude, up to 2500-3000 m ASL, the circulation appears to result from the channelling of the synoptic wind into the valleys. For episode $\mathrm{I}_{6}$ (Figure 4D), this wind comes from the north-west in this range of altitudes. It is noticeable that this direction is opposed to, or at least very different from, that of the four winds just described, which flow below $1500 \mathrm{~m}$ ASL.

It appears from the five simulations that these local dynamics set in during a persistent inversion below $1500 \mathrm{~m} \mathrm{ASL}$, and are almost independent of the episode. The circulation inside the Grenoble valley can be decomposed into four winds, which are all associated with along-valley winds flowing almost horizontally at different altitudes, resulting in a vertical layering of the ABL. Large-scale vortices also form over the entrance of the Grésivaudan valley and along the flank of the Belledonne range.

\subsubsection{Influence of the Synoptic Wind}

Following Whiteman and Doran (1993), the direction of the wind in the Grésivaudan and Voreppe valleys is plotted vs. the direction of the synoptic wind for all five episodes in Figure 6. More precisely, for the Grésivaudan wind, an average of the wind direction from the ground to an altitude of $400 \mathrm{~m}$ ASL on all grid points of this valley is computed every hour of the simulated episode (see Table 3). The same average is applied to compute the Voreppe wind direction, the points considered being now in the Voreppe valley, from the ground to an altitude of $600 \mathrm{~m}$ ASL. These grid points are indicated in Figure 7A.

Figure 6 clearly shows that the wind keeps the same direction in either valley whatever the episode and, therefore, whatever the synoptic wind direction. For the Voreppe valley and episode $\mathrm{I}_{6}$ for instance, the valley-wind flows toward the north-west, namely in the opposite direction to the synoptic wind. The same decoupling with the synoptic wind is observed for the Drac and Romanche valleys (not shown). This allows us to conclude that these winds are of thermal origin and result from the radiative cooling of the ground in a complex topography.

Note that the other processes leading to the formation of winds in the ABL, discussed by Whiteman and Doran 

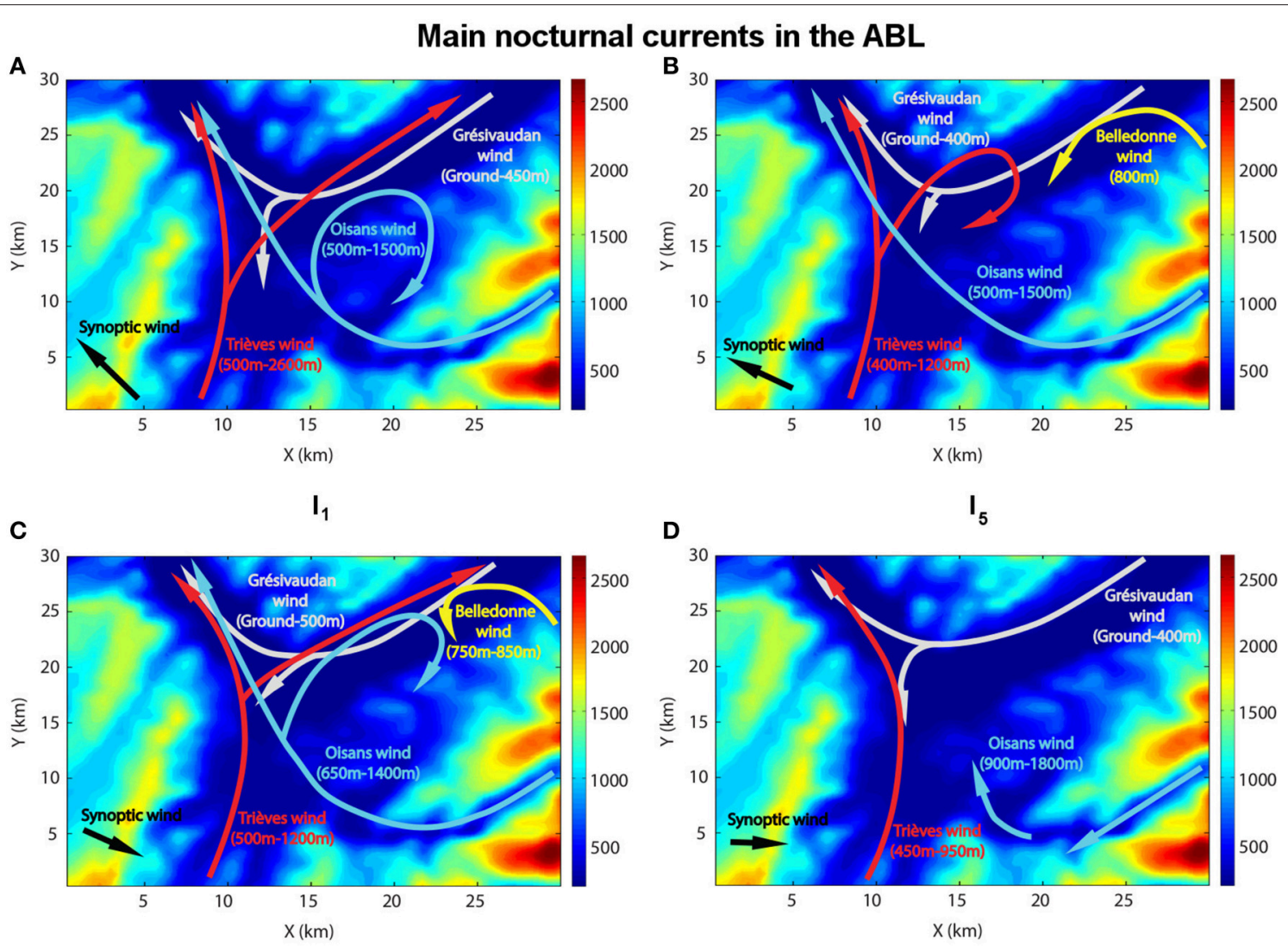

$I_{6}$

$\mathrm{I}_{7}$

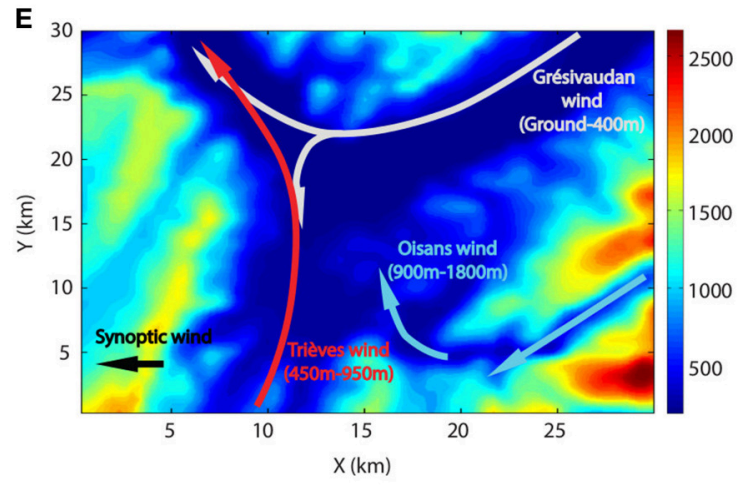

$I_{8}$

FIGURE 5 | Sketch of the main nocturnal currents during the heart of episode. (A) $I_{1}$ (November 7) ; (B) $I_{5}$ (December 15); (C) $I_{6}$ (December 28); (D) $I_{7}$ (January 13); (E) l (February 3).

(1993), are very unlikely and would have another signature. Indeed, a downward transport of horizontal momentum from above the valley, which would rather occur under unstable or neutrally stable conditions, would result in a linear dependency between the valley-wind direction and synoptic-wind direction (as indicated by the dashed dotted line in Figure 6). The channeling of the synoptic wind into the valleys (for a strong enough wind for instance) would lead to a step function (dashed line in Figure 6).
It follows that, during a wintertime persistent inversion, a unique organization of the valley-scale circulation within the Grenoble valleys sets in in the ABL, which does not depend upon the episode, because it is decoupled from the synoptic wind. This circulation is controlled by the local topography and the radiative cooling of the ground. Hence, a single episode may be considered as being representative of any persistent inversion of the winter when the circulation in the valley is considered. 


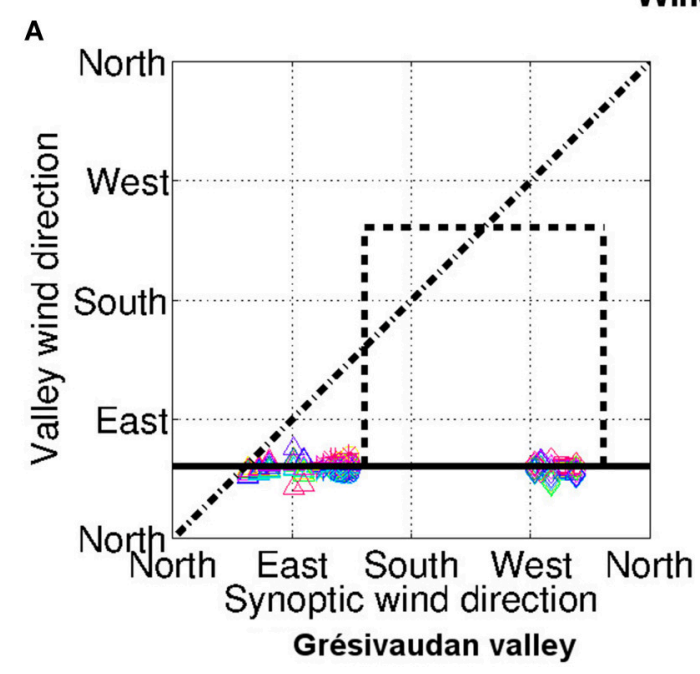

Wind direction diagrams

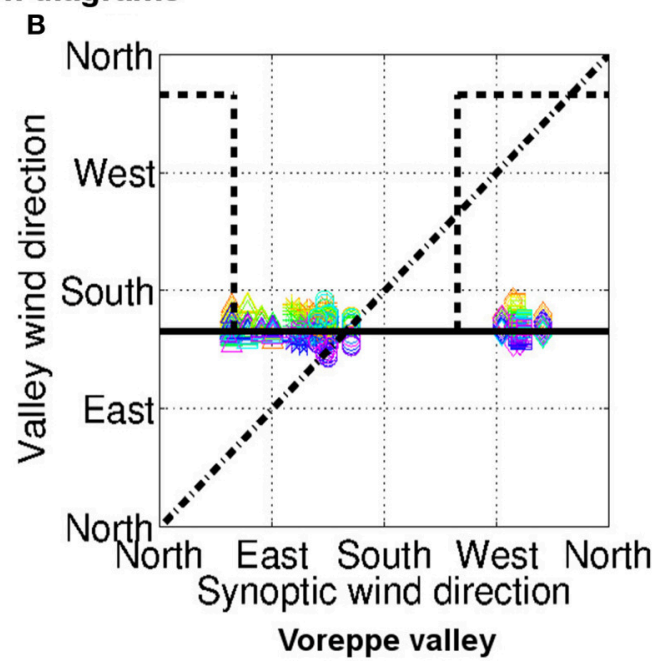

FIGURE 6 | Direction of the valley-wind vs. that of the synoptic wind. The valley-wind direction is plotted every hour of the C1 configuration, each hour having a different color, and each episode is represented by a symbol: $\circ: I_{1}, \star: I_{5}, \square: I_{6}, \diamond: I_{7}, \Delta: I_{8}$. The horizontal black line is the direction of the valley close to the Grenoble basin. (A) Grésivaudan valley; (B) Voreppe valley. The valley-wind direction is averaged over the horizontal grid points displayed in Figure 7A, from the ground up to $400 \mathrm{~m}$ for the Grésivaudant valley and up to $600 \mathrm{~m}$ for the Voreppe valley. See text for details.

\section{SPATIAL STRUCTURE OF THE ATMOSPHERIC BOUNDARY LAYER}

In the following focus is made on the heart of episode $\mathrm{I}_{6}$, simulated with the finest resolution of the $\mathrm{C}_{2}$ numerical configuration and for a longer period of 2 days (see Table 3 ).

The choice of episode $\mathrm{I}_{6}$ results from several considerations:

- $I_{6}$ is the strongest and the longest persistent inversion of the considered winter (see Table 2).

- The heart of episode $I_{6}$ is associated with a very low synoptic wind which flows from the north-west, namely in the direction opposite to most thermal winds of the Grenoble valleys, thereby waiving any doubt on the physical origin of the latter winds.

- The valley-scale circulation in the Grenoble valleys during episode $I_{6}$ is representative of the circulation during the other simulated episodes. This episode also clearly reveals the presence of the Belledonne wind, which is weaker in the other episodes.

\subsection{Vertical Structure of the Nocturnal Winds}

Vertical profiles of the wind speed and direction are displayed in Figure 7 for the Voreppe valley (Figure 7C) and for the Grésivaudan valley (Figure 7D). Results are shown at the end of the night (06:00 UTC) and are representative of the nocturnal vertical structure of the wind during the heart of the episode. The wind field is horizontally averaged over grid cells of the considered valley (displayed in Figure 7A) and the resulting mean speed and mean direction are computed as a function of altitude. The averaged vertical temperature for the Grésivaudan valley is also displayed in Figure 7B.

Figure 7D yields a refined view of the vertical layering described in the previous section for the Grésivaudan valley:

- The first layer (numbered as 1 in Figure 7D) lies above the ground (at about $220 \mathrm{~m} \mathrm{ASL}$ ), up to $\simeq 500 \mathrm{~m} \mathrm{ASL}$, and is associated with the Grésivaudan wind. This wind blows from the north-east and is of rather large amplitude, up to $6 \mathrm{~m} \mathrm{~s}^{-1}$ reached at about $300 \mathrm{~m}$ ASL. Figure 7B shows that this layer is strongly stable, the average vertical potential temperature gradient $\partial \bar{\theta} / \partial z$ being equal to $22 \mathrm{~K} \mathrm{~km}^{-1}$ in the layer.

- The second layer (numbered as 2 in Figure 7D) surmounts layer 1 and is associated with a mean mass flux from the southwest, which is therefore up-valley and opposed in direction to the Grésivaudan valley. This layer is associated with the Trièves and Oisans winds which overlap in this range of altitudes. The layer is very inhomogeneous horizontally as it involves large scale vortices (of size close to half the valley width). The wind direction therefore strongly varies, implying a weak average wind speed. Like layer 1, layer 2 is strongly stable $\left(\partial \bar{\theta} / \partial z \simeq 21 \mathrm{~K} \mathrm{~km}^{-1}\right)$.

- An intermediate layer (denoted as 2') may be noticed between 800 and $950 \mathrm{~m}$, which corresponds to the Belledonne wind. The mass flux flows here from the Belledonne to the Chartreuse range, with a direction comprised between 120 and $170^{\circ}$ (namely from the south-east). This wind is weak, of less than $2 \mathrm{~m} \mathrm{~s}^{-1}$.

- In layer 3 , the mean mass flux is associated with the Trièves wind, blowing from the south-west. It has a clear jet type structure, with maximum value of about $2.7 \mathrm{~m} \mathrm{~s}^{-1}$ reached at $1250 \mathrm{~m}$. This layer extends from about 1000 to $1500 \mathrm{~m}$ and 

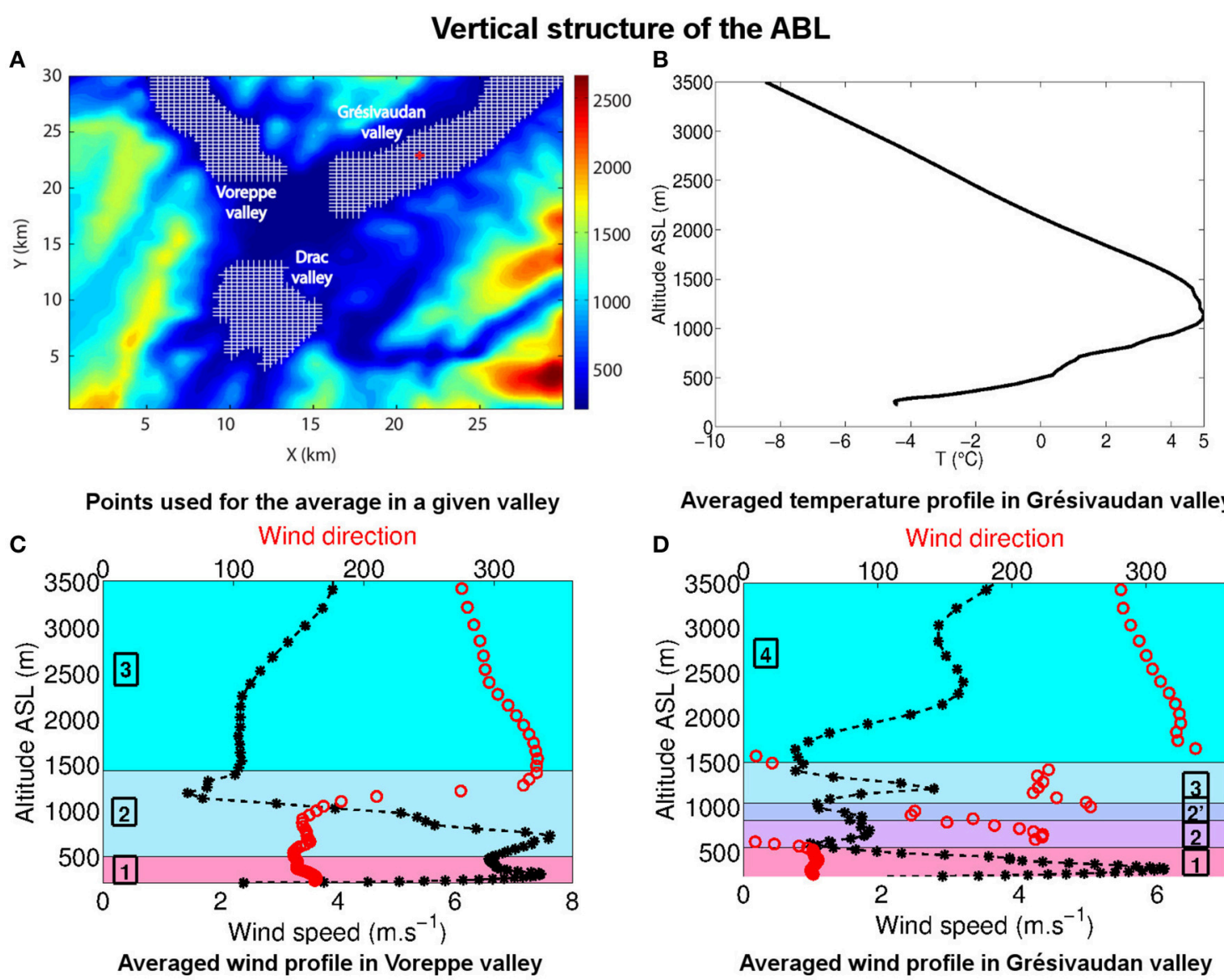

Averaged temperature profile in Grésivaudan valley

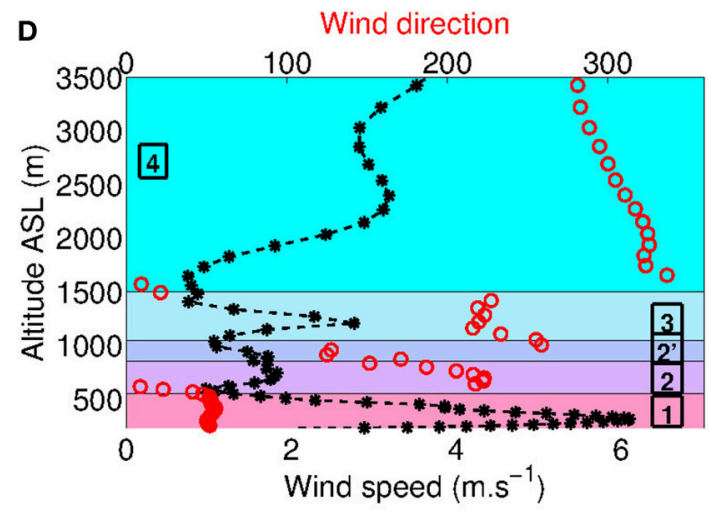

Averaged wind profile in Grésivaudan valley

FIGURE 7 | (A) The hatched zones are those over which the average for the Voreppe valley, the Grésivaudan valley and the Drac valley are performed. The point Gr, referred to in Section 5, is indicated with a red + symbol. (B) Vertical profile of temperature averaged over the Grésivaudan valley. (C) Vertical profile of wind speed ( $\star$ ) and wind direction (o) averaged over the Voreppe valley. (D) Same as (C) in the Grésivaudan valley. The averages are performed over the horizontal grid points displayed in frame (A). Profiles are shown at 06:00 UTC on December 28.

is stable with a nearly uniform temperature (corresponding to $\left.\partial \bar{\theta} / \partial z \simeq 10 \mathrm{~K} \mathrm{~km}^{-1}\right)$.

- Finally, the fourth layer (number 4 in Figure 7D) may be considered as a buffer zone between the top of the inversion layer and the bottom of the free troposphere. The synoptic wind is recognized here, being orographically channelled. It flows from the north-west with a slightly veering direction with altitude due to the shape of the mountain ranges. The stability of this layer is weaker, with $\partial \bar{\theta} / \partial z \simeq 3.6 \mathrm{~K} \mathrm{~km}^{-1}$, which is that of the free troposphere above.

The complex wind structure in the Grésivaudan valley, where the signature of the different winds can still be clearly recognized, contrasts with that in the Voreppe valley displayed in Figure 7C. There, only three main layers can be distinguished, the lowest two layers being associated with thermal winds channelled into this valley (toward the north-west) after their coming out in the Grenoble basin. The first layer corresponds to the Grésivaudan wind, up to $500 \mathrm{~m}$ ASL. The second layer, from $500 \mathrm{~m}$ to about $1100 \mathrm{~m}$, results from the overlapping of the Trieves and Oisans winds (locally a third maximum can be observed around $1000 \mathrm{~m}$, associated with the Oisans wind). The maxima of these jets reach a higher value than in the Grésivaudan valley, of $7.5 \mathrm{~m} \mathrm{~s}^{-1}$, due a strong pressure gradient between the Grenoble basin and the plain located northwest of the Voreppe valley, which accelerates the wind (not shown). The wind direction veers above $1000 \mathrm{~m}$ to align with that of the synoptic wind, marking the beginning of the buffer zone, which forms the third layer.

The Voreppe valley thus plays an important role in the ventilation of the Grenoble basin, in draining away the thermal winds. The air masses associated with these winds would otherwise partly accumulate in the Grenoble basin, possibly leading to an even higher pollution level during these wintertime persistent inversions.

The thermal winds and cooling of the ground at the bottom of the valley shape the temperature field, once anticyclonic conditions have set in. As a result, an inversion layer develops from the ground up to $1500 \mathrm{~m}$, close to the altitude of the valley top as discussed in Section 2. Figure 7B thus shows that the mean temperature profile is nearly linear, the mean temperature 
at a height of $1000 \mathrm{~m}$ above the valley bottom being almost $10^{\circ} \mathrm{C}$ higher than at the bottom. The temporal evolution of this profile and associated structure of the inversion layer are analyzed further in Section 5.

\subsection{Diurnal Circulation}

On December 28 at the latitude of Grenoble, the astronomical sunrise is at 07:18 UTC, the sunset being at 16:02 UTC. Due to orographic shading, the local sunrise is delayed by $1 \mathrm{~h}$ in the major part of the valley bottom, and similarly, the local sunset is advanced by about $1 \mathrm{~h}$ in most of the Voreppe and Drac valley (but this is not the case in the Grésivaudan valley, which has an almost full sky view factor in the west direction, and is therefore not significantly shaded in the late afternoon by the Vercors range located westward). In the Grenoble basin, whereas the duration of the day is theoretically of $8 \mathrm{~h} 45$, the duration of the period with a positive incoming insolation is then reduced to about $6 \mathrm{~h} 45$. This implies that the diurnal cycle is dominated by its nocturnal component, which lasts almost $18 \mathrm{~h}$.

Moreover, as will be shown in Section 5, due to both the low value of the wintertime insolation (further reduced by the orographic shading) and the strong stability over a significant depth of the inversion layer, daytime warming only affects a very shallow layer from the ground up to about $50 \mathrm{~m}$ above it. In this shallow layer, daytime convection occurs and triggers an upvalley wind in the main valleys of the site (not shown). The upper layers of the $\mathrm{ABL}$, from $50 \mathrm{~m}$ above the ground up to the inversion top, are not affected by the daytime warming and the valley-scale circulation herein remains the same as during nighttime.

Consequently, on a diurnal cycle, the valley-scale circulation inside the $\mathrm{ABL}$ is dominated by the nighttime valley winds detailed in the previous section. This nighttime wind organization indeed prevails in all the ABL during the cooling stage (from about 15:00 UTC to 09:00 UTC), and above the shallow convective layer of thickness $50 \mathrm{~m}$ during the warming stage (from about 09:00 UTC to 15:00 UTC).

The nocturnal wind circulation described in Section 4.1 can therefore be considered as representative of the ABL kinematic structure all along the heart of a persistent inversion.

\subsection{Recirculation and Stagnation Areas in the Grenoble Valleys}

In this section, focus is made on the first $50 \mathrm{~m}$ above the ground in order to analyse the ventilation of the surface layer. The main motivation is to determine the areas where no ventilation occurs, referred to as stagnation areas, which have an increased impact on air quality and human health. For this purpose, we use the quantification method developed by Allwine and Whiteman (1994) for the Colorado plateau basin. This method relies on two indices, a stagnation index $S$ and a recirculation index $R$, which are defined from the knowledge of the horizontal wind field components. The stagnation index, computed between two instants $t_{0}$ and $t_{1}$, represents the distance travelled by a fluid particle during this time interval. The recirculation index is also built with the wind field components, and with the stagnation index and takes values between 0 and 1 : for $R=0$, the fluid particle does not recirculate while for $R=1$, the fluid particle exactly returns to its $t_{0}$-position at $t_{1}$. Allwine and Whiteman (1994) further introduce critical thresholds from which they define the state of the flow around a measurement station to be in a stagnation, recirculation or a ventilation state. The stagnation state is defined by $S \leq 130 \mathrm{~km}$, the recirculation state by $R \geq 0.6$ and the ventilation state by the two conditions $S \geq 250 \mathrm{~km}$ and $R \leq 0.2$. Of course, the lower and upper bounds for $S$ depend on the wind speed. This method has been used by numerous authors, both for in situ measurements (f.i., Venegas and Mazzeo, 1999) and numerical modeling (f.i., Levy et al., 2009).

The indices $S$ and $R$ have been computed for configuration $C_{2}$ in all points of the boundary layer. Averaging the indices over the first $50 \mathrm{~m}$ above the ground and over the 2 days of the simulation yields the maps of Figure $\mathbf{8}$, which displays the stagnation index in Figure 8A and the recirculation index in Figure 8B. Well-ventilated areas appear, which are the Voreppe valley, the Romanche valley and, to a lesser extent, the Grésivaudan valley. By contrast the Drac valley, the south-east of the Grenoble basin, and the flank of the east-face of the Chartreuse range, for which both $S \leq 130 \mathrm{~km}$ and $R \geq 0.6$, appears as very critical areas for both stagnation and recirculation and, therefore, for the trapping of pollutants which would be emitted there. These results are consistent with the very weak wind field in the Drac valley shown on Figure 4A and with the persistent vortex structure which is present over the south-east of the Grenoble basin as shown on Figure 4B.

\section{ANALYSIS OF THE TEMPERATURE INVERSION DURING THE STAGNATION STAGE OF A PERSISTENT INVERSION EPISODE}

\subsection{Overall Evolution of a Persistent Episode}

As discussed in the Introduction, the formation of the wintertime persistent inversion is controlled by the arrival of a warm air mass at mid-altitude (around $1500 \mathrm{~m} \mathrm{ASL}$ ), associated with very low wind speed; as shown in Section 3, the circulation of the air layer below becomes decoupled from the synoptic circulation and controlled by the thermal winds. The combined effect of cold air advected toward the bottom of the valley by the down-slope and valley winds and of radiative cooling leads to the progressive built-up of an inversion layer, the temperature gradient changing from adiabatic (and therefore negative) values to positive values in a day or two. As shown in Section 4, the inversion layer eventually fills up the whole valley, up to an altitude close to the top of the valley. This evolution is described in details in Largeron and Staquet (2016) and is similar to the one reported by Whiteman et al. (1999a) for the Colorado basin and by Reeves and Stensrud (2009) for valleys and basins in the Western United States.

The destruction of the inversion occurs when a cold front or trough, associated with strong winds and often with precipitation, reaches the region. The lower part of the atmospheric boundary layer becomes affected by the penetration of channelled winds which disturb and eventually destroy the 

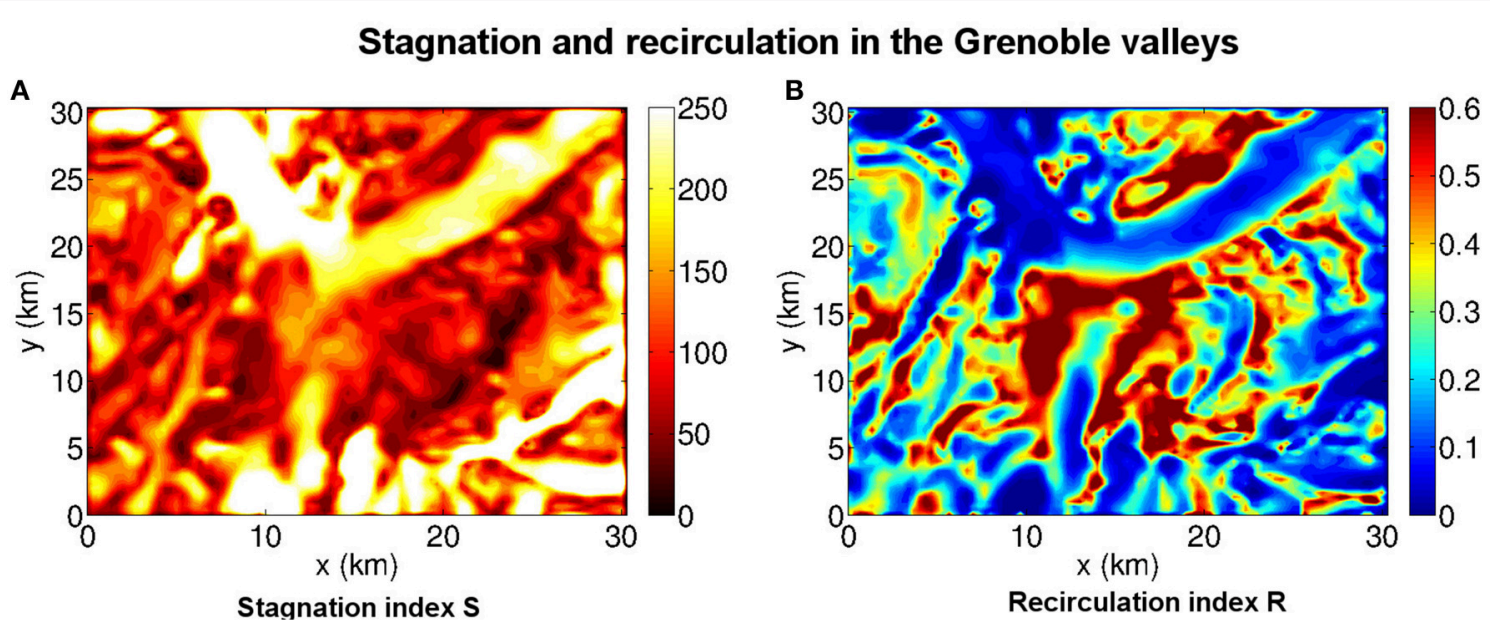

FIGURE 8 | (A) Stagnation index S (in km) averaged over the first $50 \mathrm{~m}$ above the ground and over the 2 days of configuration C2. Stagnation areas appear in red and black. (B) Recirulation index $\mathrm{R}$ averaged as $\mathrm{S}$. Recirculation areas appear in dark red. Ventilated areas $(S \geq 250 \mathrm{~km}, R \leq 0.2)$ correspond to areas where $S$ appears in white and $\mathrm{R}$ in blue.

inversion. This process is much more rapid than the formation of the inversion, as it involves mechanical and turbulent mixing processes, as opposed to the built-up of the inversion which results from radiative processes (Largeron and Staquet, 2016).

In the following, we focus on the thermal structure of the inversion during the stagnation stage of episode $\mathrm{I}_{6}$. All results are shown at one point of the Grésivaudan valley, displayed in Figure 7A, and denoted as point Gr. This point, and the temperature profiles along the vertical line going through it, appear to be representative of the properties of the temperature field in the Grésivaudan valley.

\subsection{Diurnal Forcing of the Atmospheric Boundary Layer}

In numerical models, the balance of the surfaces fluxes is usually expressed as

$$
R_{n}-G-L E-H=0,
$$

where $R_{n}$ is the net incoming radiative flux, $G$ the incoming heat flux into the ground, $L E$ the latent heat flux and $H$ the sensible heat flux (f.i., Stull, 1988).

In the SURFEX model, the surface temperature $T_{s}$ obeys the equation (Noilhan and Planton, 1989)

$$
\frac{\partial T_{s}}{\partial t}=C_{T}\left(R_{n}-L E-H\right),
$$

where $C_{T}$ is the heat capacity of the ground.

The four fluxes $R_{n}, L_{E}, H$ and $G$ are plotted vs. time in Figure 9A from 27 December 18:00 UTC to 29 December 20:00 UTC. The dashed lines correspond to the astronomical sunrise and sunset times (without taking into account orographic shading). In the context of the present study, no measurement of the surface fluxes are available so that the model performance can not be explicitly evaluated. Moreover, imbalances are frequently found in observations (e.g., Cuxart et al., 2015), pointing out some conceptual issues concerning the physical parameterizations of surface-atmosphere interactions currently used in numerical models (Foken, 2008). Nevertheless, simulated values suggest that:

- The sensible heat flux and the latent heat flux are much smaller than the radiative flux during both daytime and nighttime;

- The sensible heat flux decreases throughout the night and becomes more and more important as the night advances, whereas the latent heat flux remains negligible all night long;

- The surface flux balance is dominated by the radiative flux during the day and in the first part of the night; but in the second part of the night (after $23 \mathrm{~h}$ UTC), turbulence becomes a non-negligible component of the energy budget and partly compensates the long-wave outcoming radiative cooling.

- A radiative warming of the ground occurs for a short period of time, between about 09:00 UTC and 15:00 UTC, the radiative cooling therefore extending over the rest of the day for about $18 \mathrm{~h}$.

The surface and 2-m air temperature responses are displayed in Figure 9B. The temperatures start to increase shortly after sunrise, up to about noon, consistent with the behavior of the radiative and sensible heat fluxes. A continuous decrease follows during the afternoon and night up to sunrise. These two stages will be referred to as the warming and the cooling stages hereafter.

\subsection{Diurnal Evolution of the Inversion}

Inversions layers are characterized by their height $h_{i}$ and their strength $\Delta \theta$, where $\theta$ is the potential temperature, but also by their valley heat deficit (Whiteman et al., 1999a), already referred to in Section 2.2, which corresponds to the heat required to destroy the inversion. If a linear vertical profile of $\theta$ is assumed, and if the density $\rho$ and the specific heat of air $c_{p}$ are assumed 


\section{Surface fluxes and temperatures time series}

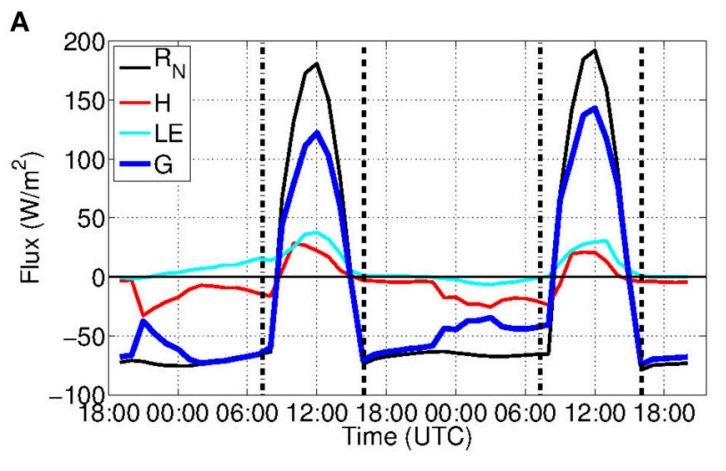

Surface fluxes

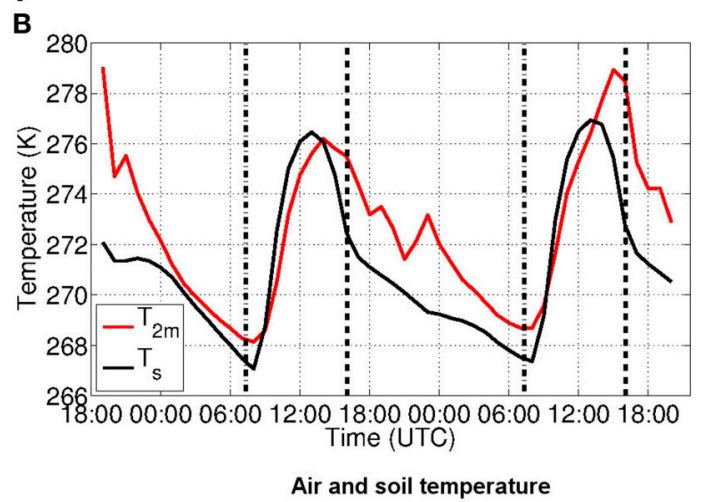

Air and soil temperature

FIGURE 9 | (A) Temporal evolution of the surface fluxes $R_{N}, H, L E, G=R_{N}-H-L E$ from 27 December, 18:00 UTC to 29 December, 20:00 UTC in the Grésivaudan valley at point $\mathrm{Gr}$ displayed in Figure 7A. The vertical dashed lines are the astronomical sunset and sunrise times for Grenoble. (B) Temporal evolution of the surface temperature $\left(T_{s}\right)$ and the $2-m$ air temperature $\left(T_{2 m}\right)$.

to be constant, it can be shown that the heat deficit can be approximated by $0.5 \rho c_{p} h_{i} \Delta \theta$ (Whiteman et al., 1999b; Largeron and Staquet, 2016).

In the present case, the height $h_{i}$ is computed as the elevation of the highest point in the ABL where $\partial \theta / \partial z>\left|\gamma_{a d i a b}\right|$ (or equivalently $\partial T / \partial z>0), \gamma_{a d i a b}$ being the adiabatic temperature gradient. The strength $\Delta \theta$ is then the difference between $\theta$ at this height and $\theta$ at the ground level.

The structure of the inversion layer in the Grésivaudan valley from 27 December, 18:00 UTC to 29 December, 20:00 UTC is illustrated in Figure 10A by a $(z, t)$ diagram of $\partial \theta / \partial z$ (above the point $G r$ ) up to $2000 \mathrm{~m}$ ASL. Each point in the figure is plotted with a color which depends on the local stability quantified by the value of $\partial \theta / \partial z$ : the points where $\partial \theta / \partial z>\left|\gamma_{\text {adiab }}\right|$, associated with an inversion, are in yellow; the points where $0<\partial \theta / \partial z<$ $\left|\gamma_{\text {adiab }}\right|$, where the layer is moderately stable with no inversion, are in orange; and the points where $\partial \theta / \partial z<0$ where the layer is locally unstable (convective) are in black. Figure 10A shows that the ABL is entirely stable all along the diurnal cycle, except in a shallow layer close to the ground (of $50 \mathrm{~m}$ depth, namely about $5 \%$ of the inversion layer thickness) during the warming stage, and at a very few localized points around $700 \mathrm{~m} \mathrm{ASL}$ at certain times, due to the presence of a strong shear. Also, in a very limited part of the $\mathrm{ABL}$, static stability is moderate with no inversion (orange color) but these zones tend to disappear during the cooling stage. The major part of the $\mathrm{ABL}$ is otherwise filled with a strong and deep inversion (yellow color), as we now discuss it.

The top of the inversion layer is underlined with a thick black line in Figure 10A. The inversion layer height little varies over the period, between 920 and $1255 \mathrm{~m}$. The lowest values are observed during the warming stage, the inversion being slightly eroded both from the bottom by the shallow convective layer and from the top by warm subsidence (this erosion was classified as type II by Whiteman, 1982). The inversion then thickens during the cooling stage. In early afternoon, when the surface air temperature is the warmest, the inversion height is still of $920 \mathrm{~m}$ implying that the inversion is far from being destroyed.

The inversion strength $\Delta \theta$, displayed in Figure 10B, varies between 10.3 and $21.3 \mathrm{~K}$ over the period. The cooling stage is associated with an intensification of the inversion strength, with a maximum value of about $21.3 \mathrm{~K}$, while the lowest values are reached during the warming stage (equal to $12 \mathrm{~K}$ on 28 December and $10.3 \mathrm{~K}$ on 29 December, at 14:00 UTC). Thus, the inversion strength decreases by nearly $50 \%$ over a day but its smallest value is still equal to $10 \mathrm{~K}$, attesting that the inversion is persistent and strong.

Computation of the heat deficit leads to huge values at sunrise, of $23.4 \mathrm{MJ} \mathrm{m}^{-2}$ on 28 December and of $20.7 \mathrm{MJ} \mathrm{m}^{-2}$ on 29 December while the values remain strong at sunset, comprised between 11.7 and $12.8 \mathrm{MJ} \mathrm{m}^{-2}$ for these 2 days. These values are close to those inferred from the ground observations (see Figure 3). They are significantly higher than those estimated by Whiteman et al. (1999a) from inversion measurements in midsize Colorado valleys $\left(1-8 \mathrm{MJ} \mathrm{m}^{-2}\right.$ at sunrise) but of the same order as the values computed for the most extreme inversions in three valleys of size comparable to that of the Grenoble valleys.

Whiteman et al. (1999a) indeed compared the characteristics of inversions observed in 20 valleys of the Rocky mountains, which are reproduced in Figure 10C. Each valley is referred to by a number in a $\left(h_{i}, \Delta \theta\right)$ diagram and the characteristics of the persistent inversion $\mathrm{I}_{6}$ estimated on December 2829 are superimposed. The heat deficit for a given valley is proportional to the area below the line joining the origin to the valley number, implying that the most intense inversions are found in the right upper corner of the diagram. These involve for instance the Bullfrog basin (number 17) located north-east of the Grand Canyon and inversion $\mathrm{I}_{6}$. $\mathrm{I}_{6}$ therefore ranges among the extreme inversions, which is consistent with this inversion to be the strongest of the winter of 20062007. 


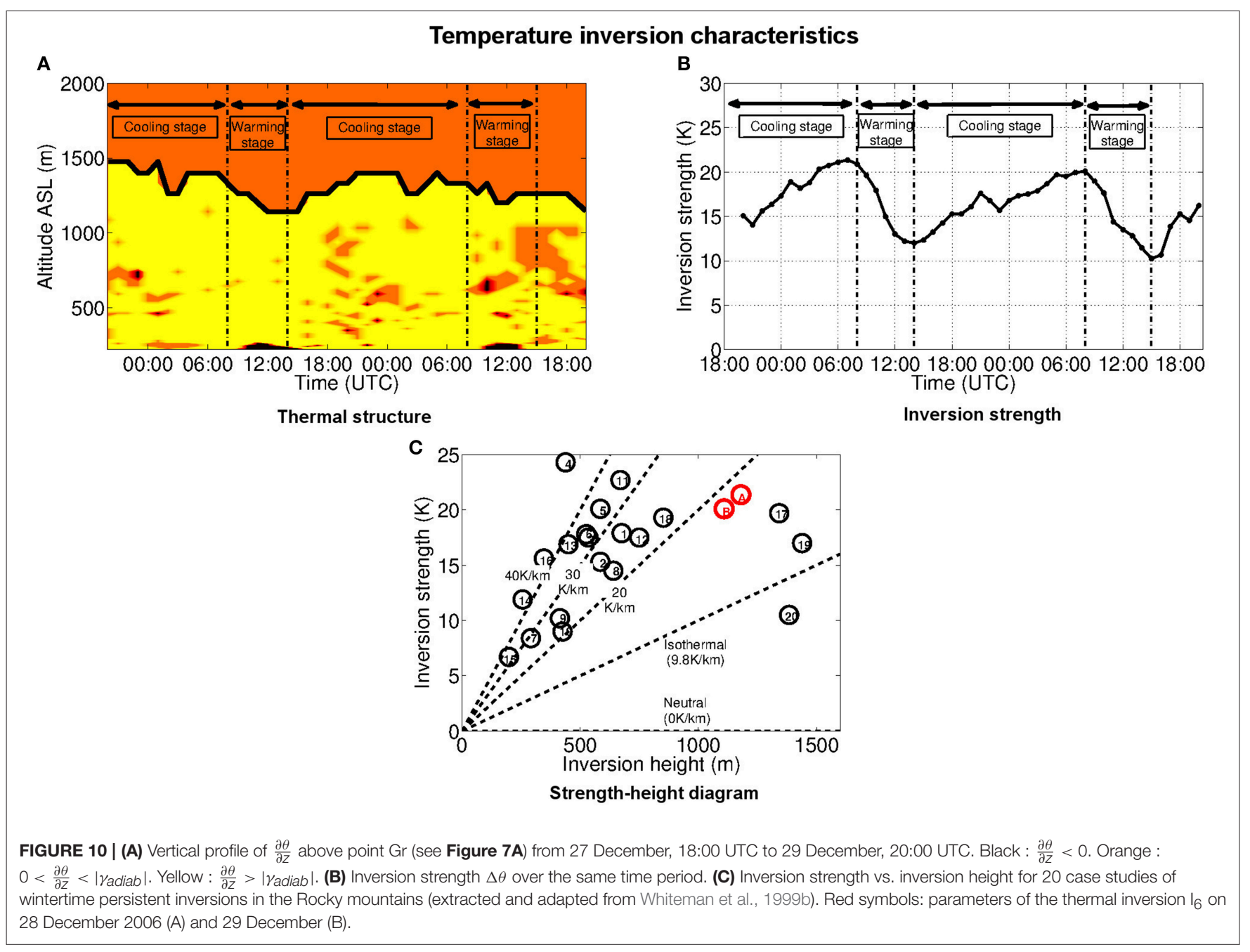

\section{SUMMARY AND CONCLUSION}

The purpose of this paper was to investigate the valleyscale circulation in the atmospheric boundary layer (ABL) of the Grenoble valleys, along with its thermodynamical structure, during persistent inversions of the 2006-2007 winter. Ground-based observations of temperature and high-resolution numerical simulations have been used for this analysis.

The first step was to detect persistent inversions from the 2-m air temperature data, a detailed analysis of which being reported in Largeron and Staquet (2016). A criterion was introduced for this purpose, based upon the temperature difference between the valley bottom and the valley top. This criterion was shown to be equivalently expressed in terms of the valley heat deficit inside the inversion layer, which represents the heat required to destroy the inversion layer. Similarly, Whiteman et al. (1999a,b) used such criterion to identify persistent cold-air pools in the Salt Lake valley. Nine episodes were detected from November to February, which all coincide with the nine strongest $\mathrm{PM}_{10}$ air-pollution episodes of that winter. These wintertime persistent inversions have a cumulated duration of one third of the total winter duration; they occur during anticyclonic conditions and are triggered by a mid-level advection of a warm air mass, as shown in Largeron and Staquet (2016). The simple method proposed here may serve as a reliable technique to detect persistent inversions from ground-based temperature data when no radio-soundings are available.

Numerical simulations were next performed with the MesoNH-SURFEX coupled model to analyse the persistent inversions, from formation to destruction. Only the stagnation stage, during which the inversion does not evolve from one day to the next, corresponding to the heart of an episode, is reported in the present paper. Several sensitivity and validation tests were performed to assess the validity of the computations, among which the sensitivity to the vertical resolution was investigated. It was found that a vertical resolution of $15 \mathrm{~m}$ at the ground allows the gross features of the atmospheric boundary-layer circulation to be reproduced, a resolution of at least $4 \mathrm{~m}$ at the ground being required for quantitative comparison with the 2-m air temperature to be obtained at the bottom of the valley. With a vertical resolution coarser than $15 \mathrm{~m}$, the inversion layer is poorly represented, with a too weak strength due to a hot bias at the 
bottom of the valley and a cold bias at the valley top. This can be explained by the need to correctly represent the down-slope winds which drive and maintain the inversion layer in inducing cold-air drainage along the slopes, from the valley top down to the valley bottom.

Numerical simulations were thus performed in two steps. Firstly, among the nine persistent inversions, the five longest and strongest inversions, which exhibit a stagnation stage, were simulated as case studies, over a 10 -h night with a vertical resolution of $15 \mathrm{~m}$ close to the ground. Focusing again upon one night in the heart of each episode, the main conclusions are that: (i) an inversion layer has formed up to an altitude of about $1500 \mathrm{~m}$, whose absolute temperature gradient is nearly constant and of about $10 \mathrm{~K} \mathrm{~km}^{-1}$. (ii) whatever the persistent inversion episode, the atmosphere can be decomposed into three layers : the free troposphere above $3000 \mathrm{~m}$ ASL, a buffer zone between 1500 and $3000 \mathrm{~m}$ ASL in which the large-scale synoptic wind is orographically channelled, and the inversion layer below $1500 \mathrm{~m}$ ASL. The inversion layer is decoupled from the atmosphere above whatever the episode, thereby recovering a common feature of wintertime persistent inversions. As a result, the atmospheric circulation in this inversion layer is controlled by thermal winds created by the nocturnal cooling of the ground; (iii) a local dynamics set in in the inversion layer, which is independent of the episode. These dynamics are characterized by a vertical layering resulting from the superposition of four main valley winds flowing from the tributary valleys of the Grenoble basin.

It follows that a detailed account of the heart of a persistent episode can be obtained by performing a high-resolution simulation of any of the five episodes. This is the second step of the numerical study. Two days during the heart of the longest and most extreme inversion were thus simulated with a $4-\mathrm{m}$ vertical resolution close to the ground. The horizontal wind field appears to have a quasi-deterministic structure, with a veering shear with altitude, the wind speed and direction at a given altitude being controlled by a down-valley wind. Large-scale vortex structures appear at the entrance of the Grésivaudan valley and along the flank of the Belledonne range. A first guess of the impact of this circulation on air quality was addressed by computing the stagnation and recirculation indices introduced by Allwine and Whiteman (1994). Stagnation regions critical for air quality were thus identified, especially south to the agglomeration and along the flank of the Belledonne range where wind speeds remain low and vortices form.

\section{REFERENCES}

Air Rhône-Alpes (2011). Suivi des Polluants Réglementés Dans la Vallée de l’Arve. Technical report, Air Rhône-Alpes.

Allwine, K., and Whiteman, C. (1994). Single-station integral measures of atmospheric stagnation, recirculation and ventilation. Atmos. Environ. 28, 713-721. doi: 10.1016/1352-2310(94)90048-5

Banta, R., Darby, L., Fast, J., Pinto, J., Whiteman, C., Shaw, W., et al. (2004). Nocturnal low-level jet in a mountain basin complex. Part I: evolution and
A detailed account of the diurnal evolution of the thermal inversion was also obtained: the height of the inversion varies between about 900 and $1200 \mathrm{~m}$, the lowest value occurring around 14:00 UTC when the inversion is eroded from below by a shallow convective layer of at most $50 \mathrm{~m}$ above the ground and from the top by a warm subsidence. The temperature difference between inversion top and bottom ranges between about $10 \mathrm{~K}$ (at 14:00 UTC) and $21 \mathrm{~K}$ (at 08:00 UTC). These features are those of a very strong and deep inversion and are actually similar to the most extreme inversions recorded by Whiteman et al. (1999a) in the Grand Canyon.

As already pointed out, no wintertime field campaign has ever been undertaken in the Grenoble valleys although this area is densely populated and strongly industrialized. Combined with the present results regarding stagnation of the atmospheric surface layer, this leads to severe human health issues. It would be very desirable to launch such a campaign, as the collected data would offer the possibility to get a better estimate of the validity of numerical simulations, regarding the quasi-deterministic pattern of the wind profiles or the thermal structure of the inversion layer we found. Field data would also allow us to get access to much finer scales than those analysed in this paper, such as surface fluxes, the knowledge of which is crucially needed to validate the ABL forcing of a surface-atmosphere coupled model or to improve the physical parameterizations. Obviously, complementary chemical and air quality studies would also benefit from acute knowledge of local fine-scale atmospheric dynamics.

\section{AUTHOR CONTRIBUTIONS}

YL: Author of the research presented in the manuscript and of the original manuscript. CS: Contribute to the manuscript.

\section{ACKNOWLEDGMENTS}

The present work was supported by a funding from région Rhône-Alpes. Computations were carried out on the machines of the French computer center IDRIS.

\section{SUPPLEMENTARY MATERIAL}

The Supplementary Material for this article can be found online at: http://journal.frontiersin.org/article/10.3389/feart. 2016.00070 effects on local flows. J. Appl. Meteorol. 43, 1348-1365. doi: 10.1175/ JAM2142.1

Chemel, C., Arduini, G., Staquet, C., Largeron, Y., Legain, D., Tzanos, D., et al. (2016). Valley heat deficit as a bulk measure of wintertime particulate air pollution in the Arve River Valley. Atmos. Environ. 128, 208-215. doi: 10.1016/ j.atmosenv.2015.12.058

Couach, O., Balin, I., Jiménez, R., Ristori, P., Perego, S., Kirchner, F., et al. (2003). An investigation of ozone and planetary boundary layer dynamics over the complex topography of Grenoble combining measurements and 
modeling. Atmos. Chem. Phys. Discuss. 3, 797-825. doi: 10.5194/acpd-3-79 7-2003

Cuxart, J. (2015). When can a high-resolution simulation over complex terrain be called LES? Front. Earth Sci. 3:87. doi: 10.3389/feart.2015.00087

Cuxart, J., Bougeault, P., and Redelsperger, J. (2000). A turbulence scheme allowing for mesoscale and large-eddy simulations. Q. J. R. Meteorol. Soc. 126, 1-30. doi: 10.1002/qj.49712656202

Cuxart, J., Conangla, L., and Jiménez, M. (2015). Evaluation of the surface energy budget equation with experimental data and the ecmwf model in the ebro valley. J. Geophys. Res. Atmos 120, 1008-1022. doi: 10.1002/2014JD0 22296

Cuxart, J., and Jiménez, M. (2007). Mixing processes in a nocturnal lowlevel jet: An les study. J. Atmos. Sci. 64, 1666-1679. doi: 10.1175/ JAS3903.1

Cuxart, J., Jiménez, M., and Martínez, D. (2007). Nocturnal meso-beta basin and katabatic flows on a midlatitude island. Month. Weather Rev. 135, 918. doi: 10.1175/MWR3329.1

Drobinski, P., Steinacker, R., Richner, H., Baumann-Stanzer, K., Beffrey, G., Benech, B., et al. (2007). Föhn in the rhine valley during map: a review of its multiscale dynamics in complex valley geometry. Q. J. R. Meteorol. Soc. 133, 897-916. doi: 10.1002/qj.70

Favez, O., El Haddad, I., Piot, C., Boréave, A., Abidi, E., Marchand, N., et al. (2010). Inter-comparison of source apportionment models for the estimation of wood burning aerosols during wintertime in an Alpine city (Grenoble, France). Atmos. Chem. Phys. Discuss 10, 559-613. doi: 10.5194/acpd-10-55 9-2010

Fernando, H. (2010). Fluid dynamics of urban atmospheres in complex terrain. Annu. Rev. Fluid Mech. 42, 365-389. doi: 10.1146/annurev-fluid-121108145459

Flamant, C., Drobinski, P., Nance, L., Banta, R., Darby, L., Dusek, J., et al. (2002). Gap flow in an alpine valley during a shallow south föhn event: Observations, numerical simulations and hydraulic analogue. Q. J. R. Meteorol. Soc. 128, 1173-1210. doi: 10.1256/003590002320 373256

Foken, T. (2008). The energy balance closure problem: an overview. Ecol. Appl. 18, 1351-1367. doi: 10.1890/06-0922.1

Gohm, A., Harnisch, F., Vergeiner, J., Obleitner, F., Schnitzhofer, R., Hansel, A., et al. (2009). Air pollution transport in an Alpine valley: results from airborne and ground-based observations. Boundary-layer Meteorol. 131, 441-463. doi: 10.1007/s10546-009-9371-9

Harnisch, F., Gohm, A., Fix, A., Schnitzhofer, R., Hansel, A., and Neininger, B. (2009). Spatial distribution of aerosols in the Inn Valley atmosphere during wintertime. Meteorol. Atmos. Phys. 103, 223-235. doi: 10.1007/s00703-0080318-3

Lafore, J., Stein, J., Asencio, N., Bougeault, P., Ducrocq, V., Duron, J., et al. (1998). The Meso-NH atmospheric simulation system. Part I: Adiabatic formulation and control simulations. Ann. Geophys. 16, 90-109. doi: 10.1007/s00585-9970090-6

Lareau, N., Crosman, E., Whiteman, C., Horel, J., Hoch, S., Brown, W., et al. (2013). The persistent cold-air pool study. Bull. Am. Meteorol. Soc. 94, 51-63. doi: 10.1175/BAMS-D-11-00255.1

Largeron, Y. (2010). Dynamics of the Stable Atmospheric Boundary Layer over Complex Terrain. Application to PM10 Pollution Episodes in Alpine Valleys. Ph.d. thesis, Université de Grenoble.

Largeron, Y., and Staquet, C. (2016). Persistent inversion dynamics and wintertime $\mathrm{PM}_{10}$ air pollution in Alpine valleys. Atmos. Environ. 135, 92-108. doi: 10.1016/j.atmosenv.2016.03.045

Levy, I., Mahrer, Y., and Dayan, U. (2009). Coastal and synoptic recirculation affecting air pollutants dispersion: a numerical study. Atmos. Environ. 43, 1991-1999. doi: 10.1016/j.atmosenv.2009.01.017

Lienhard, J., and van Atta, C. (1990). The decay of turbulence in thermally stratified flows. JFM 210, 57-112. doi: 10.1017/S0022112090001227

Mahrt, L. (2014). Stably stratified atmospheric boundary layers. Annu. Rev. Fluid Mech. 46, 23-45. doi: 10.1146/annurev-fluid-010313-1 41354

Martínez, D., Jiménez, M., Cuxart, J., and Mahrt, L. (2010). Heterogeneous nocturnal cooling in a large basin under very stable conditions. Boundary-layer Meteorol. 137, 97-113. doi: 10.1007/s10546-010-9522-z
Masson, V. (2000). A physically-based scheme for the urban energy budget in atmospheric models. Bound. Layer Meteor. 94, 357-397. doi: 10.1023/A:1002463829265

Masson, V., Champeaux, J., Chauvin, F., Meriguet, C., and Lacaze, R. (2003). A global data base of land surface parameters at $1 \mathrm{~km}$ resolution in meteorological and climate model. J. Appl. Meteor. Climatol. 16, 1261-1282. doi: 10.1175/15200442-16.9.1261

Masson, V., Le Moigne, P., Martin, E., Faroux, S., Alias, A., Alkama, R., et al. (2013). The surfexv7.2 land and ocean surface platform for coupled or offline simulation of earth surface variables and fluxes. Geosci. Model Dev. 6, 929-960. doi: 10.5194/gmd-6-929-2013

Neff, W., and King, C. (1989). The accumulation and pooling of drainage flows in a large basin. J. Appl. Meteor. 28, 518-529.

Noilhan, J., and Planton, S. (1989). A simple parameterization of land surface processes for meteorological models. Month. Weather Rev. 117, 536.

Pope, C. A., Burnett, R. T., Thun, M. J., Calle, E. E., Krewski, D., Ito, K., et al. (2002). Lung cancer, cardiopulmonary mortality, and long-term exposure to fine particulate air pollution. JAMA 287, 1132-1141. doi: 10.1001/jama.287. 9.1132

Price, J., Vosper, S., Brown, A., Ross, A., Clark, P., Davies, F., et al. (2011). COLPEX: field and numerical studies over a region of small hills. Bull. Am. Meteorol. Soc. 92, 1636-1650. doi: 10.1175/2011BAMS3032.1

Reeves, H. D., and Stensrud, D. J. (2009). Synoptic-scale flow and valley cold pool evolution in the western united states. Weather Forecast. 24, 1625-1643. doi: 10.1175/2009WAF2222234.1

Schnitzhofer, R., Norman, M., Dunkl, J., Wisthaler, A., Gohm, A., Obleitner, F., et al. (2007). Vertical distribution of air pollutants in the Inn Valley atmosphere in winter 2006. Geophys. Res. Abstr.

Silcox, G. D., Kelly, K. E., Crosman, E. T., Whiteman, C. D., and Allen, B. L. (2012). Wintertime PM 2.5 concentrations during persistent, multi-day cold-air pools in a mountain valley. Atmos. Environ. 46, 17-24.

Stull, R. (1988). An Introduction to Boundary Layer Meteorology. Kluwer.

Sun, J., Nappo, C., Mahrt, L., Belusic, D., Grisogono, B., Stauffer, D., et al. (2015). Review of wave-turbulence interactions in the stable atmospheric boundary layer. Rev. Geophys. 53, 956-993. doi: 10.1002/2015RG0 00487

Venegas, L., and Mazzeo, N. (1999). Atmospheric stagnation, recirculation and ventilation potential of several sites in Argentina. Atmos. Res. 52, 43-57. doi: 10.1016/S0169-8095(99)00030-7

Vosper, S., Hugues, J., Lock, A., Sheridan, P., Ross, A., Jemmett-Smith, A., et al. (2014). Cold-pool formation in a narrow valley. Q. J. R. Meteorol. 140, 699-714 doi: 10.1002/qj.2160

Wei, L., Pu, Z., and Wang, S. (2013). Numerical simulation of the life cycle of a persistent wintertime inversion over salt lake city. Bound. Layer Meteor. 148, 399-418. doi: 10.1007/s10546-013-9821-2

Whiteman, C. (1982). Breakup of temperature inversions in deep mountain valleys: Part I. observations. J. Appl. Meteorol. 21, 270-289.

Whiteman, C. (2000). Mountain Meteorology: Fundamentals and Applications. Oxford University Press, USA.

Whiteman, C., Bian, X., and Zhong, S. (1999a). Wintertime evolution of the temperature inversion in the Colorado Plateau Basin. J. Appl. Meteorol. 38, 1103-1117.

Whiteman, C., and Doran, J. (1993). The relationship between overlying synopticscale flows and winds within a valley. J. Appl. Meteorol. 32, 1669-1682.

Whiteman, C., Muschinski, A., Zhong, S., Fritts, D., Hoch, S., Hahnenberger, M., et al. (2008). Metcrax 2006. Bull. Am. Met. Soc. 89, 1665-1680. doi: 10.1175/ 2008bams 2574.1

Whiteman, C., Zhong, S., and Bian, X. (1999b). Wintertime boundary layer structure in the Grand Canyon. J. Appl. Meteorol. 38, 1084-1102.

Whiteman, C., Zhong, S., Shaw, W., Hubbe, J., Bian, X., and Mittelstadt, J. (2001). Cold pools in the Columbia Basin. Weather Forecast. 16, 432-447. doi: 10.1175/ 1520-0434(2001)016<0432:CPITCB > 2.0.CO;2

Whiteman, C. D., Eisenbach, S., Pospichal, B., and Steinacker, R. (2004). Comparison of vertical soundings and sidewall air temperature measurements in a small alpine basin. J. Appl. Meteorol. 43, 1635-1647. doi: 10.1175/JAM2168.1

Wolyn, P., and McKee, T. (1989). Deep stable layers in the intermountain western united states. Mon. Wea. Rev. 117, 461-472. 
Zhong, S., Whiteman, C., Bian, X., Shaw, W., and Hubbe, J. (2001). Meteorological processes affecting the evolution of a wintertime cold air pool in the Columbia basin. Month. Weather Rev. 129, 2600-2613. doi: 10.1175/15200493(2001)129<2600:MPATEO>2.0.CO;2

Zhou, B., and Chow, F. (2013). Nighttime turbulent events in a steep valley: a nested large-eddy simulation study. J. Atmos. Sci. 70, 3262-3276. doi: 10.1175/JAS-D-13-02.1

Conflict of Interest Statement: The authors declare that the research was conducted in the absence of any commercial or financial relationships that could be construed as a potential conflict of interest.
The reviewer VM declared a shared affiliation, though no other collaboration, with one of the authors YL to the handling Editor, who ensured that the process nevertheless met the standards of a fair and objective review.

Copyright (c) 2016 Largeron and Staquet. This is an open-access article distributed under the terms of the Creative Commons Attribution License (CC BY). The use, distribution or reproduction in other forums is permitted, provided the original author(s) or licensor are credited and that the original publication in this journal is cited, in accordance with accepted academic practice. No use, distribution or reproduction is permitted which does not comply with these terms. 


\section{OPEN ACCESS}

Edited by:

Miguel A. C. Teixeira,

University of Reading, UK

Reviewed by:

Peter F. Sheridan,

Met Office, UK

Lev Ingel,

Institute of Experimental Meteorology SPA "Typhoon", Russia

*Correspondence: Ivan Güttler

ivan.guettler@cirus.dhz.hr

Specialty section: This article was submitted to Atmospheric Science, a section of the journal Frontiers in Earth Science

Received: 31 August 2015 Accepted: 17 June 2016 Published: 06 July 2016

Citation:

Güttler I, Marinović I, Večenaj Ž and Grisogono B (2016) Energetics of

Slope Flows: Linear and Weakly Nonlinear Solutions of the Extended Prandtl Model. Front. Earth Sci. 4:72. doi: 10.3389/feart.2016.00072

\section{Energetics of Slope Flows: Linear and Weakly Nonlinear Solutions of the Extended Prandtl Model}

\author{
Ivan Güttler ${ }^{1 *}$, Ivana Marinović ${ }^{2,3}$, Željko Večenaj $^{2}$ and Branko Grisogono ${ }^{2}$ \\ ${ }^{1}$ Meteorological and Hydrological Service of Croatia, Zagreb, Croatia, ${ }^{2}$ AMGI, Department of Geophysics, Faculty of Science, \\ University of Zagreb, Zagreb, Croatia, ${ }^{3}$ Department of Environmental Sciences, University of Virginia, Charlottesville, VA, USA
}

The Prandtl model succinctly combines the 1D stationary boundary-layer dynamics and thermodynamics of simple anabatic and katabatic flows over uniformly inclined surfaces. It assumes a balance between the along-the-slope buoyancy component and adiabatic warming/cooling, and the turbulent mixing of momentum and heat. In this study, energetics of the Prandtl model is addressed in terms of the total energy (TE) concept. Furthermore, since the authors recently developed a weakly nonlinear version of the Prandtl model, the TE approach is also exercised on this extended model version, which includes an additional nonlinear term in the thermodynamic equation. Hence, interplay among diffusion, dissipation, and temperature-wind interaction of the mean slope flow is further explored. The TE of the nonlinear Prandtl model is assessed in an ensemble of solutions where the Prandtl number, the slope angle and the nonlinearity parameter are perturbed. It is shown that nonlinear effects have the lowest impact on variability in the ensemble of solutions of the weakly nonlinear Prandtl model when compared to the other two governing parameters. The general behavior of the nonlinear solution is similar to the linear solution, except that the maximum of the along-the-slope wind speed in the nonlinear solution reduces for larger slopes. Also, the dominance of $P E$ near the sloped surface, and the elevated maximum of $K E$ in the linear and nonlinear energetics of the extended Prandtl model are found in the PASTEX-94 measurements. The corresponding level where KE>PE most likely marks the bottom of the sublayer subject to shear-driven instabilities. Finally, possible limitations of the weakly nonlinear solutions of the extended Prandtl model are raised. In linear solutions, the local storage of TE term is zero, reflecting the stationarity of solutions by definition. However, in nonlinear solutions, the diffusion, dissipation and interaction terms (where the height of the maximum interaction is proportional to the height of the low-level jet by the factor $\approx 4 / 9$ ) do not balance and the local storage of TE attains nonzero values. In order to examine the issue of non-stationarity, the inclusion of velocity-pressure covariance in the momentum equation is suggested for future development of the extended Prandt| model.

Keywords: katabatic flow, anabatic flow, Prandtl model, nonlinear solution, total energy 


\section{INTRODUCTION}

Katabatic and anabatic winds are downslope and upslope flows that form when a density difference between the air near the slope and the nearby atmosphere develops at the same height. This type of flow is often observed in regions of complex orography and substantially affects the weather and climate in these regions (e.g., Poulos and Zhong, 2008). The topic of katabatic and anabatic wind is being actively explored and the work on its understanding includes the application of numerical models (direct numerical simulations (DNS): (e.g., Shapiro and Fedorovich, 2008); large eddy simulations (LES): e.g., Skyllingstad, 2003; Smith and PortéAgel, 2013); mesoscale models: (e.g., Smith and Skyllingstad, 2005; Zammett and Fowler, 2007); and analytical models (e.g., Prandtl, 1942; Defant, 1949; Grisogono and Oerlemans, 2001; Zardi and Serafin, 2014). Continued interest in katabatic and anabatic winds stems from the important effects of this type of orographic flows on visibility and fog formation, air pollutant dispersion, agriculture and energy use, fire-fighting operations, sea-ice formation, etc. (e.g., Shapiro and Fedorovich, 2014, and references therein). Katabatic winds develop in stably stratified planetary boundary layers (PBLs), adding an additional level of complexity to the problem of understanding and modeling this specific type of PBLs (e.g., Mahrt, 1998; Holtslag et al., 2013; Sandu et al., 2013; Mahrt, 2014; Sun et al., 2015). In reality, a strong surface heat surplus may contribute to a high Rayleigh number and initiation of free convection over the horizontal plane (e.g., Princevac and Fernando, 2007). This condition may limit the general applicability of the Prandtl model and its extensions to the case of anabatic flow for a large surface temperature surplus. However, Defant (1949) and Fedorovich and Shapiro (2009a,b) as well as several other authors, show clearly that the Prandtl model is applicable, at least qualitatively, to anabatic flow. Although the latter authors state that turbulent anabatic flows differ more, in a mean qualitative sense, from its Prandtl model version for katabatic flows, they still show and claim the overall applicability of the Prandtl model (at least qualitatively) to both flow types. In parallel to current theoretical and numerical modeling efforts, large observational campaigns and programs over complex orography should be of a high priority in order to better understand the nature of thermally driven slope flows (e.g., Poulos and Zhong, 2008; Fernando et al., 2015; Grachev et al., 2015).

In the model of Prandtl (1942), katabatic flow is the result of a balance between the along-slope buoyancy force and adiabatic warming/cooling, and normal-to-slope turbulent fluxes of momentum (i.e., friction) and heat (i.e., diffusion), respectively, in an otherwise motionless and statically stable background atmosphere. This paper starts with the classical theoretical model of slope flows developed by Prandtl (1942), somewhat modified and verified by Defant (1949), who deployed it specifically for anabatic flow (see also Zardi and Whiteman, 2013), and an extended Prandtl model that includes weakly nonlinear effects as done in Grisogono et al. (2015). It includes the standard concepts of potential, kinetic and total energy, now for katabatic and anabatic flows. In the energetics framework, wind speed and temperature perturbations are linked in one equation (i.e., the total energy equation) and the conservation and conversion properties of energy components are of special concern in various research problems (e.g., the effect of turbulent mixing may be parameterized in terms of kinetic energy). The energy approach applied here is motivated by the total turbulent energy concept developed by e.g., Mauritsen et al. (2007), where kinetic energy is related to turbulent wind perturbations, while potential energy is related to turbulent potential temperature perturbations. In our case, we focus only on mean katabatic and anabatic flows that are present over sloped surfaces. The difference, when compared to Mauritsen et al. (2007), is in our focus not being on the turbulent part of the flow but on the wind and temperature finite amplitude deviations from the background state coming from katabatic/anabatic flows. In this sense, our approach is similar to the energy framework of katabatic winds applied by Smith and Skyllingstad (2005). While, Smith and Skyllingstad (2005) define kinetic energy in the same way as Mauritsen et al. (2007), their potential energy is defined as a linear function of both temperature perturbations and the height above the slope. Although there are some differences in the literature concerning the definition of potential energy, it is typically a function of potential temperature perturbations. Potential temperature perturbations, under the assumptions of hydrostatic and adiabatic motion, include the effects of absolute temperature perturbations and changes in the distance from the surface (e.g., DeCaria, 2007). The total energy is then the sum of kinetic and potential contributions.

We limit ourselves only to the linear and weakly nonlinear solution of the (extended) Prandtl model. A detailed description of the extended Prandtl model is presented in Grisogono et al. (2015; their Section 2). The new term that extends the original Prandtl model is presumably weak and regulated by the nonlinearity parameter $\varepsilon$. Our approach is relatively simple and general, and may be applied to solutions of Prandtl-type models that include 3D effects (e.g., Burkholder et al., 2009; Shapiro et al., 2012), effects of the Coriolis force (e.g., Stiperski et al., 2007), time-dependent types of solutions (e.g., Zardi and Serafin, 2014), effects of vertically varying turbulent mixing coefficients (e.g., Grisogono and Oerlemans, 2001; Grisogono et al., 2015), etc. To sum up, this study combines the work of Mauritsen et al. (2007) and Grisogono et al. (2015), i.e., the energy concept and weak nonlinearity, respectively, to shed more light on the physics of simple slope flows.

This study is independent but based on the work of Grisogono et al. (2015). There it was shown that with the weakly nonlinear Prandtl model one obtains solutions with stronger near-surface stratification and weaker katabatic wind speed (with both constant and variable eddy heat conductivity). However, although more realistic, the solutions of the weakly nonlinear model were not superior to the linear solutions when compared to limited observations. The nonlinearity affected low-level jet strength and elevation in katabatic, but also anabatic, flows. In anabatic flow, in contrast to katabatic flow, it enhanced the lowlevel jet. The consequences of the introduced nonlinearity on the model energetics will be explored in this paper.

The goal of this study is to evaluate an ensemble of linear and weakly nonlinear solutions of the (extended) Prandtl model for katabatic and anabatic flows, and to examine the model energetics related to these solutions. In order to explore the 
sensitivity of our results to several model assumptions, we present a set of solutions where three governing parameters are perturbed: (1) the turbulent Prandtl number $\operatorname{Pr}$, (2) the slope angle $\alpha$, and (3) the so-called nonlinearity parameter $\varepsilon$ as defined in Grisogono et al. (2015). We will present certain characteristics of the solutions of the Prandtl model, the vertical profiles of kinetic $K E$, potential $P E$ and total energy $T E$, and the governing terms in the total energy TE equation.

The structure of the paper is as follows. In Section Methodology, we present the governing equations of our model and define the ensemble of solutions. In Section Results, the solutions of the (extended) Prandtl model are described, with a specific focus on the variability in the ensemble of solutions and impacts on the model energetics. Some specific differences between the nonlinear and linear solutions, as well as the limitations of our extended Prandtl model are discussed in Section Discussion. The paper is finalized in Section Summary and Conclusions, where the summary and outlook are presented.

\section{METHODOLOGY}

We first present the governing equations of the Prandtl model and develop a simple, basic energy framework where wind and potential temperature are linked with the concepts of kinetic, potential and total energy. The full description of the system would include the energy components of not only the mean slope flow, but of the background atmosphere and the turbulent part of the slope flow, and their interactions. We limit our analysis only to the part of the slope flow described by the Prandtl model, i.e., the mean slope flow with relatively large eddy diffusivity and conductivity; hence, the model may emulate a simple turbulent slope flow (Defant, 1949; Stiperski et al., 2007; Grisogono et al., 2015).

\section{Governing Equations}

Potential temperature and wind can be decomposed into $\theta=$ $\theta_{r}+\bar{\theta}+\theta^{\prime}$ and $u=u_{r}+\bar{u}+u^{\prime}$, where $\theta_{r}=\theta_{0}+\gamma z$ is the potential temperature of the background atmosphere having the vertical gradient $\gamma$ (in true vertical coordinate $Z$ ), and $\theta_{0}$ is the surface potential temperature in a statically stable background atmosphere (e.g., Zardi and Serafin, 2014). The background atmosphere is motionless: $u_{r}=0$. Next, $u^{\prime}$ and $\theta^{\prime}$ are turbulent perturbations of the wind speed and potential temperature of the slope flow, while $\bar{u}$ and $\bar{\theta}$ represent the mean finite-amplitude wind speed and potential temperature (here, averaging is defined in the Reynolds sense).

The governing equations of the Prandtl model, including the weak nonlinearity extension (without invoking the steady-state assumption for the moment) are:

$$
\begin{gathered}
\frac{\partial \bar{u}}{\partial t}=g \frac{\bar{\theta}}{\theta_{0}} \sin (\alpha)+K \operatorname{Pr} \frac{\partial^{2} \bar{u}}{\partial z^{2}} \\
\frac{\partial \bar{\theta}}{\partial t}=-\left(\gamma+\varepsilon \frac{\partial \bar{\theta}}{\partial z}\right) \bar{u} \sin (\alpha)+K \frac{\partial^{2} \bar{\theta}}{\partial z^{2}}
\end{gathered}
$$

where $g$ is acceleration due to gravity, $K$ is the eddy heat conductivity, $\operatorname{Pr}$ is the turbulent Prandtl number (all assumed constant in this study), and $z$ is the coordinate perpendicular to the constant slope surface with the slope angle $\alpha$. Parameter $\varepsilon$ controls the feedback of the flow-induced potential temperature gradient on to the corresponding background gradient, $\gamma$, because the former, below the low-level jet, can be 20-50 times stronger (in the absolute sense) than the latter (e.g., Grisogono and Oerlemans, 2001; Grisogono et al., 2015). $\varepsilon$ is an external parameter, roughly limited by the model input parameters, not by the model dynamics, and it pertains to the regular perturbation analysis used here [(Bender and Orszag, 1978; Grisogono et al., 2015); see also Supplementary Materials 1 in this study]. After multiplying Equation (1) by $\bar{u}$, multiplying Equation (2) by $\bar{\theta} g /\left(\theta_{0} \gamma\right)$ or $\bar{\theta} a$, and adding the resulting two equations, the energy equation of the extended Prandtl model is attained:

$$
\begin{aligned}
& \frac{\partial}{\partial t}\left(\frac{\bar{u}^{2}+a \bar{\theta}^{2}}{2}\right)= \\
& \underbrace{K \frac{\partial^{2}}{\partial z^{2}}\left(\frac{\operatorname{Pr} \bar{u}^{2}+a \bar{\theta}^{2}}{2}\right)}_{D I F}-K[\underbrace{\left[r\left(\frac{\partial \bar{u}}{\partial z}\right)^{2}+a\left(\frac{\partial \bar{\theta}}{\partial z}\right)^{2}\right]}_{D I S} \\
& -\underbrace{\operatorname{a\varepsilon sin}(\alpha)\left(\bar{u} \bar{\theta} \frac{\partial \bar{\theta}}{\partial z}\right)}_{I N T}
\end{aligned}
$$

where the left side term is the local storage term of $T E$ of the mean slope flow defined as the sum of kinetic $K E=\overline{u^{2}} / 2$ and potential energy $P E=a \bar{\theta}^{2} / 2$ per unit mass (cf. Smith and Skyllingstad, 2005; Mauritsen et al., 2007). The three terms on the right side are described as diffusion (DIF), dissipation (DIS), and interaction (INT) terms: DIF represents the diffusion of TE by the turbulent flow, DIS represents the dissipation of TE, and INT represents the interaction of the slope flow with the background atmosphere in the case of the weakly nonlinear model. Note that INT is equal to $\varepsilon \sin (\alpha)\left(\bar{u} \frac{\partial P E}{\partial z}\right)$, which can be interpreted as the slope-normal (i.e., nearly vertical) transport of potential energy. This term does not exist in the linear model.

Four types of steady-state solutions of Equations (1) and (2) are analyzed in Grisogono et al. (2015). They include linear and weakly nonlinear solutions with turbulent mixing coefficients either constant or vertically varying. In this paper, a subset of these solutions is analyzed (from now on, the overbar is removed from potential temperature $\bar{\theta}$ and wind speed $\bar{u}$ of katabatic/anabatic flow): (1) the linear solution with the constant turbulent diffusivity profile $\theta_{\text {LIN }}$ and $u_{\text {LIN }}$, and (2) the nonlinear solution with the constant turbulent diffusivity profile $\theta_{N O L I N}$ and $u_{\text {NOLIN }}$. Initial results concerning the vertical variability of $K$ and its impact on energy distribution show sensitivity to the formulation of $K(z)$ and strong non-stationarity even in the linear case; thus, a detailed analysis of this subset of solutions is left for future study. For simplicity, here we show only the classical solutions of the Prandtl model $\theta_{\text {LIN }}$ and $u_{L I N}$ [for the nonlinear 
solutions please refer to Grisogono et al. (2015)]:

$$
\begin{aligned}
& \theta_{\text {LIN }}=\operatorname{Cexp}\left(\frac{-z}{h_{P}}\right) \cos \left(\frac{z}{h_{P}}\right) \\
& u_{\text {LIN }}=-\mu \operatorname{Cexp}\left(\frac{-z}{h_{P}}\right) \sin \left(\frac{z}{h_{P}}\right)
\end{aligned}
$$

Following, e.g., Grisogono et al. (2015), $C$ is the surface potential temperature deficit $\theta_{\text {LIN }}(z=0)=C<0$ for the katabatic flow (or the corresponding temperature surplus in anabatic flow $\left.\theta_{\text {LIN }}(z=0)=C>0\right), \mu=\left[g /\left(\gamma \theta_{0} \operatorname{Pr}\right)\right]^{1 / 2}, h_{P}=2^{1 / 2} / \sigma$ ( $h_{P}$ can be interpreted as a characteristic depth of the Prandtl layer $), \sigma=\left[N \sin (\alpha) /\left(\operatorname{KPr}^{1 / 2}\right)\right]^{1 / 2}(\sigma$ can be interpreted as a characteristic inverse length scale), $N^{2}=\gamma g / \theta_{o}$ is background buoyancy frequency squared, and $K$ is the average eddy heat conductivity [in our case $K=$ const in Equations (1) and (2)]. The slope flow is assumed to be no-slip (i.e., $u_{L I N}(z=0)=0$ ).

In the case of linear and stationary flow, Equation (3) simplifies to:

$$
0=\frac{\partial^{2}}{\partial z^{2}}\left(\frac{\operatorname{Pr}_{L I N}^{2}+a \theta_{L I N}^{2}}{2}\right)-\left[\operatorname{Pr}\left(\frac{\partial u_{L I N}}{\partial z}\right)^{2}+a\left(\frac{\partial \theta_{L I N}}{\partial z}\right)^{2}\right]
$$

and one can easily check the equality by inserting Equations (4) and (5) into Equation (6). In the rest of the paper, we determine vertical derivatives using finite differences in the case of both linear and weakly nonlinear types of solution.

\section{Ensemble of Solutions}

We evaluate the sensitivity of our solutions to the slope angle $\alpha$, the value of the Prandtl number $\operatorname{Pr}$ and the nonlinearity parameter $\varepsilon$. Based on Grisogono et al. (2015), the basic values of these model parameters are $\alpha=-0.1 \mathrm{rad}, \operatorname{Pr}=2$ and $\varepsilon$ $=0.005 / 0.03$ (katabatic/anabatic flow) where the justification of these parameter choices is discussed in more detail by Grisogono et al. (2015). The starting values of all three parameters are taken from Grisogono et al. (2015), where linear and nonlinear solutions reproduced well the observations from the PASTEX-94 experiment (van den Broeke, 1997a,b; Oerlemans and Grisogono, 2002). An ensemble of solutions is generated by evaluating them for this basic set of parameters and also when they change in amplitude by $\pm 25 \%$ (this adds up to 27 solutions in the case of nonlinear katabatic and anabatic flows, and 9 solutions in the case of linear katabatic and anabatic flows). This ensemble will be used to examine the sensitivity of our solutions to moderate variations in the basic model assumptions. Other model parameters follow those from Grisogono et al. (2015): $\gamma=3 \mathrm{~K} / \mathrm{km}, \theta_{o}=273.2 \mathrm{~K}, C$ $=-6 \mathrm{~K}(+6 \mathrm{~K})$ in katabatic (anabatic) flow, and $K=0.06 \mathrm{~m}^{2} / \mathrm{s}$ $\left(3.0 \mathrm{~m}^{2} / \mathrm{s}\right)$ in katabatic (anabatic) flow.

\section{RESULTS}

\section{Katabatic Flow}

(a) Linear case

The vertical profiles of $\theta_{\text {LIN }}$ and $u_{\text {LIN }}$ for katabatic flow are shown in Figure 1A. The potential temperature profile reveals a statically stable profile, with $\theta_{\text {LIN }}$ increasing in the first $30 \mathrm{~m}$ above the slope. At the same time, $u_{\text {LIN }}$ starts from the no-slip condition at the sloped surface, attains a local maximum (i.e., a low-level jet is formed at the height $h_{j}$ ) and slows progressively upwards. The corresponding vertical profiles of kinetic $K E$, potential $P E$ and total $T E=K E+P E$ energy for the katabatic flow in the case of the linear solution are shown in Figure 1B. Near the surface, TE is dominated by $P E$ and surface forcing (quantified through the surface temperature deficit $C$ ). There is a perfect balance between DIF and DIS in the energy budget, Figure $1 \mathrm{C}$. The wind speed $u_{\text {LIN }}$ profile leads to a corresponding kinetic energy profile with its maximum in the first $15 \mathrm{~m}$. We proceed next with the evaluation of the sensitivity of the ensemble of solutions for the katabatic flow described by the linear model.

The following three heights are of interest to us:

(1) The height of the low-level jet $h_{j}$. This is the maximum of $u(z)$ which occurs at $h_{j}=\pi / 4 h_{P}$ in the linear solutions, i.e., it increases with increasing $P r$ and decreases with an increasing slope (see also, Figure 1D). At the same time, the maximum $u_{\text {LIN }}$ is insensitive to the slope angle and decreases with increasing $\operatorname{Pr}$ (this can be shown by inserting $h_{j}$ in Equation 5) as is confirmed in Figure S3.1A (please note that lines are shifted by the amount \pm 0.5 from the reference slope angle for presentation purposes).

(2) The depth of the stable (in the anabatic case, unstable) layer. At the top of the stable layer $d \theta / d z=0$, and this height equals $3 h_{P}$. It also increases with increasing $P r$ and decreases with an increasing slope in the linear solution (Figure 1E).

(3) The level where $K E$ starts to dominate over $P E$ (TE is primarily governed by $P E$ close to the surface, while $K E$ becomes larger than $P E$ somewhere above $h_{j}$ ). For the linear solutions of katabatic (and anabatic) flow, one can show (by setting the condition $K E / P E=1$ ) that the height where $K E$ starts to dominate equals $h_{P} \cos ^{-1}\left([1 /(1+P r)]^{1 / 2}\right)$; i.e., it also increases with increasing $\mathrm{Pr}$ and decreases with an increasing slope angle (Figure 1F). This level is directly linked, though in a nonlinear way, to the gradient Richardson number, which is significantly smaller than 1 , and the consequent onset of dynamic flow instabilities (e.g., Grisogono 2003). At the same time, the amplitude at which $K E$ starts to dominate is insensitive to the choice of slope (Figure S3.1E; lines are shifted by the amount \pm 0.5 from the reference slope angle for presentation purposes). This behavior of the $K E$ is by definition directly linked to the behavior of $u_{L I N}$. More details about this measure are presented in Supplementary Materials 2.

\section{(b) Nonlinear case}

The deviation of the nonlinear from the linear solution for katabatic flow is presented in Figure 2. The general characteristics of $\theta_{\text {NOLIN }}$ and $u_{\text {NOLIN }}$ profiles are equivalent to $\theta_{L I N}$ and $u_{L I N}$, and their corresponding $K E, P E$, and TE profiles are also similar. The nonlinear solution has slightly lower wind speeds and higher potential temperature (i.e., lower potential temperature anomalies; Figure $\mathbf{2 A}$ ) and this leads to lower $K E$, 

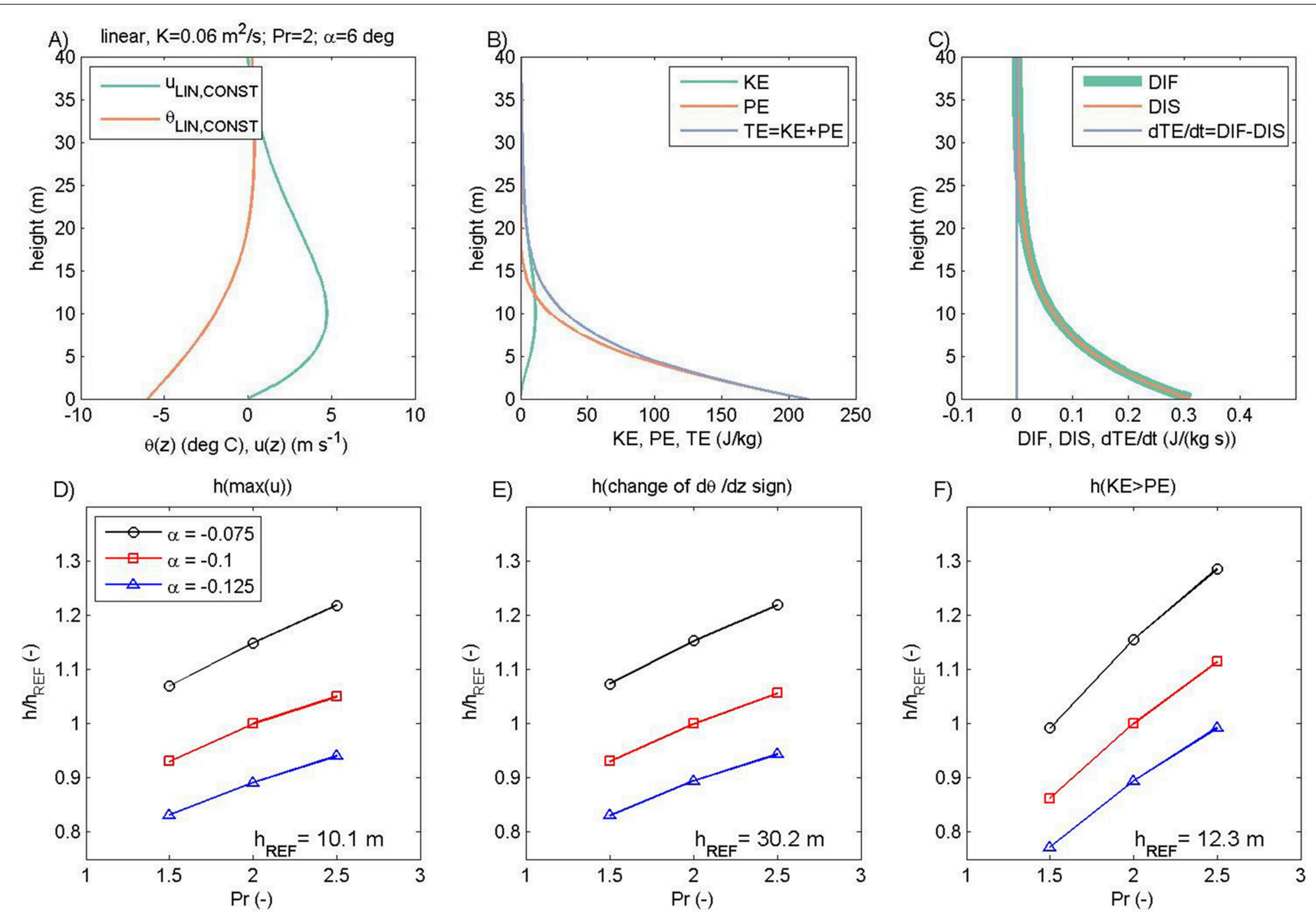

FIGURE 1 | Vertical profiles of potential temperature $\theta$ and wind speed $u$ in the linear solution of the Prandtl model for the katabatic flow (A), corresponding kinetic energy KE, potential energy PE and total energy TE (B), and diffusion DIF, dissipation DIS and storage terms $\partial T E / \partial t$ (C). The height of the low-level jet $h_{j}(\mathbf{D})$, height of the stability change $(\mathbf{E})$ and height at which $K E$ becomes larger than $P E(\mathbf{F})$ as function of Prandtl number $\operatorname{Pr}(\mathrm{x}$ axis) and slope angle $\alpha$ (different color). Heights in (D-F) are relative to the reference heights $h_{R E F}$ from the solutions when $\operatorname{Pr}=2$ and $\alpha=-0.1 \mathrm{rad}$.

$P E$, and TE (Figure $2 \mathbf{B}$ ). However, the vertical profiles of DIS and DIF do not overlap as in linear cases and are slightly larger in the nonlinear case (Figure 2C). Also, in the nonlinear case, the interaction term INT is present. Its amplitude is much lower than the other two governing terms in the energy equation. More importantly, the TE storage term is nonzero and this will be discussed later, in Section Discussion.

We also examine the sensitivity of the nonlinear solution to the choices of $\operatorname{Pr}$ and $\alpha$. Additionally, we examine the impact of the nonlinearity term $\varepsilon$, starting with $\varepsilon=0.005$ and modifying this value by $\pm 25 \%$. Nonlinear effects have the lowest impact on variability in the ensemble of 27 solutions of the weakly nonlinear Prandtl model when compared to the other two governing parameters (Figures 2D-F). The general behavior of the nonlinear solution is similar to that of the linear solution, except that the maximum $u_{N O L I N}$ (and the corresponding maximum $K E$ ) is moderately reduced for larger slopes (while the maximum $u_{\text {LIN }}$ is constant; cf. Figures S3.1A,E vs. Figures $\mathrm{S} 3.1 \mathrm{~B}, \mathrm{~F})$. This aspect of the low-level jet in the nonlinear solution is shared by LES simulations in e.g., Grisogono and Axelsen
(2012) and will be explored in future studies. The increase in $\varepsilon$ reduces all three heights (Figures $2 \mathrm{D}-\mathrm{F}$ ) and amplitudes of the maximum wind speed and KE (Figures S3.1B,F).

\section{Anabatic Flow}

In this subsection we present a general overview of anabatic flow solutions from the linear and weakly nonlinear Prandtl model. The main difference when compared to katabatic flow is the existence of the surface temperature surplus that induces the anabatic flow (now $+6 \mathrm{~K}$; cf. Defant, 1949). This change in the surface boundary condition is related to the corresponding increase in eddy heat conductivity from $K=0.06 \mathrm{~m}^{2} / \mathrm{s}$ to $K=3.0 \mathrm{~m}^{2} / \mathrm{s}$ and the increase of the nonlinearity parameter from $\varepsilon=0.005$ to $\varepsilon=0.03$, as explained in Grisogono et al. (2015): since $\max (\varepsilon) \sim \gamma h_{P} /|C|$, then $\varepsilon_{\text {Anabatic }} / \varepsilon_{\text {Katabatic }} \sim$ $\left(K_{\text {Anabatic }} / K_{\text {Katabatic }}\right)^{1 / 2}$. With this choice of $\varepsilon$, perturbations to the linear solution are present, but the general structure of the solution does not change. Although anabatic upslope winds are generally deeper than typical katabatic flows, in our comparisons the same amplitude of potential temperature deviations at the 

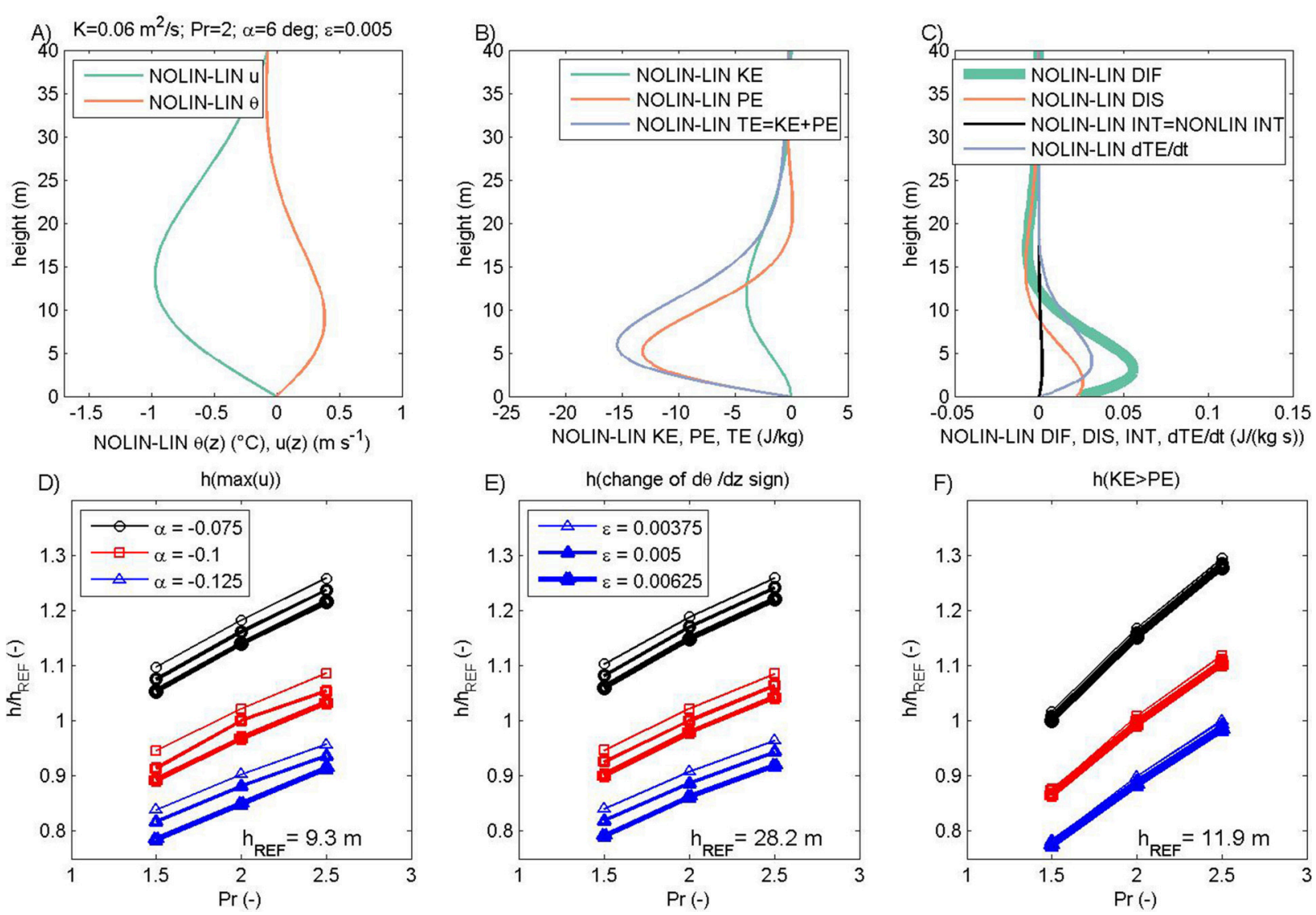

FIGURE 2 | (A-C) Differences between nonlinear and linear (Figure 1) solutions of the (extended) Prandtl model (cf. Grisogono et al., 2015). (D-F) are equivalent to (D-F) in Figure 1. Also, sensitivity to the nonlinearity parameter $\varepsilon$ (with increasing line thickness as $\varepsilon$ is increased) is included in (D-F), while (C) includes the vertical profile of the interaction term INT that is equal to zero in the linear case. The reference heights $h_{R E F}$ are based on the solutions when $\operatorname{Pr}=2, \alpha=-0.1$ rad and $\varepsilon=$ 0.005 .

surface is set so that the same potential energy of the slope flow $P E$ is found at the surface. This is also reflected in the similar range of amplitudes of the analyzed measures in subsections Katabatic Flow and Anabatic Flow, but for anabatic flow the maximum values of the analyzed heights are typically an order of magnitude larger.

\section{(a) Linear case}

The vertical profiles of the upslope wind $u_{L I N}$, potential temperature deviations $\theta_{L I N}, K E, P E$, and TE, and, finally, the terms in the total energy equation related to diffusion $D I F$, dissipation DIS and local storage $\partial T E / \partial t$ of $T E$ are shown in Figures 3A-C. All vertical profiles are equivalent to their katabatic counterpart in terms of the general structure (cf. Figure 1). The sensitivity of the low-level jet height, the level where the change in the local static stability occurs, and the level where $K E$ starts to dominate over $P E$ are equivalent to those in the linear katabatic case (cf. Figures 1D-F vs. Figures 3D-F).

(b) Nonlinear case

The nonlinear solution of anabatic flow is described in this subsection. When compared to its katabatic counterpart, the vertical profiles of along-the-slope wind speed and potential temperature have the same general structure and this is also the case for kinetic, potential and total energy of the nonlinear vs. linear solution. However, all three energy components $(K E$, $P E$, and $T E$ ) are increased in the nonlinear anabatic solution, when compared to the linear solution (Figure 4B). This is a consequence of the increased wind speeds (in absolute terms) and increased potential temperature of anabatic flow (Figure 4A). As for the linear case of anabatic flow, the nonlinear anabatic flow is extended over a deeper layer, so both the low-level jet and inversion height are higher than in the corresponding katabatic flow. As discussed later, the increase in the basic $\varepsilon$ up to $\varepsilon=$ 0.03 is the reason for the substantial rise in the magnitude of the interaction INT and total energy TE local storage terms $\partial T E / \partial t$ (Figure 4C). In contrast to katabatic flow, the TE diffusion DIF now departs from the dissipation DIS toward lower values. Also, while in katabatic flow the small amplitude of INT and the imbalance between DIF and DIS makes $\partial T E / \partial t$ become nonzero, in anabatic flow it is the sign and amplitude of the interaction term INT that dominates the production of TE.

The sensitivity of the selected height measures to $\mathrm{Pr}$ and slope angle is the same as for the linear anabatic case (and also for both katabatic types of solutions; Figures 4D-F). The main difference is found concerning the selection of $\varepsilon$. In contrast 

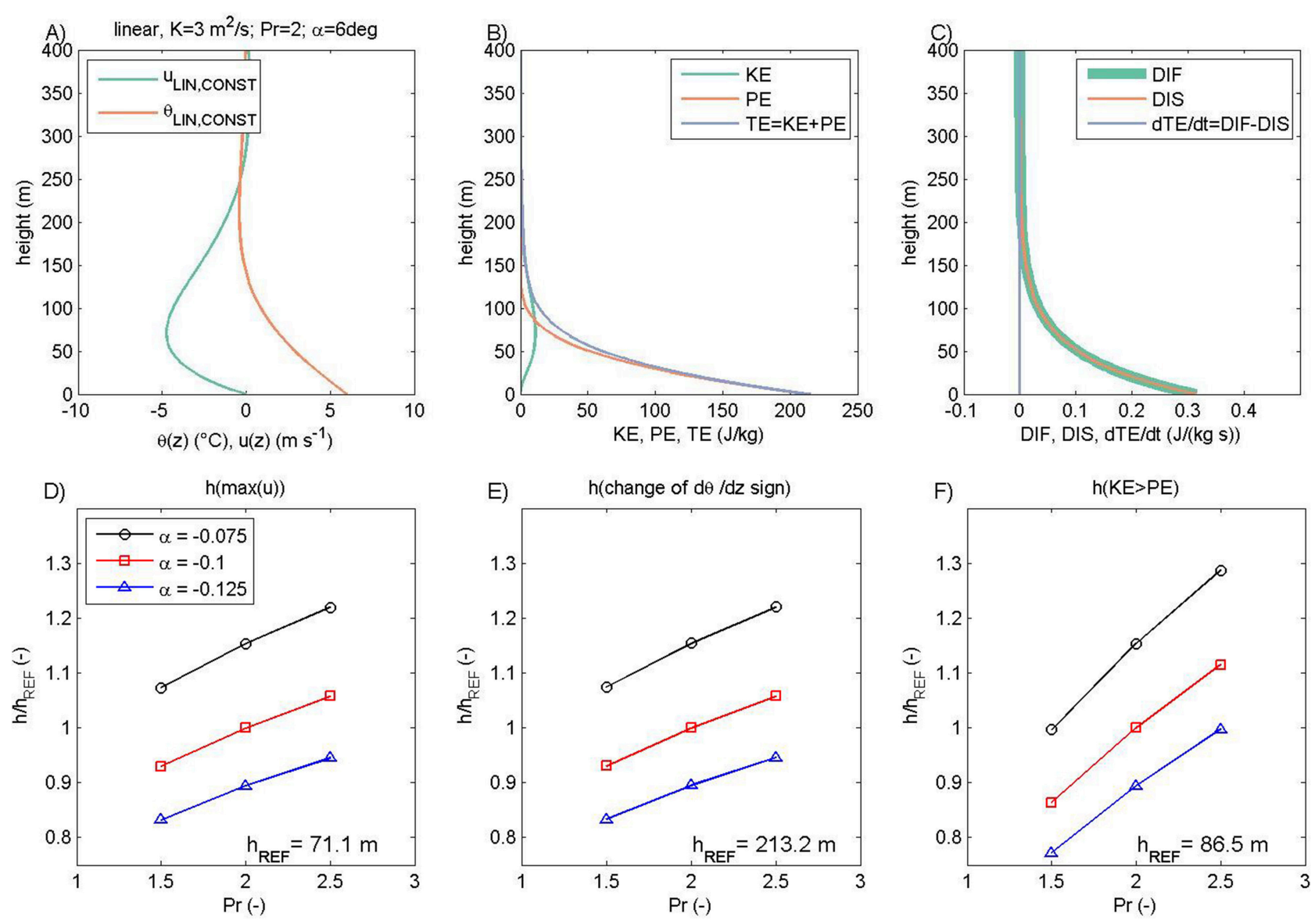

FIGURE 3 | Same as Figure 1 but for anabatic flow.

to the katabatic nonlinear case, in the anabatic nonlinear case the increase in $\varepsilon$ leads to: (1) a rise in the low-level jet height and speed (Figure 4D), (2) a rise in the inversion height (in anabatic flow a transition occurs from statically unstable to stable conditions) that is not substantial for the selected range of control parameters (Figure 4E), (3) low sensitivity of the height where $K E$ dominates over $P E$ to the nonlinearity parameter $\varepsilon$ (which can be neglected for the purposes of this study; Figure 4F).

Common to all previous solutions, while the maximum in $K E$ is attained at levels of maximum along-the-slope wind speed, $K E$ becomes larger than $P E$ above this level of maximum $K E$ (cf. Figure 4F vs. Figure 5D). At the same time, the amplitude at which $K E$ starts to dominate increases slightly as the slope increases (Figure $\mathrm{S} 3.1 \mathrm{H}$ ). The increase in $\varepsilon$ also increases the amplitude of $K E$ where it becomes larger than $P E$ (in contrast to katabatic nonlinear flow), and this sensitivity to $\varepsilon$ is comparable to the sensitivity to the slope angle $\alpha$ (Figure $\mathrm{S} 3.1 \mathrm{H})$. In summary, $K E$ dominates over $P E$ above $h_{p} \cos ^{-1}\left[1 /(1+P r)^{1 / 2}\right]$ and this height is usually between $h_{j}$ and $2 h_{j}$. It is related to the corresponding gradient Richardson number, which compares the vertical gradients of $P E$ vs. $K E$. When the Richardson number falls substantially below 1 , dynamic instabilities might occur in the corresponding sublayer (see also Supplementary Materials 2).

\section{Energetics: Katabatic and Anabatic Flows}

The potential energy maximum (PEmax) and total energy maximum (TEmax) are found at the lowest level in linear and nonlinear solutions for both anabatic and katabatic flows (insensitive to the choice of $\alpha, \operatorname{Pr}$, and $\varepsilon$ ). Also, the amplitude of PEmax and TEmax equals $215.4 \mathrm{~J} / \mathrm{kg}$ in all cases (Figures 1-4, panel B). The same amplitude of PEmax and TEmax in both katabatic and anabatic flows is a result of the same temperature anomaly at the surface (but with a different sign, i.e., $\pm 6 \mathrm{~K}$ in this study).

At the same time, the kinetic energy maximum (KEmax) is sensitive to choices in our parameter space and set of solutions (Figure 5). The height of KEmax (equivalent to the low-level jet height $h_{j}$ ) increases when $\operatorname{Pr}$ varies from $\operatorname{Pr}=1.5$ to $\operatorname{Pr}=$ 2.5, and it decreases when $|\alpha|$ is increased (Figures 5A-D). In the case of katabatic flow, the height of KEmax is within a similar range for both the linear (Figure 5A) and nonlinear case (the solutions are only slightly sensitive to $\varepsilon$; Figure $5 B$ ). In the case of anabatic flows, a similar structure of solutions is found, only over deeper layers than for katabatic flows (Figure 5C). 


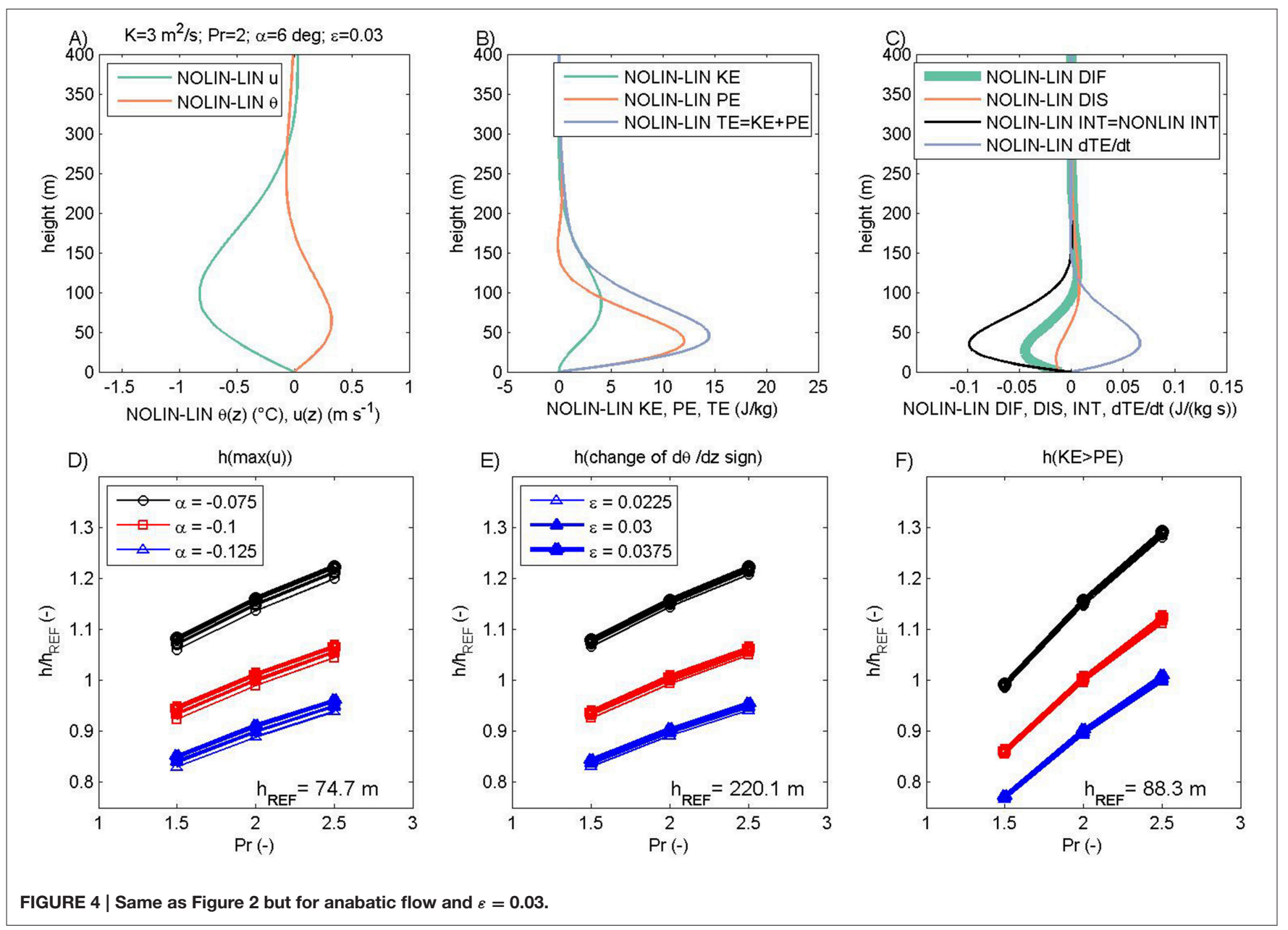

While all solutions behave in a consistent way with respect to $\operatorname{Pr}$ and $\alpha$, there is a contrasting response to the increase in $\varepsilon$ in nonlinear solutions: as $\varepsilon$ is increased, the height and amplitude of KEmax reduce in katabatic flow (Figures $5 \mathbf{B}, \mathbf{F}$ ), while they rise in anabatic flow (Figures 5D,H). The latter contrast occurs because the low-level jet height and amplitude, i.e., $h_{j}$ and $u_{\text {max }}$, show a similar sensitivity to $\varepsilon$. Grisogono et al. (2015) showed that an $\varepsilon$ increase leads to an $h_{j}$ and $u_{\max }$ decrease in the nonlinear katabatic solution, and an $h_{j}$ and $u_{\max }$ increase in the nonlinear anabatic solution.

The amplitude of KEmax in linear solutions (both katabatic and anabatic) is the only function of the $\operatorname{Pr}$ (it collapses to approximately the same values for different slopes and, more interestingly, the same structure is present for both katabatic and anabatic solutions; Figures 5E,G for presentation purposes the lines are shifted \pm 0.5 from the reference slope angle). However, in the nonlinear case, sensitivity to all three parameters is present: (1) the reduction of KEmax with increasing $P r$, which is classical Prandtl model behavior, (2) the reduction of KEmax when increasing $\alpha$ and $\varepsilon$ in katabatic flow (Figure 5F), as explained just above, but (3) an increase in KEmax when increasing the nonlinearity in anabatic flow (Figure 5H). The sensitivity of KEmax to $\operatorname{Pr}$ and $\alpha$ is expected from the formulation of KEmax in the linear solution (Equation 5) and the similarity of the linear and nonlinear vertical profiles.

The diffusion maximum (DIFmax) and dissipation maximum (DISmax) are found at the surface level in linear and nonlinear solutions for both anabatic and katabatic flows (insensitive to the choice of $\alpha, \operatorname{Pr}$, and $\varepsilon$ ). This is the simple consequence of the more intense wind and temperature vertical changes near the slope surface. In contrast to PEmax and TEmax, and similar to KEmax, the amplitude of both DIFmax and DISmax is sensitive to the Prandtl number $P r$, slope angle $\alpha$ and the nonlinearity parameter $\varepsilon$ : DIFmax and DISmax (1) reduce when Pr increases, (2) increase when the slope increases, and (3) are only slightly sensitive to increasing $\varepsilon$. DIFmax and DISmax vary in a common range in the linear and nonlinear solutions for both anabatic and katabatic flows (Figure S3.2).

In the case of nonlinear solutions for anabatic and katabatic flow, the additional interaction term is present. Both the amplitude and height of the interaction term maximum INTmax are functions of all three parameters (see Figures 6A,B for INTmax height and Figures 6E,F for INTmax amplitude). The sensitivity of the amplitude and height of INTmax shows a behavior similar to KEmax: in katabatic flow, the height of INTmax varies from $\sim 3 \mathrm{~m}$ to $\sim 5 \mathrm{~m}$, while in anabatic flow from 

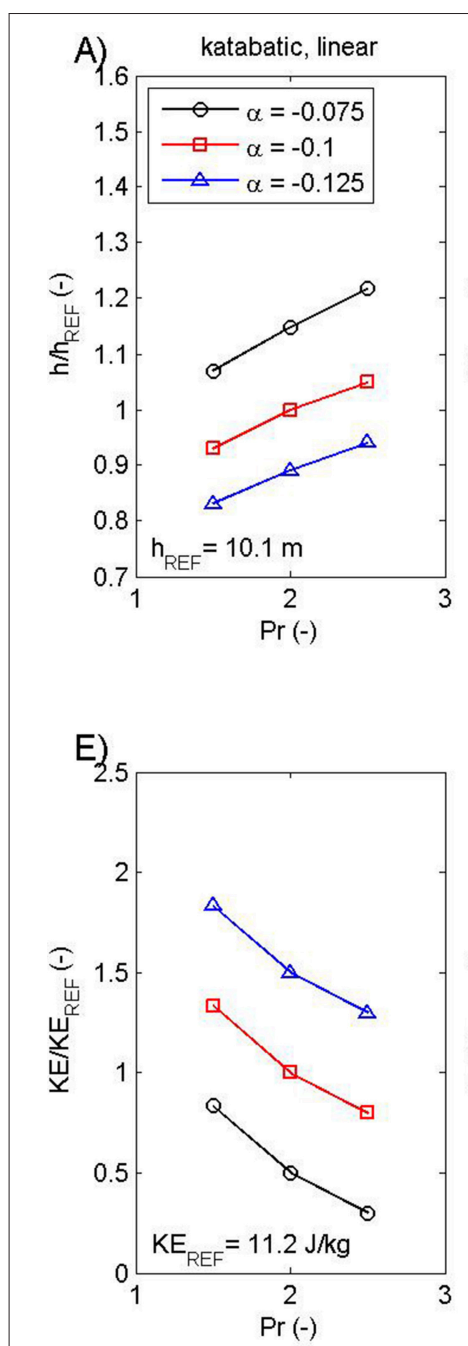
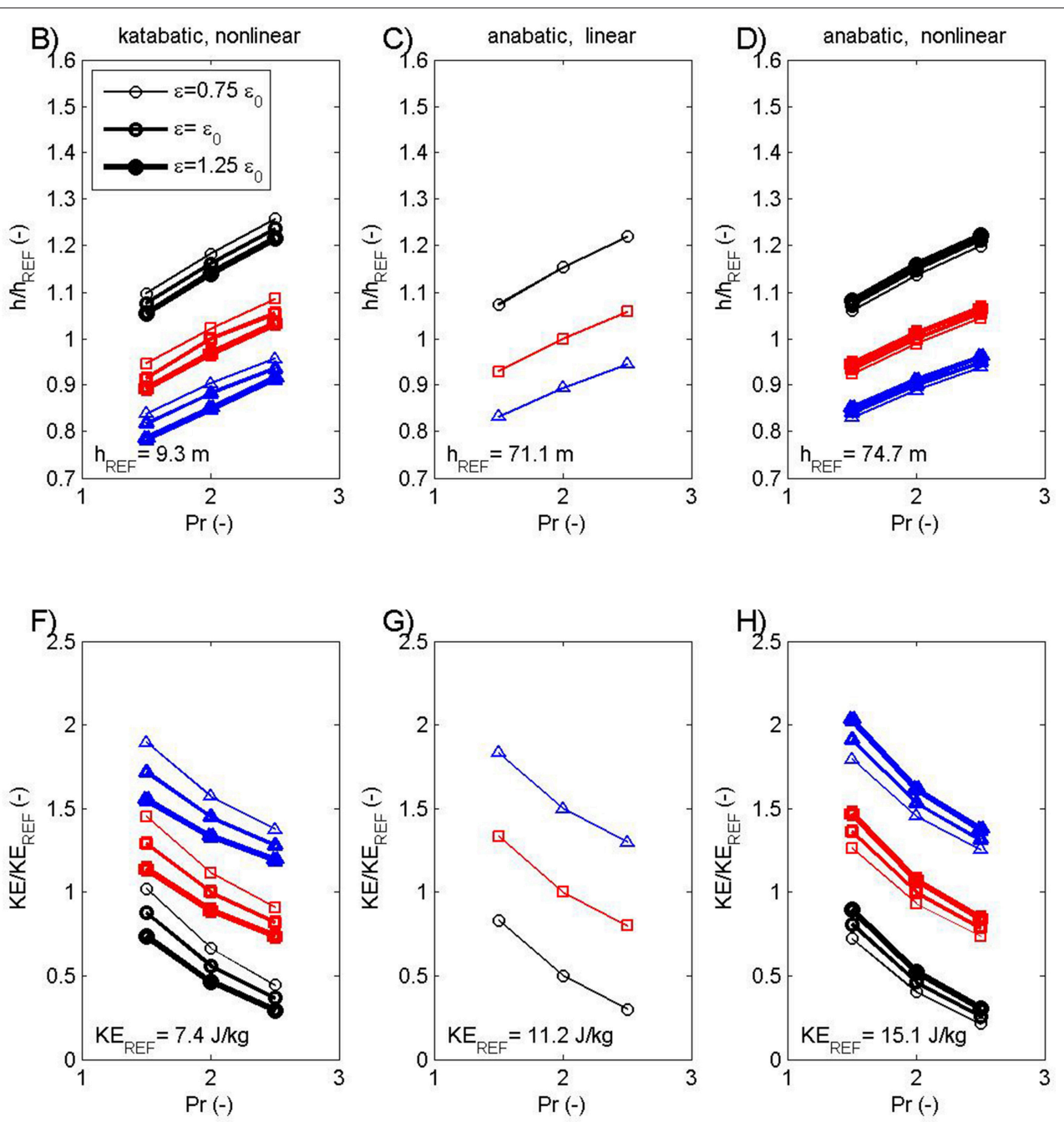

FIGURE 5 | The height of the maximum of kinetic energy $K E(A-D)$ and the maximum $K E$ value (E-H) for katabatic (A,B,E,F) and anabatic (C,D,G,H), linear (A,C,E,G), and nonlinear (B,D,F,H) cases. Selected measures are determined as functions of the Prandtl number Pr (X axis), slope angle $\alpha$ (different line color) and nonlinearity parameter $\varepsilon$ (different line thickness) in the case of nonlinear solutions. Heights in (A-D) are relative to the corresponding $h_{R E F}$. For presentation purposes, the lines in $\mathbf{( E - H )}$ are shifted \pm 0.5 from the referentce $\alpha=-0.1$ line (otherwise, exact overlap is present in $(\mathbf{E}, \mathbf{G})$, and approximate overlap is present in $\mathbf{( F , H ) . ~}$

$\sim 28 \mathrm{~m}$ to $\sim 43 \mathrm{~m}$. Also, INTmax varies from $\sim 1 \cdot 10^{-3} \mathrm{~J} / \mathrm{kg} / \mathrm{s}$ to $\sim 4 \cdot 10^{-3} \mathrm{~J} / \mathrm{kg} / \mathrm{s}$ in katabatic flow, while it is negative and varies from $\sim-0.22 \mathrm{~J} / \mathrm{kg} / \mathrm{s}$ to $\sim-0.04 \mathrm{~J} / \mathrm{kg} / \mathrm{s}$ in anabatic flow. Also, by examining the maximum of the triple product in INT (Equation 3 ), one can estimate the height where INTmax occurs: this is approximately $4 / 9 h_{j}$ (this result can be derived by using the linear solutions to find numerically the local maximum of the triple product; a more precise estimation would include the use of the nonlinear solutions $u_{\text {NOLIN }}$ and $\left.\theta_{\text {NOLIN }}\right)$. This means that INTmax, i.e., the maximum of the slope-normal transport of potential energy, occurs at about $1 / 2 h_{j}$, which is one of the new results of this study.

The last quantity examined in this subsection is the tendency of total energy $\partial T E / \partial t$. In linear solutions for anabatic and katabatic flow, this quantity is zero, reflecting the stationarity of our solutions by definition. However, in nonlinear solutions, the diffusion, dissipation and interaction terms do not balance, so $\partial T E / \partial t$ can attain nonzero values. For nonlinear katabatic flow, and based on the specific selection of model parameters, maximum values of $\partial T E / \partial t$ range from $\sim 0.01$ $\mathrm{J} / \mathrm{kg} / \mathrm{s}$ to $\sim 0.07 \mathrm{~J} / \mathrm{kg} / \mathrm{s}$ at heights reaching from $\sim 3 \mathrm{~m}$ to $\sim 5 \mathrm{~m}$ (Figures 6C,G). The amplitude/height of maximum $\partial T E / \partial t$ in the katabatic solution decreases/increases with increasing $\mathrm{Pr}$, increases/decreases with increasing $\alpha$ (because $\mid$ INT $|\sim| \alpha \mid$ ), and increases/decreases with increasing $\varepsilon$ (Figures 6C,G). For the nonlinear anabatic flow, maximum values of $\partial T E / \partial t$ range from $\sim 0.03 \mathrm{~J} / \mathrm{kg} / \mathrm{s}$ to $\sim 0.15 \mathrm{~J} / \mathrm{kg} / \mathrm{s}$ at heights ranging from $\sim 30 \mathrm{~m}$ to $\sim 45 \mathrm{~m}$ (Figures 6D,H). The amplitude and height of maximum $\partial T E / \partial t$ in the anabatic solution behave in a similar manner as in their katabatic counterpart (Figures 6D,H). The only difference is found for the case of the height of maximum $\partial T E / \partial t$, where now an $\varepsilon$ increase is linked with a $\partial T E / \partial t$ increase. Again, 

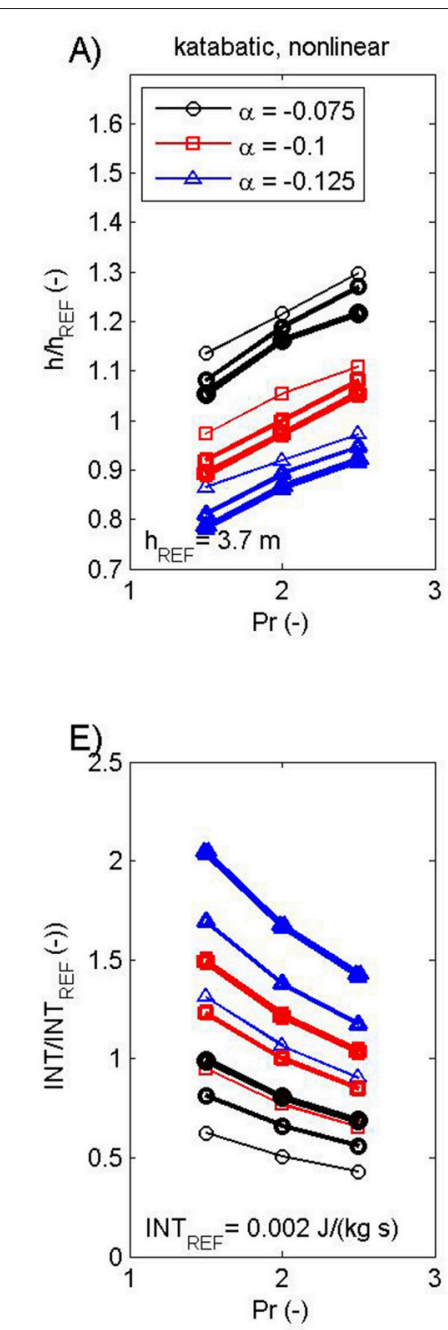
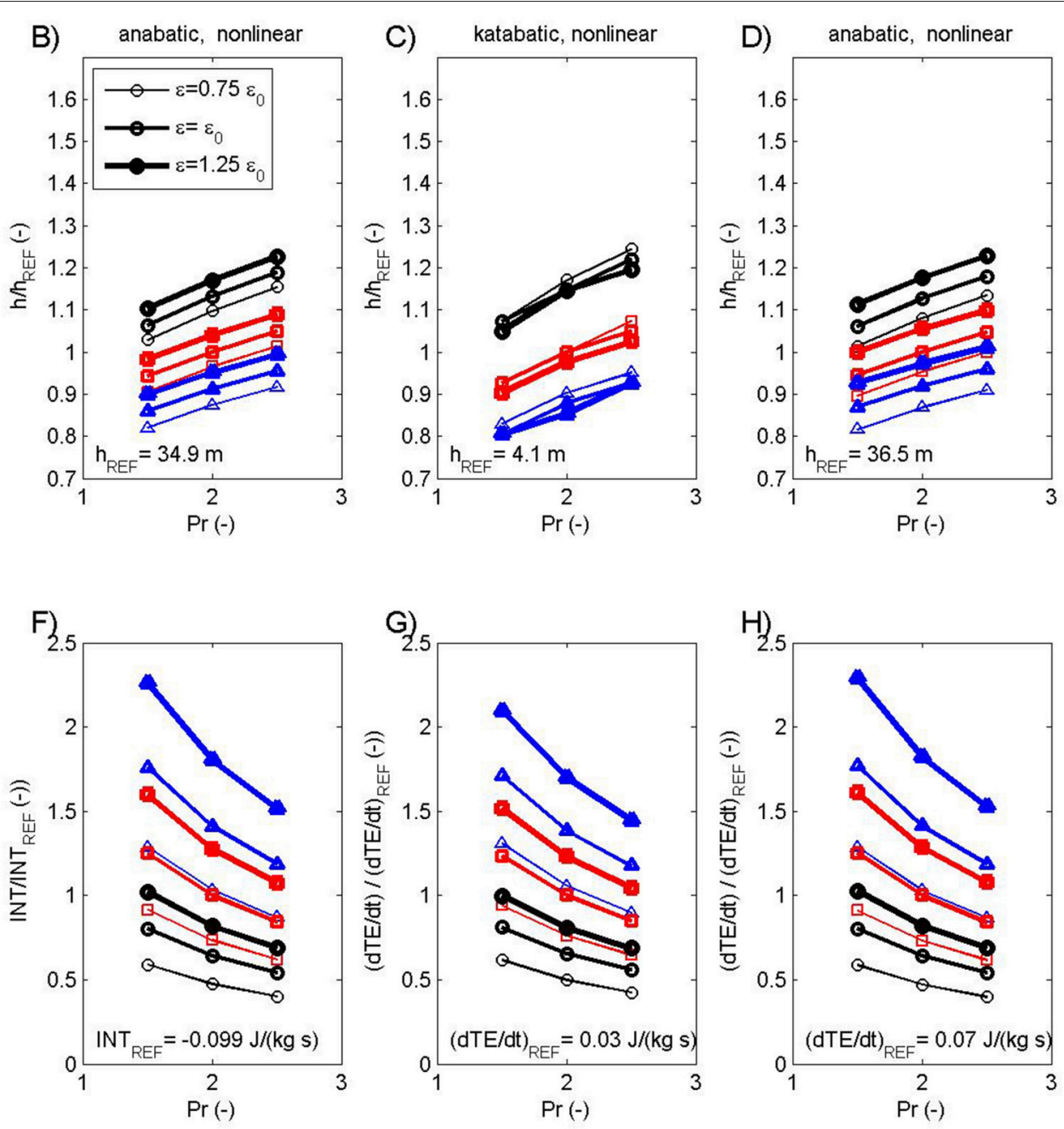

FIGURE 6 | The height of the maximum of the interaction term INT (A,B), the height of the maximum of the storage term $\partial T E / \partial t$ (C,D), the maximum

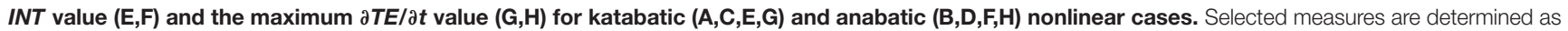
functions of Prandtl number $\operatorname{Pr}$ (x axis), slope angle $\alpha$ (different color) and nonlinearity parameter $\varepsilon$ (different line thickness). Values in panels are relative to the corresponding $h_{R E F}(\mathbf{A}-\mathbf{D}), I N T_{R E F}(\mathbf{E}, \mathbf{F})$ and $\partial T E / \partial t_{R E F}(\mathbf{G}, \mathbf{H})$.

nonzero profiles of $\partial T E / \partial t$, due to nonlinearity, imply that the stationarity of solutions is not satisfied, and depends on the joint effect of DIF, DIS, and INT terms.

\section{DISCUSSION}

In this section, we briefly discuss some of the results where references to LES studies and the issue of the non-stationarity present in our weakly nonlinear solutions are addressed.

The reduction of $h_{j}$ with an increasing slope is a wellknown feature of katabatic flows (in both LES results and the Prandtl model, see e.g., Grisogono and Axelsen, 2012). Also, with increasing $\mathrm{Pr}$, katabatic flow is characterized by an increase in the momentum mixing when compared to the heat mixing, pushing and spreading the low-level jet upwards. At the same time, the maximum $u_{\text {LIN }}$ is insensitive to the choice of slope angle but reduces for increasing $\operatorname{Pr}$ (Figure S3.1A; cf. Grisogono and Axelsen, 2012). However, in LES simulations (in contrast to the classical Prandtl model) the maximum $u$ reduces with an increasing slope angle. This is also found in the nonlinear solution of our extended Prandtl model. Future studies may explore the behavior of the LES and nonlinear solutions in detail.

Conceptually, there are no crucial differences (besides the vertical extent) in $K E, P E$, and $T E$ in anabatic and katabatic flows, since all energy measures are quadratic quantities and the same amplitude of the temperature deficit/surplus is set as a lower boundary condition. For both the anabatic and katabatic nonlinear solutions, variability due to perturbations in $\varepsilon$ is lower than variability due to the $\operatorname{Pr}$ and $\alpha$. The actual range of $\varepsilon$ is discussed in detail in Grisogono et al. (2015; their subsection 2.3). In short, the value of $\varepsilon$ should not introduce first-order corrections that modify the general structure of the zero-order solutions, and this is also confirmed by our study in terms of TE, $P E$, and TE. 
Another important difference between the linear and nonlinear katabatic (and anabatic) solutions is the nonzero $\partial T E / \partial t$ in the nonlinear case. In terms of the interaction between wind speed and potential temperature with the background atmosphere, the absolute value of the interaction term, i.e., $|I N T|$ decreases with increasing $P r$. This indicates a weaker coupling between the turbulent mixing of momentum and heat, i.e., a decrease of the slope-normal transport of potential energy; hence, the covariance between wind speed and temperature in INT weakens (note that the latter term is made of a triplet product). At the same time, as $|I N T|$ decreases with increasing $\operatorname{Pr}, \partial T E / \partial t$ also weakens with increasing $\operatorname{Pr}$. The existence of nonzero $\partial T E / \partial t$ in the nonlinear solution indicates deviations from stationarity of the total energy in the system, and reflects a leakage of energy from the background atmosphere to slope flows. It may be expected that in a more realistic flow there would be interplay among the energy terms, yielding a quasiperiodic behavior and generation of waves (most jets are unstable to small perturbations). In a more realistic model, which would allow not only for time dependency but also for vertical velocitypressure covariance, the kind of imbalance that we found in this study would immediately generate wave-like perturbations (e.g., Stiperski et al., 2007; Zhong and Whiteman, 2008; Axelsen, 2010; Largeron et al., 2010; Sun et al., 2015). Furthermore, this suggests that an extended and more comprehensive model than that presented in Grisogono et al. (2015) should allow for time dependency and/or velocity-pressure covariance. Also, slight to moderate imbalance among the energy terms in this nonlinear model may suggest that there is perhaps no real steady-state nonlinear slope flow; thus, excursions from pure steadiness could occur in nonlinear thermally driven flows. To add a point, Axelsen (2010; his Figures 3.5, 3.6) shows with an LES that pure katabatic flow is unsteady even under idealized conditions (constant slope, etc.). In his idealized simulation, internal and external gravity-wave modes are launched from the low-level katabatic jet. In short, the existence of non-stationarity in the nonlinear solution may reflect real non-stationarity in nonlinear models, LES simulations and observations, and/or limitations in the extended Prandtl model, where for the latter an inclusion of the additional nonlinear term in the momentum equation might close the energy budget. Again, this will require future study.

Lastly, the question is how the results of this study are comparable to the real atmosphere. While high-resolution observations over long gentle slopes and specific background atmospheric conditions are hard to acquire, we estimate $K E, P E$, and TE from the PASTEX-94 observations of glacier wind (van den Broeke, 1997a,b; Oerlemans and Grisogono, 2002; Figure S3.3). These results should only be considered indicative, but they do show the dominance of $P E$ near the sloped surface, and the elevated maximum of $K E$, followed by the level where $K E$ starts to dominate over PE (Figure S3.3B): all in accordance with our analysis of linear and nonlinear energetics of the (extended) Prandtl model. Interestingly, for this observation case DIF and $D I S$ do not balance either, so nonzero $\partial T E / \partial t$ is found (Figure $\mathrm{S} 3.3 \mathrm{C}$ ). The latter result suggests the existence of either nonlinear effects or other important processes in the real atmosphere, which are not taken into account in our model. However, for stronger claims and conclusions, much larger observational datasets need to be analyzed and a more comprehensive evaluation must be performed.

\section{SUMMARY AND CONCLUSIONS}

In this study, we have evaluated the energetics of the linear and weakly nonlinear solutions of the (extended) Prandtl model from Grisogono et al. (2015). From an ensemble of solutions where three controlling parameters were perturbed (i.e., the Prandtl number $P r$, the slope angle $\alpha$ and the nonlinearity parameter $\varepsilon$ ), $K E, P E$, and $T E$ profiles were estimated for both katabatic and anabatic flows. Also, the governing terms in the prognostic total energy equation were examined in four groups of solutions (linear/nonlinear, katabatic/anabatic).

The nonlinearity effect induced small to moderate variations in the total energy TE. These variations caused the nonstationarity of $T E$, which is in conflict with the initially assumed stationarity of along-the-slope wind speed and potential temperature. This suggests the need for joining nonlinear and time-dependent effects in katabatic/anabatic flow as a way of circumventing the limitations of the weakly nonlinear Prandtl model as developed by Grisogono et al. (2015). At the same time, the maximum of the wind speed (and kinetic energy) in the nonlinear solution is found to be sensitive to the slope angle (this is not present in the linear solution), and is in this way comparable to LES simulations in e.g., Grisogono and Axelsen (2012). Since the time-dependent solution to the linear Prandtl model is already quite complicated (e.g., Grisogono, 2003; Zardi and Serafin, 2014), it seems unlikely that a corresponding weakly nonlinear time-dependent analytic solution to the problem could be found in an elegant form. Yet, there are indications that there might be no steady-state nonlinear solution to thermally driven slope flows (Axelsen, 2010), which agrees with our findings.

We have limited our analysis to the energy terms and prognostic total energy equation of the mean slope flow only. It is shown that the strongest interaction between the $\theta$ - and $u$ profiles occurs at a height of around $4 / 9 h_{j}$, with $h_{j}=\pi / 4 h_{p}$, i.e., about half the height of the low-level jet. Moreover, kinetic energy dominates over potential energy above $h_{p} \cos ^{-1}[1 /(1+$ $\mathrm{Pr})^{1 / 2}$, which is typically between $h_{j}$ and $2 h_{j}$. Thus, this is the sublayer where dynamic instabilities might occur. It is directly, although nonlinearly, related to the corresponding gradient Richardson number, which compares the differential change of potential energy vs. kinetic energy of the flow. This number falls significantly below 1 in that sublayer. However, the height where $K E$ starts to dominate over $P E$ is not the height of the maximum $K E$. The latter is trivially the same as the height of the low-level jet, and always below the height where $K E>P E$.

A more complete energy framework would include an estimation of the potential and kinetic energy contributions from the basic state, turbulence and possibly mesoscale components (e.g., waves) in the system. Since there is still no satisfactory approach that would include the effects of sub-grid slope flows in the form of parameterizations in mesoscale and large-scale 
weather and climate models, greater effort should be made in order to increase the applicability of these types of models in complex orography regions (e.g., Bornemann et al., 2010).

Finally, the results of our simple small-ensemble exercise may be compared with observations (where care is needed to ensure high-resolution measurements in order to correctly estimate the first and second vertical derivatives in the total energy equation). A second approach to an independent evaluation of our analytical model includes the construction of the total energy budget from an ensemble of LES simulations (e.g., Grisogono and Axelsen, 2012), where non-stationarity and energetics of katabatic and anabatic flows can be further explored.

\section{AUTHOR CONTRIBUTIONS}

All authors listed, have made substantial, direct and intellectual contribution to the work, and approved it for publication.

\section{REFERENCES}

Axelsen, S. L. (2010). Large-Eddy Simulation and Analytical Modeling of Katabatic Winds. Ph.D., dissertation, IMAU, Utrecht University, the Netherlands, 164.

Bender, C. M., and Orszag, S. A. (1978). Advanced Mathematical Methods for Scientists and Engineers. New York, NY: McGraw-Hill Book Company.

Bornemann, J., Lock, A., Webster, S., Edwards, J., Weeks, M., Vosper, S., et al. (2010). "Understanding cold valleys in convective scale models," in 32nd EWGLAM and 17th SRNWP Meetings. Available online at: srnwp.met.hu/ Annual_Meetings/2010/ (Accessed 07-01-2016)

Burkholder, B. A., Shapiro, A., and Fedorovich, E. (2009). Katabatic flow induced by a cross-slope band of surface cooling. Acta Geophys. 57, 923-949. doi: 10.2478/s11600-009-0025-6

DeCaria, A. J. (2007). Relating static energy to potential temperature: a caution. J. Atmos. Sci. 64, 1410-1412. doi: 10.1175/JAS3906.1

Defant, F. (1949). Zur theorie der Hangwinde, nebst bemerkungen zur Theorie der Berg- und Talwinde. Arch. Meteor. Geophys. Biokl. Ser. A1, 421-450. doi: 10.1007/BF02247634

Fedorovich, E., and Shapiro, A. (2009a). Structure of numerically simulated katabatic and anabatic flows along steep slopes. Acta Geophys. 57, 981-1010. doi: 10.2478/s11600-009-0027-4

Fedorovich, E., and Shapiro, A. (2009b). Turbulent natural convection along a vertical plate immersed in a stably stratified fluid. J. Fluid Mech. 636, 41-57. doi: $10.1017 /$ S0022112009007757

Fernando, H., Pardyjak, E., Di Sabatino, S., Chow, F., De Wekker, S., and Hoch, S., et al (2015). THE MATERHORN - unraveling the intricacies of mountain weather. Bull. Am. Meteorol. Soc. 96, 1945-1967. doi: 10.1175/BAMS-D-1300131.1

Grachev, A. A., Leo, L. S., Di Sabatino, S., Fernando, H. J. S., Pardyjak, E. R., and Fairall, C. W. (2015). Structure of turbulence in katabatic flows below and above the wind-speed maximum. Boundary Layer Meteorol. 159, 469-494. doi: 10.1007/s10546-015-0034-8

Grisogono, B. (2003). Post-onset behaviour of the pure katabatic flow. Boundary Layer Meteorol. 107, 157-175. doi: 10.1023/A:1021511105871

Grisogono, B., and Axelsen, S. L. (2012). A note on the pure katabatic wind maximum over gentle slopes. Boundary Layer Meteorol. 145, 527-538. doi: 10.1007/s10546-012-9746-1

Grisogono, B., Jurlina, T., Večenaj, Ž., and Güttler, I. (2015). Weakly nonlinear Prandtl model for simple slope flows. Q. J. R. Meteorol. Soc. 141, 883-892. doi: 10.1002/qj.2406

Grisogono, B., and Oerlemans, J. (2001). A theory for the estimation of surface fluxes in simple katabatic flows. Q. J. R. Meteorol. Soc. 127, 2725-2739. doi: 10.1002/qj.49712757811

\section{FUNDING}

IG was supported by the Croatian Science Foundation, CARE project, No. 2831; IM, ŽV, and BG were supported by the Grant Agency of the Czech Science Foundation under the GAČR project 14-12892S and by the Croatian Science Foundation, CATURBO project, No. 09/151.

\section{ACKNOWLEDGMENTS}

The authors wish to thank the reviewers and the editor for providing detailed and constructive comments that have substantially improved the initial versions of the paper.

\section{SUPPLEMENTARY MATERIAL}

The Supplementary Material for this article can be found online at: http://journal.frontiersin.org/article/10.3389/feart. 2016.00072

Holtslag, A. A. M., Svensson, G., Baas, P., Basu, S., Beare, B., and Beljaars, A. C. M., et al. (2013). Stable atmospheric boundary layers and diurnal cycles: challenges for weather and climate models. Bull. Am. Meteorol. Soc. 94, 1691-1706. doi: 10.1175/BAMS-D-11-00187.1

Largeron, Y., Staquet, C., and Chemel, C. (2010). Turbulent mixing in a katabatic wind under stable conditions. Meteorol. Z. 19, 467-480. doi: 10.1127/09412948/2010/0346

Mahrt, L. (1998). Stratified atmospheric boundary layers and breakdown of models. Theoret. Comput. Fluid Dyn. 11, 263-279. doi: 10.1007/s0016200 50093

Mahrt, L. (2014). Stably stratified atmospheric boundary layers. Annu. Rev. Fluid Mech. 46, 23-45. doi: 10.1146/annurev-fluid-010313-141354

Mauritsen, M., Svensson, G., Zilitinkevich, S. S., Esau, I., Enger, L., and Grisogono, B. (2007). A total turbulent energy closure model for neutrally and stably stratified atmospheric boundary layers. J. Atmos. Sci. 64, 4113-4126. doi: 10.1175/2007JAS2294.1

Oerlemans, J., and Grisogono, B. (2002). Glacier wind and parameterization of the related surface heat flux. Tellus 54A, 440-452. doi: 10.1034/j.16000870.2002.201398.x

Poulos, G., and Zhong, S. (2008). An observational history of small-scale katabatic winds in mid-latitudes. Geogr. Compass 2, 1798-1821. doi: 10.1111/j.17498198.2008.00166.x

Prandtl, L. (1942). Führer durch die Strömungslehre. Braunschweig: Vieweg and Sohn, 648.

Princevac, M., and Fernando, H. J. S. (2007). A criterion for the generation of turbulent anabatic flows. Phys. Fluids. 19, 105102. doi: 10.1063/ 1.2775932

Sandu, I., Beljaars, A., Bechtold, P., Mauritsen, T., and Balsamo, G. (2013). Why is it so difficult to represent stably stratified conditions in numerical weather prediction (NWP) models? J. Adv. Model. Earth Syst. 5, 117-133. doi: 10.1002/jame.20013

Shapiro, A., Burkholder, B., and Fedorovich, E. (2012). Analytical and numerical investigation of two-dimensional katabatic flow resulting from local surface cooling. Boundary Layer Meteorol. 145, 249-272. doi: 10.1007/s10546-0119685-2

Shapiro, A., and Fedorovich, E. (2008). Coriolis effects in homogeneous and inhomogeneous katabatic flows. Q. J. R. Meteorol. Soc. 134, 353-370. doi: $10.1002 / q j .217$

Shapiro, A., and Fedorovich, E. (2014). A boundary-layer scaling for turbulent katabatic flow. Boundary Layer Meteorol. 153, 1-17. doi: 10.1007/s10546-0149933-3

Skyllingstad, E. D. (2003). Large-eddy simulation of katabatic flows. Boundary Layer Meteorol. 106, 217-243. doi: 10.1023/A:1021142828676 
Smith, C. M., and Porté-Agel, F. (2013). An intercomparison of subgrid models for large eddy simulation of katabatic flows. Q. J. R. Meteorol. Soc. 140, 1294-1303. doi: $10.1002 /$ qj.2212

Smith, C. M., and Skyllingstad, E. D. (2005). Numerical simulation of katabatic flow with changing slope angle. Monthly Weather Rev. 133, 3065-3080. doi: 10.1175/MWR2982.1

Stiperski, I., Kavčič, I., Grisogono, B., and Durran, D. R. (2007). Including Coriolis effects in the Prandtl model for katabatic flows. Q. J. R. Meteorol. Soc. 133, 101-106. doi: 10.1002/qj.19

Sun, J., Nappo, C. J., Mahrt, L., Belušić, D., Grisogono, B., Stauffer, D. R., et al. (2015). Review of wave-turbulence interactions in the stable atmospheric boundary layer. Rev. Geophys. 53, 956-993. doi: 10.1002/2015RG000487

van den Broeke, M. R. (1997a). Structure and diurnal variation of the atmospheric boundary layer over amid-latitude glacier in summer. Boundary Layer. Meteorol. 83, 183-205.

van den Broeke, M. R. (1997b). Momentum, heat and moisture budgets of the katabatic wind layer over amid-latitude glacier in summer. J. Appl. Meteorol. 36, 763-774.

Zammett, R. J., and Fowler, A. C. (2007). Katabatic winds on ice sheets: a refinement of the Prandtl model. J. Atmos. Sci. 64, 2707-2716. doi: 10.1175/ JAS3960.1
Zardi, D., and Serafin, S. (2014). An analytic solution for time-periodic thermally driven slope flows. Q. J. R. Meteorol. Soc. 141, 1968-1974. doi: 10.1002/ qj. 2485

Zardi, D., and Whiteman, C. D. (2013). "Diurnal mountain wind systems," in Mountain Weather Research and Forecasting, eds F. K. Chow, S. F. J. de Wekker, and B.-J. Snyder (Dordrecht: Springer), 35-119.

Zhong, S., and Whiteman, C. D. (2008). Downslope flows on a low-angle slope and their interactions with valley inversions. Part II: numerical modeling. J. Appl. Meteorol. Climatol. 47, 2039-2057. doi: 10.1175/2007 JAMC1670.1

Conflict of Interest Statement: The authors declare that the research was conducted in the absence of any commercial or financial relationships that could be construed as a potential conflict of interest.

Copyright (C) 2016 Güttler, Marinović, Večenaj and Grisogono. This is an open-access article distributed under the terms of the Creative Commons Attribution License (CC $B Y)$. The use, distribution or reproduction in other forums is permitted, provided the original author(s) or licensor are credited and that the original publication in this journal is cited, in accordance with accepted academic practice. No use, distribution or reproduction is permitted which does not comply with these terms. 


\title{
When Can a High-Resolution Simulation Over Complex Terrain be Called LES?
}

\author{
Joan Cuxart * \\ Group of Meteorology, Department of Physics, University of the Balearic Islands, Palma de Mallorca, Spain
}

The ceaseless rise of computational power leads to a continuous increase of the resolution of the numerical models of the atmosphere. It is found today that operational models are run at horizontal resolutions near $1 \mathrm{~km}$ whereas research exercises for flows over complex terrain use resolutions at the hectometer scale. Horizontal resolutions of $100 \mathrm{~m}$ or finer have been used to perform Large-Eddy Simulations (LES) for some specific regimes like, e.g., the atmospheric boundary-layer in idealized configurations. However, to use the name "Large-Eddy Simulation" implies to be able to resolve at least the largest turbulent energetic eddies, which is almost impossible to reach with resolutions of the

OPEN ACCESS

Edited by:

Haraldur Ólafsson,

University of Iceland, Iceland and

University of Bergen, Norway and Icelandic Meteorological Office,

Iceland

Reviewed by:

Miriam Sinnhuber. Karlsruhe Institute of Technology,

Germany

Alexandre Silva Lopes, Universidade do Porto, Portugal

*Correspondence: Joan Cuxart joan.cuxart@uib.cat

Specialty section:

This article was submitted to Atmospheric Science, a section of the journal Frontiers in Earth Science

Received: 27 August 2015 Accepted: 07 December 2015 Published: 23 December 2015

Citation:

Cuxart J (2015) When Can a High-Resolution Simulation Over Complex Terrain be Called LES?

Front. Earth Sci. 3:87 doi: 10.3389/feart.2015.00087 order of $100 \mathrm{~m}$ for a real case, where many different processes occur linked to different scales, many of them even smaller than $100 \mathrm{~m}$. Therefore, LES is an inappropriate denomination for these numerical exercises, that may simply be called High-Resolution Mesoscale Simulations.

Keywords: complex terrain, LES, VLES, mesoscale, high-resolution mesoscale simulations, turbulence, Inertial Subrange

\section{INTRODUCTION}

Continuous increase of the spatial resolution in mesoscale modeling is allowing currently horizontal resolutions finer than $1 \mathrm{~km}$. This allows modelers to activate the turbulence scheme in three-dimensional (3D) mode and to compute the statistics from the model results. This implies that, sometimes, by an abuse of language, the corresponding simulations are called "Large-Eddy Simulations" (LES), since the traditional tools of the LES community are used. This denomination is seldom found in the written literature [an early example was the one of Chow et al. (2006), that had resolutions of $150 \mathrm{~m}$ ], probably due to the correcting effect of the review process, but it is often heard in conferences and seminars, followed by unavoidable terminological discussions.

LES have been used by the atmospheric boundary-layer and turbulence scientific community to explore idealized flow configurations at the highest possible resolution. The largest and most energetic eddies must be well resolved, likewise most smaller eddies resulting from non-linear interaction among them, in the so-called energy cascade process that takes place in the Inertial Subrange of the spectrum of motions. The unresolved motions are expected to be homogeneous and isotropic and parameterized accordingly. The simulated regimes are usually run using homogeneous surfaces or simple patterns in the surface heterogeneities, as in Dörnbrack and Schumann (1993) for a wavy surface or Couvreux et al. (2005) and Van Heerwaarden et al. (2014), for terrain discontinuities. A good description of the resolution requirements to perform LES is given in Brasseur and Wei (2010), and a large number of references is listed there referring to other important issues concerning this type of simulations. 
The complex-terrain community has made recently a number of LES studies, mainly concerning the Convective BoundaryLayer (CBL) over slopes and in valleys, using resolutions of the order of $50 \mathrm{~m}$ and idealized terrain configurations, which fulfill the LES requirements, as in Serafin and Zardi (2010), Schmidli (2013) or Wagner et al. (2014), since the larger and most energetic eddies in this regime have sizes of several hundreds of meters. On the contrary, high-resolution simulations of stably stratified cases over complex terrain, even with resolutions of $100 \mathrm{~m}$ like in Vosper et al. (2013) for a valley cold pool, are not called LES, because the largest eddies in that regime are only of the order of some decameters with prescribed realistic surface conditions.

When Chow et al. (2006) called LES a high-resolution simulation case using hectometric resolution, they justified the use of this denomination referring to Wyngaard (2004). In that work Wyngaard suggested that the LES technique could be applied to coarser resolutions that those of LES to address the challenge of the so-called "terra incognita" or "gray zone," as the horizontal resolutions become finer than $1 \mathrm{~km}$. However, Wyngaard always made a clear distinction between what he labelled high-resolution LES and high-resolution mesoscale modeling, and indicated that the LES technique is another way of parameterizing subgrid motions. The subject of the "gray zone" has been a subject of intense research in the recent years (see Honnert et al., 2011, as an example).

The aim of the paper is clarifying when a complex terrain simulation could be called LES. It will first introduce the concept of the Inertial Subrange of turbulence in Section 2, followed in Section 3 by a discussion about the fact that an LES simulation must have a resolution falling in the Inertial Subrange. Section 4 will explore the scales of some relevant phenomena in complex terrain and see if they are able to be simulated in LES mode with the current numerical capabilities. Finally section 5 will provide the main conclusions of this paper, proposing the more convenient denomination of "High-Resolution Mesoscale Simulations" when using resolutions at the hectometer scale.

\section{THE INERTIAL SUBRANGE OF TURBULENCE}

The spectrum tensor of turbulence (see Tennekes and Lumley, 1972, for instance) is the Fourier transform of the covariance tensor of the velocity field $\left(R_{i j}(\mathbf{r}, \mathbf{x}, t)=\overline{u_{i}(\mathbf{x}, t) u_{j}(\mathbf{x}+\mathbf{r}, t)}\right.$, the overline corresponding to the Reynolds average),

$$
\psi_{i j}(\mathbf{k}, \mathbf{x}, t)=\int_{R^{3}} R_{i j}(\mathbf{r}, \mathbf{x}, t) e^{-i \mathbf{k r}} d \mathbf{r}
$$

and $\psi_{i i}(k)$ represents the amount of kinetic energy related to motions of wave number $\mathrm{k}$

$$
\frac{1}{2} \overline{u_{i}^{2}}=\frac{1}{2} \int_{R^{3}} \psi_{i i}(\mathbf{k}) d \mathbf{k}=\int_{0}^{\infty} E(k) d k
$$

where $E(k)=\frac{1}{2} \int_{S^{2}} \psi_{i i}(\mathbf{k}) d S(\mathbf{k})$ is the scalar energy spectrum, that represents the contribution to the kinetic energy of motions of wave number $\mathrm{k}$, regardless of direction. An evolution equation can be written for this quantity and, assuming that the fluid motion is in statistical equilibrium, a relation can be found for the wave numbers that lie between the scales that bring energy to the flow and those in which this energy is dissipated, but not too close to them, assuming that turbulent motions are isotropic and homogeneous (Kolmogorov, 1941).

$$
E(k) \approx \epsilon^{2 / 3} k^{-5 / 3} ;\left(\Lambda^{-1}<<k<<\eta^{-1}\right)
$$

in which $\Lambda$ is the scale at which energy is supplied to the turbulence, usually scaling well with the dimensions of the largest turbulent eddies, and $\eta$ is the so-called "Kolmogorov length," corresponding to the smallest scales in a turbulent flow, where viscosity dominates and turbulent energy is dissipated into heat (Figure 1A). The energy is transferred by non-linear interaction from the large to the small dissipative scales in what is known as "energy cascade." Introducing Equation (3) in Equation (2) and integrating, we reach the following expression for the dissipation of the turbulent kinetic energy (TKE, $e=\frac{1}{2} \overline{u_{i}^{2}}$ )

$$
\epsilon \approx \frac{e^{3 / 2}}{\Lambda}
$$

\section{LES: RESOLUTION FALLING INTO THE INERTIAL SUBRANGE}

A Large-Eddy Simulation (LES) uses a numerical model of a turbulent flow, with the aim of explicitly representing the largest and most energetic scales of turbulence. This way the upper part of the Inertial Subrange is captured and, if the later is well defined until the dissipation scales, the Kolmogorov theory is of application. The Smagorinsky-Lilly closure can be applied in first order models (see in Wyngaard, 2004) and the dissipation formula (4) can be used in models using a TKE-equation (1.5 order models, as in Cuxart et al., 2000), since in both cases it is implicitly assumed that resolved energy production and parameterized dissipation are in equilibrium.

It is therefore clear that an LES must be capturing explicitly at least the largest turbulent eddies of the Inertial Subrange. This is extremely difficult even for the simplest configurations. Let us illustrate the problem taking a simple atmospheric CBL for discussion. Assuming that the surface heating is able to generate a CBL of a height approximately of $1000 \mathrm{~m}$, the well-developed thermals may have vertical dimensions of several hundreds of meters. The non-linear interaction between convective eddies will generate smaller eddies due to the energy cascade described above. This process can be successfully simulated using resolutions of tens of meters in which the smallest resolved eddies will have scales of at most $100 \mathrm{~m}$, eddies that probably will already be homogeneous and isotropic, allowing the full application of the Kolmogorov theory.

However, even in this simple case there are two layers where the resolution is usually not able to capture the largest turbulent eddies in them: the surface layer near the ground and the entrainment zone at the top of the CBL. In the first case the eddies are smaller as they are closer to the surface and are not captured by the aforementioned resolution of some tens of 

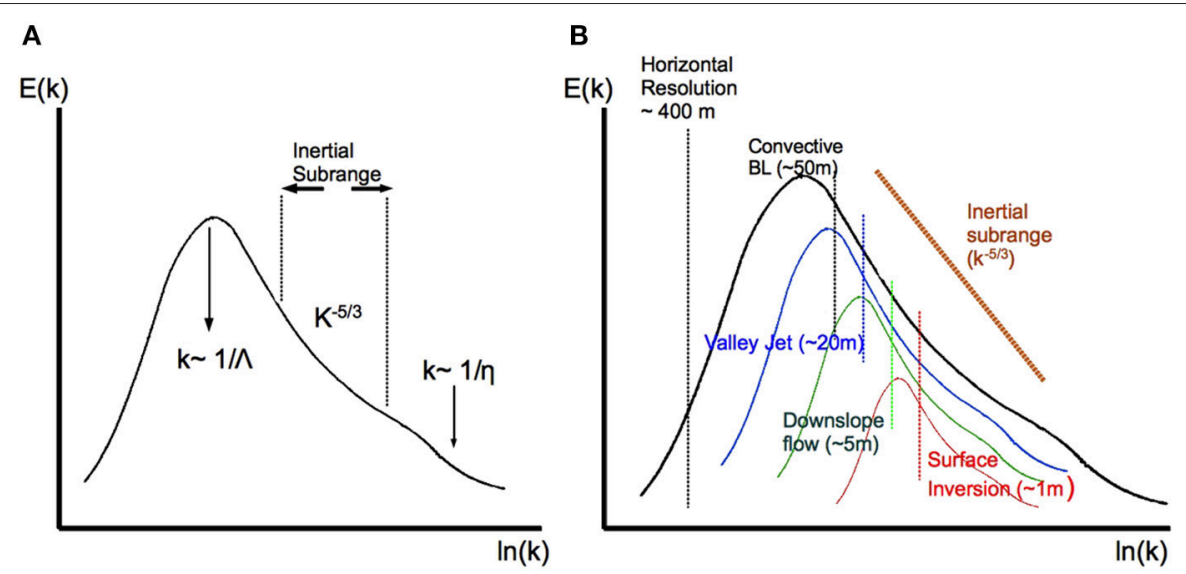

FIGURE 1 | (A) Spectrum for a turbulent flow that has a scale of entrance of energy $(\Lambda)$, a dissipation scale $(\eta)$ and a well defined Inertial Subrange. (B) Conceptual representation of the Inertial Subranges corresponding to different structures in a valley flow. The vertical lines indicate the typical scale of the upper size of the Inertial Subrange.

meters (see a detailed discussion in Brasseur and Wei, 2010). The classical solution in this case is to impose the phenomenological knowledge that the similarity theory provides to overcome this limitation or to use hybrid approaches (Bechmann and Sorensen, 2010). In the case of the entrainment zone, stable stratification limits the vertical size of the eddies and introduces anisotropy in the turbulence. The solutions would be either to increase the resolution-and, as long as the latter is too low, the numerical results may not match well with the available observations (Sullivan et al., 1998) — or to introduce a parameterization for this effect in the layer.

In a general case, as the size of the most energetic eddies of a regime becomes smaller, the finer must be the resolution of the LES. For instance a weakly stably stratified nocturnal boundary layer has the more energetic eddies that have typical sizes below $100 \mathrm{~m}$, slightly anisotropic, and that need resolutions of the order of 5 to $10 \mathrm{~m}$ to be well resolved, still having the problem of the surface layer (Beare et al., 2006). For a more stable nocturnal boundary layer, eddies are typically of few meters, even for slope flows, and the requested resolutions for a LES must be clearly finer than $5 \mathrm{~m}$ (Jimenez and Cuxart, 2005).

When a simulation is using a resolution that is not capturing the most energetic turbulent eddies of all the different configurations that it contains, then there is a range of energetic motions that cannot be parameterized using the Kolmogorov theory. We would say that in this case the resolution falls to the left of the beginning of the Inertial Subrange (see Figure 1). If this limitation happens extensively for a simulation, then it cannot be called LES anymore, and the $3 \mathrm{D}$ subgrid turbulence scheme is just a parameterization of the unresolved motions not sustained on the Kolmogorov theory and therefore, conceptually similar to a pure one-dimensional approach.

There is also the issue of the anisotropy of the grid mesh. LES must have a grid that captures energetic eddies and those not resolved are supposed to be isotropic and homogeneous. A largely anisotropic grid would allow anisotropic eddies to be unresolved and it is advisable to refrain using grids that depart too much from isotropy [customarily never more than a 1 to 4 ratio between vertical and horizontal resolution, Baggett et al. (1997)]. This requirement is sometimes relaxed near the surface, where similarity theory is used.

In the next section we will discuss about how a real case simulation for complex terrain is very often not fulfilling the LES requirements, and why, in consequence, they cannot be called LES.

\section{CHARACTERISTIC SCALES OF THE REGIMES OVER COMPLEX TERRAIN}

The examples cited in the previous section were mostly for flat terrain Boundary-Layer regimes and the LES resolution requirements became evident, especially near the surface and in stably stratified conditions.

Figure 2 is a vertical cross section from a simulation for La Cerdanya Valley in the Pyrenees (Martinez-Villagrasa et al., 2015), using a horizontal resolution of $400 \mathrm{~m}$ and a vertical resolution of $5 \mathrm{~m}$, with a 1.5 order one-dimensional turbulence scheme activated, a setup identical to the one described in Jimenez and Cuxart (2014) for a narrow valley in the Northern Pyrenees. We call this a high-resolution mesoscale simulation, as it is capturing phenomena with scales between one and $40 \mathrm{~km}$ (mesogamma and mesobeta structures). This approach, with very anisotropic grid elements, cannot be called LES by definition, since the grid structure should not depart too much from isotropy, and because many relevant eddies have scales well below $400 \mathrm{~m}$ and could never be captured by this discretization setup. However, the dynamic equations and the one-dimension turbulence scheme are able to provide a description of the flows that is very close to reality.

Some of the main structures taking place in a wide mountainous valley at night can be seen in Figure 2, where the wind direction is shown. The valley bottom has near $5 \mathrm{~km}$ width and $25 \mathrm{~km}$ length, whereas the distance between the tops of the 


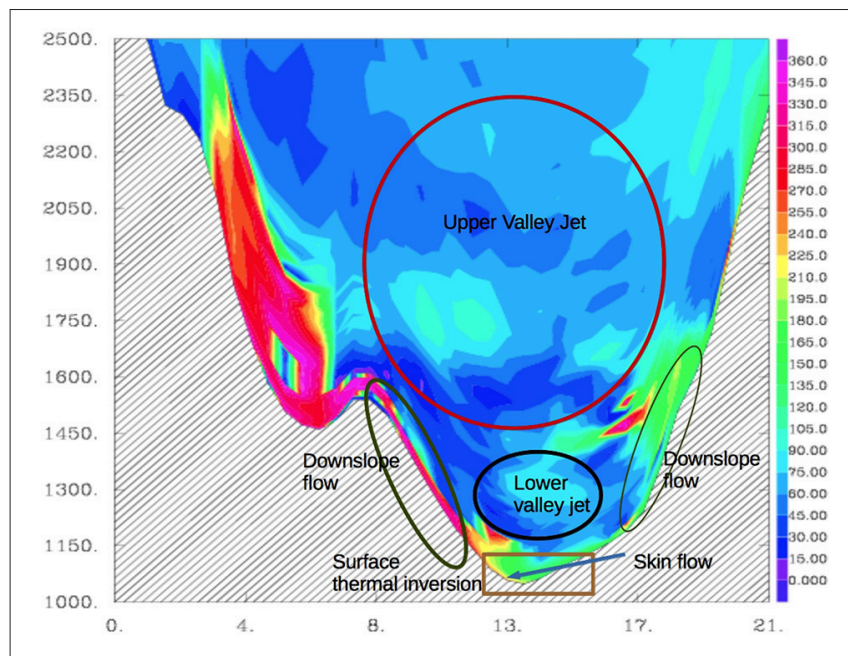

FIGURE 2 | Vertical cross-section of the wind direction for a simulation of La Cerdanya valley in the Pyrenees, for a cross valley direction at night. Some main structures are indicated. The vertical scale is in meters and the horizontal scale in kilometers. surrounding mountain ranges is close to $20 \mathrm{~km}$ and the vertical distance from peaks to valley is $1500 \mathrm{~m}$. Five main structures have been signaled in the Figure and some relevant scales are listed in Table 1. A conceptual graph indicating a typical start of the Inertial subrange for each structure is given in Figure 1B. For a real flow containing all these structures the spectrum may be a superposition of the above-mentioned spectra and, consequently, it may not show an inertial subrange.

The upper valley jet is a mesobeta structure, covering usually the whole length of the valley and many times connected to adjacent valley structures, that can exist at day and at night. Its depth is of several hundred meters and a simulation with a resolution near $100 \mathrm{~m}$ could resolve well the largest eddies and perform a LES of this structure. The lower valley jet results from the accumulation of cold air at night flowing downvalley, many times above a shallow thermal inversion, with some intermittent mixing events between the two structures during the night. Typically they have a depth of 50-100 $\mathrm{m}$ and a LES of them could be made with resolutions of the order of $10-20 \mathrm{~m}$.

All the other important structures signaled in Figure 2 have much smaller scales and it is currently not possible to be able to capture the most energetic eddies related to them with the setups that we are discussing here. All of them are shallow and in contact with the surface, therefore they adapt to the topographical characteristics of the terrain. These are usually broken structures, anisotropic (shallow and elongated) with energetic eddies that have a short size in the vertical. To make an LES of them, resolutions of the order of 5-10 m would be a minimum requirement. Furthermore, no generally accepted similarity expressions exist, due to the high influence of the local effects, although work is in progress for some simple configurations, such as in Grisogono et al. (2007), but these approaches seem still not to be well adjusted to general observations (Nadeau et al., 2013).
TABLE 1 | Valley flow structures: order of magnitude of sizes and of related inertial subrange.

\begin{tabular}{lccc}
\hline Structure & Horizontal scale & Vertical scale & Inertial Subrange \\
\hline Upper valley jet & $O(10 \mathrm{~km})$ & $\mathrm{O}(1 \mathrm{~km})$ & $\mathrm{O}(100 \mathrm{~m})$ \\
Lower valley jet & $\mathrm{O}(1 \mathrm{~km})$ & $\mathrm{O}(100 \mathrm{~m})$ & $\mathrm{O}(10 \mathrm{~m})$ \\
Downslope flow & $\mathrm{O}(100 \mathrm{~m})$ & $\mathrm{O}(10 \mathrm{~m})$ & $\mathrm{O}(1 \mathrm{~m})$ \\
Skin flow & $\mathrm{O}(100 \mathrm{~m})$ & $\mathrm{O}(1 \mathrm{~m})$ & $\mathrm{O}(0.1 \mathrm{~m})$ \\
Surface inversion & $\mathrm{O}(1 \mathrm{~km})$ & $\mathrm{O}(100 \mathrm{~m})$ & $\mathrm{O}(1 \mathrm{~m})$ \\
Shallow surface inversion & $\mathrm{O}(1 \mathrm{~km})$ & $\mathrm{O}(10 \mathrm{~m})$ & $\mathrm{O}(0.1 \mathrm{~m})$ \\
\hline
\end{tabular}

Specifically, for downslope flows and deep surface inversions, any LES study of them would require vertical resolutions of the order of at least $5 \mathrm{~m}$, keeping in mind that grid meshes must not be too anisotropic. When addressing skin flows or shallow inversions, which are cases of strongly stably stratified regimes, the resolution should be of the order of $1 \mathrm{~m}$ or finer.

\section{THE CURRENT STATE: HIGH-RESOLUTION MESOSCALE SIMULATIONS}

Arguments have been given above about why activating a 3D turbulence scheme does not directly imply that the simulation becomes an LES. The latter should capture the most energetic eddies of all the structures present in the simulated field. If it is a real case, it will include daytime, nighttime and the morning and evening transitions, with a large variety of structures of different scales, many of them with sizes of only a few meters. Besides, to perform an LES implies using a quasi-isotropic grid mesh, since it is necessary that the resolved eddies belong to the upper part of the Inertial Subrange. The only usual exception is to impose the phenomenological knowledge close to the surface by use of the similarity theory, not well established yet over complex terrain for strongly stratified conditions.

To use resolutions of some hundreds of meters has been a hot research topic in the last decade, since it is still unclear when it is more convenient to use a parameterized $1 \mathrm{D}$ turbulence scheme or to activate a $3 \mathrm{D}$ turbulence scheme that may resolve part of the most energetic eddies, in what is called the "gray zone" area. With these resolutions, for turbulence eddies of scales of $100 \mathrm{~m}$ or smaller, a 3D scheme will not add much to the performance of a 1D scheme, because the horizontal gradients will already be taken into account by the advection scheme of the model. On the other hand, energetic eddies of several hundreds of meters will be resolved by the model and highly parameterized $1 \mathrm{D}$ turbulence schemes may also account for their effects, leading to what is known as the "double-counting" problem (Honnert et al., 2011).

A good practice is to inspect very carefully the characteristics of the regime that is the subject of study before fixing the key options in the simulation setup. In complex terrain daytime real flows, eddies will be of relatively large dimension and the choice of a 3D turbulence scheme may be advisable, 
even if the resolution is as low as some hundreds of meters. In this case we would be in the frame of what is called "Very-Large Eddy simulation" (VLES, Smolarkiewicz and Prusa, 2002), for which the resolution falls outside but near the Inertial subrange and the $3 \mathrm{D}$ scheme is still just a parameterization.

For the morning and evening transitions, as well as for the nighttime, most eddies are of scales of some decameters. This resolution is currently not affordable for the vast majority of numerical studies over real complex terrain. Furthermore, many of these eddies are strongly anisotropic, having a horizontal dimension much larger than the vertical one. Therefore, many of the horizontal characteristics will be approximately well captured by the dynamical equations. Instead, the turbulence exchanges will be of small dimension, sometimes generated by vertical gradients and many times in an intermittent manner. Therefore, here it is perhaps more advisable to choose a 1D-turbulence scheme to save computational resources, although a $3 \mathrm{D}$ scheme with a highly parameterized mixing length will probably be acting equivalently, due to the very small values of the horizontal gradients. In this case we would be talking about a highresolution mesoscale simulation, since the horizontal resolution is clearly coarser than the scales of the larger eddies at the beginning of the Inertial Subrange.

Summarizing, for simulations of real flows over complex terrain:

- A simulation over complex terrain with horizontal resolution of some hectometers with a $3 \mathrm{D}$ turbulence scheme is not an

\section{REFERENCES}

Baggett, J. S., Jimenez, J., and Kravchenko, A. G. (1997). Resolution Requirements in Large-eddy Simulations of Shear Flows. Stanford: Turbulence Research Center.

Beare, R. J., Macvean, M. K., Holtslag, A. A., Cuxart, J., Esau, I., Golaz, J. C., et al. (2006). An intercomparison of large-eddy simulations of the stable boundary layer. Boundary Layer Meteorol. 118, 247-272. doi: 10.1007/s10546-00 4-2820-6

Bechmann, A., and Sorensen, N. N. (2010). Hybrid RANS/LES method for wind flow over complex terrain. Wind Energy 13, 36-50. doi: 10.1002/we.346

Brasseur, J. G., and Wei, T. (2010). Designing large-eddy simulation of the turbulent boundary layer to capture law-of-the-wall scaling. Phys. Fluids 22, 021303. doi: $10.1063 / 1.3319073$

Chow, F. K., Weigel, A. P., Street, R. L., Rotach, M. W., and Xue, M. (2006). High-resolution large-eddy simulations of flow in a steep Alpine valley. Part I: methodology, verification, and sensitivity experiments. J. Appl. Meteorol. Climatol. 45, 63-86. doi: 10.1175/JAM2322.1

Couvreux, F., Guichard, F., Redelsperger, J. L., Kiemle, C., Masson, V., Lafore, J. P., et al. (2005). Water-vapour variability within a convective boundarylayer assessed by large-eddy simulations and IHOP 2002 observations. Q. J. R. Meteorol. Soc. 131, 2665-2693. doi: 10.1256/qj.04.167

Cuxart, J., Bougeault, P., and Redelsperger, J. L. (2000). A turbulence scheme allowing for mesoscale and large-eddy simulations. Q. J. R. Meteorol. Soc. 126, 1-30. doi: 10.1002/qj.49712656202

Dörnbrack, A., and Schumann, U. (1993). Numerical simulation of turbulent convective flow over wavy terrain. Boundary Layer Meteorol. 65, 323-355.

Grisogono, B., Kraljevic, L., and Jericevic, A. (2007). Notes and correspondencethe low-level katabatic jet height versus MoninObukhov height. Q. J. R. Meteorol. Soc. 133, 2133-2136. doi: 10.1002/qj.190
LES, since the resolved motions do not belong to the Inertial Subrange of the flow.

- In the daytime, with prevailing convective motions, simulations with resolutions of the order of $50 \mathrm{~m}$ or finer can be considered LES, whereas coarser resolutions of a few hectometers correspond to Very-Large Eddy Simulations (VLES), as the smaller resolved motions are into the Inertial Subrange.

- For other regimes, especially at night, simulations at the hectometer resolution have resolved eddies that are far from the Inertial Subrange. The turbulence is highly parameterized, either with a $1 \mathrm{D}$ scheme or with a 3D scheme. Even finer resolutions fail to capture the inertial subrange of shallow structures near the surface. In this case an LES is usually not possible with the computational capabilities at hand of most research teams. These numerical exercises should be called more properly "High-Resolution Mesoscale Simulations."

\section{FUNDING}

This article has received financial support of the Spanish Government, supplement with European FEDER funds, through Grant CGL2012-37416-C04-01.

\section{ACKNOWLEDGMENTS}

Maria Antonia Jiménez is acknowledged for providing the vertical cross-section used to build Figure 2.
Honnert, R., Masson, V., and Couvreux, F. (2011). A diagnostic for evaluating the representation of turbulence in atmospheric models at the kilometric scale. $J$. Atmos. Sci. 68, 3112-3131. doi: 10.1175/JAS-D-11-061.1

Jimenez, M. A., and Cuxart, J. (2005). Large-eddy simulations of the stable boundary layer using the standard Kolmogorov theory: range of applicability. Boundary Layer Meteorol. 115, 241-261. doi: 10.1007/s10546-0043470-4

Jimenez, M. A., and Cuxart, J. (2014). A study of the nocturnal flows generated in the north side of the Pyrenees. Atmos. Res. 145, 244-254. doi: 10.1016/j.atmosres.2014.04.010

Kolmogorov, A. N. (1941). "Dissipation of energy in locally isotropic turbulence," in Doklady Akademii Nauk SSSR, Vol. 32 (Moscow), $16-18$.

Martinez-Villagrasa, D., Conangla, L., Tabarelli, D., Jimenez, M. A., Miro, J. R., Zardi, D., et al. (2015). "Slope and valley flows at the Cerdanya valley in the Pyrenees," in 33rd International Conference on Alpine Meteorology (Innsbruck).

Nadeau, D. F., Pardyjak, E. R., Higgins, C. W., and Parlange, M. B. (2013). Similarity scaling over a steep alpine slope. Boundary Layer Meteorol. 147, 401-419. doi: 10.1007/s10546-012-9787-5

Schmidli, J. (2013). Daytime heat transfer processes over mountainous terrain. J. Atmos. Sci. 70, 4041-4066. doi: 10.1175/JAS-D-13-083.1

Serafin, S., and Zardi, D. (2010). Daytime heat transfer processes related to slope flows and turbulent convection in an idealized mountain valley. J. Atmos. Sci. 67, 3739-3756. doi: 10.1175/2010JAS3428.1

Smolarkiewicz, P. K., and Prusa, J. M. (2002). VLES modelling of geophysical fluids with nonoscillatory forward-in-time schemes. Int. J. Numer. Methods Fluids 39, 799-819. doi: 10.1002/fld.330

Sullivan, P. P., Moeng, C. H., Stevens, B., Lenschow, D. H., and Mayor, S. D. (1998). Structure of the entrainment zone capping the convective 
atmospheric boundary layer. J. Atmos. Sci. 55, 3042-3064. doi: 10.1175/15200469(1998)055<3042:SOTEZC > 2.0.CO;2

Tennekes, H., and Lumley, J. L. (1972). A First Course in Turbulence. Cambridge, MA: MIT press.

Van Heerwaarden, C. C., Mellado, J. P., and De Lozar, A. (2014). Scaling laws for the heterogeneously heated free convective boundary layer. J. Atmos. Sci. 71, 3975-4000. doi: 10.1175/JAS-D-13-0383.1

Vosper, S., Carter, E., Lean, H., Lock, A., Clark, P., and Webster, S. (2013). High resolution modelling of valley cold pools. Atmos. Sci. Lett. 14, 193-199. doi: $10.1002 /$ asl 2.439

Wagner, J. S., Gohm, A., and Rotach, M. W. (2014). The impact of horizontal model grid resolution on the boundary layer structure over an idealized valley. Mon. Weather Rev. 142, 3446-3465. doi: 10.1175/MWR-D-14-0 0002.1
Wyngaard, J. C. (2004). Toward numerical modeling in the Terra Incognita. J. Atmos. Sci. 61, 1816-1826. doi: 10.1175/15200469(2004)061<1816:TNMITT > 2.0.CO;2

Conflict of Interest Statement: The author declares that the research was conducted in the absence of any commercial or financial relationships that could be construed as a potential conflict of interest.

Copyright (๑) 2015 Cuxart. This is an open-access article distributed under the terms of the Creative Commons Attribution License (CC BY). The use, distribution or reproduction in other forums is permitted, provided the original author(s) or licensor are credited and that the original publication in this journal is cited, in accordance with accepted academic practice. No use, distribution or reproduction is permitted which does not comply with these terms. 


\section{Influence of the Details of} Topography on Weather Forecast Evaluation of HARMONIE Experiments in the Sochi Olympics Domain over the Caucasian Mountains

\section{OPEN ACCESS}

Edited by:

Daniel J. Kirshbaum

McGill University, Canada

Reviewed by:

Juan Pedro Montávez,

Murcia University, Spain Ivana Stiperski,

University of Innsbruck, Austria

*Correspondence:

Laura Rontu

laura.rontu@fmi.fi

Specialty section:

This article was submitted to Atmospheric Science, a section of the journal

Frontiers in Earth Science

Received: 25 August 2015 Accepted: 27 January 2016 Published: 12 February 2016

Citation:

Rontu L, Wastl C and Niemelä S (2016) Influence of the Details of Topography on Weather Forecast Evaluation of HARMONIE Experiments

in the Sochi Olympics Domain over

the Caucasian Mountains.

Front. Earth Sci. 4:13.

doi: 10.3389/feart.2016.00013

\author{
Laura Rontu $^{1 *}$, Clemens Wastl ${ }^{2}$ and Sami Niemelä ${ }^{1}$ \\ ${ }^{1}$ Finnish Meteorological Institute, Helsinki , Finland, ${ }^{2}$ Zentralanstalt für Meteorologie und Geodynamik, Vienna, Austria
}

New fine-resolution surface elevation data was implemented into HARMONIE-AROME-SURFEX Numerical Weather Prediction (NWP) system. The grid-scale mean orography, used as a basis of the model's terrain-following vertical coordinate, as well as variables for suggested new parameterizations of radiation and momentum fluxes were derived. Validation against the surface observations from the Sochi Winter Olympic Games 2014, provided by the WMO FROST-2014 program at the Caucasian mountains, showed minor degradation of the screen-level temperature forecast when only the source orography was updated. Implementation of the orographic radiation parameterizations allowed to alleviate the degradation of scores. Detailed sensitivity studies, done by using three-dimensional and single-column experiments, showed that substantial and physically realistic changes in the downweling short- and long-wave radiation fluxes took place locally. However, their influence on the the simulated screen-level temperature remained small. Comparison of the simulated and observed radiation fluxes would offer a reliable alternative for validation of NWP models. Unfortunately, surface-level radiation observations were not made during the Sochi Olympics.

Keywords: orographic effects, radiation flux, Numerical Weather Prediction, parametrization, FROST-2014

\section{INTRODUCTION}

As the resolution of the NWP models improves toward the kilometer-scale, simulation of the local effects, in particular those related to mountains, becomes increasingly important. On the other hand, new sources of high resolution orography, such as SRTM (Shuttle Radar Topography Mission, Jarvis et al., 2008) offer possibilities to improve the description of the orography features and thus the treatment of all scales of orographic effects also in the finest-resolution NWP models. This study reports implementation and testing of a new fine-resolution orography and parameterizations of orographic effects on radiation in SURFEX, which is an externalized surface parametrization and data assimilation system for NWP and climate models (Masson et al., 2013). SURFEX is coupled to the HARMONIE-AROME forecast system (Seity et al., 2011). The suggested 
parameterizations have been developed earlier and applied to the operational weather forecast model HIRLAM (Undén et al., 2002).

Accounting for the variability of downweling short- and long-wave radiation fluxes due to the slopes, shadowing obstacles and limited sky view has been shown important for a correct local temperature forecast. For example, verification of the fine-resolution operational NWP models of the Austrian meteorological service has indicated systematic errors of the screen-level temperature up to $2^{\circ}$ during the morning hours in narrow Alpine valleys when shadowing effects are not taken into account. Since the pioneering work by Kondratyev (1977), parameterizations of orographic effects on radiation have been developed for NWP and climate models by Müller and Scherer (2005), Senkova et al. (2007), Manners et al. (2012), Helbig and Löwe $(2012,2014)$ and applied in WRF, HIRLAM, UKMO, and COSMO models.

The location of this study was chosen over Caucasian mountains near the city of Sochi, Russia. The 2014 winter Olympic games were held in Sochi on 8-23 February 2014. As a part of the event, the World Meteorological Organization (WMO) organized both research development and forecast demonstration projects. The FROST-2014 (Forecast and Research: the Olympic Sochi Testbed) project focused in particular on the development and demonstration of modern short-term NWP and nowcasting systems over mountainous regions in winter-time conditions and on the assessment of practical use of this information. In order to do so, the project deployed several additional observation sites in the area. Temperature measurements provided by these automatic meteorological station (AMS) observations were used for comparison and validation in our study.

To understand how the choice of basic orography influences the model results, we first rerun HARMONIE Sochi Olympic forecasts using the default input data for calculation of the gridscale surface elevation. We then replaced the default source by finer-resolution surface elevation data and compared the results of experiments which used the horizontal resolution of $1.5 \mathrm{~km}$, applying the standard verification methods and using the extended observations provided by FROST-2014. Next, we implemented new orographic radiation parameterizations to HARMONIE and derived the needed orographic variables from the fine-resolution surface elevation data. Our aim was to study the overall impact of the parameterizations and to test alternative formulations related to the view of the sky. For this purpose, we performed three-dimensional and single-column model simulations during February 2014 and evaluated the results in terms of the predicted radiation fluxes and near-surface temperatures. We also compared the three-dimensional experiment results to the AMS screen-level temperature observations. The aim was to reveal the differences and also to evaluate how suitable and sufficient the available observations were for this kind of validation.

The rest of this study is organized as follows: Section 2 introduces HARMONIE NWP system and the suggested orographic radiation parameterizations. Section 3 presents the source data and preparation of orography-related variables for the model. Special attention is paid to the definition of sky view factor for the radiation parameterizations. Section 4 defines the numerical experiments and Section 5 introduces the observations and validation methods. Results of the threedimensional and single-column experiments are presented and discussed in Section 6. Conclusions and outlook in Section 7 finish the study.

\section{MODELS AND METHODS}

\subsection{HARMONIE-AROME-SURFEX}

We focus on development and testing of orographic radiation parameterizations in the framework of HIRLAM-ALADIN Regional Mesoscale Operational NWP In Europe (HARMONIE) system. The HARMONIE NWP system combines elements from the global IFS/Arpege model (Déqué et al., 1994) with the ALADIN non-hydrostatic dynamics (Bénard et al., 2010) and physical parameterizations of AROME (Seity et al., 2011) and HIRLAM (Undén et al., 2002). Surface-related processes are treated by the externalized parametrization and data assimilation system SURFEX (Masson et al., 2013). In this study we implemented new schemes for parametrization of the orographic radiation fluxes into SURFEX, testing them in the full HARMONIE-AROME.

\subsection{Parametrization of Orographic Effects on Radiation}

A parametrization scheme for orographic effects on radiation (hereafter referred to as ororad) was introduced to HARMONIEAROME following Senkova et al. (2007) (hereafter referred to as SRS7). Slope $\left(\delta_{s l}\right)$ and shadow $\left(\delta_{s h}\right)$ factors (see Section 3.2) were derived and applied to modify downweling shortwave radiation (SWD) flux at the surface level. These factors modify the direct SWD by taking into account the different slope angles and directions with respect to the current solar position and estimate the relief shadows due to the neighboring obstacles. Restricted visibility of the sky is described by the sky-view factor $\left(\delta_{s v}\right)$, which is applied to modify the diffuse SWD and downweling longwave radiation (LWD) fluxes.

An essential feature of the SRS7 approach, different from the approach by e.g., Müller and Scherer (2005) and the recent Manners et al. (2012), is the derivation of these orographic factors from the most detailed subgrid-scale surface elevation information independently of the grid-scale mean orography. In the parametrization, radiation fluxes should be averaged, not the orography. Directional slopes and their fractions as well as directional local horizon angles were defined for every NWP model gridpoint. In this way, the parameterizations become scale-independent, flexibly adapting to any NWP model domain, space and time resolution, which is important for the operational usage.

Ororad parametrization was originally developed for the synoptic-scale HIRLAM with a typical horizontal resolution of $10-20 \mathrm{~km}$. Application of the methods in the kilometerresolution HARMONIE NWP system required derivation of the needed orographic parameters from fine resolution SRTM 
(Shuttle Radar Topography Mission, Jarvis et al., 2008) data, see Section 3.1 for the details.

In the current HARMONIE (version 38h1), the default atmospheric radiation scheme is based on the radiative transfer code in the Integrated Forecast System (IFS cycle 25R1, European Centre for Medium-Range Weather Forecast implementation in 2002), see ECMWF (2012) and Mascart and Bougeault (2011). An alternative radiation scheme originates in ALADIN, Mašek et al. (2015). The radiation scheme of HIRLAM, based on Savijärvi (1990), see also Nielsen et al. (2014), is available for experimentation. The suggested ororad parameterizations are applicable for preparation of the downweling solar and terrestrial radiation fluxes for SURFEX independently of the atmospheric radiation scheme. Without loss of generality, we limit the testing of ororad to the case of IFS scheme.

\section{OROGRAPHY DATA AND MODEL VARIABLES}

\subsection{Fine Resolution Data on Surface Elevation}

Grid-scale and subgrid-scale orography variables for the new $1.5-\mathrm{km}$ resolution experiments were derived from 3-arc-second resolution Shuttle Radar Topography Mission surface elevation data (SRTM 3", Jarvis et al., 2008). In our experiments, surface elevation from SRTM 3" replaced that from GTOPO30 (USGS, 1998), used by default in SURFEX. The horizontal resolution of $3^{\prime \prime}$ corresponds to ca. $90 \mathrm{~m}$ at the Equator, while one GTOPO30 pixel represents a size of ca. $900 \mathrm{~m}$. Figure 1 illustrates different resolution grids in the surroundings of the highest peak of Europe Mt. Elbrus (43.355 N, 42.439 E, $5642 \mathrm{~m}$ ), located on the Caucasian mountains, in a rectangle of $\sim 15 \mathrm{~km}$ width, laid over a map based on Google Earth (Wikipedia, 2012). The rectangle would represent a typical resolution of a synoptic scale NWP model with only one gridbox over the area in Figure 1A. Figure $\mathbf{1 B}$ shows $1 \mathrm{~km}$ resolution,

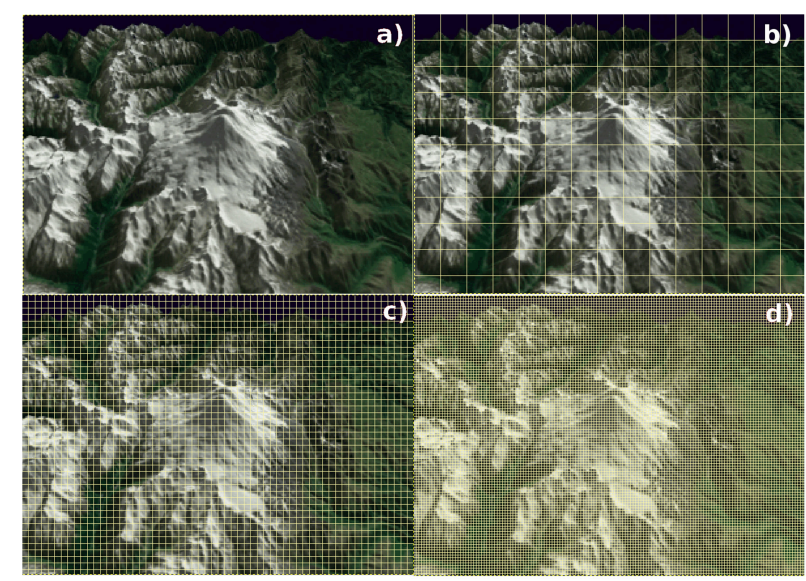

FIGURE 1 | Schematic illustration of grids of different resolutions laid over a Mt. Elbrus map: (A) $15 \mathrm{~km}$, (B) 1 km, (C) $250 \mathrm{~m}$, (D) $100 \mathrm{~m}$. corresponding e.g., the GTOPO30 data or the finest resolution Olympic HARMONIE grid. Figures 1C,D represent roughly the resolutions of the Global Multi-resolution Terrain Elevation 7.5arc-second data (GMTED2010, USGS (2010)) and SRTM 3" data, respectively. GTOPO30, SRTM and GMTED2010 are all used as fine-resolution source data for derivation of surface-elevation dependent variables in various NWP models. GTOPO30 would provide a synoptic-scale model with more than two hundred pixels for calculation of subgrid-scale statistics within each gridcell. SRTM 3" resolution is needed to acquire the same amount of source pixels for our $1.5-\mathrm{km}$ resolution experiments.

\subsection{Orography Variables in the Model Grid}

Mean surface elevation for the forecast model and data assimilation as well as various subgrid-scale orography parameters for radiation and momentum flux parameterizations (Table 1) were derived from the fine-resolution source orography (Section 3.1) for a Lambert conical conformal projection grid with horizontal resolutions from 1 to $2.5 \mathrm{~km}$. Calculations were done within the SURFEX framework by using its physiography definition (PGD) subroutines.

The difference between the average surface elevation $\mathrm{H}_{\Delta x}$ derived from GTOPO30 and SRTM 3 " for the $1.5-\mathrm{km}$ resolution experiments is shown in Figure 2. The local differences vary from -386 to $+453 \mathrm{~m}$, but the average difference is small, $+3 \mathrm{~m}$. The maximum grid-average surface elevation over the area was 5040 or $5050 \mathrm{~m}$, in both cases about $600 \mathrm{~m}$ lower than the top of Mt.Elbrus according the fine-resolution SRTM $3^{\prime \prime}$ estimate. As calculated from SRTM $3^{\prime \prime}$ to the $1.5 \mathrm{~km}$ grid, the mean $\sigma_{s s o}$ over the land area of the Caucasian domain was $44 \mathrm{~m}$, with maxima of $350 \mathrm{~m}$ (not shown). The corresponding values based on GTOPO30 were 33 and $482 \mathrm{~m}$. Due to the coarse resolution of the GTOPO30 data with respect to the chosen model grid, $\sigma_{\text {sso }}$ values look somewhat random (see also Table 3 for selected locations). The standard deviation over each gridpoint area was based only on ca. 5 GTOPO30 elevation values.

Preprocessing of the SRTM $3^{\prime \prime}$ surface elevation data was necessary for derivation of the constant-in-time variables for orographic radiation parameterizations (For the operational NWP, this preprocessing will be replaced by processing within the SURFEX PGD system - Alexandre Mary, personal communication). Preprocessing was done in the SRTM $3^{\prime \prime}$ latitude-longitude grid. Slope angle and direction were calculated by using Geospatial Data Abstraction Library software (GDAL, 2014). Treatment of the local horizon and sky view was modified as compared to the original SRS7 method, and is documented in the next section.

\subsection{Local Horizon and Sky View}

Following the method suggested by SRS7, the local horizon angle $h_{h, i}$, defined as a height angle from the surface, was estimated at each SRTM $3^{\prime \prime}$ gridpoint from the difference between the surface elevation at the point and its 50 neighbors (i.e., within a distance $<5 \mathrm{~km}$ ) in each $1^{\circ}$ sector. These $1^{\circ} h_{h, i}$ values were averaged over eight directional sectors, each representing $45^{\circ}$ centered at N, NE, E, SE, S, SW, W, NW directions. 
TABLE 1 | Orography-related parameters within grid resolution.

\begin{tabular}{|c|c|c|c|c|}
\hline Parameter & Description & Unit & Usage & Remarks \\
\hline $\mathrm{H}_{\Delta x}$ & Mean surface elevation & $\mathrm{m}$ & Dynamics & Smoothed \\
\hline$\sigma_{S S O}$ & Subgrid-scale standard deviation & $\mathrm{m}$ & Momentum & \\
\hline$s_{s s o}$ & Mean subgrid-scale slope angle & $\mathrm{rad}$ & - & Eigenvalue of gradient correlation tensor \\
\hline$h_{m, i}$ & Slope angle in direction $i$ & $\mathrm{rad}$ & Radiation & \\
\hline$f_{j}$ & Fraction of slope in direction $i$ & - & Radiation & \\
\hline$h_{h, i}$ & Local horizon in direction $i$ & $\mathrm{rad}$ & Radiation & \\
\hline$\delta_{S V}$ & Sky view factor & - & Radiation & Derived, runtime \\
\hline$\delta_{S l}$ & Slope factor & - & Radiation & Derived, runtime \\
\hline$\delta_{\text {sh }}$ & Shadow factor & - & Radiation & Derived, runtime \\
\hline
\end{tabular}

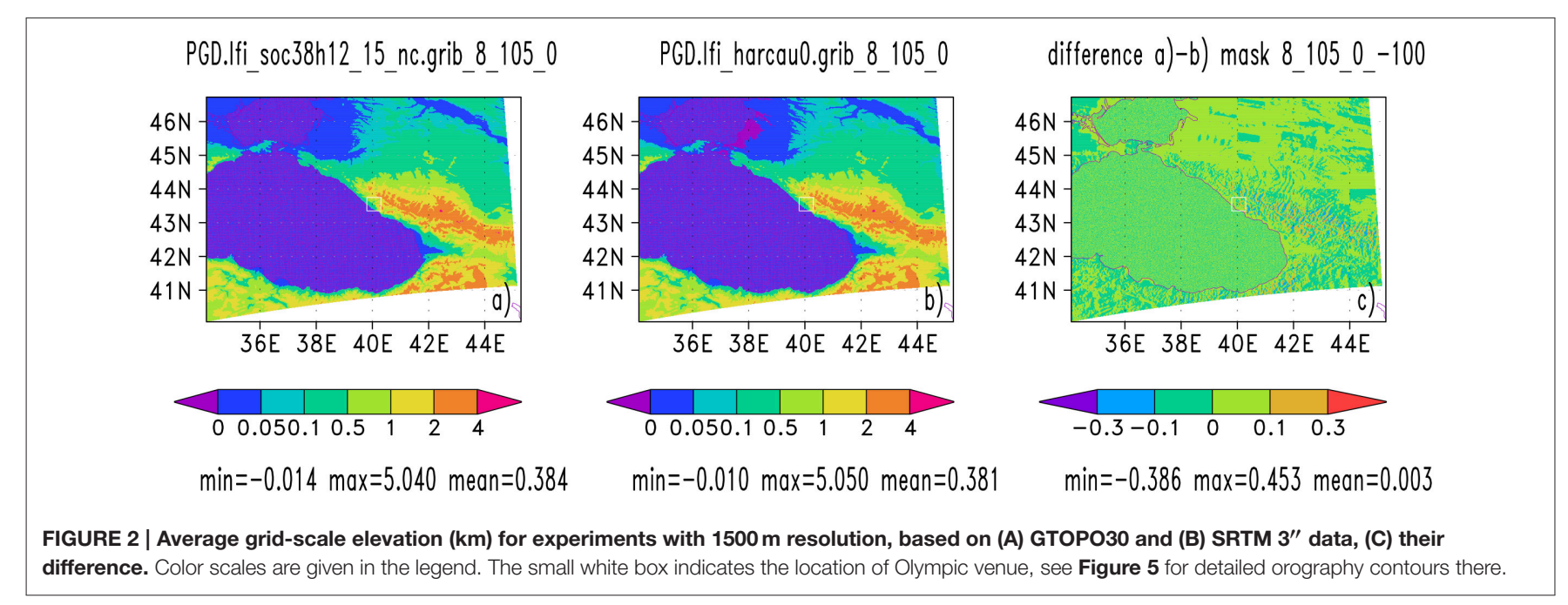

Originally, the elevation differences within each $1^{\circ}$ sector around a central point were weighted according to the squared distance from the center and a mean value obtained. Based on comparison of observed and calculated local horizon angles around several Alpine stations, we omitted the weighting and used instead the maximum local horizon angle. For example, the Alpine station St. Leonhard/Pitztal (47.027 N, 10.865 E) lies in a very narrow south-north oriented valley in Tyrol, Western Austria, and suffers from significant shadowing by surrounding mountains. Figure 3 shows a comparison between the observed horizon and the two different methods for calculation of the local horizon angle. The blue dots, which refer to the updated method, are clearly closer to the observations than the red dots, which refer to the weighted angles as suggested by SRS7. The result shown in Figure 3 represents the local horizon at the station location on the SRTM 3 " grid.

The sky view factor $\delta_{s v}$ was calculated by averaging the local horizon angle over all directions $\left(360^{\circ}\right)$. A definition of $\delta_{s v}$ as the average of $1-\sin \left(h_{h, i}\right)$ was suggested by Müller and Scherer (2005) and applied by SRS7. Following SRS7, we calculated $\delta_{s v}$ at each fine resolution source grid point during the preprocessing. A different approach for calculation of the sky factor was suggested by Manners et al. (2012), based on Helbig et al. (2009). Manners et al. (2012) derived and applied an equation in the NWP model grid (their Equation 14, see also their Figure 5; note that their horizon angle is defined from zenith toward the obstacles). The aim was to approximate the proportion of radiant flux from the visible sky falling on the inclined surface as a fraction of the flux that would have been received from an unobstructed hemisphere.

We tested the Manners et al. (2012) formulation but adapted the method to the subgrid-scale statistical approach by SRS7. A modified $\delta_{s v}^{\prime}$ was obtained by summation of the sectorial mean $h_{h, i}$ values during the aggregation of the values to the NWP grid. The mean directional slope $h_{m, i}$ in each of the eight directional sectors and the directional fraction of the slopes $f_{i}$ in each model gridsquare were taken into account:

$$
\delta_{s v}^{\prime} \approx \sum_{i=1}^{8} \frac{f_{i} \cos ^{2}\left(h_{h, i}+h_{m, i}\right)}{\cos \left(h_{m, i}\right)}
$$

Here we assumed implicitly that it is sufficient to account for the local horizon $h_{h, i}$ in the same direction where a portion $f_{i}$ of the slopes $h_{m, i}$ is facing to. Including scaling with the slope $\cos \left(h_{m, i}\right)$ when calculating $\delta_{s v}^{\prime}$ allowed to avoid scaling by the ambiguous grid-average slope during the actual forecast run. (However, usage of $\delta_{s v}^{\prime}$ during runtime leads to a slight inconsistency in 


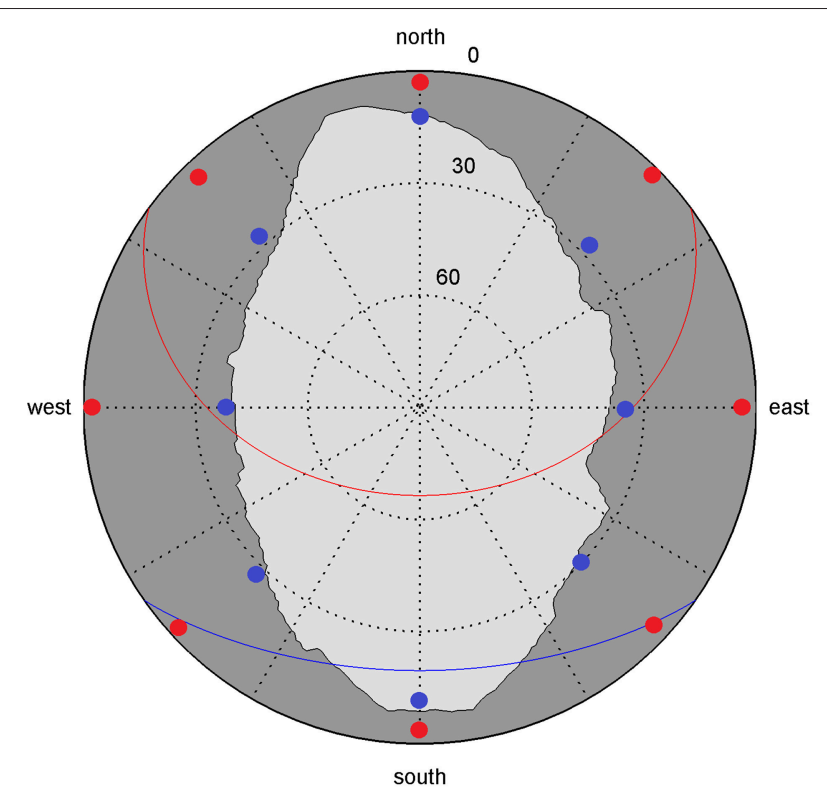

FIGURE 3 | Observed horizon (gray shaded area) and calculated local horizon angles (blue dots: without weighting, red dots: weighted) around the Alpine station St. Leonhard/Pitztal, Austria. Red and blue lines show the path of the sun at the winter (blue) and summer (red) solstice.

calculation of a second-order term related to the reflection of shortwave radiation by the surrounding terrain.) The modified $\delta_{s v}^{\prime}$ was calculated by using the SURFEX PGD tools during the aggregation, while the original $\delta_{s v}$ was obtained in the fineresolution SRTM 3" grid and only averaged during aggregation. By modifying Equation (1) further, it is possible to apply it also to approximation of $\delta_{s v}$ as an average of the sectorial $1-\sin \left(h_{h, i}\right)$ values suggested by SRS7. There exist even more possible ways to approximate the sky view factor at the source level, during aggregation or in the model grid, all based on the local horizon angle calculated in the source grid.

We compared four approximations, which led to somewhat different grid-scale sky-view factor values. As illustrated by Figure 4, the overall distributions of $\delta_{s v}$, estimated by the different methods, look fairly similar, but the minimum values and local features can be significantly different. The maps represent the Sochi Olympic venue in the model grid of 1.5$\mathrm{km}$ resolution (area shown in Figure 5). The area-mean of $\delta_{s v}^{\prime}$ based on Equation (1) over the Olympic venue was the smallest, 0.87 (87\%; Figure 4C), while the values for the SRTMlevel (Figure 4B) and aggregation-level (Figure 4D) SRS7 $\delta_{s v}$ were 0.90 and 0.91 , correspondingly. These numbers can be compared to the evidently overestimated average $\delta_{s v}=0.98$, derived from the original weighted $h_{h, i}$ (Figure 4A). Over the flat areas, the sky view factor is defined to be unity (100\%), and all values were included in the calculation of the area means. Spatial correlations of $\delta_{s v}$ were 0.88 between the SRTMlevel SRS7 and Equation (1), 0.92 between the aggregationlevel SRS7 and Equation (1), 0.91 between the SRTM-level and aggregation-level SRS7. The influence of the $\delta_{s v}$ differences in the parameterized long-wave and diffuse short-wave radiation fluxes will be discussed in Section 6.

\section{DEFINITION OF HARMONIE EXPERIMENTS}

Two series of three-dimensional HARMONIE experiments were designed (Table 2) for the purpose of examining the influence of different parameterizations and the introduction of the SRTM $3{ }^{\prime \prime}$ based surface elevation. For the reference, the series of Olympic experiments (soc38h12) was set up similar to the real-time forecast applied by the Finnish Meteorological Institute during the Sochi Olympic games 2014. Here, three different model domains with the horizontal resolution of 2500, 1500, and $1000 \mathrm{~m}$ and 65 levels in vertical (65L) were defined over a CaucasianBlack Sea domain, shown by the full area of Figure 2 for the main $1500 \mathrm{~m}$ experiments. As the number of gridpoints was kept constant $(640 \times 500)$, the domain covered by the $2500 \mathrm{~m}$ $(1000 \mathrm{~m})$-resolution experiment was larger (smaller) than this. Above the surface, the lowest model level was centered at the elevation of ca. $14 \mathrm{~m}$. GTOPO30 (USGS, 1998) 30" resolution data set was used as a source for surface elevation in all control experiments. No orography-related parameterizations for radiation or momentum fluxes were applied. A multilayer parametrization for the natural and urban canopy (so-called CANOPY scheme, Masson and Seity, 2009) was applied for treatment of the turbulent momentum fluxes in all but one of the reference experiments.

The second series of experiments (harcau) was designed to test the orographic parameterizations. These experiments were defined using $1500 \mathrm{~m} / 65 \mathrm{~L}$ resolution and based on the SRTM 3 " surface elevation data. A control experiment harcau0 introduced the SRTM 3 " elevation for calculation of the gridscale mean orography and its variance only, without application of the orography-related parameterizations. This experiment is comparable to the Olympic experiment soc38h12_15_nc, because the only difference between them is the source of the fine resolution orography.

Preliminary experiments were run separately with orographic parameterizations only for radiation and only for momentum fluxes. They showed that the two parameterizations in practice acted independently, i.e., the radiation parameterizations did not influence wind and momentum fluxes and the (weak) momentum flux parameterizations did not influence the temperature and radiation fluxes. Consequently, experiments harcau3, harcau6, and harcau8 were defined, containing both parameterizations and only differing with respect to the calculation of local horizon angle and sky view factor (see Section 3.2). Further we will focus on the evaluation of the radiation parameterizations.

In all experiments, HARMONIE version $38 \mathrm{~h} 1.2$ was applied with assimilation of near-surface (SYNOP) observations only. Horizontal boundary data were obtained from European Centre for Medium Range Weather Forecasts (ECMWF). Boundary data was used also as the initial state for the atmospheric variables. Default options for fine-scale dynamics and physical 


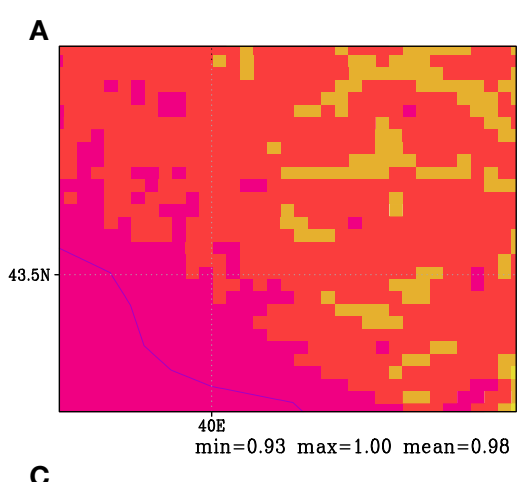

C

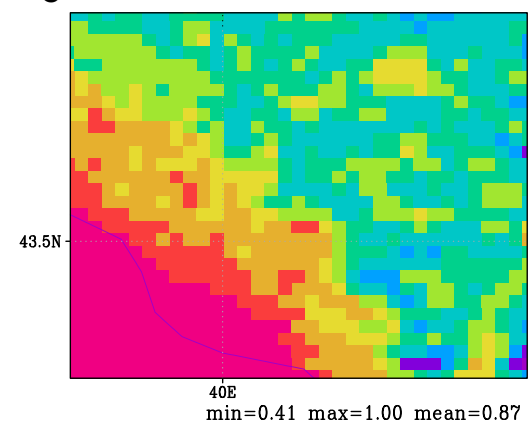

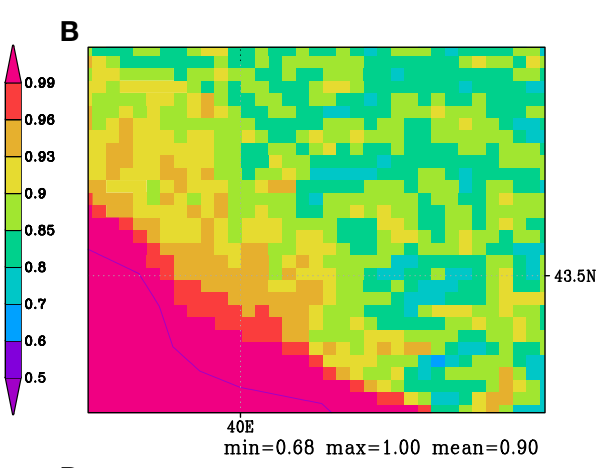

D

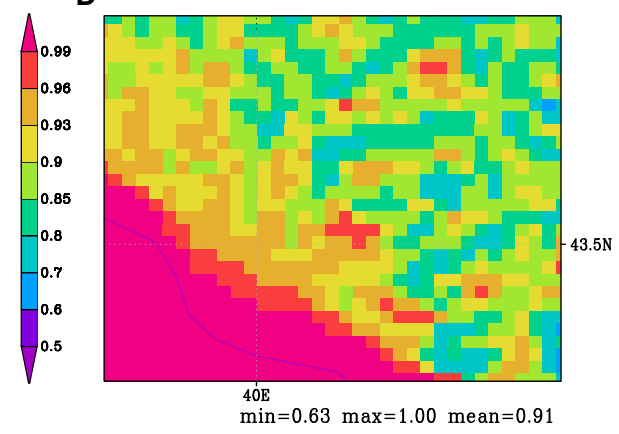

FIGURE 4 | Sky view factor $\delta_{S v}$ (fraction from 0 to 1 shown by the color scale) zoomed to the Olympic venue: (A) based on local horizon angle obtained with the distance-dependent weighting, (B) STRM-level estimate without weighting of the horizon angle, (C) $\delta_{\text {sv }}^{\prime}$ estimated from Equation (1), (D) aggregation-level estimate according to SRS7 definition. The approximate coastline is indicated in the lower left part of the figures, latitude $43.5 \mathrm{~N}$ and longitude $40 \mathrm{E}$ are marked into the maps.

parameterizations were used, except those described above. The analysis-forecast cycles with forecast lead time up to $+27 \mathrm{~h}$ were initiated at 00, 06, 12, and 18 UTC for February 2014.

\section{DIAGNOSTICS AND VALIDATION}

\subsection{Observations}

The Sochi Olympic area was located on the eastern coast of the Black Sea. The events took place in two specific areas, in the coastal and mountain clusters. The coastal cluster hosted indoor sports, whereas all the weather-dependent events, such as ski jumping, cross-country, and alpine skiing, took place in the mountain cluster. Therefore, most of the new observations were deployed in the mountain cluster. The distance from the mountain cluster to the coast of Black sea was about $40 \mathrm{~km}$ along the road following a $3-6 \mathrm{~km}$ wide valley, surrounded by high mountains peaking $\sim 2000-2500 \mathrm{~m}$ from the sea surface.

The main village in the mountain cluster was Krasnaya Polyana, which is located in the valley about $550 \mathrm{~m}$ above the sea surface. The sport events were organized in a close vicinity at the nearby mountains on both sides of the valley. The ski stadium, alpine skiing and ski jumping venues were at the heights of 1400,2200 , and $600 \mathrm{~m}$ above the sea surface, respectively. An official WMO SYNOP weather station (37107) provides regular near-surface weather observations in Krasnaya Polyana together with the coastal Adler station (37171). All sporting venues were equipped with several AMS measuring basic meteorological variables such as the screen-level temperature and relative humidity and anemometer-level wind speed/ direction.

The placement of the AMS observations allowed estimation of the temperature differences between the northern and southern side of the valley and within the valley itself. However, radiation measurements were not made and are not, to our knowledge, available elsewhere over the Caucasian mountains. Table 3 summarizes the observations used in this study. The dataset was divided into three categories based on the geographical location: mountain, valley and coastal stations. Figure 5 shows the location of the stations. Most of the mountain stations were located in the southern side of the valley (north-facing slope), whereas one station (39043) was located on a south-facing slope. All valley stations were distributed along the valley from the mountain cluster to the coast of Black Sea. Note that in some cases, the elevation of the station differs significantly from the $1.5-\mathrm{km}$ resolution grid-average model surface elevation because of the unresolved orography features.

\subsection{HARMONIE Station Verification}

The two series of experiments (Table 2) were first validated using the HARMONIE standard station verification system and the Olympic surface-based observations (Section 5.1). In HARMONIE station verification, values from the closest gridpoints are bilinearly interpolated to station locations. For 
the screen-level temperature, it is also possible to take into account in the verification the difference between the grid-scale mean elevation and the elevation reported by each station by assuming moist adiabatic lapse rate between these levels. This is only a rough estimate. Interpolations of the temperature and surface elevation as well as the height adjustment depend on the grid-scale mean surface elevation and on the model's horizontal and vertical resolution, which brings some ambiguity to the validation process over the complex topography. Validation over the Olympic venue was done separately for the mountain, valley and coastal clusters during February 2014. In addition, the experiments were validated against all SYNOP station measurements available over the whole Caucasian model domain (39-51 measurements, depending on the observed variable and time of the day). We will focus on the screen-level temperature and mention also the $10-\mathrm{m}$ wind verification (Section 6.1).

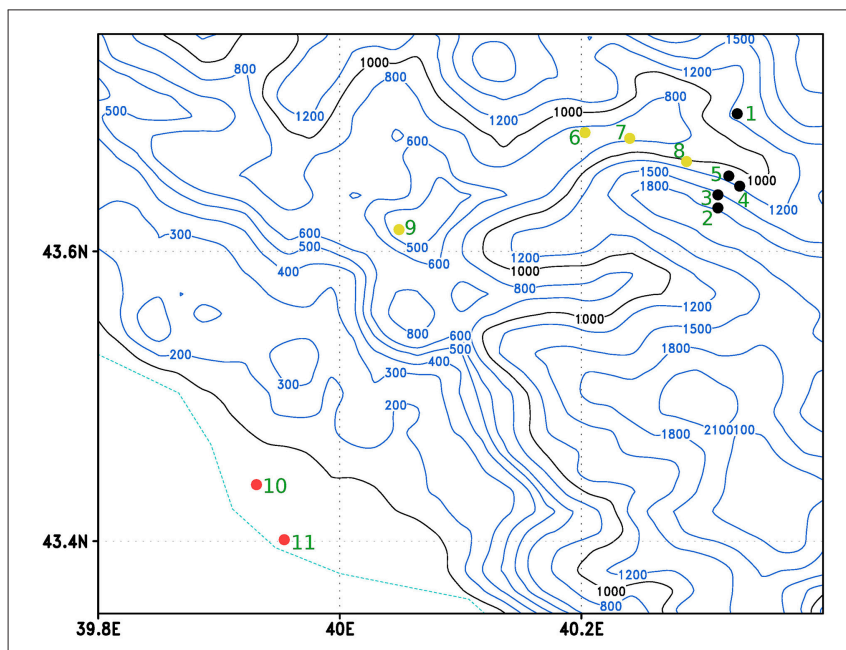

FIGURE 5 | Location of the observation sites. Mountain, valley, and coastal stations are marked with black, yellow, and red dots, respectively. Green numbers refer to the list of stations in Table 3. The contours represent average orographic height (meters) in Harmonie model with $1.5 \mathrm{~km}$ grid size, based on SRTM $3^{\prime \prime}$ data. Dashed cyan line on the lower left denotes coastline.

TABLE 2 | Three-dimensional HARMONIE experiments.

\begin{tabular}{|c|c|c|c|c|}
\hline Name & $\begin{array}{l}\text { Resolution } \\
\text { (km/65L) }\end{array}$ & $\begin{array}{l}\text { Sfc } \\
\text { elevation }\end{array}$ & Parameterizations & Remarks \\
\hline soc38h12_25 & 2.5 & GTOPO30 & CANOPY & \\
\hline soc38h12_15 & 1.5 & GTOPO30 & CANOPY & \\
\hline soc38h12_10 & 1.0 & GTOPO30 & CANOPY & \\
\hline soc38h12_15_nc & 1.5 & GTOPO30 & - & \\
\hline harcau0 & 1.5 & SRTM $3^{\prime \prime}$ & - & \\
\hline harcau3 & 1.5 & SRTM $3^{\prime \prime}$ & ororad, orotur & Weighted $h_{h, i}$ \\
\hline harcau6 & 1.5 & SRTM $3^{\prime \prime}$ & ororad, orotur & Maximum $h_{h, i}$ \\
\hline harcau8 & 1.5 & SRTM $3^{\prime \prime}$ & ororad, orotur & Equation 1 \\
\hline
\end{tabular}

\subsection{Intercomparison of Model Variables at Selected Locations}

To see more local details of the radiation fluxes and temperatures, values of selected predicted model variables were picked from +6-h forecasts of the SRTM 3"-based experiments at the observation locations (Table 3). Results will be presented in Section 6.2. Every day, all four forecasts initialized at 00, 06, 12 , and 18 UTC were included. The nearest gridpoint values were selected without interpolation to observation locations in order to minimize uncertainties related to interpolation over the complex topography. Screen-level temperatures with hourly interval were available for comparison. For the other variables like radiation fluxes and surface temperature, the different experiments were intercompared without observations.

\subsection{Sensitivity Experiments with the Single-Column Model}

MUSC, the single column version of HARMONIE, based on Malardel et al. (2006), was used for testing of sensitivity of the predicted radiation fluxes and the near-surface temperature to the ororad parameterizations. The atmospheric profiles, near-surface initial data and the surface properties for MUSC experiments were selected from the three-dimensional experiments for chosen locations and short experiments run up to 1 day. A vertical resolution of 65 pressure-based hybrid coordinate levels was used, corresponding to that in the full model.

The single-column model offers a tool to analyze the behavior of the full three-dimensional experiments in a controllable environment. In the single-column framework, any of the orographic variables (see Section 3.2) can be modified for a selected location. Different formulations of the physical parameterizations can be tested. However, the single-column experiment results are not directly comparable with observations, mainly because the model three-dimensional dynamics is not included in the setup. In the present experiments, the atmospheric initial state was kept unchanged during the single-column runs but the surface state was updated by the surface-related parameterizations. These experiments were performed in order to understand the interactions in the model and roughly estimate the effects caused either by the different surface properties or by modifications of the physical parameterizations.

\section{RESULTS AND DISCUSSION}

\subsection{Validation of Screen-Level Temperature and Anemometer-Level Wind against SYNOP Observations}

The first validations of the real-time Olympic HARMONIE forecasts revealed a problem of nighttime screen-level temperatures: in some situations they were strongly underestimated (Niemelä et al., 2014). The finest-resolution experiments seemed to suffer from this problem most. This can be clearly seen also in the verification time-series which compare the reference Olympic experiments, run for the present study, 
with the observations of the mountain cluster during the first week of February 2014 (Figure 6). This figure even indicates the reason and solution of the problem as one of the reference experiments, the one without the surface sublayer (CANOPY) parameterizations, compares to the observations clearly better than the others. It was found that the scheme, which treats turbulence in the surface layer based on Masson and Seity (2009), may in clear and calm nights result in decoupling of the atmosphere from the surface. Hence, in the second series of experiments used for testing the fine-resolution surface orography and the proposed orographic parameterizations, the surface sublayer scheme was excluded. A similar comparison of the time-series by these experiments (not shown) confirms that this particular temperature problem was solved in all of them.

All HARMONIE experiments of the second series, which relied on SRTM 3" source orography and used $1500 \mathrm{~m}$ resolution, showed a negative bias of $T_{2 m}$ especially during day. Experiment soc38h12_15_nc, based on GTOPO30 source orography, showed the smallest bias of (height-adjusted) $T_{2 m}$. In verification against all Sochi AMS observations, the experiment harcau0 showed $0.3-0.4^{\circ}$ larger bias than soc38h12_15_n. The corresponding difference was $0.1-0.2^{\circ}$ when validated against the regular SYNOP station observations over the whole Caucasian domain in February 2014. The verification result of the height-adjusted $T_{2 m}$ is illustrated for the Olympic valley cluster by Figure 7. The experiments harcau3 (not shown), harcau6 and harcau8, which included orographic parameterizations, were consistently but very little better than harcau0.

In the valley, the height adjustment only influenced the magnitude of the screen-level temperature bias but not the relations between the experiments. In the mountain cluster, the adjustment increased the negative bias of soc38h12_15_nc $T_{2 m}$ to the same level (ca. $-1^{\circ}$ for all forecast lead times) as that of the harcau experiments. All the valley stations were located somewhat lower than indicated by the mean elevation of the corresponding gridpoint, based on both SRTM $3^{\prime \prime}$ and GTOPO30. The situation was the opposite in the mountain cluster where only one station lay lower in the model than in the nature (Table 3). This is because the orography details were not fully resolved even within the $1500 \mathrm{~m}$ model resolution. We will continue analysing the differences in Section 6.2.

Wind observations over the Olympic venue and also over the whole Caucasian experiment domain indicated weak winds during February 2014. In particular, the Olympic observations often reported zero winds, which are never predicted by the model. In these conditions, wind speeds were overestimated by the model as compared to the observations, typically up to $1 \mathrm{~m} / \mathrm{s}$ over the whole Caucasian area and by $0.5-1 \mathrm{~m} / \mathrm{s}$ over the Olympic mountain and valley clusters (not shown). Orographic momentum flux parameterizations led to a slight decrease of this positive bias as compared to the default setup where these were not applied. Nevertheless, the Olympic wind speed observations, set up to serve the Olympic competitions, were found insufficiently representative for the validation of orographic parameterizations and will not be discussed further in this study.

\subsection{Comparison of Three-Dimensional Model Experiments}

Monthly mean values of predicted downweling shortwave radiation $(\mathrm{SWD}=$ global radiation, i.e., the sum of direct and diffuse components) and longwave (LWD) radiation fluxes with and without (experiments harcau6 and harcau0) ororad parameterizations are shown in Figure 8. The average fluxes were calculated from $+24 \mathrm{~h}$ forecasts starting at 00 UTC. Differences up to $\pm 50 \mathrm{Wm}^{-2}$ of SWD can be found around the mountain ridge in the NW part of the Olympic venue while the average difference harcau6 - harcau0 was only $-2 \mathrm{Wm}^{-2}$. The local differences in LWD were smaller, between -3 and $20 \mathrm{Wm}^{-2}$ but the area-averaged LWD of harcau6 was by $8 \mathrm{Wm}^{-2}$ larger than that of harcau0. The monthly mean SWD fluxes were typically less than half of the LWD fluxes, about 120 vs. $260 \mathrm{Wm}^{-2}$. Length of day from sunrise to sunset in February in this location is $\sim 10 \mathrm{~h}$. February 2014 was predominantly cloud-free over the Olympic venue.

TABLE 3 | Summary of the observation sites used in the study.

\begin{tabular}{|c|c|c|c|c|c|c|c|}
\hline & Name & Number & Location & Height (m) & Height $\pm \sigma_{\text {sso }} \mathbf{S}^{*}$ & Height $\pm \sigma_{\text {sso }} \mathbf{G}^{*}$ & Group \\
\hline 1 & Ski stadium & 39043 & $43.695 \mathrm{~N}, 40.329 \mathrm{E}$ & 1483 & $1307 \pm 167$ & $1403 \pm 154$ & Mountain \\
\hline 2 & RKHU-2 & 00008 & $43.630 \mathrm{~N}, 40.313 \mathrm{E}$ & 2137 & $1890 \pm 180$ & $1814 \pm 145$ & Mountain \\
\hline 3 & RKHU-4 & 00011 & $43.639 \mathrm{~N}, 40.313 \mathrm{E}$ & 1580 & $1506 \pm 200$ & $1421 \pm 142$ & Mountain \\
\hline 4 & RKHU-7 & 00065 & $43.645 \mathrm{~N}, 40.331 \mathrm{E}$ & 980 & $1229 \pm 132$ & $1106 \pm 129$ & Mountain \\
\hline 5 & Freestyle-1080 & 39048 & 43.652 N, $40.322 \mathrm{E}$ & 1077 & $990 \pm 136$ & $912 \pm 119$ & Mountain \\
\hline 6 & Krasnaya Polyana & 37107 & 43.682 N, $40.203 \mathrm{E}$ & 564 & $786 \pm 124$ & $762 \pm 111$ & Valley \\
\hline 7 & Ski Jump-650 & 39040 & $43.678 \mathrm{~N}, 40.240 \mathrm{E}$ & 628 & $712 \pm 115$ & $721 \pm 14$ & Valley \\
\hline 8 & Sledge-830 & 39046 & $43.662 \mathrm{~N}, 40.287 \mathrm{E}$ & 835 & $824 \pm 128$ & $836 \pm 114$ & Valley \\
\hline 9 & Kepsha & 37100 & $43.615 \mathrm{~N}, 40.049 \mathrm{E}$ & 180 & $392 \pm 96$ & $405 \pm 7$ & Valley \\
\hline 10 & Adler & 37171 & $43.439 \mathrm{~N}, 39.931 \mathrm{E}$ & 12 & $9 \pm 3$ & $22 \pm 5$ & Coastal \\
\hline 11 & Imeretinka & 37095 & $43.401 \mathrm{~N}, 39.954 \mathrm{E}$ & 6 & $-2 \pm$ none & $-0 \pm$ none & Coastal \\
\hline
\end{tabular}

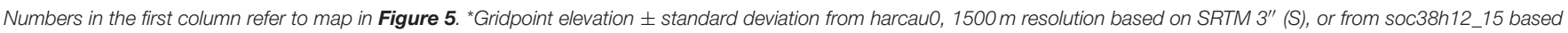
on GTOPO3O (G). 


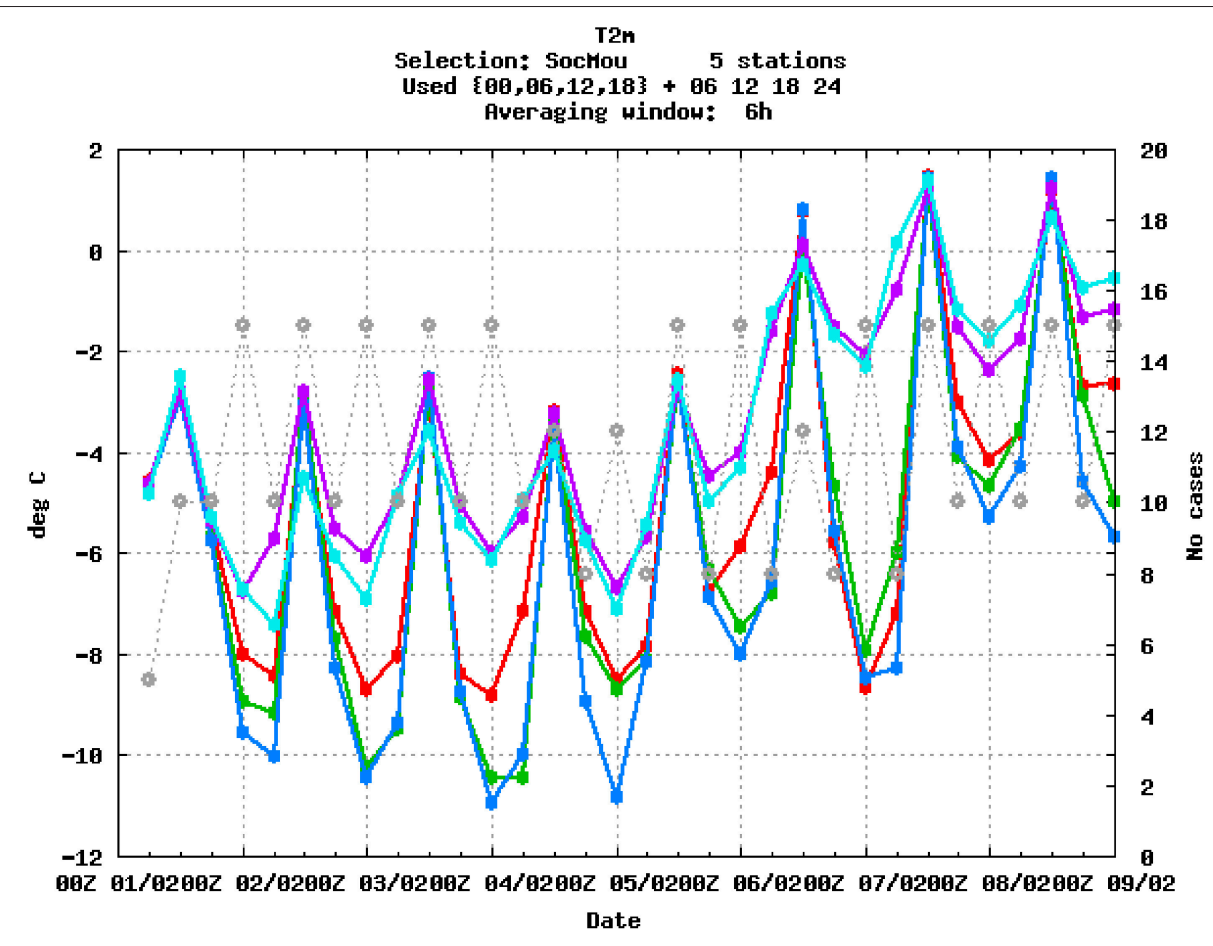

FIGURE 6 | Comparison of screen-level temperatures over the Olympic mountain cluster stations during the first week of February 2014: observed (light blue), the reference Olympic experiments with $2500 \mathrm{~m}$ (red), $1500 \mathrm{~m}$ (green), $1000 \mathrm{~m}$ (dark blue) resolution and $1500 \mathrm{~m}$ without surface sublayer scheme (violet). Number of cases (model-observation pairs) used for validation are shown with gray dots.

Downweling radiation fluxes influence the surface energy budget over different surface types, from which the grid-average surface (skin) temperature $T_{s}$ is calculated by SURFEX. Thus, $T_{s}$ should show the impact of the radiation parameterizations, although it is not directly comparable to available observations (because only $T_{2 m}$ was observed). Figure 9 shows the difference between harcau6 and harcau0 in terms of the monthlymean nighttime and daytime land surface temperatures, based on $+24 \mathrm{~h}$ forecasts starting at 00 and $12 \mathrm{UTC}$, respectively. The difference between the colder northern and warmer southern slopes shows up when comparing the daytime $T_{s}$ values. The maximum differences varied from -3 to $+2^{\circ}$ in NW part of the venue, where also the maximum differences in SWD fluxes were seen. The average $T_{s}$ at 12 UTC over the Olympic venue area was $0.3^{\circ}$ larger in harcau6 than in harcau0.

During night, the experiment harcau6 with ororad parameterizations showed up to $1^{\circ}$ warmer surface temperatures in valleys with limited sky view (see distribution of $\delta_{s v}$ in Figure 4) and corresponding larger LWD flux (Figure 8), as compared to the reference experiment harcau0. The average $T_{S}$ at 00 UTC over the Olympic venue was $0.2^{\circ}$ higher in harcau6 than in harcau 0 . The corresponding maximum difference of $T_{s}$ at 00 UTC between the experiments harcau 8 and harcau6, that used different approximations for calculation of $\delta_{s v}$, was also $1^{\circ}$, and on the average harcau8 was $<0.1^{\circ}$ warmer than harcau6 (not shown). This difference was due to the smaller mean sky view factor in harcau8 $(0.87)$ compared to harcau6 (0.90; see Section 3.3).
The systematic increase of the area mean daytime and nighttime $T_{s}$ in harcau6 and harcau8 compared to harcau0 might be interpreted to be mainly due to the systematic increase of LWD as the average SWD change was small. During daytime, the surface temperature differences between harcau3 and harcau0 were similar to the difference between harcau6 and harcau0 but clearly smaller during night (not shown) because of the open sky views in harcau3, where the average $\delta_{s v}$ was 0.98 .

In HARMONIE, the screen-level temperature $T_{2 m}$, which was verified against observations in Section 6.1, is diagnosed from the lowest model layer temperature $T_{\text {nlev }}$ (at ca. $14 \mathrm{~m}$ according to the 65 level configuration of the present experiments) and from the surface temperature $T_{s}$ by applying interpolation which takes into account the surface layer stability. Thus, $T_{2 m}$ is not a direct prognostic variable in the model. The lowest model level temperature $T_{n l e v}$ depends mainly on the large-scale atmospheric flow, and is less influenced by the local conditions. The observed $T_{2 m}$ showed more local variability and typically clearly higher maximum values than any of the HARMONIE experiments. This underestimation of the daytime screen-level temperature led to the negative bias shown by the station verification (Section 6.1). With the radiation parameterizations, we were able to influence $T_{s}$ typically $< \pm 1^{\circ}$. The impact of the parameterizations to the $T_{2 m}$ values was much smaller, on average $+0.1^{\circ}$ and varying from -0.6 to $+0.6^{\circ}$.

For examination of the local effects, the diurnal cycles of radiation fluxes, screen-level and surface temperatures were 


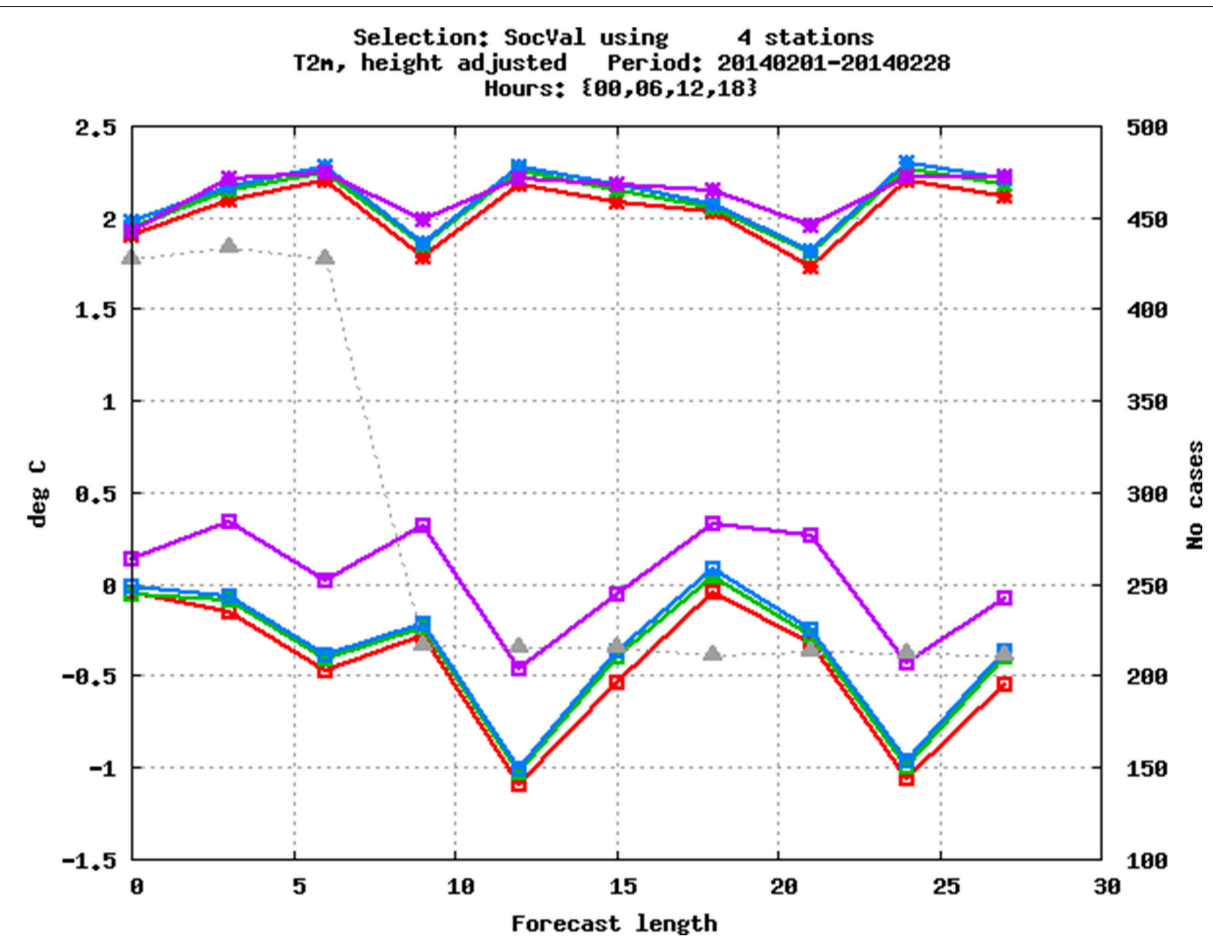

FIGURE 7 | Standard deviation (upper curves) and bias (lower curves) of the height-adjusted screen-level temperatures $T_{2 m}$ as a function of forecast length (hours) against observations of the Olympic valley stations during February 2014: harcau0 (red), harcau6 (green), harcau8 (dark blue), soc38h12_15_nc (violet), number of cases (gray triangles).

depicted at two Olympic stations, 37107 and 00011, based on $+6 \mathrm{~h}$ forecasts by the experiments harcau0, harcau3, harcau6, harcau8, and soc38h12_15_nc (Figures 10, 11). At the former location, the gridpoint nearest to the Krasnaya Polyana valley station, $90 \%$ of the slopes looked southward while at the latter the northern slopes prevail so that no SE-S-SW-looking slopes were present in the gridpoint around this station. Local shadowing obstacles were found mostly east from 00011 . The sky view factor was $0.85 / 0.92 / 0.98$ for the location of 37107 and $0.90 / 0.86 / 0.98$ for 00011 in the experiments harcau6/harcau8/harcau3, correspondingly.

At these two stations, all experiments with and without the orographic parameterizations underestimated the maximum daytime screen-level temperature $T_{2 m}$ up to several degrees as compared to the hourly observations. This was true especially at 37107 , which is located deeper in a valley than indicated by the mean gridpoint elevation around it. The minimum $T_{2 m}$ was typically somewhat higher than observed (Figures 10A, 11A). The maximum $T_{s}$ at the northward-looking station 00011 (Figure 11B) was typically $0.5-1^{\circ}$ higher in the reference experiment harcau0 than in the ororad experiments harcau 6 and harcau3, while it was slightly lower at 37107 (Figure 10B). The difference between experiments in the minimum (nighttime) $T_{s}$ values was small, harcau0 showing the coldest values. The lowest model level temperatures $T_{\text {nlev }}$ were practically the same for all experiments except the Olympic reference soc38h12_15_nc, based on GTOPO30 surface elevation, where the values tended to be slightly higher, corresponding to the somewhat higher $T_{s}$ and $T_{2 m}$ in this experiment (not shown).

Corresponding global SWD radiation fluxes are shown in Figures 10C, 11C. During mostly clear days, the maximum direct SWD flux was typically up to one hundred $\mathrm{Wm}^{-2}$ larger at 37107 and up to two hundred $\mathrm{Wm}^{-2}$ smaller at 00011 in harcau6, harcau3 and harcau 8 as compared to harcau0. The difference was due to the direct radiation (not shown). This may explain the differences in maximum $T_{s}$ values between the harcau experiments with and without ororad parameterizations. During the few mostly cloudy days, SWD fluxes differed less between these experiments.

LWD (Figures 10D, 11D) was smaller in the reference harcau0 than in the ororad experiments. However, the difference was smaller than in the the case of SWD, typically around $10 \mathrm{Wm}^{-2}$. The differences between harcau6, harcau3, and harcau8 (harcau8 not shown), which were related to calculation of the local horizon and sky view factor, showed up mostly in LWD fluxes which increased with decreasing $\delta_{s v}$. The diffuse SWD and thus the global downweling SWD decreased slightly with decreasing $\delta_{s v}$. The fluxes by harcau3 were close to those by harcau0, while those by harcau8 were slightly larger (smaller) for 00011 (37107) compared to harcau6.

\subsection{Single-Column Sensitivity Studies}

When solar angles are low in winter, orography may significantly influence SWD fluxes not only in the morning and evening but 


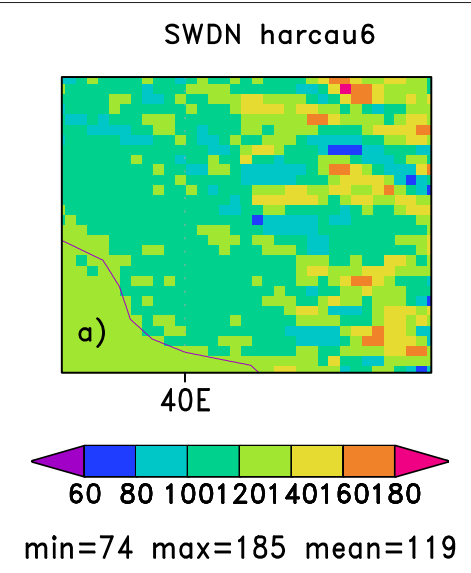

LWDN harcau 6
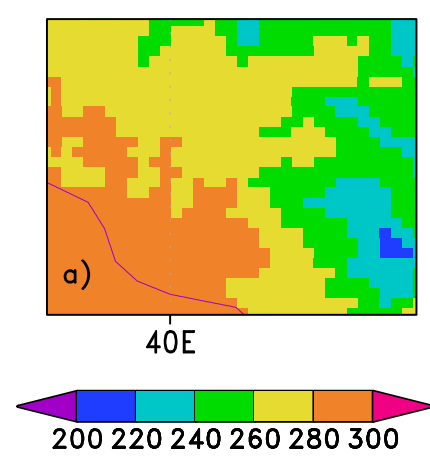

$\min =216 \max =286$ mean $=266$
SWDN harcau0

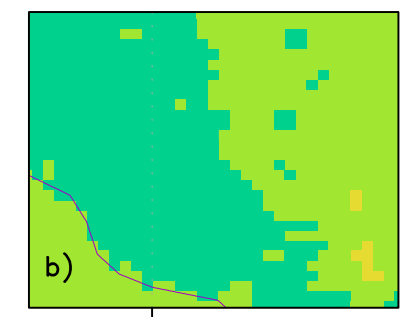

$40 \mathrm{E}$

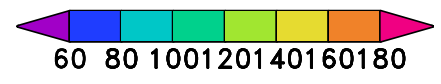

$\min =108 \max =142$ mean $=121$

LWDN harcau0

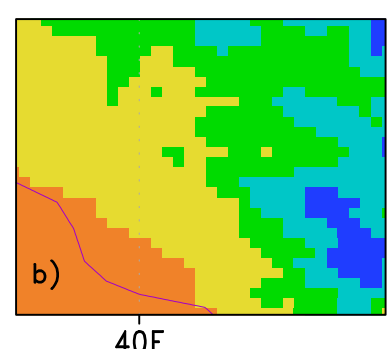

$40 \mathrm{E}$

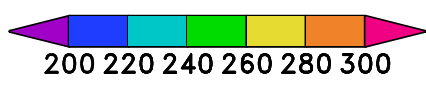

$\min =209 \max =286$ mean $=258$
SWDN harcau6 - harcau0

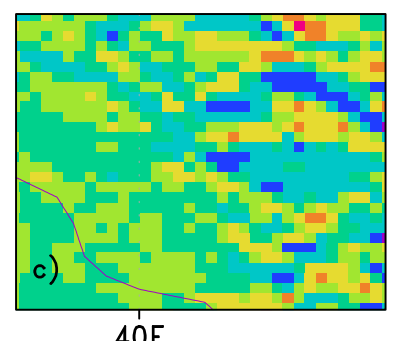

$40 E$

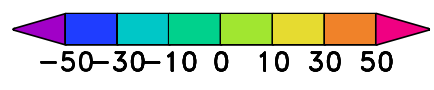

$\min =-53$ max $=53$ mean $=-2$

FIGURE 8 | Monthly average SWD (upper panel) and LWD (lower panel), unit: Wm ${ }^{-2}$, based on accumulated fluxes from 00 UTC + 24 h forecasts 1-28 February 2014 with and without ororad parameterizations: (A) harcau6, (B) harcau0, and (C) their difference, map zoomed to the Olympic venue area. Color scales are shown in the figures. See Table $\mathbf{2}$ for the experiment definitions and Figure $\mathbf{5}$ for the surface elevation contour map of the Olympic venue area.

also during the day, as indicated by the previous figures. In order to study in detail the behavior of the slope, shadow and sky view factors (Section 3.2) and ororad parameterizations (Section 2.2), we chose atmospheric profiles from the harcau6 experiment on the 8th February 00 UTC $+6 \mathrm{~h}$ to be used as initial state for $+24 \mathrm{~h}$ MUSC experiments. Surface temperatures given by different experiments are compared in Figure $\mathbf{1 2}$ for the same stations as in Figures 10, 11. MUSC experiments were run with all, none or partial ororad parameterizations using $\delta_{s v}, \delta_{s l}$, and $\delta_{s h}$ derived from the SRTM $3^{\prime \prime}$ source data for the gridpoints closest to these stations.

It is evident from Figure 12 that the minimum $T_{\mathcal{S}}$ was unrealistically low as compared to the three-dimensional results (Figures 10B, 11B), which seem to be closer to the observed minimum screen-level temperatures (Figures 10A, 11A). This is a problem specific to the single-column experiments. There are several possible reasons, including the constant in time clear-sky atmospheric state, different role of vertical mixing in the full model and in the single-column experiments etc. It is important to remember that MUSC experiment results are not directly comparable to observations. However, comparison between the different model setups can give valuable information even when the results do not validate well against the observations.

The main conclusion from Figure 12 is the key role of the slope factor, controlling the direct short-wave radiation (SWdir), at the chosen locations. Due to the slope effect, SWdir increased by $199 \mathrm{Wm}^{-2}$ (not shown) and the maximum $T_{s}$ by $2.1 \mathrm{~K}$ at the southward-looking station 37107. Corresponding decrease of SWdir and maximum $T_{s}$ at the northward-looking 00011 reached $-291 \mathrm{Wm}^{-2}$ and $-4.1 \mathrm{~K}$. Another conclusion is that the restricted sky view led to an increase of $T_{s, \max }$ of the order of $1-2^{\circ}$, mostly because of the increased LWD but also by the increased diffuse SW flux. The shadow effect only showed up limited time in the morning and afternoon when it led to a small decrease of SWdir and $T_{s}$. MUSC results are consistent with the three-dimensional model results over the selected locations and the Olympic domain. They explain the impact of different components of the ororad parameterizations also to the monthly averages. However, in the full model the local effects smooth out when the monthly mean values are averaged also over a larger area. 

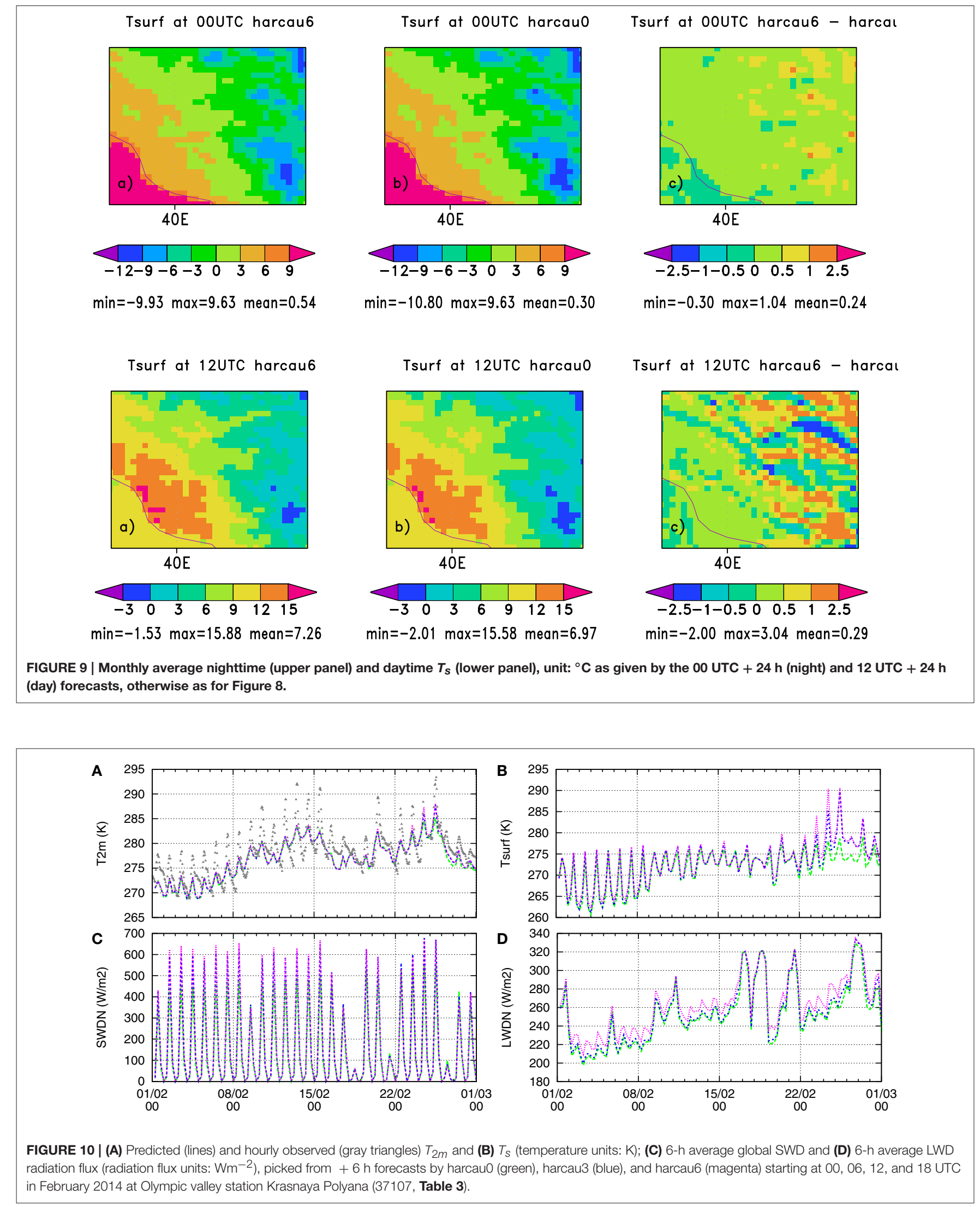

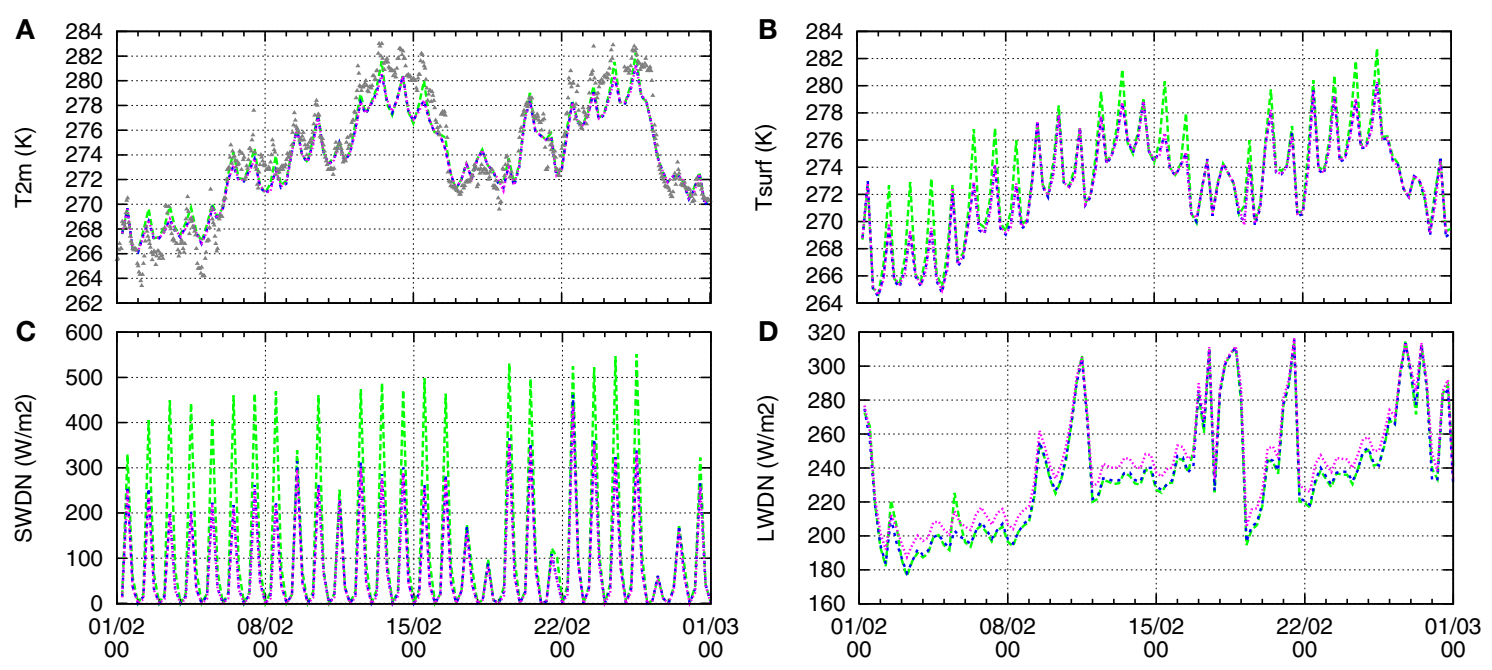

FIGURE 11 | As in Figure 10 but at Olympic mountain station 11. Note the different scales on y-axes.

Additional single-column sensitivity experiments were performed by using $\delta_{s v}$ values resulting from the different formulations discussed in Section 3.3. The nighttime $T_{s}$ temperature increased with decreasing sky view factor at both stations, the maximum increase being of the order of $1^{\circ}$ when $\delta_{s v}$ decreased from 1 to 0.85 . This agrees with similar results of the $1 \mathrm{D}$ and $3 \mathrm{D}$ experiments reported above.

\section{CONCLUSIONS AND OUTLOOK}

In this study, new fine-resolution source orography and new parameterizations for orographic radiation fluxes were introduced to the mesoscale NWP system HARMONIEAROME-SURFEX. The needed orographic variables for the NWP experiments were derived from the SRTM $3^{\prime \prime} 90 \mathrm{~m}$ resolution surface elevation data set. Synoptic observations of the Sochi winter Olympic games 2014 were used for validation of the three-dimensional model experiments. Analysis of the verification results and model-observation comparison at selected locations was completed by single-column model sensitivity experiments.

Olympic HARMONIE produced sufficiently good forecasts already when run in real time during the Sochi Olympic games 2014. A preliminary comparison of the reproduced Olympic experiments, which were based on GTOPO30 surface elevation and used no orographic parameterizations, against the FROST2014 AMS screen-level temperature observations showed that a problem of underestimated screen-level temperature, which was revealed during clear and calm nights at the mountain cluster, was solved by excluding the surface sublayer parameterizations (so called CANOPY scheme). Further experiments were performed excluding this scheme, and the experiment without CANOPY was used as a reference for the comparisons.
Comparison of the reference GTOPO30-based experiment to the reference SRTM 3"-based experiment without orography parameterizations showed an increased negative screen-level temperature bias in the standard verification at all FROST2014 AMS when the new orography was applied in the model. The change was smaller according to the verification against all SYNOP stations over a larger Caucasian domain. The increase of the negative bias was related to the change of the source orography only. However, significance and the exact reasons of this remain to be understood.

The change of source orography changed the mean surface elevation of each land-area gridpoint, i.e., led to an adjustment of the model's surface-pressure based vertical coordinate. The assumptions in the standard verification (interpolations, height adjustment of the screen-level temperature) and in the diagnosis of the screen-level temperature from the lowest model level and surface temperatures in the model are influenced by the surface elevation. In this study, the source of orography description was changed without specific adaptation to the coarser-resolution surface physiography description (land-sea distribution, soil and surface type are given with ca. 1-km resolution). Also the surface data assimilation and all surface parameterizations (including those related to snow) remained untouched. Implicit scaledependencies related to the surface elevation in the model dynamics, turbulence and cloud microphysics parameterizations and their interactions may have influenced the model results and diagnostics. It is thus necessary to study systematically all aspects - data assimilation, model dynamics, parameterizations, verification-related to the change of source orography in the HARMONIE-AROME NWP system using different horizontal and vertical resolutions, before suggesting such a change for application in the operational forecasting.

Comparison of the experiments with orographic parameterizations to the reference SRTM 3"-based experiment showed a consistent but very small improvement of this negative 


\section{Station 37107}
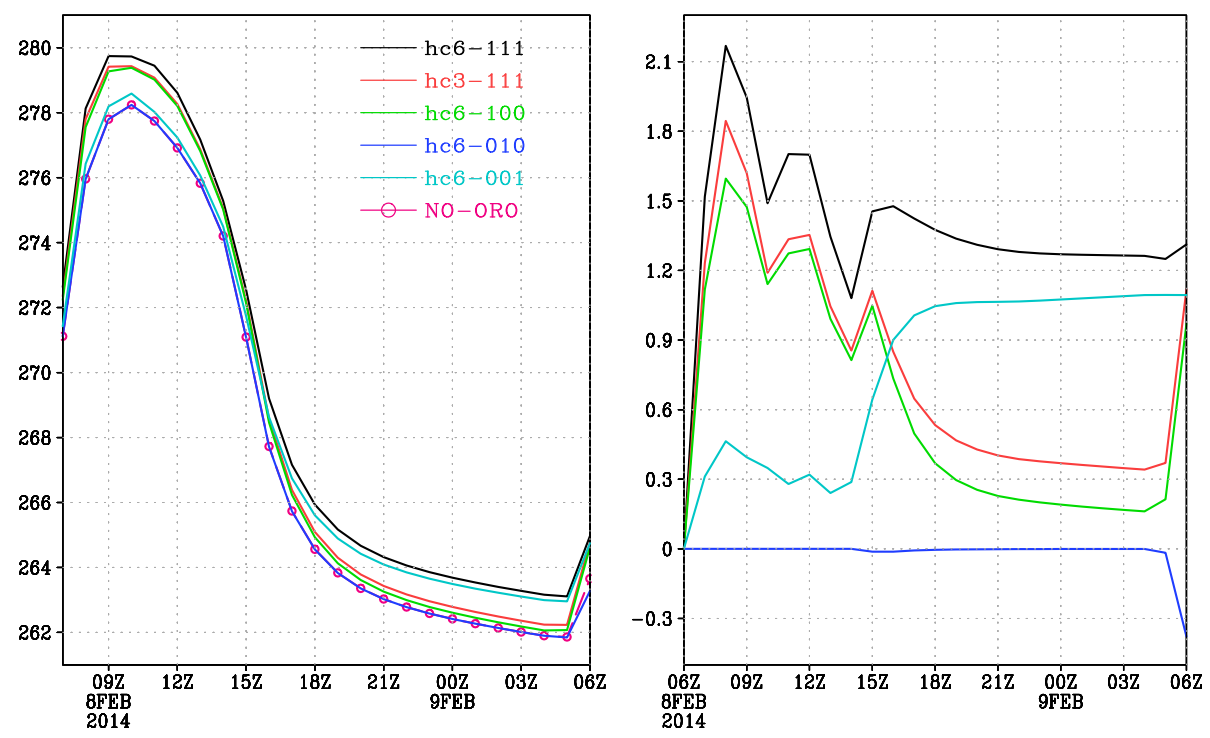

Station 00011
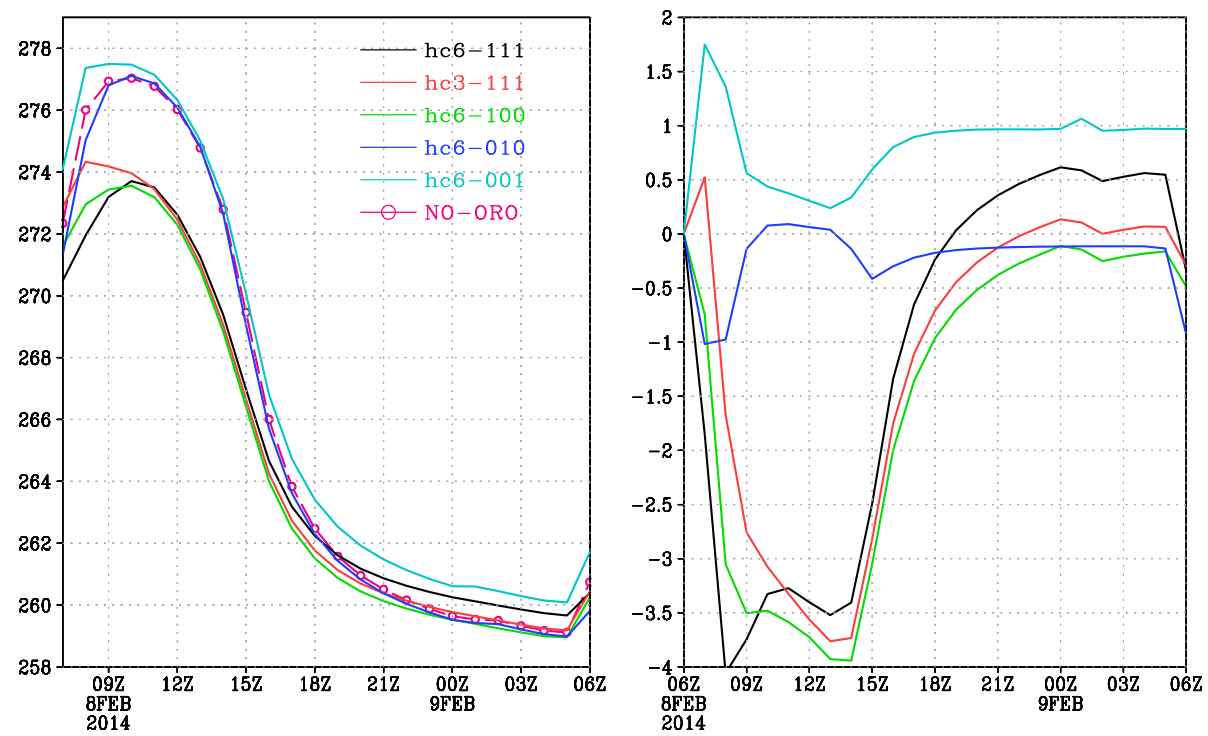

FIGURE 12 | Surface temperature $T_{s}$ (K, y-axis) as a function of time (x-axis) for the stations 37107 (upper panel) and 00011 (lower panel) from MUSC experiments initiated at $\mathbf{0 6}$ UTC the 8th of February 2014. Values on the left, difference from the reference (no ororad) on the right. Experiment name is given in the legend as experiment-ororad: hc6, ororad variables from harcau6; hc3, ororad variables from harcau3; 000, no ororad; 111, all ororad; 100, slope effect only; 010, shadow effect only; 001, sky view effect only.

screen-level temperature bias. The three-dimensional and singlecolumn sensitivity studies showed that the new orographic radiation parameterizations behaved as expected. The largest impact was due to the slope effect, i.e., the direction of the slopes with respect to the incoming direct solar radiation. However, averaged over a larger domain, the related variations in downweling SW radiation smoothed out. Limited sky view enhanced the downweling long-wave radiation systematically in valleys so that the average monthly LWD increased locally by up to $10 \mathrm{Wm}^{-2}$. The smaller was the average sky view factor, the larger was the overall increase of the flux. The nighttime minimum surface temperatures increased locally up to $1-2^{\circ}$. The daytime maxima changed between -2 and $+3^{\circ}$. However, the change of the surface temperature was reflected only little in the screen-level temperature and insignificantly in the lower model level temperature values. 
The method of calculation of the local horizon around a given point was improved compared to the method suggested by Senkova et al. (2007) and shown to be reliable by comparing to the observed horizons in several Alpine valleys. However, the different approximations in calculation of the grid-scale sky view factor from the local horizon at the level of the fine-resolution source orography, during aggregation to the model grid or even within the NWP model resolution led to somewhat different results, with mean values varying from 0.87 to 0.91 over the Olympic venue. The related uncertainty showed up mainly on the longwave radiation fluxes where the differences were small on average but systematic. None of the approximations can be considered absolutely correct or incorrect. Further studies could be devoted to quantification of these uncertainties within different model resolutions.

For a realiable validation of the radiation parameterizations, and the NWP results in general, the measurements of the downweling short- and long-wave radiation fluxes at the surface would be very useful. The observed fluxes and those predicted by the model correspond to each other more closely than for example the usually validated screenlevel temperature, anemometer-level wind, cloud cover, or mean sea level pressure. We would suggest to make radiation observations available during the 2018 winter

\section{REFERENCES}

Bénard, P., Vivoda, J., Mašek, J., Smolková, P., Yessad, K., Smith, C., et al. (2010). Dynamical kernel of the Aladin-NH spectral limited-area model: revised formulation and sensitivity experiments. Q. J. R. Meteorol. Soc. 136, 155-169. doi: $10.1002 / q j .522$

Déqué, M., Dreveton, C., Braun, A., and Cariolle, D. (1994). The ARPEGE/IFS atmosphere model: a contribution to the French community climate modelling. Clim. Dyn. 10, 249-266.

ECMWF (2012). IFS Documentation cy38r1, Part IV: Physical Processes. Available online at: https://software.ecmwf.int/wiki/display/IFS/CY38R1+Official+IFS+ Documentation

GDAL (2014). Geospatial Data Abstraction Library. Available online at: www.gdal. org (Accessed Januaary 01, 2014).

Helbig, N., and Löwe, H. (2012). Shortwave radiation parameterization scheme for subgrid topography. J. Geophys. Res. Atmos. 117, 2156-2202. doi: 10.1029/2011JD016465

Helbig, N., and Löwe, H. (2014). Parameterization of the spatially averaged sky view factor in complex topography. J. Geophys. Res. Atmos. 119, 4616-4625. doi: 10.1002/2013JD020892

Helbig, N., Löwe, H., and Lehning, M. (2009). Radiosity approach for the shortwave surface radiation balance in complex terrain. J. Atmos. Sci. 66, 2900-2912. doi: 10.1175/2009JAS2940.1

Jarvis, A., Reuter, H. I., Nelson, A., and Guevara, E. (2008). Hole-Filled SRTM for the Globe Version 4. Available online at: srtm.csi.cgiar.org (Accessed Januaary 01, 2014).

Kondratyev, K. Y. (1977). Radiation Regime of Inclined Surfaces. WMO Technical Report 152, World Meteorological Organization, Geneva.

Malardel, S., Lac, C., Pinty, J.-P., Thouron, O., Bouteloup, Y., Bouyssel, F., et al. (2006). "Representation of clouds in AROME", in Proceedings of the ECMWF Workshop on Parametrization of Clouds in Large-Scale Models (Reading: ECMWF).

Manners, J., Vosper, S. B., and Roberts, N. (2012). Radiative transfer over resolved topographic features for high-resolution weather prediction. Q. J. R. Meteorol. Soc. 138, 720-733. doi: 10.1002/qj.956
Olympic games in Pyeongchang on the Korean Taebaek mountains.

\section{AUTHOR CONTRIBUTIONS}

All authors listed, have made substantial, direct and intellectual contribution to the work, and approved it for publication.

\section{FUNDING}

Support by the International HIRLAM and LACE programs and Meteo France is gratefully acknowledged.

\section{ACKNOWLEDGMENTS}

We thank Nora Helbig for reminding us about the different ways to calculate the sky view factor. The extensive and careful critical comments by the two anonymous reviewers helped to improve our manuscript substantially. Our thanks are due to Yann Seity and Alexandre Mary from Meteo France for discussions and guidance in the implementation of orographic radiation parameterizations into SURFEX. Observational data from the WMO FROST project were available for this study via the Finnish Meteorological Institute.

Mascart, P. J., and Bougeault, P. (2011). The Meso-NH Atmospheric Simulation System: Scientific Documentation. Technical Report, Meteo France, Toulouse, France.

Masson, V., Le Moigne, P., Martin, E., Faroux, S., Alias, A., Alkama, R., et al. (2013). The surfex v7.2 land and ocean surface platform for coupled or offline simulation of earth surface variables and fluxes. Geosci. Model Dev. 6, 929-960. doi: 10.5194/gmd-6-929-2013

Masson, V., and Seity, Y. (2009). Including atmospheric layers in vegetation and urban offline surface schemes. J. Appl. Meteorol. Clim. 48, 1377-1397. doi: 10.1175/2009JAMC1866.1

Mašek, J., Geleyn, J.-F., Brožková, R., Giot, O., Achom, H. O., and Kuma, P. (2015). Single interval shortwave radiation scheme with parameterized optical saturation and spectral overlaps. Q. J. R. Meteorol. Soc. 142, 304-326. doi: 10.1002/qj.2653

Müller, M. D., and Scherer, D. (2005). A grid- and subgrid-scale radiation parametrization of topographic effects for mesoscale weather forecast models. Mon. Weather Rev. 133, 1431-1442. doi: 10.1175/MWR 2927.1

Nielsen, K. P., Gleeson, E., and Rontu, L. (2014). Radiation sensitivity tests of the HARMONIE 37h1 NWP model. Geosci. Model Dev. 7, 1433-1449. doi: 10.5194/gmd-7-1433-2014

Niemelä, S., Näsman, S., and Nurmi, P. (2014). FROST-2014 - Performance of Harmonie $1 \mathrm{~km}$ during Sochi Olympics. ALADIN-HIRLAM Newsl. 3, 79-86. Available online at: http://www.cnrm.meteo.fr/aladin/IMG/pdf/aladin-hirlamnewsletter_no3_september2014.pdf

Savijärvi, H. (1990). Fast radiation parameterization schemes for mesoscale and short-range forecast models. J. Appl. Meteor. 29, 437-447.

Seity, Y., Brousseau, P., Malardel, S., Hello, G., Bénard, P., Bouttier, F., et al. (2011). The AROME-France convective-scale operational model. Mon. Weather Rev. 139, 976-991. doi: 10.1175/2010MWR 3425.1

Senkova, A., Rontu, L., and Savijärvi, H. (2007). Parametrization of orographic effects on surface radiation in HIRLAM. Tellus 59A, 279-291. doi: 10.1111/j.1600-0870.2007.00235.x 
Undén, P., Rontu, L., Järvinen, H., Lynch, P., Calvo, J., Cats, G., et al. (2002). The HIRLAM-5 Scientific Documentation, December 2002. Available online at: http://hirlam.org

USGS (1998). GTOPO30, Global 30 Arc Second Elevation Data Set. U.S. Geological Survey. Available online at: https://ta.cr.usgs.gov/ GTOPO30

USGS (2010). GMTED 2010, Global Multi-resolution Terrain Elevation Data 2010 (GMTED2010). U.S. Geological Survey. Available online at: http://topotools.cr. usgs.gov/gmted_viewer/

Wikipedia (2012). Elbrus. Available online at: https://en.wikipedia.org/wiki/File: Elbrus_3D_version_1.gif (Accessed Januaary 01, 2014).
Conflict of Interest Statement: The authors declare that the research was conducted in the absence of any commercial or financial relationships that could be construed as a potential conflict of interest.

Copyright (c) 2016 Rontu, Wastl and Niemelä. This is an open-access article distributed under the terms of the Creative Commons Attribution License (CC $B Y)$. The use, distribution or reproduction in other forums is permitted, provided the original author(s) or licensor are credited and that the original publication in this journal is cited, in accordance with accepted academic practice. No use, distribution or reproduction is permitted which does not comply with these terms. 
OPEN ACCESS

Edited by:

Daniel J. Kirshbaum,

McGill University, Canada

Reviewed by:

Ana María Durán-Quesada,

University of Costa Rica, Costa Rica

Eric Hendricks,

National Park Service, USA

${ }^{*}$ Correspondence:

Yuh-Lang Lin

ylin@ncat.edu

Specialty section:

This article was submitted to

Atmospheric Science,

a section of the journal

Frontiers in Earth Science

Received: 08 September 2015

Accepted: 01 February 2016

Published: 19 February 2016

Citation:

Liu L, Lin Y-L and Chen S-H (2016)

Effects of Landfall Location and Approach Angle of an Idealized

Tropical Cyclone over a Long Mountain Range.

Front. Earth Sci. 4:14. doi: 10.3389/feart.2016.00014

\section{Effects of Landfall Location and Approach Angle of an Idealized Tropical Cyclone over a Long Mountain Range}

\author{
Liping Liu ${ }^{1}$, Yuh-Lang Lin ${ }^{2,3 *}$ and Shu-Hua Chen ${ }^{4}$ \\ ${ }^{1}$ Department of Mathematics, North Carolina A\&T State University, Greensboro, NC, USA, ${ }^{2}$ Department of Physics, North \\ Carolina A\&T State University, Greensboro, NC, USA, ${ }^{3}$ Department of Energy and Environmental Systems, North Carolina \\ A\&T State University, Greensboro, NC, USA, ${ }^{4}$ Department of Land, Air and Water Resources, University of California, Davis, \\ CA, USA
}

Effects of landfall location and approach angle on track deflection associated with a tropical cyclone (TC) passing over an idealized and Central Appalachian Mountain is investigated by a series of idealized numerical experiments. When the TC landfalls on the central portion of the mountain range, it is deflected to the south upstream, passes over the mountain anticyclonically, and then moves westward downstream. The TC motion is steered by the positive vorticity tendency (VT) which is dominated by horizontal vorticity advection upstream and downstream, but with additional influence from the stretching and residual terms, which are mainly associated with diabatic heating and frictional effects. The track deflection mechanism upstream and downstream is similar to the dry flow in previous study, but is very different in the vicinity of the mountain. When the TC landfalls near the northern (southern) tip, it experiences less (more) southward deflection due to stronger (weaker) vorticity advection around the tip. When the TC approaches the mountain range from the southeast and landfalls on the northern tip, center, or southern tip, the track deflections are similar to those embedded in an easterly flow but with weaker orographic blocking. These results are similar to the cases simulated in the dry flow in previous study, except that there is no track discontinuity due to the weaker orographic blocking associated with strong TC convection. When a TC moves along the north-south mountain range from the south, it tends to deflect toward the mountain and then crosses over to the other side at later time. In these cases, the positive VT is influenced by all horizontal vorticity advection, vorticity stretching (diabatic heating), and residual (friction) terms due to longer and stronger interaction with the mountain range. The vorticity stretching is mainly caused by diabatic heating in the moist flow, instead of by lee slope vorticity stretching in the previous study for dry flow.

Keywords: tropical cyclones, orographic effects, TC track deflection, idealized simulations, approach angle, landfalling location 


\section{INTRODUCTION}

When a tropical cyclone (TC) passes over a mountain range, its steering flow and cyclonic circulation are often strongly modified by the orography. This tends to enhance the precipitation associated with the storm's rainbands (e.g., Lin, 2007 for a brief review). During the summer and fall seasons, floods over the Appalachians are often a product of enhanced heavy rainfall from hurricanes. This is analogous to flooding associated with the passage of typhoons over Taiwan's Central Mountain Range (CMR) (Lin, 2007). One example is Hurricane Camille (1969), which dumped up to $686 \mathrm{~mm}$ of rainfall at one location along the slopes of the Appalachian Mountains in central Virginia (Schwarz, 1970). Another example is Hurricane Ivan (2004) (Stewart, 2004). On September 16, 2004 in Macon County, North Carolina, Ivan produced a debris flow $3.6 \mathrm{~km}$ downslope along Peeks Creek to the Cullasaja River, causing five deaths and two injuries along with destruction of 16 buildings. The heavy rainfall areas are closely related to storm tracks, which tend to be strongly influenced by orography. Therefore, it is important to make accurate predictions of the orographic effects on track deflection and understand the underlying mechanisms.

TC track deflections due to orography have occurred over several mesoscale mountain ranges. In particular, deflections have been well documented for typhoons passing over Taiwan's CMR and the Cordillera Central of northern Luzon in the Philippines (e.g., Brand and Blelloch, 1974; Wang, 1980), TC's passing over Madagascar and La Reunion mountains (Rakotomavo et al., 2011), and hurricanes passing over the Cordillera Central of Hispañola (Bender et al., 1987) and the Sierra Madre Mountains of Mexico (Zehnder, 1993; Zehnder and Reeder, 1997). Similar track deflections have also been observed with mid-latitude cyclones passing over the Appalachians (e.g., O'Handley and Bosart, 1996). Effects of orography on TC tracks have been studied extensively for typhoons passing over Taiwan's CMR and the Cordillera Central of northern Luzon in the Philippines.

Due to complicated interactions between orographic and thermal forcing, both idealized numerical simulations (e.g., Chang, 1982; Bender et al., 1987; Yeh and Elsberry, 1993a,b; Lin et al., 1999, 2005, denoted as L05 hereafter, Lin and Savage, 2011; Tang and Chan, 2013, 2014; Wu et al., 2015), real-case simulations (e.g., Wu, 2001; Lin et al., 2006; Jian and Wu, 2008; Huang et al., 2011; Hsu et al., 2013; Wang et al., 2013; Wu et al., 2015), and observations (Brand and Blelloch, 1974; Wang, 1980; Hsu et al., 2013) have shown that when a typhoon approaches Taiwan's CMR, its track may deflect either to the north or south upstream of the mountain. It has been proposed that the track deflection may be due to mean cyclonic circulation (e.g., Chang, 1982; Bender et al., 1987), channeling mechanism (e.g., Lin et al., 1999; Jian and $\mathrm{Wu}, 2008$; Huang et al., 2011), blocking effect (e.g., Yeh and Elsberry, 1993a,b, L05, Lin and Savage, 2011), latent heating (e.g., Hsu et al., 2013; Tang and Chan, 2013, 2014; Wang et al., 2013), terrain-induced gyes (Tang and Chan, 2013, 2014), approaching angle and landing location (Lin and Savage, 2011; Tang and Chan, 2014), and midtropospheric northerly asymmetric flow (Wu et al., 2015). Thus, the basic dynamics deserve further investigation. In this study, we will focus on the effects of landfall location and impinging angle on TC track deflection in an idealized environment.

In addition to the above mechanisms, the landfall location and approach angle also play important roles in the track deflection of a TC passing over Taiwan's CMR (Wang, 1980). Based on the 6-h Atlantic hurricane best track (HUDAT) of 28 landfalling TCs that impinged on the Appalachians during 1979 and 2006, Harville (2009) (denoted as H09 hereafter) classified the tracks into four types (Figure 1): (1) approximately perpendicular to the mountains from east to west (Type A), (2) parallel on the eastern side of the mountain (Type B), (3) parallel on the western side of the mountains (Type C), and (4) similar to Type A, but from west to east. From idealized simulations of a drifting vortex over an idealized version of Taiwan's CMR (Lin and Savage, 2011; denoted as LS11 hereafter), found that the local vorticity generation is dominated more by vorticity advection upstream of the mountain range, vorticity stretching over the lee side and its immediate downstream area, and vorticity advection again far downstream of the mountain as it steers the vortex back to its original direction of movement. The vorticity advection upstream of the mountain range is explained by the flow splitting associated with orographic blocking. Based on a vorticity budget analysis, it was found that jumps in the vortex path are largely governed by stretching on the lee side of the mountain, with the maximum stretching and associated track jump located on the faster side of the vortex. The present study is an extension of Lin and Savage (2011) by adding latent heating and the planetary boundary layer to the numerical simulations.

In idealized simulations, Tang and Chan (2013) found that a pair of terrain-induced gyres is formed when an idealized TC moves closer to Taiwan's CMR. This pair of gyres rotates cyclonically around the TC and the gyre-associated flow near the TC center causes a northward deflection of the TC track prior to landfall. An examination of the potential vorticity tendencies (PVTs) suggests that, in addition to the PVT produced by this flow, the PVT from diabatic heating cannot be ignored in explaining the TC track during landfall. Topography also altered the asymmetric diabatic pattern of the TC by three major mechanisms: an incursion of dry air from the mountain, lowlevel convergence induced by the terrain-altered wind field in the southwestern part of the TC and the development of convergence cyclonically inward from the eastern side of the mountain to the TC core. In the second part of this series of studies, Tang and Chan (2014) examined the effects of Taiwan's CMR on the tracks of several TCs approaching Taiwan from southeast at different latitudes. They found that TCs approaching the south of Taiwan slow down and are first deflected southwestward and then northward. Moreover, a sharp northward deflection occurs for a TC passing further south of Taiwan, but the deflection is small for a TC passing further north of Taiwan. Although, Tang and Chan $(2013,2014)$ have advanced our understanding of orographic effects on TC tracks, the TCs inserted in their numerical simulations were steered by the pair of beta gyres far upstream, instead of advected by the southeasterly basic flow. In this study, we will be investigating the orographic effect 

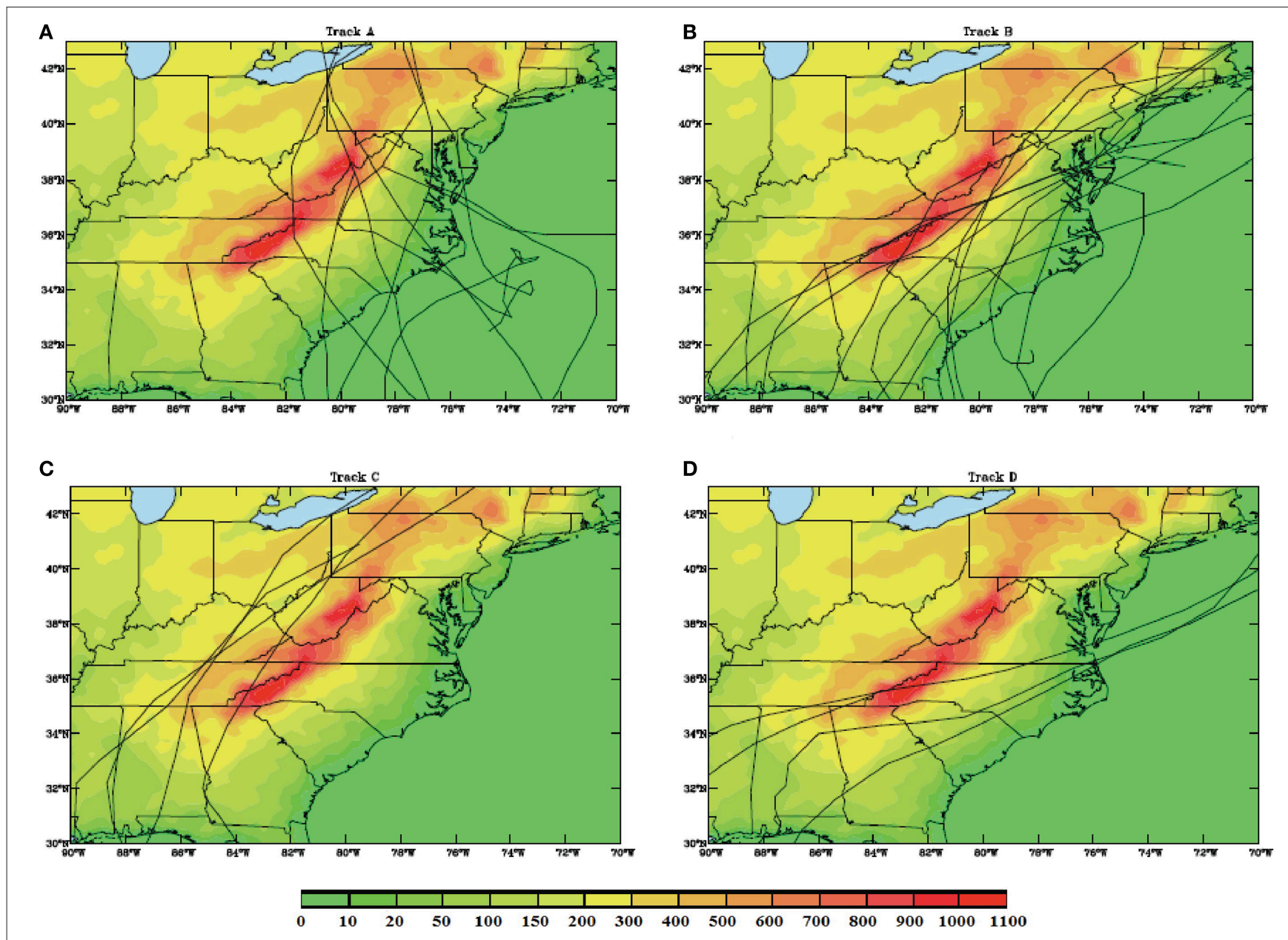

FIGURE 1 | Classification of track categories (A-D) for hurricanes passing over the South-Central Appalachian (SCA) mountain range (Harville, 2009-H09).

on the basic flow and its impact on the TC track. In term of idealized simulations, our current study differs from Huang et al. (2011) and Wu et al. (2015) in focusing on effects of landing location and impinging angle and also including vorticity budget analysis.

In this paper we will extend the study of LS11 to simulate a TC over an idealized Southern and Central Appalachians (SCA) mountain range by using a more realistic, state-ofart numerical weather prediction model. In addition, unlike a dry cyclone vortex used in LS11 and L05, a more realistic bogus TC is initiated in a conditionally unstable atmosphere with land surface, moist and planetary boundary layer (PBL) processes included. A vorticity budget analysis is adopted to help understand the effects of combined forcing of orography and convection, instead of the potential vorticity tendency (PVT) diagnostic approach taken by some previous studies (e.g., Wu and Wang, 2000; Chan et al., 2002; Hsu et al., 2013; Tang and Chan, 2013, 2014; Wang et al., 2013). The direct diabatic heating effects will be investigated along with the rainfall distributions.
The numerical model and its experimental design will be described in Section The Numerical Model and Experimental Design. In Section The Mechanisms for Track Deflection, a vorticity budget analysis will be performed to help understand the fundamental dynamics of the track deflection associated with a numerically simulated TC traversing over an elongated mountain range. Sensitivity of the track deflection to landfall locations and approach angles will be presented in Section Effects of landfall Locations and approach Angles. The concluding remarks can be found in Section Concluding Remarks.

\section{THE NUMERICAL MODEL AND EXPERIMENTAL DESIGN}

The model used for this study is the Advanced Research Weather Research and Forecasting (ARW) model version 3.4.1 (Skamarock et al., 2008). The ARW model is a threedimensional, fully compressible, non-hydrostatic model using terrain-following vertical coordinates. The governing equations for ARW are written in flux-form with conserved mass and 
dry entropy. In this study, the Runge-Kutta third-order time difference scheme is employed, and the fifth- and third-order advection schemes are used for the horizontal and vertical directions respectively.

The physics parameterization or representation schemes that are chosen for all simulations in this study include: Kain-Fritsch cumulus parameterization scheme (Kain and Fritsch, 1993; Kain, 2004), Purdue-Lin microphysics parameterization scheme (Lin et al., 1983; Chen and Sun, 2002), YSU PBL parameterization scheme (Hong and Pan, 1996), Monin-Obukov surface layer scheme, Unified NOAH land-surface processes scheme, Secondorder diffusion term on coordinate surfaces for turbulence and mixing processes, and Horizontal Smagorinsky first-order closure for eddy coefficient option. The Purdue-Lin scheme (Lin et al., 1983; Chen and Sun, 2002) was chosen due to its capability in simulating mixed-phase microphysical processes for practical applications, recommended by Skamarock et al. (2008), as they can account for the interaction of water and ice particles. In idealized simulations, Fovell et al. (2009) found that the PurdueLin scheme (Chen and Sun, 2002) produced relatively better results. The Kain-Fritsch cumulus parameterization scheme (Kain and Fritsch, 1993; Kain, 2004) is chosen since it is CAPE based, thus is more suitable in triggering the deep convection in a conditionally unstable atmosphere, such as the one used in the current study. The YSU scheme (Hong and Pan, 1996) is chosen due to the non-local closure, which does not rely on or too sensitive to localized heat, moisture, and momentum fluxes. No longwave or shortwave radiation parameterizations are applied to any simulations. Details of these schemes and their relevant references can be found in the ARW user manual (Skamarock et al., 2008). An idealized "moist tropical sounding" of Dunion (2011) is given to provide the moisture (see Figure 2 of Nolan, 2011).

A preexisting, mid-level, bogus vortex with peak tangential wind speed of $20.8 \mathrm{~ms}^{-1}$ at a radius of maximum winds of $90 \mathrm{~km}$ is set at $(5385 \mathrm{~km}, 3240 \mathrm{~km})$. The vertical structure of the initial vortex is in the radiative-convective-equilibrium state (Nolan, 2011; Nolan et al., 2013). Rather than using the fairly narrow tangential wind profile generated by a Gaussian vorticity distribution, our simulations use a modified Rankine vortex with decay parameter as 0.4 , which is more realistic for the development stage (Mallen et al., 2005). The SST is fixed to $28^{\circ} \mathrm{C}$. The domain-wide wind profile is purely zonal, with $U(\mathrm{p})=-5 \mathrm{~ms}^{-1}$ from the surface to $850 \mathrm{hPa}$, increasing with the shape of a cosine as a function of log-pressure height to $5 \mathrm{~ms}^{-1}$ at $200 \mathrm{hPa}$, then remaining at this value for all greater heights. The zonal wind is assumed to be in thermal wind balance. The model derives the geopotential height using iterative method and then calculates other fields.

All simulations use one single domain $(6480 \mathrm{~km}, 6480 \mathrm{~km})$ with a $15-\mathrm{km}$ horizontal resolution. The number of the horizontal grid intervals is 433 in both the $x$ and $y$ directions. In the vertical, grids are stretched from the surface to the model top $(20 \mathrm{~km})$ with a total of 41 levels. The time interval is $45 \mathrm{~s}$. A 4-km deep sponge layer is set from 16 to $20 \mathrm{~km}$ to reduce the wave reflection from the model top. The periodic lateral boundary condition is applied to the $y$-direction and the open boundary conditions are applied to both east and west side boundaries. The model is integrated for 12 days for all cases simulated in this study.

In the control case (CNTL or A2), the westward-moving bogus TC approaches the center of an idealized bell-shaped SCA mountain island from the east. This is the characteristic of track type A2 (see Figure 2A). The idealized mountain (see Figure 2B), which is oriented in the north-south direction, is approximately $1400 \mathrm{~km}$ long and $200 \mathrm{~km}$ wide, and the maximum mountain height $(h)$ is $1 \mathrm{~km}$, which mimics the SCA Mountains. The maximum tangential wind $\left(V_{\max }\right)$ of the TC vortex is approximately $60 \mathrm{~m} / \mathrm{s}$ after it is fully developed and the basic mean wind $(U)$ is $5 \mathrm{~m} / \mathrm{s}$. The basic flow Froude number $(U / N h)$ is approximately 0.5 , which represents moderate orographic blocking on the basic flow. On the other hand, the vortex Froude number $\left(V_{\max } / N h\right)$ is approximately 6 , indicating weak orographic blocking on the outer circulation of the TC vortex (L05). The initial bogus vortex has $V_{\max }=20.8 \mathrm{~m} / \mathrm{s}$ and is located $1100 \mathrm{~km}$ from the eastern boundary in the $x$ direction and centered in the $y$ direction.

The following is the formula for the geometry of the idealized SCA mountain range,

$$
h(x, y)= \begin{cases}\frac{h_{0}}{\left(1+\frac{\left(x-x_{0}\right)^{2}}{a^{2}}\right)^{3 / 2}}, & \left|y-y_{0}\right| \leq b \\ \frac{h_{0}}{\left(1+\frac{\left(x-x_{0}\right)^{2}}{a^{2}}+\frac{\left(y-y_{c}\right)^{2}}{a^{2}}\right)^{3 / 2}}, & \left|y-y_{0}\right|>b\end{cases}
$$

where $h_{0}=1 \mathrm{~km}, x_{0}=2600 \mathrm{~km}, y_{0}=3240 \mathrm{~km}, a=50 \mathrm{~km}, b=$ $600 \mathrm{~km}, y_{c}=y_{0}+b$ for $y \geq y_{0}+b$ and $y_{c}=y_{0}-b$ for $y \leq y_{0}-b$. The mountain is inserted in the beginning of the simulations, as part of the terrain-following coordinates.

The track types proposed by $\mathrm{H} 09$ are reclassified to track types $\mathrm{A} 1-3, \mathrm{~B}, \mathrm{AB} 1-3, \mathrm{C}$, and D1-2, and used to study the sensitivity to landfall locations and approach angles (Figure 2A). Types A1-A3 are similar to Track A of $\mathrm{H} 09$ except with landfall locations on the northern, central, and southern part of the SCA differentiated. Types $\mathrm{AB} 1-\mathrm{AB} 3$ are similar to $\mathrm{A} 1-\mathrm{A} 3$ respectively, but from south-southeast. Types D1 and D2 are similar to Track D of H09 except the former crosses over the SCA, while the latter skirts around the southern tip of the mountains. Types $\mathrm{B}$ and $\mathrm{C}$ are the same as those of H09. This study focuses on track types A1$\mathrm{A} 3, \mathrm{AB} 1-\mathrm{AB} 3, \mathrm{~B}$, and $\mathrm{C}$. Their associated flow and orographic parameters are summarized in Table $\mathbf{1}$.

\section{THE MECHANISMS FOR TRACK DEFLECTION}

Figure 3A shows the $258 \mathrm{~h}$ accumulated rainfall for case CNTL (A2). Since the accumulated rainfall coincides with the TC track (Figure 3B), it can also be used as a proxy of the TC path. Note that the TC is deflected to the south upstream, passing over the mountain anticyclonically (clockwise) and westward downstream from the mountain at a latitude south of its original latitude. The continuous track and the southward deflection is similar to the behavior of a drifting TC vortex embedded in a dry, stably stratified fluid flow as found in Lin et al.'s previous 

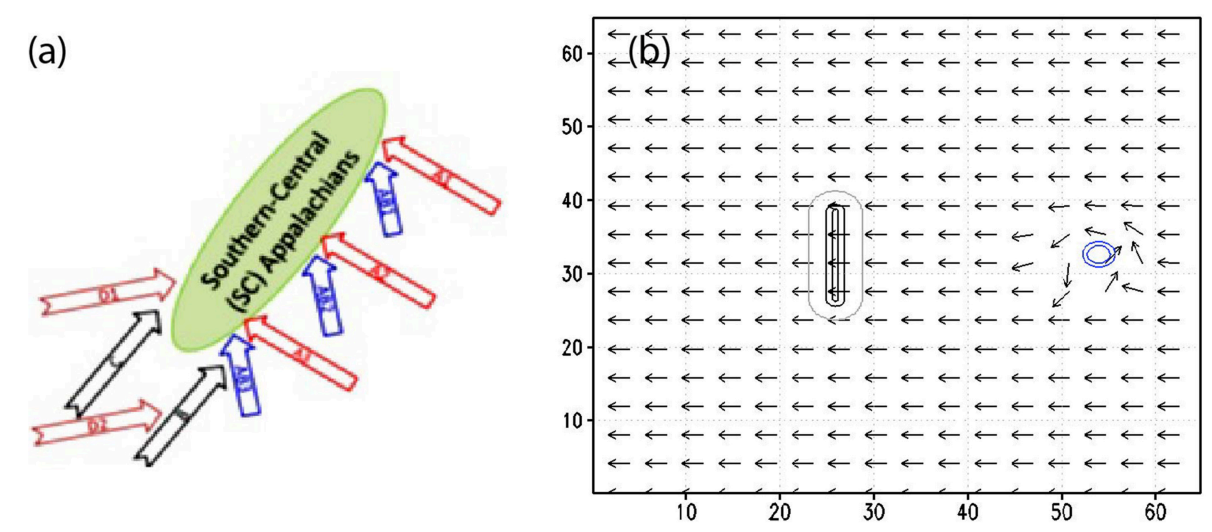

FIGURE 2 | (A) Detailed classification of tropical cyclone (TC) track types over the South-Central Appalachian (SCA) mountain range. (B) The entire computation domain with the mountain range, the wind flow and the initial bogus vortex. The units of the coordinates in the $x$ and $y$ directions are $100 \mathrm{~km}$. The mountain contours are 5, 400, and $600 \mathrm{~m}$. The vorticity contours are $5 \times 10^{-5} s^{-1}$, and $1 \times 10^{-4} s^{-1}$.

studies $(1999,2005)$ and the typhoons observed and simulated by Jian and $\mathrm{Wu}$ (2008) and Huang et al. (2011). In a dry flow, they can be explained by the conservation of potential vorticity due to orographic blocking on a drifting vortex embedded in a dry, stably stratified fluid flow (Lin, 2007, Figure 5.36). The mechanisms which control the track deflection in the CNTL case are much more complicated due to the inclusion of moist and PBL processes. In the following, they will be explored by performing a vorticity budget analysis.

It is well-known that a TC tends to move toward an area with a positive vorticity tendency (VT) (i.e., $\partial \zeta / \partial t>0$; e.g., Holland, 1983, see Lin, 2007, for a brief review). Thus, a vorticity budget analysis of the individual terms of the vorticity equation provides an appropriate approach to reveal the mechanisms which control the track change. In order to do so, we analyze the individual terms of the following vorticity equation on an $f$-plane in the pressure vertical coordinates, which may be written as below.

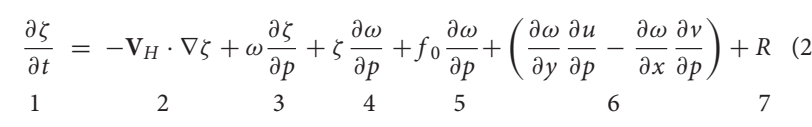

Here $\mathbf{V}_{H}$ is the horizontal wind vector $(u, v)$, $\omega$ the omega vertical motion, $f_{0}$ the constant Coriolis parameter $\left(f_{0}=5.0 \times\right.$ $10^{-5} s^{-1}$ ). The right-hand side of Equation (2) represents the horizontal vorticity advection (term 2), vertical vorticity advection (term 3), vorticity stretching associated with preexisting relative vorticity (term 4 ), vorticity stretching associated with pre-existing planetary vorticity (term 5), tilting term (term 6 ), and residual term (term 7 , mainly contributed by friction). The residual term is obtained by subtracting the VT (term 1) by terms 2-6. The 950-500 hPa layer-averaged VT, horizontal vorticity advection, vorticity stretching with pre-existing relative vorticity, and residual term (i.e., terms 1, 2, 4, and 7, respectively) and the flow and TC fields are shown when the TC is located upstream at $132 \mathrm{~h}$ (Figure 4), right before crossing the mountain at $168 \mathrm{~h}$ (Figure 5), just over the mountain at $183 \mathrm{~h}$ (Figure 6), and downstream from the mountain at $216 \mathrm{~h}$ (Figure 7 ). Other terms are not shown because they are insignificant compared to the above terms.

At $132 \mathrm{~h}$, the TC is located at about $700 \mathrm{~km}$ upstream of the mountain ridge, it has already started moving southwestward (Figure 3) toward the area with maximum positive VT (Figure 4A). This area with positive VT is accompanied by an area with negative VT located to the northeast (Figure 4A). Compared to other significant terms (Figures 4B-D), the positive VT (Figure 4A) is mainly contributed by the horizontal vorticity advection (Figure 4B) with additional influence from vorticity stretching (Figure 4C) and residual terms (Figure 4D). The southwestward advection of the vorticity can be explained by the anticyclonic turning of a continuously stratified flow over a mesoscale mountain due to the generation of high pressure over the mountain (Smith, 1979), which forces the flow to turn southward upstream for an easterly basic flow (see Figure 5.36 of Lin, 2007). This southwestward basic flow then advects the vertical vorticity associated with the TC vortex to give a negative-positive VT dipole toward southwest as shown in Figure 4B. Note that in Figure 4B could be interpreted as a wavenumber-2 pattern if one takes into account of the small area of positive vorticity advection embedded in the much larger area of negative vorticity advection, however, the overall distribution is dominated by the negative-positive dipole.

The area with maximum vorticity stretching is dominated by the TC convection, especially the diabatic heating associated with the eyewall convection, thus it forms a ring collocated with the eyewall, as approximately represented by the rainfall distribution. The residual term (Figure 4D) has multiple (three more specifically) maxima of the same order of magnitude, located at different azimuthal angles, thus it is less certain about its contribution to the VT. Part of the reason is that the residual term may involve some complicated physical processes which are related to viscosity, planetary boundary layer parameterization, turbulent mixing, etc. and numerical smoothing or diffusion. These processes may influence the vorticity tendency differently. For example, viscosity tends to spin down the vertical vorticity, but on the other hand, turbulence mixing may cause turbulence 

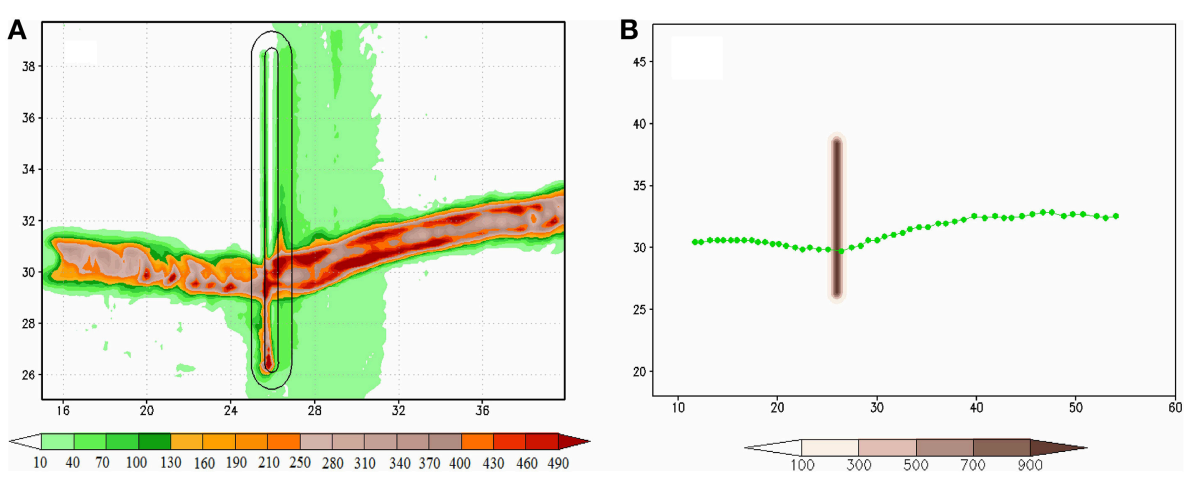

FIGURE 3 | (A) Accumulated rainfall (shaded, in mm), and (B) TC track for every $6 \mathrm{~h}$ starting at $0 \mathrm{~h}$. The units of the coordinates in the $x$ and $y$ directions are $100 \mathrm{~km}$.
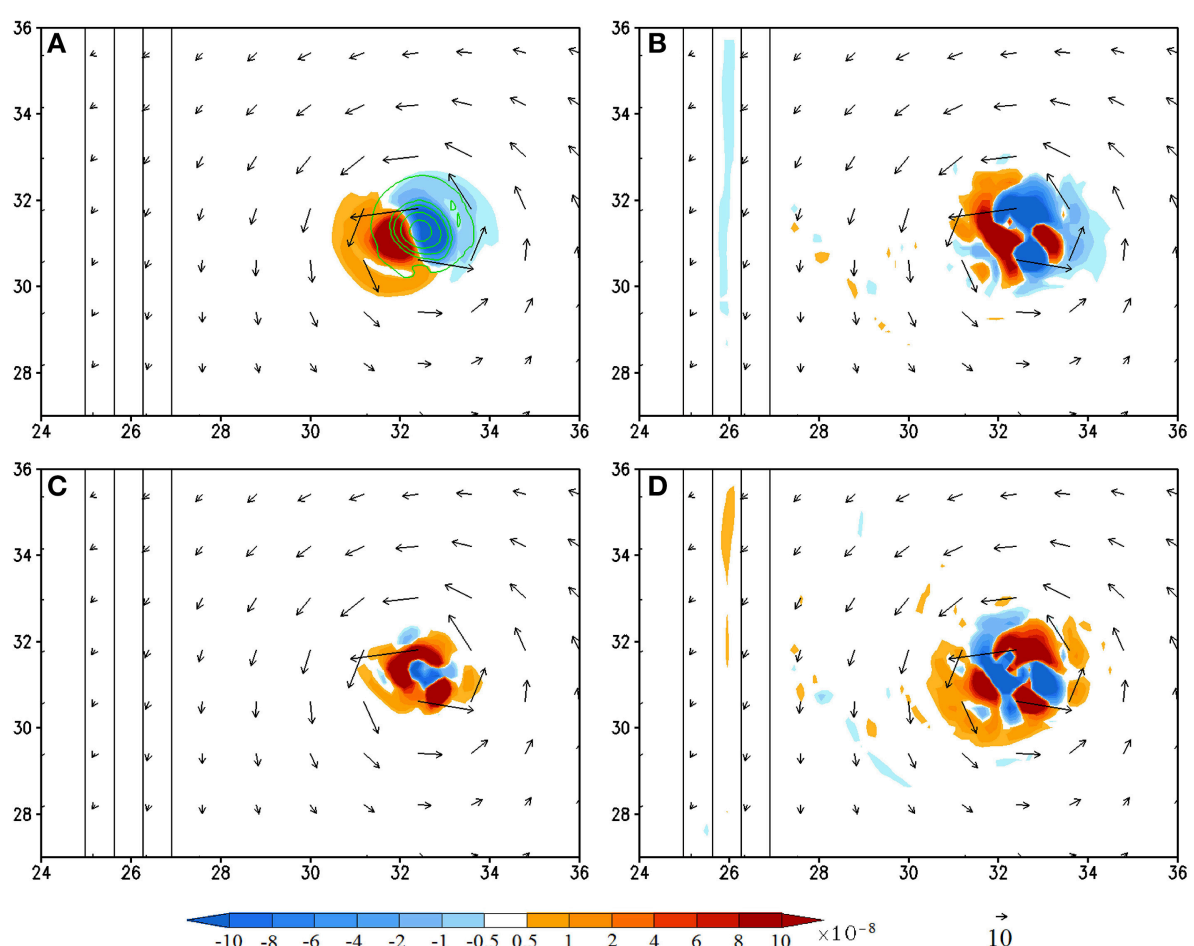

FIGURE 4 | (CNTL/A2) Vorticity budget analysis averaged for the 950-500 hPa layer at $132 \mathrm{~h}$ (shaded): (A), vorticity tendency (VT); (B), horizontal vorticity advection; (C), relative vorticity stretching, and (D), residual term. The vertical vorticity field is depicted by contours (0.0001, $0.0005,0.001,0.002$, $\left.0.003 \mathrm{~s}^{-1}\right)$ in $(\mathbf{A})$. The residual term is obtained by subtracting $(\mathbf{A})$ by the sum of $(\mathbf{B}, \mathbf{C})$, vertical advection, planetary vorticity stretching, and tilting terms. The last three terms are not shown since their magnitudes are negligible compared to terms (A-C). The units of the coordinates in the $x$ and $y$ directions are $100 \mathrm{~km}$.

dissipation leading to the generation of potential vorticity (e.g., Smith, 1989; Schär and Durran, 1997) and relative vorticity. This problem deserves further investigation, but is out of scope of the present study. In summary, we found that the upstream track deflection is dominated by the vorticity advection with additional contributions from vorticity stretching and viscosity. This is consistent with the studies with a dry flow except the effects of diabatic heating and viscosity (LS11). For real cases, this implies that a TC's motion is mainly steered by the orographically influenced basic flow but slightly modified by convective heating (mainly represented in the vorticity stretching term) and friction (mainly represented by the residual term).

At $168 \mathrm{~h}$, the TC has moved to the foothill (Figure 5) and it turns slightly toward west-southwestward before passing over the mountain (Figure 3). Similar to that at $132 \mathrm{~h}$, the VT field shows a positive VT area located to the west-southwest of the TC vortex center, accompanied by a negative VT area to the east-northeast (Figure 5A). The area of this VT couplet is expanded slightly larger and exhibits a banded structure to the south of the TC center. The positive VT is mainly contributed 

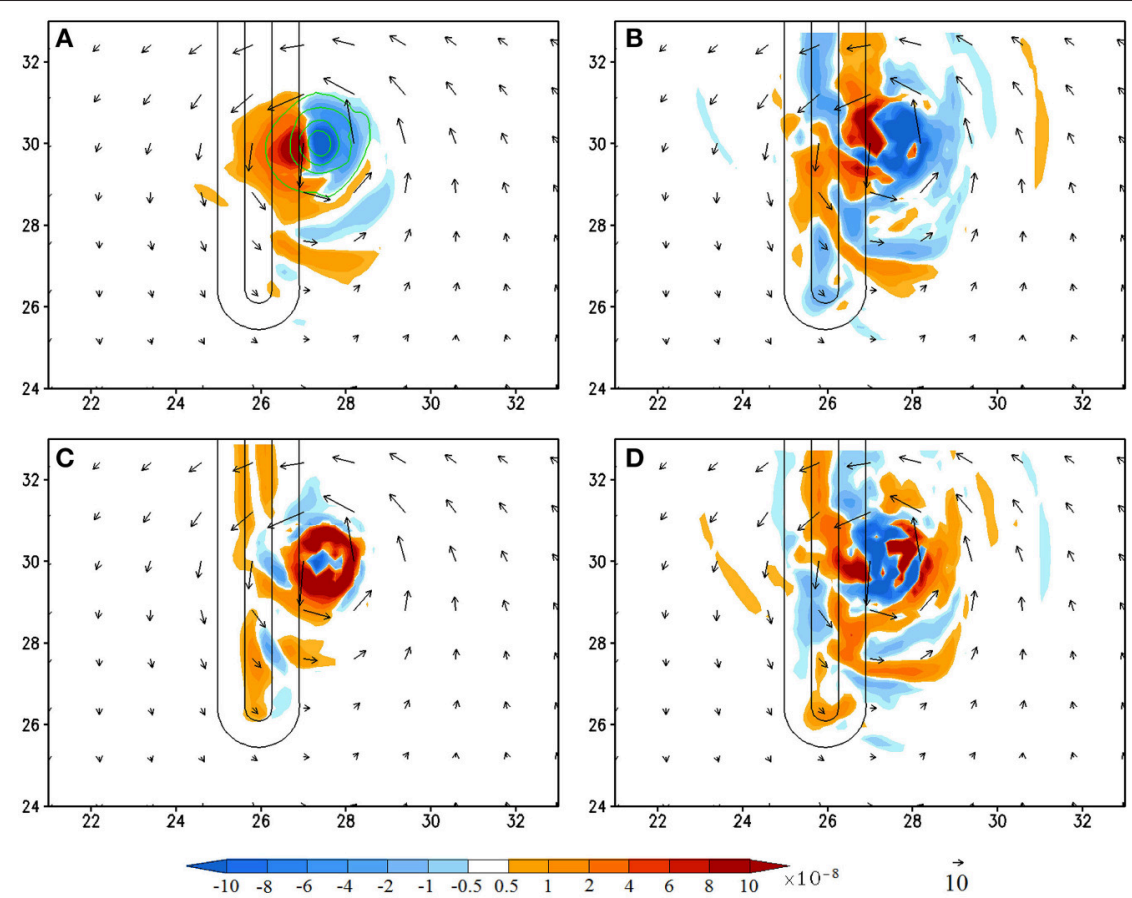

FIGURE 5 | (CNTL/A2) Vorticity budget analysis averaged for the 950-500 $\mathrm{hPa}$ layer at $\mathbf{1 6 8 \mathrm { h }}$ (shaded): (A), vorticity tendency (VT); (B), horizontal vorticity advection; (C), relative vorticity stretching, and (D), residual term. The vertical vorticity field is depicted by contours $(0.0001,0.0005,0.001,0.002$, $0.003 \mathrm{~s}^{-1}$ ) in (A). The residual term is obtained by subtracting (A) by the sum of (B,C), vertical advection, planetary vorticity stretching, and tilting terms. The last three terms are not shown since their magnitudes are negligible compared to terms (A-C). The units of the coordinates in the $x$ and $y$ directions are $100 \mathrm{~km}$.
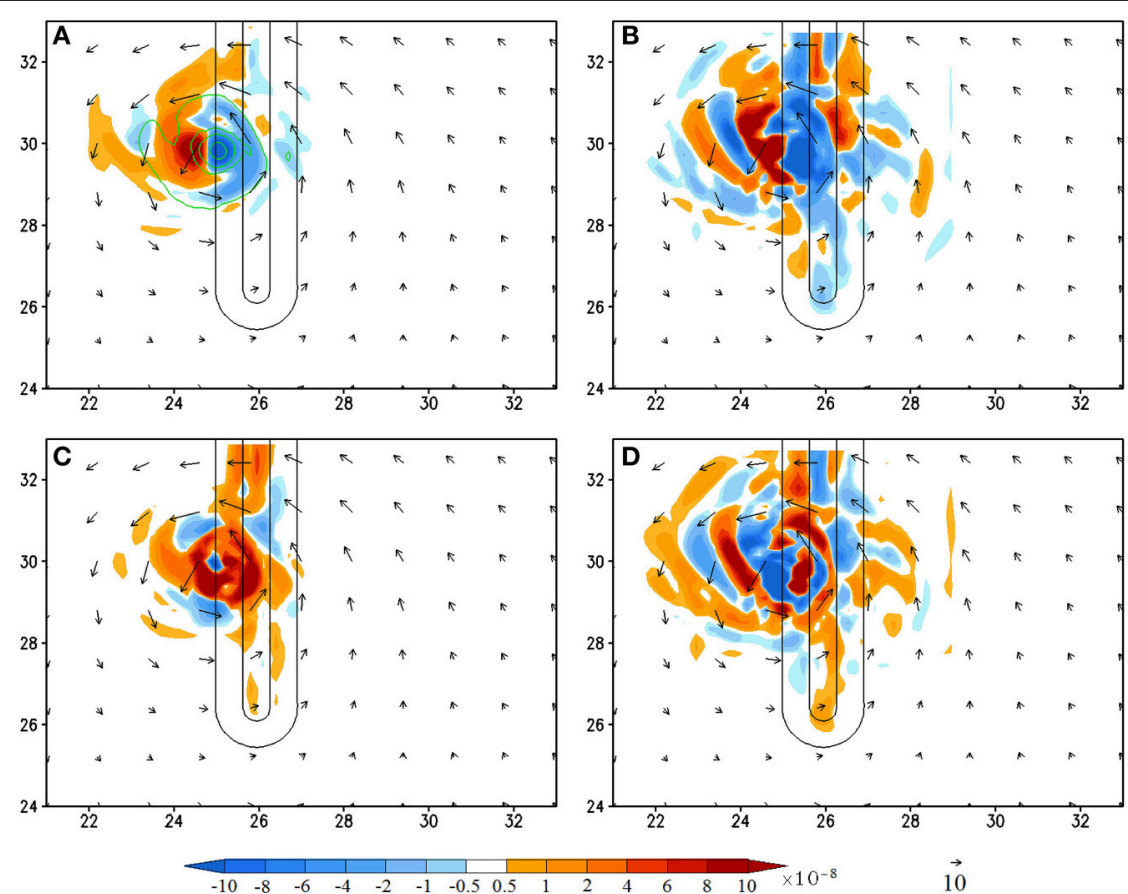

FIGURE 6 | (CNTL/A2) Vorticity budget analysis averaged for the 950-500 hPa layer at $180 \mathrm{~h}$ (shaded): (A), vorticity tendency (VT); (B), horizontal vorticity advection; (C), relative vorticity stretching, and (D), residual term. The vertical vorticity field is depicted by contours $(0.0001,0.0005,0.001,0.002$, $\left.0.003 \mathrm{~s}^{-1}\right)$ in (A). The residual term is obtained by subtracting (A) by the sum of $(\mathbf{B}, \mathbf{C})$, vertical advection, planetary vorticity stretching, and tilting terms. The last three terms are not shown since their magnitudes are negligible compared to terms (A-C). The units of the coordinates in the $x$ and $y$ directions are $100 \mathrm{~km}$. 

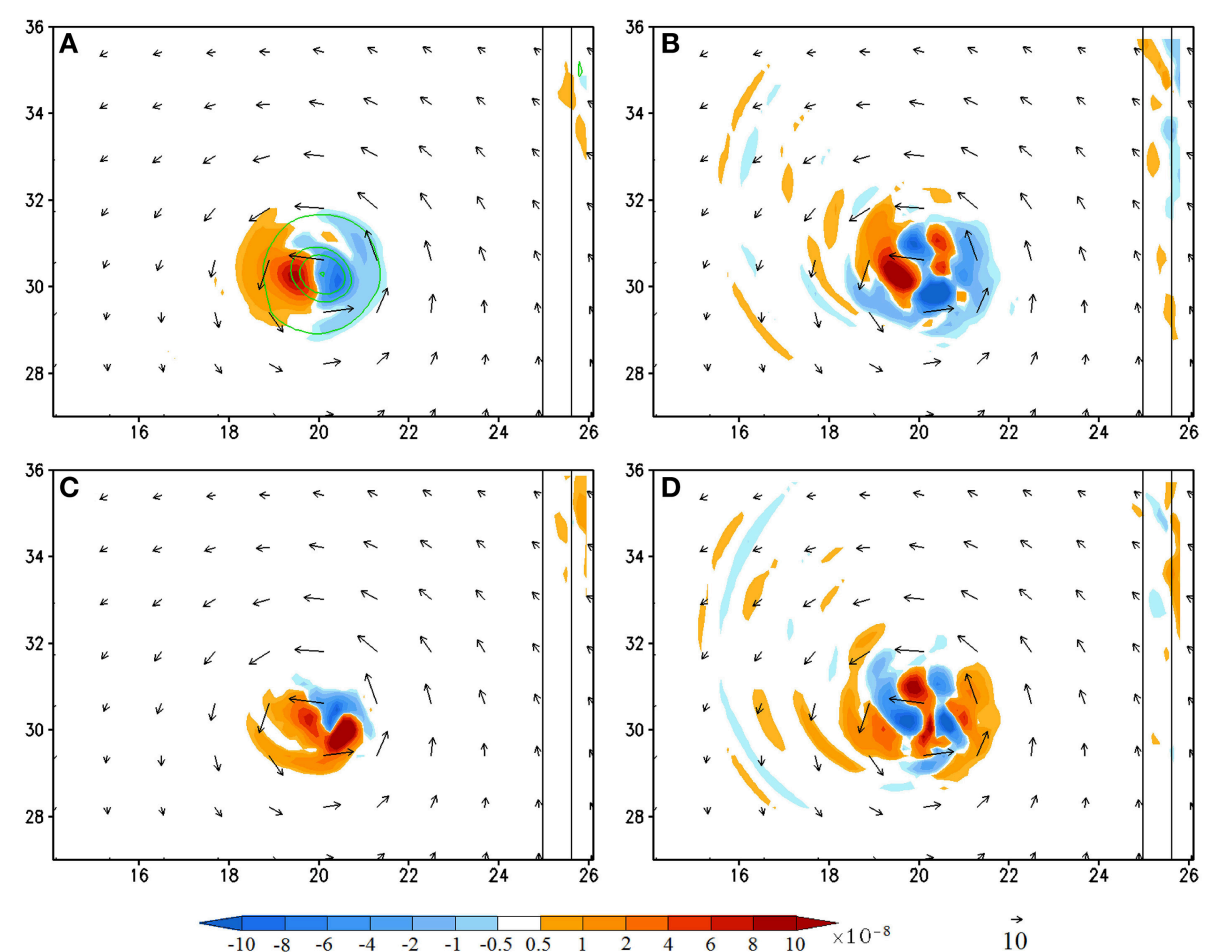

FIGURE 7 | (CNTL/A2) Vorticity budget analysis averaged for the 950-500 hPa layer at 216 h (shaded): (A), vorticity tendency (VT); (B), horizontal vorticity advection; (C), relative vorticity stretching, and (D), residual term. The vertical vorticity field is depicted by contours (0.0001, $0.0005,0.001,0.002$, $0.003 \mathrm{~s}^{-1}$ ) in (A). The residual term is obtained by subtracting $(\mathbf{A})$ by the sum of $(\mathbf{B}, \mathbf{C})$, vertical advection, planetary vorticity stretching, and tilting terms. The last three terms are not shown since their magnitudes are negligible compared to terms (A-C). The units of the coordinates in the $x$ and $y$ directions are $100 \mathrm{~km}$.

by the horizontal vorticity advection, vorticity stretching, and viscosity (residual term). The distribution of positive horizontal vorticity advection is also expanded to a larger area and exhibits a much more complicated pattern (Figure 5B) compared to that at $132 \mathrm{~h}$ (Figure 4B), which, apparently, is caused by orographic forcing. The positive vorticity stretching (Figure 5C) and residual term located near the maximum VT, i.e., near $(x, y)=(2700$, $3000 \mathrm{~km}$ ) (Figure 5D), have made contributions to the positive VT (Figure 5A). Note that the residual term may play more essential contribution than the horizontal vorticity advection to local positive VT. Similar to those at $132 \mathrm{~h}$ (Figure 4), the vorticity stretching is associated with the eyewall convection (Figures 5C, 8B) and the positive residual term may be associated with viscosity and turbulence mixing. Thus, we may conclude that the maximum positive VT when the TC moves to the foothill is dominated by the horizontal vorticity advection but with additional contributions from vorticity stretching and viscosity.

At $180 \mathrm{~h}$ when the TC passes over the mountain peak to lee side of the mountain (Figure 6), the area with maximum positive VT is shifted to the west. This indicates that the TC will be moving westward, which indeed is consistent with the simulated TC track (Figure 3B). This differs significantly from the dry simulations of L05 in which the TC resumes its westward movement at the same latitude of the initial bogus vortex. It appears that the anticyclonic (clockwise) turning over the mountain requires a longer period of time, which is not
TABLE 1 | Names, landfall locations, and approach angles of simulated cases.

\begin{tabular}{lllc}
\hline Case & $\begin{array}{l}\text { Approach direction (from) } \\
\text { and location }\end{array}$ & $\begin{array}{c}\text { Approach angle (between } \\
\text { approaching and mountain } \\
\text { directions) }\end{array}$ \\
\hline A2 (CNTL) & Easterly & On Center & $90^{\circ}$ \\
A1 & & On North & \\
A3 & On South & \\
\hline AB1 & Southeasterly & On South & $45^{\circ}$ \\
AB2 & & On Center \\
AB3 & & On North \\
\hline B & Southerly along & East Side \\
C & West Side & \\
\hline
\end{tabular}

applicable for a relatively narrower mountain as adopted by the CTNL experiment. Unlike that at 132 and $168 \mathrm{~h}$, the maximum positive VT is mainly contributed by the vorticity advection and stretching (Figures 6B,C). Specifically, the vorticity stretching contributing to the positive VT (or say, the TC movement) is the diabatic heating associated with the western portion of the eyewall convection, instead of the convection over the lee slope (upslope with respect to the TC circulation) induced by 

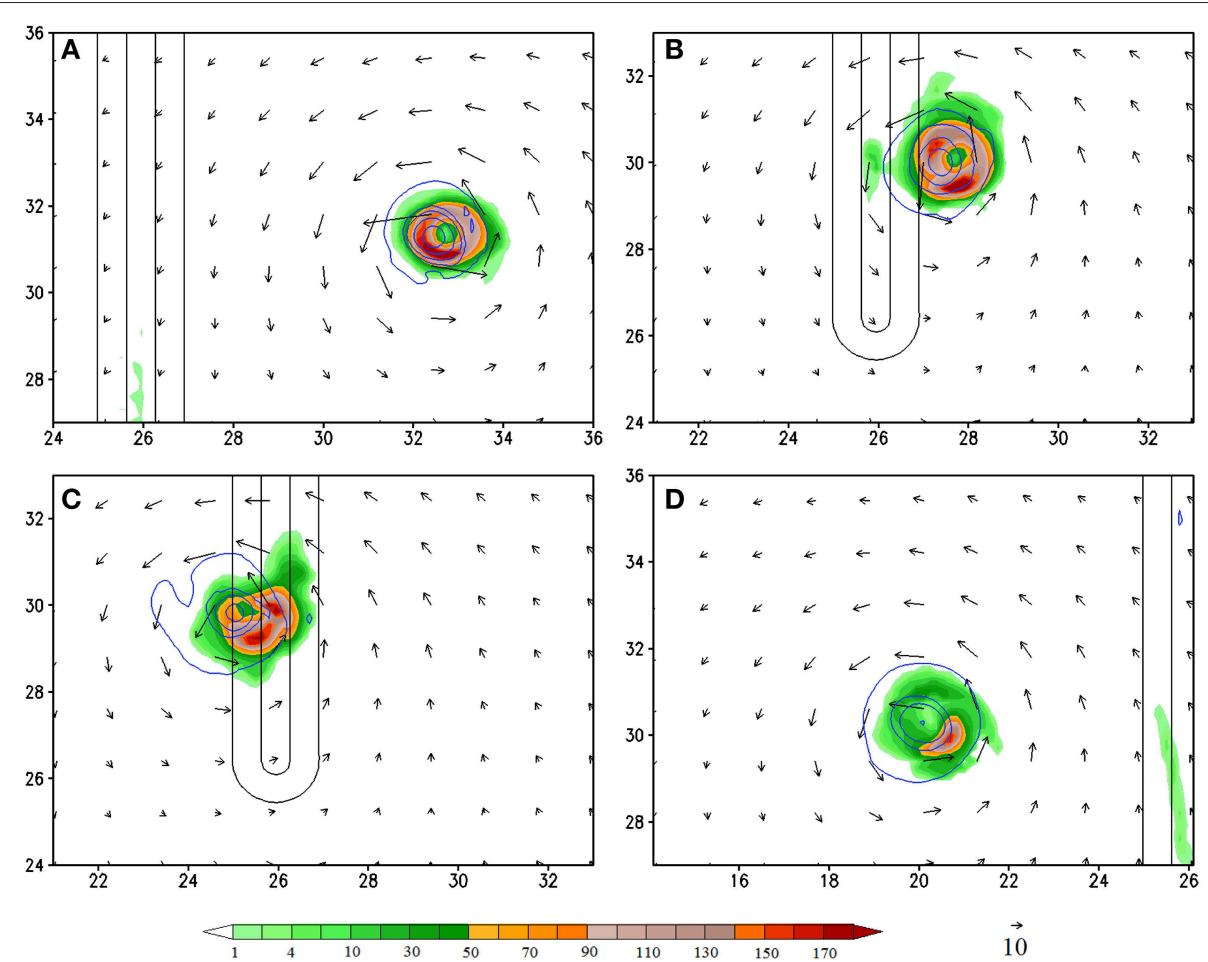

FIGURE 8 | (CNTL/A2) Accumulated $3 \mathrm{~h}$ rainfall (shaded, in $\mathrm{mm} / \mathbf{3} \mathrm{h}$ ) at (A) $\mathbf{1 3 2} \mathrm{h}$, (B) $\mathbf{1 6 8} \mathrm{h}$, (C) $\mathbf{1 8 0} \mathrm{h}$, and (D) $\mathbf{2 1 6} \mathrm{h}$. The units of the coordinates in the $x$ and $y$ directions are $100 \mathrm{~km}$. The relative vorticity fields are depicted by contours $\left(0.0001,0.0005,0.001,0.002,0.003 \mathrm{~s}^{-1}\right)$.

the orography (Figures $\mathbf{6 C}, \mathbf{8 C}$ ). The residual term makes a negative contribution to the VT (Figures 6A,D), indicating that the frictional effect is spinning down the vorticity over the lee slope. Note that, due to a relatively narrower mountain range, the TC track on the lee side is to the south of the original latitude. This differs significantly from the dry simulations of L05. The anticyclonic (clockwise) turning over the mountain area requires a substantial contribution from the TC convection, which produces strong vorticity stretching over a significant period of time.

At $216 \mathrm{~h}$, the TC vortex moves far away from the mountain to about $(x, y)=(2000,3000 \mathrm{~km})$ (Figure 7). At this time, the maximum positive VT is dominated by vorticity advection, enhanced slightly by vorticity stretching (Figures 7B,C), but with almost no contribution from the frictional effects (Figure 7D). Note that the diabatic heating does make significant contribution to the vorticity stretching to the southeast of the eyewall (Figures 7C, 8D), but not the area with maximum positive VT.

\section{EFFECTS OF LANDFALL LOCATIONS AND APPROACH ANGLES}

In this section, the effects of landfall location on track deflection are investigated by performing sensitivity experiments with vorticity budget analyses of the TC making landfall at the northern tip (A1) and the southern tip (track A3) of the mountain. These results are then compared with those of track

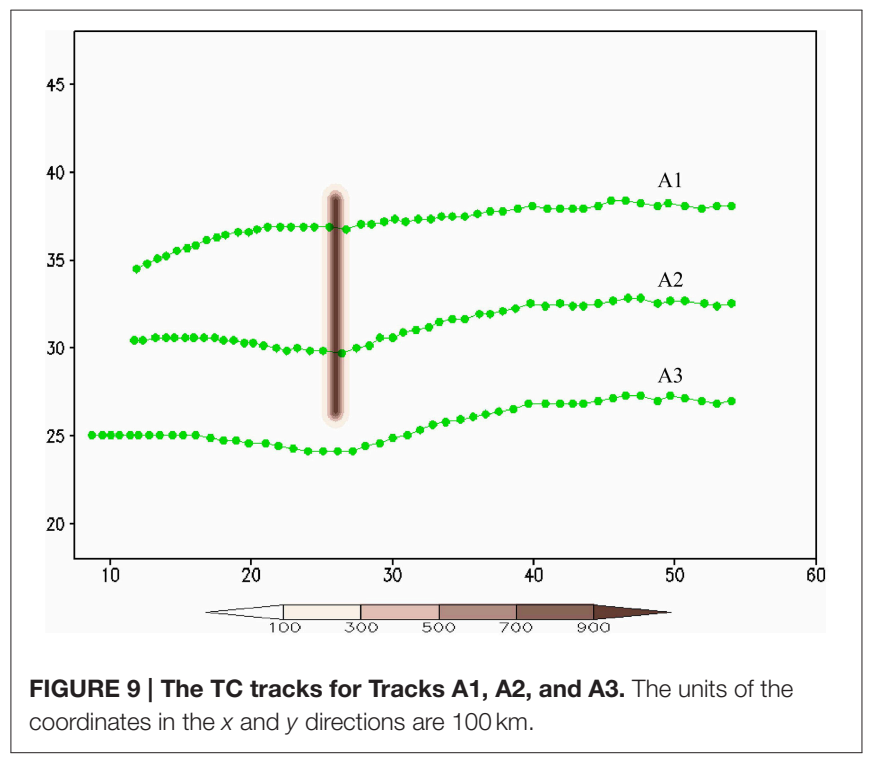

A2 (case CNTL). Figure 9 shows the simulated tracks A1, A2, and A3 (track A2 has been discussed and analyzed in the previous section). Note that compared to track A2 (Figure 3), track A1 (Figure 9) has less southward deflection when it passes over the mountain. Near the foothill, the maximum positive VT is dominated by the vorticity advection without much contribution from vorticity stretching and frictional effects 

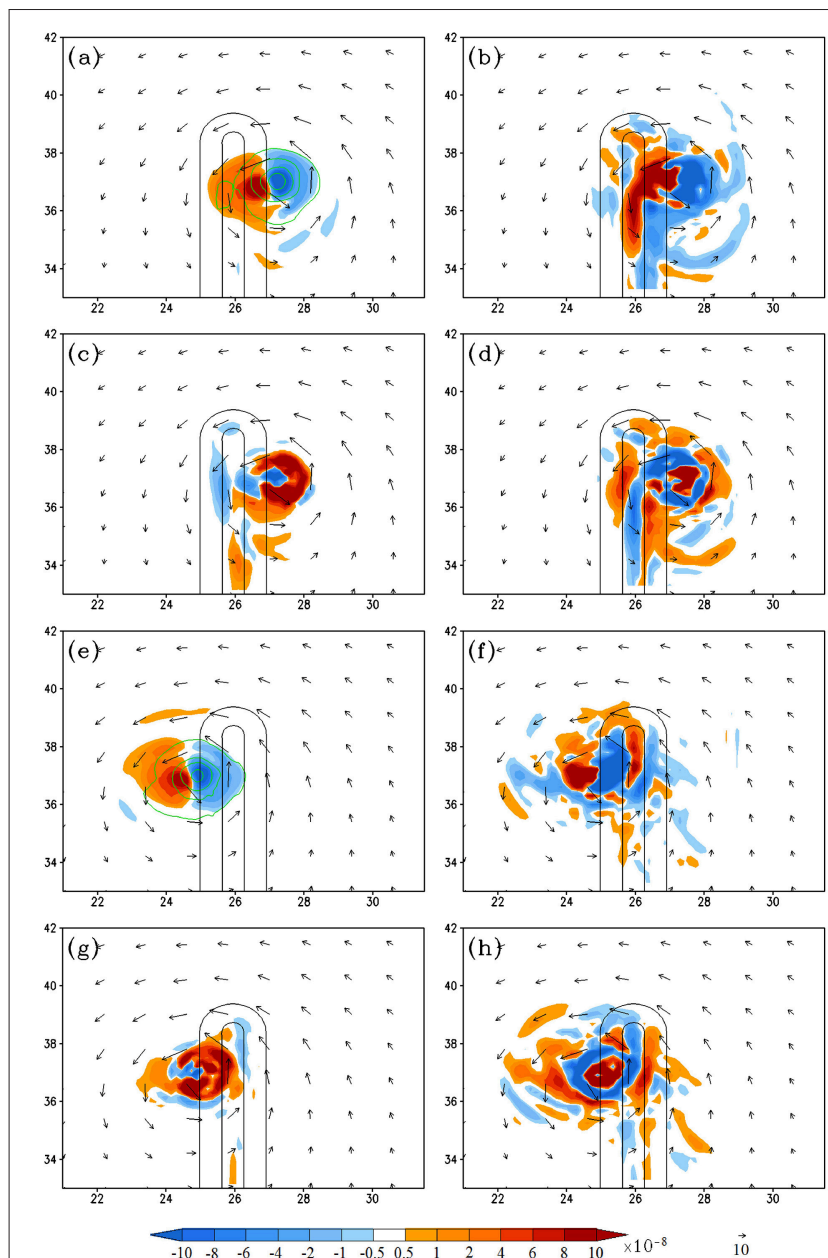

FIGURE 10 | (Track A1) The vorticity budget analysis for Track A1: (A-D) are the $950-500 \mathrm{hPa}$ layer-averaged vorticity tendency (VT), horizontal vorticity advection, relative vorticity stretching, and residual term, respectively, at $\mathbf{1 7 1} \mathbf{h}$. (E-H) Are the same as (A-D), respectively, but at $186 \mathrm{~h}$. The units of the coordinates in the $x$ and $y$ directions are $100 \mathrm{~km}$. The vertical vorticity fields are depicted by contours $(0.0001,0.0005,0.001,0.002$, $\left.0.003 s^{-1}\right)$ in $(\mathbf{A}, \mathbf{E})$

(Figures 10A-D). This is mainly due to stronger vorticity advection toward the northwest induced by the basic easterly flow and the cyclonic TC circulation around the northern mountain tip. On the lee side, the maximum positive VT (Figure 10E) is dominated by the vorticity advection, enhanced slightly by vorticity stretching (Figures 10F,G), but has almost no contribution from the frictional effect (Figure 10H). The dominance of vorticity advection of the maximum positive VT and TC movement is similar to the dry flow (case N of LS11).

Figure 11 shows the vorticity budget analysis when the TC is passing over the southern tip (case A3). When the TC moves to the southeast tip of the mountain, the maximum positive VT (Figure 11A) is mainly contributed by the frictional effects (Figure 11D) and slightly contributed by the vorticity stretching (Figure 11C), but, unlike the above cases (cases A2 and A1), no contributions from vorticity advection. The frictional effects may
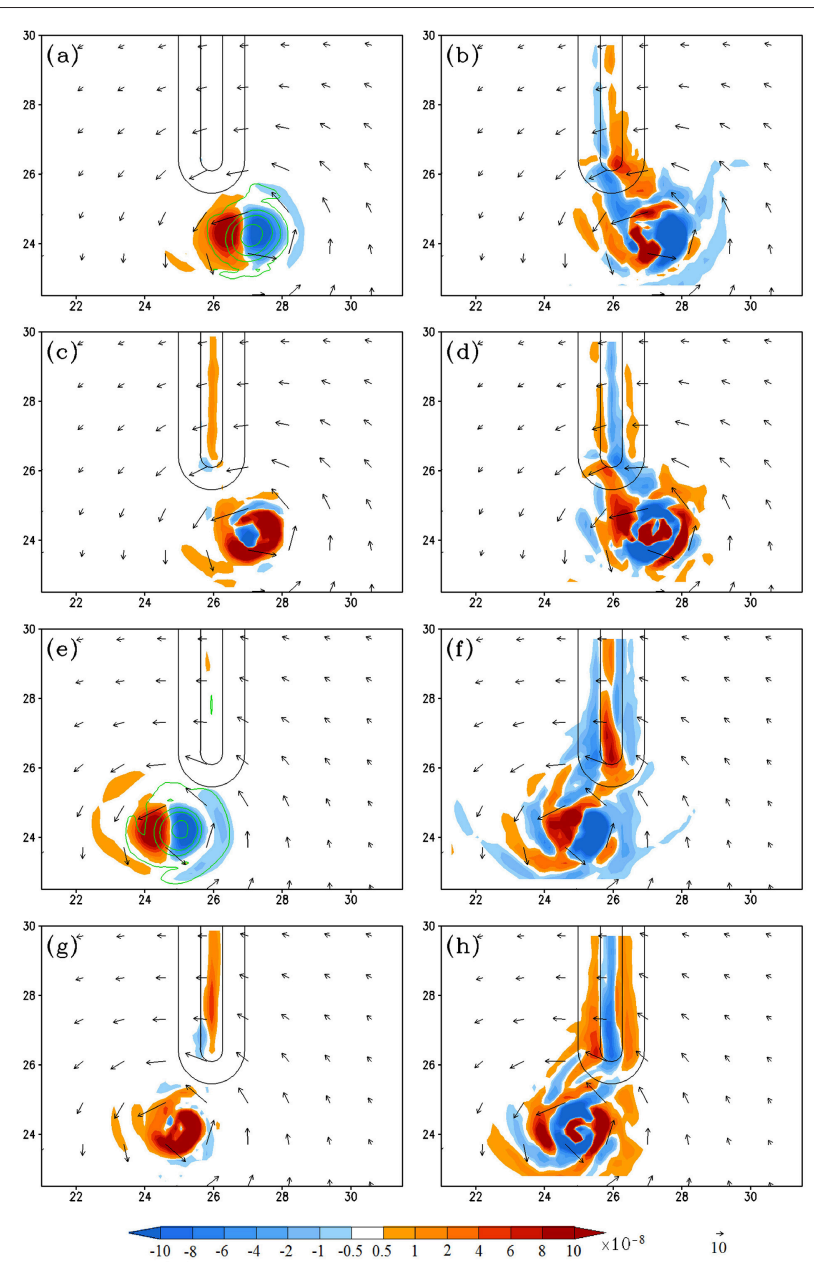

FIGURE 11 | (Track A3) The vorticity budget analysis for Track A3: (A-D) are the $950-500 \mathrm{hPa}$ layer-averaged vorticity tendency (VT), horizontal vorticity advection, relative vorticity stretching, and residual term, respectively, at $\mathbf{1 7 1} \mathbf{h}$. (E-H) Are the same as (A-D), respectively, but at $186 \mathrm{~h}$. The units of the coordinates in the $x$ and $y$ directions are $100 \mathrm{~km}$. The vertical vorticity fields are depicted by contours $(0.0001,0.0005,0.001,0.002$, $\left.0.003 s^{-1}\right)$ in $(\mathbf{A}, \mathbf{E})$.

be caused by stronger interaction of the northern part of the TC circulation and the southern mountain tip. After passing over the mountain, the track curves northwestward back to its original direction, which is dominated by the vorticity advection, like earlier cases.

In order to investigate the effects of approach angle and landfall locations on track deflection, three sensitivity experiments mimicking track types $\mathrm{AB} 1, \mathrm{AB} 2$, and $\mathrm{AB} 3$ have been performed and the results are shown in Figure 12. All of the simulated TCs approach the south-north oriented mountain range from the southeast or $135^{\circ}$. The TC makes landfall near the northern tip, center, and southern tip in cases $A B 1, A B 2$, and $A B 3$, respectively. The mechanisms of the track deflection for tracks $A B 1, A B 2$, and $A B 3$ are similar to those for tracks $A 1$, $\mathrm{A} 2$, and $\mathrm{A} 3$ as discussed above. Tracks AB2 and AB3 are less curvy compared to those of A2 an A3 due to less orographic 


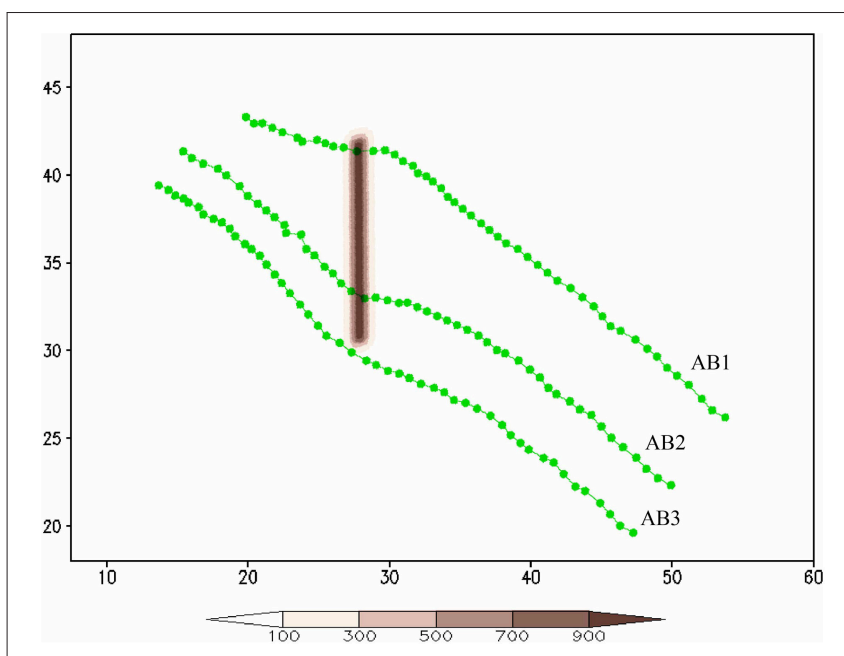

FIGURE 12 | The TC tracks for AB1, AB2, and AB3. The units of the coordinates in the $x$ and $y$ directions are $100 \mathrm{~km}$.

blocking caused by approach angle. It is interesting to observe that track $\mathrm{AB} 1$ is curved opposite to tracks $\mathrm{AB} 2$ and $\mathrm{AB} 3$ (i.e., turning cyclonically when the $\mathrm{TC}$ is passing over the northern tip). This is consistent with that found in dry experiments of LS11 where the vorticity advection upstream of the mountain range is caused by the flow splitting of the basic flow. Track AB1 may be used to explain most of the cyclonic track over northern Taiwan experienced by typhoons (e.g., Wang, 1980; Chang, 1982; see Figure 1A of L05) since the Central Mountain Range of Taiwan has a relatively shorter scale in the north-south direction and most of the typhoons approached the CMR from the southeast, instead of east. Thus, a TC tends to go around the corner due to basic flow splitting and the dominant control of positive vorticity tendency (VT) by vorticity advection.

As classified by $\mathrm{H} 09$ and illustrated in Figure 2, hurricanes making landfall on the Gulf coast may transverse along the SCA to the east (type B) or west (type C). This means the approach angle is turned to $180^{\circ}$ from the south (southwest) for a south-to-north (southwest-to-northwest) oriented mountain range. Figure 13 shows the TC tracks for sensitivity experiments $\mathrm{B}$ and $\mathrm{C}$. These track deflections are quite interesting. For both tracks, the TC turns toward the mountain in the southern portion of the mountain range, crosses over the mountain peak to the foothill on the lee side near the center (track B) or the northern end (track C) of the mountain range, wobbles near the northern tip of the mountain range, and then resumes its original track parallel but to the west of the mountain.

The mechanisms of the track deflection in case B can also be explained by the vorticity budget analysis. Figure 13A shows the TC continuously moving northward along the mountain. When the TC moves along the eastern flank in the southern portion of the mountain range, the vorticity budget analysis at $150 \mathrm{~h}$ indicates that the positive VT is influenced by all major terms, including vorticity advection, vorticity stretching, and residual terms (Figures 14A-D). The vorticity stretching is mainly associated with the latent heat released by the eyewall convection,

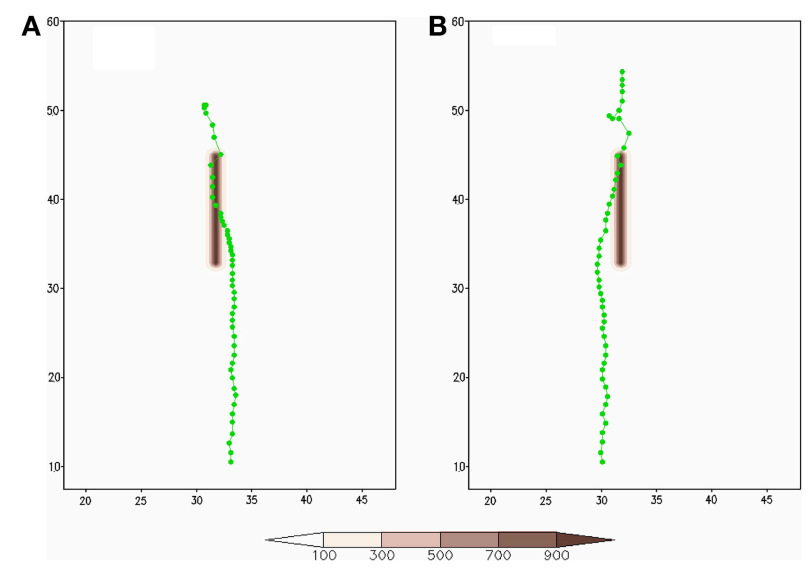

FIGURE 13 | The TC tracks for (A) Track B and (B) Track C. The units of the coordinates in the $x$ and $y$ directions are $100 \mathrm{~km}$.

as evidenced by the 3-h rainfall field (Figure 14I). When the TC moves northward to $y=3300 \mathrm{~km}$ at $204 \mathrm{~h}$ (Figures 14E-H,J), it also moves westward simultaneously toward the eastern slope around $x=3000 \mathrm{~km}$. During this period, the maximum positive VT is also influenced by the combined effects of vorticity advection, stretching and viscosity. Since the positive VT is located toward the northwest of the TC center, the TC is deflected toward northwest while it moves northward along the mountain ridge. This continues to be the case as the TC moves farther northward at later time (not shown).

For track $\mathrm{C}$, the track behavior, vorticity budget analysis, and mechanisms for track deflection are similar to those for track B. The simulated track $\mathrm{C}$, which is deflected toward the mountain and crosses over the mountain peak, is similar to the track of Hurricane Ivan (2004) although Ivan's track might have also been influenced by its synoptic environment.

\section{CONCLUDING REMARKS}

In this study, a series of idealized numerical experiments are performed using the ARW model to investigate the effects of landfall location and approach angle on track deflection of a tropical cyclone (TC) passing over an idealized South-Central Appalachian (SCA) Mountain. A bogus TC is initialized with a climatological tropical sounding with land surface, moist and PBL processes. A series of sensitivity experiments and vorticity budget analysis are performed to help understand the effects of landfall locations and approach angles on the track deflection.

When the TC landfalls on the central portion of the mountain range (Track A2), it is deflected to the south upstream, passes over the mountain anticyclonically, and then moves westward downstream. The TC motion is steered by the positive vorticity tendency (VT). When the TC is away from the mountain, the positive VT is dominated by horizontal vorticity advection upstream and downstream, but with additional influence from the stretching and residual terms. Physically, the stretching and residual terms are mainly associated with diabatic heating 

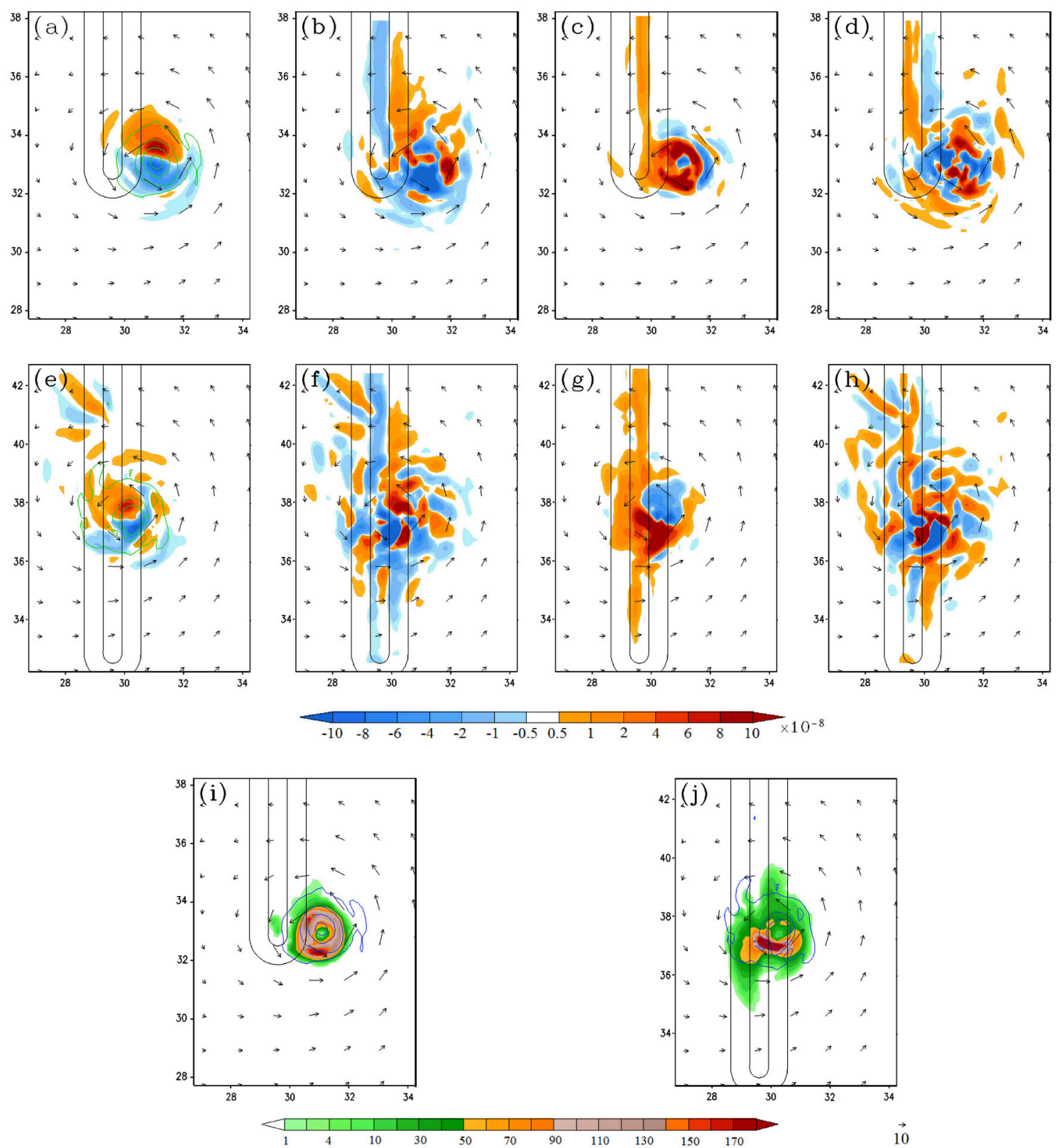

$\overrightarrow{10}$

FIGURE 14 | (Track B) The vorticity budge analysis for Track B: (A-D) are the 950-500 hPa layer-averaged vorticity tendency (VT), horizontal vorticity advection, relative vorticity stretching, and residual term, respectively, at $\mathbf{1 5 0} \mathbf{~ h}$. (E-H) Are the same as (A-D) respectively, but at $204 \mathrm{~h}$. (I,J) Are the $3 \mathrm{~h}$ accumulated rainfall at $150 \mathrm{~h}$ and $204 \mathrm{~h}$ (in $\mathrm{mm} / 3 \mathrm{~h}$ ), respectively. The units of the coordinates in the $x$ and $y$ directions are $100 \mathrm{~km}$. The vertical vorticity field is depicted by contours $\left(0.0001,0.0005,0.001,0.002,0.003 \mathrm{~s}^{-1}\right)$ in (A).

and friction effects, respectively. Near the foothill, the TC turns slightly toward west-southwestward before passing over the mountain, contributed by horizontal vorticity advection, vorticity stretching, and residual terms. When the TC passes over the mountain peak to the lee (western) side, it resumes the westward movement. At this time, the area with maximum positive VT is dominated by the horizontal vorticity advection and stretching. When the TC moves farther away from the mountain, the maximum positive VT is dominated by vorticity advection, enhanced slightly by vorticity stretching. Note that the track deflection upstream and downstream of the mountain, which are dominated by vorticity advection, is similar to the dry flow. The present results are similar to that found in LS 11. However, it is very different in the vicinity of the mountain. In LS11, the vorticity stretching occurs mainly over the lee side and its immediate downstream area, while in this study it makes contribution to the VT over both the upslope and downslope of the mountain due to the diabatic heating associated with moist 
convection. In addition, the vorticity advection upstream of the mountain is mainly caused by the flow splitting in LS11, which is less significant in the moist flow as simulated in this study. This can be explained by the weaker orographic blocking associated with strong TC convection in the moist flow.

Effects of landfall location on track deflection are investigated by performing a series of sensitivity experiments of the TC making landfall at the northern tip (A1) and the southern tip (A3) of the mountain. When the TC landfalls near the northern (southern) tip (Track A1 (A3)), it experiences less (more) southward deflection due to stronger (weaker) vorticity advection around the tip. The track deflections in the present cases are similar to those in the dry flow (LS11), except that there exists no track discontinuity due to the weaker orographic blocking associated with strong TC convection. The combined effects of approach angle and landfall location on track deflection are investigated by performing three sensitivity experiments, $\mathrm{AB} 1, \mathrm{AB} 2$, and $\mathrm{AB} 3$, in which the TC approaches the south-north oriented mountain range from the southeast (or $135^{\circ}$ ) and makes landfall on the northern tip, center, and southern tip, respectively. The mechanisms of the track deflection for AB1-AB3 are similar to those for A1-A3, but are less curvy due to weaker orographic blocking. The track deflection of case AB2 is similar to that simulated in a dry flow (case SE) in LS11.

For TCs going along the mountain range along the eastern flank (track B) and the western flank (track C), both of them are deflected toward the mountain range, cross over the mountain peak to the other side of the mountain, and then resume their northward movement. In these cases, the positive VT is influenced by all horizontal vorticity advection, vorticity stretching, and residual terms, which are associated with diabatic heating and frictional effects, due to longer and stronger interaction with the mountain range. The major difference of this study from the dry flow is that the vorticity stretching is mainly caused by diabatic heating in the current moist flow, instead of by lee slope vorticity stretching in LS11.

\section{REFERENCES}

Bender, M. A., Tuleya, R. E., and Kurihara, Y. (1987). A numerical study of the effect of island terrain on tropical cyclones. Mon. Wea. Rev. 115, 130-155. doi: 10.1175/1520-0493(1987)115<0130:ANSOTE > 2.0.CO;2

Brand, S., and Blelloch, J. W. (1974). Changes in the characteristics of typhoons crossing the island of Taiwan. Mon. Wea. Rev. 102, 708-713.

Chan, J. C. L., Ko, F. M. F., and Lei, Y. M. (2002). Relationship between potential vorticity tendency and cyclone motion. J. Atmos. Sci. 59, 1317-1336. doi: 10.1175/1520-0469(2002)059<1317:RBPVTA > 2.0.CO;2

Chang, S. W.-J. (1982). The orographic effects induced by an island mountain range on propagating tropical cyclones. Mon. Wea. Rev. 110, 1255-1270.

Chen, S.-H., and Sun, W.-Y. (2002). A one-dimensional time-dependent cloud model. J. Meteor. Soc. Japan 80, 99-118. doi: 10.2151/jmsj.80.99

Dunion, J. P. (2011). Re-writing the climatology of the Tropical North Atlantic and Caribbean Sea atmosphere. J. Clim. 24, 893-908. doi: 10.1175/2010JCLI3496.1

Fovell, R. G., Corbosiero, K. L., and Kuo, H.-C. (2009). Cloud microphysics impact on hurricane track as revealed in idealized experiments. J. Atmos. Sci. 66, 1764-1778. doi: 10.1175/2008JAS2874.1

Harville, S. L. (2009). (H09): Effects of Appalachian Topography on Precipitation from Landfalling Hurricanes. MS thesis, North Carolina State University. Available online at: http://repository.lib.ncsu.edu/ir/handle/1840.16/2849
Overall, the TC is steered by the maximum positive VT, which is dominated by the vorticity advection far away from the mountain. The vorticity stretching and residual terms, which are dominated by the diabatic heating and frictional effects, respectively, make additional influences on track deflection when the TC is near or over the mountain area. Note that in the present study, we found that the vorticity tendency (VT) approach is appropriate since the TC track is consistent with the VT, similar to that applied to TC motion, such as northwestward beta drift (e.g., Holland, 1983) and shear effects (e.g., Wu and Emanuel, 1993). The PV Tendency (PVT) diagnostic approach, as originally proposed by $\mathrm{Wu}$ and Wang (2000) and Chan et al. (2002) and applied to real-case simulations (e.g., Hsu et al., 2013; Tang and Chan, 2013, 2014; Wang et al., 2013), appears to be an attractive method and will be considered in our future research.

\section{AUTHOR CONTRIBUTIONS}

LL: Design and simulate cases, analyze the simulated results, and write the paper. YL: Design the experiments, analyze the simulated results and write the paper. SC: Install the idealized TC model, help design the idealized experiments, run cases, and proofreading the paper.

\section{ACKNOWLEDGMENTS}

The help from Dr. David S. Nolan's on idealized WRF modeling with a bogus vortex and the proofreading of the manuscript by Guy Oldaker IV are highly appreciated. The anonymous reviewers' comments have improved the quality of the paper significantly. The first two authors are supported by the NSF Award AGS-1265783, OCI-1126543, and CNS-1429464, while the third author is supported by NSF AGS-1015910.

Holland, G. J. (1983). Tropical cyclone motion: environmental interaction plus a beta effect. J. Atmos. Sci. 40, 328-342.

Hong, S.-Y., and Pan, H.-L. (1996). Non-local boundary layer vertical diffusion in Medium-Range Forecast model. Mon. Wea. Rev. 124, 1215-1238.

Hsu, L.-H., Kuo, H.-C., and Fovell, R. G. (2013). On the geographic asymmetry of typhoon translation speed across the mountainous island of Taiwan. J. Atmos. Sci. 70, 1006-1022. doi: 10.1175/JAS-D-12-0173.1

Huang, Y.-H., Wu, C.-C., and Wang, Y. (2011). The influence of island topography on typhoon track deflection. Mon. Wea. Rev. 139, 1708-1727. doi: 10.1175/2011MWR3560.1

Jian, G.-J., and Wu, C.-C. (2008). A numerical study of the track deflection of Super-Typhoon Haitang (2005) prior to its landfall in Taiwan. Mon. Wea. Rev. 136, 598-615. doi: 10.1175/2007MWR2134.1

Kain, J. S. (2004). The Kain-Fritsch convective parameterization: an update. J. Appl. Meteor. 43, 170-181. doi: 10.1175/15200450(2004)043<0170:TKCPAU>2.0.CO;2

Kain, J. S., and Fritsch, J. M. (1993). Convective parameterization for mesoscale models: the Kain-Fritsch scheme. Represent. Cumulus Conv. Numerical Models Meteor. Monogr. Amer. Meteor. Soc. 24, 165-170. doi: 10.1007/978-1-93570413-3_16

Lin, Y.-L. (2007). Mesoscale Dynamics. Cambridge, UK: Cambridge University Press. doi: 10.1017/cbo9780511619649 
Lin, Y.-L., Chen, S.-Y., Hill, C. M., and Huang, C.-Y. (2005). (L05): Control parameters for tropical cyclones passing over mesoscale mountains. J. Atmos. Sci. 62, 1849-1866. doi: 10.1175/JAS3439.1

Lin, Y.-L., Farley, R. D., and Orville, H. D. (1983). Bulk parameterization of the snow field in a cloud model. J. Clim. Appl. Meteor. 22, 1065-1092.

Lin, Y.-L., Han, J., Hamilton, D. W., and Huang, C.-Y. (1999). Orographic influence on a drifting cyclone. J. Atmos. Sci. 56, 534-562. doi: 10.1175/15200469(1999)056<0534:OIOADC>2.0.CO;2

Lin, Y.-L., and Savage, L. C. (2011). (LS11): Effects of landfall location and the approach angle of a cyclone vortex encountering a mesoscale mountain range. J. Atmos. Sci. 68, 2095-2106. doi: 10.1175/2011JAS3720.1

Lin, Y.-L., Witcraft, N. C., and Kuo, Y.-H. (2006). Dynamics of track deflection associated with the passage of tropical cyclones over a mesoscale mountain. Mon. Wea. Rev. 134, 3509-3538. doi: 10.1175/MWR3263.1

Mallen, K. J., Montgomery, M. T., and Wang, B. (2005). Reexamining the nearcore radial structure of the tropical cyclone primary circulation: implications for vortex resiliency. J. Atmos. Sci. 62, 408-425. doi: 10.1175/JAS3377.1

Nolan, D. S. (2011). Evaluating environmental favorableness for tropical cyclone development with the method of point-downscaling. J. Adv. Model. Earth Syst. 3, M08001-M08028. doi: 10.1029/2011ms000063

Nolan, D. S., Atlas, R., Bhatia, K. T., and Bucci, L. R. (2013). Development and validation of a hurricane nature run using the Joint OSSE Nature Run and the WRF model. J. Adv. Earth. Model. Syst. 5, 1-24. doi: 10.1002/jame.20031

O'Handley, C., and Bosart, L. F. (1996). The impact of the Appalachian Mountains on cyclonic weather systems. Part I: A climatology. Mon. Wea. Rev. 124, 1353-1373. doi: 10.1175/1520-0493(1996)124<1353:TIOTAM>2.0.CO;2

Rakotomavo, Z. A. P. H., Raholijao, N., and Lin, Y.-L. (2011). Effects of Madagascar Mountain Range on tropical cyclone tracks. Part I: Classification of cylone tracks reaching the East Coast. 41. Available online at: http://mesolab.ncat.edu/ publications\%20\%28web\%29/2011_Rakotomavo\%20et\%20al._Effects\%20of \%20Madagascar\%20mountains\%20on\%20TC\%20tracks.pdf or http://mesolab. ncat.edu

Schär, C., and Durran, D. R. (1997). Vortex formation and vortex shedding in continuously stratified flows past isolated topography. J. Atmos. Sci. 54, 534-554.

Schwarz, F. K. (1970). The unprecedented rains in Virginia associated with the remnants of Hurricane Camille. Mon. Wea. Rev. 98, 851-859. doi: 10.1175/ 1520-0493(1970)098<0851:TURIVA > 2.3.CO;2

Skamarock, W. C., Klemp, J. B., Dudhia, J., Gill, D. O., Barker, D. M., Duda, M. G., et al. (2008). A Description of the Advanced Research WRF version 3. NCAR technical note. Available online at: http://www2.mmm.ucar.edu/wrf/ users/docs/arw_v3.pdf

Smith, R. B. (1979). The influence of mountains on the atmosphere. Adv. Geophys. 21, 87-230. doi: 10.1016/S0065-2687(08)60262-9

Smith, R. B. (1989). "Hydrostatic airflow over mountains," in Advances in Geophysics, Vol. 31., ed B. Saltzman (New York, NY: Academic Press), 1-41.

Stewart, S. R. (2004). Hurricane Ivan, 2-24 September 2004. Tropical Cyclone Report, National Hurricane Center, NOAA, 1-44. Available online at: http:// www.nhc.noaa.gov/data/tcr/AL092004_Ivan.pdf
Tang, C. K., and Chan, J. C. (2013). Idealized simulations of the effect of Taiwan and Philippines topographies on tropical cyclone tracks. Q. J. Roy. Meteor. Soc. 140, 1578-1589. doi: 10.1002/qj.2240

Tang, C. K., and Chan, J. C. (2014). Idealized simulations of the effect of local and remote topographies on tropical cyclone tracks. Q. J. Roy. Meteor. Soc. 141, 2045-2056. doi: 10.1002/qj.2498

Wang, C.-C., Chen, Y.-H., Kuo, H.-C., and Huang, S.-Y. (2013). Sensitivity of typhoon track to asymmetric latent heating/rainfall induced by Taiwan topography: a numerical study of Typhoon Fanapi (2010). J. Geophys. Res. Atmos. 118, 3292-3308. doi: 10.1002/jgrd.50351

Wang, S.-T. (1980). Prediction of the Movement and Strength of Typhoons in Taiwan and its Vicinity. Research Report, 108. Taipei: National Science Council.

Wu, C.-C. (2001). Numerical simulation of Typhoon Gladys (1994) and its interaction with Taiwan terrain using the GFDL hurricane model. Mon. Wea. Rev. 129, 1533-1549. doi: 10.1175/1520-0493(2001)129<1533:NSOTGA $>$ 2.0.CO;2

Wu, C.-C., and Emanuel, K. A. (1993). Interaction of a baroclinic vortex with background shear: application to hurricane movement. J. Atmos. Sci. 50, $62-76$.

Wu, C.-C., Li, T.-H., and Huang, Y.-H. (2015). Influence of mesoscale topography on tropical cyclone tracks: further examination of the channeling effect. J. Atmos. Sci. 72, 3032-3050. doi: 10.1175/JAS-D-14-0168.1

$\mathrm{Wu}$, L., and Wang, B. (2000). A potential vorticity tendency diagnostic approach for tropical cyclone motion. Mon. Wea. Rev. 128, 1899-1911. doi: 10.1175/1520-0493(2000)128<1899:APVTDA > 2.0.CO;2

Yeh, T.-C., and Elsberry, R. L. (1993a). Interaction of typhoons with the Taiwan topography. Part I: upstream track deflection. Mon. Wea. Rev. 121, 3193-3212.

Yeh, T.-C., and Elsberry, R. L. (1993b). Interaction of typhoons with the Taiwan topography. Part II: continuous and discontinuous tracks across the island. Mon. Wea. Rev. 121, 3213-3233.

Zehnder, J. A. (1993). The influence of large-scale topography on barotropic vortex motion. J. Atmos. Sci. 50, 2519-2532. doi: 10.1175/1520-0469(1993)050<2519: TIOLST $>2.0 . \mathrm{CO} ; 2$

Zehnder, J. A., and Reeder, M. J. (1997). A numerical study of barotropic vortex motion near a large-scale mountain range with application to the motion of tropical cyclones approaching the Sierra Madre. Meteor. Atmos. Phys. 64, 1-19. doi: 10.1007/BF01044127

Conflict of Interest Statement: The authors declare that the research was conducted in the absence of any commercial or financial relationships that could be construed as a potential conflict of interest.

Copyright (c) $2016 \mathrm{Liu}$, Lin and Chen. This is an open-access article distributed under the terms of the Creative Commons Attribution License (CC BY). The use, distribution or reproduction in other forums is permitted, provided the original author(s) or licensor are credited and that the original publication in this journal is cited, in accordance with accepted academic practice. No use, distribution or reproduction is permitted which does not comply with these terms. 


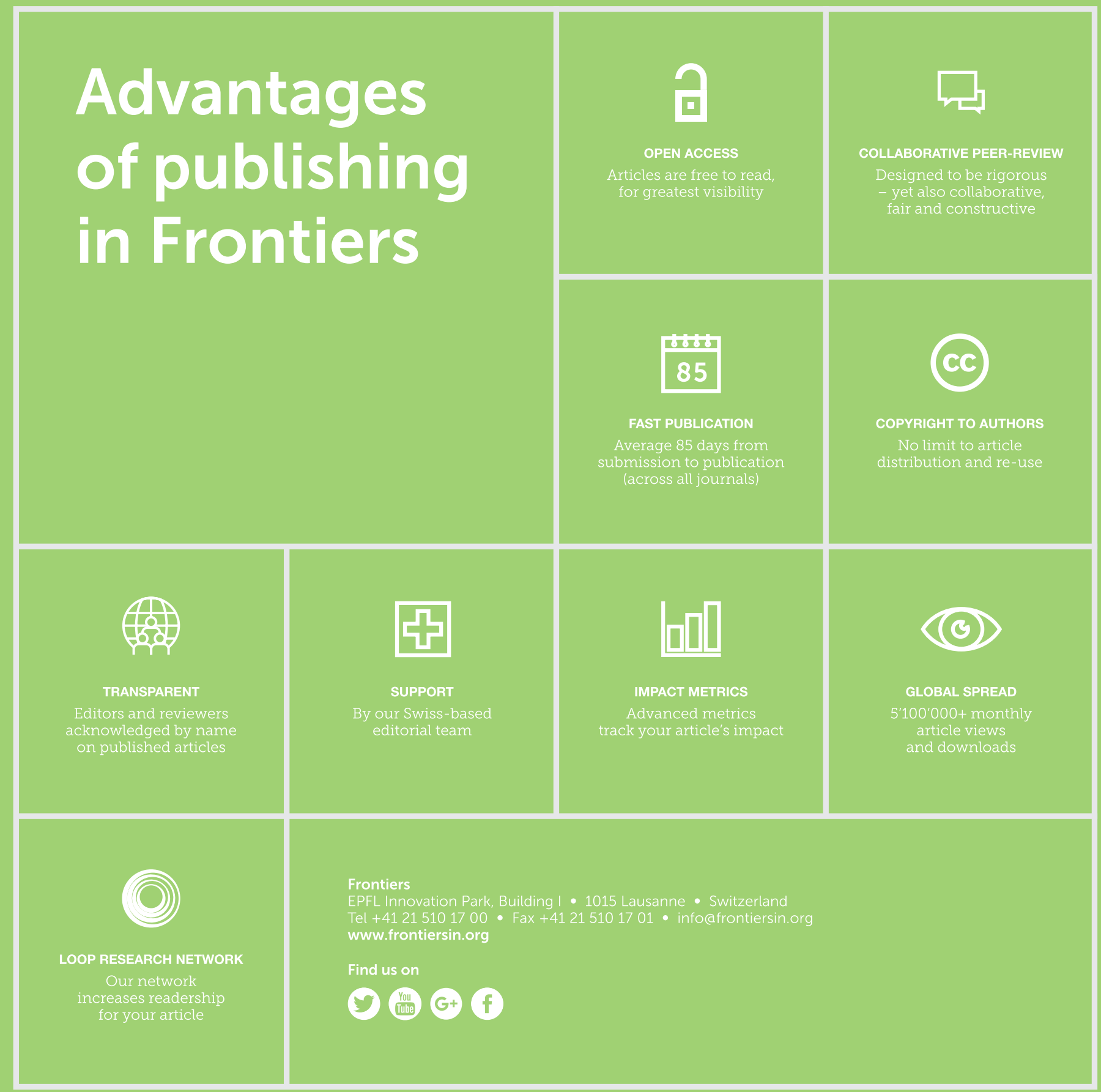

